
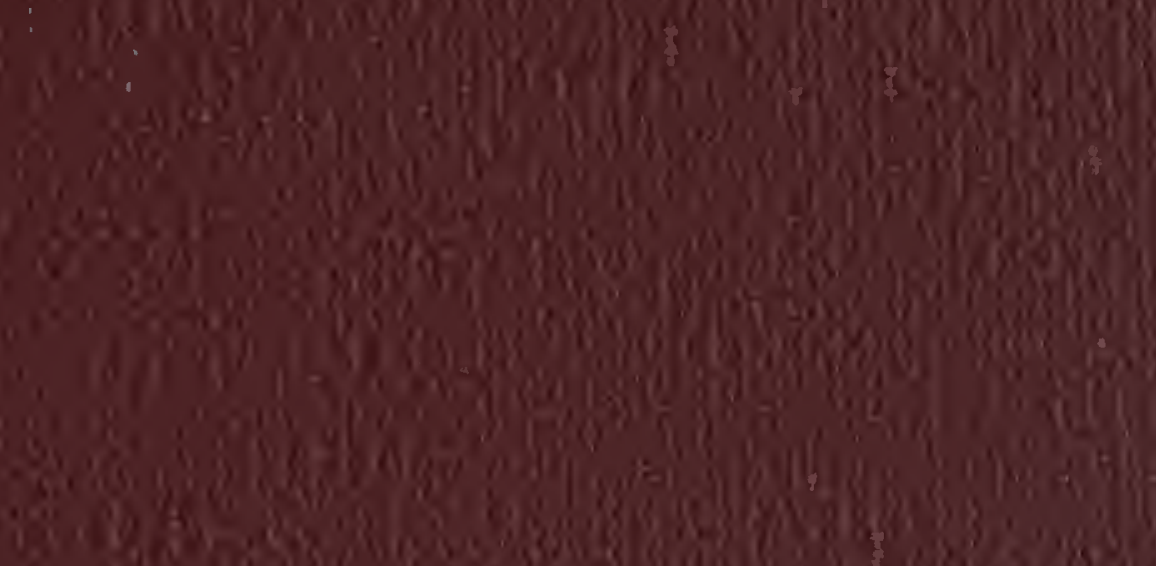

28

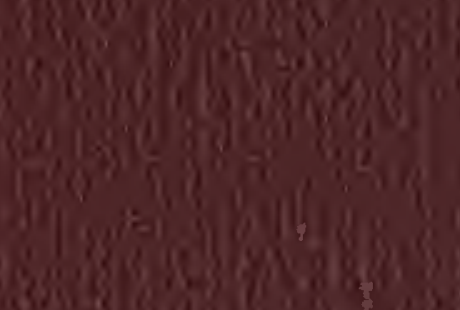
r.s.

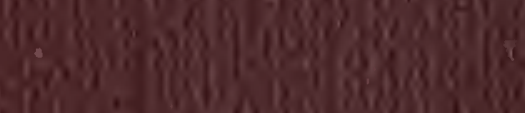
alu is

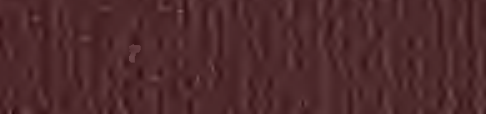
a songengong

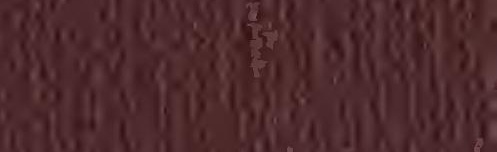

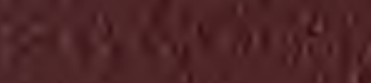

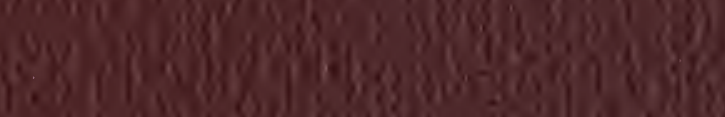

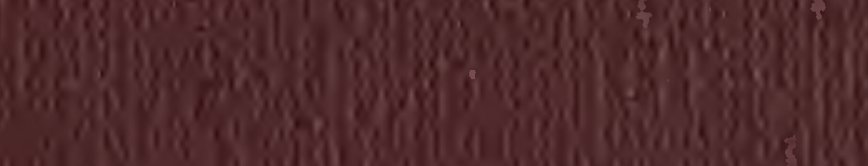

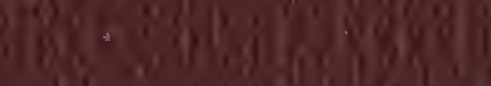

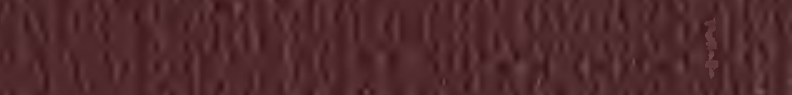

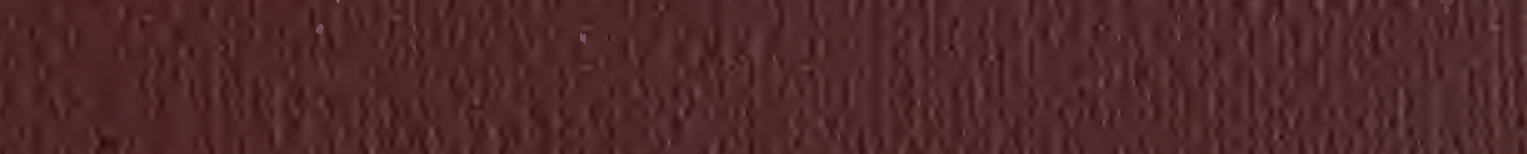

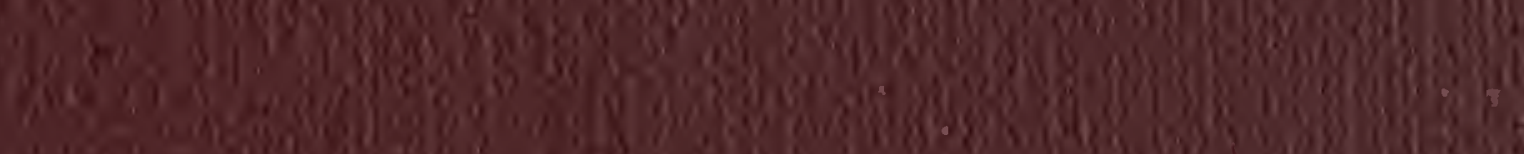
-

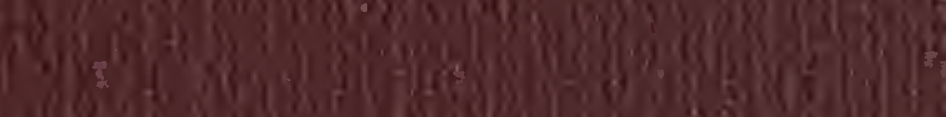

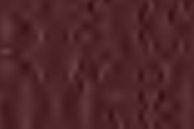

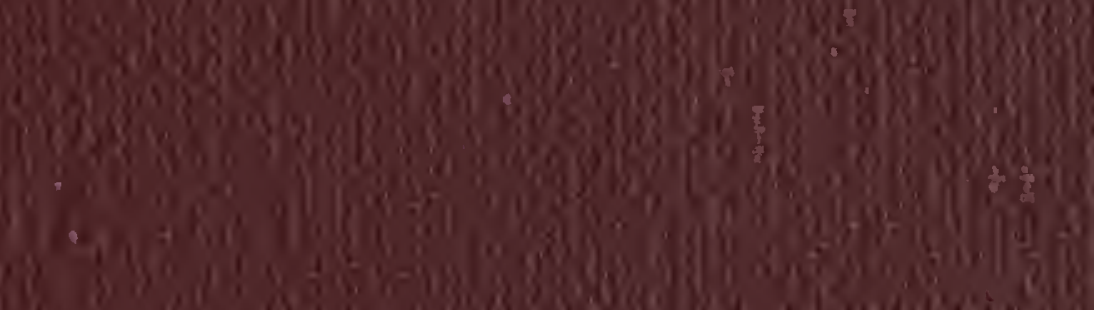
Bis not

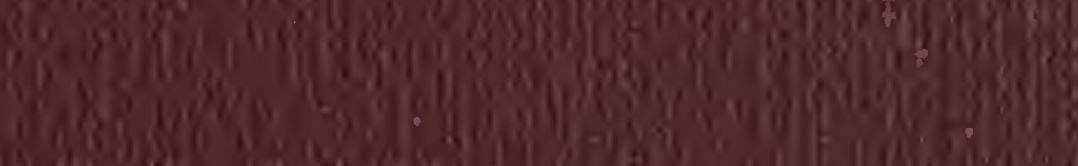

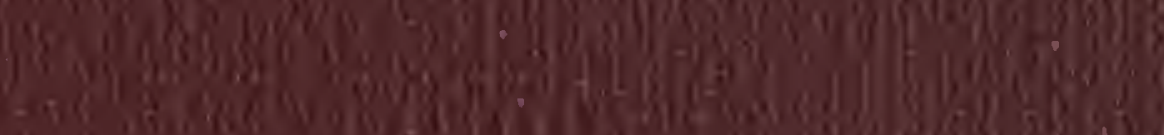

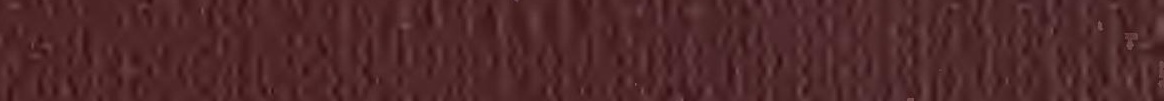

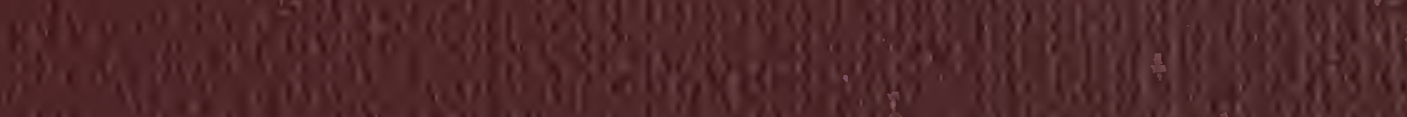

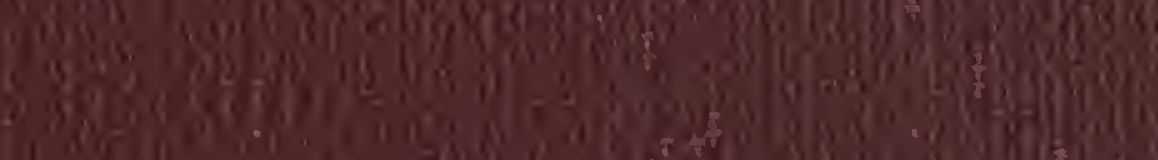
(0.5)

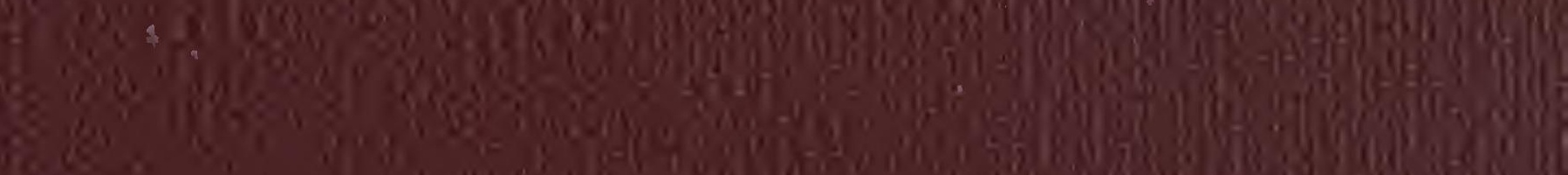

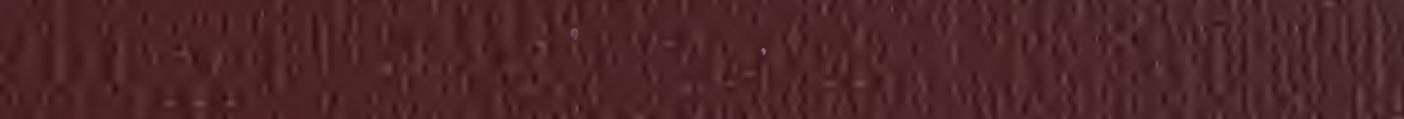

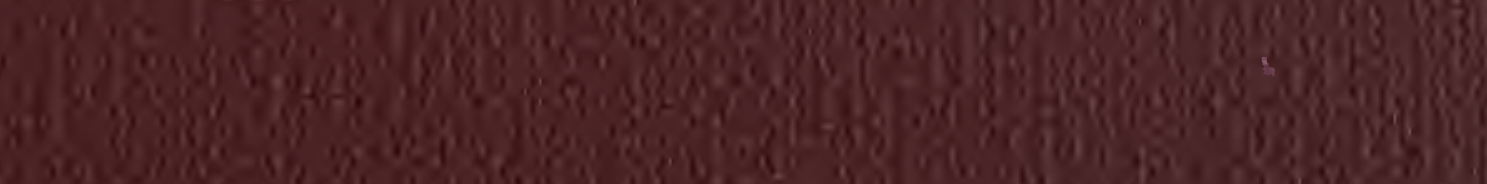


?;

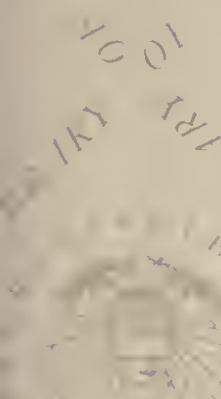

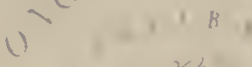

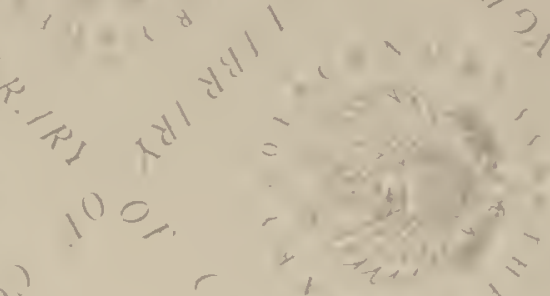

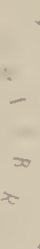

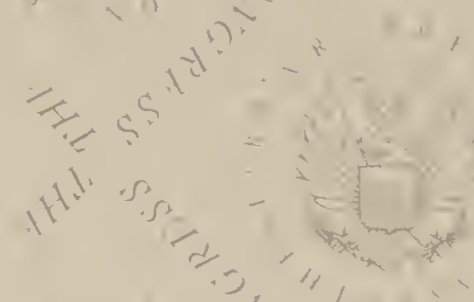$$
\text { eft? }
$$

$$
\begin{aligned}
& \begin{array}{l}
0,0 \\
00
\end{array} \\
& \text { if }
\end{aligned}
$$

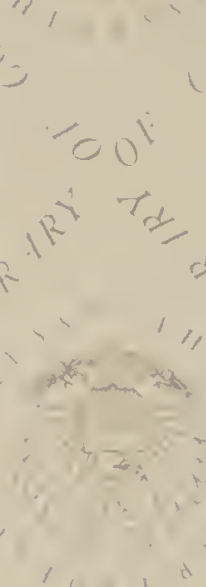

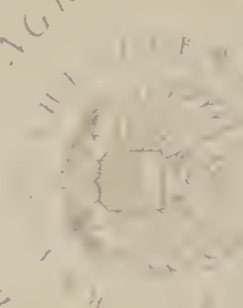
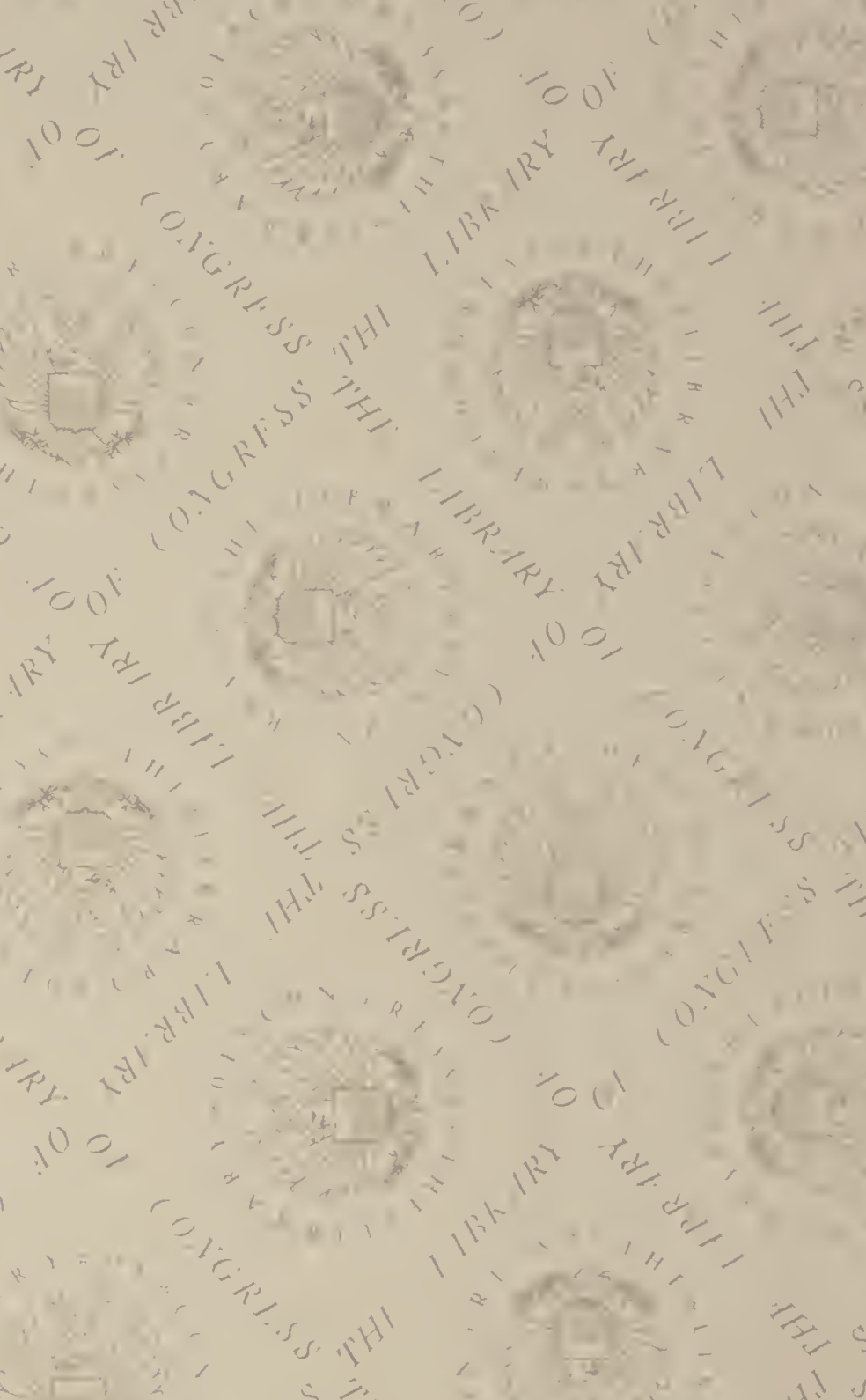

$11 x^{x}$

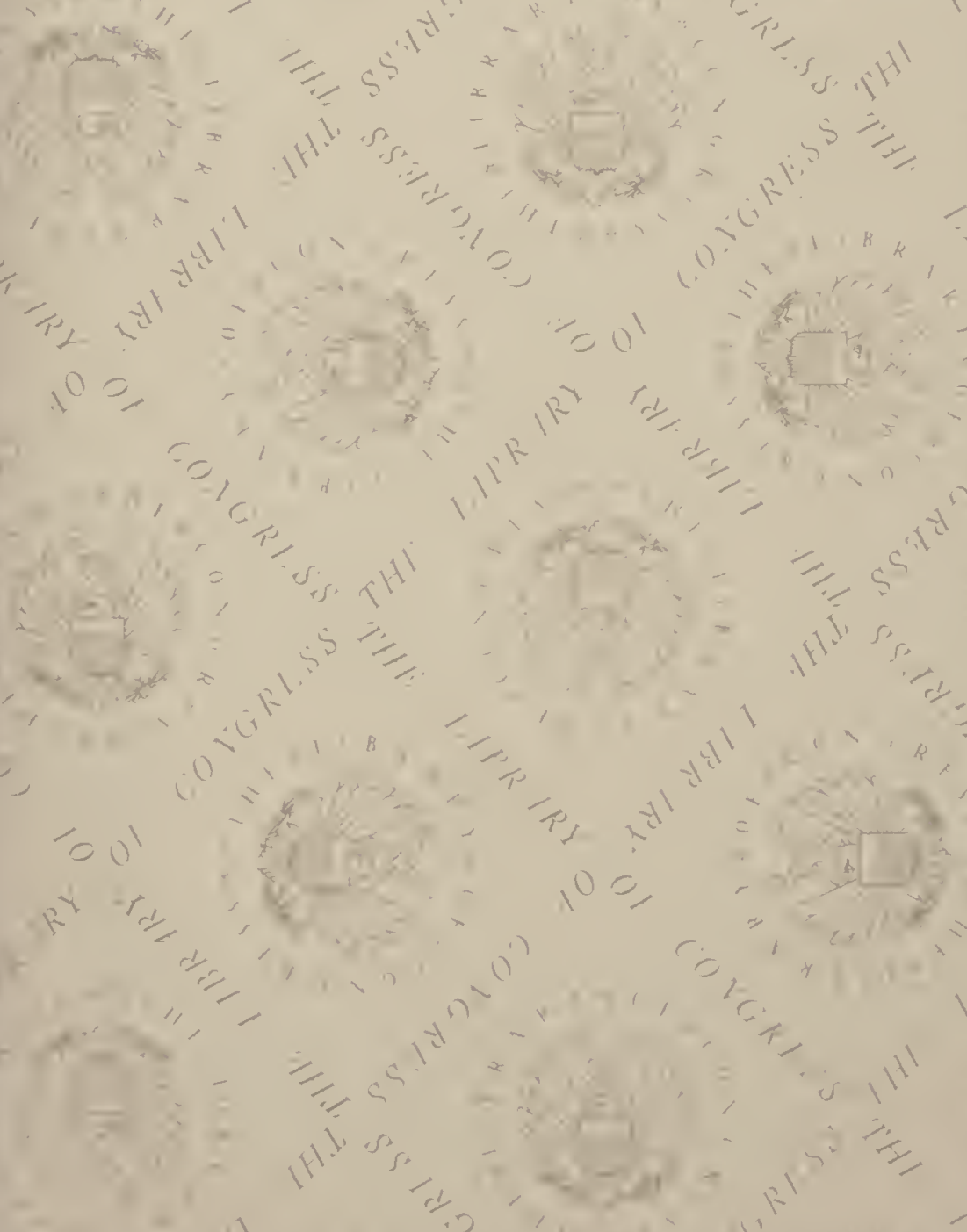







\title{
COMPARATIVE ZOOLOGY
}

\author{
STRUCTURAL AND SYSTEMATIC
}

FOR USE IN

\section{\$chools anto Colleges}

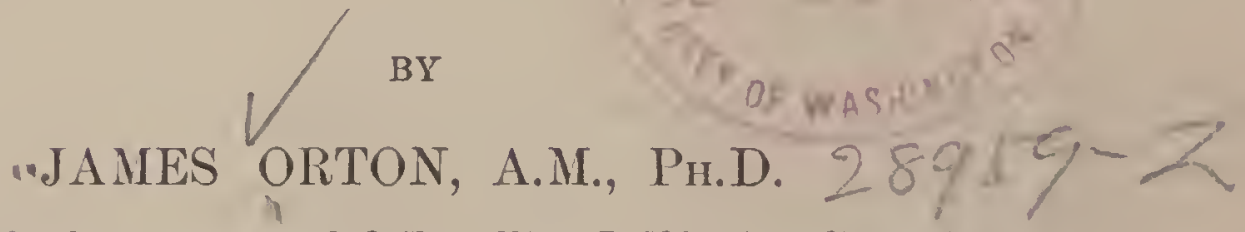

LATF PROFESSOI OF NATURAL IISTORY IN VASSAR OOLIEGE, CORRESPONDING

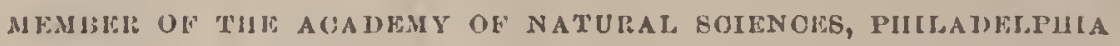
ANI) OF TIIE LYOHUM WF NATULAL HBTORY, N. Y., ETU.

\section{NEW EDITION, REVISED BY}

CHARLES WRIGHT DODGE, M.S.

PROFUSBOL OF BLOLOGY IN TUE UNIVERSITY OF LOCHIESTER

"The cducation of a naturalist now cmisists chiefly in learning how to compare."-AGAssiz

NEW YORK

H A R ER \& BROTHERS PUBLISHERS 


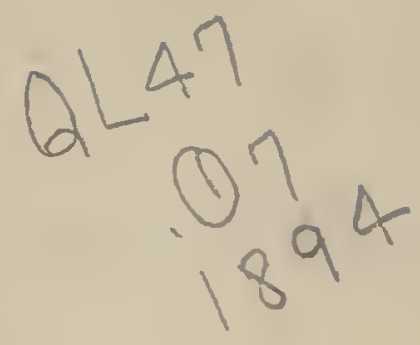

Entered according to Act of Congress, in the year 1876, by HARPER \& BROTHERS,

In the Office of the Librarian of Congress, at Washington.

Copyright, 1883, by Harper \& Brothers.

Copyright, 1894, by Harpre \& Brothers. 


\section{PREFA C E.}

THE distinctive character of this work consists in the treatment of the whole Animal Kingdom as a unit; in the comparative study of the development and variations of organs and their functions, from the simplest to the most complex state; in withholding Systematic Zoology until the student has mastered those structurai affinities upon which true classification is founded; and in being fitter for High Schools̀ and Mixed Schools by its language and illustrations, yet going far enough to constitute a complete grammar of the science for the undergraduate course of any College.

It is designed solely as a mannal for instruction. It is not a work of reference, nor a treatise. So far as a book is encyclopedic, it is unfit for a text-book. This is prepared on the principle of "just enongh, and no more." It aims to present clearly, and in a somewhat new form, the established facts and principles of Zoology. All theoretical and debatable points, and every fact or statement, however valuable, which is not absolutely necessary to a clear and adequate conception of the leading principles, are omitted. It is written in the light of the most recent pliase of the science, but not in the interest of any particular theory. To have given an exhaustive survey of animal life wonld have been not only undesirable, but impossible. Even Cuvier's great work must be supple- 
mented by museums, monographs, and microscopes. Natural History has outgrown the limits of a single book. Trial has proved the folly of giving the student so many things to learn that he has no time to understand, and the error of condemning the student to expend his strength upon the details of classification, which may change in the coming decade, instead of upon structure, which is permanent. Of course, specialists will miss many things, and find abundant room for criticism in what they regard as deficiencies; but the work should be judged by what it does contain, rather than by what it does not.

What is claimed, in the language of inventors, is the selection and arraugement of essential principles and typical illustrations from the standpoint of the teacher. The synthetic method is employed, as being the most natural: to begin with complex Man, instead of the simplest forms, wonld give a false idea. Man is not a model, but a monstrosity, the nlost modified of Vertebrates. But these outlines must be filled up, on the part of the teacher, by lectures, and by the exhibition of specimens; and, on the part of the student, by observation (noting, above all, the characteristic habits of animals), and by personal work with the knife and microscope. No text-book can take the place of nature, or supersede oral instruction from a competent teacher.

Suggestions and corrections from naturalists and teachers will be thankfully received.

In a work of this character, which is but a compound of the labors of all naturalists, it would be superfluons to make acknowledgments. The works referred to on page 397 have been specially consulted. 


\section{REVISER'S NOTE.}

In this revision of Professor Orton's work only such changes have been introdnced into the text as have been miade necessary by recent progress in Zoology. Some errors of statement have been corrected, and the classification has been slightly clianged. The principal addition consists of an Appendix composed of directions and suggestions for the performance of simple physiological experiments, and for the examination of certain animals representative of the more important groups. These experiments and dissections are so elenentary that they may easily be performed by either teacher or pupil. Very little apparatus, few instruments, and no special skill are required. The Appendix is designed to make the book of more practical value than formerly, and thus adapt it to the laboratory rather than the literary method of teaching.

An asterisk at the head of the chapter indicates that its subject-matter may be illustrated by practical work, for which directions will be fonnd in the Appendix.

Charlese Wright Dopge.

University of Rochester. 



\section{CONTENTS.}

IN'TRODUCTION.

Definition of Zoology, and its Place among the Sciences............. Historical Sketch................................ 14

PART I.-STRUCTURAL ZOOLOGY.

CHAP'TER I.

Minerals and Organized Bodies Distinguishled............ 13 CHAPTER II.

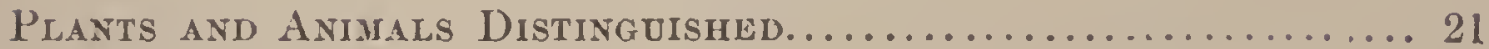

CHAP'TER III.

Relation betwelen Minerals, Plants, and Animat........... 27

CHAPTER IV.

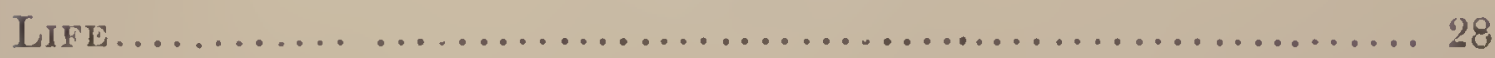

CHAPTER V.

Organization.................................. 30

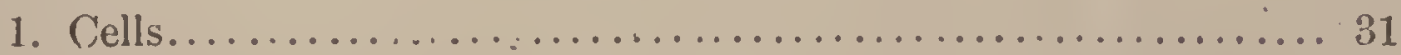

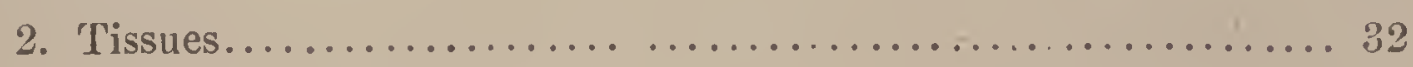

3. Organs, and their Functions...................... 41

CHAPTER VI.

Nutrition....................................... 45

CHAP'TER VII.

The Food of Anmals.............................. 47 


\section{CHAPTER VIII.}

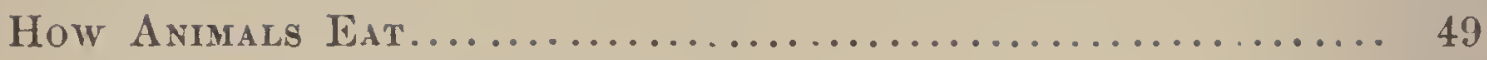

1. The Prehension of Food...................... 49

2. The Mouths of Animals........................ 55

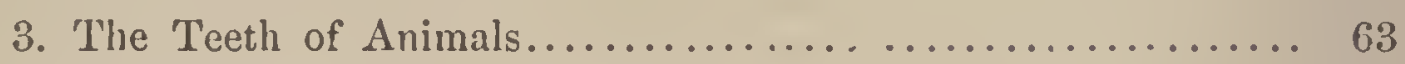

4. Deglutition, or How Animals Swallow.............. 72

CHAPTER IX.

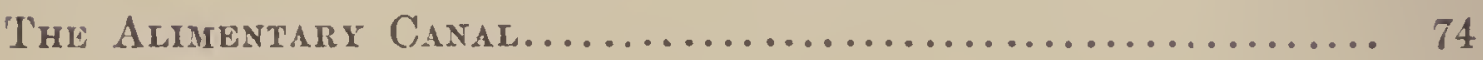

CHAPTER $\mathrm{X}$.

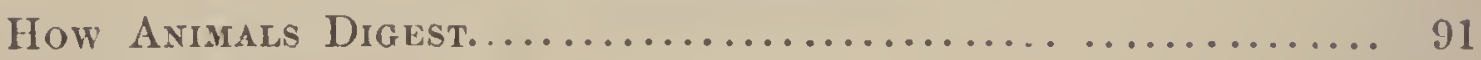

CHAPTER XI.

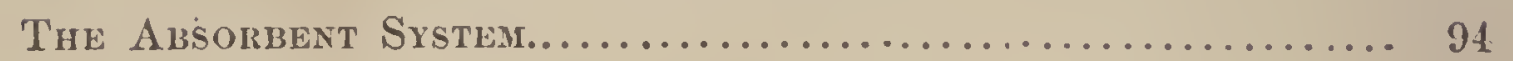

CHAP'TER XII.

The Blood of Animals .............................. 97

CHAPTER XIII.

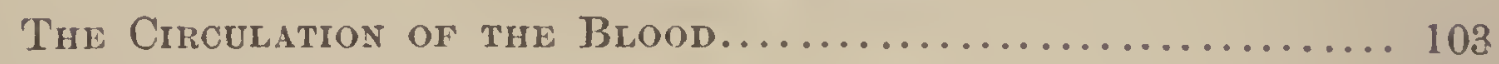

CHAPTER XIV.

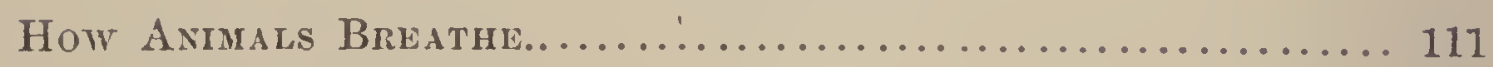

CHAP'TER XV.

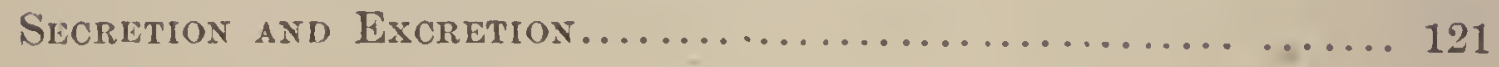

CHAP'TER XVI.

The Sikin and Skezeton.

CHAPTER XVII.

How Ammats Move.............................. 154

1. Muscle................................ 155

2. Locomotion................................ 157

CHAP'TER XVIII.

The Nervous System........................... 166

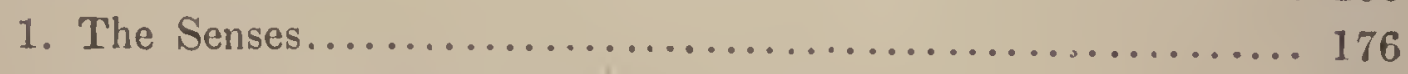

2. Instinct and Intelligence...................... 184

3. The Voices of Animals...................... 188 


\section{CHAPTER XIX.}

\section{CHAPTER XX.}

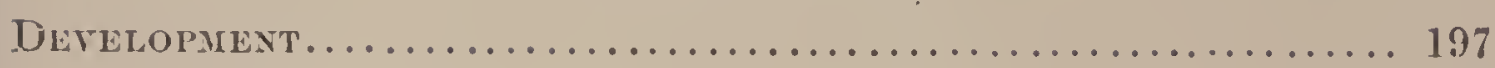

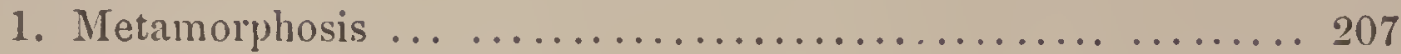

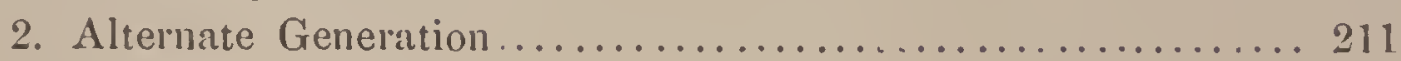

3. Growth and Repair............................. 214

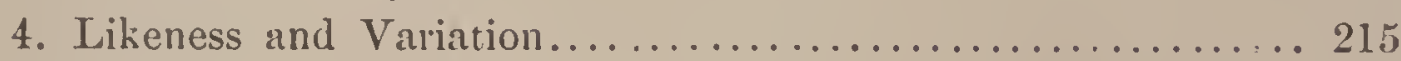

5. Homology, Analogy, and Correlation ............... 217

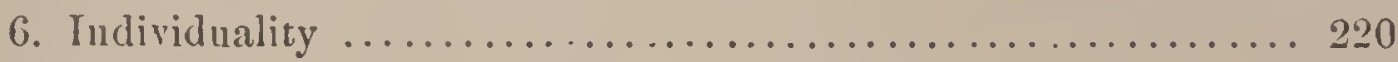

7. Relations of Number, Size, Form, and Rank........... 221

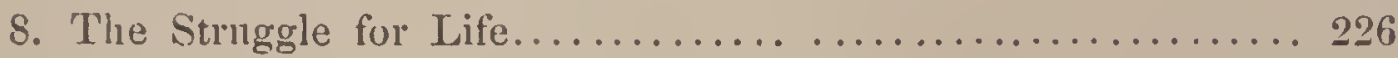

\section{PART II.-SYSTEMATIC ZOOLOGY.}

\section{CHAP'TER XXI.}

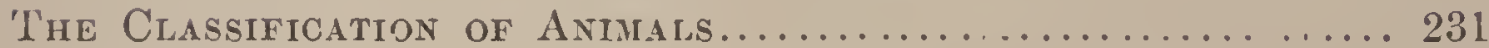

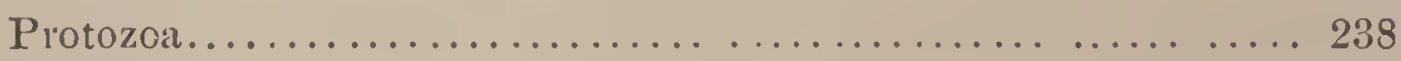

Porifera........................................ 244

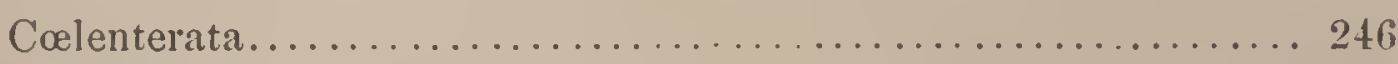

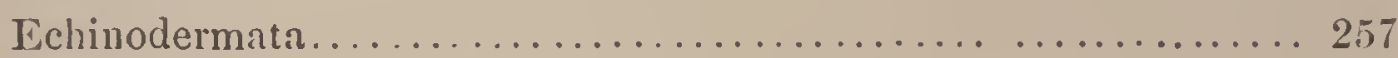

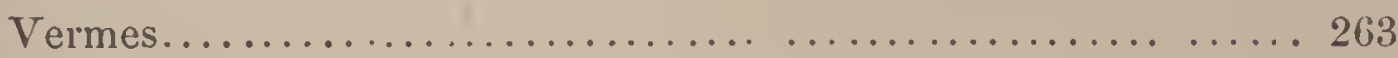

Mollusca.................................... 269

Arthropoda................................ 281

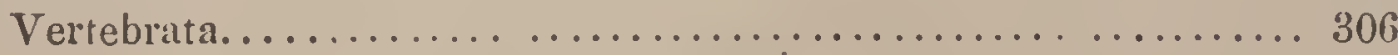

\section{CHAPTER XXII.}

Systematic Arraygemext of Representative Forms......... 362

CHAPTER XXIII.

The Distribution of Axmals......................... 371

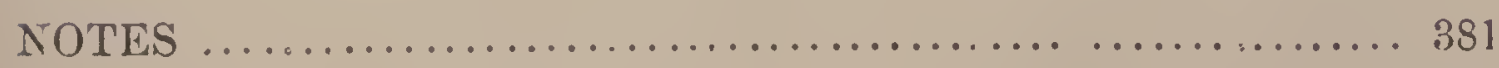

THE NATURALIST'S IIBRARY.................. 397

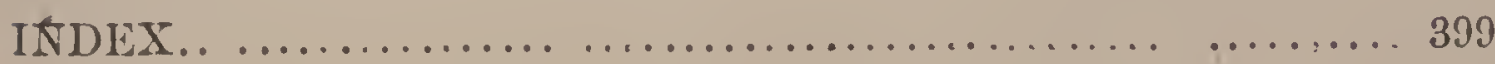


The first thing to be determined about a new specimen is not its name, but its most prominent character. Until you know an animal, care not for its name.-AGassiz.

The great benefit which a scientific education bestows, whether as training or as knowledge, is dependent upon the extent to which the mind of the student is brought into immediate contact with facts-upon the degree to which he learns the habit of appealing directly to Nature.-HuxukY. 


\section{N T R O D U C T I O N.}

1. Definition of Zoology, and its Place among the Sciences.-The province of Natural History is to describe, compare, and classify natural objects. These objects have been divided into the "organic" and the "inorganic," or those which are, and those which are not, the products of life. Biology is the science of the former, and Mineralogy the science of the latter. Biology again separates into Botany, or the Natural History of Plants, and Zoology, or the Natural History of Animals; while Mineralogy divides into Mineralogy proper, the science of mineral species, and Litholory, the science of mineral aggregates or rocks. Geology is that comprehensive knowledge of the earth's structure and development which rests on the whole doctrine of Natural History.

If we examine a piece of chalk, and determine its physical and chemical characters, its mode of occurrence and its uses, so as to distinguish it from all other forms of matter, we have its Mineralogy. But chalk occurs in vast natural beds : the examination of these masses-their origin, structure, position, and relation to other rocks-is the work of the Lithologist. Further, we observe that while chalk and marble are chemically alike, they widely differ in another respect. Grinding a piece of chalk so thin that we can see through it, and putting it under a microscope, we find imbedded in it innumerable bodies, about the hundredth of an inch in diameter, having a well-defined, symmetrical shape, and chambered like a Nautilus. We cannot say these are accidental aggregations, nor are they crystals : if the oyster-shell is formed by an oyster, these also must be the products of life. Indeed, the dredge brings up similar microscopic skeletons from the bottom of the Atlantic. So we conclude that chalk is but the dried mud of an ancient sea, the cemetery of count- 
less animals that lived and died long ago. 'The consideration of their fossil remains belongs to Paleontology, or that part of Biology which describes the relics of extinct forms of life. To study the stratigraphical position of the chalkbed, and by the aid of its Paleontology to determine its age and part in the world's history, is the business of Geology.

Of all the sciences, Zoology is the most extensive. Its field is a world of varied forms-hundreds of thousands in number. To determine their origin and development, their structure, habits, distribution, and mutual relations, is the work of the Zoologist. But so many and far-reaching are the aspects under which the animal creation may be contemplated, that the general science is beyond the grasp of any single person. Special departments have, therefore, arisen; and Zoology, in its comprehensive sense, is the combined result of the labors of many workers, each in his own line of research.

Structural Zoology treats of the organization of animals. There are two main branches: Anatomy, which considers the constitution and construction of the animal frame; and Physiology, which is the study of the apparatus in action. The former is separated into Embryology, or an account of the successive modifications through which an animal passes in its development from the egg to the adult state; and Morphology, which includes all inquiries concerning the form of mature animals, or the form and arrangement of their organs. The microscopical examination of any part, especially the tissues, belongs to Histology. Comparative Zoology is the comparison of the anatomy and physiology of all animals, existing and extiuct, to discover the fundamental likeness underneath the superficial differences, and to trace the adaptation of organs to the habits and spheres of life. It is this comparative science which has led to such grand generalizations as the unity of structure amidst the diversity of form in the animal creation, and by revealing the degrees of affinity between species has enabled us to classify them in natural groups, and thus laid the foundation of Systematic Zoology. When the study of structure is limited to a particular class or species of animals, or to a particular organ or part, monographic sciences are created, as Ornithotomy, 
or anatomy of birds; Osteology, or the science of bones; and Odontography, or the natural history of teeth.

Systematic Zoology is the classification or grouping of animals according to their structural and developmental relations. The systematic knowledge of the several classes, as Insects, Reptiles, and Birds, has given rise to subordinate sciences, like Entomology, Herpetology, or Omithology. ${ }^{1 *}$

Distributive Zoology is the knowledge of the successive appearance of animals in the order of time (Paleontology in part), and of the geographical and physical distribution of animals, living or extinct, over the surface of the earth.

Theoretical Zoology includes those provisional modes of grouping facts, and interpreting them, which still stand waiting at the gate of science. They may be true, but we cannot say that they are true. The evidence is incomplete. Such are the theories which attempt to explain the origin of life and the origin of species.

Suppose we wish to understand all about the Horse. Our first object is to study its structure. The whole body is enclosed within a hide, a skin covered with hair ; and if this hide be taken off, we find a great mass of flesh or muscle, the substance which, by its power of contraction, enables the animal to move. On removing this, we have a series of bones, bound together with ligaments, and forming the skeleton. Pursuing our researches, we find within this framework two main cavities: one, begimning in the skull and running through the spine, containing the brain and spinal marrow; the other, commencing with the mouth, contains the gullet, stomach, intestines, and the rest of the apparatus for digestion, and also the heart and lungs. Examinations of this character would give us the Anatomy of the Horse, or, more precisely, Hippotomy. The study of the bones alone would be its Osteology; the knowledge of the nerves would belong to Neurotomy. If we examined, under the microscope, the minute structure of the hair, skin, flesh, blood, and bone, we would learn its Histology. The consideration of the manifold changes undergone in developing from the egg to the full-grown animal, would be the Embry-

* Numbers like this refer to the Notes at the end of the volume. 
ology of the Horse; and its Morphology, the special study of the form of the adult animal and of its internal organs.

Thus far we have been looking, as it were, at a steamengine, with the fires out, and nothing in the boiler; but the body of the living Horse is a beautifully formed, active machine, and every part has its different work to do in the working of that machine, which is what we call its life. The science of such operations as the grinding of the food in the complex mill of the mouth; its digestion in the laboratory of the stomach; the pumping of the blood through a vast system of pipes over the whole body; its purification in the lungs; the process of growth, waste, and repair ; and that wondrous telegraph, the brain, receiving impressions, sending messages to the muscles, by which the animai is endowed with voluntary locomotion-this is Physiology. But Horses are not the only living creatures in the world; and if we compare the structures of various animals, as the Horse, Zebra, Dog, Monkey, Eagle, and Codfish, we shall find more or less resemblances and differences, enough to enable us to classify them, and give to each a description which will distinguish it from all others. This is the work of Systematic Zoology. Moreover, the Horses now living are not the only kinds that have ever lived; for the examination of the earth's crust - the great burial-ground of past ages-reveals the bones of numerous horse-like animals : the study of this pre-adamite race belongs to Paleontology. The chronological and geographical distribution of species is the department of Distributive Zoology. Speculations about the origin of the modern Horse, whether by special creation, or by development from some allied form now extinct, are kept aloof from demonstrative science, under the head of Theoretical Zoology.

2. History.-The Greek philosopher Aristotle (B.c. 384322 ) is called the "Father of Zoology." Certainly, he is the only great representative in ancient times, though his frequent allusions to familiar works on anatomy show that something had been done before him. His "History of Animals," in nine books, displays a wonderful knowledge of external and internal structure, habits, instincts, and uses. His descriptions are incomplete, but generally exact, so far 
as they go. Alexander, it is said, gave him nine hundred talents to collect materials, and put at his disposal several thousand men, for hunting specimens and procuring information.

The Romans accomplished little in natural science, though their military expeditions furnished unrivalled opportunities. Nearly three centuries and a half after Aristotle, Pliny (A.D. 23-79) wrote his "Natural History." He was a voluminous compiler, not an observer : he added hardly one new fact. He states that his work was extracted from over two thousand volumes, most of which are now lost.

During the Middle Ages, Natural History was studied in the books of the ancients; and at the close of the fifteenth century it was found where Pliny had left it, with the addition of many vague hypotheses and silly fancies. Albertus Magnus, of the thirteenth century, and Conrad Gesner and Aldrovandus, of the sixteenth, were voluminous writers, not naturalists. In the latter half of the sixteenth century, men began to observe nature for themselves. The earliest noteworthy researches were made on Fishes, by Rondelet (15071556) and Belon (1517-1564), of France, and Salviani (15141572 ), of Italy. They were followed by valuable observations upon Insects, by Redi (1626-1698), of Italy, and Swammerdam (1637-1680), of Holland; and towards the end of the same century, the Dutch naturalist, Leeuwenhoeck (1632-1723), opened a new world of life by the use of the microscope.

But there was no real advance of Systematic Zoology till the advent of the illustrious John Ray (1628-1705), of England. His "Synopsis," published in 1693, contained the first attempt to classify animals according to structure. Ray was the forerunner of "the immortal Swede," Linnæus (1707$1778)$, "the great framer of precise and definite ideas of natural objects, and terse teacher of the briefest and clearest expressions of their differences." His chief merit was in defining generic groups, and inventing specific names. ${ }^{2}$ Scarcely less important, however, was the impulse which he gave to the pursuit of Natural History. The spirit of inquiry, which his genius infused among the great, produced voyages of rescarch, which led to the formation of national museums. 
'The first expedition was sent forth by George III. of England, in 1765. Réaumur (1683-1757) made the earliest zoological collection in France; and the West Indian collections of Sir Hans Sloane (1660-1752) were the nucleus of the British Museum. The accumulation of specimens suggested comparisons, which eventually resulted in the highest advance of the science.

The brilliant style of Buffon (1707-1788) made Zoology popular not only in France, but throughout Europe. While the genius of Linnæus led to classification, that of Buffon lay in description. He was the first to call attention to the subject of Distribution. Lamarck (1745-1829), of Paris, was the next great light. The publication of his "Animaux sans Vertèbres," in 1801, was an epoch in the history of the lower animals. He was also the first prominent advocate of the transmutation of species.

But the brightest luminary in Zoology was George Cuvier (1769-1832), a German, born on French soil. Before his time, "there was no great principle of classification. Facts were accumulated, and more or less systematized, but they were not yet arranged according to law ; the principle was still wanting by which to generalize them and give meaning and vitality to the whole." It was Cuvier who found the key. He was the first so to interpret structure as to be ablo from the inspection of one bone to reconstruct the entire animal, and assign its position. His anatomical investigations revealed the natural affinities of animals, and led to the grand generalization, that the most comprehensive groups in the kingdom were based, not on special character's, but on different plans of structure. Palissy had long ago (1580) asserted that petrified shells were of animal origin; but the publication of Cuvier's "Memoir on Fossil Elephants," in 1800 , was the beginning of those profound researches on the remains of ancient life which created Paleontology. The discovery of the true relation between all animals, living and extinct, opened a boundless field of inquiry; and from that day the advance of Zoology has been unparalleled. Special studies of particular parts or classes of animals have so rapidly developed, that the history of Zoology during the last tifty years is the history of many sciences. ${ }^{3}$ 


\section{PART I.}

STRUCTURAL ZOOLOGY. 



\section{COMPARATIVE ZOOLOGY.}

\section{CHAPTER I.}

MINERALS AND ORGANIZED BODIES DISTINGUISHED.

Nature may be separated into two great kingdomsthat of mere dead matter, and that of matter under the influence of life. ${ }^{4}$ These differ in the following points:

(1) Composition.-While most of the chemical elements are found in different living beings, by far the greater part of their substance is composed of three or four-carbon, oxygen, and hydrogen; or these three with the addition of nitrogen. Next to these elements, sulphur and phosphorus are most widely distributed, though always found in very small quantities. The organic compounds belong to the carbon series, and contain three, four, or five elements. The former class, comprising starch, sugar, fat, etc., are relatively stable. The latter, possessing the three elements named, with nitrogen and sulphur or phosphorus, are very complex, containing a very large number of atoms to the molecule, and are usually unstable. Here belong albumen, myosin, chondrin, etc., the constituents of the living tissues. The formula for albumen is said to be $\mathrm{C}_{72} \mathrm{H}_{112} \mathrm{~N}_{18} \mathrm{SO}_{22}$, or some multiple of this formula. These compounds also contain more or less water, and usually exist in a jelly-like condition, neither solid nor fluid. All organic compounds are formed through the chemical activities of protoplasm, which is the only living substance. Inorganic may, under its influence, be changed 
to organic matter, and vice versâ; dead matter which enters the body of organized beings in the form of nutriment is changed in to living substance, which, after serving its purpose, passes again as waste to the inorganic world.

(2) Structure.-Minerals are homogeneous, while organized bodies are usually heterogeneous; $i$. e., composed of different parts, called tissues and organs, having peculiar uses and definite relations to one another. The tissues and organs, again, are heterogeneous, consisting inainly of microscopic cells, structures developed only by vital action. All the parts of an organism are mutually dependent, and reciprocally means and ends, while each part of a mineral exists for itself. The smallest fragment of marble is as much marble as a mountain-mass; but the fragment of a plant or animal is not an individual.

(3) Size and Shape.-Living bodies gradually acquire determinate dimensions; so do minerals in their perfect or crystal condition. But uncrystallized, inorganic bodies have an indefinite bulk. Most minerals are amorphous; crystals have regular forms, bounded, as a rule, by plane surfaces and straight lines; plants and animals are circumscribed by curved surfaces, and rarely assume accurate geometrical forms. ${ }^{5}$

(4) Phenomena.-Minerals remain internally at rest, and increase by external additions, if they grow at all. Living beings are constantly changing the matter of which they are composed, and grow by taking new matter into themselves and placing it among the particles already present. Organized bodies, moreover, pass through a cycle of changes-growth, development, reproduction, and death. These phenomena are characteristic of living as opposed to inorganic bodies. All living bodies grow from within, constantly give up old matter and replace it by new, reproduce their kind, and die; and no inorganic body shows any of these phenomena. 


\section{CHAPTER II.*}

\section{PLANTS AND ANIMALS DISTINGUISHED.}

IT may seem an easy matter to draw a line between plants and animals. Who cannot tell a Cow from a Cabbage? Who would confound a Coral with a Mushroom? Yet it is impossible to assign any absolute, distinctive character which will divide the one mode of life from the other. The difficulty of defining an animal increases with our knowledge of its nature. Linnæus defined it in three words; $\nmid$ a century later, Owen declared that a definition of plants which would exclude all animals, or of animals which would not let in a single plant, was impossible. Each different character used in drawing the boundary will bisect the debatable ground in a different latitude of the organic world. Between the higher animals and higher plants the difference is apparent; but when we reflect how many characters the two have in common, and especially when we descend to the lower and minuter forms, we discover that the two "kingdoms" touch, and even dissolve into, each other. This border-land has been as hotly contested among naturalists as many a disputed frontier between adjacent nations. Its inhabitants have been taken and retaken several times by botanists and zoologists; for they have characters that lead on the one side to plants, and on the other to animals. To solve the difficulty, some eminent naturalists, as Häckel and Owell, propose a fourth "kingdom," that of the Protista, to receive those living beings which are organic, but not distinctly vegetable or animal. But a greater difficulty arises in attempting to fix its precise limits.

* See Appendix.

+ "Minerals grow; plunts grow and live; animals grow, live, and feel." 
The drift of modern research points to this: that there are but two kingdoms of nature, the mineral and the organized, and these closely linked together; that the latter must be taken as one whole, from which two great branches rise and diverge. "There is at bottom but one life, which is the whole life of some creatures, and the common basis of the life of all; a life of simplest moving and feeling, of feeding and breathing, of producing its kind and lasting its day: a life which, so far as we at present know, has no need of such parts as we call organs. Upon this general foundation are built up the manifold special characters of animal and vegetable existence; but the tendency, the endeavor, so to speak, of the plant is one, of the animal is another, and the unlikeness between them widens the higher the building is carried up. As we pass along the series of either [branch] from low to high, the plant becomes more vegetative, the animal more animal." $"$

Defining animals and plants by their prominent characteristics, we may say that a living being which has cellwalls of cellulose, and by deoxidation and synthesis of its simple food-stuffs produces the complicated organic substances, is a plant; while a living being which has albuminous tissues, and by oxidation and analysis reduces its complicated food-stuffs to a simpler form, is an animal. But both definitions are defective, including too many forms, and excluding forms that properly belong to the respective kingdoms. No definition is possible which shall include all animals and exclude all plants, or vice versî.

(1) Origin.-Both branches of the tree of life start alike: the lowest of plants and animals consist of a single cell. In fact, the cycle of life in all living beings begins in a small, round particle of matter, a cell-in the higher plants called an ovule, in the higher animals an ovum. 
This cell consists mainly of a semi-fluid substance called protoplasm. In the very simplest forms the protoplasm is not enclosed by a membrane or cell-wall. In most plants the cell-wall is present, and consists of cellulose, a substance akin to starch; in animals, with few exceptions, the wall is a pellicle of firmer protoplasm, $i$. e., albuminous.

(2) Composition.-Modern research has broken down the partition between plants and animals, so far as chemical nature is concerned. The vegetable fabric and secretions may be ternary or binary compounds; but the essential living parts of plants, as of animals, are quaternary, consisting of four elements-carbon, hydrogen, oxygen, and nitrogen. Cellulose (woody fibre), starch, and chlorophyl (green coloring matter) are eminently vegetable products, but not distinctive; for cellulose is wanting in some plants, as some Fungi, and present in some animals, as Tunicates; starch, under the name of glycogen, is found in the liver and brains of Mammals, and chlorophyl gives color to the fresh - water Polyp. Still, it holds good, generally, that plants consist mainly of cellulose, dextrin, and starch; while animals are mainly made up of albumen, fibrin, and gelatin; that nitrogen is more abundant in animal tissues; while in plants carbon is predominant.

(3) Form.-No outline can be drawn which shall be common to all animals or all plants. The lowest members of each group have no fixed shape. The spores. of Confervæ can hardly be distinguished from animalcules; the compound and fixed animals, Sea-mat and Sea-moss (Polyzoa), and Corals, often resemble vegetable forms, although in structure widely removed from plants. Similar conditions of life are here accompanied by an external likeness. In free-living animals this resemblance is not found.

(4) Structure.-A plant is the multiplication of the unit -a cell with a cellulose wall. Some simple animals have 
a similar simple cellular structure; and all animal tissues, while forming, are cellular. But this character, which is permanent in plants, is generally transitory in animals. In the more highly organized tissues the cells are so united as partly or wholly to lose their individuality, and the characteristic part of the tissue is the intercellular substance, while the cells themselves are small and unimportant, or else the cells are fused together and lose their dividing walls, as in striated muscles and in nerves. Excepting the lowest forms, animals are more composite than, plants, $i . e$. , their organs are more complex and numerous, and more specially devoted to particular purposes. Repetition of similar parts is a characteristic of plants; and when found in animals, as the Angle-worm, is called vegetative repetition. Differentiation and specialization are characteristic of animals. Most animals, moreover, have fore-and-aft polarity; in contrast, plants are up-and-down structures, thongh in this respect they are imitated by radiated animals, like the Star-fish. Plants are continually receiving additional members; most animals soon become perfect.

(5) Physiology.-In their modes of nutrition, plants and animals stand widest apart. A plant in the seed and an animal in the egg exist in similar conditions: in both cases a mass of organic matter accompanies the germ. When this supply of food is exhausted, both seek nourishment from without. But here analogy ends: the green plant feeds on mineral matter, the animal on organic. Some plants have the power to form chlorophyl, the green coloring matter of leaves, which uses the energy of the sunlight to form starch ont of the inorganic substances-carbondioxide and water. They are able also to form albuminoid matter out of inorganic substances. A very few animals which have a substance identical with or allied to chlorophyl have the same power, but in general animals are de- 
pendent for their food on the compounds put together in plants. Colorless plants, as Fungi, possessing no chlorophyl, feed, like animals, on organic compounds. No living being is able to combine the simple elements-carbon, oxygen, hydrogen, and nitrogen-into organic compounds.

The food of plants is gaseons (carbon-dioxide and ammonia) or liquid (water containing substances in solution), that of animals usually more or less solid, though solid substances must be changed to liquids before being capable of absorption into the tissues. The plant, then, absorbs these foods through its outer surface, while the animal takes its nourishment in larger or smaller masses, and digests it in a special cavity. A few exceptions, however, occur on both sides. Certain moulds seem to swallow their food, ${ }^{7}$ and certain animals, as the tape-worm, liave no digestive tract.

Plants are ordinarily fixed, their food is brought to them, and a large share of their work, the formation of organic compounds, is done by the energy of the sunlight; while animals are usnally locomotive, must seek their food, and are unable to utilize the general forces of nature as the plant does. The plant is thus able to grow much more than the animal, as very little of the nourishment received is used to repair waste, while in most animals the time soon comes when waste and repair are approximately equal. But in both all work done is paid for by waste of substance already formed.

In combining carbon-dioxide and water to form starch the plant sets oxygen free $\left(6\left(\mathrm{CO}_{2}\right)+5\left(\mathrm{H}_{2} \mathrm{O}\right)=\mathrm{C}_{6} \mathrm{H}_{10} \mathrm{O}_{5}+\right.$ $\left.6\left(\mathrm{O}_{2}\right)\right)$ : in oxidizing starch or other food the animal uses oxygen and sets carbon-dioxide free. The green plant in the sunlight, then, gives off oxygen and nses carbon-dioxide, while plants, which have no chlorophyl, at all times, and all plants in the darkness, use oxygen and give off carbon-dioxide, like an animal. Every plant begins life 
like an animal - a consumer, not a producer: not till the young shoot rises above the soil, and unfolds itself to the light of the sun, at the touch of whose mystic rays chlorophyl is developed, does real, constructive vegetation begin; then its mode of life is reversed-carbon is retained and oxygen set free.

Most plants, and many animals, multiply by budding and division; on both we practise grafting; in both the cycle of life comes round again to the ovule or ovum. Do annuals flower but to die? Insects lay their eggs in their old age.

Both animals and plants liave sensibility. This is one of the fundamental physiological properties of protoplasm. But in plants the protoplasm is scattered and buried in rigid structures: feeling is, therefore, dull. In animals, the protoplasin is concentrated into special organs, and so feeling, like electricity lammed into Leyden jars, goes off with a flash. ${ }^{8} \quad$ Plants never possess conscionsness or volition, as the higher animals do.

The self-motion of animals and the rooted state of plants is a very general distinction; but it fails wlere we need it most. It is a cliaracteristic of living things to move. The protoplasm of all organisms is unceasingly active. ${ }^{9}$ Besides this internal movement, myriads of plants, as well as animals, are locomotive. Rambling Diatons, writhing Oscillaria, and the agile spores of Cryptogams crowd our waters, their orgaus of motion (cilia) being of the very same character as in microscopic animals; while Sponges, Corals, Oysters, and Barnacles are stationary. A contractile vesicle is not exclusively an animal property, for the fresh-water Volvox and Gonium have it. The muscular contractions of the highest animals and the sensible motions of plants are both due to changes in the protoplasm in their cells. The ciliary movements of animals and of microscopic plants are precisely similar, and in neither case indicate consciousness or self-determining power. 
Plants, as well as animals, need a season of repose. Both have their epidemics. On both, narcotic and acrid poisons produce analogous results. Are some animals warm-blooded? In germination and flowering, plants erolve heat-the stamens of the Arum, e. g., showing a rise of $20^{\circ} \mathrm{F}$. In a sense, an Oak has just as much heat as an Elephant, only the miserly tree locks up the sunlight in solid carbon.

At present, any boundary of the Animal Kingdom is arbitrary. "We cannot distinguish the vegetable from the animal kingdom by any complete and precise definition. Although ordinary observation of their usual representatives may discern little that is common to the two, yet there are many simple forms of life which hardly rise high enough in the scale of being to rank distinctively either as plant or animal ; there are undoubted plants possessing faculties which are generally deemed characteristic of animals; and some plants of the lighest grade share in these endowments." 10

\section{CHAPTER III.}

\section{RELATION BETWEEN MINERALs, PLAN'TS, AND ANIMALS.}

There are no independent members of creation: all things touch upon one another. The matter of the living world is identical with that of the inorganic. The plant, feeding on the minerals, carbon-dioxide, water, and ammonia, builds them up into complex organic compounds, as starch, sugar, gum, cellulose, albumen, fibrin, casein, and gluten. When the plant is eaten by the animal, these substances are used for building up tissues, supplying energy, repairing waste, laid up in reserve as glycogen and fat, or oxidized in the blood to produce heat. The albuminoids are essential for the formation of tissues, like muscle, nerve, 
cartilage; but the ternary compounds help in repairing waste, while both produce heat. When oxidized, whether for work or warmth, these complex compounds break up into the simple compounds - water, carbon dioxide, and (ultimately) anmonia, and as such are returned to earth and air from the animal. Both plant and animal end their life by going back to the mineral world: and thus the circle is complete-from dust to dust. Carbonate of ammonia and water, a blade of grass and a horse, are but the same elements differently combined and arranged. Plants compress the forces of inorganic nature into chemical compounds; animals liberate them. Plants produce; animals consume. The work of plants is synthesis, a building-up; the work of animals is analysis, or destruction. The tendency in plants is deoxidation; the tendency in animals is oxidation. Without plants, animals would perish; without animals, plants had no need to be. There is no plant which may not serve as food to some animal.

\section{CHAPTER IV.*}

\section{LIFE.}

ALL forces are known by the phenomena which they cause. So long as the animal and plant were supposed to exist in opposition to ordinary physical forces or independently of them, a vital force or principle was postulated by which the work of the body was performed. It is now known that most, if not all, of the phenomena manifested by a living body are due to one or more of the ordinary physical forces - heat, chemical affinity, electricity, etc. There is no work done which demands a vital force.

The common modern view is that vitality is simply a * See Appendix. 
collective name for the sum of the phenomena displayed by living beings. It is neither a force nor a thing at all, but is an abstraction, like goodness or sweetness; or, to use Huxley's expression, to speak of vitality is as if one should speak of the horologity of a clock, meaning its time-keeping properties.

A third theory is still possible. The combination of elements into organic cells, the arrangement of these cells into tissues, the grouping of these tissues into organs, and the marshalling of these organs into plans of structure, cail for some further shaping, controlling power to effect such wonderful co-ordination. Moreover, the manifestation of feeling and consciousuess is a inystery which no plysical hypothesis has cleared up. The simplest vital phenomenon has in it something over and above the known forces of the laboratory. ${ }^{11}$ If the vital machine is given, it works by physical forces; but to produce it and keep it in order needs, so far as we now know, more than mere physical force. To this controlling power we may apply the name vitality.

Life is exhibited only under certain conditions. One condition is the presence of a physical basis called protoplasm. This substance is found in all living bodies, and, so far as we know, is sinilar in all - a viscid, transparent, homogeneous, or minutely granular, albuminoid matter. Life is inseparable from this protoplasm; but it is dormant unless excited by some external stimulants, such as heat, light, electricity, food, water, and oxygen. Thus, a certain temperature is essential to growth and motion; taste is induced by chemical action, and sight by luminous vibrations.

The essential manifestations of animal life may be reduced to four: contractility; irritability, or the peculiar power of receiving and transmitting impressions; the power of assimilating food; the power of reproduction. All these powers are possessed by protoplasm, and so by 
all animals: all move, feel, grow, and multiply. But some of the lowest forms are without the slightest trace of organs; they seem to be as perfectly homogeneous and structureless as a drop of jelly. They could not be more simple. They are devoid of muscles, nerves, and stomach; yet they have all the fundamental attributes of life-moving, feeling, eating, and propagating their kind. It has been supposed that the muscular and nervous matter is diffused in a molecular form; but all we can say is, that the highest power of the microscope reveals no organized structure whatever-i.e., there are no parts set apart for a particular purpose, but a fragment is as good as the whole to perform all the functions of life. The animal series, therefore, begins with forms that feel without nerves, move without muscles, and digest without a stomach, protoplasm itself having all these properties: in other words, life is the cause of organization, not the result of it. Animals do not live because they are organized, but are organized because they are alive.

\section{CHAPTER V.*}

ORGA NIZATION.

$\mathrm{W}_{\mathrm{E}}$ have seen that the simplest life is a formless speck of protoplasm, withont distinctions of structure, and therefore without distinctions of function, all parts serving all purposes - mouth, stomach, limb, and lung - indiscriminately. There is no separate digestive cavity, no separate respiratory, muscular, or nervous systems. Every part will successively feed, feel, move, and breathe. Just as in the earliest state of society all do everything, each does all. Every man is his own tailor, architect, and lawyer. But in the progress of social development the principle of

* See Appeudix. 
the division of labor emerges. First comes a distinction between the governing and governed classes; then follow and multiply the various civil, military, ecclesiastical, and industrial occupations.

In like manner, as we advance in the animal series, we find the body more and more heterogeneous and complex by a process of differentiation, i.e., setting apart certain portions of the body for special duty. In the lowest forms, the work of life is carried on by very simple apparatus. $^{12}$ But in the higher organisms every function is performed by a special organ. For example, contractility, at first the property of the entire animal, becomes centred in muscular tissue; respiration, which in simple beings is effected by the whole surface, is specialized in lungs or gills; sensibility, from being cominon to the whole organism, is handed over to the nerves. An animal, then, whose body, instead of being uniform throughout, is made up of different parts for the performance of particular functions, is said to be organized. And the term is as applicable to the slightly differentiated cell as to complex Man. Organization is expressed by single cells, or by their combination into tissues and organs.

1. Cells.-A cell is the simplest form of organized life. In general, it is a microscopic globule, consisting of a delicate membrane enclosing a minute portion of protoplasm. The very simplest kinds are without granules or signs of circulation; but usually the protoplasm is granular, and contains a defined separate mass called the nucleus, within which are sometimes seen one or two, rarely more, dark, round specks, named nucleoli. The enveloping membrane is

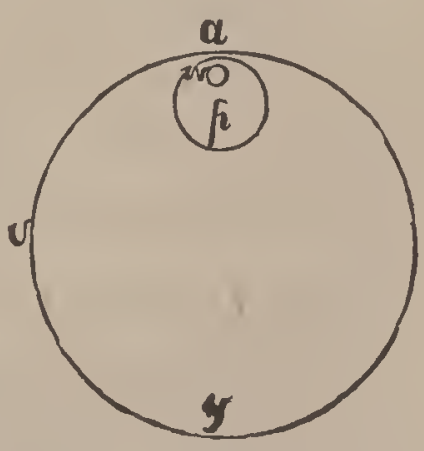

Frg. 1.-Parts of a Cell: $a, v, y$, cell-wall ; $p$, uucleus; $w$, nucleolus. extremely thin and transparent, and structureless: it is only an excretion of dead matter acting as a boundary to 
the cell-contents. ${ }^{13}$ The nuclens generally lies near the centre of the protoplasm, and is the centre of activity.

Cells vary greatly in size, but are generaily invisible to the naked eye, ranging from $\frac{1}{500}$ to $\frac{1}{10000}$ of an inch in diameter. Abont 4000 of the smallest would be necessary to cover the dot of this letter $i$. The natural form of isolated cells is spherical; but when they crowd each other, as seen in bone, cartilage, and muscle, their outlines become angular, either hexagonal or irregular.

Within the narrow boundary of a simple sphere, the cell-membrane, are exhibited all the essential phenomena of life - growth, developinent, and reproduction. The physiology of these minute organisms is of peculiar interest, since all animal structure is but the multiplication of the cell as a unit, and the whole life of an animal is that of the cells which compose it: in them and by them all its vital processes are carried on. ${ }^{14}$

The structure of an animal cell can be seen in bloodcorpuscles, by diluting with a weak (.5 per cent.) solution of salt a drop of blood from a Frog, and placing it under the microscope. (See Fig. 63.) With this may be compared vegetable cells as seen in a drop of fluid yeast or a drop of water into which pollen grains from some flower lave been dusted.

2. Tissues.-There are organisms of the lowest grade (as Paramecium) which consist of a single cell, living for and by itself. In this case, the animal and cell are identical: the Paramecium is as truly an individual as the Elephant. But all animals, save these unicellular beings, are mainly aggregations of cells: for the varions parts of a body are not only separable by the knife in to bones, muscles, nerves, etc., but thcse are susceptible of a finer analysis by the microscope, which shows that they arise from the development and union of cells. These cellular fabrics, called tissues, differ from one another both chemically and structurally, but agree in being permeable to liquids-a property 
which secures the flexibility of the organs so essential to animal life. Every part of the human body, for example, is moist: even the hairs, nails, and cuticle contain water. The contents as well as the shape of the cells are usually modified according to the tissue which they form: thus, we find cells containing earthy matter, iron, fat, mucus, etc.

In plants, the cell generally retains the characters of the cell; but in animals (after the embryonic period) the cell usually undergoes such modifications that the cellular form disappear's. The cells are connected together or en veloped by an intercellular substance (blastema), which may be watery, soft, and gelatinous, firmer and tenacions, still more solid and hyaline, or hard and opaque. In the fluids of the body, as the blood, the cells are separate; i. e., the blastema is fluid. But in the solid tissues the cells coalesce, being simply connected, as in the epidermis, or united into fibres and tubes.

In the lowest forms of life, and in all the higher animals in their earliest embryonic state, the cells of which they are composed are not transformed into differentiated tissues: definite tissues make their first appearance in the Sponges, and they differ from one another more and more widely as we ascend the scale of being. In other words, the bodies of the lower and the immature animals are more uniform in composition than the higher or adult forms. In the Vertebrates only are all the following tissues found represented:

(1) Epithelial Tissue.-This is the simplest form of cellular structure. It covers all the free surfaces of the body, internal and external, so that an animal may be said to be contained between the walls of a double bag. That which is internal, lining the mouth, windpipe, lungs, blood-vessels, gullet, stomach, intestines-in fact, every cavity and canal - is called epithelium. It is a very delicate skin, formed of flat or cylindrical cells, and in some parts (as in the wind-pipe of air-breathing animals, and along the gills 
of the Oyster) is covered with cilia, or minute hair-like portions of protoplasm, about $\frac{1}{600}$ of an inch long, which

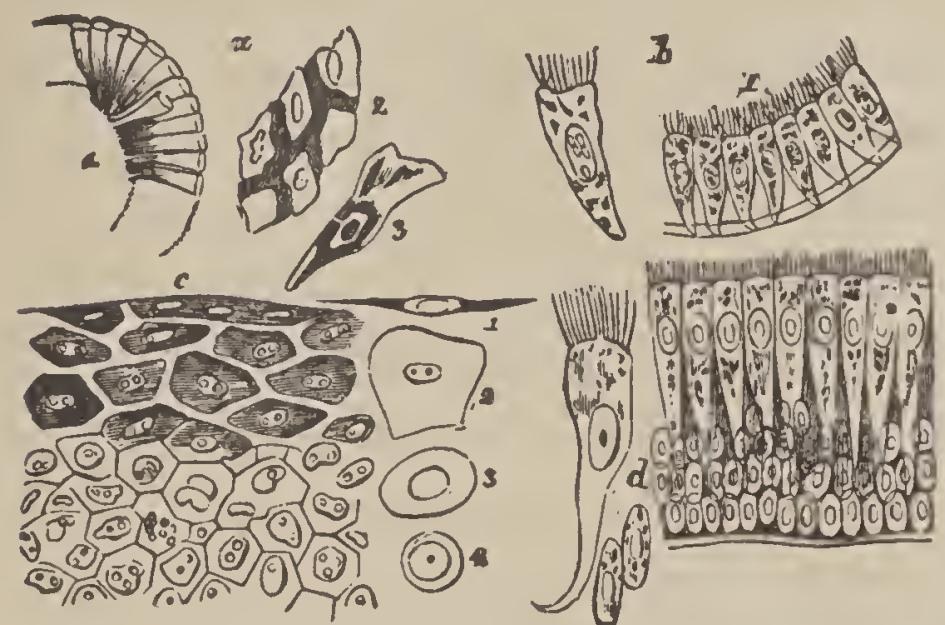

Frg. 2. - Various kinds of Epithelium Cells: $a$, columnar, from small intestine; 3 , a single cell, showing nucleus; $b$, ciliated, from one of the small airtubes; $d$, the same, from the windpipe, with single cell magnified about 200 times; $c$, squamous, from eyelid of a calf, showing changes of form, from the deep to superficial cells, 1 being the scurf. are incessantly moving. Continuous with thisinner lining of the body (as seen on the lip), and covering the outside, is the epidermis, or cuticle. It is the outer layer of the "skin," which we can remove by a blister, and in Man varies in thickness from $\frac{1}{800}$ of an inch on the cheek to $\frac{1}{10}$ on the sole of the foot. It is constantly wearing off at the surface, and as constantly being replenished from the deeper portion; and in the process of growth and passage outward, the cells change from the spherical form to dead horny scales (seen in scurf and dandruff). In the lower layer of the cuticle we find the pigment cells, characteristic of colored races. Neither the epidermis nor the corresponding tissue within (epithelium) has any blood-vessels or nerves. The epithelial tissue, then, is simply a superficial covering, bloodless and insensible, protecting the more delicate parts underneath. Hairs, horns, hoofs, nails, claws, corns, beaks, scales, tortoise-shell, the wings of Insects, etc., are modifications of the epidermis.

The next three sorts of tissue are characterized by a great development of the intercellular substance, while the cells themselves are very slightly modified.

(2) Connective Tissue.-This is the most extensive tissue in animals, as it is the great connecting medium by which the different parts are held together. Could it be taken 
out entire, it would be a complete mould of all the organs. It surrounds the bones, muscles, blood-vessels, nerves, and glands, and is the substance of the ligaments, tendons, "true skin," mucous membrane, etc. It varies in character, being soft, tender, and elastic, or dense, tough, and generally unyielding. In the former state, it consists of innumerable fine white and yellow fibres, which interlace in all directions, leaving irregular spaces, and form-

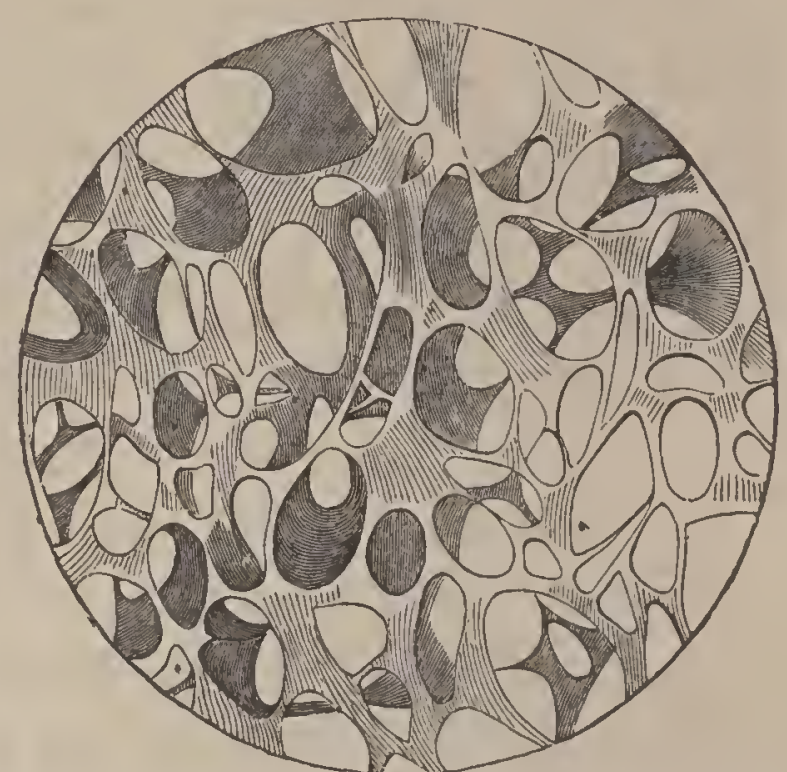

Frg. 3.-Connective Tissue, showing areolar structure, $\times 25$. ing a loose, spongy, moist web. In the latter, the fibres

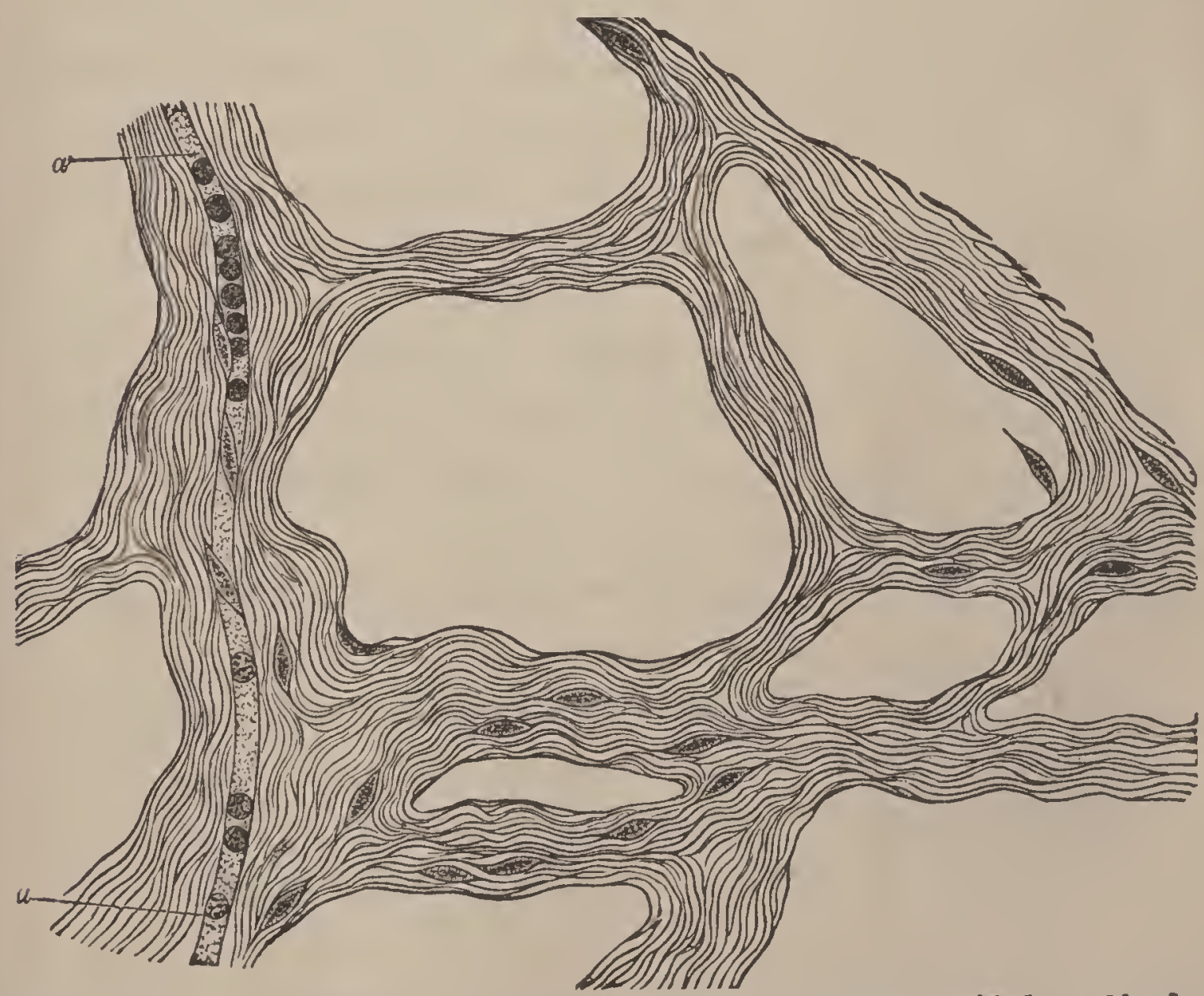

FrG. 4-Connective Tissue from human peritoneum; highly magnifled; $a$, bloodvessel. 
are condensed into sheets or parallel cords, having a wavy, glistening appearance. Such structures are the fasciæ and tendons. Connective tissue is not very sensitive. It contains gelatin - the matter which tans when hide is made into leather. In this tissue the intercellular substances take the form of fibres. The white fibres are inelastic, and from $\frac{1}{40000}$ to $\frac{1}{24000}$ of an inch in diameter. They are best seen in the tendons. The yellow fibres are

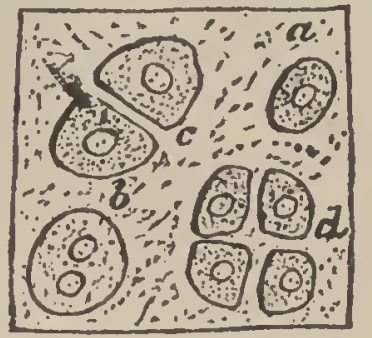

FrG. 5.-Hyaline Cartilage, Diagram: $\alpha$, cartilage cell ; $b$, cell about to divide; $c$, cell divided into two; $d$, into four parts. The space between the cells is filled with transparent intercellular substance; highly magnified.

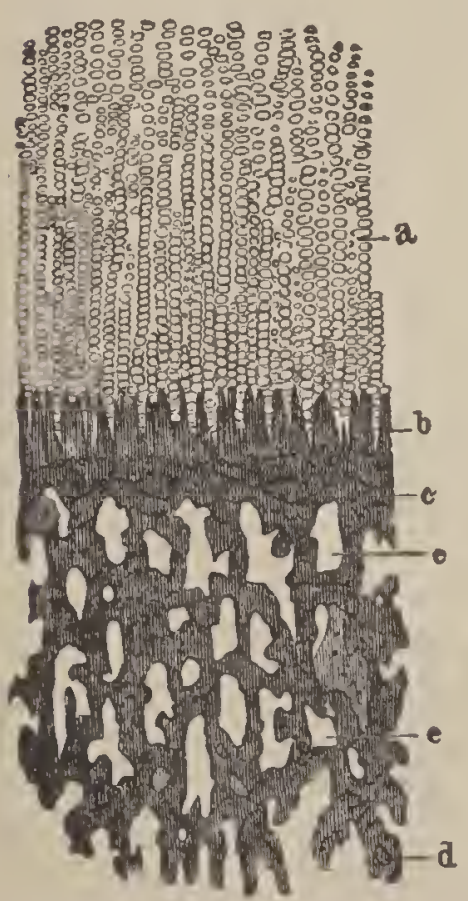

Fig. 6. - Ossifying Cartilage, $\times 10 ; \alpha$, cartilage cells, passing into compact bone, $c$, and then spongy bone, $e$. elastic, curled at the ends, very long, and from $\frac{1}{21000}$ to $\frac{1}{4000}$ of an inch in diameter. They are shown in the hinge-ligament of an Oyster. Connective tissue appears areolar, $i$. e., shows interspaces, only under the inicroscope.

(3) Cartilaginous Tissue.-This tissue, known also as "gristle," is composed of cells imbedded in a granular or hyaline substance, which is dense, elastic, bluish - white, and translucent. It is found where strength, elasticity, and insensibility are wanted, as at the joints. It also takes the place of the long bones in the embryo. When cartilage is mixed with connective tissue, as in the ear, it is called fibro-cartilage.

(4) Osseous Tissue.-This hard, opaque tissue, called "bone," differs from the former two in having the intercellular spaces or meshes filled with phosphate of lime and other earths, instead of a hyaline or fibrous substance. It may be called petrified tissue-the quantity of earthy matter, and therefore the brittleness of the bone, increasing with the 
age of the animal. If a chicken-bone be left in dilute muriatic acid several days, it may be tied into a knot, since the acid has dissolved the lime, leaving nothing but cartilage and connective tissue. If a bone be burned, it becomes light, porous, and brittle, the lime alone remaining. ${ }^{15}$

Bone is a very vascular tissue; that is, it is traversed by minute blood-vessels and nerves, which pass through a

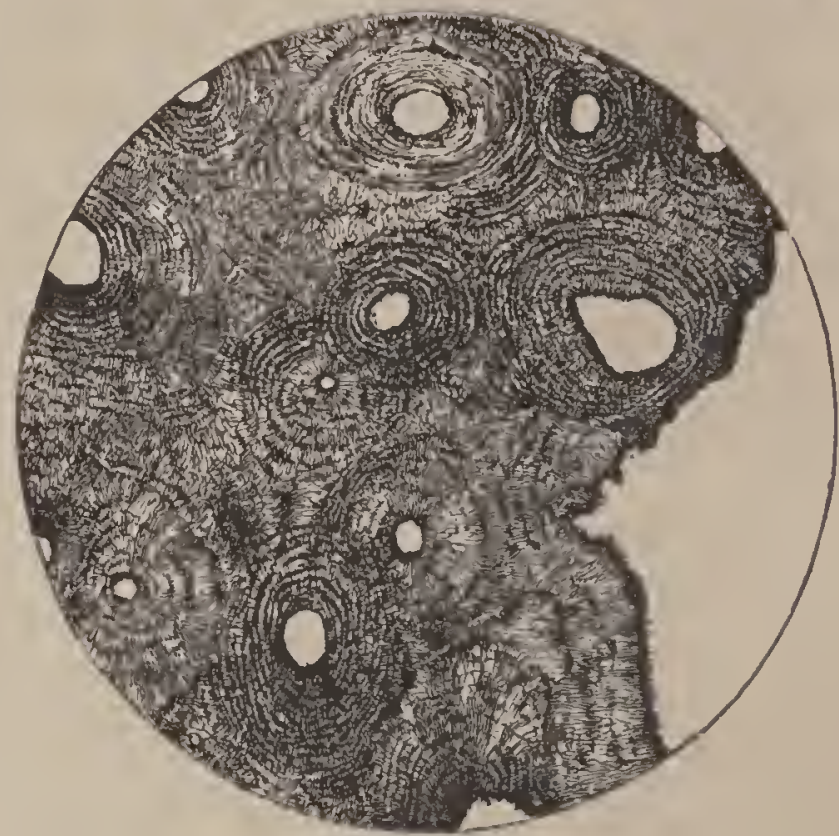

Fig. 7. - Transverse section of a Bone (Human Femur), $\times 50$, showing Haversian cauals. net-work of tubes, called Haversian canals. The canals average $\frac{1}{1000}$ of an inch, being finest near the surface of the bone, and larger further in, where they form a cancellated or spongy structure, and finally merge (in the long

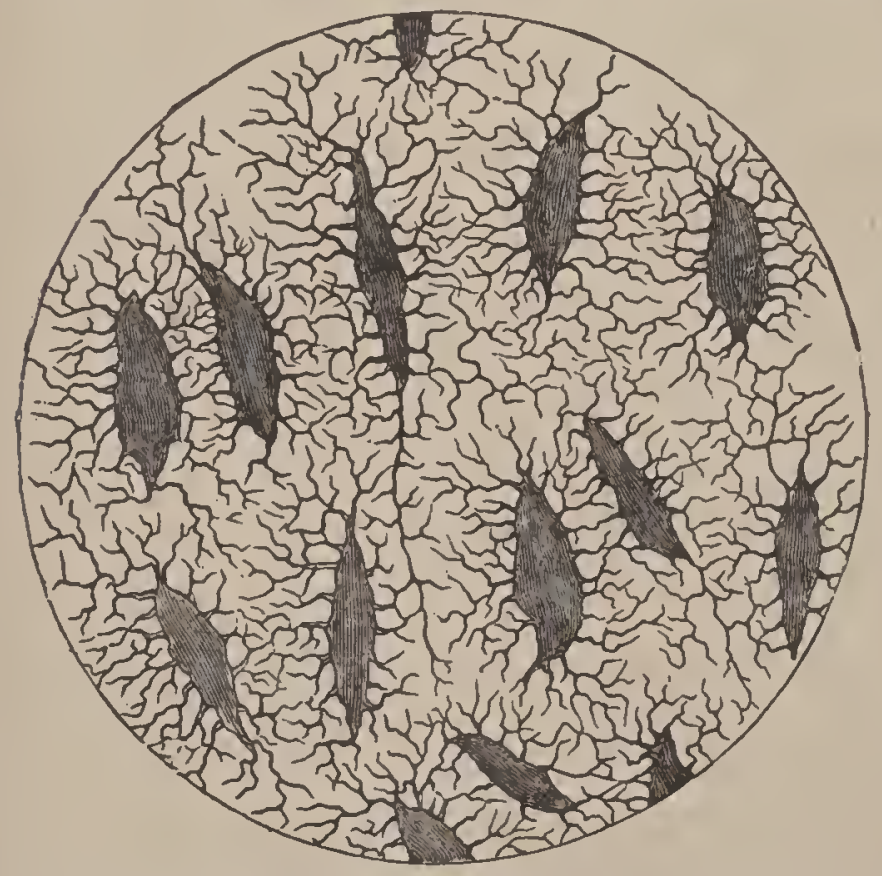

Fig. S. - Frontal Bone of Human Sknll nnder the microscope, showing lacnnæ and canaliculi. bones) into the central cavity, containing the marrow. Under the microscope, each canal appears to be the centre of a multitude of lamince, or plates, arranged around it. Lying between these plates are little cavities, called lacunoe, which are connected by exceedingly finetubes, or canaliculi. These represent the spaces occupied by the original cells of the bone, and differ in shape and size in different animals. 
True bone is found only in Vertebrates, or back-boned animals.

(5) Dental Tissue.-Like bone, a tooth is a combination of earthy and animal matter. It may be called petrified skin. In the higher animals, it consists of three parts: dentine, forming the body of the tooth, and always present; enamel, capping the crown; and cement, covering the fangs (Fig. 31). The last is true bone, or osseous tissue.

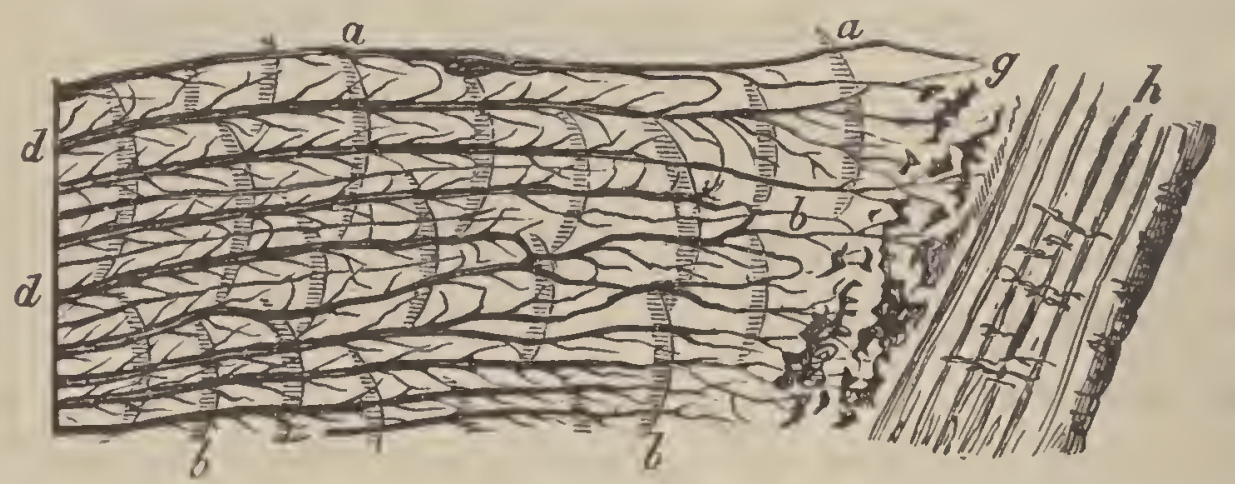

Fxa. 9. - Highly magnified section of Dentine and Cement, from the fang of a Human Molar: $a, b$, marks of the original dentinal pulp; $d$, dentinal tubes, terminating in the very sensitive, modified layer, $g ; h$, cement.

Dentine resembles bone, but differs in having neither lacunæ nor (save in Shark's teeth) canaliculi. It shows, in place of the former, innumerable parallel tubes, reaching from the outside to the pulp-cavity within. The "ivory" of Elephants consists of dentine. Enamel is the hardest substance in the body, and is composed of minute six-sided

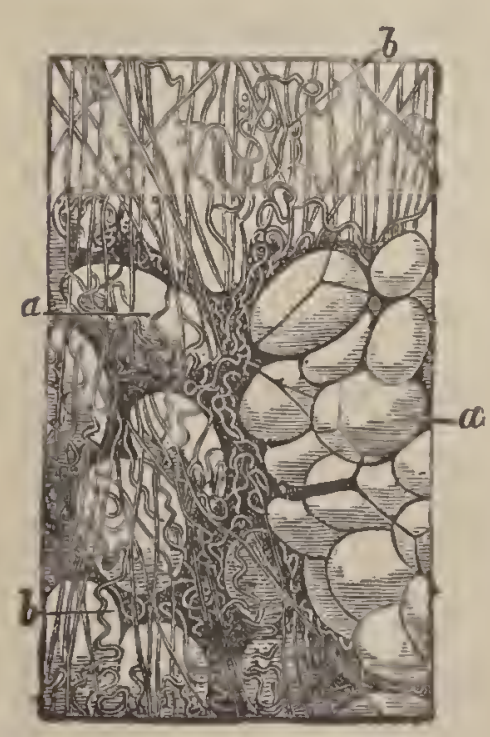

Fig. 10.-Adipose 'lissue, $a$; with tibres of connective tissue, $b$. fibres, set closely together. It is wanting in the teeth of most Fishes, Snakes, Sloths, Armadillos, Sperm-whales, etc.

True dental tissue is confined to Vertebrates.

(6) Adipose Tissue.-Certain cells become greatly enlarged and filled with fat, so that the original protoplasm occupies a very small part of the space within the cell-membrane. These cells are united into masses by connective tissue, in the skin (as in the "blub. 
ber" of whales), between the muscles (as in "streaky" meat), or in the abdominal cavity, in the omentum, mesentery, or about the kidneys." The marrow of bones is an example. Globules of fat occur in many Molluses and Insects; but true adipose tissue is found only in backboned animals, particularly the herbivorous. In the average Man, it constitutes about $\frac{1}{20}$ part of his weight, and a single Whale has yielded 120 tons of oil. The fat of animals has the different names of oil, lard, tallow, suet, spermaceti, etc. It is a reserve of nutriment in excess of consumption, serving also as a packing material, and as a protection against cold.

(7) Muscular Tissue.-If we examine a piece of lean meat, we find it is made up of a number of fasciculi, or bundles of fibres, placed side by side, and bound together by connective tissue. The microscope informs us that each fibre is itself a bundle of smaller fibres; and when one of these is more closely examined, it is found to be enclosed in a delicate, smooth tube, called the sarcolemma. This tube is filled with very minute, parallel fibrils, averaging $\frac{1}{10000}$

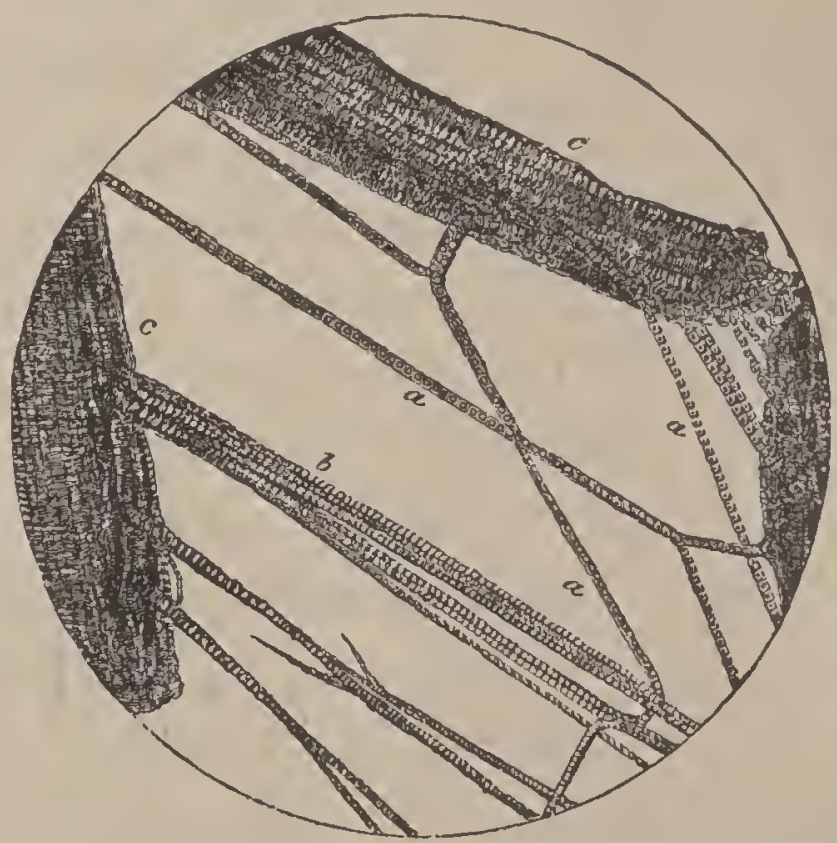

Fra. 11.-Striated Muscular Fibre (of the Pig), $\times 200$. The constituent fibres are seen at $a$; $c$ is a fasciculus, or bundle.

of an inch in diameter, and having a striated aspect. Tissue of this description constitutes all ordinary muscle, or "lean meat," and is marked by regular cross-lines, or strice.

Besides this striated muscular tissue, there exist, in the coats of the stomach, intestines, blood-vessels, and some other parts of Vertebrates, smooth muscular fibres, or mem. 
branes, which show a nucleus under the microscope, and do not break up into fibrils (Fig. 122). The gizzards of

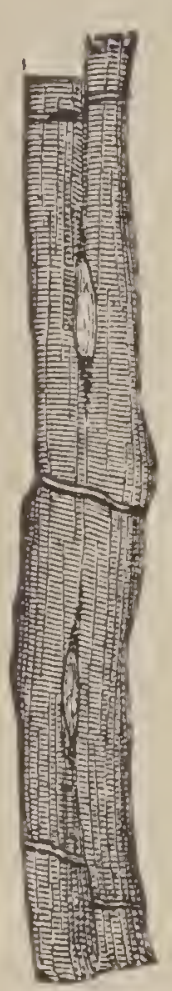

Fic. 12. - Striated Muscular Fibres, from the heart of Man, divided by transverse septa into separate nucleated portions. fowls exhibit this form.

All muscle has the property of shortening itself when excited; but the contraction of the striated kind is under the control of the will, while the movement of the smooth fibres is involuntary. ${ }^{16}$ Muscles are well supplied with arteries, veins, and nerres; but the color is due to a peculiar pigment, not to the blood.

Muscular tissue is found in all animals from the Coral to Man.

(8) Nervous Tissue.-Nervous matter exists under three forms: First-the cellular, consisting of nucleated cells, varying from $\frac{1}{6000}$ to $\frac{1}{20} \overline{0}$ of an inch in diameter, and found in the nerve-centres (Fig. 132), the gray portion of the brain, spinal cord, and other ganglia. Second-the fibrous, consisting of pale, flat, extremely fine filaments. They abound in the sympathetic nerves, and are the only nerves found in the Invertebrates. Third-the tubular. These are much larger than the fibrous, the coarsest being $\frac{1}{1200}$ of an inch in diameter. They consist of tubes enclosing a transparent fibre and a fatty substance called the nerve-marrow. ${ }^{17}$ The delicate tube itself is called neurilemma, analogous to the sarcolemma of muscular tissue. Nerve-tubes are found only in back-boned animals, in the white substance of the brain, spinal cord, and in the nerves.

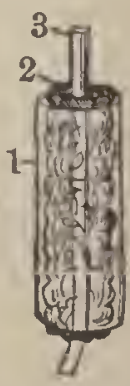

Fra. 13.-Structure of a Nerve: 1 , sheath, or neurilemma; 2 , mednllary substance of Schwann : 3, axis cylinder, or primitive band.

A bundle of fibrous or tubular nervous matter, sur. rounded by connective tissue, constitutes a nerve. 


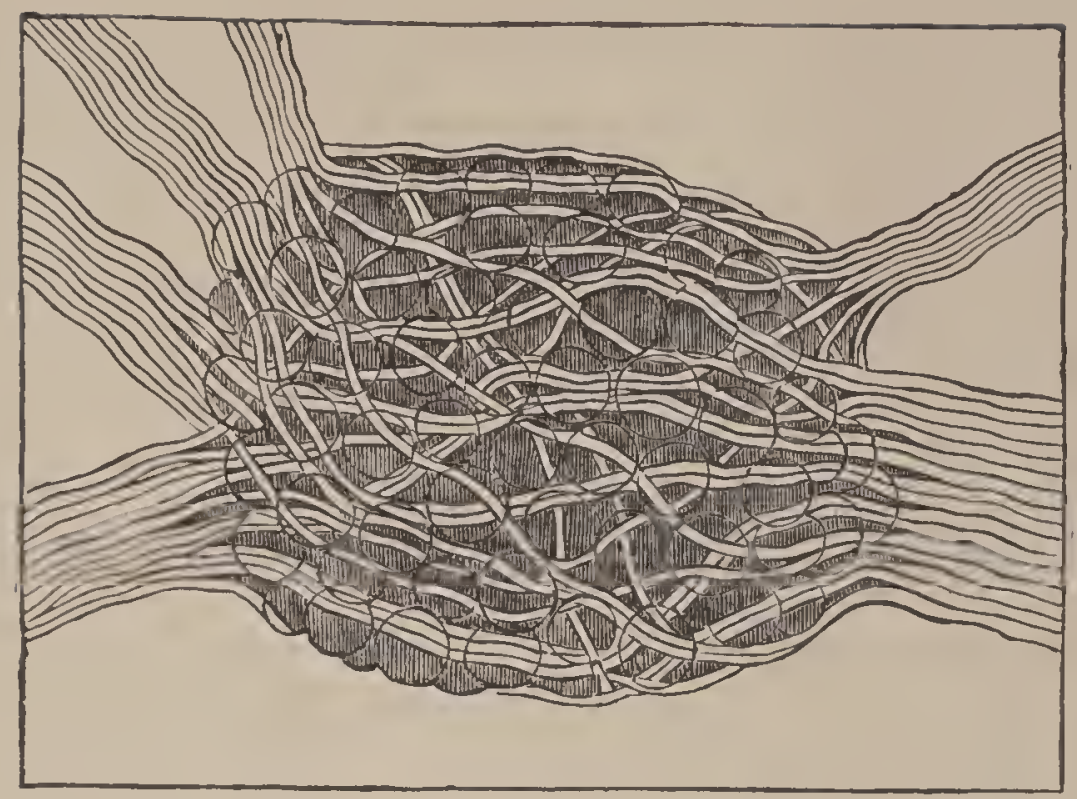

Fra. 14.-A Ganglion of the Sympathetic Nerve of a Mouse.

3. Organs, and their Functions.-Animals, like Plants, grow, feel, and move; these three are the capital facts of every organism. Besides these there may be some peculiar phenomena, as motion and will.

Life is manifested in certain special operations, called functions, performed by certain special parts, called organs. Thus, the stomach is an organ, whose function is digestion. A single organ may manifest vitality, but it does not (save in the very lowest forms) show forth the whole life of the animal. For, in being set apart for a special purpose, an organ takes upon itself, so to speak, to do something for the benefit of the whole animal, in return for which it is absolved from doing many things. The stomach is not called upon to circulate or purify the blood.

There may be functions without special organs, as the Amœba digests, respires, moves, and reproduces by its general mass. But, as we ascend the scale of animal life, we pass from the simple to the complex: groups of cells or tissues, instead of being repetitions of each other, take on a difference, and become distinguished as special parts with specific duties. The higher the rank of the animal, the more complicated the organs. The more complicated the structure, the more complicated the functions. But in 
all animals, the functions are performed under conditions essentially the same. Thus, respiration in the Sponge, the Fish, and in Man has one object and one means, though the methods differ. A function, therefore, is a group of similar phenomena effected by analogous structures.

The life of an animal consists in the accumulation and expenditure of force. The tissues are storehouses of power, which, as they waste, is given off in various forms. Thus, the nervous tissue generates nerve-force; the muscles, motion. If we contemplate the phenomena presented by a Dog, the most obvious fact is his power of moving from place to place, a power produced by the interplay of muscles and bones. We observe, also, that his motions are neither mechanical nor irregular; there is method in his movement. He has the power of willing, seeing, hearing, feeling, etc.; and these functions are accomplished by a delicate apparatus of nerves.

But the Dog does not exhibit perpetual motion. Sooner or later he becomes exhausted, and rest is necessary. Sleep gives only temporary relief. In every exercise of the muscles and nerves there is a consumption or waste of their substance. The blood restores the organs, but in time the blood itself needs renewal. If not renewed, the animal becomes emaciated, for the whole body is laid under contribution to furnish a supply. Hence the feelings of hunger and thirst, impelling the creature to seek food. Only this will maintain the balance between waste and repair. We notice, therefore, an entirely different set of functions, involving, however, the use of motion and will. The Dog seizes a piece of meat, grinds it between its teeth, sends it into the stomach, where it is digested, and then into the intestine, where it is further changed; there the nourishing part is absorbed, and carried to the heart, which propels it through tubes, called blood-ressels, all over the body. In this process of digestion, certain fluids 
are required, as saliva, gastric juice, and bile: these are secreted by special organs, called glands. Moreover, since not all the food eaten is fitted to make blood, and as the blood itself, in going around the body, acts like a scavenger, picking up worn-out particles, we have another function, that of excretion, or removal of useless matter from the system. The kidneys and lungs do much of this; but the lungs do something else. They expose the blood to the air, and introduce oxygen, which, we shall find, is essential to the life of every animal.

These centripetal and centrifugal movements in the body-throwing in and throwing out-are so related and involved, especially in the lower forms, that they cannot be sharply defined and classified. It has been said that every Dog has two lives - a vegetative and an animal. The former includes the processes of digestion, circulation, respiration, secretion, etc., which are common to all life; the functions of the other, as motion, sensation, and will, are peculiar to animals. The heart is the centre of the vegetative life, and the brain is the centre of the animal life. The aim of the vegetative organs is to nourish the individual, and reprodnce its kind; the organs of locomotion and sense establish relations between the individual and the world without. The former maintain life; the others express it. The former develop, and afterwards sustain, the latter. The vegetative organs, however, are not independent of the animal; for without muscles and nerves we could not procure, masticate, and digest food. The closer the connection and dependence between these two sets of organs, the higher the rank. ${ }^{18}$

All the apparatus and phenomena of life may be in. cluded under the heads of

Nutrition,

Motion,

Sensation.

Reproduction. 
These fomr are possessed by all animals, but in a variety of ways. No two species have exactly the same mechanism and method of life. We must learn to distinguish between what is vital and what is only accessory. That only is essential to life which is common to all forms of life. Our brains, stomachs, livers, hands, and feet are lnxuries. They are necessary to make us human, but not living, beings. Half of our body is taken up with a complicated system of digestion; but the Amœba has neither month nor stomach. We have an elaborate apparatus of motion; the adult Oyster cannot stir an inch.

Nutrition, Motion, and Sensation indicate three steps up the grade of life. Thus, the first is the prominent function in the Coral, which simply "vegetates," the powers of moving and feeling being very feeble. In the higher Insect, as the Bee, there is great activity with simple organs of nutrition. In the still higher Mammal, as Man, there is less power of locomotion, though the most perfect nutritive system; but both functions are subordinate to sensation, which is the crowning development.

In studying the comparative anatomy and physiology of the animal kingdom, our plan will be to trace the various organs and functions, from their simplest expression upward to the highest complexity. Thus Nutrition will begin with absorption, which is the simplest method of taking food; going higher, we find digestion, but in no particular spot in the body; next, we see it confined to a tube; then to a tube with a sac, or stomach; and, finally, we reach the complex arrangement of the higher animals. 


\section{CHAPTER VI.}

NUTRITION.

Nutrition is the earliest and most constant of vital operations. So prominent is the nutritive apparatus, that an animal has been likened to a moving sac, organized to convert foreign matter into its own likeness, to which the complex organs of animal life are but auxiliaries. Thus, the bones and muscles are levers and cords to carry the body about, while the nervous system directs its motions in quest of food.

The objects of nutrition are growth, repair, and propagation. The first object of life is to grow, for no animal is born finished. Some animals, like plants, grow as long as they live ${ }^{19}$ but the majority soon attain a fixed size. In all animals, however, without exception, food is wanted for another purpose than growth, namely, to repair the waste which is constantly going on. For every exercise of the muscles and nerves involves the death and decay of those tissues, as shown by the excretions. The amount of matter expelled from the body, and the amount of nourishment needed to make good the loss, increase with the activity of the animal. The supply must equal the demand, in order to maintain the life of the individual; and as an organism can make nothing, it must seek it from without. Not only the muscles and nerves are wasted by use, but every organ in the body; so that the whole structure needs constant renewal. An animal begins to die the moment it begins to live. The function of nutrition, therefore, is constructive, while motion and sensation are destructive. 
Another source of demand for food is the production of germs, to propagate the race, and the nourishment of such offspring in the egg and infantile state. This reproduction and development of parts which can maintain an independent existence is a vegetative phenomenon (for plants have it), and is a part of the general process of Nutrition. But it will be more convenient to consider it hereafter (chapters xix., xx.). Still another necessity for aliment among the higher animals is the maintenance of bodily heat. This will be treated under the head of Respiration.

For the present, we will study Nutrition, as manifested in maintaining the life of an adult individual.

In all animals, this process essentially consists in the introduction of food, its conversion into tissue, its oxidation, and the removal of worn-out material.

1. The food must be procured, and swallowed. tion.)

(Inges-

2. The food must be dissolved, and the nutritious parts separated into a fluid. (Digestion.)

3. The nutritive fluid must be carefully taken up, and then distributed all over the body. (Absorption and Circulation.)

4. The tissues must repair their parts wasted by use, by transforming a portion of the blood into living matter like themselves. (Assimilation.)

5. Certain matters must be eliminated from the blood, some to serve a purpose, others to be cast out of the system. (Secretion and Excretion.)

6. In order to produce work and heat, the food must be oxidized, either in the blood or in the tissues, after assimilation. The necessary oxygen is obtained through exposure of the blood to the air in the lungs. (Respiration in part.)

7. The waste products of this oxidation taken up by the blood must be got rid of; some from the lungs (car- 
bon dioxide, water), some from the kidneys (water, urea, mainly), some from the skin (water, salines). (Respiration in part, Excretion.)

The mechanism to accomplish all this in the lowest forms of life is exceedingly simple, a single cavity and surface performing all the functions. But in the majority of animals the apparatus is very complicated: there is a set of organs for the prehension of food; another, for aigestion ; a third, for absorption; a fourth, for distribution; and a fifth, for purification.

\section{CHAPTER VII. \\ THE FOOD OF ANIMALS.}

THE term food includes all substances which contribute to nutrition, whether they simply assist in the process, or are actually appropriated, and become tissue. With the food is usually combined more or less indigestible matter, which is separated in digestion.

Food is derived from the mineral, vegetable, and animal kingdoms. Water and salt, for example, are inorganic. The former is the most abundant, and a very essential article of food. Most of the lower forms of aquatic life seem to live by drinking: their real nourishment, however, is present in the water in the form of fine particles. The Earthworm, some Beetles, and certain savage tribes of Men swallow earth; but this, likewise, is for the organic matter which the earth contains. As no animal is produced immediately from inorganic matter, so no animal can be sustained by it.

Nutritious or tissue-forming food comes from the organic world, and is albuminous, as the lean meat of ani- 
mals and the gluten of wheat; oleaginous, as animal fat and vegetable oil; or saccharine, as starch and sugar. The first is the essential food-stuff; no substance can serve permanently for food-that is, can permanently prevent loss of weight in the body-unless it contains albuminous matter. As stated before, all the living tissues are albuminous, and therefore albuminous food is required to supply their waste. Albumen contains nitrogen, which is necessary to the formation of tissue; fats and sugars are rich in carbon, and therefore serve to maintain the heat of the body, and to repair part of the waste of tissues. Warm-blooded animals feed largely on farinaceous or starchy substances, which in digestion are converted into sugar. But any animal, of the higher orders certainly, whether herbivorous or carnivorous, would starve, if fed on pure albumen, oil, or sugar. Nature insists upon a mixed diet; and so we find in all the staple articles of food, as milk, meat, and bread, at least two of these principles present. As a rule, the nutritive principles in vegetables are less abundant than in animal food, and the indigestible residue is consequently greater. The nutriment in flesh increases as we ascend the animal scale; thus, Oysters are less nourishing than Fish; Fish, less than Fowl; and Fowl, less than the flesh of Quadrupeds.

Many animals, as most Insects and Manmals, live solely on vegetable food, and some species are restricted to particular plants, as the Silk-worm to the white mulberry. But the majority of animals feed on one another; such ale hosts of the microscopic forms, and nearly all the radiated species, marine Mollusks, Crustaceans, Beetles, Flies, Spiders, Fishes, Amphibians, Reptiles, Birds, and clawed Quadrupeds.

A few, as Man himself, are omnivorous, $i . e$, are maintained on a mixture of animal and vegetable food. The use of fire in the preparation of food is peculiar to Man, 
who has been called "the cooking animal." A few of the strictly herbivorous and carnivorous animals have shown a capacity for changing their diet. Thus, the Horse and Cow may be brought to eat fish and flesh; the Sea-birds can be habituated to grain; Cats are fond of alligatorpears; and Dogs take naturally to the plantain. Certain animals, in passing from the young to the mature state, make a remarkable change of food. Thus, the Tadpole feeds upon vegetable matter; but when it becomes a Frog it lives on Insects.

Many tribes, especially of Reptiles and Insects, are able to go without food for months, or even years. Insects in the larval, or caterpillar, state are very voracions; but upon reaching the perfect, or winged, state, they eat little -some species taking no food at all, the mouth being actually closed. The males of some Rotifers and other tribes take no food from the time of leaving the egg until death.

In general, the greater the facility with which an animal obtains its food, the more dependent is it upon a constant supply. Thus, carnivores endure abstinence better than herbivores, and wild animals than domesticated ones.

\section{CHAPTER VIII.}

H O W A I M L S EAT.

1. The Prehension of Food.-(1) Liquids.-The simplest method of taking nourishment, thongh not the method of the simplest animals, is by absorption through the skin. The Tape-worm, for example, living in the intestine of its host, has neither mouth nor stomach, but absorbs the digested food with which its body is bathed (Fig. 216). Many other animals, especially Insects, live upon liquid food, but obtain it by suction through a special orifice or 
tube. Thus, we find a mouth, or sucker, furnished with teeth for lancing the skin of animals, as in the Leech; a bristle-like tube fitted for piercing, as in the Mosquito; a sharp sucker armed with barbs, to fix it securely during the act of sucking, as in the Louse; and a long, flexible proboscis, as in the Butterfly (Fig. 23). Bees have a hairy, channelled tongue (Fig. 22), and Flies have one terminating in a large fleshy knob, with or without little "knives" at the base for cutting the skin (Fig. 24); both lap, rather than suck, their food.

Most animals drink by suction, as the Ox; and a iew by lapping, as the Dog; the Elephant pumps the water up with its trunk, and then pours it into its throat; and Birds (excepting Doves) fill the beak, and then, raising the head, allow the water to run down.

Many aquatic animals, whose food consists of small particles diffused through the water, have an apparatus for creating currents, so as to bring such particles within their reach. This is particularly true of low, fixed forms, which are unable to go in search of their food. Thus, the Sponge draws nourishment from the water, which is made to circulate through the system of canals traversing its body by the vibration of minute hairs, or cilia, lining parts of the canals (Fig. 189). The microscopic Infusoria have cilia surrounding the mouth, with which they draw or drive into the body little currents containing nutritious particles. Bivalve mollusks, as the Oyster and Clam, are likewise dependent upon this method of procuring food, the gills being covered with cilia. So the singular fish, Amphioxus (the only example among Vertebrates), employs ciliary action to obtain the minute organisms on which it feeds (Fig. 282). The Greenland Whale has a mode of ingestion somewhat unique, gulping great volumes of water into its mouth, and then straining ont, through its whalebone sieve, the small animals which the water may contain (Fig. 342). 
(2) Solids. -When the food is in solid masses, whether floating in water or not, the animal is usually provided with prehensile appendages for taking hold of it. The jellylike Amœba has neither mouth nor stomach, but extemporizes them, seizing its food by means of its soft body. The food then passes through the denser, outer portion of the body into the softer interior, where it is digested.

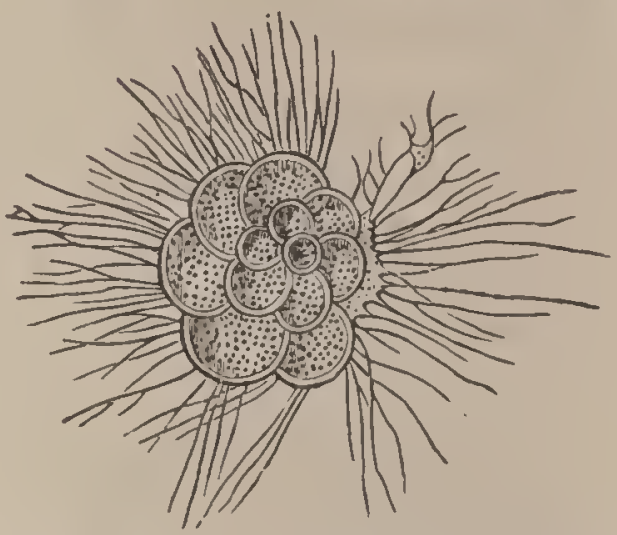

Fra.15.-A Rhizopod (Rotalia Veneta), with pseudopodia extended, $\times 30$. The waste particles are passed out in a similar way. In the Foraminifers, thread-like projections (pseudopodia) of the body are thrown out which adhere to the prey. The soft jelly-like substance of the body then flows toward and collects about the food, and digests it (Fig. 15).

A higher type is seen in Polyps and Jelly-fishes, which have hollow tentacles around the entrance to the stomach (Figs. 38 and 193). These tentacles are contractile, and some, moreover, are covered with an immense number of minute sacs, in each of which a highly elastic filament is coiled up spirally (lasso-cells, nettle-cells). When the tentacles are touched by a passing animal, they seize it, and at the same moment throw ont their myriad filaments, like so many lassos, which penetrate the skin of the victim, and probably also emit a fluid, which paralyzes it; the mouth, meanwhile, expands to an extraordinary size, and the creature is soon engulfed in the digestive bag.

In the next stage, we find no tentacles, but the food is brought to the month by the flexible lobes of the body, commonly called "arms," which are covered with hundreds of minute suckers; and if the prey, as an Oyster, is too large to be swallowed, the stomach protrudes, like a proboscis, and sucks it out of its shell. This is seen in the Star-fish (Fig. 126). 
A great advance is shown by the Sea-urchin, whose mouth is provided with five sharp teeth, set in as many jaws, and capable of being projected so as to grasp, as well as to masticate, its food (Figs. 214, 28).

In Mollusks having a single shell, as the Snail, the chief organ of prehension is a strap-like tongue, covered with ininute recurved teeth, or spines, with which the animal

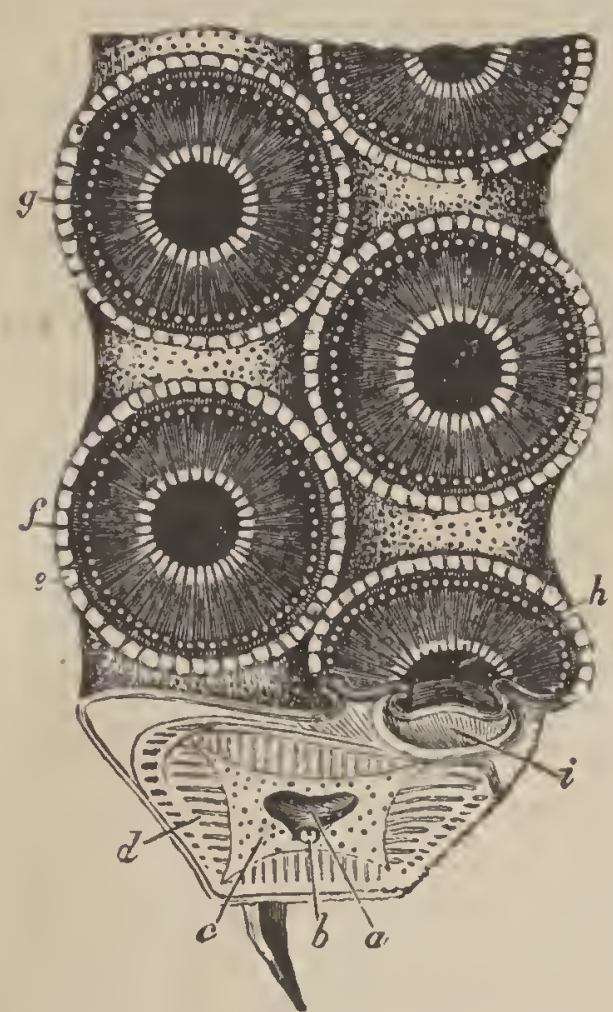

Fig. 16.-Suckers on the Tentacles of a Cuttle-fish : $a$, hollow axis of the arm, containing nerve and artery; $c$, cellular tissue ; $d$, radiating tibres; $h$, raised margin of the disk around the aperture $f, g$, which contains a retractile membrane, or "pistou," $i$.

rasps its food, while the upper lip is armed with a sharp, horny plate (Fig. 29). In many marine species, as the Whelk, the tongue is situated at the end of a retractile proboscis, or muscular tube. In the Cuttle-fish, we see the sudden development of an elaborate system of prehensile organs. Besides a spinous tongue, it has a pair of hard mandibles, resembling the beak of a Parrot, and working vertically; and around the mouth are eight or ten powerful arms furnished with numerous cup-like suckers. So perfect is the adhesion of these suckers, that it is easier to tear away a limb than to detach it from its hold.

The Earth-worm swallows earth containing particles of decaying vegetable matter, which it secures with its lips, the upper one being prolonged. Other worms (as Nereis) are so constructed that the gullet, which is frequently armed with teeth and forceps, can be turned inside out, to form a proboscis for seizing prey.

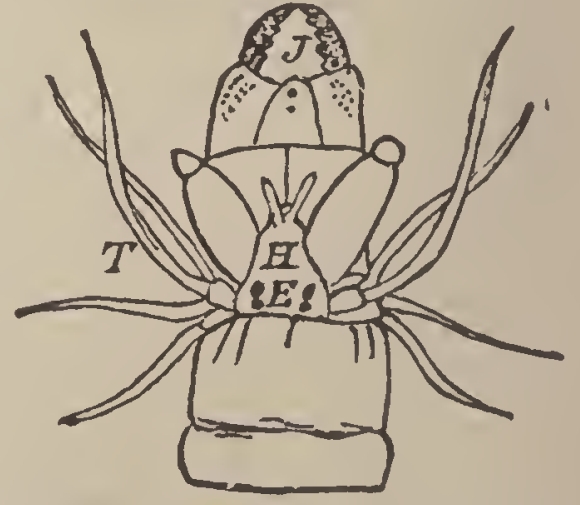

Fig. 17.-Nereis - hend, with extended proboscis: $J$, jaws: $T$, tentacles; $H$, head; $E$, eyes. 
The Arthropoda exhibit a great variety of means for procuring nourishment, in addition to the suctorial contrivances already mentioned, the innumerable modificar tions of the mouth corresponding to the diversity of food. Millepedes, Caterpillars, and Grubs have a pair of horny jaws moving horizontally. The Centipede has a second pair of jaws, which are really modified feet, terminated by curved fangs containing a poison-duct. The Horseshoe Crab uses its feet for prehension, and the thighs, or basal joints of its legs, to masticate the food and force it into the stomach. The first six pairs of legs in the Lobster and Crab are likewise appropriated to conveying food into the mouth, the sixth being enormously developed, and furnished with powerful pincers. Scorpions have a similar pair of claws for prehension, and also a pair of small forceps for holding the food in contact with the mouth. In their relatives, Fro. 18. - One of the Fangs, or Perforated the Spiders, the claws are

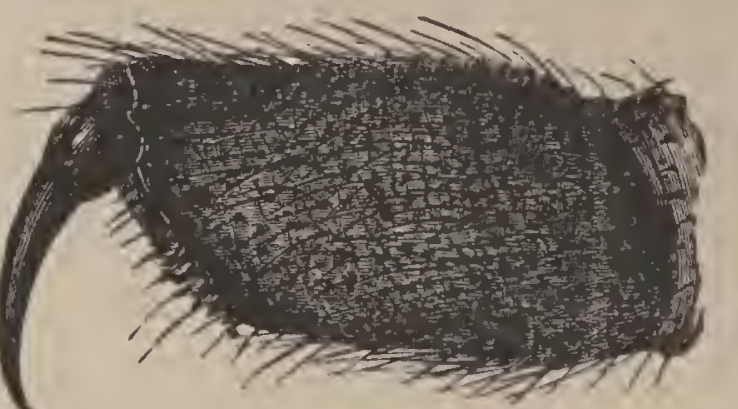
wanting, and the forceps end in a fang, or hook, which is perforated to convey venom. ${ }^{20}$

The biting Insects, as Beetles and Locusts, have two pairs of horny jaws, which open sidewise, one above and the other below the oral orifice. The upper pair are called mandibles; the lower, maxillæ. The former are armed with sharp teeth, or with cutting edges, and sometimes are fitted, like the molar's of quadrupeds, to grind the food. The maxillæ are usually composed of several parts, some of which serve to hold the food, or to help in dividing it, while others (palpi) are both sensory and prehensile. There is generally present a third pair of jaws-the labium-which are united in the middle line, and serve as a lower lip. They also bear palpi. The Mantis seizes its prey with its long 
fore-legs, crushes it between its thighs, which are armed with spines, and then delivers it up to the jaws for mastication. All Arthropods move their jaws horizontally.

The back-boned animals generally apprehend food by means of their jaws, of which there are two, moving vertically. The toothless Sturgeon draws in its prey by powerful suction. The Hag-fish has a single tooth, which it plunges into the sides of its victim, and, thus securing a firm hold, bores its way into the flesh by means of its sawlike tongue. But Fishes are usually well provided with teeth, which, being sharp and curving inward, are strictly prehensile. The fins and tongue are not prehensile. A mouth with horny jaws, as in the Turtles, or bristling with teeth, as in the Crocodile, is the only means possessed by nearly all Amphibians and Reptiles for securing food. The Toad, Frog, and Chameleon capture insects by darting out the tongue, which is tipped with glutinous saliva. The constricting serpents (Boas) crush their prey in their coils before swallowing; and the venomous Snakes have poison-fangs. No reptile has prehensile lips. All Birds use their toothless beaks in procuring food, but birds of prey also seize with their talons, and Woodpeckers, Hummers, and Parrots with their tongues. The beak varies greatly in shape, being a hook in the Eagle, a probe in the Woodpecker, and a shovel in the Duck.

Among the Quadrupeds we find a few special contrivances, as the trunk of the Elephant, and the long tongues of the Giraffe and Ant-eater; but, as a rule, the teeth are the chief organs of prehension, always aided more or less by the lips. Ruminants, like the Ox, having hoofs on their feet, and no upper front teeth, employ the lips and tongue. Such as can stand erect on the hind-legs, as the Squirrel, Bear, and Kangaroo, use the front limbs for holding the food and bringing it to the mouth, but never one limb alone. The clawed animals, like the Cat and Lion, 
make use of their feet in securing prey, all four limbs being furnished with curved retractile claws; but the food is conveyed into the month by the movement of the head and jaws. Man and the Monkeys employ the hand in bringing food to the mouth, and the lips and tongue in taking it into the cavity. The thumb on the human hand is longer and more perfect than that of the Apes and Monkeys; but the foot of the latter. is also prehensile.

2. The Mouths of Animals. -In the Parasites, as the Tapeworm, which absorb nourishment throngh the skin, and Insects, as the May-fly and Bot-fly, which do all their eating in the larval state,

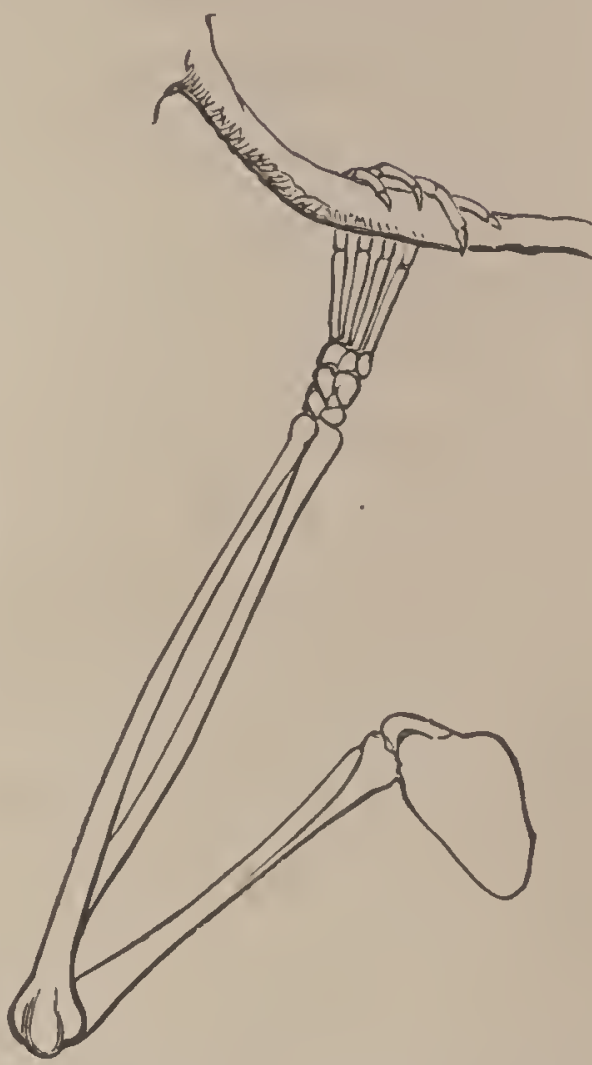

Fig. 19.-Arm of the Thumbless Monkey (Ateles). the mouth is either wanting or rudimentary. The Amœba, also, has no mouth proper, its food passing through the firmer outside part of the bit of protoplasm which constitutes its body. Month and anus are thus extemporized, the opening closing as soon as the food or excrement has passed through.

In the Infusoria the mouth is a round or oval opening leading through the cuticle and outer layer of protoplasm to the interior of the single cell which makes their body. It is usually bordered with cilia, and situated on the side or at one end of the animal.

An elliptical or quadrangular orifice, surrounded with tentacles, and leading directly to the stomach, is the ordinary mouth of the Polyps and Jelly-fishes. In those which are fixed, as the Actinia, Coral, and Hydra, the mouth looks upward or downward, according to the position in which the animal is attached (Figs. 38, 191, 207): 
in those which freely move about, as the Jelly-fish, it is generally underneath, the position of the animal being reversed (Fig. 193). In some, the margin, or lip, is protruded like a proboscis; and in all it is exceedingly dilatable.

The mouth of the Star-fish and Sea-urchin is a simple round aperture, followed by a very short throat. In the Star-fish, it is enclosed by a ring of hard tubercles and a membrane. In the Sea-urchin, it is surrounded by a muscular membrane and minute tentacles, and is armed with five sharp teeth, set in as many jaws, resembling little conical wedges (Fig. 28).

Among the headless Mollusks, the oral apparatus is very simple, being inferior to that of some of the radiated animals. In the Oyster and Bivalves generally, the mouth is an unarmed slit-a mere inlet to the œsophagus, situated in a kind of hood formed by the union of the gills at their origin, and between two pairs of delicate lips. These lips make a furrow, along which pass the particles of food drawn in by the cilia, borne by cells which cover the surface of the lips.

Of the higher Mollusks, the little Clio (one of the Pteropods) has a triangular mouth, with two jaws armed with sharp horny teeth, and a tongue covered with spiny hooklets all directed backward. Some Univalves have a simple fleshy tube. Others, as the Whelk, have an extensible proboscis, which unfolds itself, like the finger of a glove, and carries within it a rasp-like tongue, which can bore

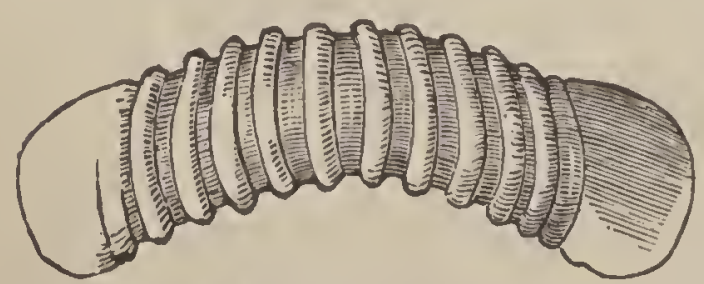

FIG. 20.—Jaw of the Common Suail (Helix albolabris). into the hardest shells. Such as feed on vegetable matter, as the Snail, have no proboscis, but on the roof of the mouth a curved horny plate fitted to cut leaves, etc., which are pressed against it by the lips, and on the floor of the mouth a small tongue covered with delicate teeth. As fast as the tongue is worn off by use, it grows out from the rost. 
The mouth of the Cuttle-fish is the most elevated type below that of the Fishes. A broad circular lip nearly conceals a pair of strong horny mandibles, not unlike the beak of a parrot, but reversed, the upper mandible being the shorter of the two, and the jaws, which are cartilaginous, are inbedded in a mass of muscles, and move vertically. Between them is a fleshy tongue covered with teeth.

The parasitic Worms, living within or on the outside of other animals, generally have a sucker at one end or underneath, serving simply for attachment, and another which is perforated. The latter is a true suctorial mouth, being the sole inlet of food. It is often surrounded with hooklets or teeth, which serve both to scarify the victim and secure a firm hold. In the Leech, the mouth is a triangular opening with thick lips, the upper one prolonged, and with three jaws. In many Worms it is a fleshy tube, which can be drawn in or extended, like the eye-stalks of the Snail, and contains a dental apparatus inside (Fig. 17).

Millepedes and Centipedes have two lateral jaws and a four-lobed lip.

In Lobsters and Crabs the mouth is situated underneath the head, and consists of a soft upper lip, then a pair of upper jaws provided with a short feeler, below which is a thin bifid lower lip; then follow two pairs of membranous under jaws, which are lobed and hairy; and next, three pairs of foot-jaws (Fig. 250). The Horse-shoe Crab has no special jaws, the thighs answering the purpose. The Barnacle has a prominent mouth, with three pairs of rudimentary jaws.

With few exceptions, the months of Insects in the larval state are fitted only for biting, the two jaws being horny shears. But in the winged, or perfect, state, Insects may be divided into the masticating (as the Beetle) and 

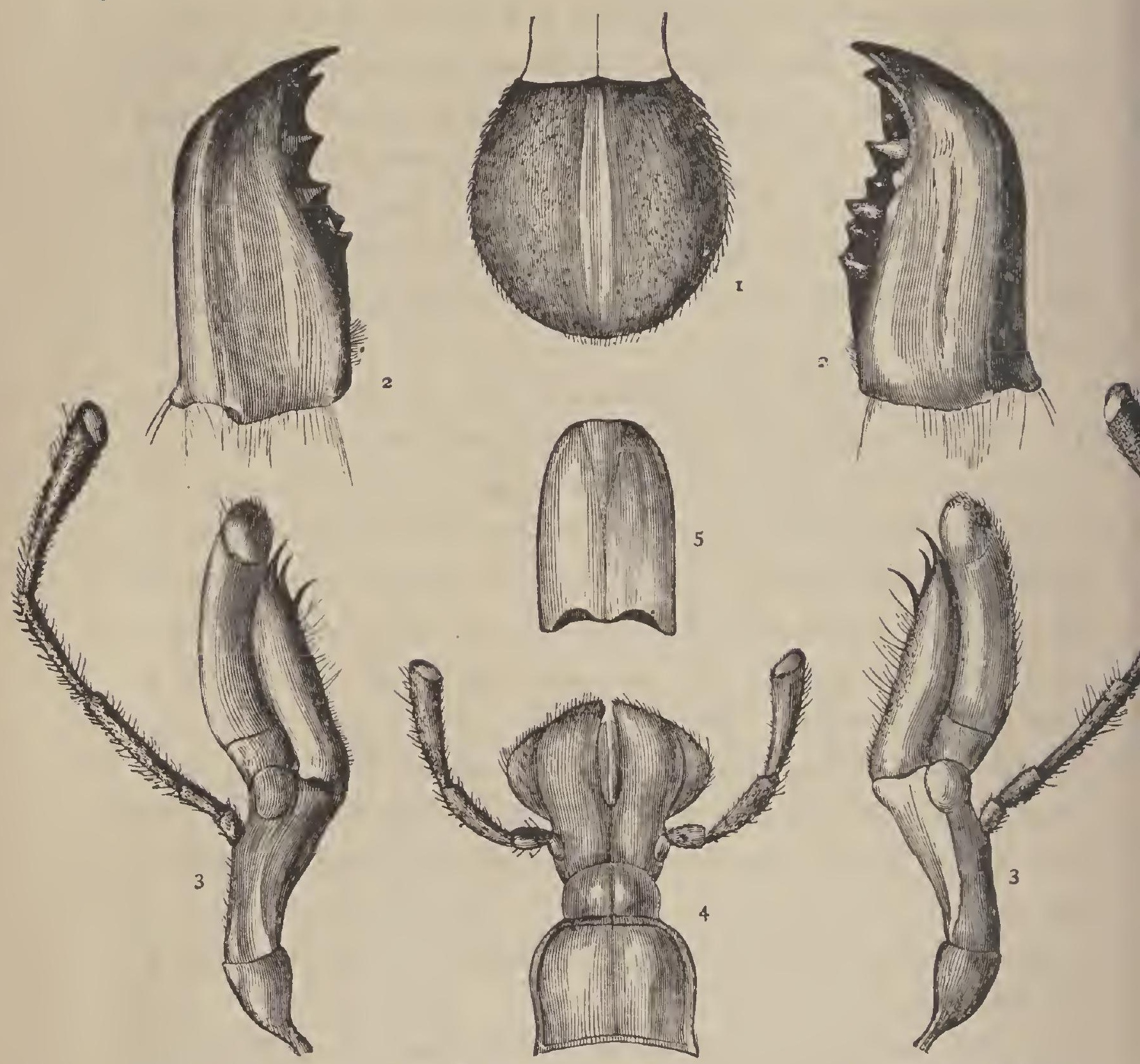

Fia. 21.-Mnuth of a Lncust dissected: 1, labrum, or upper lip; 2, mandibles; 3, jaws; 4, labium, or lower lip; 5 , tongue. The appendages to the maxillæ and lower lip are palpi.

the suctorial (as the Butterfly). In the former group, the oral apparatus consists of two pairs of horny jaws (mandibles and maxillce), which work horizontally between an upper (labrum) and an under (labium) lip. The maxillæ and under lip carry sensitive jointed feelers (palpi). The front edge of the labium is commonly known as the tongue (ligula). ${ }^{21} \quad$ In such a month, the mandibles are the most important parts; but in passing to the suctorial Insects, we find that the mandibles are secondary to the maxillæ and labium, which are the only means of taking food. In 
the Bee tribe, we have a transition between the biting and the sucking Insects-the mandibles "supply the place of trowels, spades, pickaxes, saws, scissors, and knives," while the maxillæ are developed into a sheath to enclose the long, slender, hairy tongue which laps up the sweets of flowers. In the suctorial Butterfly, the lips, mandibles, and palpi are reduced to rudiments, while the maxillæ are the only useful oral organs. These are excessively lengthened into a proboscis, their edges locking by means of minute teeth, so as to form a central canal, through which the liquid food is pumped up into the mouth. Seen un-

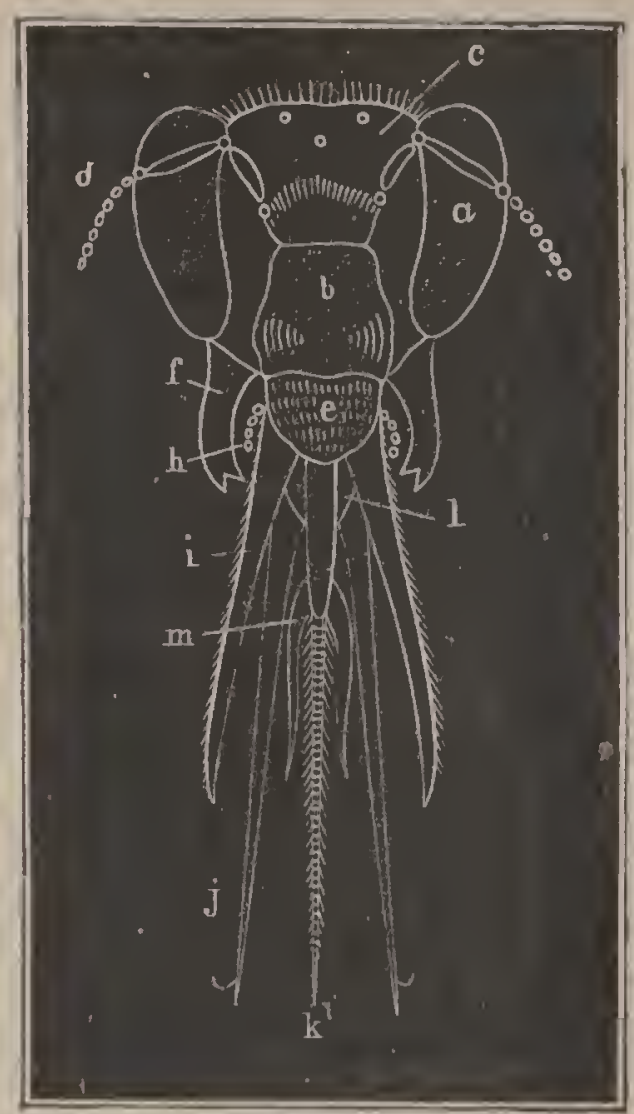

Fla. 22.-Head of a Wild Bee $(A n-$ thophora retusa), front view: $a$, compound eyes; $b$, clypeus; $c$, three simple eyes; $d$, antennæ; $e_{\text {, }}$ labrum : $f$, maudibles; $i$, maxillæ; $h$, maxillary palpi ; $l$, palpifer ; $j$, labial palpi; $m$, paraglossæ; $k$, ligula. der the microscope, the proboscis is made up of innumerable rings interlaced with spiral muscular fibres. The

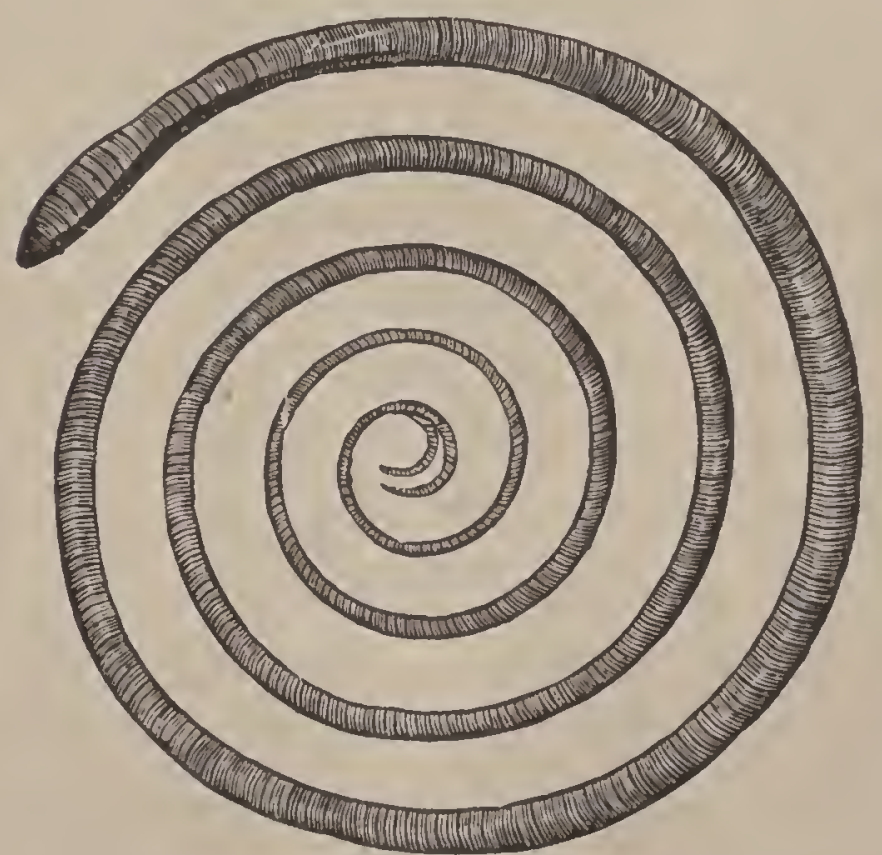

F1G. 23.-Proboscis of a Butterfly, maguified. proboscis of the Fly is a modified lowel lip; that of the Bugs and Mosquitos, fitted both for piercing and suction, is formed by the union of four bristles, which are the mandibles and maxillæ strangely altered, and encased in the labinm when not in use. 


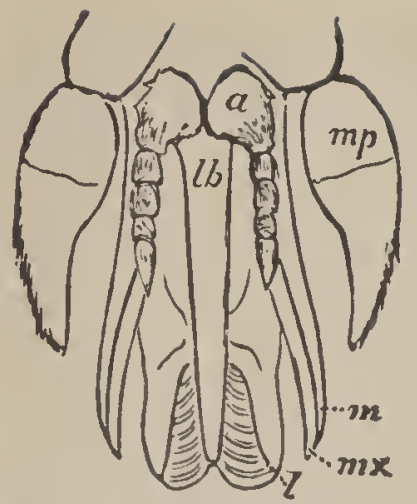

Fig. 24.-Mouth of the Horse-fly ('T'abanus lin$e o l a): a$, antennæ; $m$, mandibles; $m x$, maxillæ; $m p$, maxillary palpi ; lb, labrum; $l$, labium, or tongue.

As most of the Arachnids live by suc. tion, the jaws are seldom used for mastication. In the Scorpion, the apparent representatives of the mandibles of an Insect are transformed into a pair of small forceps, and the palpi, so small in Insects, are developed into formidable claws: both of these organs are prehensile. In Spiders, the so-called mandibles, which move more or less vertically, end in a fang; and the club-like palpi, often resembling legs, have nothing to do with ingestion or locomotion. Both Scorpions and Spiders have a soft upper lip, and a groove within the mouth, which serves as a canal while sucking their prey. The tongue is external, and situated between a pair of diminutive maxillæ.

In the Ascidians the first part of the alimentary canal is enormously enlarged and modified to serve as a gillsac. At the bottom of this sac, and far removed from its external opening, lies the entrance to the digestive tract proper. Into it the particles of food entering with the water are conveyed (Fig. 279).

The mouth of Verte-

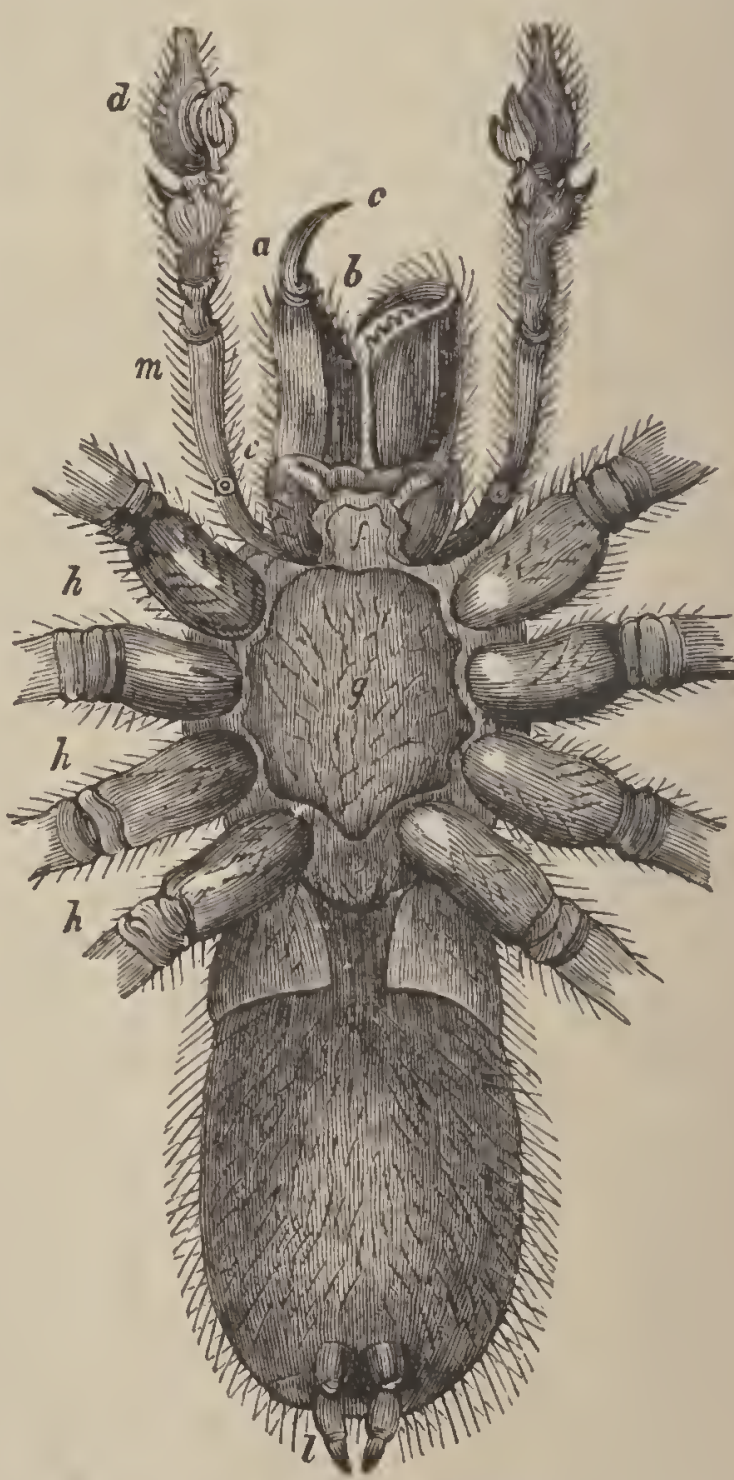

Fra. 25.-Under Surface of Male Spider: $a$, $c$, poisoll-fang; $b$, teeth on interior margin of mandible, $e ; f$, labium: $g$, thorax ; $h$, limbs; $i$, abdomen; $l$, spinuerets; $m$, maxillary palpus; $d$, dilated terminal joint. 
brates is a cavity with a fixed roof (the hard palate) and a movable floor (the tongue and lower jaw), having a transverse opening in front, ${ }^{22}$ and a narrow outlet behind, leading to the gullet. Save in Birds and some others, the cavity is closed in front with lips, and the margins of the jaws are set with teeth.

In Fishes the mouth is the common entry to both the digestive and respiratory organs; it is, therefore, large, and complicated by a mechanism for regulating the transit of the food to the stomach and the aërated water to the gills. The slits leading to the gills are provided with rows of processes which, like a sieve, prevent the entrance of food, and with valves to keep the water, after it has entered the gills, from returning to the mouth. So that the mouths of Fishes may be said to be armed at both ends with teeth-bearing jaws. A few Fishes, as the Sturgeon, are toothless; but, as a class, they have an extraordinary dental apparatus-not only the upper and lower jaws, but even the palate, tongue, and throat being sometimes studded with teeth. Every part of the mouth is evidently designed for prehension and mastication. Lips are usually present; but the tongue is often absent, or very small, and as often aids respiration as ingestion.

Amphibians and Reptiles have a wide mouth; even the insect-feeding Toads and the Serpents can stretch theirs enormously. True fleshy lips are wanting; hence the savage aspect of the grinning Crocodile. With some exceptions, as Toads and Turtles, the jaws are armed with teeth. Turtles are provided with horny beaks. The tongue is rarely absent, but is generally too thick and short to be of much use. In the Toad and Frog it is singularly extensile: rooted in front and free behind, it is shot from the mouth with such rapidity that the insect is seized and swallowed more quickly than the eye can follow. The Chameleon's tongue is also extensile. Snakes 


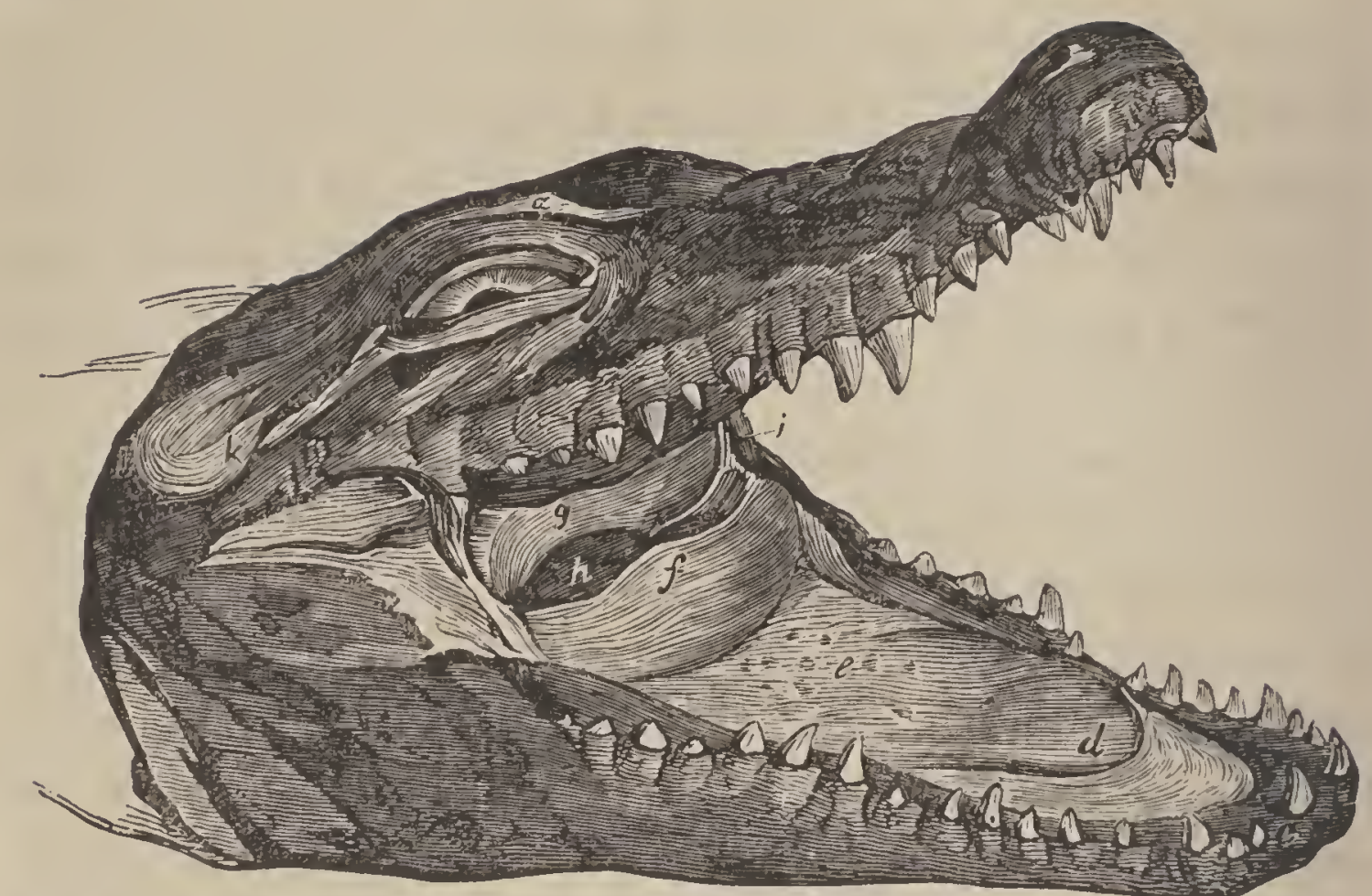

Frg. 26.-Mouth of the Crocodile: $d$, tongue; $e$, glands; $f$, inferior, and $g$, superior, valve, separating the cavity of the mouth from the throat, $h$.

have a slender forked tongue, consisting of a pair of muscular cylinders, which is solely an instrument of touch.

Birds are without lips or teeth, the jaws being covered with horn forming a beak. This varies greatly in shape, being extremely wide in the Whippoorwill, remarkably long in the Pelican, stout in the Eagle, and slender in the Hummer. It is hardest in those that tear or bruise their food, and softest in water-birds. The tongue is also corered with a horny sheath, and generally spinous, its chief function being to secure the food when in the mouth. It is proportionally largest and most fleshy in the Parrots.

The main characteristics of the mammalian mouth are flesh lips and mobile cheeks. ${ }^{23}$ In the duck-billed Monotremes lips are wanting, and in the Porpoises they are barely represented. But in the herbivorous quadrupeds they, with the tongue, are the chief organs of prehension; in the carnivorous tribes they are thin and retractile; while in the Whale the upper lip falls down like a curtain, overlapping the lower jaw several feet. As a rule, the mouth is terminal; but in the Elephant, Tapir, Hog, 
and Shrew, the upper lip blends with the nose to form a proboscis, or snout. The mouth is comparatively small in the Elephant and in gnawing animals like the Squirrel, wide in the Carnivores, short in the Sloth, and long in the Ant-eater. Teeth are usually present, but vary in form and number with the habits of the animal. The Ant-eater is toothless, and the Greenland Whale has a sieve made of horny plates. The tongue conforms in size and shape with the lower jaw, and is a muscular, sensitive organ, which serves many purposes, assisting in the prehension, mastication, and swallowing of food, besides being an organ of taste, touch, and speech. Its surface is covered with minute prominences, called papilloe, which are arranged in lines with mathematical precision. In the Cats, these are developed into recurved spines, which the animal uses in cleaning bones and combing its fur. Similar papillæ occur on the roof and sides of the mouth of the $\mathrm{Ox}$ and other Ruminants. In some animals, as the Hamster

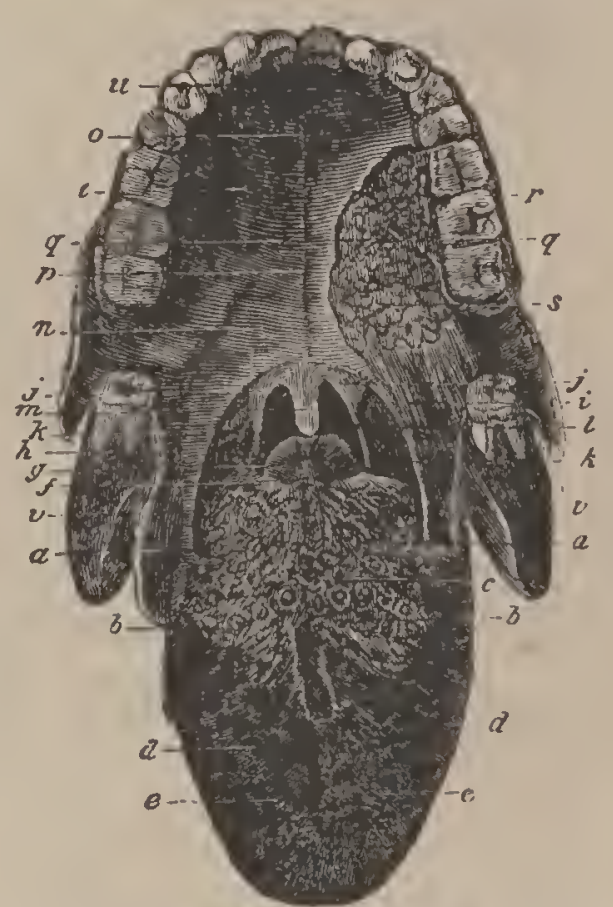

Frg. 27.-Human Tongne and adjacent parts: $a$, lingual papillæ: $b$, papillæ forming $V$-shaped lines; $d$, fungiform papillx; $e$, filiform papillæ; $g$, epiglottis; $m$, uvula, or conical process, hanging from the soft palate, $n$; $o$, hard palate; $r$, palatiue glands, the mucous membrane being removed; $v$, section of the lower jaw. and Gopher, the cheeks are developed into pouches in which the food may be carried. These may be lined with hair. The tongue is remarkably long in the Ant-eater and Giraffe, and almost immovable in the Gnawers, Elephants, and Whales.

3. The Teeth of Animals. - Nearly all animals have certain hard parts within the mouth for the prehension or trituration of solid food. If these are wanting, the legs are often armed with spines, or pincers, to serve the same 
purpose, as in the Horse-shoe Crab; or the stomach is lined with "gastric teeth," as in some marine Snails; or the deficiency is supplied by a muscular gizzard, as in Birds, Ant-eaters, and some Insects. Even the Lobster and Crab, in addition to their complicated oral organs, lave the stomach furnished with a powerful set of teeth.

The Sea-urchin is the first of animals, and almost the only one below Worms and Mollusks, which exhibits

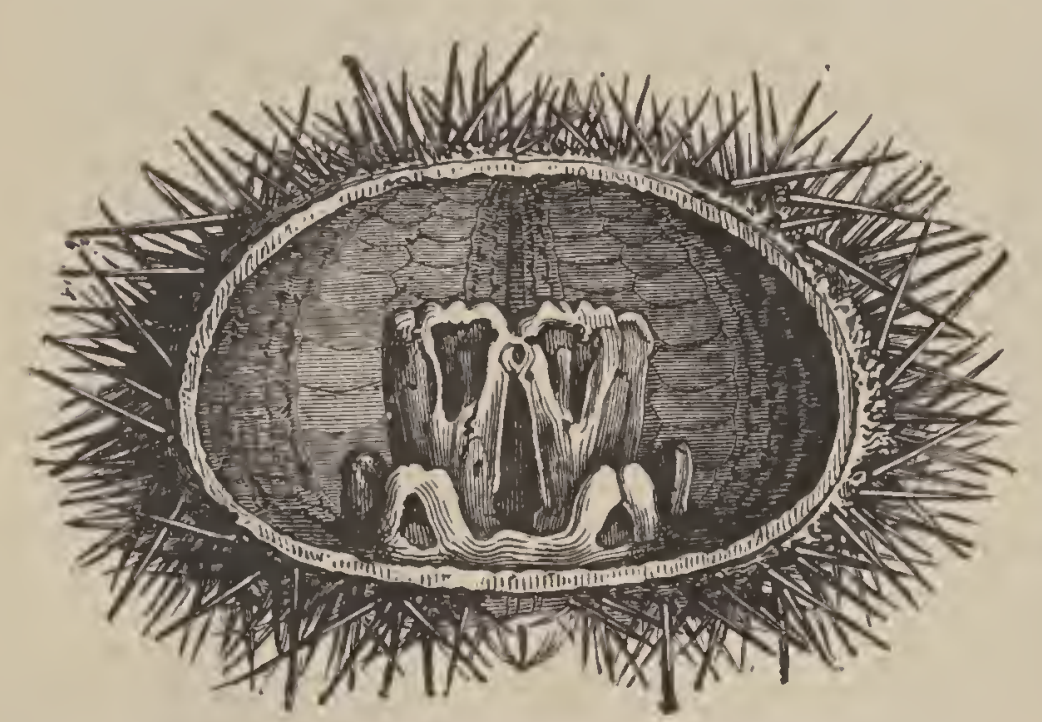

Fid. 28. -Sea-urchiu bisected, showing masticating apparatus. anything like a dental apparatus. Five calcareous teeth, having a wedge - shaped apex, each set in a triangular pyramid, or "jaw," are moved upon each other by a complex arrangement of levers and muscles. Instead of moving up and down, as in Vertebrates, or from right to left, as in Arthropods, they converge towards the centre, and the food passes between ten grinding surfaces.

The Rotifers (a group of minute Worms) have a curious pair of horny jaws. That which answers to the lower jaw is fixed, and called the "anvil." The upper jaw consists of two pieces called "hammers," which are sharply notched, and beat upon the "anvil" between them (Fig. 219).

The horny-toothed mandibles of Insects, already mentioned, are prehensile, and also serve to divide the food.

The three little white ridges in the month of the Leech are the convex edges of horny semicircles, each bordered by a row of nearly a hundred hard, sharp teeth. When the mouth, or sucker, is applied to the skin, a sawing 
movement is given to the horny ridges, so that the "bite" of the Leech is real. ly a saw-cut.

The dentition of the univalve Mollusks, or the Snails, is generally lingual, i. e., it consists of

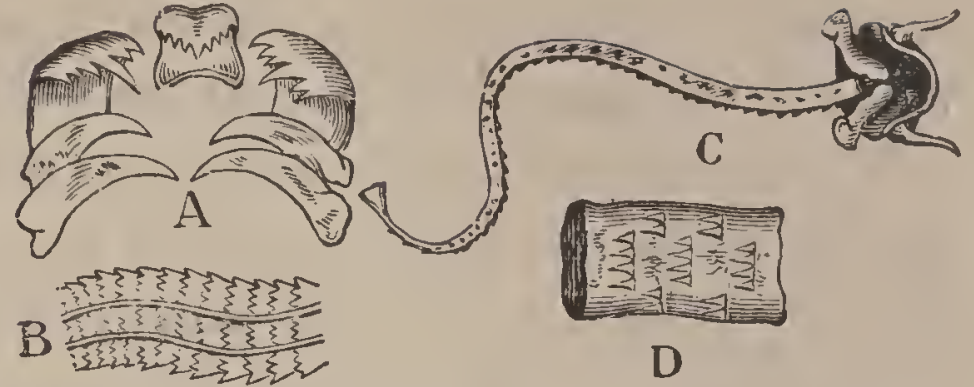

Fig. 2\%.-Teeth and Masticatory Apparatus of Gaster()pods : A, portion of odontophore, or "tongne," of Velutina, enlarged; B, portion of odontophore of Whelk (Buccinum undatum), magnified - the entire toingue has 100 rows of teeth; $C$, head and odoutophore of Limpet (Patella vulgata) ; D, portion of same, greatly magnified, to show the transverse rows of siliceous teeth. microscopic teeth, usually siliceous and amber-colored,

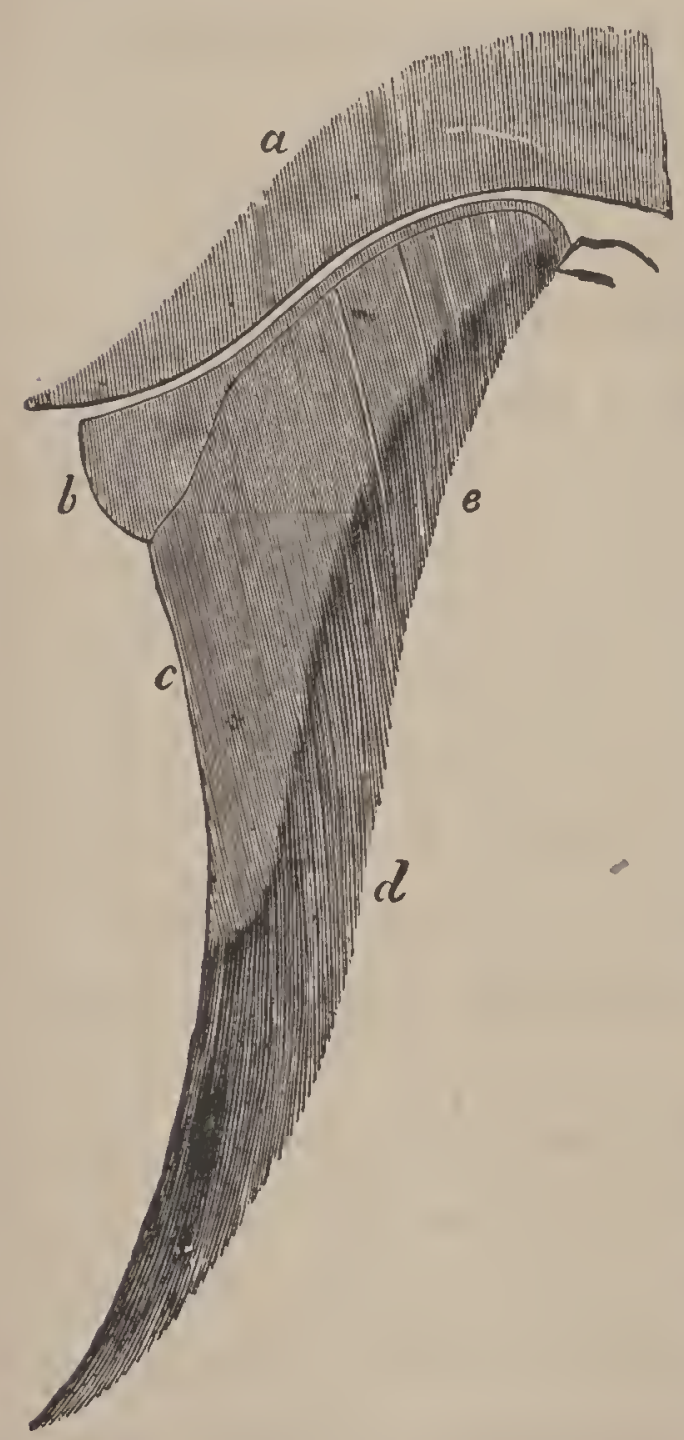

EIG. 30.-.Section of one half of the Upper Jaw of a Whale (Balcenoptera), showing baleen-plates: $a$, superior maxillary bone; $b$, ligamentous gum plates, triangular in shape, and attaching the horny body of the ba- plates, triangular in shape, and leen-plate, $c$; $d$, fringe of bristles; $e$, fringed on the inner side, hang
smaller plates. 
in rows from the gums of the upper jaw. In some Whales there are about 300 plates on each side. ${ }^{25}$

True teeth, consisting mainly of a hard, calcareous substance called dentine, are found only in back-boned animals. They are distinct from the skeleton, and differ

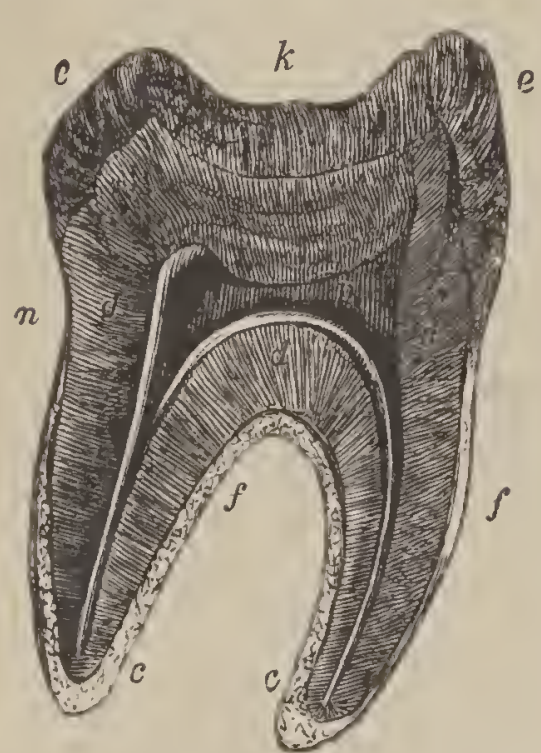

Frg. 31.-Section of Human Molar, enlarged: $k$, crown; $n$, neck: $f$, fang; $e$, enamel; $d$, dentine; $c$, cement; $p$, pulpcavity.

particles of blood. One modification of it is ivory, seen in the tusks of Elephants. Enamel is the hardest tissue of the body, and contains not more than two per cent. of animal matter. It consists of six-sided fibres set side by side, at right angles to the surfaces of the dentine. Cement closely resembles bone, and is present only in the teeth of the higher animals.

Teeth are usually confined to the jaws; but the number, size, form, structure, position, and mode of attachment vary with the food and habits of the animal. As a rule, animals developing large numbers of teeth in the back part of the month are inferior to those having fewer teeth, and those nearer the lips. The teeth of Mammals only have fangs.

'The teeth of Fishes present the greatest variety. In number, they range from zero to hundreds. The Hag. 
fish (Myxine) has a single tooth on the roof of the mouth, and two serrated plates on the tongue; while the mouth of the Pike is crowded with teeth. In some we find teeth short and blunt, in the shape of cubes, or prisms, arranged like mosaic work. Such pavement-teeth (seen in some Rays) are fitted for grinding sea-weed and crushing shell-fish. But the cone is the most common form: sometimes so slender and close as to resemble plush, as in the Perch; or of large size, and flattened like a spear-head with serrated edges, as in the Shark; but more often like the canines of Mammals, curved

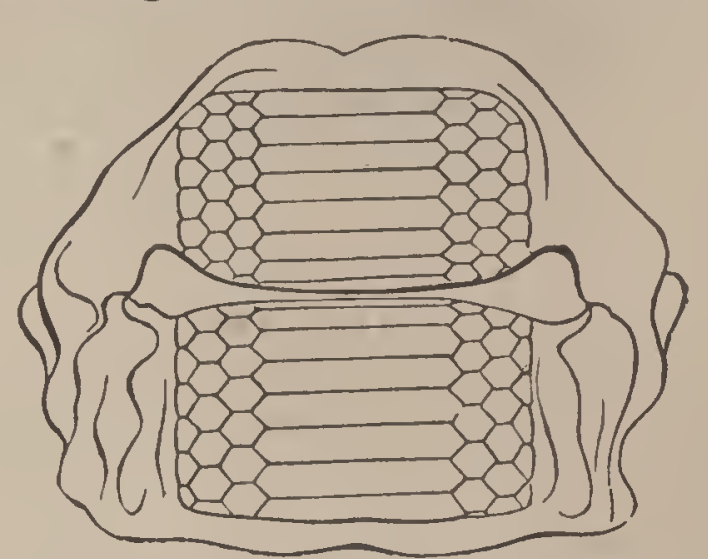

FIG. 32.-Jaws and Pavement-teeth of a Ray (Myliobates).

inward to fit them for grappling. In the Shark, the teeth are confined to the fore-part of the month; in the Carp, they are all situated on the bones of the throat; in the Parrot-fish, they occupy both back and front; but in most Fishes the teeth are developed also on the roof, or palate, and, in fact, on nearly every bone in the month. They seldom appear (as in the Salmon) on the upper maxillary. As to mode of attachment, the teeth are generally anchylosed (fastened by bony matter) to the bones which support them, or simply bound by ligaments, as in the Shark. In a few Fishes, the teeth consist of flexible cartilage; but almost invariably they are composed of some kind of dentine, enamel and cement being absent.

Of Amphibians and Reptiles, Toads, Turtles, and Tortoises are toothless; Frogs have teeth in the upper jaw only; Snakes have a more complete set, but Saurians possess the most perfect dentition. The number is not fixed even in the same species: in the Alligator it varies from 72 to 88 . The teeth are limited to the jawbones in the higher forms (Saurians); but in others, as the Serpents, 
they are plantea also in the roof of the month. With few exceptions, they are conical and curved (Fig. 33). In the Serpents they are longest and sharpest; and the venomous species have two or more fangs in the upper jaw.

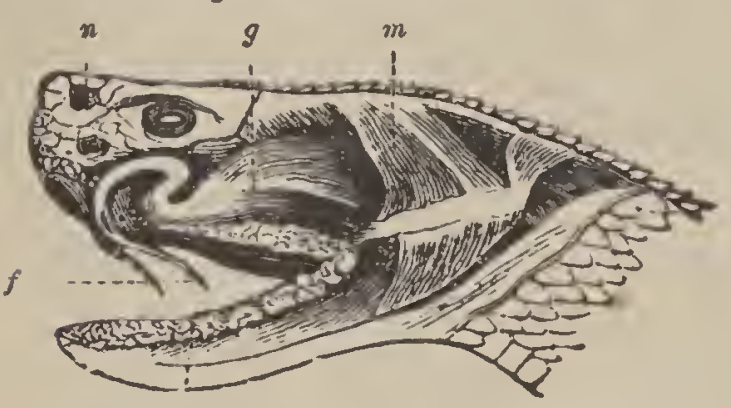

These fangs contain a canal, through which the poison is forced by muscles which compress the gland. The bones to which they are attached are movable, and the

Fra. 33.- Poison Apparatus of the Rattlesnake: $g$, glaud, with duct, leading to the fang, $f ; m$, elevator muscles of the jaw, which, in contracting, compress the gland ; $s$, salivary glands on the edge of the jaws; $n$, uostril.

fangs ordinarily lie flat upon the gums, but are brought into a vertical position in the act of striking. As a rule, the teeth of Reptiles are simply soldered to the bone which supports them, or lodged in a groove; but those of Crocodiles are set in sockets. Reptilian teeth are made of dentine and a thin layer of cement, to which is added in most Saurians a coat of enamel on the crown.

In the majority of Mammals, the teeth are limited in number and definite in their forms. The number ranges from 1 in the Narwhal (but the longest tooth in the kingdom) to 220 in the Dolphin. The average is 32, occurring in Ruminants, Apes, and Man; but 44 (as in the Hog and Mole) is called the typical or normal number, and this number is exceeded only in the lower groups. When very numerons, the teeth are of the Reptilian type, small, pointed, and of nearly equal size, as in the Porpoise. In the higher Mammals, the teeth are comparatively few, and differ so much in size, shape, and use, that they can be classed into incisors, canines, premolars, and molars. Such a dental series exhibits a double purpose, prehension and mastication. The chisel-shaped front teeth are fitted for cutting the food, and hence called incisors. These vary in number: the Lion has six in each jaw; the Squir. 
rel has two in each jaw, but remarkably developed; the $\mathrm{Ox}$ has none in the upper jaw, and the Elephant none in the lower ; while the Sloth has none at all. ${ }^{20}$ The canines, so called because so prominent in the Dog, are conical, and, except in Man, longer than the other teeth. They are designed for seizing and tearing; and they are the most formidable weapons of the wild carnivores. There

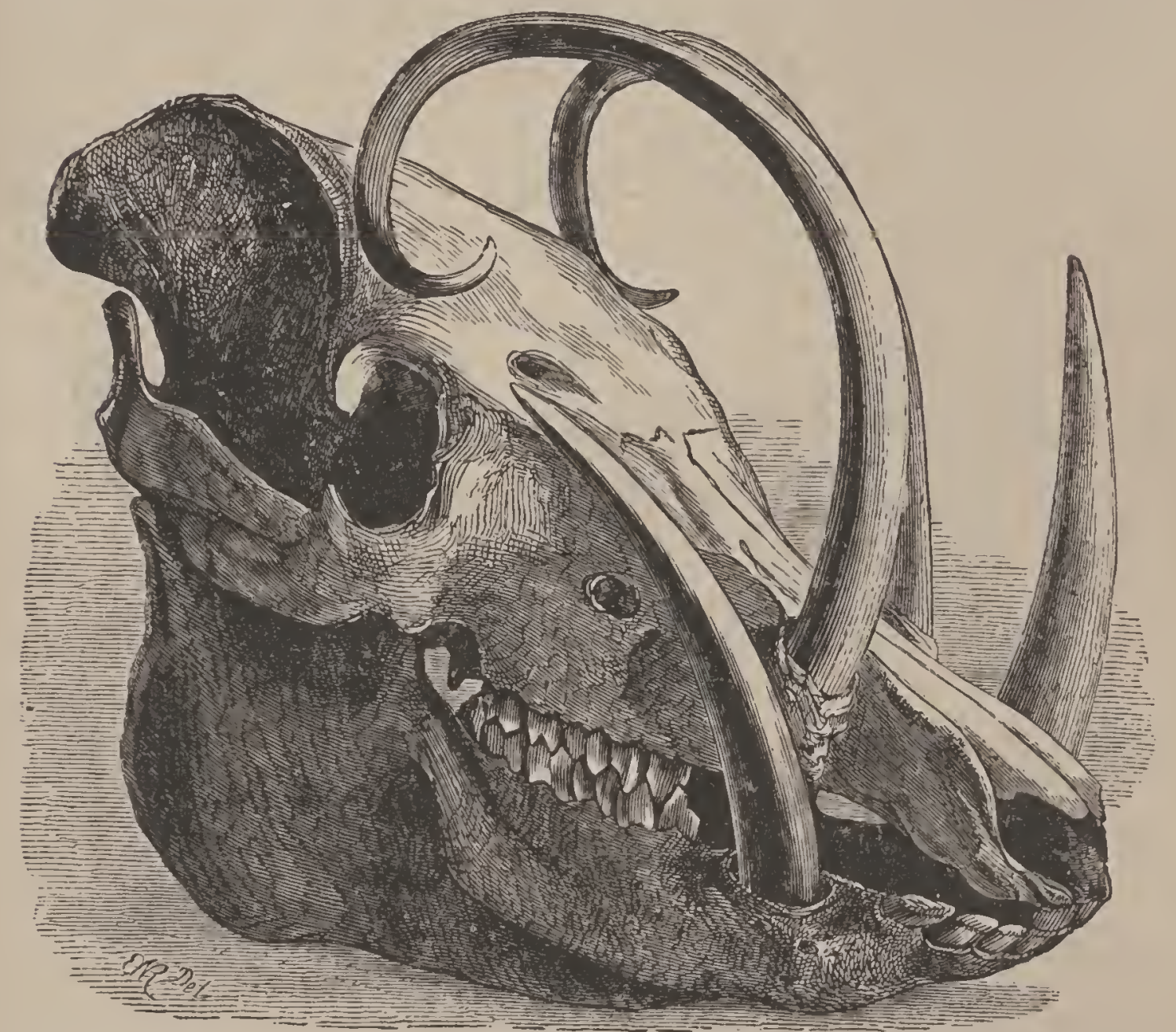

Fig. 34.-Skull of the Babirusa, or Malayan Hog, showing growth and curvature of the canines.

are never more than four. They are wanting in all Rodents, and in nearly all herbivorous quadrupeds. The molars, or grinders, vary greatly in shape, but closely correspond with the structure and habits of the animal, so that a single tooth is sufficient to indicate the mode of life and to identify the species. ${ }^{27}$ In the Ruminants, Rodents, Horses, and Elephants, the summits of the molars are flat, like mill-stones, with transverse or curving ridges 
of enamel. In the Cats and Dogs, they are narrow and sharp, passing by each other like the blades of scissors, and therefore cutting, rather than grinding, the food. The more purely carnivorous the species, and the more it feeds upon living prey, the fewer the molars. In animals living on mixed diet, as the Hog and Man, the crowns have blunt tubercles. Premolars, or bicuspids, are those which were preceded by milk-teeth; the true, or back, molars had no predecessors.

The dentition of Mammals is expressed by a formula, which is a combination of initial letters and figures in

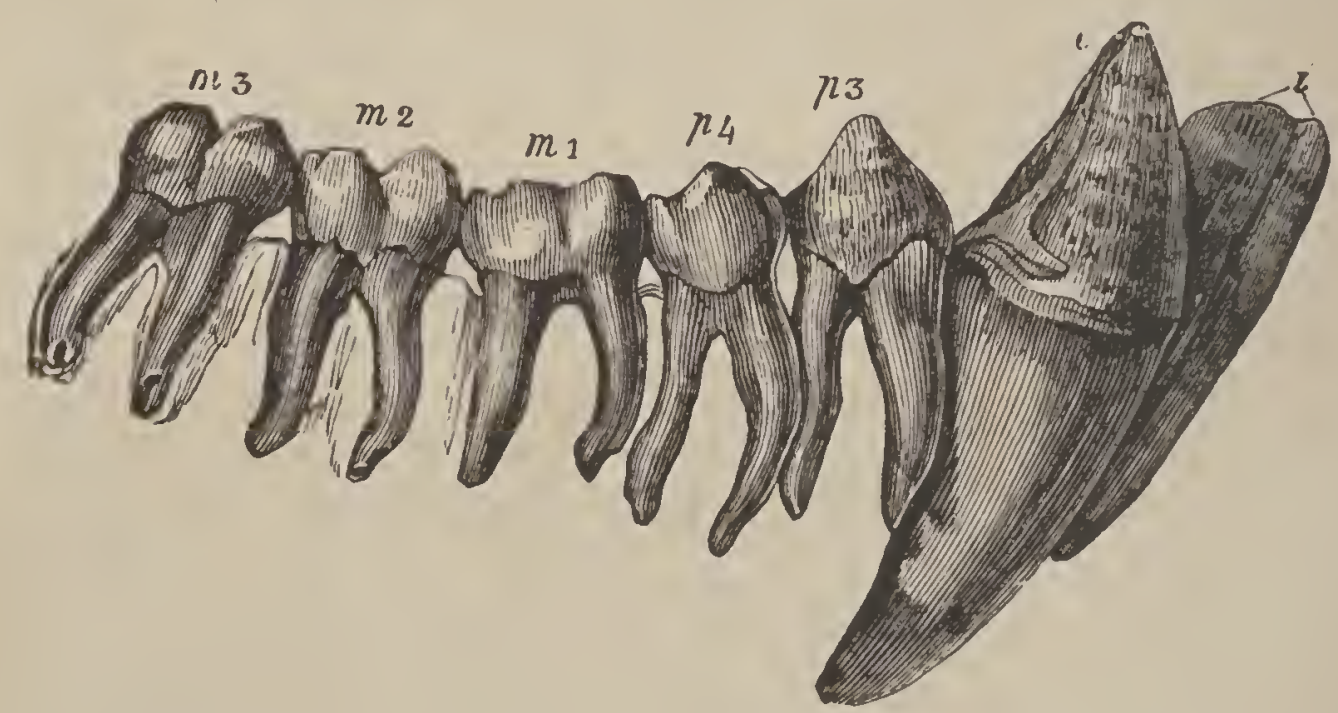

FiG. 35. - Teeth of the right lower jaw of adult male Chimpanzee (Troglodytes niger), natural size. The molar series does not form a curve, as in Man.

fractional form, to show the number and kind of teeth on each side of both jaws. Thus, the formula for Man is : $i, \frac{2-2}{2-2} ; c, \frac{1-1}{1-1} ; p, \frac{2-2}{2-2} ; m, \frac{3-3}{3-3}=32$.

The teeth of Mammals are always restricted to the margins of the jaws, and form a single row in each. But they rarely form an unbroken series. ${ }^{28}$ The teeth inplanted in the premaxillary bone, and in the corresponding part of the lower jaw, whatever their number, are incisors. The first tooth behind the premaxillary, if sharp and projecting, is a canine.

Each tooth has its particular bony socket. ${ }^{20}$ The molars 
nay be still further strengthened by having two or more diverging fangs, or roots, a feature peculiar to this class. The incisors and canines have but one fang; and those that are perpetually growing, as the incisors of Rodents and Elephants, have none at all. The teeth of flesh-eating Mammals usually consist of hard dentine, surrounded on the root with cement and capped with enamel. In the herbivorous tribes, they are very complex, the enamel and cement being inflected into the dentine, forming folds, as in the molar of the $\mathrm{Ox}$, or plates, as in the compound tooth of the Elephant. This arrangement of these tissues, which differ in hardness, secures a surface with prominent

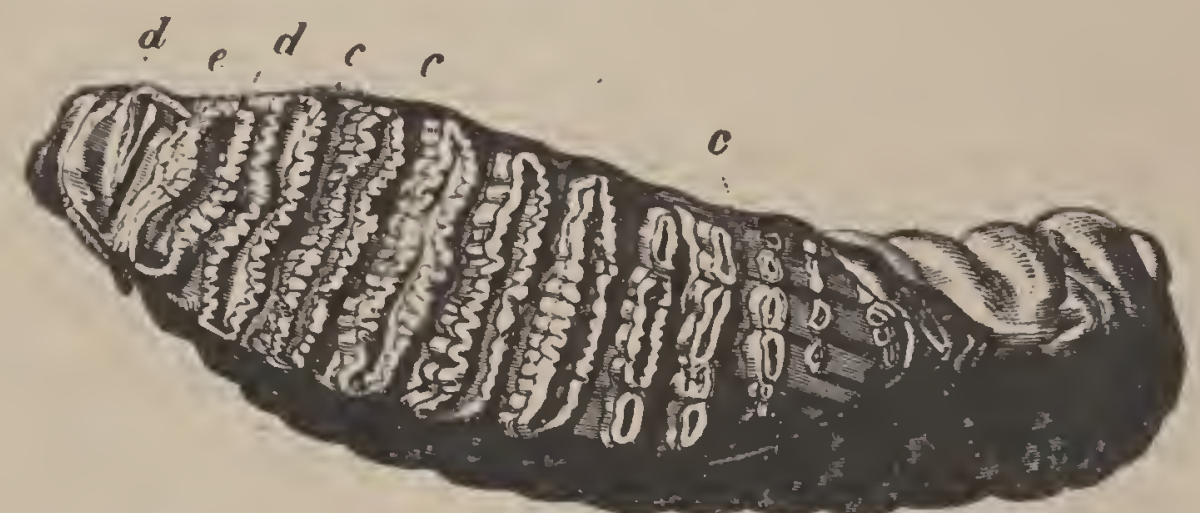

Fia. 36. - Upper Molar Tooth of Indian Elephant (Elephas Indicus), showing transverse arrangement of dentine, $d$, with festooned border of euamel plates, $e ; c$, cement; one-third natural size.

ridges, well adapted for grinding. The cutting teeth of the Rodents consist of dentine, with a plate of enamel on the anterior surface, and the unequal wear preserves a chisel-like edge. Enamel is sometimes wanting, as in the molars of the Sloth and the tusks of the Elephant.

In Fishes and Reptiles, there is an almost unlimited succession of teeth; but Mammalian teeth are cast and renewed but once in life.

Vertebrates use their teeth for the prehension of food. as weapons of offence or defence, as aids in locomotion, and as instruments for uprooting or cutting down trees. But in the higher class they are principally adapted for dividing or grinding the food. ${ }^{30}$ While in nearly all other 
Vertebrates the food is bolted entire, Mammals masticate it before swallowing. Mastication is more essential in the digestion of vegetable than of animal food; and hence we find the dental apparatus most efficient in the herbivorous quadrupeds. The food is most perfectly reduced by the Rodents.

'Teeth, as we shall see, are appendages of the skin, not of the skeleton, and, like other superficial organs, are especially liable to be modified in accordance with the habits of the creature. They are, therefore, of great zoological value; for, such is the harmony between them and their uses, the naturalist can predict the food and general structure of an animal from a sight of the teeth alone. For the same reason, they form important guides in the classification of animals; while their durability renders them available to the paleontologist in the determination of the nature and affinities of extinct species, of which they are often the sole remains. Even the structure is so peculiar that a fragment will sometimes suffice.

4. Deglutition, or How Animals Swallow.-In the lowest forms of life, the mouth is but an aperture opening immediately into the body-substance, and the food is drawn in by ciliary currents. But in the majority of animals, a muscular tube, called the gullet, or œsophagus, intervenes between the mouth and stomach, the circular fibres of which contract, in a wave-like manner, from above downward, propelling the morsel into the stomach. ${ }^{31}$ In the higher Mollusks, Arthropods, and Vertebrates, deglutition is generally assisted by the tongue, which presses the food backward, and by a glairy juice, called saliva, which facilitates its passage through the gullet. ${ }^{32}$ Vertebrates have a cavity behind the mouth, called the throat, or pharynx, which may be considered as a funnel to the osophagus. ${ }^{33}$ In air-breathers, it has openings leading to the windpipe, nose, and ears. In Man, as in Mammals generally, the 
process of deglutition is in this wise : the food, masticated by the teeth and lubricated by the saliva, is forced by the tongue and cheeks into the pharynx; the soft palate keeping it out of the nasal aperture, and the valve-like epiglottis falling down to form a bridge over the opening to the windpipe. The moment the pharynx receives the food, it is firmly grasped, and, the muscular fibres contracting above it and left lax below it, it is rapidly thrust into the œsophagus. Here, a similar movement (the peristaltic) strips the food into the stomach. ${ }^{34}$ The rapidity of these contractions transmitted along the œsophagus may be observed in the neck of a Horse while drinking.

Deglutition in the Serpents is painfully slow, and somewhat peculiar. For how is an animal, without limbs or molars, to swallow its prey, which is often much larger than its own body? The Boa-constrictor, e.g., seizes the

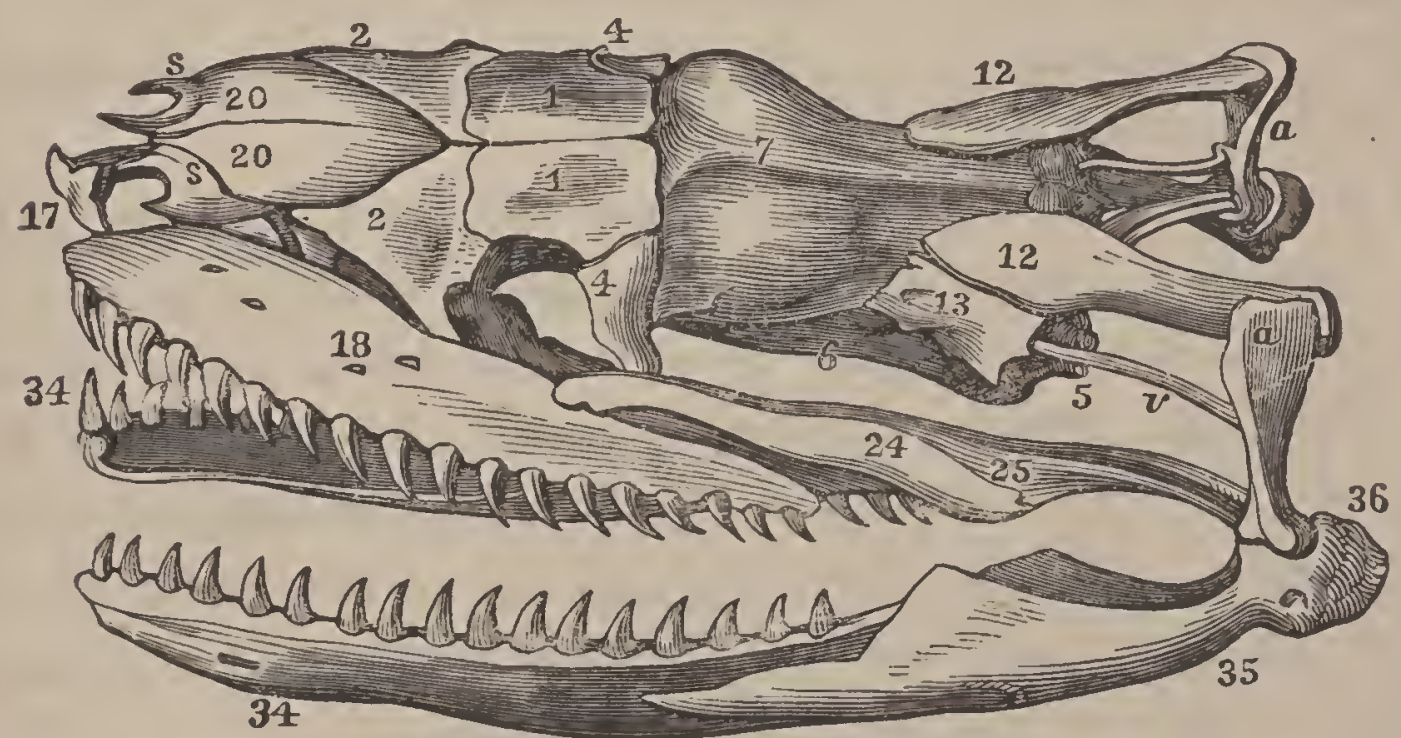

Fra. 37.-Skull of Boa-constrictor: 1, frontal ; 2, prefrontal ; 4, postfrontal ; 5, basioccipital; 6 , sphenoid; 7 , parietal; 12 , squamosal; 13 , proötic; 17 , premaxillary; 18 , maxillary : 20, nasal ; 24 , transverse ; 25 , internal pterygoid; 34 , dentary, lower jaw ; 35 , angular ; 36 , articular; $\alpha$, quadrate; $s$, prenasal; $v$, petrosal.

lead of its victim with its sharp recurving teeth, and crushes the body with its overlapping coils. Then, slowly uncoiling, and covering the carcass with a slimy mucus, it thrusts the head into its mouth by main force, the mouth stretching marvellously, the skull being loosely put 
together. One jaw is then unfixed, and the teeth withdrawn by being pushed forward, when they are again fastened farther back upon the animal. The other jaw is then protruded and refastened; and thus, by successive movements, the prey is slowly and spirally drawn into the wide gullet.

\section{CHAPTER IX. \\ THE ALIMENTARY CANAL.}

The Alimentary Canal is the great route by which nutritive matter reaches the interior of the body. It is the most universal organ in the animal kingdom, and the rest are secondary or subservient to it. In the higher animals, it consists of a mouth, pharynx, gullet, stomach, and intestine.

It is a general law, that food can be introduced into the living system only in a fluid state. While plants send forth their roots to seek nourishment from without, animals, which may be likened to plants turned outside in, have their roots (called absorbents) directed inward along the walls of a central tube or cavity. This cavity is for the reception and preparation of the food, so that animals may be said to carry their soil about with them. The necessity for such a cavity arises not only from the fact that the food, which is usually solid, must be dissolved, so as to make its way through the delicate walls of the cavity into the system, but also from the occurrence of intervals between the periods of eating, and the consequent need of a reservoir. For animals, unlike plants, are thrown upon their own wits to procure food. 
The Protozoa, as the Amœba and Infusoria, can hardly be said to have a digestive canal. The animal is here composed of a single cell, in which the food is digested. The jelly-like Anneba passes the food through the firmer outer layer (ectosarc) into the more fluid inner part (endosarc), where it is digested. The Infusoria, which have a cuticle, and so a more definite form, possess a mouth, or opening, into the interior of their cell-body, and at least a definite place where the excrement is passed out. But we cannot call this cell-cavity a digestive tract.

In the higher animals, the alimentary canal is a continuation of the skin, which is reflected inward, as we turn the finger of a glove. ${ }^{35}$ We find every grade of this reflection, from the sac of the Hydra to the long intestinal tube of the Ox. So that food in the stomach is still outside of the true body.

The simplest form of such a digestive tract is seen in the Hydra (Fig. 191). Here the body is a simple bag, whose walls are composed of two layers of cells (ectoder'm and endoderm). A mouth leads into the cavity, and serves as well for the outlet of matter not wanted. The endodermal cells furnish the juices by which the food is digested and absorb the nutritious portions of

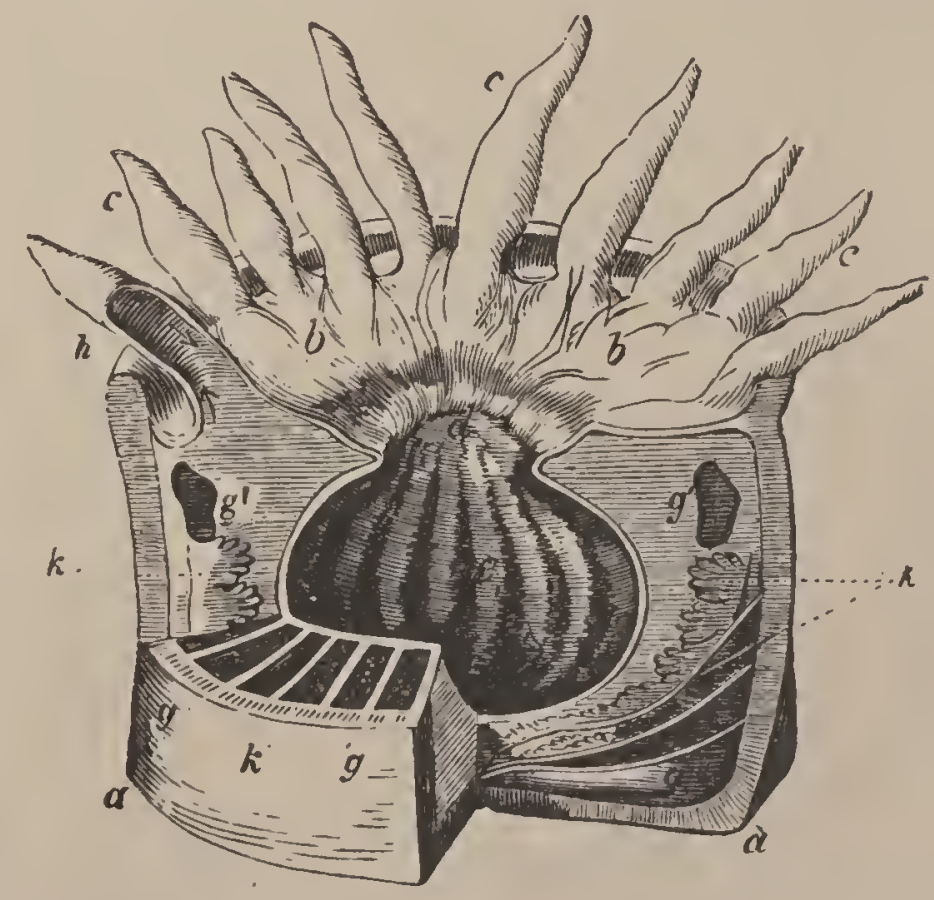

Fra. 3S.-Dissected Actinia: $a$, the thick opaque skin consisting of ectoderm, lined with muscular fibres; $c$, the tubular tentacles communicating with the interspaces, $k$, between the membranous vertical folds; $y, g^{\prime}$, orifices in the walls allowing passige of respiratory water from one compartment to another; $d$, mouth leadiug to gastric cavity, $c$. 
it. The Polyps have also but one external opening; but from this hangs down a short tube, open at both ends, raaching about half-way to the bottom of the bodycavity. Such an arrangement would be represented by a bottle with its neck turned inward. In this suspended sac, which is somewhat constricted at the extremities, digestion takes place; but the product passes freely into all the surrounding chambers, along with the water for respiration (Fig. 38). The Medusæ, or Jelly-fishes, preserve the same type of a digestive apparatus; but the sac is cut off from the general cavity, and numerous canals radiate from it to a circular canal near the margin of the disk (Fig. 196). In the Star-fishes (Fig. 126), we find a great advance. The sac-like stomach sends off two glandular branches to each arm, which doubtless furnish a fluid to aid in digestion (socalled hepatic cœca). There is also an anus present in some forms, but it hardly serves to pass off the waste matter.

Thus far we have seen but one opening to the digestive cavity, rejected portions returning by the same road by which they enter. But a true alimentary canal should have an anal aperture distinct from the oral. The simplest form of such a canal is exhibited by the Sponge, in its system of absorbent pores for the entrance of liquid, and of several main channels for its discharge. The apparatus, however, is not marked off from the general cavity of the body, and digestion is not distinct from circulation. ${ }^{36}$

The Sea-urchin presents us with an important advance -one cavity with two orifices; and the complicated apparatus of higher animals is but the developinent of this type. This alimentary canal begins in a mouth well provided with teeth and muscles, and extends spirally to its outlet, which generally opens on the upper, or opposite, 
surface. Moreover, while in some of the Worms the canal is a simple tube running through the axis of the cylindrical body from oral orifice to anal aperture, the canal of the Sea-urchin shows a distinction of parts, foreshadowing the pharynx, gullet, stomach, andintestines. Both mouth and vent have muscles for constriction and expansion; and, as the vent is on the summit of the shell, and the latter is covered with spines, the ejected particles are seized by delicate forks (pedicellarice), and passed on from one to the other down

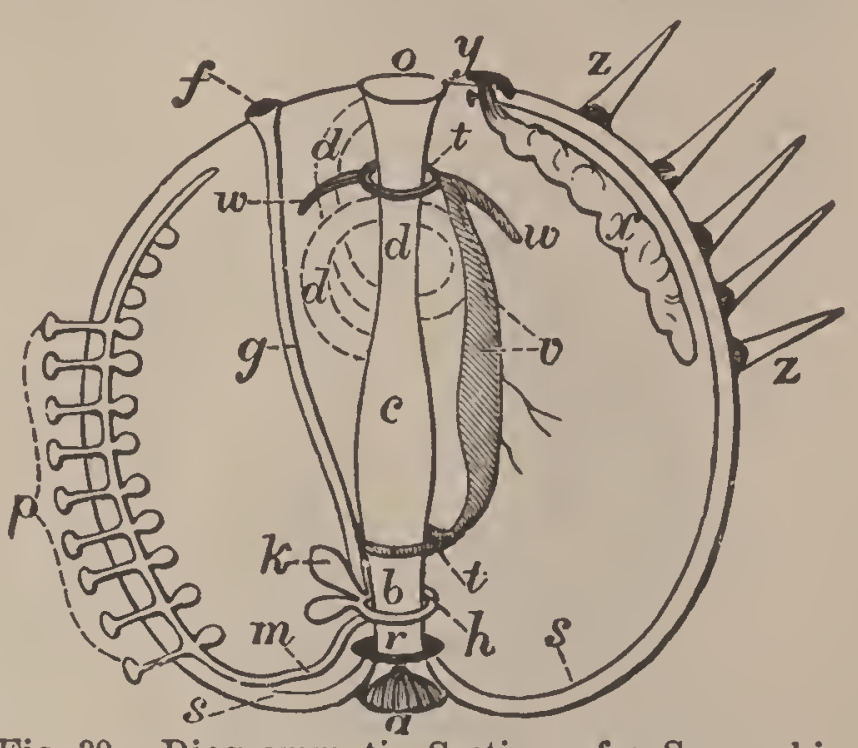

Fig. 39.-Diagrammatic Section of a Sea-urchin (E'chinus): $a$, mouth; $b$, œsophagus; $c$, stomach ; $d$, intestine: $f$, madreporiform tubercle; $g$, stone-canal; $h$, ambulacral ring; $\left.k, \mathrm{P}_{0}\right)$ lian vesicles, which are probably reservoirs of fluid; $m$, ambulacral tube; $o$, anus; $p$, ambulacra, with their contractile vesicles: $r$, nervous ring around the gullet; $s$, two nervous trunks, the right terminating, at anal pole, in a small ganglion; $t$, blond-vascular rings counected by $v$, the contractile heart; $w$, two arterial trunks radiatirg from the anal ring: $x$, an ovary openivg at the anal pole in a genital plate, $y ; z$, spines, with their tubercles. the side of the body, till they are dropped off into the water. ${ }^{37}$

The Worms present us with a great range of structure in the digestive tract. It is sometimes almost as simple as that of the Hydra-a mere sac. The Earth-worm has a tube running straight through the body, divided into pharynx, œsophagus, crop, gizzard, and sacculated intestine. The Leech has large sacs on each side of the intes. tine. The Sea-worms have the pharynx armed with teeth, and some have glandular cœeca attached to the intestine. The plan is that of a straight tube extending from mouth to anus. In Myriapods and larvæ of Insects, the same general plan is continued, the canal passing in a straight line from one extremity to the other, but showing a division into gullet, stomach, and intestine. ${ }^{88}$ Crustacea, like the 
Lobster, have a sliort gullet leading to a large cavity, situated in the front of the animal, which is a gizzard, rather

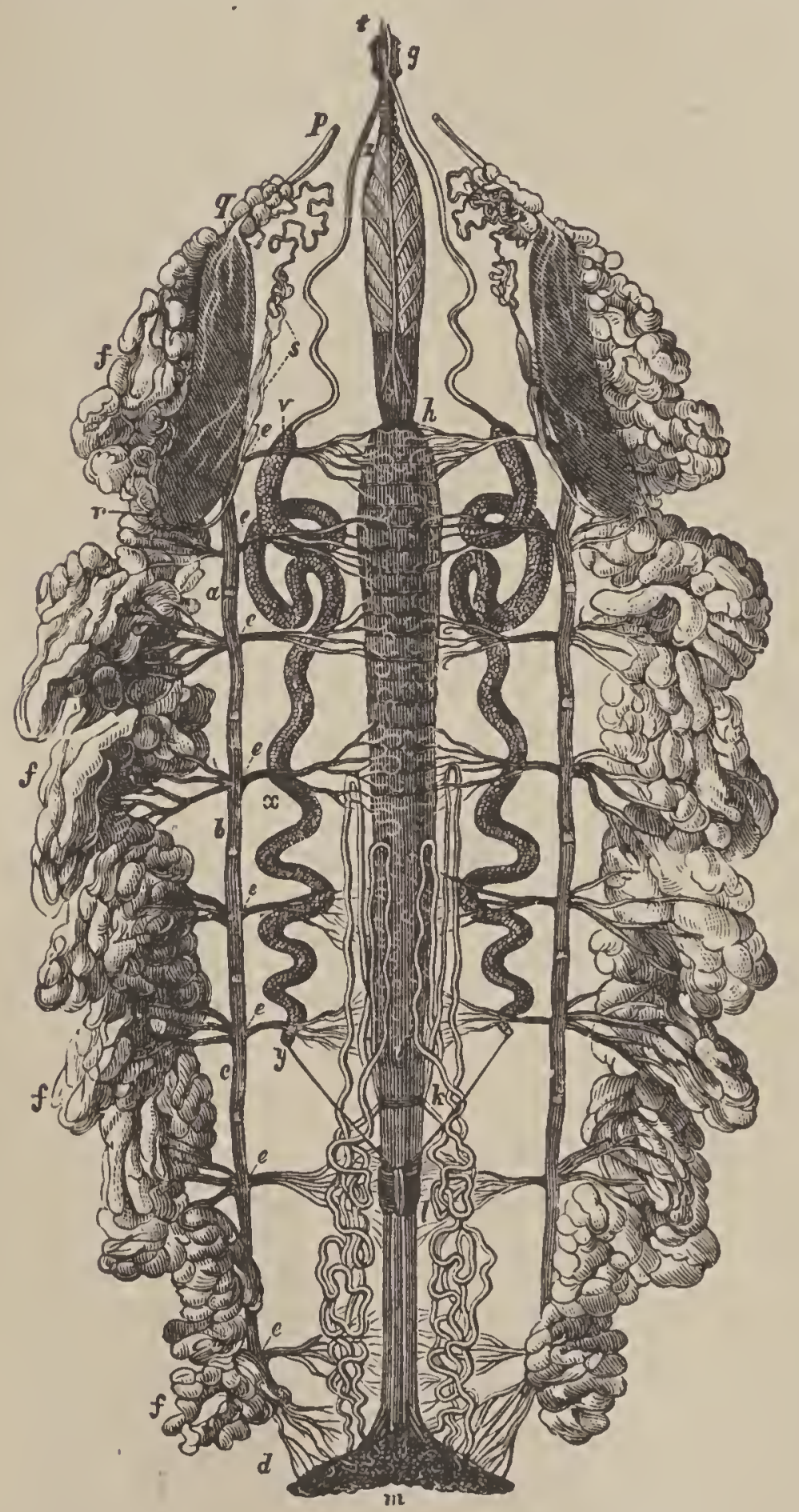

Fra. 40.-Anatomy of a Caterpillar: $g, h$, œsophagus; $h$, $i$, stomach ; $h$, hepatic vessels ; $l, m$, intestine; $q, r$, salivary glands; $p$, salivary duct: $a, b, c$, longitudinal tracheal tronks; $d, e$, air-tubes distributed to the viscera ; $f$, fat-mass; $v, x, y$, silk-secretors; $z$, their excletory ducts, terminating in $t$, the spinneret, or $f u$ sulue. than stomach, as it has thick muscular walls armed with teeth. A wellmarked constriction separates this organ from the intestine. The "liver," really a pancreas, is highly developed; instead of numerous follicles, there is a large bilaterally symmetrical organ, divided into three lobes on each side, pouring its secretion into the upper part of the intestine, which is the true stomach.

Among Insects, there is great variation in the form and length of the canal. The following parts can generally be distinguished: gullet, crop, gizzard, stomach, and large and small intestines, with many glandular appendages. The crop, gizzard, and large intestine are sometimes absent, especially in the carnivorous 
species. In Bees, the crop is called the "honey-bag." The gizzard is found in Insects having mandibles, and is

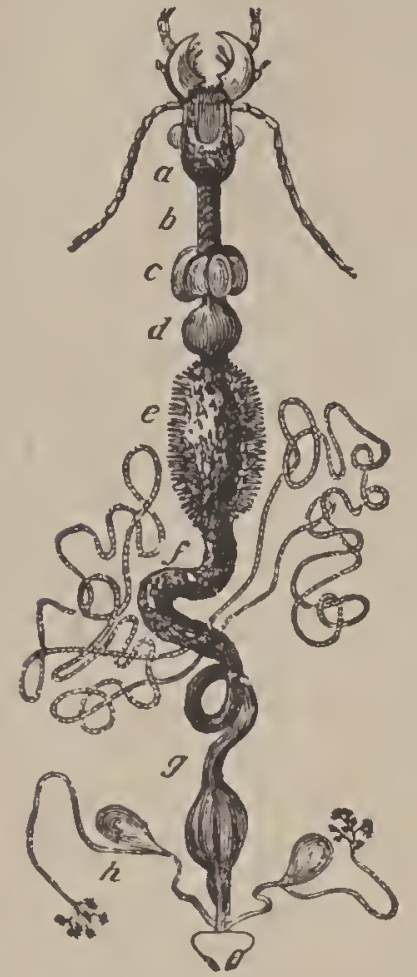

Fig. 41.-Alimentary canal of a Beetle: $a$, pharynx; $b$, gullet, leading to crop, $c$, gizzard, $d$, and stomach, $e ; f$, delicate urinary tubes; $g$, intestine; $h$, other secreting organs.

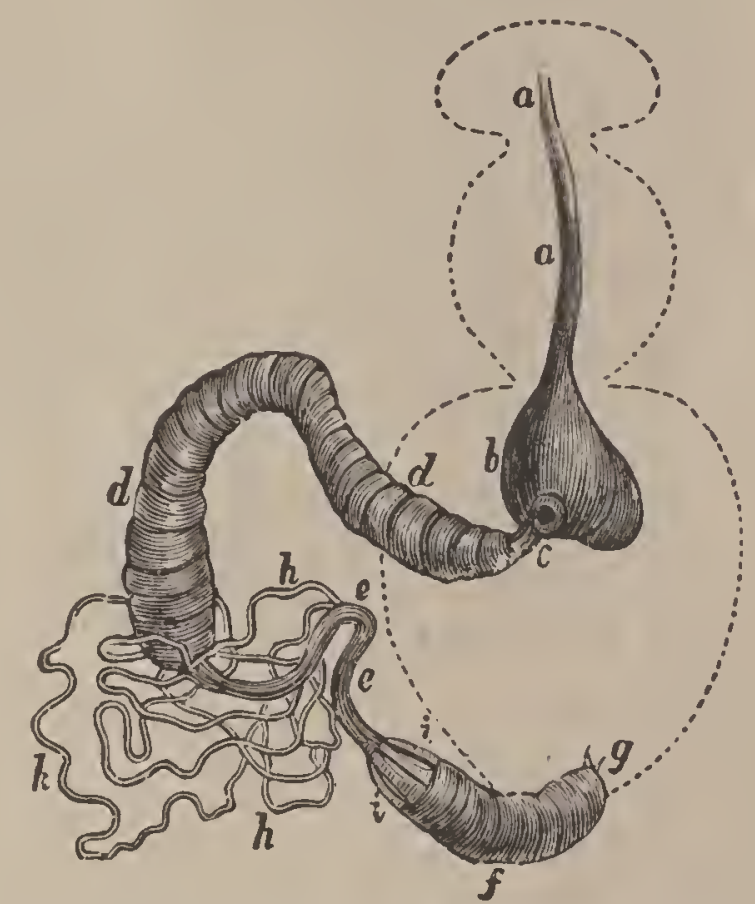

Fig. 42. - Alimentary Caual of the Bee (A pis mellifica) : $a$, gullet; $b$, crop; $c, d$, stomach; $e$, small intestine; $f$, large intestine; $g$, anal orifice; $h$, urinary ves. sels; $i$, auxiliary glands.

frequently lined with rows of horny teeth, which are specially developed in Grasshoppers, Crickets, and Locusts. The intestines are remarkable for their convolutions. Insects have no true liver; but its functions are performed by little cell-masses on the inside of the stomach. ${ }^{39}$

The alimentary canal of Spiders is short and straight, the pharynx and gullet being very minute. The stomach is characterized by sending out tubular prolongations, and

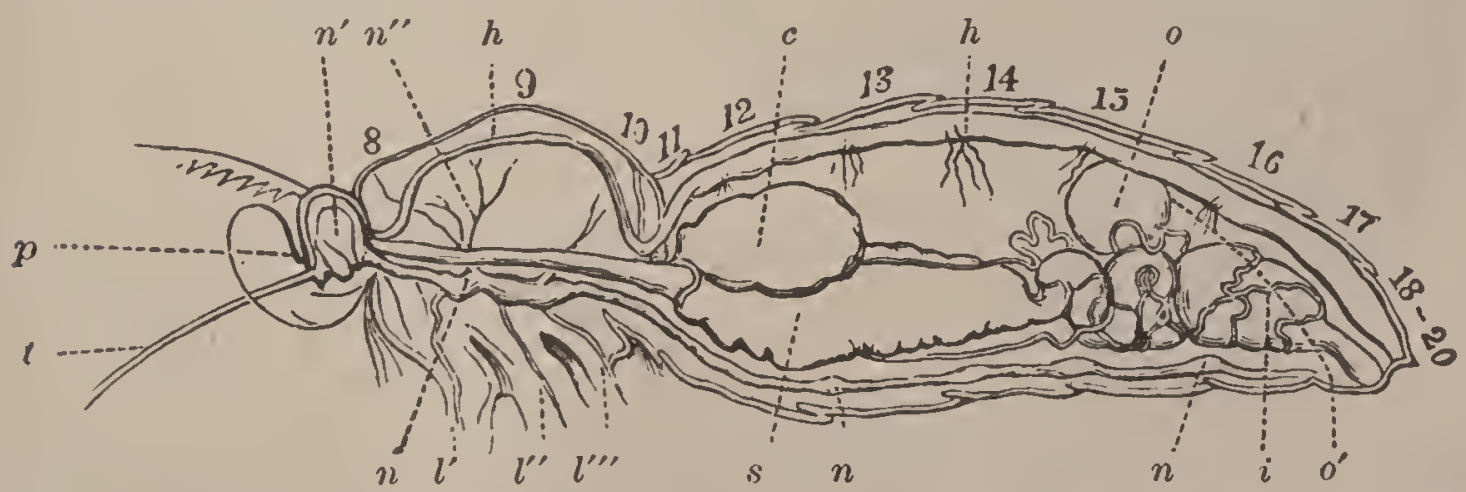

Fig 43.-Anatomy of a Sphinx Moth : $n$, nervous cord; $n^{\prime}$, brain sending off nerves to the legs, $l^{\prime}, l^{\prime \prime}, l^{\prime \prime \prime}$, and for the wings at $n^{\prime \prime} ; h$, dorsal vessel, or heart; $c$, crop; $s$, stomach ; $i$, intestines; 0 , reproductive orgaus; $o^{\prime}$, oviduct ; $8-20$, segments. 
the intestines end in a large bladder-like expansion. Scorpions have no stomachal cavity - a straight intestine passes directly through the body.

In bivalve Mollusks, like the Clam, the mouth opens into a short œsophagus which leads into the stomach, which lies imbedded in a large liver, and the intestine, describing a few turns, passes directly through the beart. ${ }^{40}$ In the univalve Mollusks, like the Snail, the gullet is long: and frequently expands into a crop; the stomach is often double, the anterior being a gizzard provided with teeth for mastication; the intestine passes through the liver, and ends in the fore-part of the body, usually on the right side.

The highest Mollusks, as the Cuttle-fish and Nantilus, exhibit a marked advance. A mouth with powerful mandibles leads to a long gullet, which ends in a strong muscular gizzard resembling that of a fowl. ${ }^{41}$ Below this is a cavity, which is either a stomach or duodenum; it receives

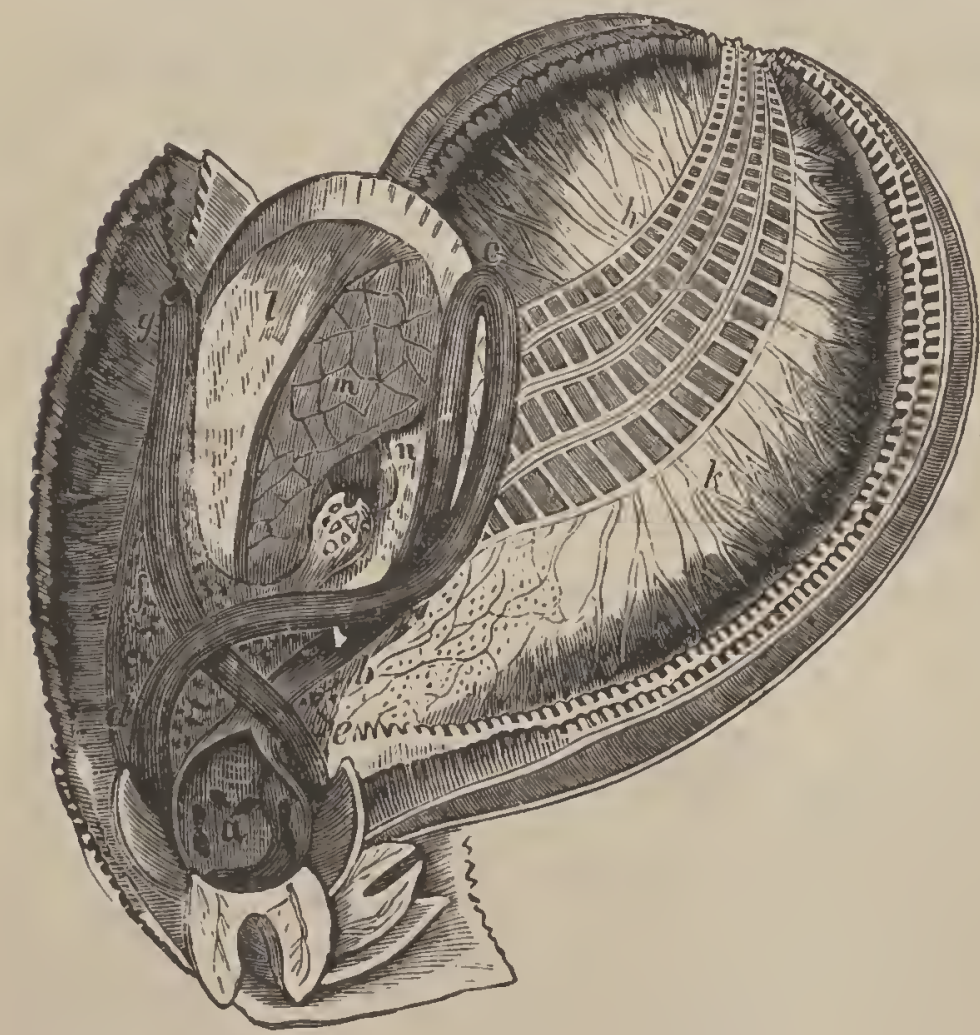

EIG. 44.-Alimentary Canal of the Oyster: $a$, stomach laid open; $d$, liver : $b, c, d, f$, convolutions of the intestine; $g$, anal aperture; $n, o$, auricle and ventricle; $l$, $m$, adductor muscle; $h, k$, lobes of mouth divided to show the venous canals at the base of the gills. the secretion from a large digestive gland or pancreas. The intestine is a tube of uniform size, which, after one or two slight curves, bends up, and opens into the "funnel" near the mouth.

Fishes have a simple, short, and wide alimentary canal. The stomach is separated from the intestine 


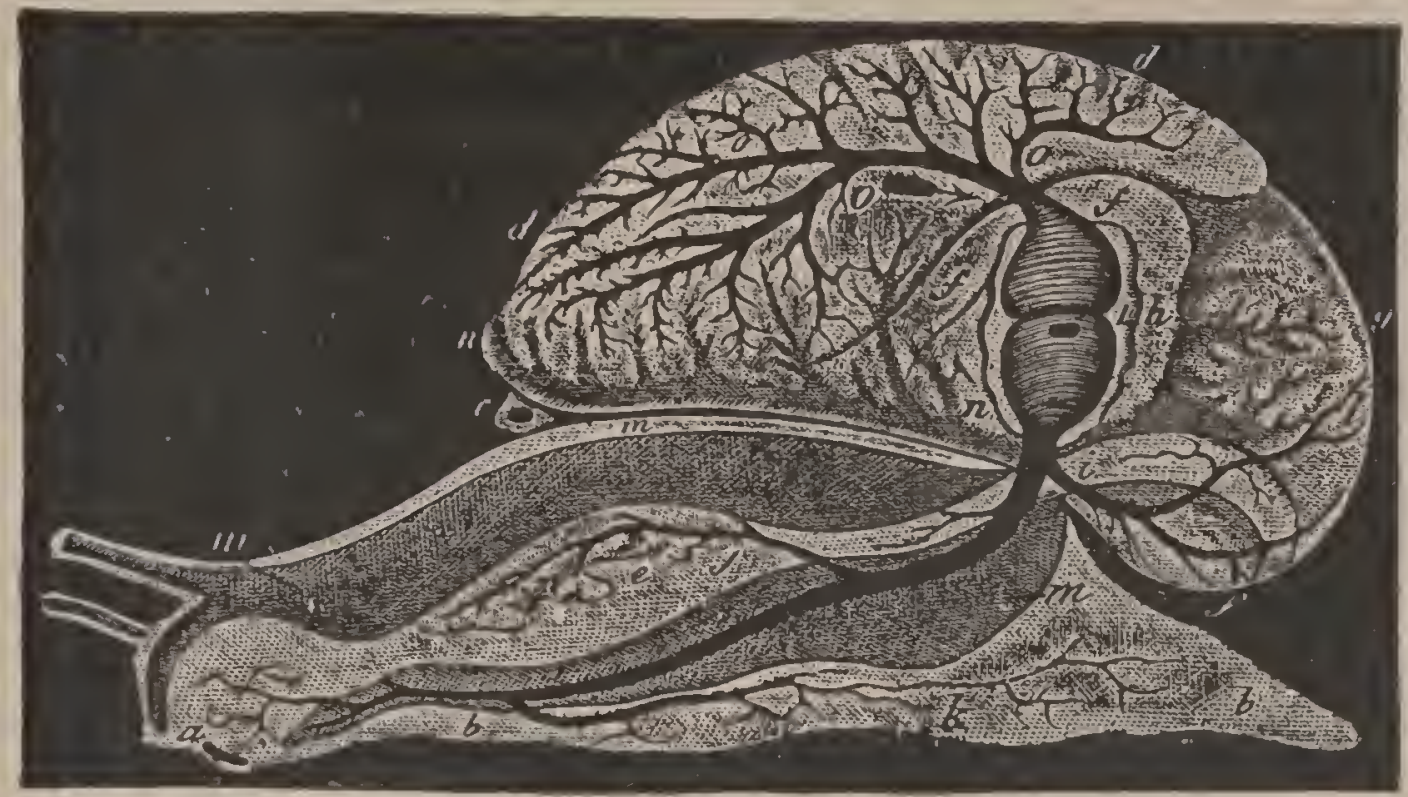

Fig. 45. -Auatomy of a Gasteropod (Snail): $a$, mouth; $b$, foot ; $c$, anus; $d$, Iung ; $e$, stomach, covered above by the salivary glands; $f$, intestine : $g$, "liver "; $h$, heart ; $i$, aorta $; j$, gastric artery $k$, artery of the foot $;$, hepatic artery; $m$, ahdominal cavity, supplying the place of a venous sinus; $n$, irregular canal communicating with the abdominal cavity, and carrying the blood to the lung; 0 , vessel carry. ing blood from the lung to the heart.

by a narrow "pyloric" orifice, or valve, but is not so clearly distinguished from the gullet, so that regurgitation is easy. ${ }^{42}$

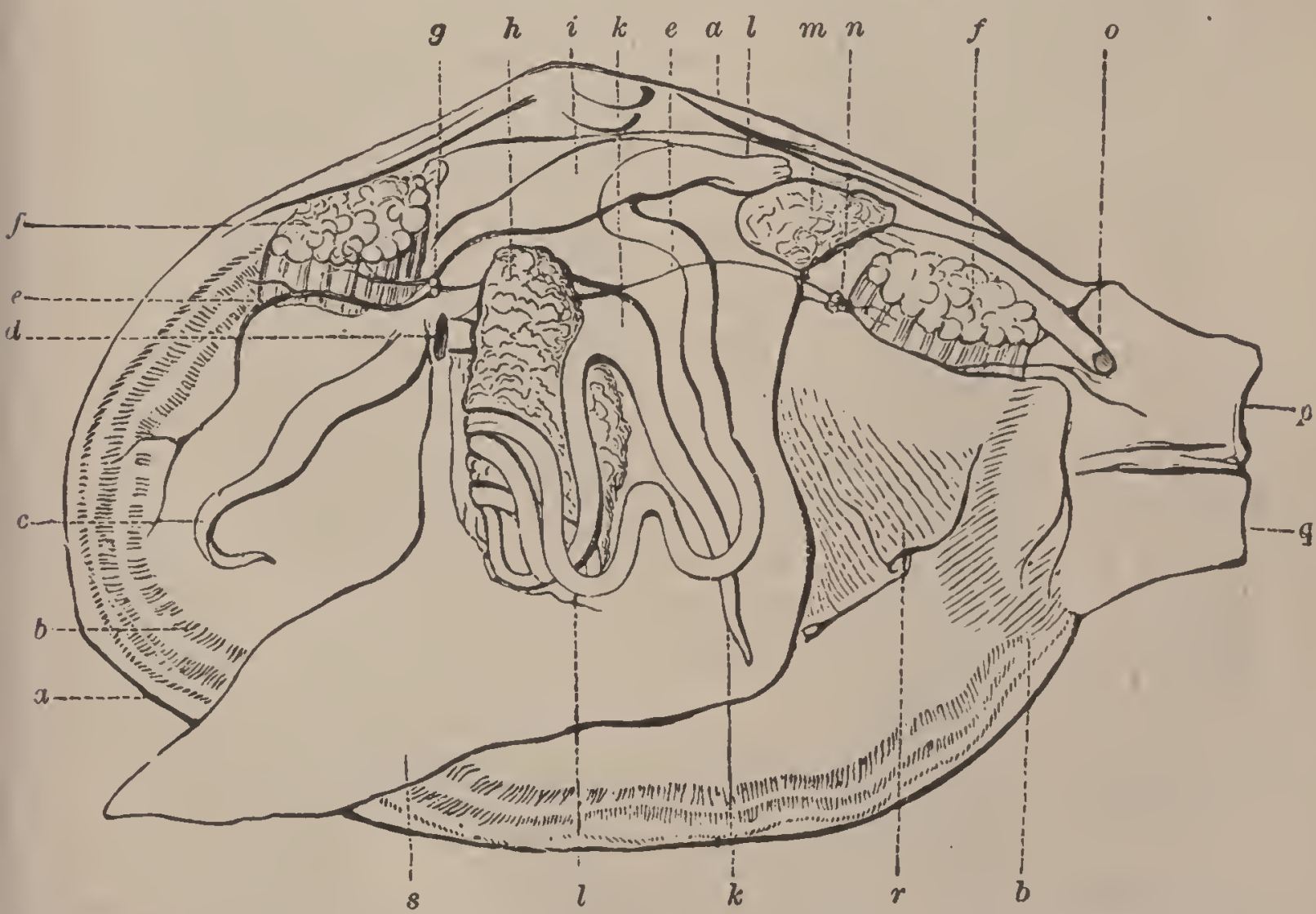

Fra. 46. -A natomy of a Lamellibranch (Mactra): $a$, shell; $b$, mantle; $c$, tentacles, or lips ; $d$, mouth ; $e$, nerves ; $f$, muscles; $g$, unterior, and $n$, posterior ganglion ; $h$, "liver" ; $i$, heart; $k$, stomach; $l$, intestiue passing through the heart; $m$, kidney; $o$, anal end of the intestine; $p$, exhalent, and $q$, inhalent respiratory tubes, or siphuns; $r$, gills; $s$, foot. 
Indeed, it is common for Fishes to disgorge the indigestible parts of their food, and some, as the Carp, send the food back to the pharynx to be masticated. The stomach is usually bent, like a siphon; but the intestine is nearly straight, and without any marked distinction into small and large. Its appendages are a large liver and a rudimentary pancreas.

In the Amphibians, as the Frogs, the digestive apparatus is very similar to that of Fishes; but the two portions of

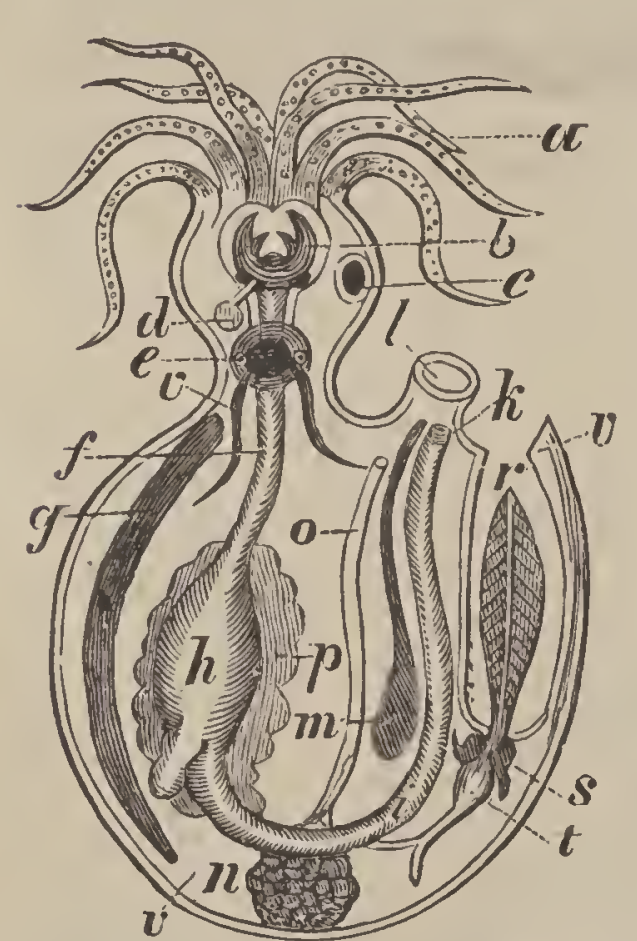
the intestine can be more readily distinguished. The Reptiles generally have a long, wide gullet, which passes insensibly into the stomach, and a short intestine (about twice the length of the body) very distinctly divided into small and large by a constriction. $^{43}$ The vegetalsle-feeding Tortoises have a comparatively long intestinal tube; and the Serpents luare a slender stomach, but little wider than the rest of Fra. 47.-Anatumy of a Cephalopod the alimentary canal.

(diagram): $a$, tentacles ; $b$, masticatory apparatus; $c$, eye; $d$, salivary gland; $e$, nervous ganglia; (Fig. 49) is more complex than
$f$, œsophagus; $g$, internal shell, or "cuttle-bove;" $h$, stomach; $i$, in- any hitherto mentioned. It re-
testine; $k$, anns; $l$, fnmmel; $m$, ink-bag: $n$, ovary; o, oviduct; $p$, sembles that of the Cuttle-fish, but "liver"; $r$, gill contained in the offer's a still more striking analogy heart; $t$, systemic heart; $v$, mantle. to the gizzard of a Bird, having very thick walls, and the muscular fibres radiating precisely in the same manner, so that, in this respect, the Crocodile may be considered the connecting link between Reptiles and Birds. ${ }^{44}$ In Crocodiles also the duodenum, with which the intestine begins, is first distinctly defined. Into this part of the intestine the liver and pancreas, or sweet-bread, pour their secretions. Furthermore, in the 
lower animals, the intestines lie more or less loose in the abdomen; but in the Crocodile, and likewise in Birds and Mammals, they are supported by a membrane called mesentery.

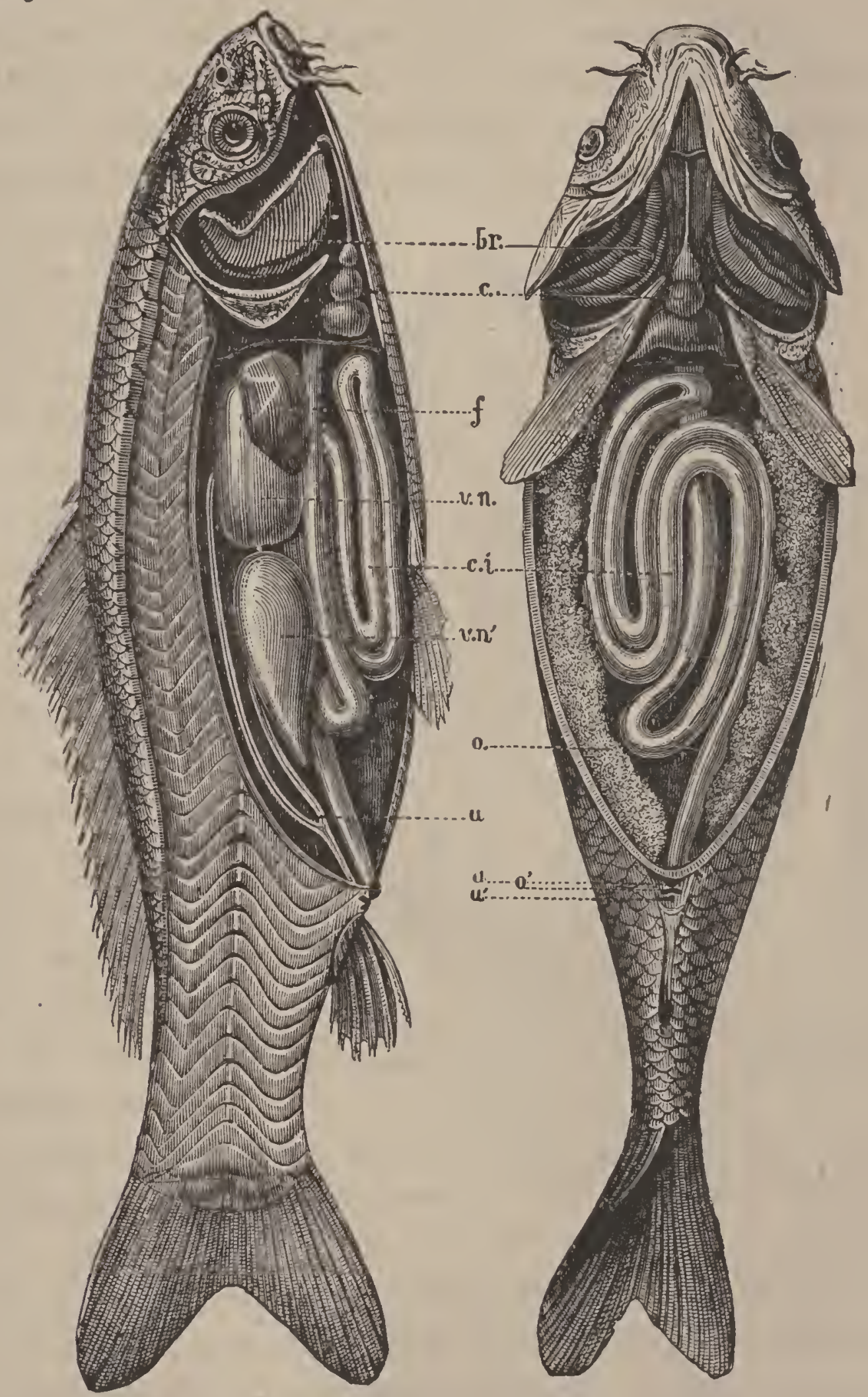

Fig. 48. - Anutomy of the Cirp: br, branclix, or gills; $c$, heart; $f$, liver; $v n, v n^{\prime}$, swimming-bladder; $c i$, intestiual canal ; $o$, ovarium; $u$, ureter ; $a$, anus ; $o^{\prime}$, genital opening: $u^{\prime}$, npening of ureter. The side-view shows the disposition of the muscles in vertical flakes. 
In Birds, the length of the alimentary canal varies with their diet, being greatest in those living on grain and fruit. The gullet corresponds in length with the neck, which is longest in the long-legged tribes, and in width with the food. In those that swallow large fish entire, the gullet is dilatable, as in Snakes. In nearly all Birds, the food is delayed in some cavity before digestion: thus, the Pelican has a bag under the lower jaw, and the Cormorant has a

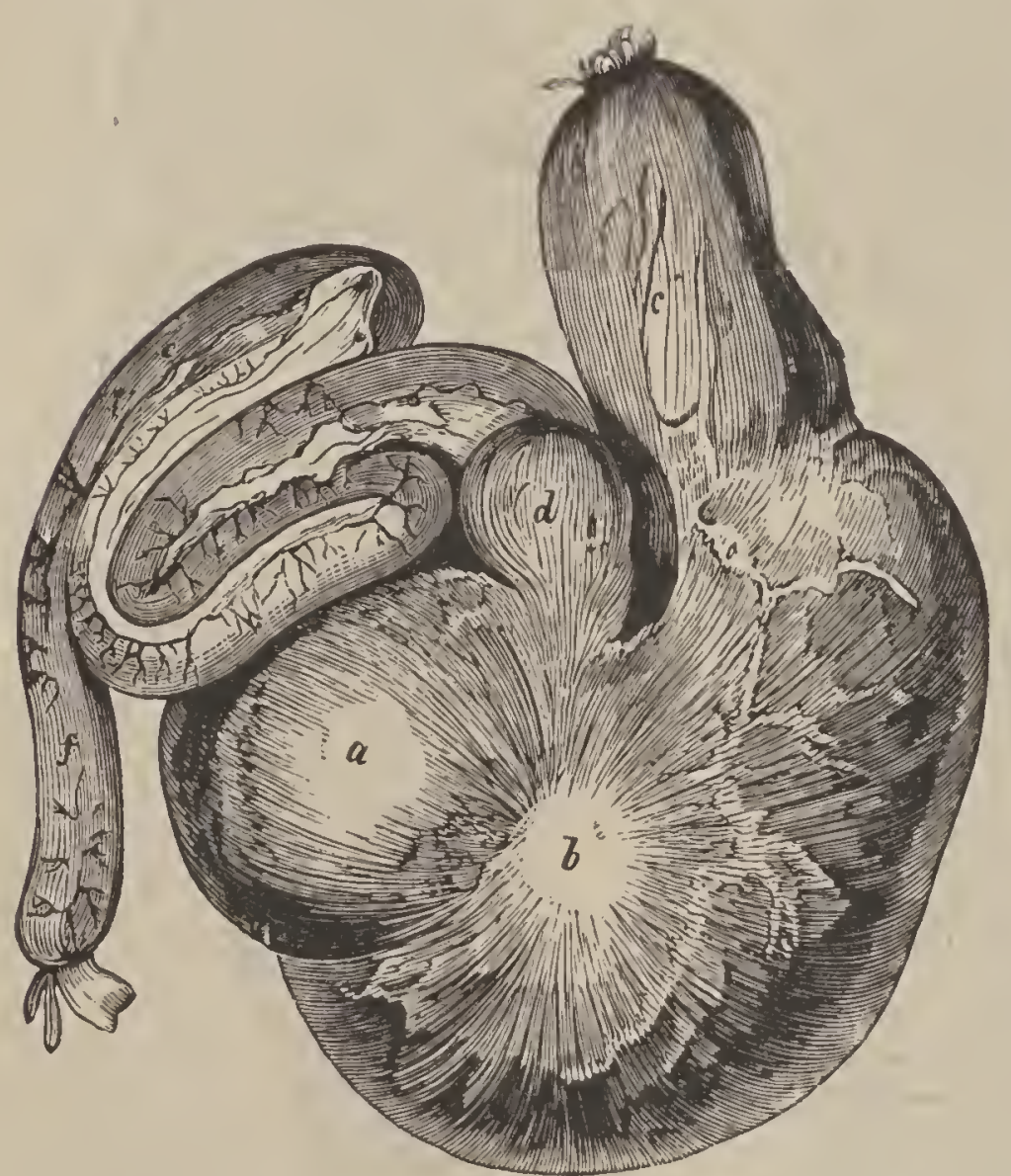

Fig. 49.-Stomach of the Crocodile: $a$, muscular fibres radiating from a central tendon, $b ; d$, commencement of duodenum ; $c$, cesuphagus : $f$, intestine. capacious gullet, where they store up fishes; while those that gorge themselves at intervals, as the Vulture, or feed on seeds and grains, as the Turkey, havea pouch, called the crop, developed near the lowel end of the gullet. ${ }^{45}$ The Ostrich, Goose, Swan, most of the Waders, and the fruit or insect-eating Birds, which find their food in tolerable abundance, and take it in small quantities, have no such reservoir. Pigeons have a double crop.

In all Birds, the food passes from the gullet into the proventriculus, or stomach proper, where it is mixed with a "gastric juice" secreted from glands on the surface. Thence it goes into the gizzard, an oval sac of highly muscular texture, and lined with a tough, horny skin." 
The gizzard is most highly developed, and of a deep-red color, in the Scratchers and flat-billed Swimmers (as Fowls and Swans); but comparatively thin and feeble in Birds of Prey (as the Eagle). The gizzard is followed by the intestines, which are longer than those of Reptiles: the small intestine begins with a loop (the duodenum), and is folded several times upon itself; the large intestine is short and straight, terminating in the sole outlet of the body, the cloaca. A liver and pancreas are always attached to the upper part of the small intestine.

The alimentary canal in Mammals is clearly separated into four distinct cavities: the pharynx, or throat; the oesophagus, or gullet; the stomach; and the intestines.

The pharynx is more complicated than in Birds. It is a funnelshaped bag, having seven openings leading into it: two from

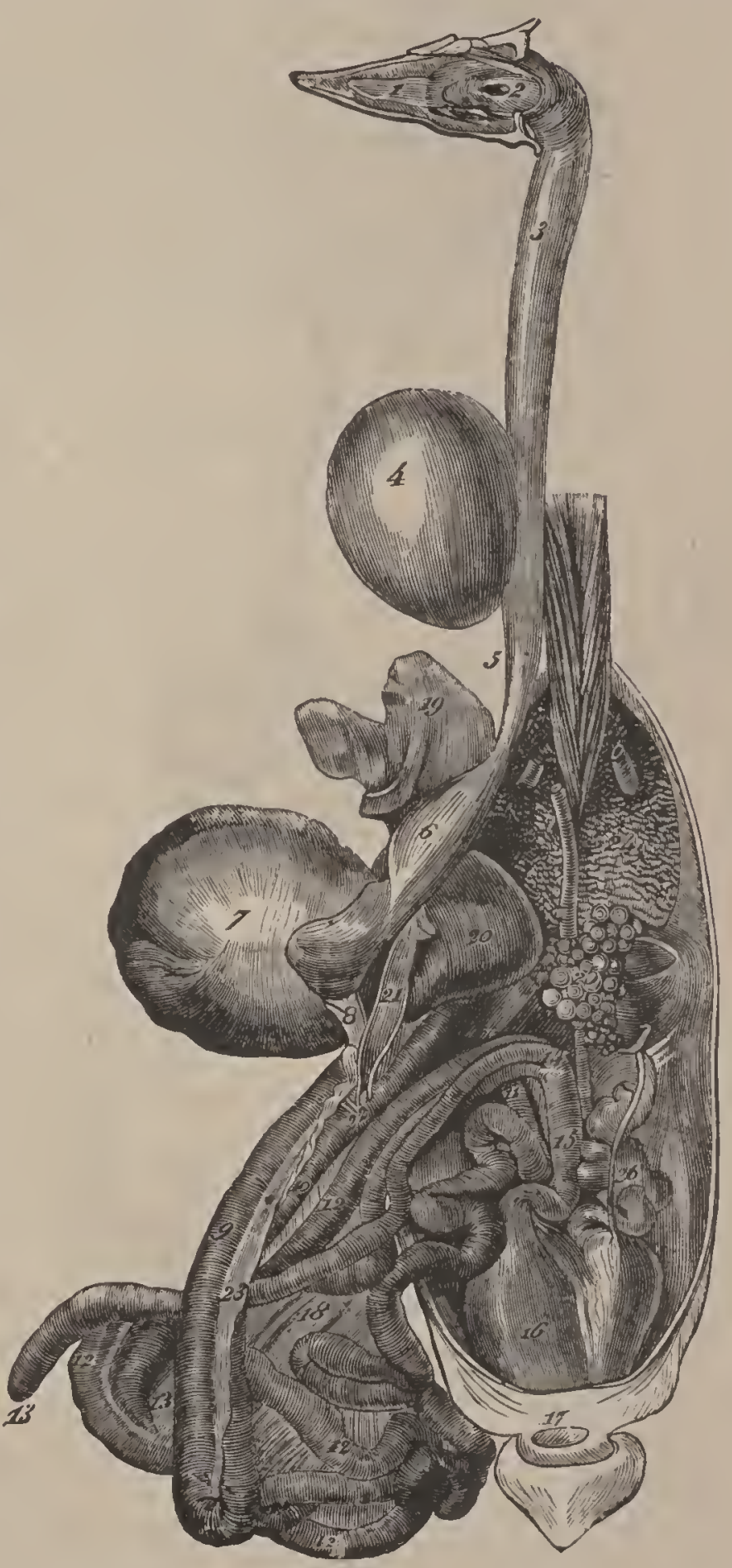

Fig. 50.-Digestive Apparatus of the Furvl: 1, tongue; 2 , pharyux; 3,5 , csophagne; 4 , crop ; 6 , proventricnlus ; 7 , gizzard ; $8,9,10$, dnodenum ; 11,12 , small intestiue; 13 , two creca (analogue of the colon of mammals); 14 , their insertion into the intestiual tube; 15 , rectum; 16 , cloaca; 17 , anus ; 18 , mesentery ; 19,20 , left and light lobes of liver: 21 , gall-bladder; 22 , insertion of pancreatic and biliary dncts ; 23 , pancreas; 24 , lung; 25 , ovaly ; 26 , oviduct. 


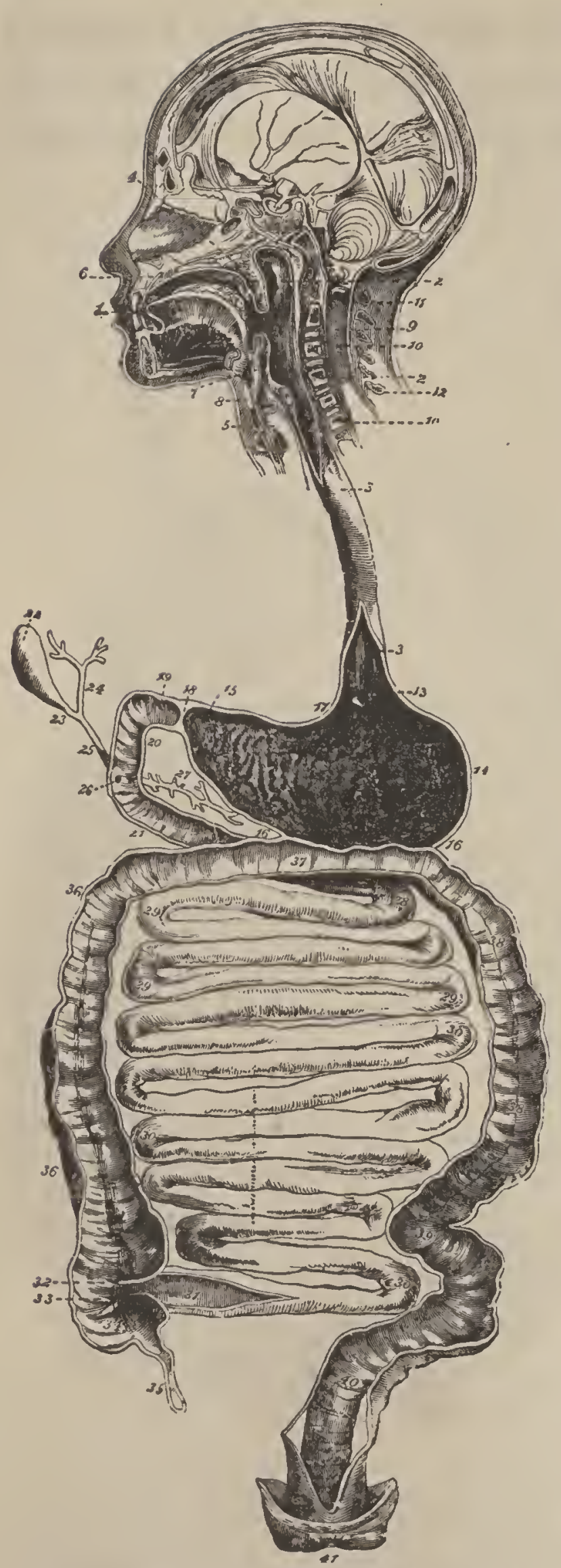

the nostrils, and two from the ears; one from the windpipe, guarded by the epiglottis; one from the mouth, with a fleshy. curtain called the soft palate; and one from the œesophagus. It is the natural passage for food between the mouth and the nesophagus, and of air between the nostrils and windpipe. Like the mouth, it is lined with a soft mucous membrane.

The œsophagus is a long and narrow tube, formed of two muscular layers: in the outer lay$\mathrm{er}$, the fibres run lengthwise; in the other, they are circular. It is lined with mucous membrane. While in all Fishes, Reptiles, and Birds the body cavity is one, in Mammals it is divided, by a partition called the diaphragm, into two cavities - the thorax, containing the heart, lungs,

FIG. 51.-Digestive Apparatus of Man (diagram): 1, tougue; 2, pharynx; 3, œsophagus ; 4 , soft palate; 5 , larynx; 6 , palate; 7 , epiglottis; 8 , thyroid cartilage; 9 , beginning of spinal marrow; $10,11,12$, vertebrr, with spinous processes; 13 , cardinc orifice of stomach; 14 , left end of stomach; 18 , pyloric valve; $19,20,21$, duodenum; 22 , gall-bladder ; 27 , dnct from pancreas ; $28,2$. , jejunum of intestine: 30 , ileum; 34 , cœcum; $36,37,38$, colon, or large intestine ; 40 , rectum. 
etc.; and the abdomen, containing the stomach, intestines, etc. The osophagus passes through a slit in the

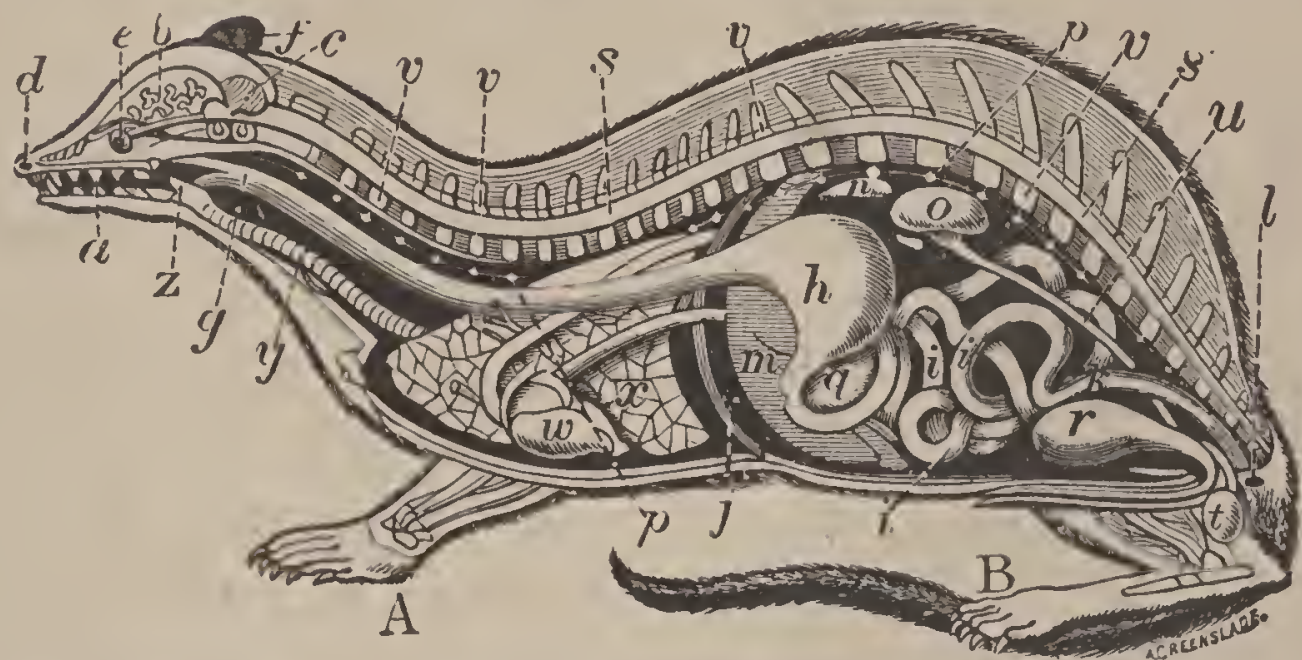

Frg. 52.-Ideal Section of a Mammalian Vertebrate: A, pectoral, or fore limb; B, pelvic, or hind limb: $a$, mouth; $b$, cercbrnm; $c$, cerebellum; $d$, nose; $e$, eye; $f$, ear; $g$, csophagus; $h$, stumach ; $i$, intestine; $j$, diaphragm, or midriff ; $k$, rectum, or termination of intestine $l$, , anus ; $m$, liver ; $u$, spleen ; $o$, kidney ; $p$, sympathetic system of nerves; $q$, pancreas $; r$, urinary bladder ; $s$, spinal cord; $u$, ureter; $v$, vertebral column; $w$, heart; $x$, lung; $y$, trachea, or windpipe; $z$, epiglottis.

diaphragm, and almost immediately expands into the stomach.

In the majority of Mammals, the stomach is a muscular bag of an irregular oval shape, lying obliquely across the abdomen. In the Flesh-eaters, whose food is easy of solution, the stomach is usually simple, and lies nearly in the course of the alimentary canal; but in proportion as the food departs more widely in its composition from the body itself, and is therefore more difficult to digest, we find the stomach increasing in size and complexity, and turned aside from the general course of the canal, so as to retain the food a longer time. The inlet, or open-

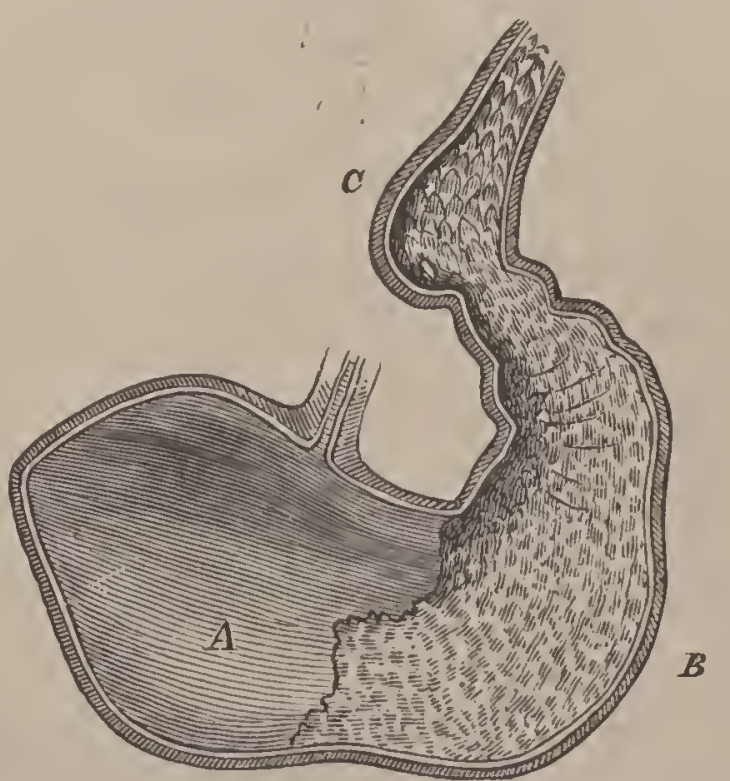

Fig. 53.-Section of Horse's Stomach : A, left sac; $B$, right sac; $C$, duodenum. ing, into the osophagus is called cardiac; the outlet, or 
opening, leading into the intestines is called pyloric. In the Carnivores, Apes, and most odd-toed quadrupeds, the

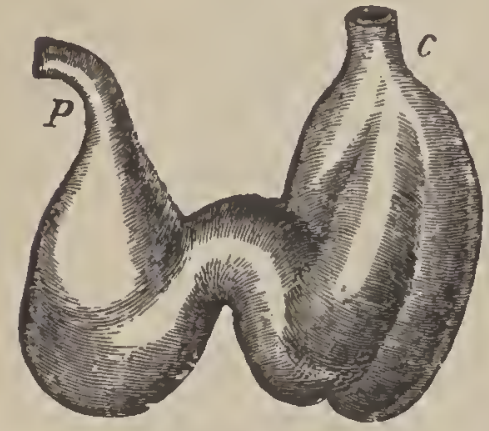

Frg. 54. - Stomach of the Porpoise : $c$, cardiac opening; $p$, pyloric opening. stomach resembles that of Man. That of the toothless Ant-eatel has the lower part turned into a kind of gizzard for crushing its food. The Elephant's is subdivided by numerous folds. In the Horse, it is constricted in the middle; and in the Rodents, Porpoises, and Kangaroos, the constriction is carried so far as to make two or three sections. But animals that chew the cud (Ruminants) have the most complex stomach. It is divided into four peculiar chambers: First, the paunch (rumen), the largest of all, receives the half-masticated food when first swallowed. The inner surface is covered with papillæ, except in the Camel, which has large cells for storing up water. Fra. 55.-Stomach of the Lion: $c$, cardiac orifice, or From this, the food entrance of esophagus; $p$, pyloric orifice.

passes into the honey-comb stomach (reticulum), so named from its structure. Liquids swallowed usually go directly to this cavity, without passing through the paunch, and

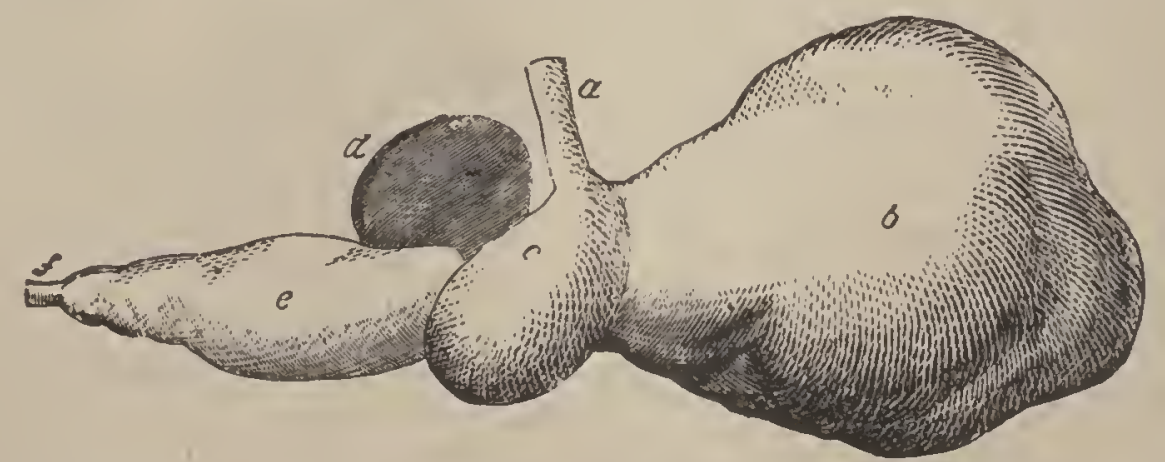

Fia. 56.-Complex Stomach of a Ruminant: $a$, gullet; $b$, rumen, or pannch ; $c$, reticnlum; $d$, psalterium, or manyplics; $e$, abomasus ; $f$, pylorus leading to dnodenum. 
hence it is sometimes called the water-bag. Here the food is made into little balls, and returned to the mouth to undergo a thorough mastication. When finally swallowed, it is directed, by a groove from the œsophagus, to the third, and smallest, cavity, the manyplies (psalterium), named from its numerous folds, which form a strainer to keep back any undivided food; and thence it passes into the true stomach (abomasus), from which, in the calf, the rennet is procured for curdling milk in the manufacture of cheese. This fourth cavity is like the human stomach in form and function, and is the only part which secretes gastric juice. The rumen and reticulum are rather dilatations of the œesophagus than parts of the stomach itself; while the latter is divided by constriction into two chambers, the psalterium and abomasus, as in many other animals.

In structure, the stomach resembles the œesophagus. The smooth outside coat (peritoneum) is a reflection of the membrane which lines the whole abdomen. The middle, or muscular, coat consists of three layers of fibres, running length-
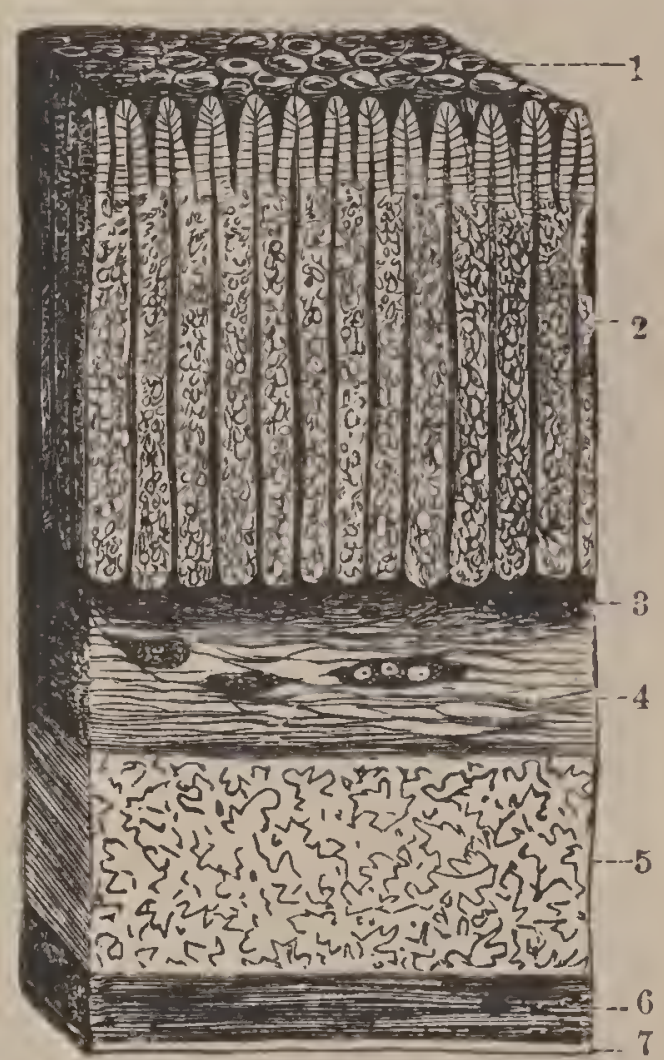

FIg. 57. - Vertical Section of the Coats of the Stomach: 1, surfice of mucous membrane, and mouths of gastric follicles; 2 , gastric tubuli, or follicles: 3 , dense connective tissue; 4, submucons tissne; 5 , transverse mnscular fibre; 6 , longitudiual muscular tibre; 7 , fibrous, or serous, crat. wise, around and obliquely. The successive contraction and relaxing of these fibres produce the worm-like motion of the stomach, called peristaltic. The innermost, or mucous, membrane, is soft, velvety, of a reddish-gray color in Man, and filled with multitudes of glands, which secrete the gastric juice. The human stomach, when distended, will 
hold about five pints; that of the Kangaroo is as long as its body.

The intestinal canal in Mammals begins at the pyloric end of the stomach, where there is a kind of valve or circular muscle. Like the stomach, it varies greatly, according to the nature of the food. It is generally longest in the Vegetable-feeders, and shortest in the Flesh-feeders. The greater length in the former is due to the fact that

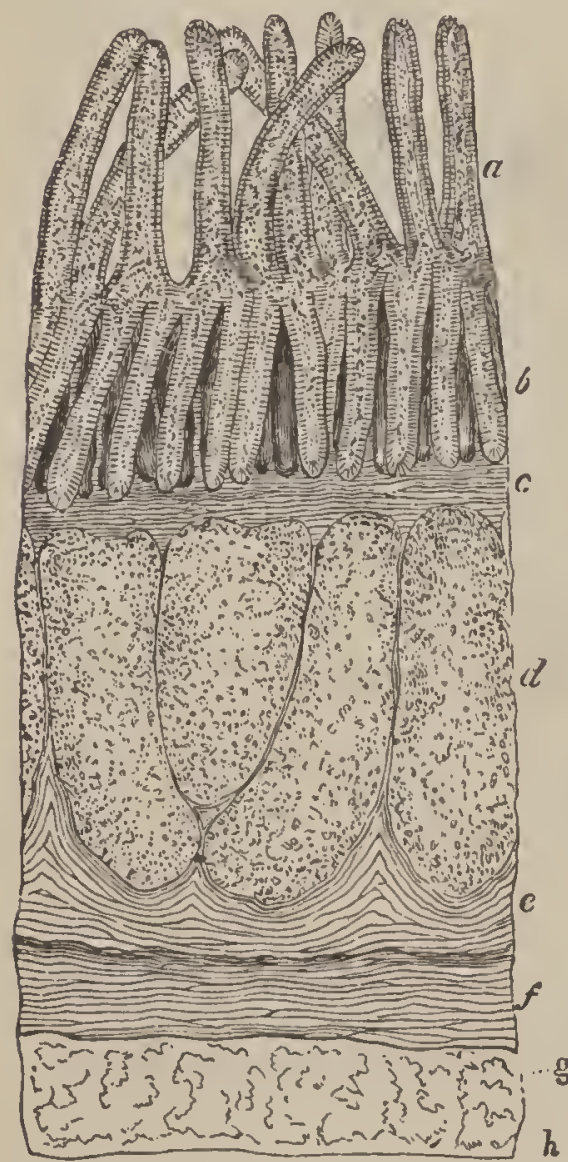
vegetable food requires a longer time for digestion, and that a greater bulk of such food is required to obtain a given quantity of nutriment. The intestines measure 150 feet in a full-grown Ox, while they are but three times the length of the body in the Lion, and six times in Man. Save in some lower forms, as the Whales, there are two main divisions, the "small" and "large" intestines, at the junction of which is a valve. The former is the longer of the two, and in it digestion is completed, and from it the most of absolption Fig. 58.-Section of the Wall of takes place. The large intestine is
the Human Intestine (ileum), $\times 50: a$, villi; $b$ and $d$, glands; inainly a tempolary lodging-place $c$ and $e$, mucons membrane; $f$, for the nseless part of the food,
circular muscles; $g, h$, lougitudinal muscles. until it is expelled from the body. The beginuing of the small intestine is called the duodenum, into which the ducts from the liver and pancreas open. The intestinal canal has the same structure as the stomach, and by a peristaltic motion its contents are propelled downward. The inside of the small intestine is covered with a host of thread-like processes (villi), resembling the pile of velvet. 
In taking this general survey of the succession of forms which the digestive apparatus presents among the principal groups of animals, we cannot fail to trace a gradual specialization. First, a simple sac, one orifice serving as inlet for food and outlet for indigestible matter; next, a short tube, with walls of its own suspended in the bodycavity; then a canal passing through the body, and, therefore, having both mouth and vent; next, an apparatus for mastication, and a swelling of the central part of the canal into a stomach, having the special endowment of secreting gastric juice; then a distinction between the small and large intestine, the former thickly set with villi, and receiving the secretions of large glands. We also notice that food, the means of obtaining it, the instruments for mastication, and the size and complexity of the alimentary canal, are closely related.

\section{CHAPTER X.*}

HOW ANIMALS DIGEST.

The object of the digestive process is the reduction of food into such a state that it can be absorbed into the system. For this purpose, if solid, it is dissolved; for fluidity is a primary condition, but not the only one. Many soluble substances have to undergo a chemical change before they can form parts of the living body. If albumen or sugar be injected into the veins, it will not be assimilated, but be cast out unaltered.

To produce these two essential changes, solution and transmutation, two agencies are used - one mechanical, the other chemical. The former is not always needed, for many animals find their food already dissolved, as the 
Butterfly; but solid substances, to facilitate their solu. tion, are ground or torn into pieces by teeth, as in Man; by jaws, as in the Lobster; or by a gizzard, as in the Turkey.

The chemical preparation of food is indispensable. ${ }^{47}$ It is accomplished by one or more solvent fluids secreted in the alimentary canal. The most important, and one always present, is the gastric juice, the secretion of which is restricted to the stomach, when that cavity exists. In the higher animals, numerous glands pour additional fluids into the digestive tube, as saliva into the upper part or month, and bile and pancreatic juice into the upper part of the intestine. In fact, the mucous membrane, which lines the alimentary canal throughont, abounds with secreting glands or cells.

The Digestive Process is substantially the same in all animals, but it is carried further in the more highly developed forms. In the Infusoria, the food is acted upon by some secretion from the protoplasm of the body, the exact nature of which is unknown. In the Star-fish and Sea-urchin, we find two solvents-a gastric juice, and another resembling pancreatic juice; but the two appear to mingle in the stomach. Mollusks and Arthropods show a clear distinction between the stomach and intestine, and the contents of the pancreas are poured in to the latter. There are, therefore, two stages in the digestive act: first, the food is dissolved by the gastric juice in the stomach, forming chyme; secondly, the chyme, upon entering the intestine, is changed into chyle by the action of the pancreatic secretion, and is then ready to be absorbed into the system.

In Vertebrates, a third solvent is added, the bile, which aids the pancreatic juice in completing digestion. But Mammals and Insects have a still more perfect and elaborate process; for in them the saliva of the month acts chemically upon the food; while the saliva in many other 
animals has no other office, so far as we know, than to moisten the food for swallowing.

Taking Man as an example, let us note the main facts in the process. During mastication, by which the relative surface is increased, the food is mixed with saliva, which moistens the food, ${ }^{48}$ and turns a sinall part of the starch into grape-sugar. Passed into the stomach, the food meets the gastric juice. This is acid, and, first, stops the action of the saliva; secondly, by means of the pepsin which it contains, and the acid, it dissolves the albumen, fibrin, and such constituents of the food. This solution of albuminoids is called a peptone, and is especially distinguished from other such solutions by its diffusibility-i. e., the ease with which it passes through a membrane. Some of these peptones, with the sugars of the food, whether original or the product of the action of the saliva, are absorbed from the stomach. The food, while in the stomach, is kept in continual motion, and, after a time, is discharged in gushes into the intestine. The name chyme is given to the pulpy mass of food in the stomach. ${ }^{49}$ In the intestine the chyme meets three fluids-bile, pancreatic juice, and intestinal juice. All of these are alkaline, and at once give the acid chyme an alkaline reaction. This change permits the action of the saliva to recommence, which is aided by the pancreatic and intestinal juices. The pancreatic juice has much more important functions. It changes albuminoid food into peptones, and probably breaks up the fats into very small particles, which are suspended in the fluid chyle. This forms an

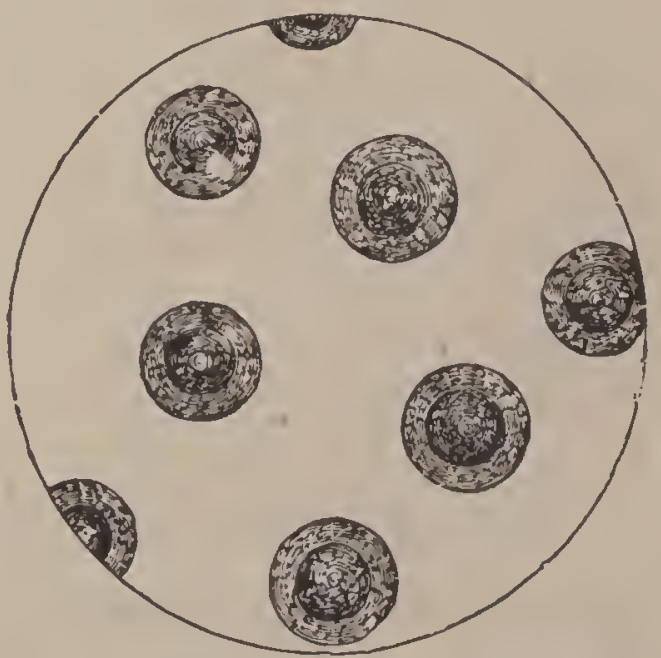

Fra. 59.-Chyle Corpuscles, $\times 500$. emulsion, like milk, and causes the chyle to appear whitish. The bile has important functions, but little under- 
stood. It emulsifies and saponifies part of the fats, so that they are dissolved, and perhaps aids in preventing the food from decomposing during the process of digestion and absorption. The chyle is slowly driven through the small intestine by the creeping, peristaltic motion of its walls, the nutritious portion being taken up by the absorbents, as described in the next chapter, while the undigested part remaining is discharged from the large intestine. ${ }^{.1}$

\section{CHAPTER XI. \\ THE ABSORBENT SYSTEM.}

The nutritive matter (chyle), prepared by the digestive process, is still outside of the organism. How shall it enter the living tissue?

In animals, like the Infusoria and Polyps, whose digestive department is not separated from the body-cavity, the food, as soon as dissolved, mingles freely with the tissues and organs it has to nourish. In the higher Invertebrates having an alimentary canal, the chyle passes, by simple transudation, through the walls of the canal directly into the soft tissues, as in Insects, or is absorbed from the canal by veins in contact with it, as in Sea-urchins, Mollusks, Worms, and Crustaceans, and then distributed through the body.

In Vertebrates only do we find a special absorbent system. Three sets of vessels are concerned in the general process by which fresh material is taken up and added to the blood: Capillaries, Lacteals, and Lymphatics. Only the two former draw material from the alimentary canal.

It is a general law that the food is absorbed as fast as 
it is dissolved, and, therefore, there is a constant loss in the passage down the canal. In the mouth and osophagus, the absorption is slight; but much of that which has yielded to the gastric juice, with most of the water, is greedily absorbed by the capillaries of the stomach, and made to join the current of blood which is rushing to the liver. Absorption by the capillaries also takes place from the skin and lungs. Medicinal or poisonous gases and liquids are readily introduced into the system by these channels.

We have seen that the oily part of the food passes unchanged from the stomach into the small intestine, where, acted upon by the pancreatic juice, it is cut up into extremely minute particles, and that the undigested albuminoids and starches are digested in the intestine. Two kinds of absorbents are present in the intestine, lacteals and blood-capillaries. Both the lymphatic and blood systems send vessels into the velvety villi $i^{\text {s1a }}$ with which the intestine is lined. The bloodcapillaries lie towards the ontside of the villus and the lacteal in the centre. The albuminoids and sugars are

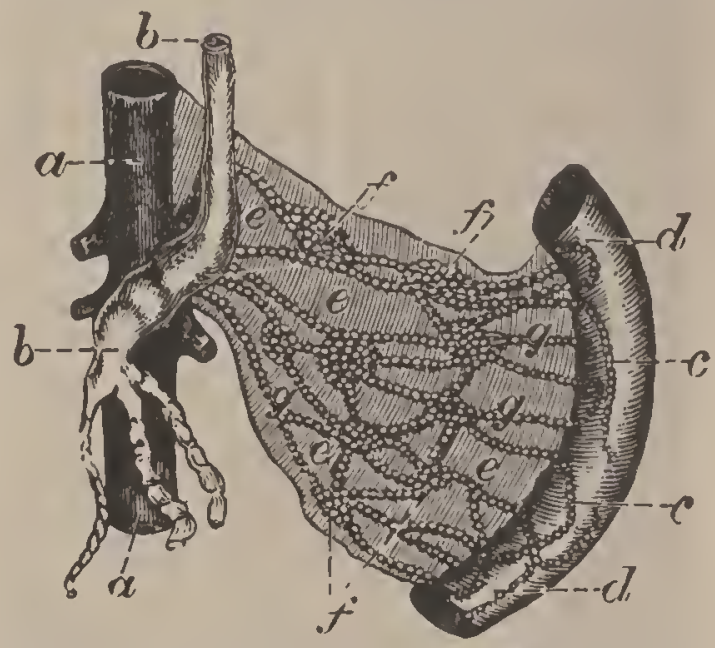

Fro. 60.-Lacteal System of Mammal : $a$. descending arorta, or principal artery : $b$, thoracic duct; $c$, origin of lacteal vessels, $g$, in the walls of the intestine, $d ; e$, mesentery, or membrane attaching the intestiuc to walls of the body; $f$, lacteal, or mesenteric, glinds. chiefly absorbed by the blood-vessels and go to the liver. The fats pass on into the lacteals, which receive their name from the milky appearance of the chyle. These lacteals unite into larger trunks, which lie in the mesentery (or membrane which suspends the intestine from the back wall of the abdomen), and these pour their contents into one large vessel, the thoracic duct, lying along the backbone, and joining the jugular vein in the neck. 
The lacteals are only a special part of the great lymphatic system, which absorbs and carries to the thoracic

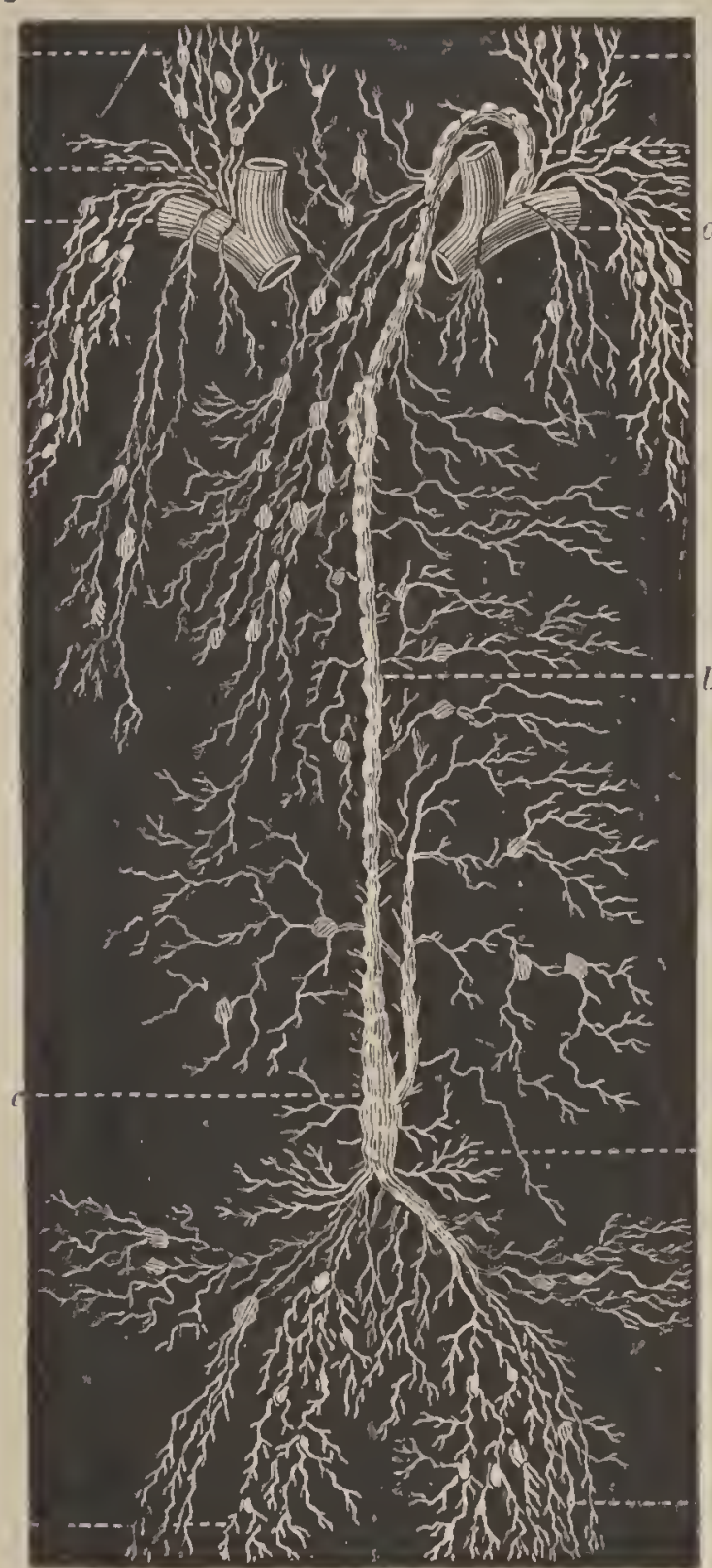

FIG.61.-Principal Lymphatics of the HIIman Body: $a$, union of left jugular and subclavian veins: $b$, thoracic duct; $c$, receptaculum chyli. The oval bodies are glands. duct matter from all parts of the body. ${ }^{52}$ The lymph is a transparent fluid having many white blood corpuscles. It is, in fact, blood, minus the red corpuscles, while chyle is the same fluid rendered milky by numerous fat-globules. During the intervals of digestion, the lacteals carry ordinary lymph. This fluid is the overflow of the blood - the plasma and white corpuscles which escape from the blood capillaries, and carry nutriment to, and waste from, those parts of the variuus tissues which are not in contact with the blood capillaries. This surphus overflow is returned to the blood by the lymphatics. The current is kept up by the movements of the body, and in many Vertebrates, as Frogs and Fishes, by lymph hearts.

Like the roots of Plants, the absorbent vessels do not commence with open mouths; but the fluid which enters them must traverse the membrane which covers their minute extremities. This membrane is, however, porous, and the fluids pass through it by the forces of filtration and diffusion. ${ }^{53}$ How the fat gets into the lacteals is not yet well understood, but the lacteals are themselves rhythmically contractile, and force the absorbed chyle towards 
the heart. The valves of the lymphatics prevent its return.

\section{CHAPTER XII.* \\ THE BLOOD OF ANIMALS.}

The Blood is that fluid which carries to the living tissues the materials necessary to their growth and repair, and removes their waste and worn-out material. The great bulk of the body is occupied with apparatus for the preparation and circulation of this vital fluid.

The blood of the lower animals (Invertebrates) differs so widely from that of Man and other Vertebrates, that the former were long supposed to be without blood. In them the blood is commonly colorless; but it has a bluish cast in Crustaceans; reddish, yellowish, or greenish, in Worms; and reddish, greenish, or brownish, in Jellyfishes. The red liquid which appears when the head of a Fly is crushed is not blood, but comes from the eyes. In Vertebrates, the blood is red, excepting the whiteblooded fish, Amphioxus. ${ }^{54}$

As a rule, the more simple the fabric of the body, the more simple the nutritive fluid. In unicellular animals (as Protozoa), in those whose cells are comparatively independent (as Sponges), and in small and lowly organized animals (like Hydra), there is no special circulating fluid. Each cell feeds itself either directly from particles of food, or from the products of digestion. In Polyps and Jelly-fishes, the blood is scarcely different from the products of digestion, although a few blood-corpuscles are pres. ent. But in the more highly organized Invertebrates the blood is a distinct tissue, coagulating, and containing white corpuscles. The blood of the Vertebrates, appar-

* See Appendix. 
ently a clear, homogeneous liquid, really consists of minute grains, or globules, of organic matter floating in a fluid.

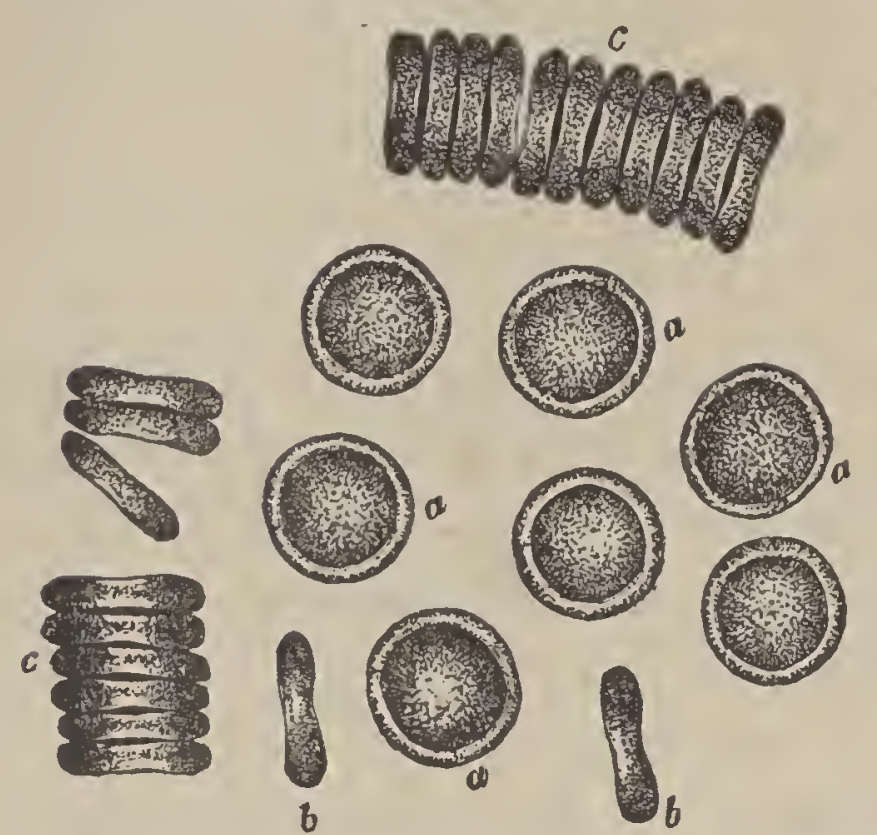

Fra. 62.-Red Blood-corpnscles of Mau : $a$, shows circular contour; $b$, a biconcave section; $c$, a group in chains.

red. The red disks have a tendency to collect together into piles; the colorless ones remain single. Meanwhile, the plasma separates into two parts by coagulating; that is, minute fibres form, consisting of fibrin, leaving a pale yellowish fluid, called serum. ${ }^{55}$ Had the blood not been filtered, the corpuscles and fibrin would have mingled, forming a jelly-like mass, known as clot. Further, the serum will coagulate if heated, dividing into hardened albumen and a watery fluid, called serosity, which contains the soluble salts of the blood.

These several parts may be expressed thus:

$$
\text { Blood } \begin{cases}\text { Corpuscles }\left\{\begin{array}{l}
\text { colored } \\
\text { colorless }
\end{array}\right\} & \text { clot. } \\
\text { Plasma } & \left\{\begin{array}{l}
\text { fibrin- } \\
\text { serum }
\end{array}\right\} \begin{array}{l}
\text { albumen. } \\
\text { serosity=water and salts. }
\end{array}\end{cases}
$$

If now we examine the nutritive fluid of the simplest animals, we find only a watery fluid containing granules. In Radiates and the Worms and Mollusks, there is a similar fluid, with the addition of a few colorless corpuscles. But 
there is little fibrin, and, therefore, it coagulates feebly or not at all. In the Arthropods and higher Mollusks, the circulating fluid contains colorless nucleated cells, and coagulates. ${ }^{56}$ InVertebrates, there are, in addition to the plasma and white corpuscles of Invertebrates, red corpuscles, to which their blood owes its peculiar hne. In Fishes, Amphibians, Reptiles, and Birds, i.e., all oviparous Vertebrates, these red corpuscles are

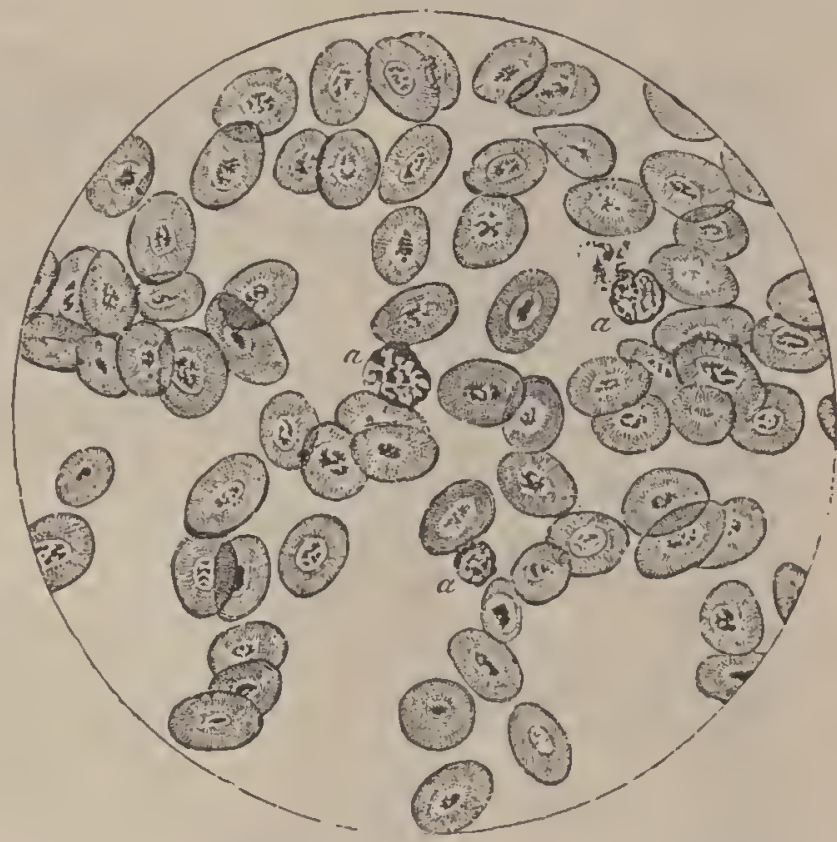

Fig. 63.-Nucleated Blood-cells of a Frog, $\times 250$. nucleated; but in those of Mammals, no nucleus has been discovered. ${ }^{57}$

All blood-corpuscles are microscopic. The colorless are more uniform in size than the red; and generally smaller

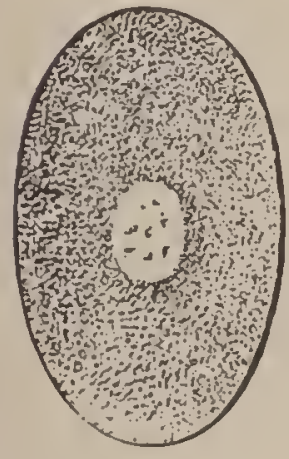
cle of the Frog, showing a white prominence at the centre. (except in Mammals), being about $\frac{1}{2500}$ of an inch in diameter. The red corpuscles are largest in Amphibians (those of Proteus being the extreme, or $\frac{1}{2} \frac{1}{5 \sigma}$ of an inch), next in Fishes, then Birds and Mammals. The smallest known are those of the Muskdeer. In Mammals, the size agrees with the size of the animal only within a natural order; but in Birds the correspondence holds good throughont the class, the largest being found in the Ostrich, and the smallest in the Humming-bird. In Man, they measure $\frac{1}{3 \frac{2}{2} \sigma}$ of an inch, so that it would take 40,000 to cover the head of a pin.

As to shape, the colorless corpuscles are ordinarily glob- 
ular, or sac-like, in all animals; but they are constantly changing. The form of the red disks is more permanent, although they are soft and elastic, so that they squeeze

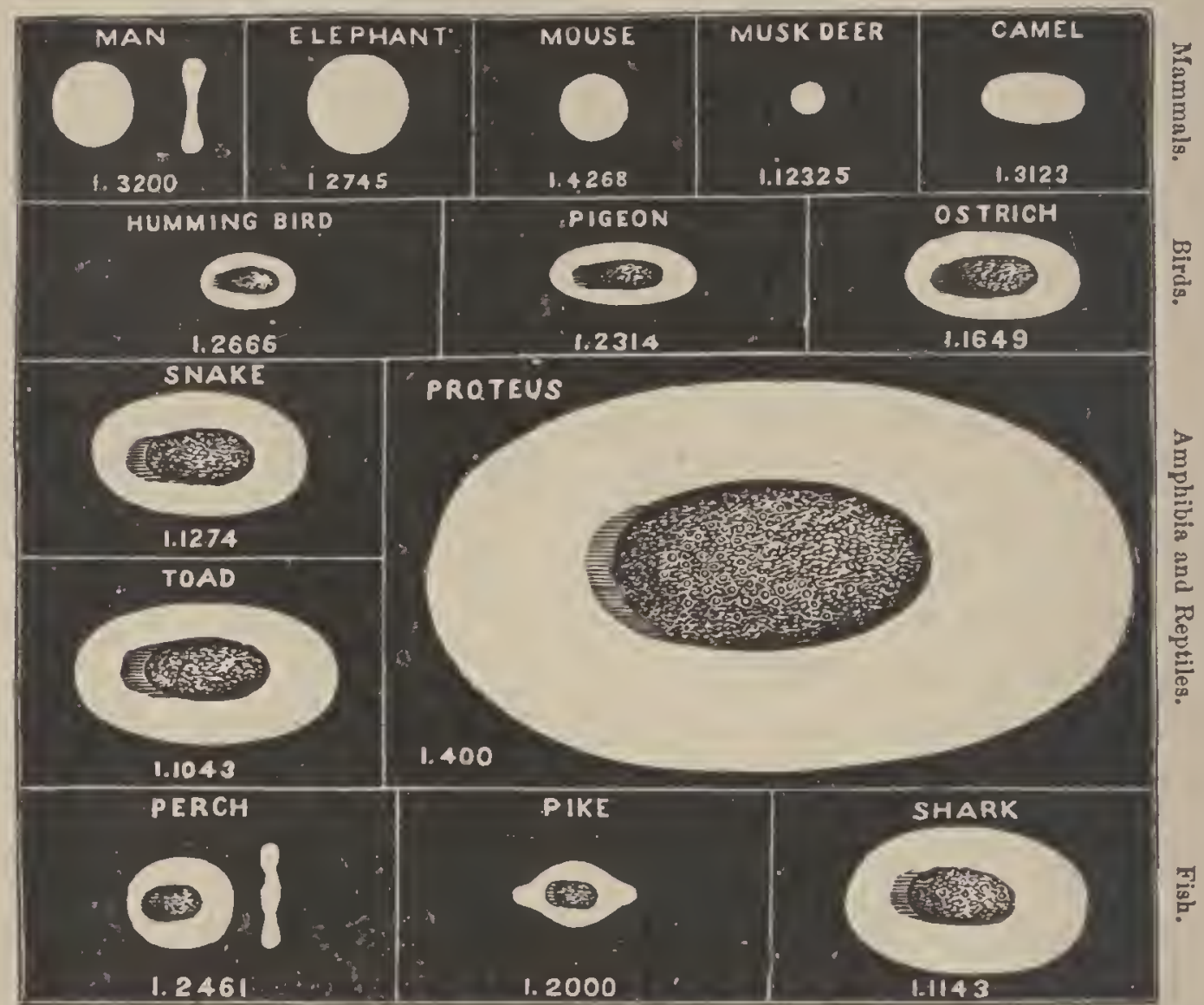

Fig. 65.-Comparative Size and Shape of the red Corpuscles of various Animals.

through very narrow passages. They are oval, circular, or angular, in Fishes; oval in Reptiles, Birds, and the Camel tribe; and circular in the rest of Mammals. They are double-convex when nucleated, and double-concare when circular and not nucleated.

Blood is always heavier than water; but is thinner in cold-blooded than in warm-blooded animals, in herbivores than in carnivores. The blood of Birds, which is the hottest known, being $10^{\circ}$ higher than Man's, is richest in red corpuseles. In Man, they constitute about one half the mass of blood. The white globules are far less numerous than the red; they are relatively more abundant in venous than arterial blood, in the sickly and ill-fed than in the healthy and vigorous, in the lower Vertebrates than in Birds and Mannmals. Their number is subject to great 
rariations, increasing rapidly after a meal, and falling as rapidly.

There is less blood in cold-blooded than in warm-blooded animals; and the larger the animal, the greater is the

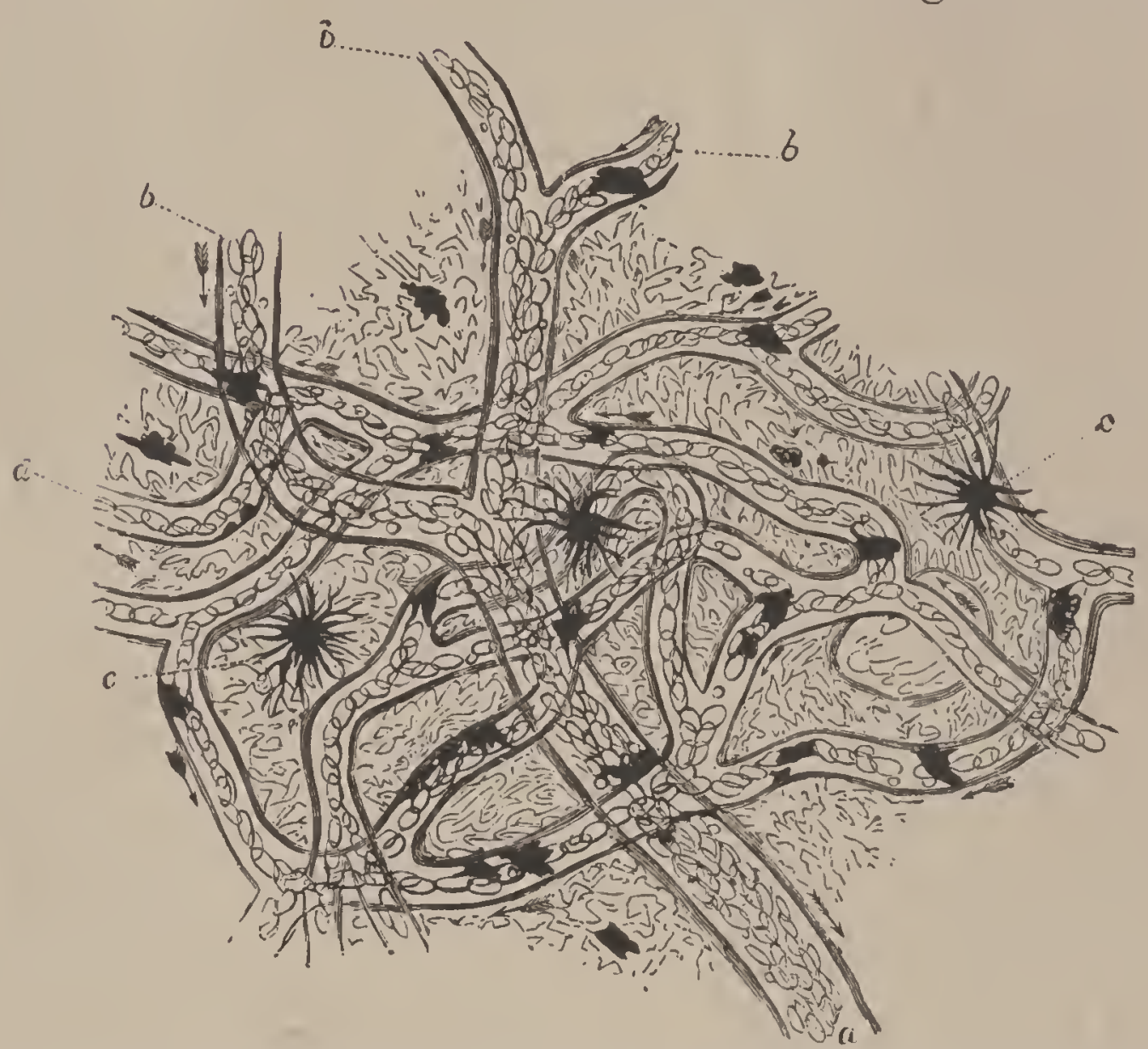

FIG. 66. - Capillary Circulation in the Web of a Frog's Foot, $\times 100: a, b$, small veins

$d$, capillaries in which the oval corpuscles are seen to follow one another in single series; $c$, pigment-cells in the skin.

proportion of blood to the body. Man has about a gallon and a half, equal to one thirteenth of his weight. The heart of the Greenland Whale is a yard in diameter.

The main Office of the Blood is to supply nourishment to, and take away waste matters from, all parts of the body. It is at once purveyor and scavenger. In its circulation, it passes, while in the capillaries, within an infinitesimal distance of the varions tissnes. Some of the plasma, carrying the nutritive matter needed, exudes through the walls of the capillary tubes; the tissue assimilates or makes like to itself whatever is suitable for its growth and repair; and the lymphatics take up the tran- 
suded fluid, and return it to the blood-vessels. At the same time, the waste products of the tissues are collected and brought through the venous capillaries, veins, and Jymphatics to the excretory organs. The special function of the several constituents of the blood is not wholly known. The colorless corpuscles in Vertebrates are supposed to be the source of the red disks. The latter are the carriers of oxygen, which is taken up by their red matter (hæmoglobin) in the lungs, and given up to the tissues. The same office is performed by the blue coloring-matter (hæmocyanin) in the blood of certain Invertebrates, as the Squid and Lobster. The carbon-dioxide is taken up mainly by the plasma.

Like the solid tissues, the blood, which is in reality a liquid tissue, is subject to waste and renewal, to growth and decay. The loss is repaired from the products of digestion, carried to the blood by the lacteals, or absorbed directly by the capillaries of the digestive tract. The white corpuscles are probably prepared in many parts of the body, especially the liver, spleen, and lymphatic glands. In the lower organisms, the nutritive food is prepared by contact with the tissues, without passing through special organs. Lymph differ's from blood chiefly in containing less albumen and fibrin, and no red disks. Chyle is lymph loaded with fat globules, and is found in the lacteals and vessels connected with them during the absorption of food containing fat. 


\section{CHAPTER XIII.*}

THE CIRCULATION OF THE BLOOD.

The Blood is kept in continual motion in order to nourish and purify the body and itself. For as life means work, and work brings waste, there is constant need of fresh material to make good the loss in every part of the system, and of the removal of matter which is no longer fit for use.

In the very lowest animals, where every part of the structure is equally capable of absorbing the digested food and is in contact with it, there is no occasion for any circulation, although even in them the digested food is not allowed to stagnate. But in proportion as the power of absorption is confined to certain parts, the mole is the need and the greater the complexity of an apparatus for conveying the nutritive fluid to the various tissues.

In nearly all animals, the nutritive fluid is conveyed to the various parts of the body by a system of tubes, called blood-vessels. The higher forms

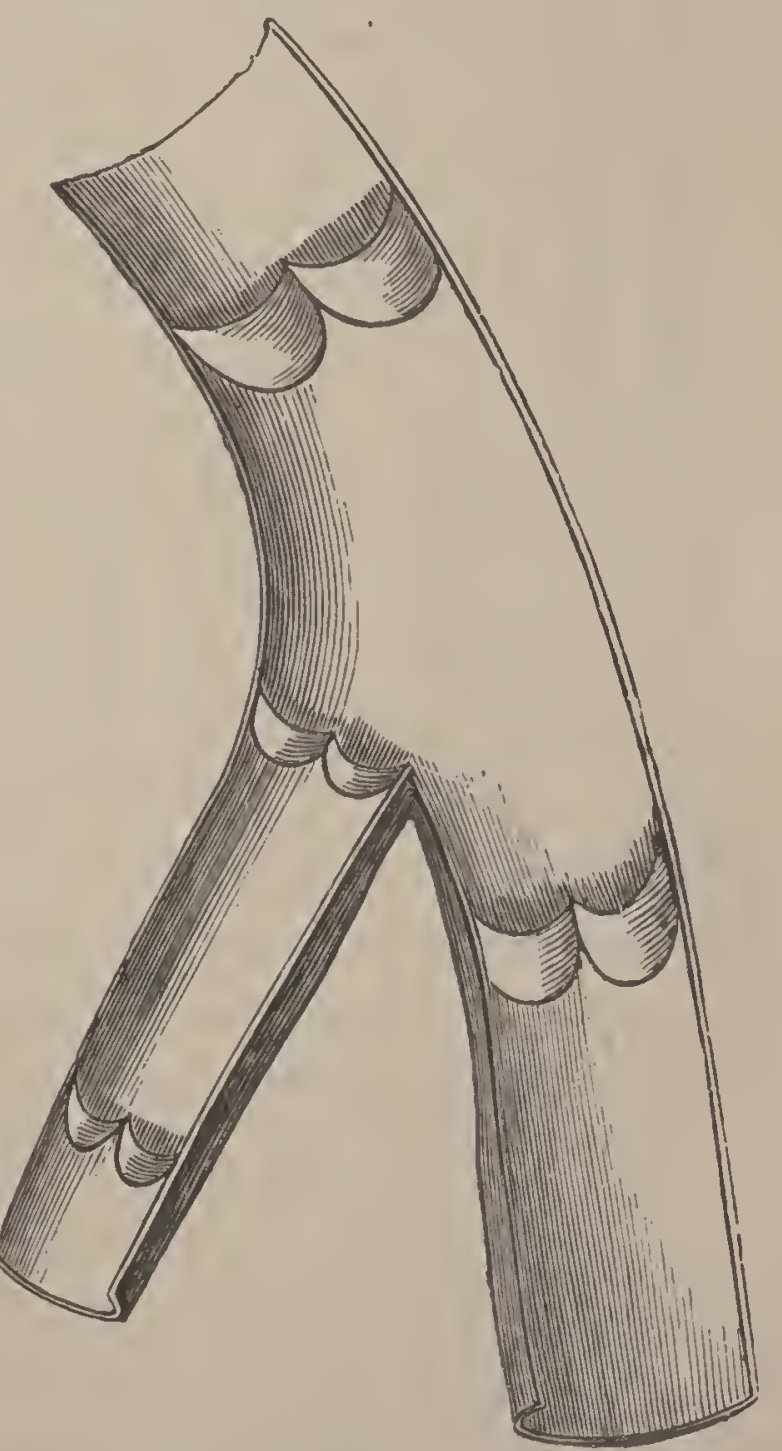

Fra. 67. - Veuous Valves. They usually occur in pairs, as represented.

* See Appendix. 
have two sets-arteries and veins, in which the blood moves in opposite directions, the former carrying blood

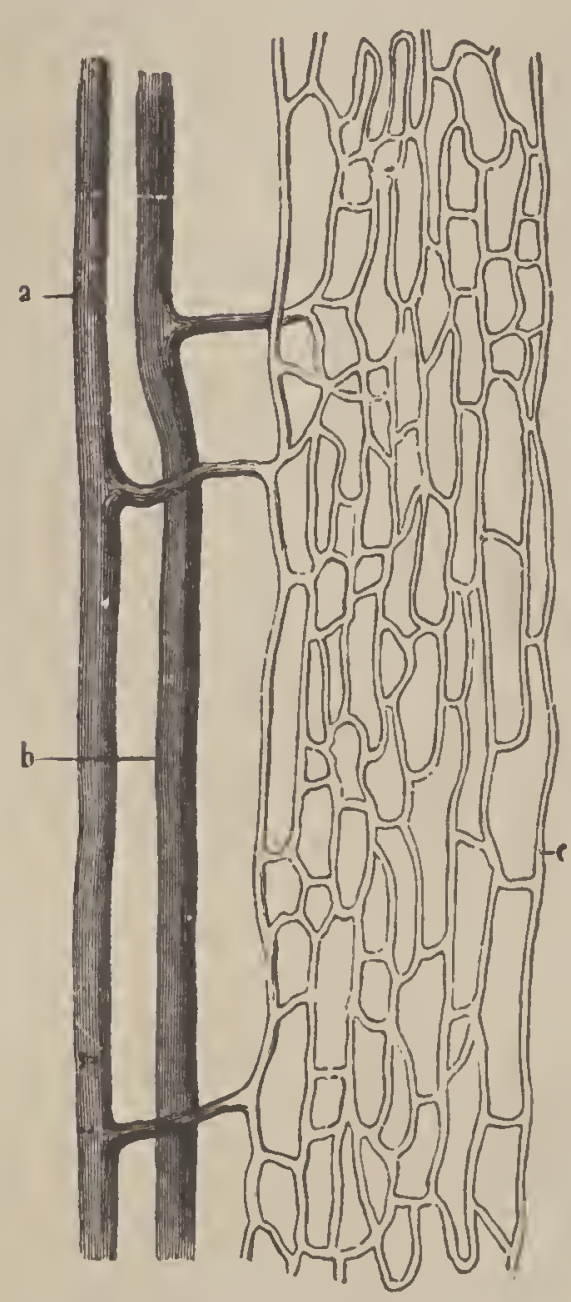
from a central reservoir or heart, the latter taking it to the heart. In the Vertebrates, the walls of these tubes are made of three coats, or layers, of tissue, the arteries being elastic, like rubber, and many of the veins being furnished with valves. ${ }^{56}$ The great artery coming out of the heart is called aorta, and the grand venous trunk, entering the heart on the opposite side, is called vena cava. Both sets divide and subdivide until their branches are finer than hairs; and joining these finest arteries and finest veins are interınediate microscopic tubes, called capillaries (in Man about $\frac{1}{3000}$ of an inch Fig. 65. - Relation of artery, $a$, in diameter). ${ }^{60}$ In these only, so
vein, $b$, and capillaries, $c$, as seen in the muscles of a Dog. thin and delicate are their walls, does the blood come in contact with the tissues or the air.

In those Vertebrates which have lungs there are two sets of capillaries, since there are two circulations-the systemic, from the heart around the system to the heart again, and the pulmonary, from the heart through the respiratory organ back to the heart. This double course may be illustrated by the figure 8 . In gill-bearing animals there are capillaries in the gills, but not a double circulation.

There is no true system of blood-vessels below the Star-fish. The simplest provision for the distribution of the products of digestion is shown by the Jelly-fish, whose stomach sends off radiating tubes (Fig. 196), through which the digested food passes directly to the various parts of 
the body instead of being carried by the agency of a circulating medium-viz., the blood.

The first Approach to a Circulatory System is made by the Star-fish and the Sea-urchin. A vein runs along the whole length of the alimentary tube, to absorb the chyle, and forms a circle around each end of the tube. These circular vessels send off branches to various parts of the body; but as they are not connected by a net-work of capillaries, there can be no circuit (Fig. 39).

A higher type is exhibited by the Insects. If we examine the back of any thin-skinned Caterpillar, a long pulsating tube is seen running beneath the skin from one end of the body to the other. This dorsal vessel, or heart, as it is called, is open at both ends, and divided by valves into compartments, permitting the blood to go forward, but not backward. Each compartment communicates by a pair of slits, guarded by valves, with the body - cavity, so that fluids may enter, but cannot escape. "Circulation" is very simple. We have seen that the chyle exudes through the walls of the alimentary canal directly into the cavity of the abdomen, where it mingles with the blood already there. This mixed fluid is drawn into the dorsal tube through the valvular openings as it expands; and upon its contraction, all the side-valves are closed, and the fluid is forced towards the head. Passing out at the front opening, it is again diffused among and

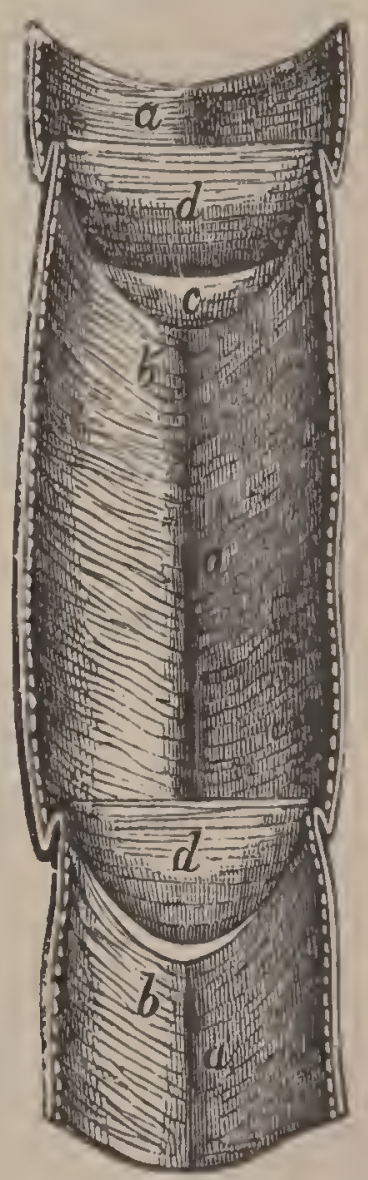

Fra. 69. - Part of the Dorsnl Vessel, or Heart, of a Cockchafer bisected: $a, b$, muscular walls: $d$, valves between the compartments ; $c$, valve defending one of the orifices communicating with the general cavily of the abdomen. between the tissues of the body. The blood, therefore, does not describe a circle in definite channels so as to return constantly to its point of departure. 
Many worms (as the Earth-worm) have a pulsating tube extending from tail to head above the alimentary canal, a similar trube on the ventral side through which the blood returns, and cross-tubes in every seginent. In the Lobster and Crab, Spider and Scorpion, the dorsal tube sends

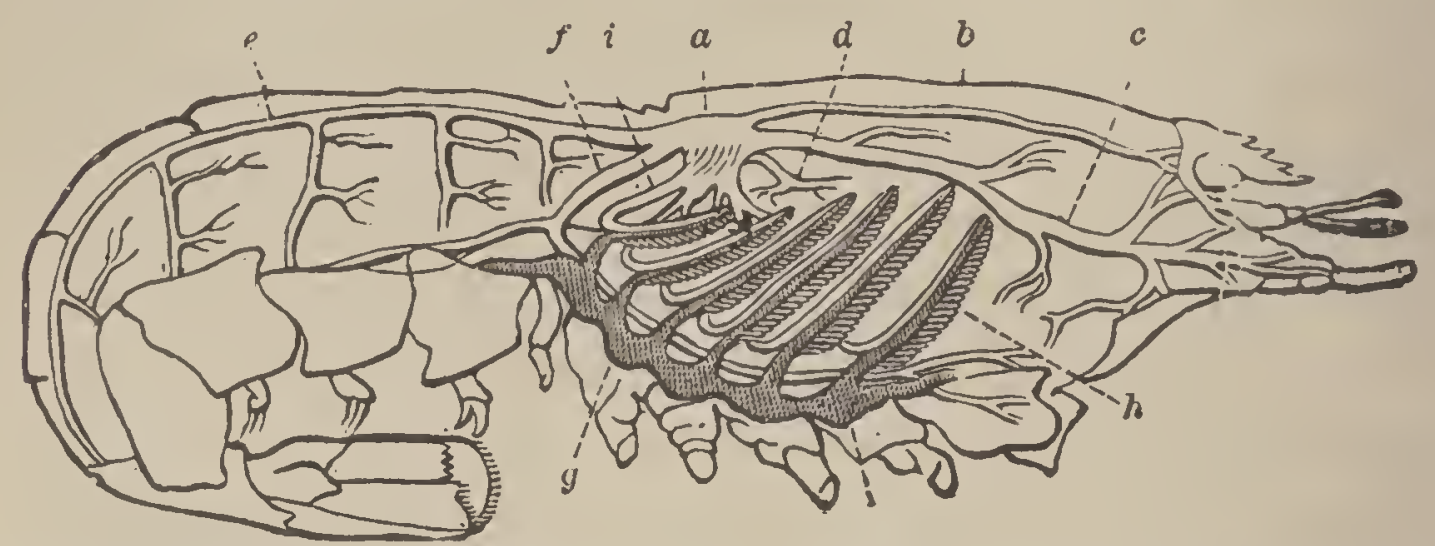

Fra. 70.-Circulation in a Lobster: $a$, heart: $b$, artery for the eyes; $c$, artery for antennæ; $d$, hepatic artery; $e$, superior abdominal artery $f$, sterual artery ; $g$, venous sinuses transmitting blood from the body to the branchia, $h$, whence it returus to the heart by the branchio-cardiac vessels, $i$.

off a system of arteries (not found in Insects); but the blood, as it leaves these tubes, escapes into the general cavity, as in other Arthropoda. The Lobster and Crab, however, show a great advance in the concentration of the propelling power into a short muscular sac.

A third development of the circulatory system is furnished by the Mollusks. Comparatively sluggish, they need a powerful force-pump in the form of a compact heart. In the Oyster and Snail (Figs. 44, 45), we find such an organ having two cavities-an auricle and a ventricle, one for receiving, and the other for distributing, the blood. The auricle injects the blood into the ventricle, which propels it by the arteries to the various organs. Thence it passes, not immediately to the veins, as in higher animals, but in to the spaces around the alimentary canal. A part of this is carried by vessels to the gills or lung, and then returned with the unpurified portion to the auricle. The whole of the blood, therefore, does not make a complete circuit. The Clam has a similar heart, but with two auricles. 
A still higher form is seen in the Cuttle-fish, the high. est of the Invertebrates. This animal has a central heart, with a ventricle and two auricles, and, in addition, the veins which collect the blood from the system to send it back to the heart by the way of the gills are furnished with two branchial hearts, which accelerate the circulation through those organs. Many of the arteries and veins are joined by capillaries, but not all; so that in no invertebrate animal is the blood returned to the heart by a continuous closed system of bloodvessels.

As a rule, in all animals having any circulation at all, the current always takes one direction. This is generally necessitated by valves. But a curious exception is presented by the Ascidians, whose tubular heart is valveless, and the contractions occur alternately at one end and then the other; so that the blood oscillates to and fro, and a given vessel is at one time a vein and at another an artery. In this respect it resembles the fœtal heart of higher animals (Fig. 279).

In Vertebrates only is the circulating current strictly confined

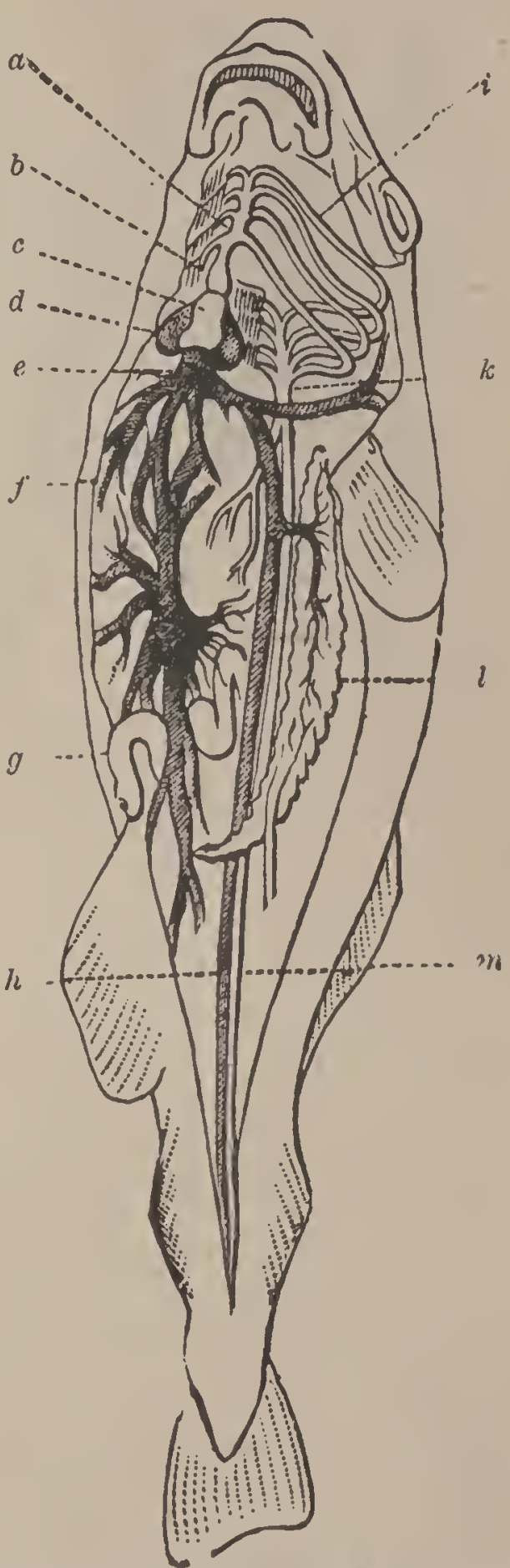

Fig. 71.-Circulating Apparatus in the Fish: $a$, branchial artery ; $b$, arterial bulb; $c$, ventricle; $d$, auricle; $e$, venous sinus; $f$, portal vein ; $g$, Intestine; $h$, vena cava ; $i$, branchial vessels ; $k$, dorsal ar. tery, or aorta; $l$, kidueys; $m$, dorsal artery. to the blood-vessels; in no case does it escape into the general cavity of the body. In other respects, there is 
no great advance in the apparatus of the lowest Vertebrates over that of the highest Mollusks. A Fish's heart

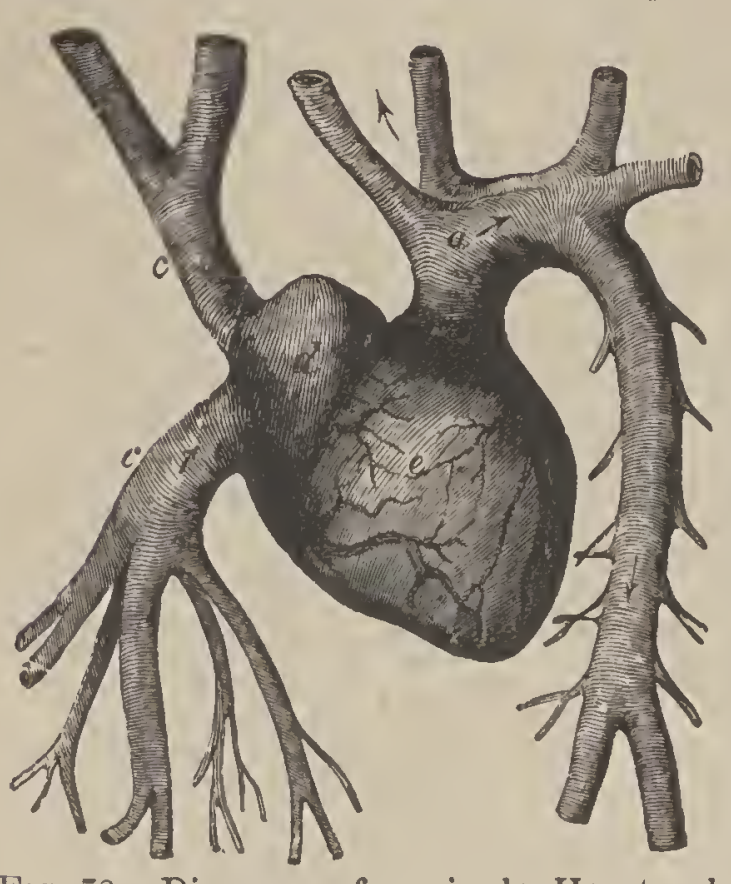

FIG. $72 .-D i a g r u m$ of a single Heart: $d$, auricle; $e$, ventricle; $c$, veins leading to auricle; $a$, aorta, or main artery. has, like that of an Oyster, two cavities, but its position is reversed. Instead of driving arterial blood over the body, it receives the returning, or venous, blood, and sends it to the gills. Recollected from the gills, the blood is passed into a large artery, or aorta, along the back, which distributes it by a complex system of capillaries among the tissues. These capillaries unite with the ends of the veins which pass the blood into the auricle $^{6 n}$ (Figs. 71, 75).

In Amphibians and in Reptiles generally (as Frogs, Snakes, Lizards, and Turtles), the heart has three carities -two auricles and one ventricle. The venous blood from the body is received into the right auricle, and the purified blood from the lungs into the left. Both throw their contents into the ventricle, which pumps the mixed blood in two directions-partly to the lungs, and partly around the system (Fig. 76). Circulation is, therefore, incomplete, since the whole current does not pass through the lungs, and three kinds of blood are found in the body-arterial, venous, and mixed. In many animals, however, arrangements exist which nearly separate the venous from the arterial blood.

The ventricle of Reptiles is partially divided by a partition. In the Crocodile, the division is complete, so that there are really four cavities-two auricles, and two ventricles. But hoth ventricles send off aortas which cross 
one another, and at that point a small aperture brings the two into communication. The venous and arterial currents are, therefore, mixed, but not within the heart, as in the other Reptiles, nor so extensively. In the structure of the heart, as well as in that of the gizzard, Crocodiles approach the Birds.

The Highest Form of the Circulating System is pussessed by the warm-blooded Vertebrates, Birds and Mammals. Not a drop of blood can inake the circuit of the body without passing through

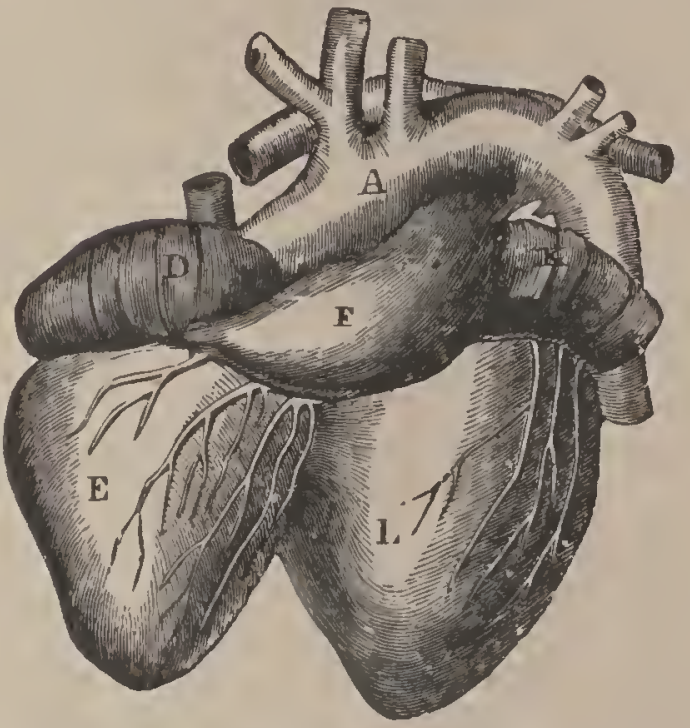

Fig. 73.-Heart of the Dugong, in fourchambered heart, the parts being more separated than in higher animals: $\mathrm{E}$, right ventricle; $L$, left veutricle; 1 , right auricle; $\mathrm{F}$, pulmonary artery: $\mathrm{K}$, left auricle; $\mathbf{A}$, aorta. the lungs, the circulation to and from those organs being as perfect as the distribution of arterial blood. The heart

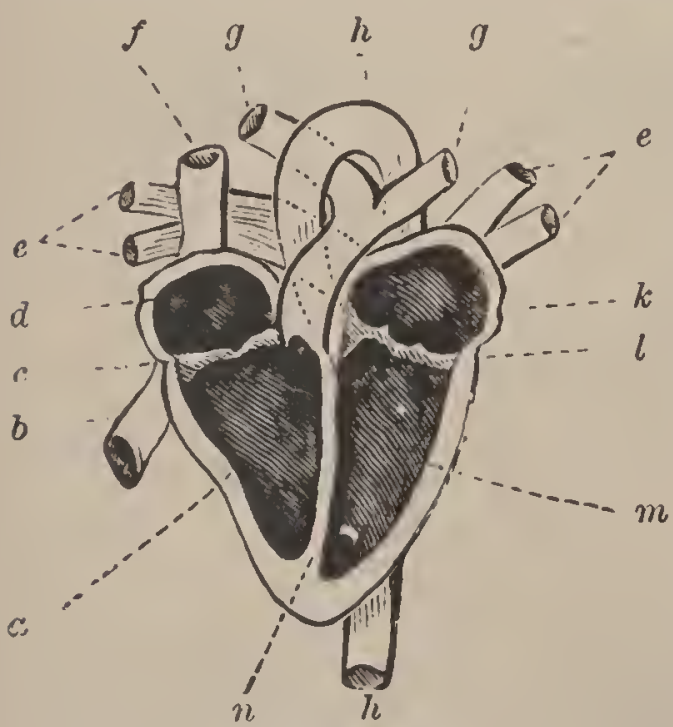

Fis. 74. - 'Theoretical Section of the Human Heart: $a$, right ventricle; $b$, iuferior vena cava; $c$, tricuspia valve; $d$, right auricie; $e$, pulmonary veins; $f$, superior vena cava; $g$, pulmonary arteries; $h$, aorta $; k$, left auricle; $l$, mitral valve; $m$, left ventricle; $n$, septum.

consists of four cavities - a right auricle and ventricle, and a left auricle and ventricle. In other words, it is a hollow muscle divided internally by a vertical partition into two distinct chambers, each of which is again divided by a valve into an auricle and a ventricle. The work of the right auricle and ventricle is to receive the blood from the veins, and send it to the lungs; while the other two receive the blood from the lungs, and propel it over the body. The left ventricle has more to do than any other cavity. The two auricles contract at the same instant; 


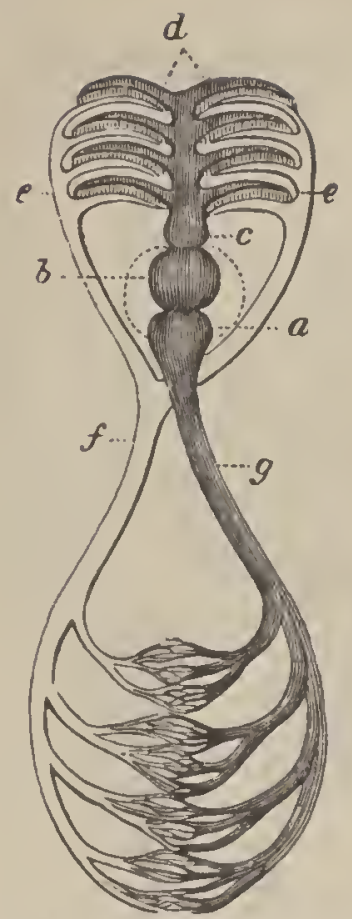

so also do the ventricles. The course of the current in Birds and Mammals is as follows: the venous blood brought from the system is discharged by two or three large trunks ${ }^{61}$ into the right auricle, which immediately forces it past a valve ${ }^{62}$ into the right ventricle. The ventricle then contracts, and the blood rushes through the pulmonary artery past its semilunar valves into the lungs, where it is changed from venous to arterial,

Fr. 75 . - Plan of Circula- returning by the pulmonary veins to tion in Fishes: $a$, auricle : $b$, ventricle : $c$, branchial artery ; $e$, branchial veins, bringing blood from the gills, $d$, and uniting iu the aorta, $f ; g$, the left auricle. This sends it past the mitral valves into the left ventricle, which drives it past the semilunar valves into the aorta, and thence, by vena cava.

its ramifying arteries and capillaries, into all parts of the body except the lungs. From the systemic capillaries, the blood, now changed from arterial to venous, is gathered by the veins, and conveyed back to the heart.

The Rate of the Blood-current generally increases with the activity of the animal, being most rapid in Birds. ${ }^{63} \quad$ In Insects, however, it is comparatively slow; but this is because the air is taken to the blood-the whole
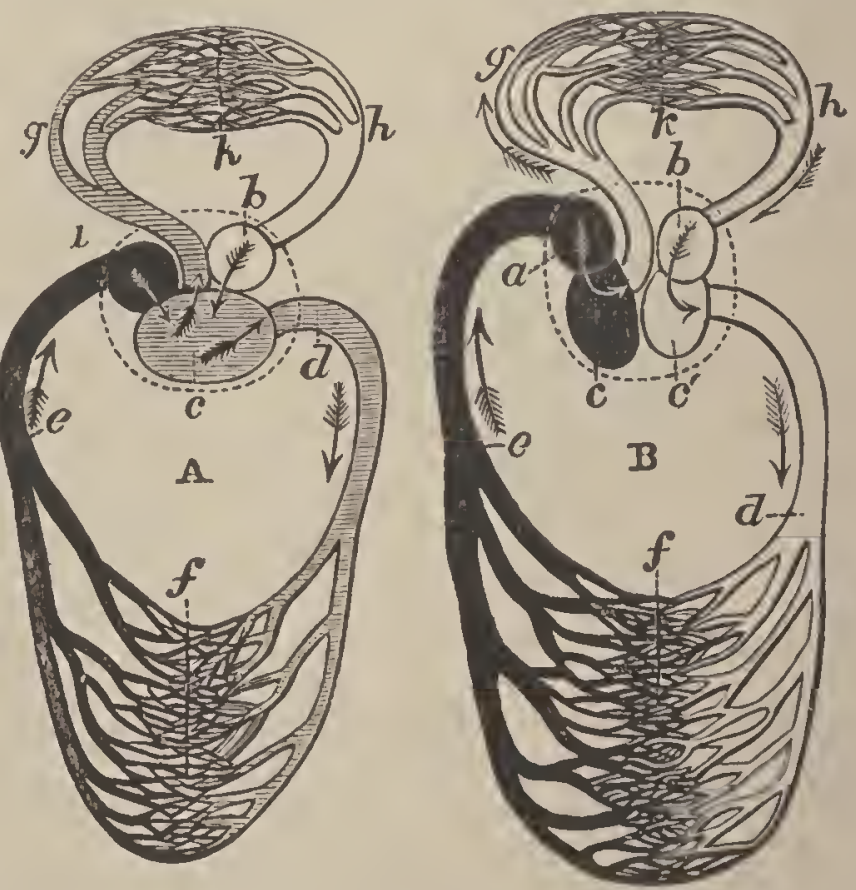

Fig. 76.-A, Plan of Circulation in Amphilia and Reptiles; B, Plan of Circulation in Birds and Mammals: $a$, right auricle receiving venous blood from the system: $b$, left auricle receiving arterial blood from the lnngs; $c, c^{\prime}$, ventricles: $d, e, f$, systemic artery, vein, and capillaries; $g$, pulmonary artery; $h, k$, vein and capillaries. 
body being bathed in air, so that the blood has no need to hasten to a special organ. However, activity nearly doubles the rate of pulsation in a Bee. The motion in the arteries is several times faster than in the veins, but diminishes as the distance from the heart increases. In the carotid of the Horse, the blood moves $12 \frac{1}{2}$ inches per second; in that of Man, 16 ; in the capillaries of Man, 1 to 2 inches per minute; in those of a Frog, 1.

- The Cause of the Blood-current may be cilia, or the contractions of the body, or pulsating tubes or hearts. In the higher animals, the impulse of the heart is not the sole means: it is aided by the contractions of the elastic walls of the arteries themselves, the movements of the chest in respiration, and the attraction of the tissues for the arterial blood in the capillaries. In the Chick, the blood moves before the heart begins to beat; and if the heart of an animal be suddenly taken out, the motion in the capillaries will continue as before. It has been estimated that the force which the human heart expends in twenty-four hours is about equivalent to lifting 217 tons one foot.

\section{CHAPTER XIV.*}

HOW ANIMALS BREATHE.

Arterial Blood, in passing through the system, both loses and gains certain substances. It loses constructive material and oxygen to the tissues. These losses are made good from the digestive tract and breathing organ. It gains also certain waste materials from the tissues, which must be got rid of. Of these waste products, one, carbon dioxide, is gaseous, and is passed off from the same organ as that where the oxygen is taken in. This exchange of * See Appendix. 
gases between the animal and its surroundings is called Respiration.

The First Object of Respiration is to convert venous into arterial blood. It is done by bringing it to the surface, so that carbon dioxide may be exhaled and oxygen absorbed. The apparatus for this purpose is analogous to the one used for circulation. In the lowest anirrals, the two are combined. But in the highest, each is essentially a pump, distributing a fluid (in one case air, in the other blood) through a series of tubes to a system of cells or capillaries. They are also closely related to each other: the more perfect the circulation, the more careful the provision made for respiration.

Respiration is performed either in air or in water. So that all animals may be classed as air-breathers or water-breathers. The latter are, of course, aquatic, and seek the air which is dissolved in the water. Land-snails, Myriapods, Spiders, Insects, Reptiles, Birds, and Mammals breathe air directly; the rest, with few exceptions, receive it through the medium of water. In the former case, the organ is internal; in the latter, it is more or less on the outside. But however varied the organs-tubes, gills, or lungs -they are all constructed on the same principle-a thin membrane separating the blood from the atmosphere.

(1) Protozoa, Sponges and Polyps have no separate respiratory apparatus, but absorb air, as well as food, from the currents of water passing throngh them or bathing the surface of their bodies.

In the Star-fish, Sea-urchin, and the like, we find the first distinct respiratory organs, although none are exclusively devoted to respiration. There are two sets of canals - one carrying the nutrient fluid, and the other, radiating from a ring around the mouth, distributing aerated water, used for locomotion as well as respiration. This may be called the "water-pipe system." Besides this, 
there are sometimes numerous gill-like fringes, which cover the surface of the body and probably aid in respiration (Fig. 39).

Fresh-water Worms, like the Leech and Earth-worm, breathe by the skin. The body is always covered by a viscid fluid, which has the property of absorbing air. The air is, therefore, brought into immediate contact with the soft skin, underneath which lies a dense net-work of blood-vessels.

But most water-breathing animals have gills. The simplest form is seen in Marine Worms: delicate veins projecting through the skin make a series of arborescent tufts along the side of the body; as these float in the water, the blood is purified. ${ }^{64} \mathrm{Bi}$ valve Mollusks have four flat gills, consisting of delicate membranes filled with bloodvessels and covered with cilia. In the Oys- Fra. 77.-Lob-worm ter, these ribbon-like folds are exposed to

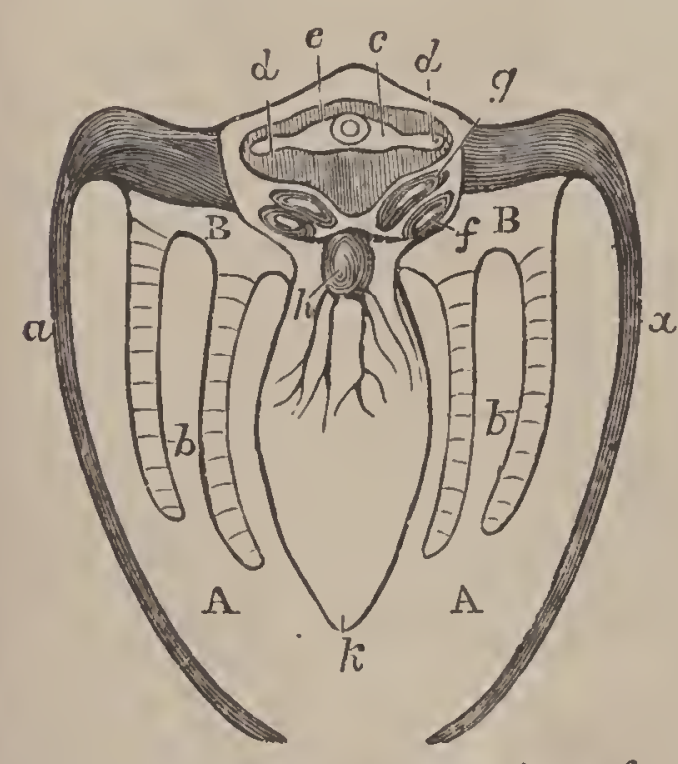

Fia. 7S.-Diagrammatic Section of a Lamellibranch (Anodon): $\alpha$, lobes of mantle; $b$, gills, showing transverse partitions; $c$, veutricle of hoart; $d$, auricles: $e$, pericardium ; $f, g$, kidneys; $h$, venons sinus; $k$, foot: $\mathrm{A}$, branchial, or pallial, chamber; $\mathrm{B}$, epibranchial chamber. the water when the shell opens; but in the Clam, the mantle en-

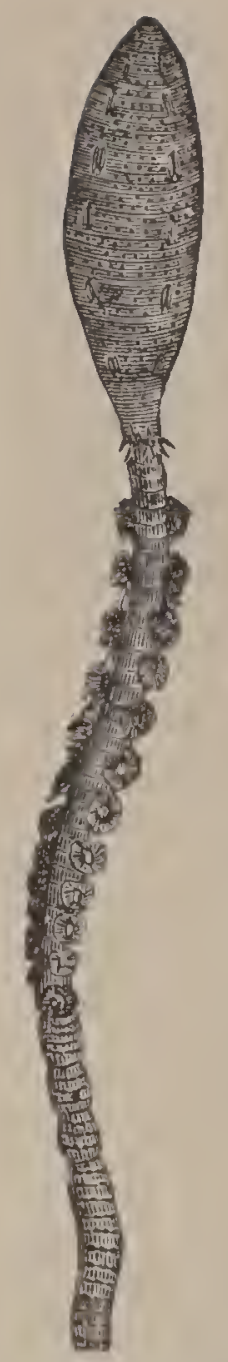

(Arenicola piscatorum), a dorsibran. cliate, showing the tufts of capit laries, or exterun! gills. The large head is without eyes or jaws. closes them, forming a tube, called siphon, through which the water is driven by the cilia. The aquatic Gasteropods (Univalves) have either tufts, like the Worms, or comblike ciliated gills in a cavity behind the head, to which the water is admitted by a siphon. The Cuttle-fish has flat gills covered by the mantle; but the 
water is drawn in by muscular contractions of the mantle instead of by cilia. The end of the siphon through which it is ejected is called the funnel. The gills of Lobsters and Crabs are placed in cavities covered by the sides of the shell (carapace); and the water is brought in from behind by the action of a scoop-shaped process attached to one of the jaws, which constantly bales the water out at the front.

The perfection of apparatus for aquatic respiration is seen in Fishes. The gills are comb-like fringes supported on four or five bony or cartilaginous arches, and contain myriads of microscopic capillaries, the object being to expose the venous blood in a state of minute subdivision to streams of water. The gills are always covered. In bony fishes they are attached to the hinder side of bony arches, all covered by a flap of the skin, supported by bones (the gill-cover, or operculum), and the water escapes from the opening left at its hinder edge. In Sharks, the gills are placed in pouches which open separately (Figs. 164 and 287). The act of "breathing water" resembles swallowing, only the water passes over the surface of the gills instead of entering the gullet.

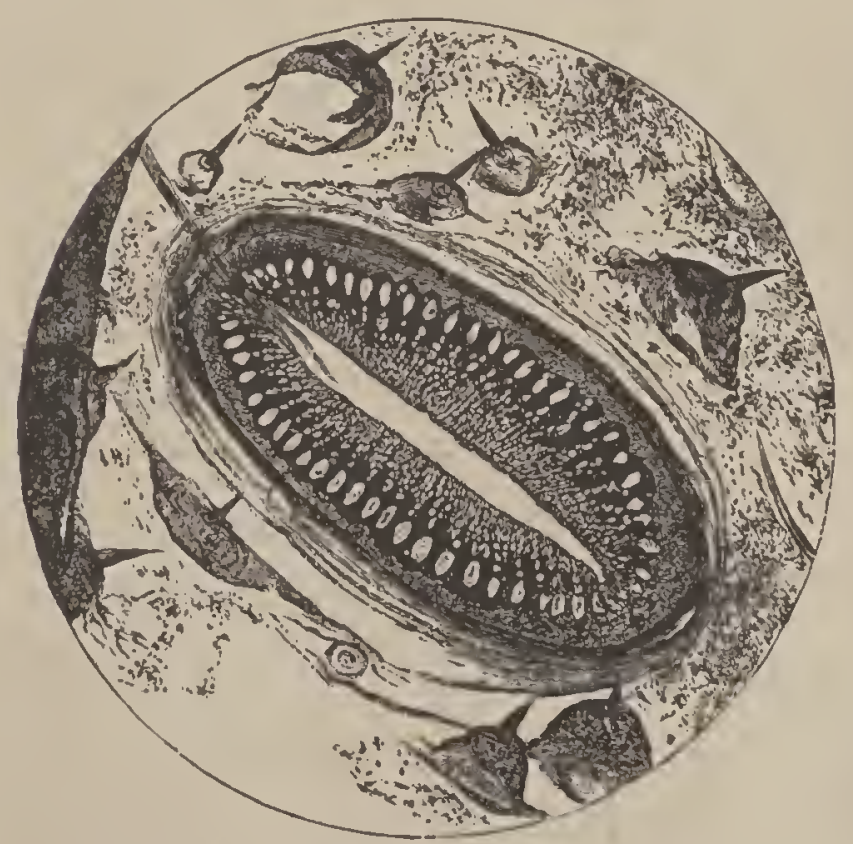

Fig. 79. - Spiracle of an Insect, $\times 75$.

(2) Air-breathers have trachece, or lungs. The former consist of two principal tubes, which pass from one end of the body to the other, opening on the surface by apertures, called spiracles, resembling a low of button-holes along the sides of the thorax and abdomen, and ramifying through the small. est and most delicate organs, so that the air may follow 
the blood wherever it circulates. To keep the pipes ever open, and at the same time leave them flexible, they are provided inside with an elastic spiral thread, like the rubber tube of a drop-light. Respiration is performed by the movements of the abdomen, as may be seen in the Bee when at rest. This "air-pipe system," as it may be termed, is best developed in Insects.

The "nerves" of an Insect's wing consist of a tube within a tube: the inner one is a trachea carrying air, and the outer one, sheathing it, is a blood-vessel. So perfect

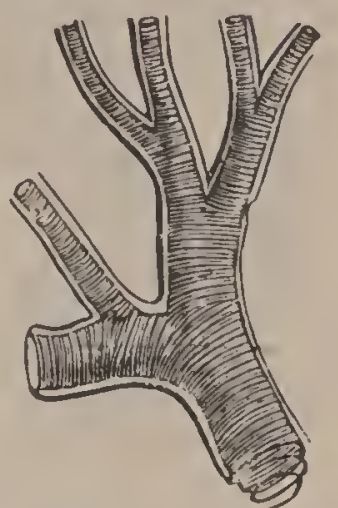

Fig. 80. - Tracheal Tube of an Insect, highly magrified, showing elastic spiral thread. is the aeration of the whole body, from brain to feet, the blood is oxygenated at the moment when, and on the spot where, it is carbonized; only one kind of fluid is,

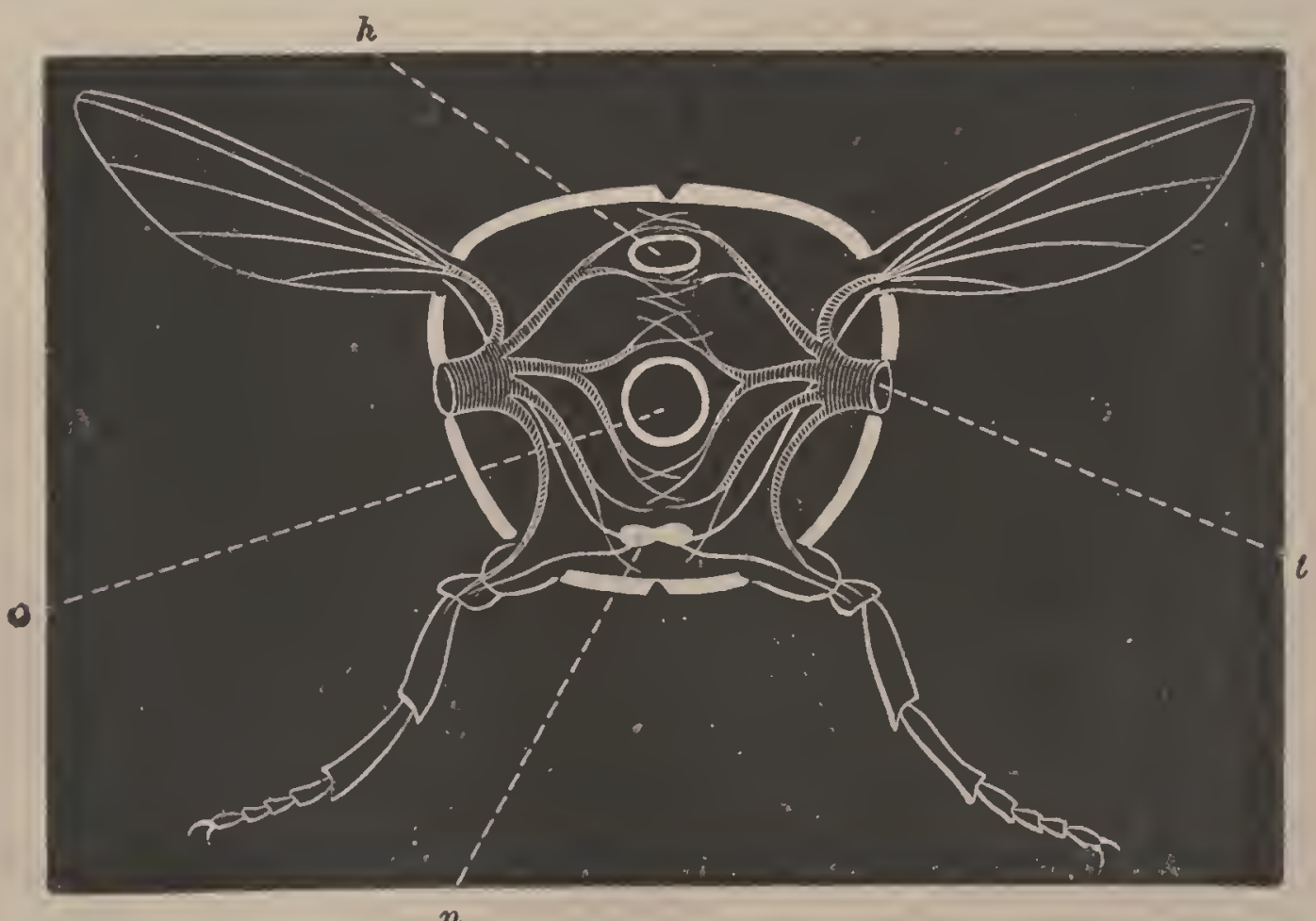

$n$

Fig. 81.-Ideal Section of a Dee: $a$, alimentary canal; $h$, dorsal vessel; $t$, trachea: $n$, nervous cord.

therefore, circulating - arterial. It is difficult to drown an Insect, as the water cannot enter the pores; but if a drop of oil be applied to the abdomen, it falls dead at once, being suffocated. The largest spiracle is usually 


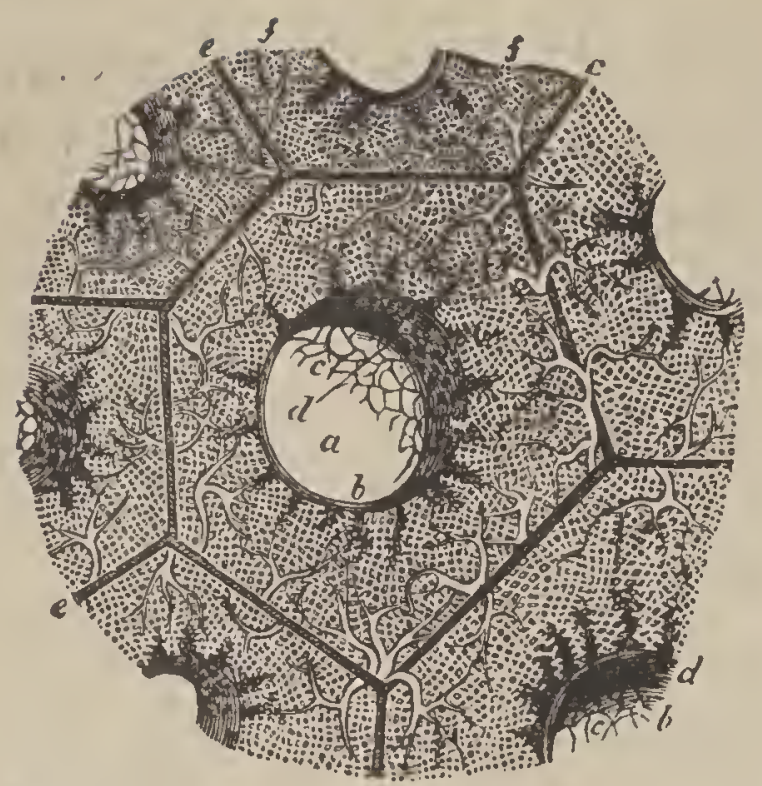

Fig. S2.-Section through' a brouchial tribe, Lung of a Bird, magnified: $a$, the cavity; $b$, its lining membrane supporting bloodressels; $c$, perforations at the orifices of the lobular passages, $d$; e, interlobular spaces, containing the terminal brauches of the pulmouary vessels supplying the capillary plexus, $f$, to the meshes of which the air rets access by the lobular passages.

found on the thorax, as under the wing of a Moth: such may be strangled by pinching the thorax.

In Millipedes and Centipedes, the spiracles open into little sacs connected together by tubes; in Spiders and Scorpions, the spiracles, usually four in number, are the months of sacs without the tubes, and the interior of the sac is gathered into folds. Landsnails have one spiracle, or aperture, on the left side of the neck, leading to a large cavity, or sac, lined with fine blood-vessels. These sacs represent the primitive idea of a ling, which is but an infolding of the skin, divided up into cells, and covered with capillary reins. ${ }^{65}$

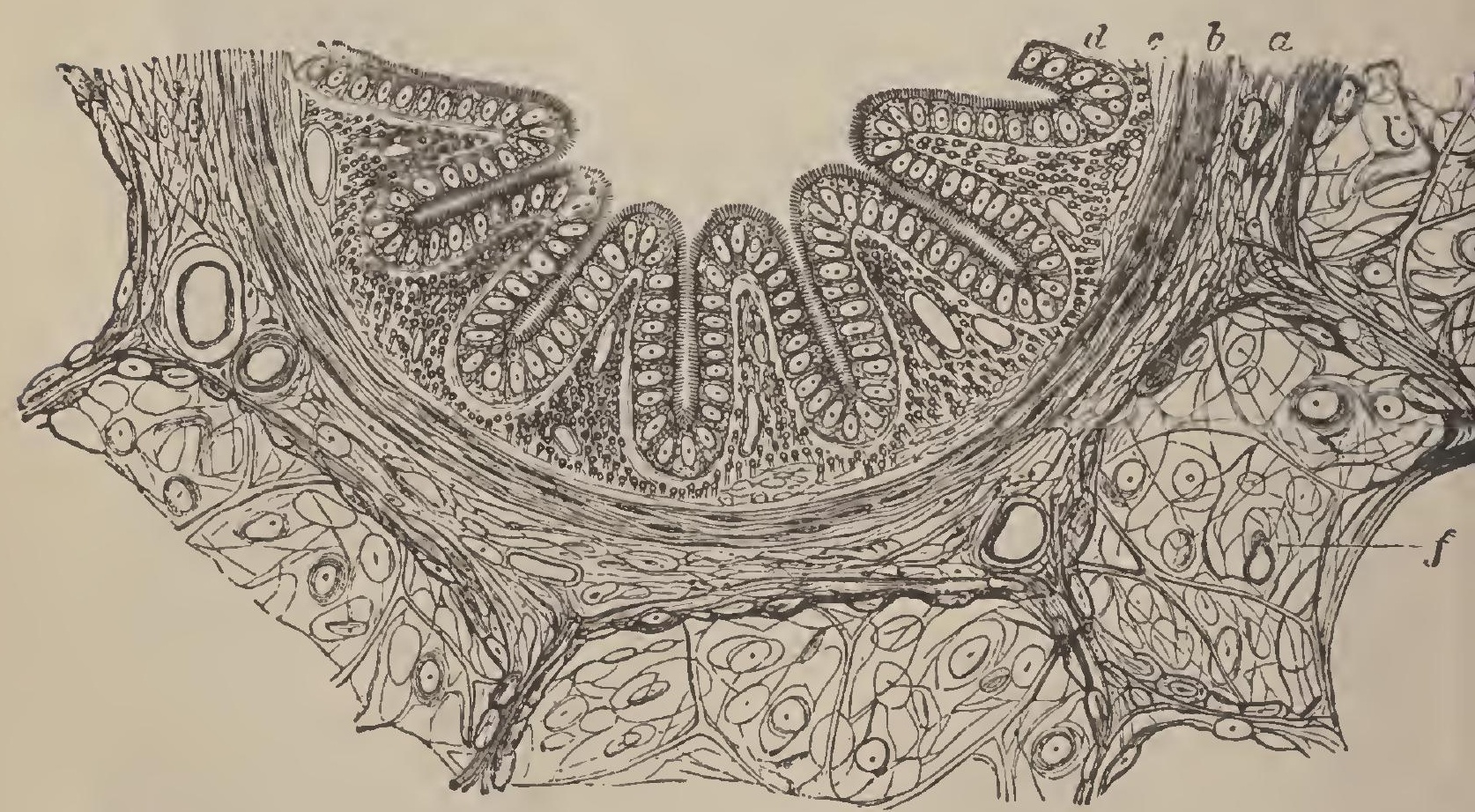

FIG. S3, - Part of a transverse section of a Pir's Bronchial Twig, $\times 24 n$ : a, outer fibrous layer; $b$, muscular layer; $c$, inner fibrous layer; $d$, epithelial layer with cilia; $f$, one of the neighboring alveoli. 
Like the alimentary canal, the lungs of an animal are really an inflected portion of the onter surface; so that breathing by the skin and breathing by lungs are one in principle. Indeed, in many animals, especially Frogs, respiration is carried on by both lungs and skin.

The lungs of Vertebrates are derived from the front part of the alimentary canal. In some Fishes, air is swallowed, which passes the whole length of the digestive tract, and is expelled from the anus. Here the whole canal serves for respiration. In Reptiles, Birds, and Mammals the hinder part of the intestine develops an outgrowth (the allantois) during embryo-life which serves as the embryo's breathing organ (Figs. $170,171)$.

All Vertebrates lave two kinds of respiratory organs in the course of their life. Fishes have gills; their lung (the air-bladder) rarely serves as a functional respiratory organ, and is sometimes wanting. Amphibians have gills while in the larval state. Some keep them throughout life; but all develop functional lungs, and also breathe by means of the skin.

In the remaining Vertebrates, the allantois is the breathing organ of the embryo, and the lung is the breathing organ of the adult. The skin is of small or no importance in respiration.

The lungs of Vertebrates are elastic membranous sacs, divided more or less into cavities (the air-cells) to increase the surface. Upon the walls of the air-cells are spread the capillary blood-vessels. The smaller the cells, the greater the extent of surface upon which the

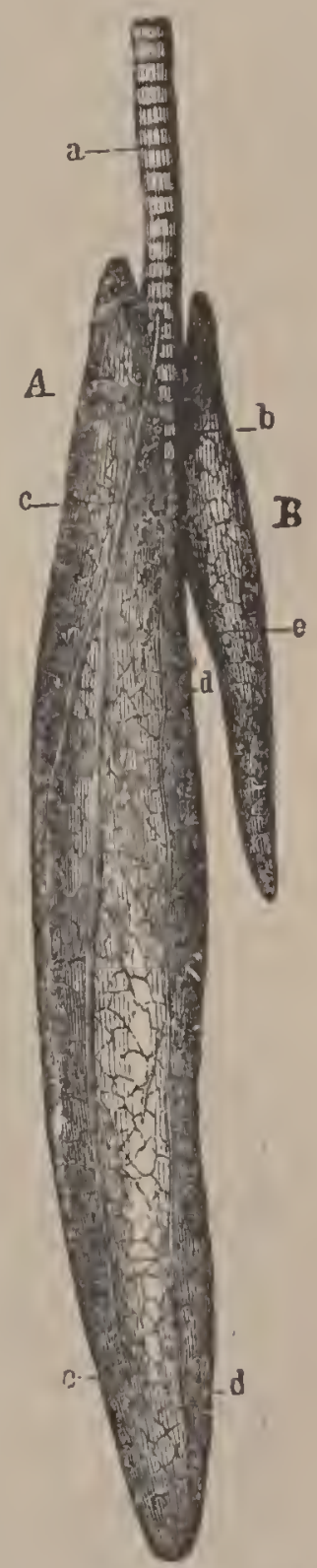

Frg. 84. - Lunge of a Suake: $a$, trachen; $b$, its bifurcation; $c$, pulmonary artery ; $d$, pulmonary vein; the lung $\mathrm{B}$, is rudimentary. 
blood is exposed to the influence of the air, and, therefore, the more active the respiration and the purer the blood. The lungs are relatively largest in Reptiles, and smallest in Mammals. But in the cold-blooded Amphibians and Reptiles, the air-cells are few and large; in the warm-blooded Birds and Mammals, they are exceedingly numerous and minute. ${ }^{66}$ In Birds and Marnmals, the blood in the capillaries is exposed to the air on all sides; in the Reptiles, on one only. Respiration is most perfect in Birds; they require, relatively to their weights, more air than Mammals or Reptiles, and most quickly die for lack of it. In Birds, respiration is not confined to the lungs; but, as in Insects, extends through a great part of the body. Airsacs connected with the lungs exist in the abdomen and under the skin of the neck, wings, and legs. Even the bones are hollow for this purpose; so that if the wind-

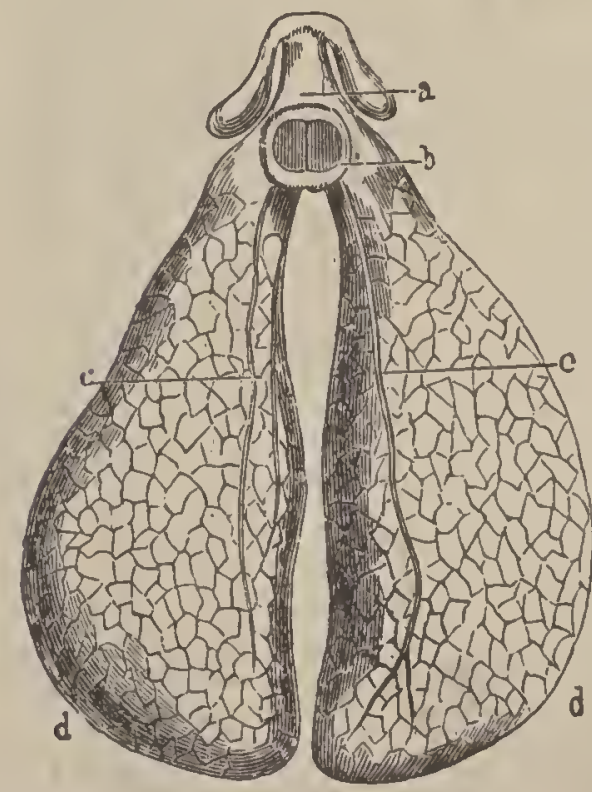

Frg. S5.-Lungs of a Frog: $a$, hyoid apparatus : $b$, cartilaginous ring at ront of the lungs; $c$, pulmonary vessels; $d$, pulmomary sacs, having this peculiarity common to all coldblooded air-breathers, that the trachea does not divide into bronchial branches, but terminates abruptly by orifices which open at once into the general cavity. A curtilaginous net-work divides the space in to littie sacs, on the walls of which the capillaries are spread.

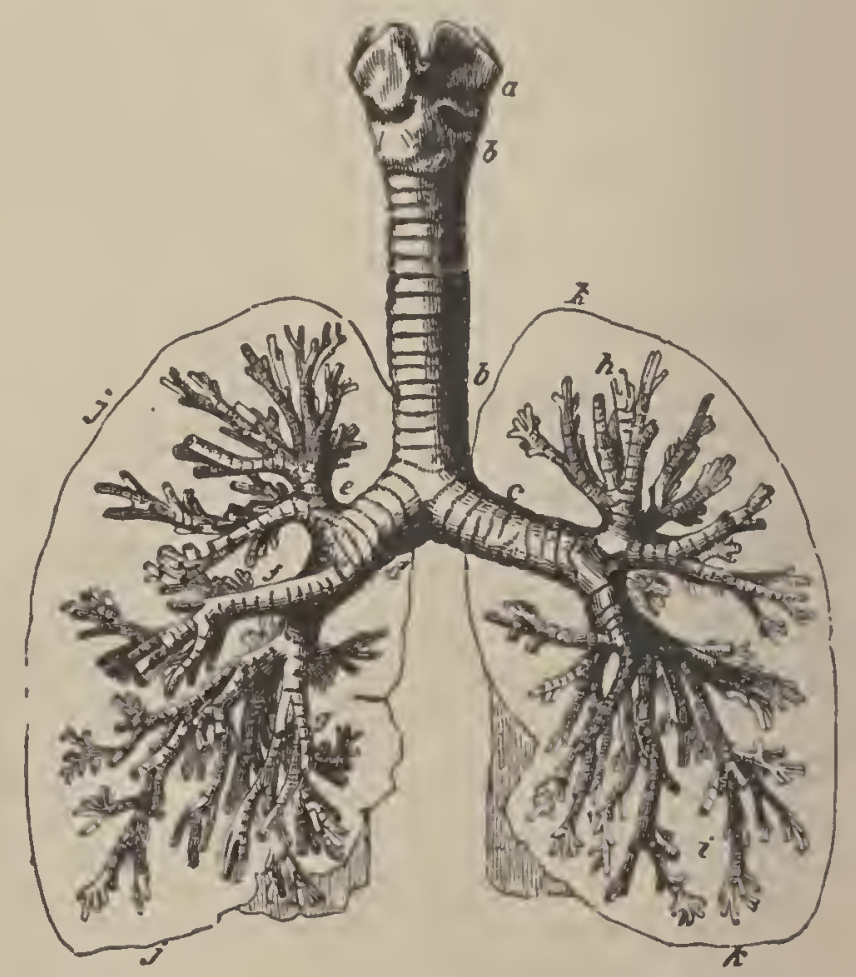

Fra. S6. - Distribution of Air - tubes in Mammalian Lungs : $a$, larynx; $b$, trachea; $c, d$, left and right brouchial tubes: $e, f, g$, the ramifications. In Man the suldivision continues until the ultimate tubes are one twenty-fifth of an inch in diameter. Each lobule represents in miniature the structure of tbe entire lung of a Frog. 
pipe be tied, and an opening be made in the wing-bone, the bird will continue to respire. The right lung is usually the larger; in some Snakes, the left is wanting entirely. In most Vertebrates, lungs are freely suspended; in Birds, they are fastened to the back.

The lungs communicate with the atmosphere by means, of the trachea, or windpipe, formed of a series of cartilaginous rings, which keep it constantly open. It begins in the back part of the mouth, opening into the pharynx by a slit, called the glottis, which, in Mammals, is protected by the valve-like epiglottis. The trachea passes along the neck in front of the cesophagus, and divides into two branches, or bronchi, one for each lung. In Birds and Mammals, the bronchial tubes, after entering the lungs, subdivide again into minute ramifications.

Vertebrates are the

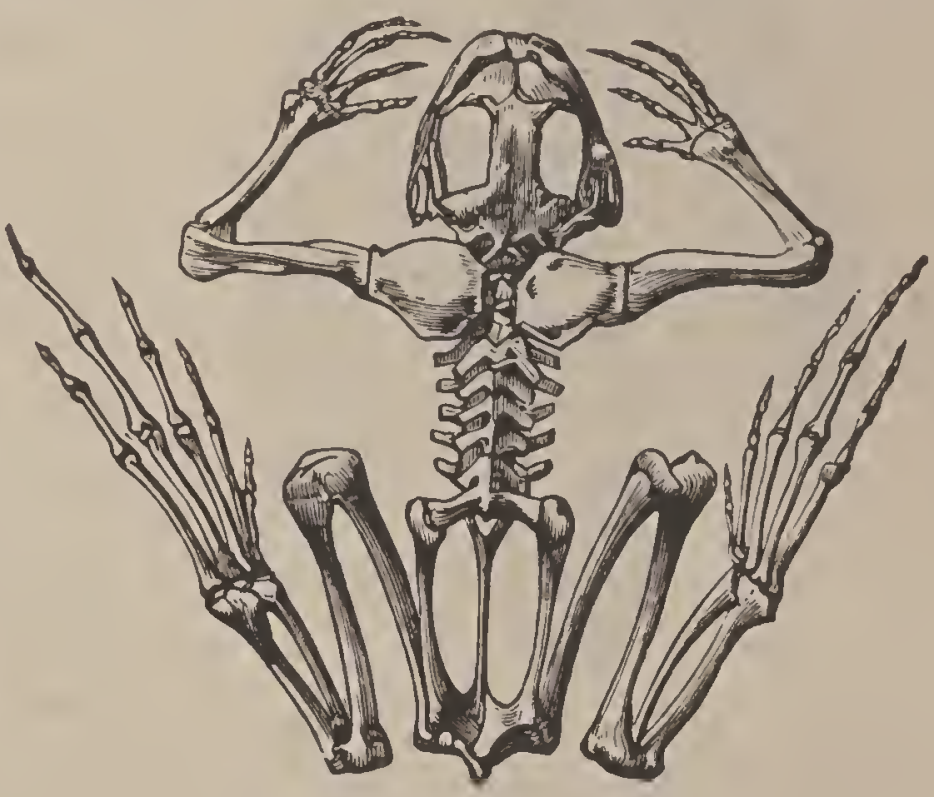

Fra. 87.-Skeleton of a Frog. only animals that breathe through the mouth or nostrils. Frogs, laving no ribs, and Turtles, whose ribs are soldered together into a shield, are compelled to swallow the air. Snakes, Lizards, and Crocodiles draw it into the lungs by the play of the ribs. ${ }^{67}$ Birds, unlike other animals, do not inhale the air by an active effort; for that is done by the springing-back of the breast-bone and ribs to their natural position. To expel the air, the breast-bone is drawn down towards the back-bone by muscles, which movement compresses the lungs.

Mammals aione have a perfect thorax-i. e., a closed cavity for the heart and lungs, with movable walls (breast 
bone and ribs) and the diaphragm, or muscular partition, separating it from the abdomen. ${ }^{68}$ Inspiration (or filling the lungs) and expiration (or emptying the lungs) are both accomplished by muscular exertion; the former, by raising the ribs and lowering the diaphragm, thus enlarging the

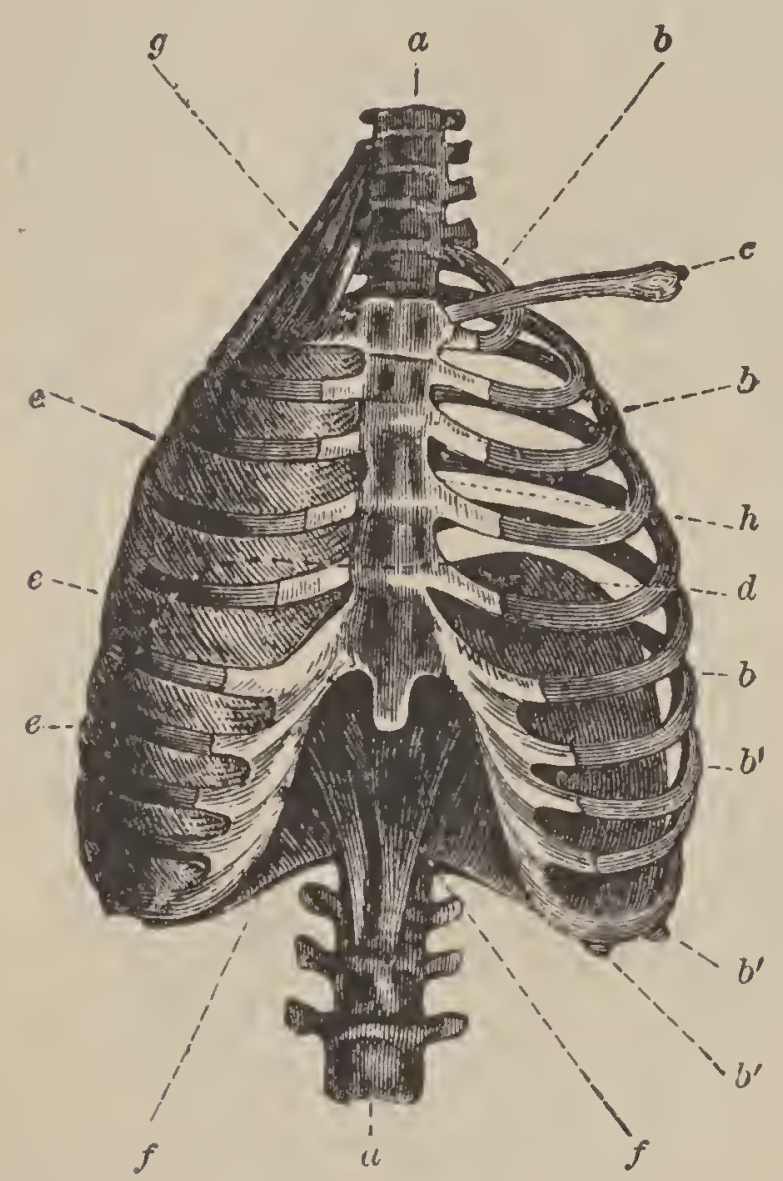

Fig. SS.-Human Thorax : $a$, vertebial column; $b, b^{\prime}$, ribs, the lower ones false; $c$, clavicle; $e$, intercostal muscles, removed on the left side to show the diaphragm, $d$; $f$, pillars of the diaphragm attached to the jumbar vertebre; $g$, muscles for elevatiug the ribs; $h$, sternum. capacity of the chest, in consequence of which the air rushes in to prevent a vacunm; the latter, by the ascent of the diaphragm and the descent of the ribs.

As a rule, the more active and more muscular an animal, the greater the demand for oxygen. Thus, warm-blooded animals live fast, and their rapidly decaying tissues call for rapid respiration; while in the cold-blooded creatures the waste is comparatively slow. Respiration is most active in Birds, and least in water-breathing animals. The sluggish Toad respires more slowly than the busy Bee, the Mollusk more slowly than the Fish. But respirations, like beats of the heart, are fewer in large Mammals than in small ones. An average Man inhales about 300-400 cubic feet of air per day of rest, and much more when at work.

Another result of respiration, besides the purification of the blood, is the production of heat. The chemical combination of the oxygen in the air with the carbon in the tissues is a true combustion; and, therefore, the more 
active the animal and its breathing, the higher its temperature. Birds and Mammals have a constant temperature, which is usually higher than that of the atmosphere $\left(108^{\circ}\right.$ and $100^{\circ} \mathrm{F}$. respectively). They are therefore called constant-temperatured or warm-blooded. Other animals do not vary greatly in temperature from that of their snrroundings, and are called changeable-temperatured or coldblooded. Still, their temperature does not agree exactly with that of the air or water. The Bee is from $3^{\circ}$ to $10^{\circ}$, and the Earth-worm and Snail from $1 \frac{1}{2}^{\circ}$ to $2^{\circ}$, higher than the air. The mean temperature of the Carp and Toad is $51^{\circ}$; of Man, $98^{\circ}$; Dog, $99^{\circ}$; Cat, $101^{\circ}$; Squirrel, $105^{\circ}$; Swallow, $111^{\circ}$.

\section{CHAPTER XV.*}

\section{SECRETION AND EXCRETION.}

In the circulation of the blood, not only are the nutrient materials deposited within the body in the form of tissue, but certain special fluids are separated and conveyed to the external or internal surfaces of the body. These fluids are of two kinds: some, like saliva, gastric juice, bile, milk, etc., are for useful purposes; others, like sweat and urine, are expélled from the system as useless or injurious. The separation of the former is called secretion; the removal of the latter is excretion. Both processes are substantially alike.

In the lower forms, there are no special organs, but secretion and excretion take place from the general surface. The simplest form of a secreting organ closely resembles that of a respiratory organ, a thin membrane separating the blood from the cavity into which the secretion is to

\footnotetext{
* See Appendix.
} 
be poured. Usually, however, the cells of the membrane manufacture the secretion from materials furnished by the blood. Even in the higher animals, there are such secreting membranes. The membranes lining the nose and ali-

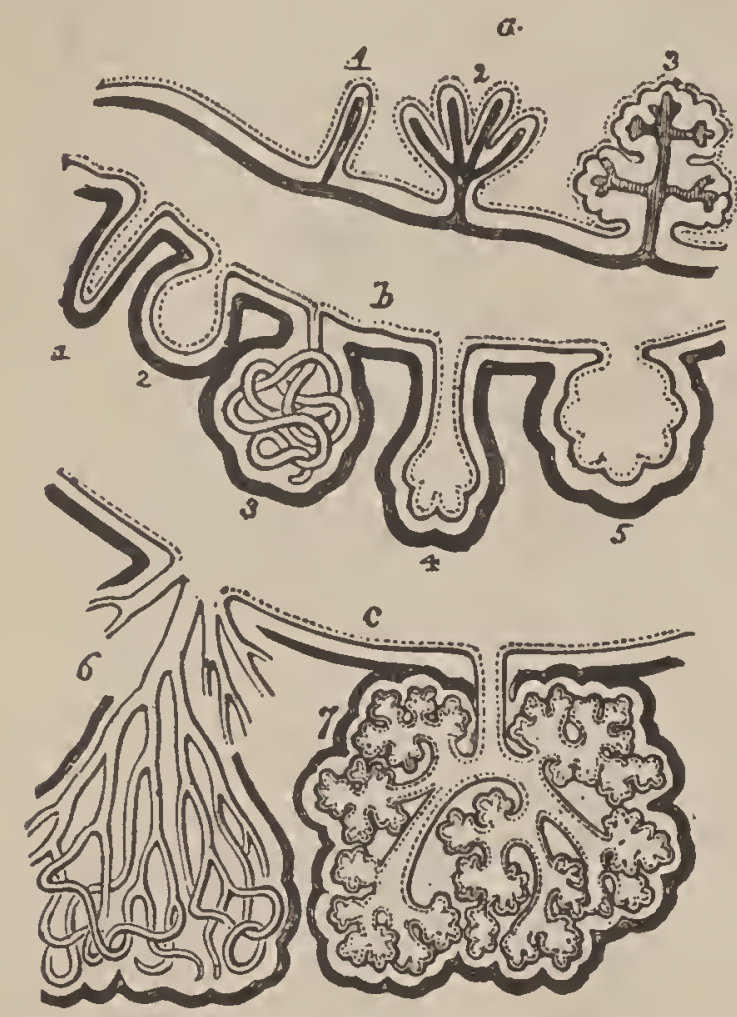

Fig. 89.-Three plans of secreting Membranes. The beavy line represents the areolar-vascular lnyer; the next line is the basement, or limiting membrane; and the dotted line the epithelial layer: $a$ shows increase of surface by simple plaited or fringed projections; $b$, five modes of increase by recesses, forming simple glands, or follicles; $c$, two forms of compound glands.

mentary canal and enclosing the lungs, heart, and joints, secrete lubricating fluids.

The infolding of such a membrane into little sacs or short tubes (follicles), each having its own outlet, is the type of all secreting and excreting organs. The lower animals have nothing more complex, and the apparatus for preparing the gastric fluid attains no further development eren in Man. When a cluster of these follicles, or sacs, discharge their contents by one common duct, we have a gland. But whether membrane, follicle, or gland, the organ is covered with a net-work of blood-vessels, and lined with epithelial cells, which are the real agents in the process.

The chief Secreting Organs are the salivary glands, gastric follicles, pancreas, and liver, all situated along the digestive tract.

1. The salivary glands, which open into the month, secrete saliva. They exist in nearly all Vertebrates, higher Mollusks, and Insects, and are most largely developed in such as live on vegetable food. The saliva serves to lubricate or dissolve the food for swallowing, and, in some Marnmals, aids also in digestion of starch.." 
2. The gastric follicles are minute tubes in the walls of the stomach secreting gastric juice. They are found in all Vertebrates, and in the higher Mollusks and Arthropods. In the lower forms, a simple membrane lined with cells serves the same purpose. Under the microscope, the soft mucous membrane of the human stomach presents a honey-comb appearance, caused by numerous depressions or cells. At the bottom of these depressions are clusters of spots, which are the orifices of the tubnlar follicles. The follicles are about $\frac{1}{2} \frac{1}{50}$ of an inch in diameter, and number millions.

3. The pancreas, or "sweetbread," so

important in the process of digestion, Stomach of a Dog, $x$ exists in all but the lowest animals. In its structure it closely resembles the sal-
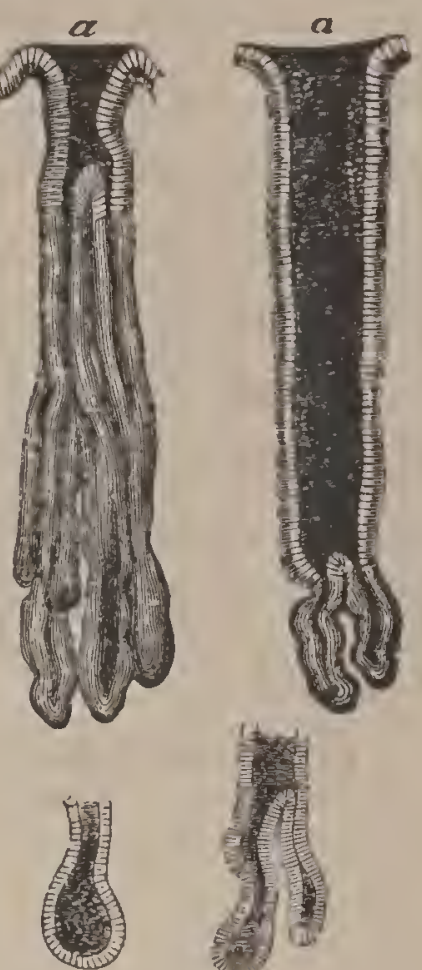
150 ; near the mouth, $a$, there is a lining of $\mathrm{co}$. lumuar epithelinm. ivary glands. In the Cuttle-fish, it is represented by a sac; in Fishes, by a group of follicles. It is proportion-

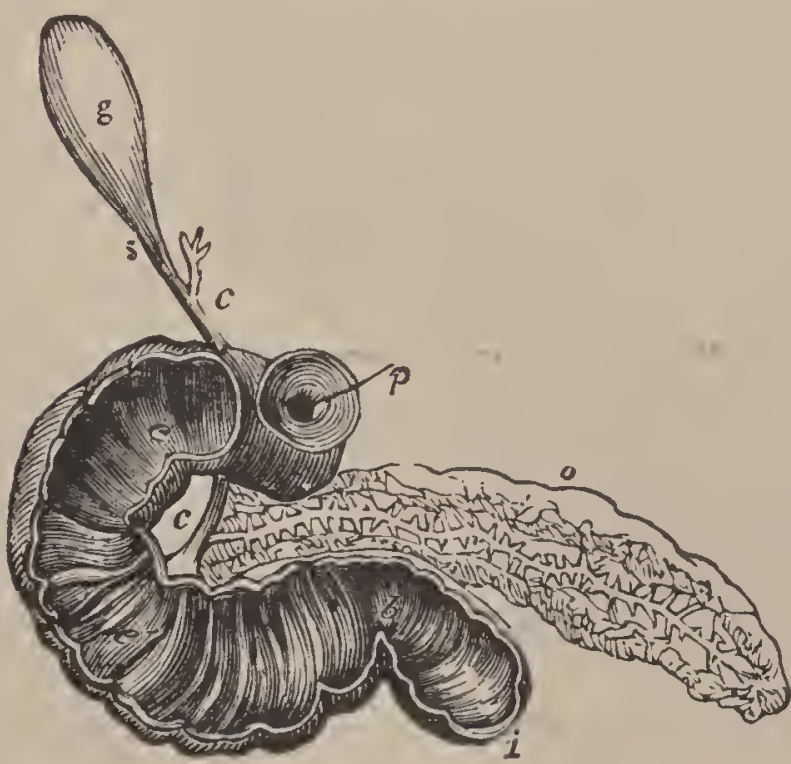

Fir. 91.-Pancreas of Man, $n ; g$, gall-bladder; $s$, cystic duct; $c$, duct from the liver; $p$, pyloric valve; $e, i$, duodenum. ally largest in Birds whose salivary glands are deficient. The pancreatic juice enters the duodenum.

4. A so-called "liver" is found in all animals having a distinct digestive cavity. In the lower animals its function has been shown to be that of a pancreas. Thus, in Polyps it is represented by yellowish cells lining the stomach; in Insects, by cells 
in the wall of the stomach; in Mollusks, by a cluster of sacs, or follicles, forming a loose compound gland. In Vertebrates, a true liver, the largest gland in the body, is well defined, and composed of a multitude of lobules (which give it a granular appearance) arranged on the capillary veins, like grapes on a stem, and containing nucleated secreting cells. It is of variable shape, but usually two, three, or five lobed, and is centrally situated - in Mammals, just below the diaphragm. In most Vertebrates, there is an appendage to the liver, called the gall-bladder, which is simply a reservoir for the bile.

The so-called liver of Invertebrates is more like the

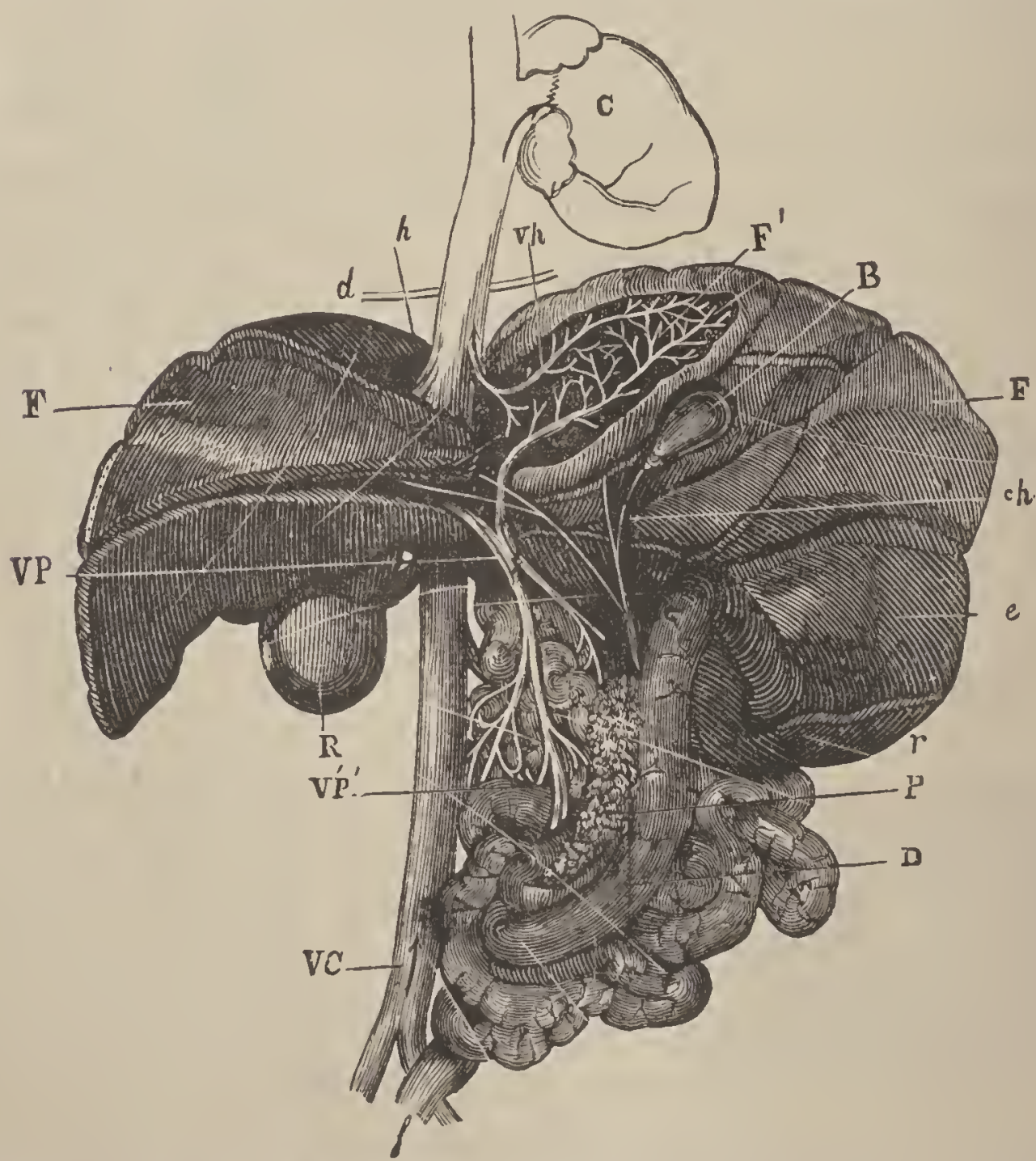

Fr6. 92.-Liver of the $\operatorname{Dog}, F, F ; D$, duodenum and intestines: $P$, pancreas; $r$, spleen: $e$, stomach, $f$, rectum; $R$, right kidney; $\mathrm{B}$, gall-bladder; ch, cystic duct; $F$, lobe of liver dissected to show-distribution of portal vein, VP, and hepatic vein, $v h$; $d$, diaphragm; VC, reua cava; $\mathrm{C}$, heart. 
pancreas of Vertebrates in function, as its secretion. digests starches and albuminoids. The liver of Vertebrates is both a secretory and an excretory organ. The bile performs an important, although ill-understood, function in digestion, and also contains some waste products. The gland also serves to form sugar from part of the digested food, and may well be called a chemical workshop for the body. In animals of slow respiration, as Crustaceans, Mollusks, Fishes, and Reptiles, fat accumulates in the liver. "Cod-liver oil" is an example.

The great Excreting Organs are the lungs, the kidneys, and the skin; and the substances which they remove from the system-carbonic acid, water, and ureaare the products of decomposition, or organic matter on its way back to the mineral kingdom. ${ }^{70}$ Different as these organs appear, they are constructed upon the same principle: each consisting of a rery thin sheet of tissue separating the blood to be purified from the atmosphere, and straining ont, as it were, the noxious matters. All, moreover, excrete the same substances, but in very different proportions: the lungs exhale carbon dioxide and water, with a trace of urea; the kidneys expel water, urea, and a little carbon dioxide; while the skin partakes of the nature of both, for it is not only respiratory, especially among the lower animals, but it performs part of the work of the kidneys when they are diseased.

1. The lungs (and likewise gills) are mainly excretory organs. The oxygen they impart sweeps with the blood through every part of the body, and unites with the tissues and with some elements of the blood. Thus are produced heat and work, whether muscular, nervous, secretory, etc. As a result of this oxidation, carbon dioxide, water, and urea or a sinilar substance, are poured into the blood. The carbon dioxide and part of the water are passed off from the respiratory organs. This process is 
more immediately necessary to life than any other: the arrest of respiration is fatal.

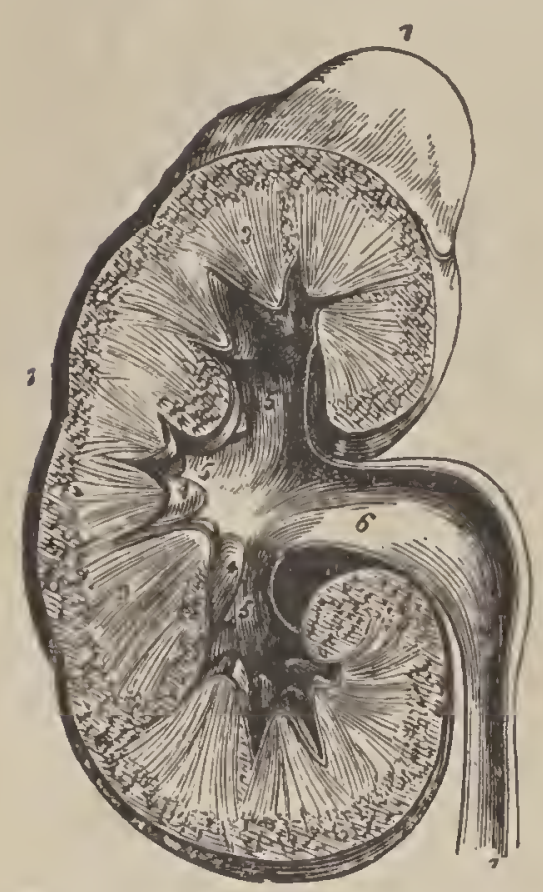

Fig. 93.-Section of Humau Kidney, showing the tubular portion, 3 , çrouped into cones: 7 , the ureter, or wutlet for the secretion.

2. While the lungs (and skin also, to a slight degree) are sources of gain as well as loss to the blood, the kidneys are purely excretory organs. Their main function is to eliminate the solid products of decay which cannot pass out by the lungs. In Mammals, they are discharged in solution; but from other animals which drink little the excretion is more or less solid. In Insects, the kidneys are groups of tubes (Figs. 41,42); in the higher Mollusks, they are represented by spongy masses of follicles (Fig. 46); in Vertebrates, they are well-developed glands, two in number, and consist of closely packed tubes.

3. The skin of the soft-skinned animals, particularly of Ainphibians and Mammals, is covered with minute pores, which are the ends of as many delicate tubes that lie coiled up into a knot within the true skin. These are the sweat-glands, which excrete water, and with it certain salts and gases.

Besides these secretions and excretions, there are others, confined to particular animals, and designed for special purposes: such are the oily matters secreted from the skin of quadrupeds for lubricating the hair and keeping the skin flexible; the tears of Reptiles, Birds, and Mammals; the milk of Mammals; the ink of the Cuttle-fish; the poison of Jelly-fishes, Insects, and Snakes; and the silk of Spiders and Caterpillars. 


\section{CHAPTER XVI.* \\ THE SKIN AND SKELETON.}

The Skin, or Integument, is that layer of tissue which covers the outer surface of the body. The term Skeleton is applied to the hard parts of the body, whether external or internal, which serve as a framework or protection to the softer organs, and afford points of attachment to inuscles. If external, as the crust of the Lobster, it is called Exoskeleton; if internal, as the bones of Man, it is called Endoskeleton. 'The former is a modification of the skin; the latter, a hardening of the deeper tissues.

1. The Skin.-In the lowest forms of life, as Amœba, there is no skin. The protoplasm of which they are composed is firmer ontside than inside, but no membrane is present. In Infusoria, there is a very thin cuticle covering the animal. They have thus a definite form, while the Amœbæ continually change. Sponges and Hydras also have no true skin. But in Polyps, the outside layer of the animal is separated into two portions-ecderon and enderon ${ }^{71}$ - which may be regarded as partly equivalent to epidermis and dermis in the higher animals. These two layers are, then, generally present. The outer is cellular, the latter fibrous, and may contain muscular fibres, blood-vessels, nerves, touch-organs, and glands. It thus becomes very complicated in some animals.

In Worms and Arthropods, the cellular layer, here called hypodermis, excretes a structureless cuticle, which may become very thick, as in the tail of the Horseshoe Crab, or may be hardened by deposition of lime-salts, as in many Crustacea. The loose skin, called the mantle,

* See Appendix. 
which envelopes the body of the Mollusk corresponds to the true skin of higher animals. The border of the manthe is surrounded with a delicate fringe, and, moreover, contains minute glands, which secrete the shell and the coloring matter by which it is adorned. The Tunicates have a leather'y epidermis, remarkable for containing, instead of lime, a substance resembling vegetable cellulose.

In Mammals, whose skin is most fully developed, the dermis is a sheet of tough elastic tissue, consisting of interlacing fibres, and containing blood-vessels, lymplatics, sweat-glands, and nerves. It is the part converted into leather when hides are tanned, and attains the extreme thickness of three inches in the Rhinoceros. The upper surface in parts of the body is covered with a vast number of minute projections, called papillae, each containing the termination of a nerve; these are the essential agents in the sense of touch (Fig. 148). ${ }^{72}$ They are best seen on the tongue of an $\mathrm{Ox}$ or Cat, and on the human fingers, where they are arranged in rows.

Covering this sensitive layer, and accurately moulded to all its furrows and ridges, lies the bloodless and nerveless epidermis. It is that part of the skin which is raised in a blister. It is thickest where there is most pressure or hard usage: on the back of the Camel it attains unusual thickness. The lower portion of the epidermis (called rete mucosum) is comparatively soft, and consists of nucleated cells containing pigment-granules, on which the color of the animal depends. Towards the surface the cells become flatteried, and finally, on the outside, are changed to horny scales (Fig. 2,c).

These scales, in the higher animals, are constantly wearing off in the for'm of scurf, and as constantly being renewed from below. In Lizards and Serpents, the old epidermis is cast entire, being stripped off from the head to the tail: in the Toad, it comes off in two picces; in the 


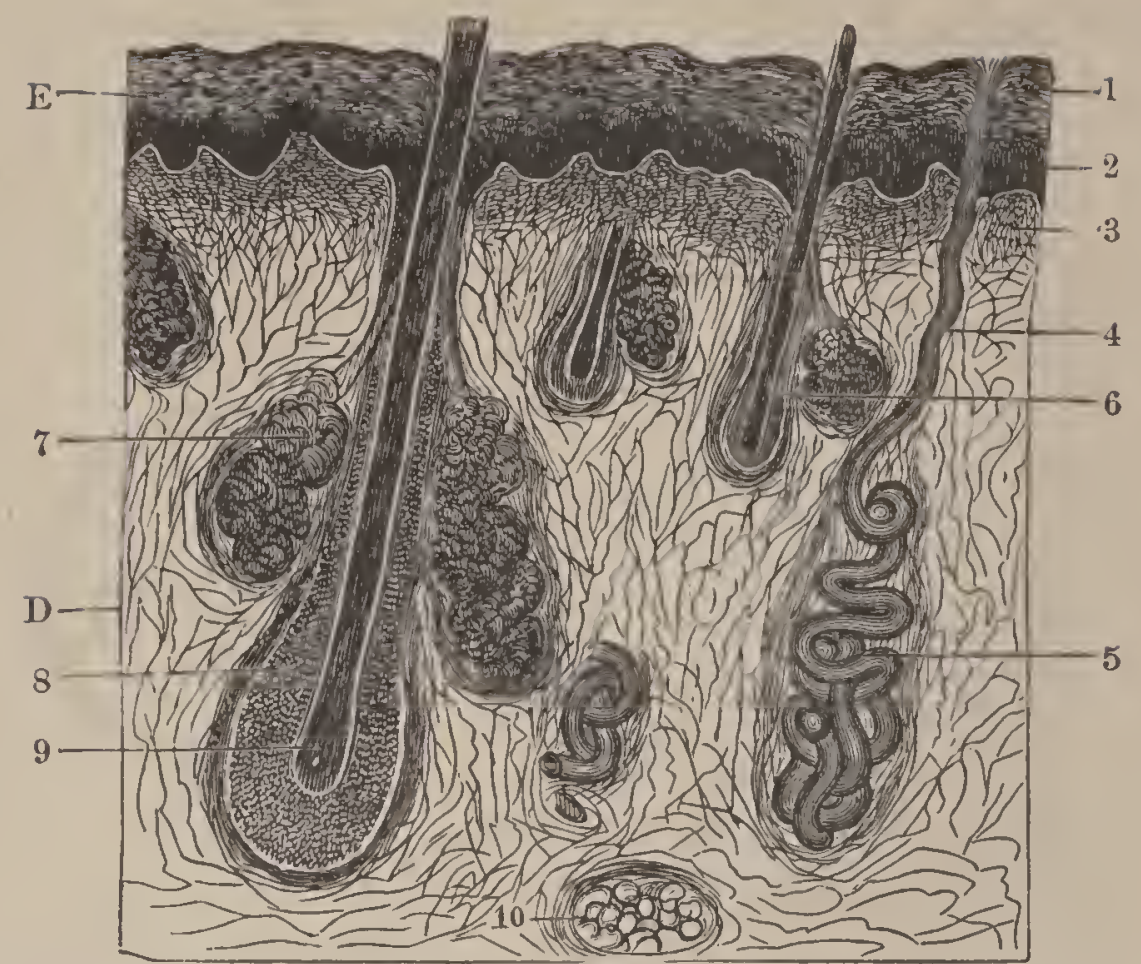

F'v. 94.-Section of Skin from Horse's Nostril : E, epidermis; D, dermis; 1, horny layer of epidermis ; 2 , rete mucosum; 3 , papillary layer of dermis; 4 , excretory duct of a sudoriparous, or sweat, gland; 5 , glomerule, or convoluted tube of the same; 6 , hair follicle; 7 , sebaceons gland; 8 , internal sheath of the hair follicle; 9 , bulb of the hair; 10 , mass of adipose tissue.

Frog, in shreds; in Fishes and some Mollusks, in the form of slime. However modified the epidermis, or whatever its appendages, the like process of removal goes on. Mammals shed their hair; Birds, their feathers; and Crabs, their shells. When the loss is periodical, it is termed moulting.

2. The Skeletons. - (1) The Exoskeleton is developed by the hardening of the skin, and, with very few exceptions, is the only kind of skeleton possessed by invertebrate animals. The usual forms are coral, shells, crusts, scales, plates, hairs, and feathers. It is horny or calcareous; while the endoskeleton is generally a deposit of earthy material within the body, and is nearly confined to the Vertebrates. The exoskeleton may be of two kinds - dermal and epidermal.

Some of the Protozoa, as Polycistina and Foraminifera, possess siliceous and calcareous shells of the most beautiful patterns. The Toilet Sponge has a skeleton 
of horny fibres, which is the sponge of commerce. Coral is the solid framework of certain Polyps. There are two kinds: one represented by the common white coral, which is a calcareous secretion within the body of
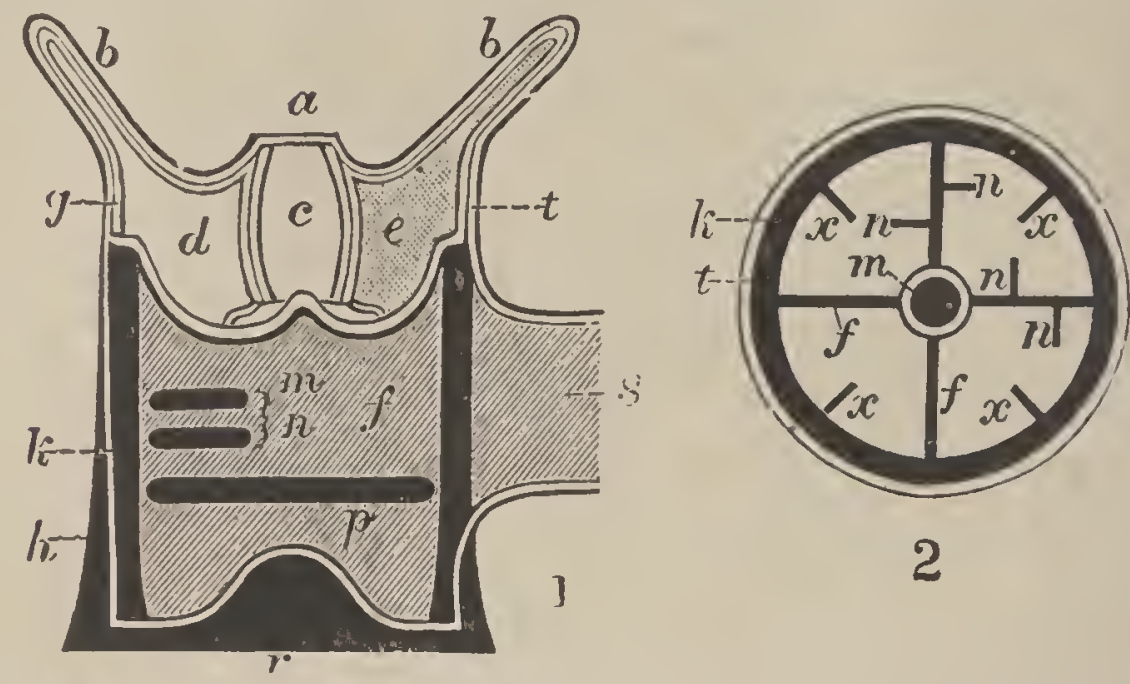

Fig. 95.-1, Vertical Section, and, 2, Transverse Section, of a sclerudermic Corallite: $a$, mouth; $b$, tentacles; $c$, stomach; $d$, internesenteric chamber; $e$, mesentery ; $f$, septum : $g$, endoderm : $h$, epitheca $k$, theca, or outer wall ; $m$, colnmella ; $u$, 6hort partitions; $p$, tabula, or transwerse partition ; $r$, sclerobase; $s$, cœneuchyma, or common substance counecting neighboring corallites; $t$, ectuderm; $x$, pali, or imperfect partitions.

the Polyp, in the form of a cylinder, with partitions radiating towards a centre (scleroderm); the other, repre. sented by the solid red coral of jewelry, is a central axis deposited by a group of Polyps on the outside (sclero-

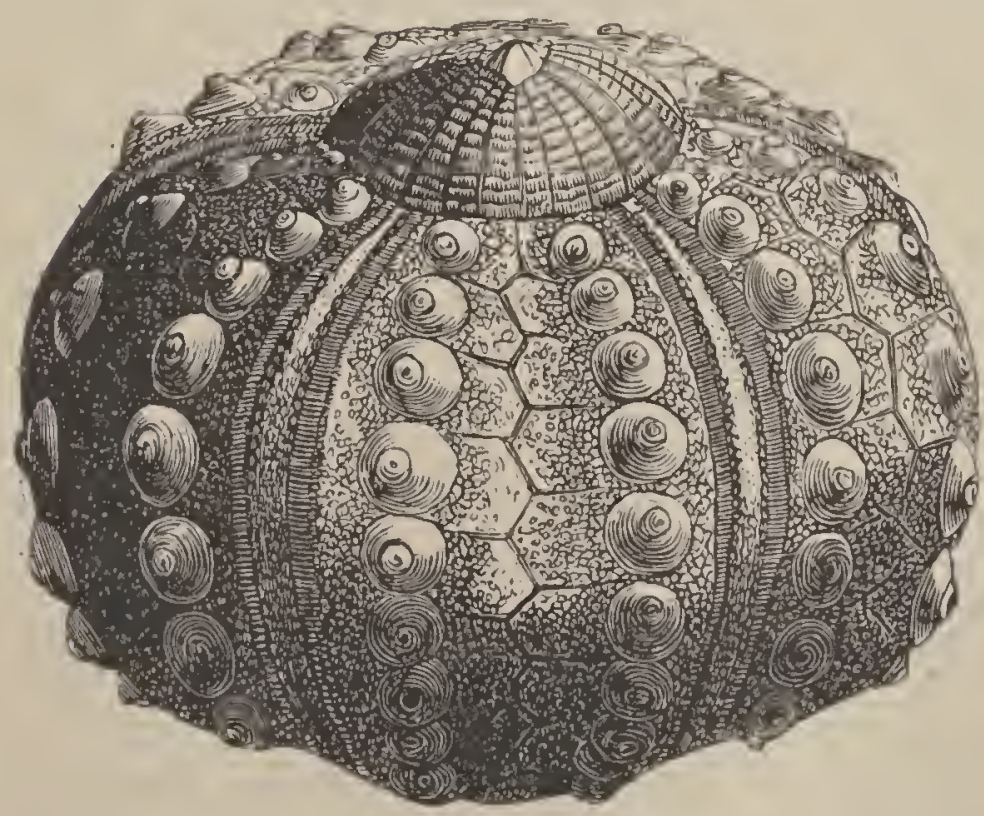

FIG. 96. -Shell of Sea-urchin (Cidaris) without its spiues. elaborate and beautiful construction. base). The first sor't is a dermal, the latter an epidermal, exoskeleton.

The skeleton of the Star-fish is a leathery skin in which are embedded calcareous particles and plates. The Sea-urchin is covered with an inflexible shell of The shell is really a 
calcified skin, being a net-work of fibrous tissne and earthy matter. It varies in shape from a sphere to a disk, and consists of hundreds of angular pieces accurately fitted together, like mosaic-work. These form ten zones, like the ribs of a melon, five broad ones alternating with five nar-

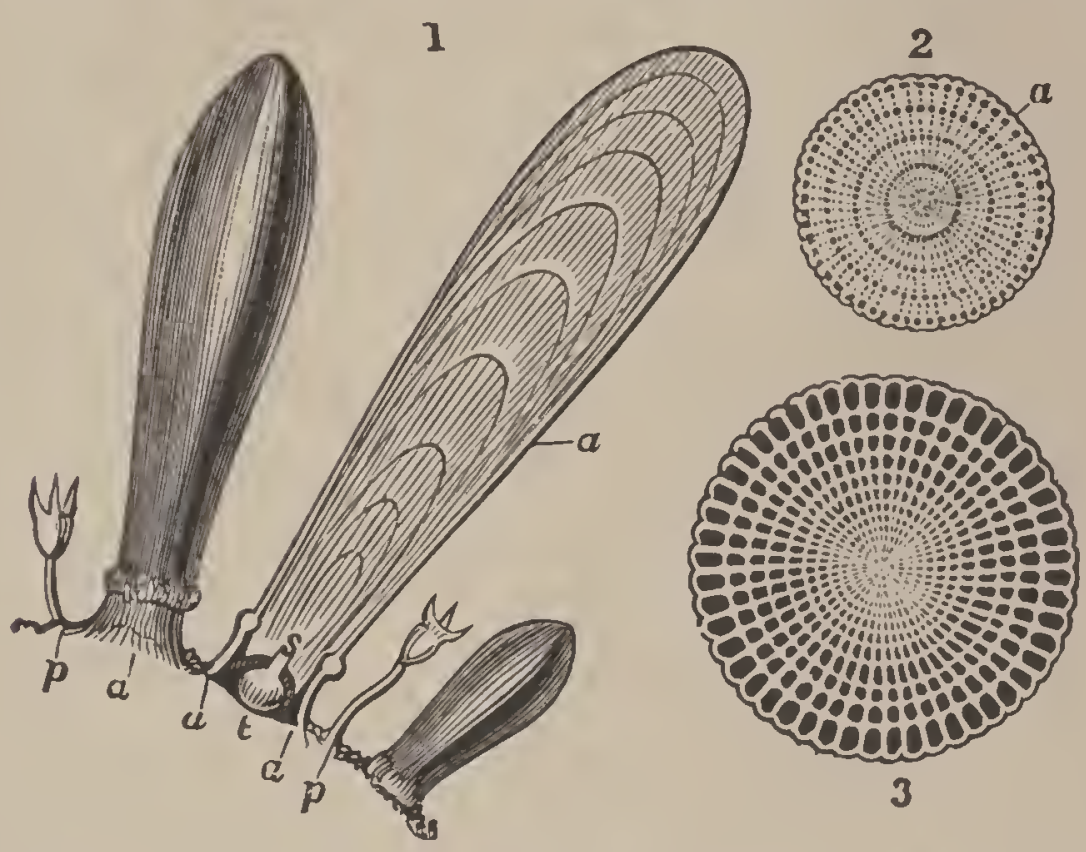

FIG. 97.-Structure of Sea-11rchins' Spines: 1, $a$, spine of Cidaris cut longitudinally : $t, s$, ball-and-socket joint ; $p$, pedicellarix ; 2,3 , transverse sections of spines of Cidaris and Echinus.

rower ones. The former (called interambulacra) are covered with tubercles bearing movable spines. The narrow zones (called ambulacra, as they are likened to walks through a forest) are pierced with small holes, through which project fleshy sucker-feet.

The skin of the Lobster is hardened by calcareons deposit into a "crust," or shell;" but, instead of forming one piece, it is divided into a series of segments, which move on each other. The number of these segments, or rings, is usually twenty-five in the head, eight in the thorax, and seven in the abdomen. In the adult, however, the rings of the head and thorax are often soldered together into one shield, called cephalo-thorax; and in the Horseshoe Crab the abdominal rings are also united. 'The shell of Crustaceans is periodicaliy cast off, for the animals continue to grow even after they have reached their 
mature form. This moulting is a very remarkable operation. How the Lobster can draw its legs from their cases

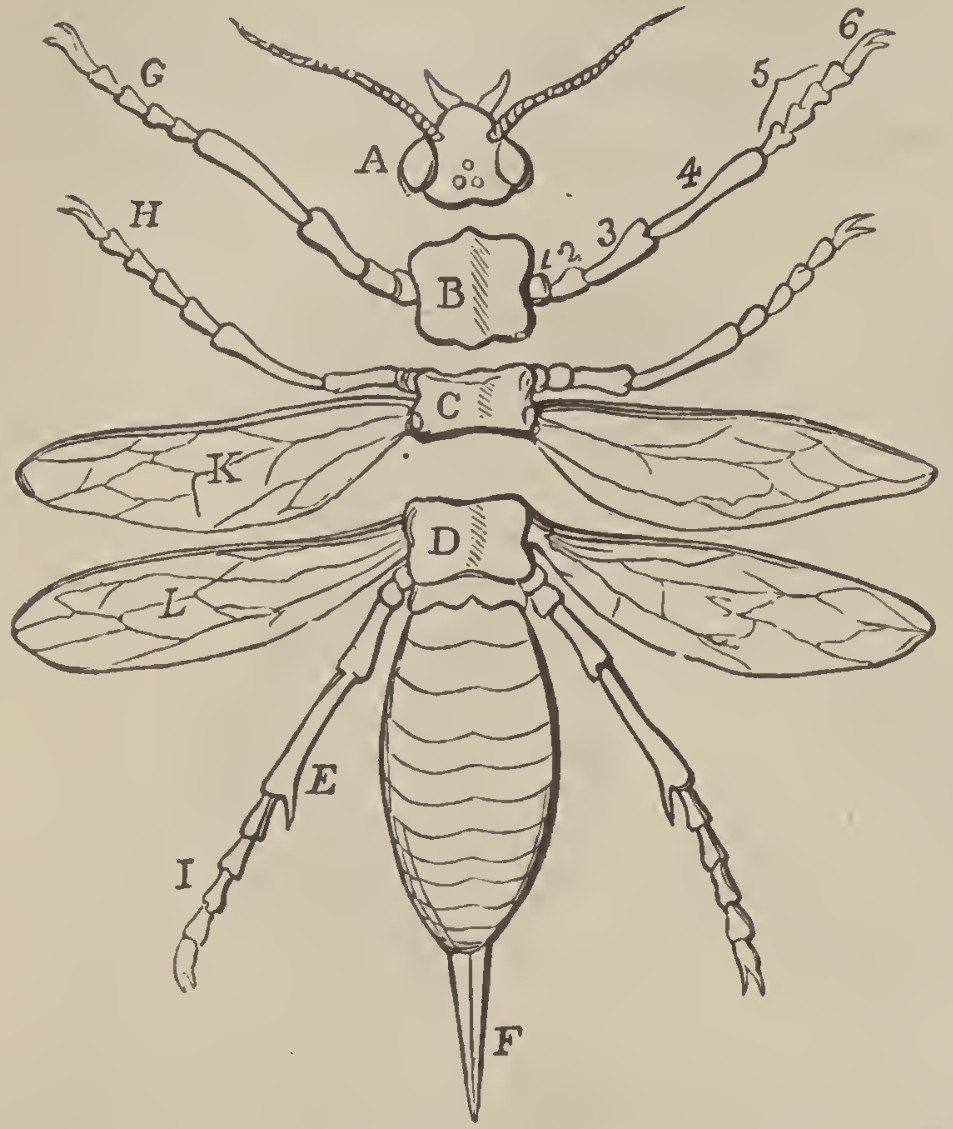

Fra. 98.-Diagram of an Insect: $A$, head bearing the eyes and antennæ: $B$, prothorax, carrying the first pair of legs; C, mesothorax, carrying the second pair of legs and frst prir of wings; $D$, metathorax, carrying the third pair of legs and second pair of wings; $\mathrm{E}$, abdomen, with ovipositor, $\mathrm{F} ; 1$, coxn, or hip; 2 , trochanter ; 3 , femur, or thigh; 4 , tibin, or shank; 5 , tarsus, or foot; 6 , claw. without unjointing or splitting them was long a puzzle. The flesh becomes soft, and is drawn through the joints, the wounds thus caused quickly healing. The castoff skeleton is a perfect copy of the animal, retaining in their places the delicate coverings of the eyes and autennæ, and even the lining membrane of the stomach with its teeth.

The horny crust of Insects differs

from that of Crustaceans in consisting mainly of a horny substance called chitin and in containing no lime. The head, thorax, and abdomen are distinct, and usually consist of fourteen visible segments-one for the head, three for the thorax (called prothorax, mesothorax, and metathorax), and ten for the abdomen. The antennæ, or feelers, legrs, and wings, as well as hairs, spines, and scales, are appendages of the skeleton. As Insects grow only during the larval, or caterpillar, state, moulting is confined to that period. These skeletons are epidermal, deposited in successive layers, from the inside, and are, therefore, capable of but slight enlargement when once formed. 
The shells of Mollusks are well-known examples of exoskeletons. The mantle, or loose skin, of these animals secretes calcareous earth in successive layers, converting the

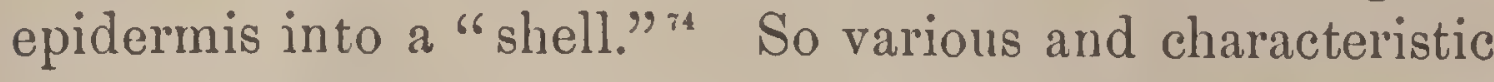
is the microscopic character of shells, that a fragment is sometimes sufficient to determine the group to which it belongs. Many shells resemble that of the Fresh-water Mussel (Unio), which is composed of three parts: the external brown epidermis, of horny texture; then the prismatic portion, consisting of minute columns set perpendicularly to the surface; and the internal nacreons layer, or " mother-of-pearl," made up of exceedingly thin plates. The pearly lustre of the last is due to light falling upon the outcropping edges of wavy laminæ. ${ }^{75}$ In many cases, the prismatic and nacreous layers are traversed by minute tubes. Another typical shell-structure is seen in the common Cone, a section of which shows three layers, besides the epidermis, consisting of minute plates set at different angles. The Nautilus shell is composed of two distinct layers: the outer one having the fracture of broken china ; the inner one, nacreous.

Most living shells are made of one piece, as the Snail; these are called "univalves." Others, as the Clam, consist of two parts, and are called "bivalves." In either case, a valve may be regarded as a hollow cone, growing in a spiral form. The ribs, ridges, or spines on the ontside of a shell mark the successive periods of growth, and, therefore, correspond to the age of the animal. The figures on the following page show the principal parts of the ordinary bivalves and univalves. The valves of a bivalve are generally equal, and the umbones, or beaks, a little in front of the centre. The valves are bound together by a ligature near the umbones, and often, also, by means of a "hinge" formed by the "teeth" of one valve interlocking into cavities in the other. The aperture of 
a univalve is frequently closed by a horny or calcareous plate, called "operculum," which the animal carries on its back, and which is a part of the exoskeleton. The shells of Mollusks are epidermal, and are, therefore, dead and incapable of true repair. When broken, they can be mender

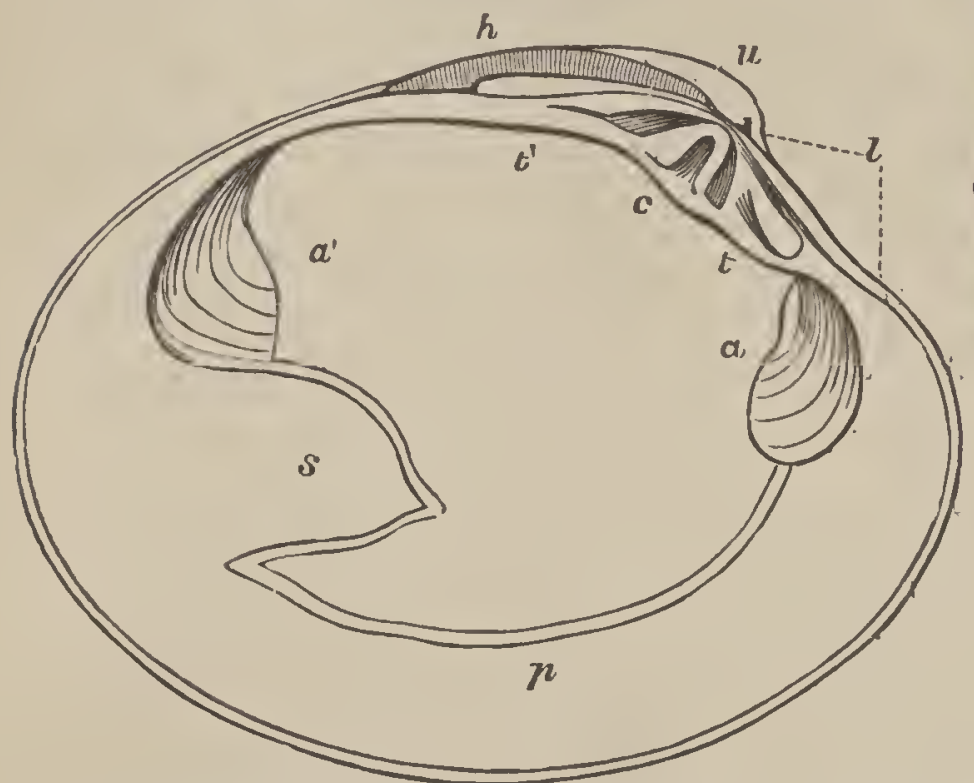

Fig. 99.-Left Valve of a Bivalve Mollusk (Cytherea chione): $h$, hinge ligament: $\iota$, umbo ; $l$, lunule ; $c$, cardiual, and $t, t^{\prime}$, lateral teeth ; $a, a^{\prime}$, impressions of the anterior and posterior adductor mus. cles ; $p$, pallial impression ; $s$, sinus, occupied by the retractor of the siphous.

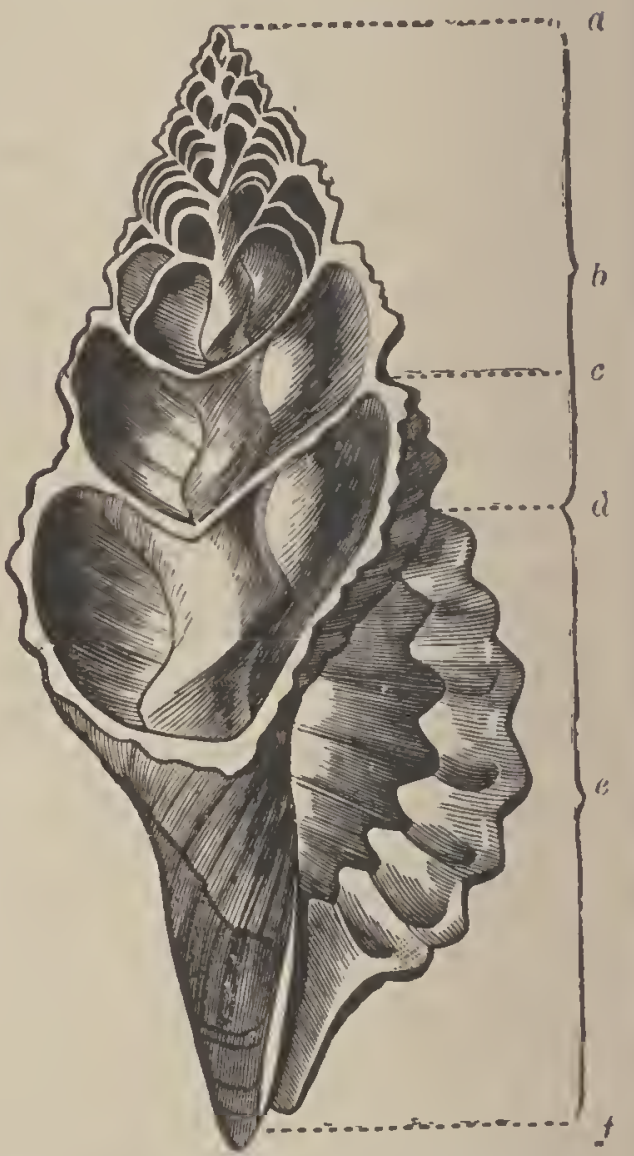

Fra. 100. - Section of a Spiral Univalve (Triton corrugatus): $a$, apex; $b$, spire; $c$, suture: $d$, posterior caual; $e$, outer lip of the aperture; $f$, anterior caual.

only by the animal pouring out lime to cement the parts together. They cannot grow together, like a broken bone. Imbedded in the back of the Cuttle-fish is a very light spongy "bone," which, as already observed, is a secretion from the skin, and therefore belongs to the exoskeleton. It has no resemblance to true bone, but is formed, like shells, of a number of calcareous plates. Nevertheless, the Cuttle-fish does exhibit traces of an endoskeleton: these are plates of cartilage, one of which surrounds the brain, and hence may be called a skull. To this cartilage, not to the "cuttle-bone," the muscles are attached.

In Vertebrates, the exoskeleton is subordinate to the endoskeleton, and is feebly developed in comparison. It 
is represented by a great valiety of appendages to the skin, which are mainly organs for protection, not for support. Some are horny developments of the epidermis, such as hairs, feathers, nails, claws, hoofs, horns, and the scales of Reptiles; othel's arise from the hardening of the dermis by calcareous matter, as the scales of Fishes, the bony $\begin{gathered}\text { Fig. 101.-Skeletal Architecture in the Armadil- } \\ \text { lo, showing the relation of the carapax to the }\end{gathered}$ plates of Crocodiles and vertebral column.

Turties, and the shield of the Armadillo.

The scales of Fishes (and likewise the spines of their vertical fins) lie imbedded in the overlapping folds of the skin, and are covered with a thin, slimy epidermis. The scales of the bony Fishes (Perch, Salmon, etc.) consist of

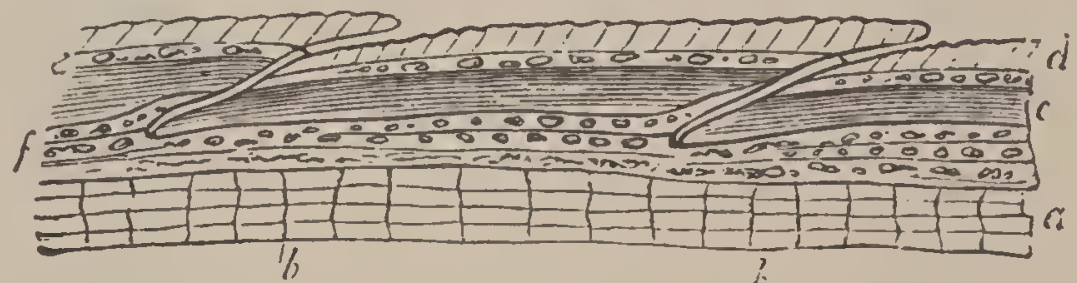

Fıa. 102.-Diagramınatic Section of the Skin of a Fish $(C a r p)$ : a, derm, showing lam. inated structure with vertical fibres, $b$; $c$, gristly layer"; $e$, liminated layer, with calcareous graunles; $d$, superficial portion developing into scales; $f$, scale-pit.

two layers, slightly calcareous, and marked by concentric and radiating lines. Those of the Shark have the structure of teeth, while the scutes, or plates, of the Crocodiles, Turtles, and Arınadillos are of true bone.

The scales of Snakes and Lizards are horny epidermal plates covering the overlapping folds of the true skin. In some Turtles these plates are of great size, and are called "tortoise-shell;" they cover the scutes. The scales on the legs of Birds, and on the tail of the Beaver and Rat, have the same structure. Nails are flattened horny plates developed from the upper surface of the fingers 


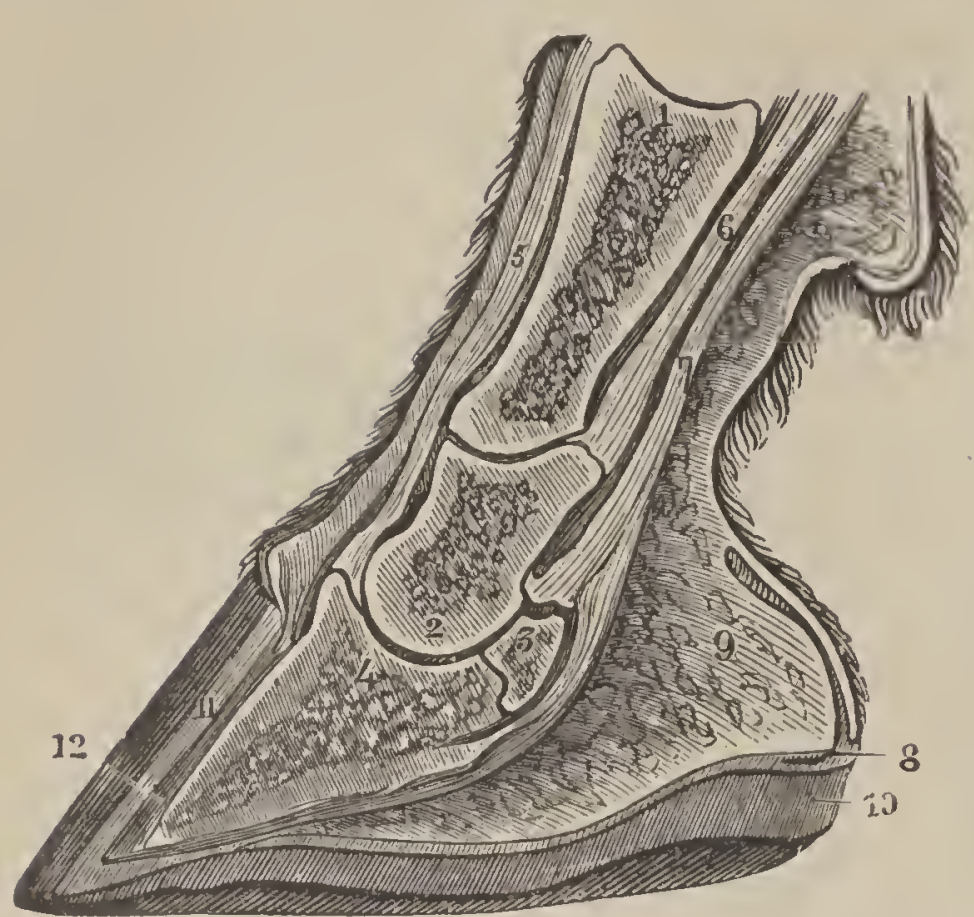

Fig. 103.-Vertical Section of the Forefoot of the Horse (middle digit): $1,2,4$, proximal, middle, and distal, or ungual, phalanges; 3 , sesamoid, or nut-bone; 5 , 6,7 , tendons; 9 , elastic tissue: 8,10 , internal and external floor of the hoof; 11,12 , interial and external walls.

rattles of the Rattlesnake, and the beaks of Turtles and Birds, are likewise epidermal.

Hairs, the characteristic clothing of Mammals, are elongated horny cones, composed of "pith" and "crust." The latter is an outer layer of minute overlapping scales, which are directed towards the point, so that rubbing a human liair or fibre of wool between the thumb and finger pushes the rootend away. The root is bulbous, and is contained in a minute depression, or sac, formed by an infolding of the skin. Hairs are usually set obliquely into the skin. Porcupine's quills and Hedgehog's spines make an easy transition to and toes. Claws are sharp conical nails, being developed from the sides as well as upper surface; and hoofs are blunt cylindrical claws. Hollow horns, as of the $\mathrm{Ox}$, may be likened to claws sheathing a bony core. The horn of the Rhinoceros is a solid mass of epidermal fibres. "Whalebone," the

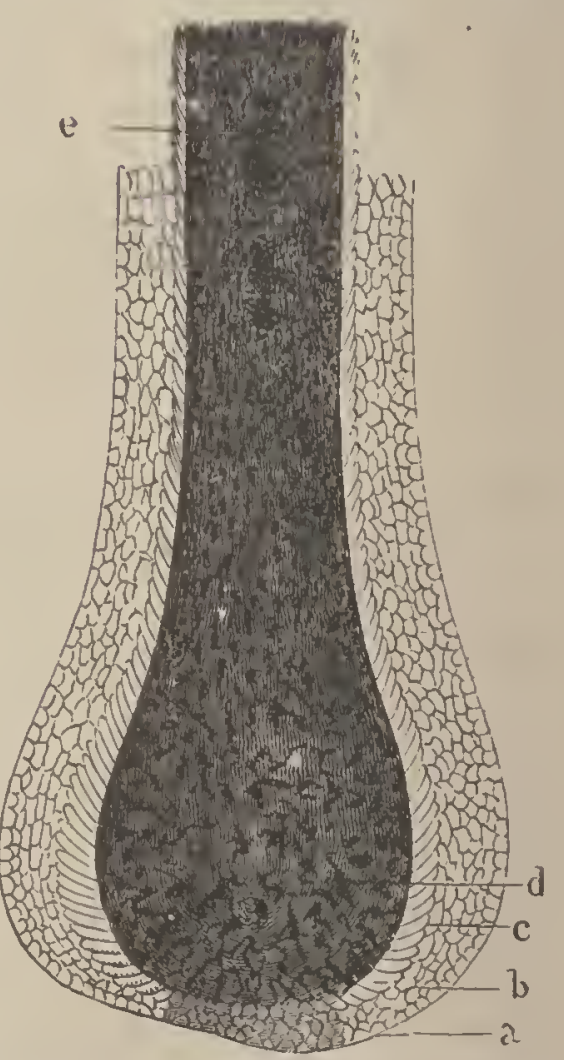

Frg.104.- Section of the Rootaud part of the Shaft of a Human Hair; it is covered with epidermic scales, the inner layer, $c$, forming the outer covering of the shaft, being inbricated; the root consists of angular cells loaded with pigment. 
feathers, which differ from hairs only in splitting up into" numerous laminæ. They are the most complicated of all the modifications of the epidermis. They consist of "a "quill" (answering to the bulb of a hair), and a "shaft," supporting the "vane," which is made up of "barbs," "barbules," and interlocking "processes." The quill alone is hollow, and has an orifice at each end. The feather is moulded on a papilla, the shaft lying in a groove on one side of it, and the vane wrapped around it. When the feather emerges from the skin, it unfolds itself. Thus shaft and vanes together resemble the quill split down one side and spread ont.

The teeth of Mollusks, Worms, and Arthropods are also epidermal structures. Those of Vertebrates are mixed in their origin, the dentine being derived from the dermis and the enamel from the epidermis. In all cases teeth belong to the exoskeleton.

(2) The Endoskeleton, as we have seen, is represented in the Cuttlefish. With this and some other exceptions, it is peculiar to Verte-

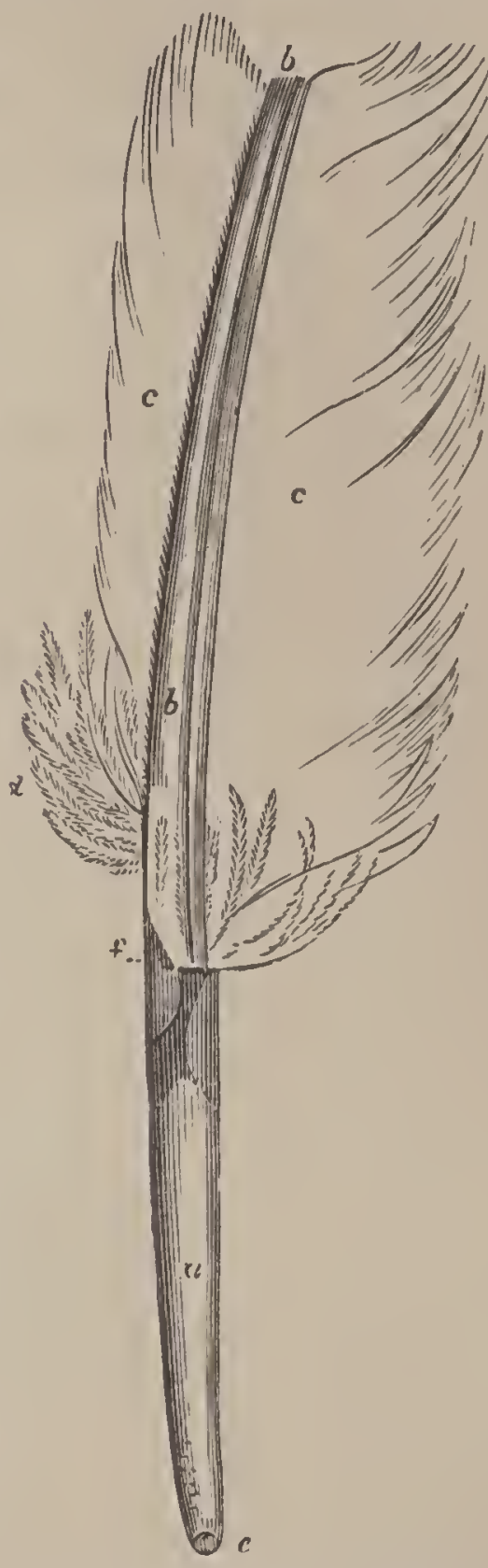

Fig. 105.-Parts of a Feather: $a$, quill, or barrel ; $b$, shaft; $c$, vane, or beard; $d$, accessory plume, or down; $e, f$, lower and upper umbilicus, or orifice, leading to the interior of the quill. brates. In the Cuttle-fish, and some Fishes, as the Sturgeon and Shark, it consists of cartilage; but in all others (when adult) it is bone or osseous tissue. Yet there is a diversity in the composition of bony skeletons; that of fresh-water Fishes contains the least earthy matter, and that of Birds the most. Hence the density and ivory- 
whiteness of the bones of the latter. Unlike the shells of Mollusks and the crust of the Lobster, which grow by the addition of layers to their borders, bones are moist, living parts, penetrated by blood-vessels and nerves, and covered with a tough membrane, called periosteum, for the attachment of muscles.

The surface of bones is compact; but the interior may be solid or spongy (as the bones of Fishes, Turtles, Sloths, and Whales), or hollow (as the long bones of Birds and the active quadrupeds). There are also cavities (called "sinuses") between the inner" and onter" walls of the skull, as is remarkably shown by the Elephant. The cavities in the long bones of quadrupeds are filled with marrow; those in the long bones of Birds and in skulls contain air.

The number of bones not only differs in different animals, but varies with the age of an individual. In very early life there are no bones at all; and ossification, or the conversion of cartilage into bone, is not completed until maturity. This process begins at a multitude of points, and theoretically there are as many bones in a skeleton as centres of ossification. But the actual number is usually much less-a result of the tendency of these centres to coalesce. Thus, the thigh-bone in youth is composed of fire distinct portions, which gradually unite. So in the lower Vertebrates many parts remain distinct which in the higher are joined into one. The occiput or hone at the base of Man's skull is the union of four bones, which are seen separate in the skull of the Fish, or of a baby.

A complete skeleton, made up of all the pieces which might enter into its composition, does not exist. Every Vertebrate has some deficiency. All, except Amphioxus, have a skull and back-bone; but in the developinent of the varions parts, and especially of the appendages, there is endless variety. Fishes possess a great number of skullbones, but have no fingers and toes. The Snake has plenty 
of ribs and tail, but no breast-bone; the Frog has a breastbone, but neither tail nor ribs. As the skeleton of a Fish is too complicated for the primary student, we will select for illustration the skeleton of a Lion-the type of quad.

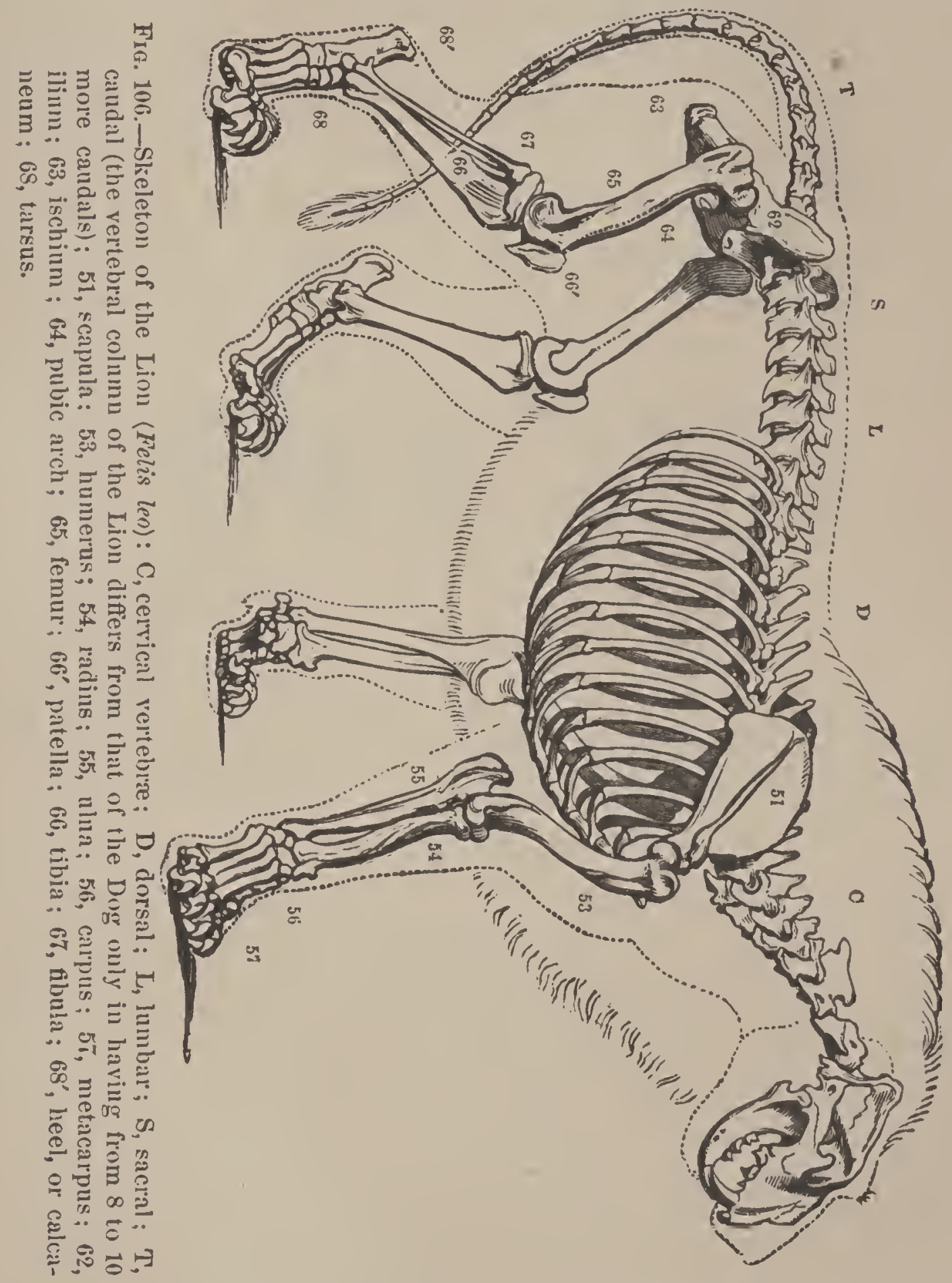

rupeds. It should be remembered, however, that all Vertebrates are formed on one plan.

In the lowest Vertebrate, Amphioxus, the only skeleton is a cartilaginous rod running from head to taii. There is no skull, nor ribs, nor limbs. In the cartilaginous Fishes, 
the backbone is only partially ossified. But usually it consists of a number of separate bones, called vertebroe, arranged along the axis of the body. They range in number from 10 in the Frog to 305 in the Boa-constrictor. The skull, with its appendages, and the vertebræ, with the ribs and sternum, make up the axial skeleton. The shoulder and pelvic girdles and the skeleton of the limbs constitute the appendicular skeleton.

A typical vertebra consists of a number of bony pieces so arranged as to form two arches, or hoops, comnected by

B.
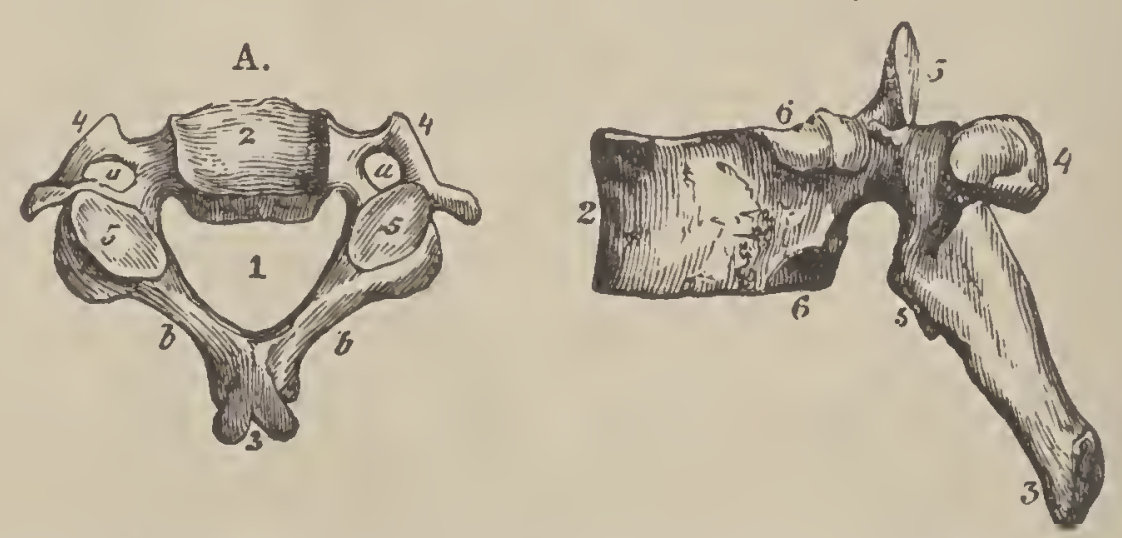

F16. 107.-Vertebræ-A, cervical ; B, dorsal; 2, centrum ; 4, transverse process, cortaining formen, $a$, for artery; 5 , articular process; 3 , spinous process, or neural spine; 1 , neural canal; 6 , facets for head of rib, the tubercle of the rib fitting in a facet on the process, $4 ; b$, laininæ, or neurapophyses.

a central bone, or centrum. ${ }^{76}$ The upper hoop is called the neural arch, because it encircles the spinal marrow; the lower hoop is called the homal arch, because it encloses the heart and the great central blood-vessels. An actual vertebra, however, is subject to so many modifications, that it deviates more or less from this ideal type. Selecting one from the middle of the back for an example, we see that the centrum sends off from its dorsal side two branches, or processes, called neurapophyses. These meet to form the nemral arch, under which is the nerural canal, and above which is a process called the neural spine. On the anterior and posterior edges of the arch are smooth surfaces, or zygapophyses, which in the natural state are covered with cartilage, and come in contact with 
the corresponding surfaces of the preceding and succeeding vertebræ. The bases of the arch are notched in front and behind, so that when two vertebræ are put together a round opening (intervertebral foramen) appears between the pair, giving passage to the nerves issuing from the spinal cord. From the sides of the arch, blunt transverse processes project outward and backward, called diapophyses. Such are the main elements in a representative vertebra. The hæmal arch is not formed by any part of the vertebra, but by the ribs and breast-bone. Theoretically, however, the ribs are considered as elongated processes from the centrum (pleurapophyses), and in a few cases a homal spine is developed corresponding to the neural spine.

The vertebræ are united together by ligaments, but chiefly by a very tough, dense, and elastic substance between the centra. The neural arches form a continuous canal which contains and protects the spinal cord; hence the vertebral column is called the neuroskeleton. The column is always more or less curved; but the beautiful sigmoid curvature is peculiar to Man. The vertebræ gradually increase in size from the head towards the end of the trunk, and then diminish to the end of the tail. The neural arch and centrum are seldom wanting; the first vertebra in the neck has no centrum. and the last in the tail is all centrum. The vertebræ of the extremities (head and tail) depart most widely from the typical form.

The vertebral column in Fishes and Snakes is divisible into three regions--head, trunk, and tail. In the higher animals there are six divisions of the vertebral column: the skull, and cervical, dorsal, lumbar, sacral, and caudal vertebra.

The skull ${ }^{n}$ is formed of bones whose shape varies greatly from that of typical vertebræ. The number of distinct bones composing the skull is greatest in Fishes, and least in Birds: this arises partly from the fact that 


\section{BONES OF THE MAMMALIAN SKULL**}

BRAIN-CASE.

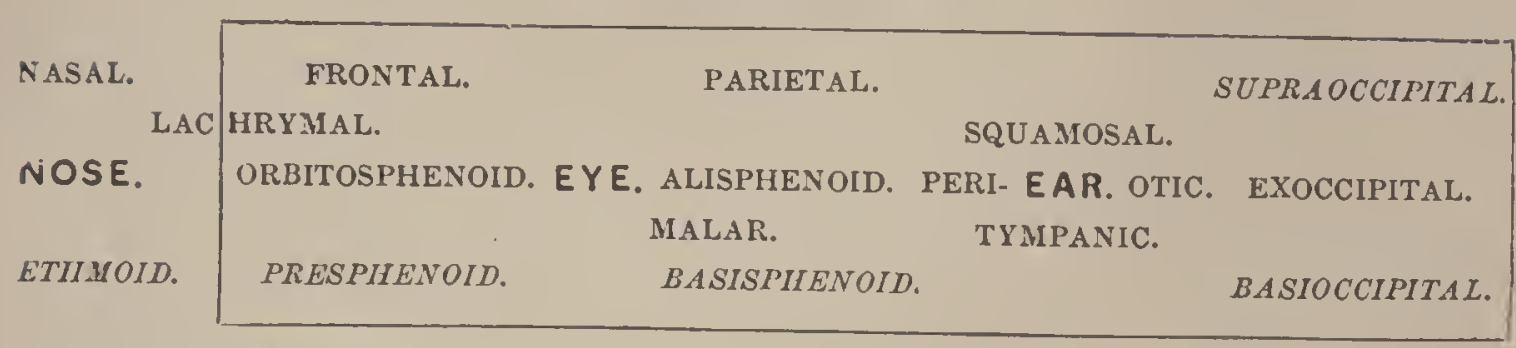

VOMER.

HYOID ARCH.

PREMAXILLA. MAXILLA. PALATINE. PTERYGOID.

LOWER JAW, OR MANDIBLE.

\section{THE SKULL OF THE DOG.}

Fig. 108.-Under surface. F1G. 109.-Upper surface. F1G. 110.-Longitudinal vertical section; one-half natural size: $S O$, supraccipital : $E x O$, exoccipital ; $B O$, basioccipital ; $I P$, interparietal ; $P a$, parietal ; $F r$, frontal ; $S q$, squamosal ; $M A$, malar; $L$, lachrymal; $M x$, maxilla ; $P N I x$, premaxilla; $N a$, nasal; $M T T$, maxilloturbillai: $E T$, ethmoturbinal; $M E$, ossitied portion of the mesethmoid; $C E$, cribriform, or sieve-like, plate of the ethmoturbinal; VO, vomer ; $P S$, presphenoid; $O S$, orbitosphenoid; $A S$, alisphenoid; $B S$, basisphenoid; $P l$, palatine ; $P t$, pterygoid; Per, periotic; 'T', tympanic bulla ; an, anterior narial aperture; $a p$, or apf, auterior palatine foramen; $p p f$, posterior palatine foramen; io, infraorbital foramen; $20 f$, postorbital process of frontal bone; $o p$, optic forameu; $s f$, sphenoidal fissure; $f r$, foramen rotundum, and anterior openiug of alisphenoid canal; $a s$, posterior opening of alisphenoid canal ; fo, foramen ovale ; flm, furamen licerum medinm; $g f$, glenoid fossa; $g p$, postglenoid process; $p g f$, postglenoid foramen; cam, external auditory meatus; sm, stylomastoid foranen; $f p$, foramen lacerum posterins ; $c f$, condylar foramen ; $p p$, paroccipital process; oc, occipital condyle ; $f m$, foranen magnum; $a$, angnlar process ; $s$, synuphysis of the mandible where it unites with the left ramus ; $i d$, inferior dental caual; $c d$, condyle; $c p$, coronoid process; the ${ }^{*}$ indicates the part of the craninm to which the condyle is articnlated when the mandible is in place; the upper border in which the teeth are implanted is called alveolar; $s h, e h, c h, b h, t h$, hyoidean apparatus, or os linguce, supporting the tongue. In the skulls of old animals, there are three ridges: occipital, behind; sagittal, median, on the upper sulface; and superorbital, across the frontal, in the region of the eyebrows. The last is highly developed in the Gorilla and other Apes.

* In this diagram, modified from Huxley's, the italicized bones are single; the rest are double. Those in the line of the Ethmoid form the Cranio-facial $A x$ s: these, with the other sphenoids and occipitals, are developed in cartilage; the rest are membraue boues. In the Humun skull, the four occipitals conlesce into on: 
the bones remain separate in the former case, while those of the chick become united together (anchylosed) in the full-grown Bird; but many bones are present in the Fish which have no representatives in the Bird. The skull consists of the brain - case and the face. The principal parts of the skull, as shown in the Dog's, are: 1. The occipital bone behind, enclosing a large hole, or foramen magnum, on each side of which are rounded prominences, called condyles, by which the skull articulates with the first cervical vertebra. 2. The two parietal bones. 3. The two frontal bones. These five form the main walls of the skull. 4. 'The sphenoid, on the floor of the skull in front of the occipital, and consisting of six pieces. 5. The two temporal bones, in which are situated the ears. In Man each temporal is a single bone; but in most animals there are three or more-the periotic, tympanic, and squamosal. 6. The malars, or "cheek-bones," each of which sends back a process to meet one from the squamosal, forming the zygomatic arch. 7. The two nasals, forming

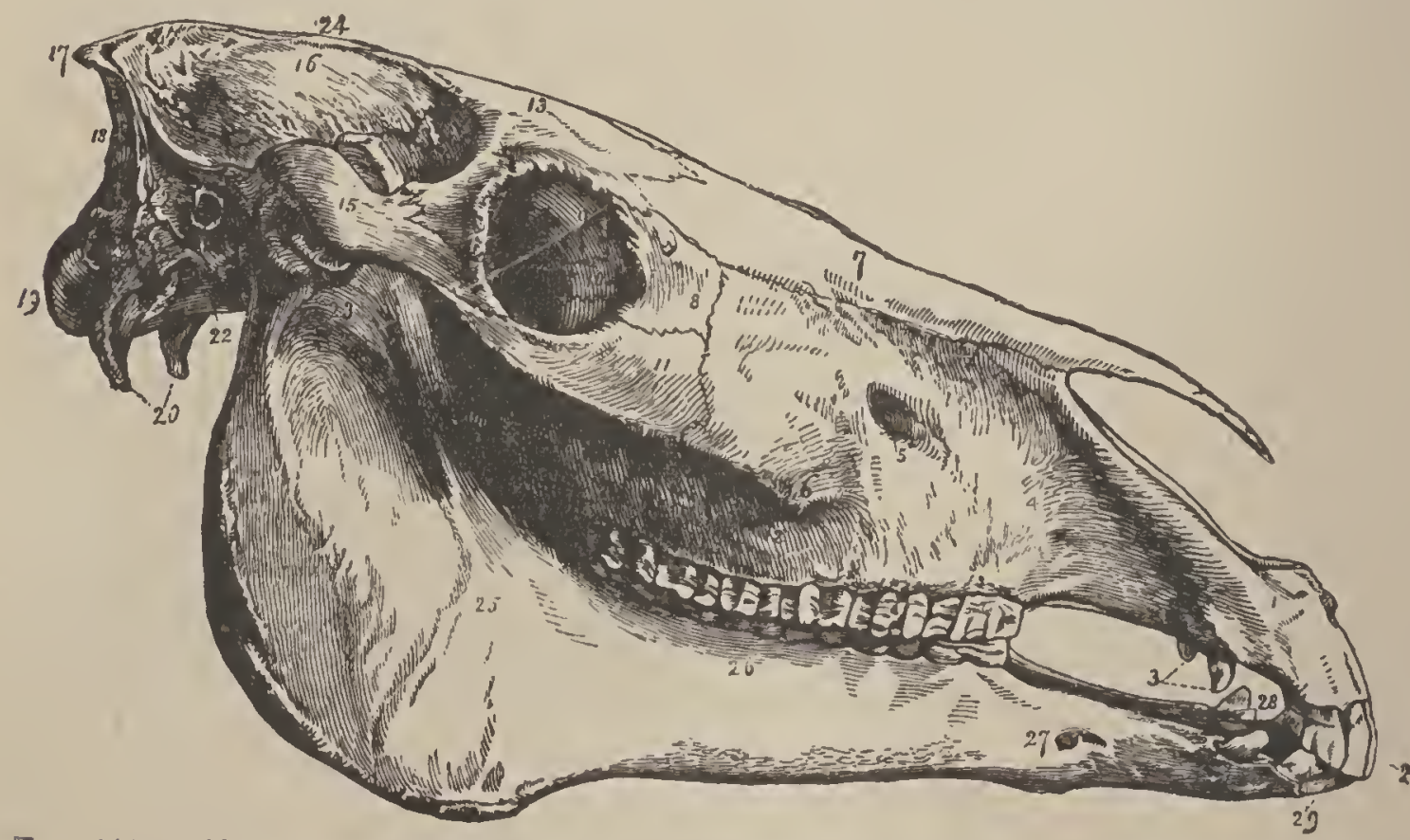

Fra. 111. - Skull of the Horse: 1, premaxillary bone; 2, npper incisors; 3, nppe* canines; 4 , superior maxillary; 5 , infraorbitai foramen; 6 , superior maxillary spine; 7 , nasal bones; 8 , lachrymal; 9 , orbital cavity; 10 , lachrymal fossa; 11 , malar; 12 , npper molars; 13 , frontal; 15 , zygomatic arch; 16 , parietal; 17 , oc. cipital protnberance; 18 , occipital crest; 19 , occipital condyles; 20 , styloid processes; 21, petrons bone; 22, basilar process; 23 , condyle of inferior maxillary : 24 , parietal crest ; 25 , inferior maxillary ; 26 , lower molars ; 27 , anterior maxillary foramen ; $2 \mathrm{~S}$, lower canines; 29 , lower incisors. 
the roof of the nose. 8. The two maxillce; that part of the upper jaw in which the canines, premolars, and molars are lodged. 9. The two premaxilloe, in which the upper incisor's are situated. 10. The two palatines, which, with the maxillary bones, form the roof of the month. There are two appendages to the skull: the mandible, or lower jaw, whose condyles, or rounded extremities, fit into a cavity (the glenoid) in the temporal bone; and the hyoid bone, situated at the root of the tongue.

The simplest form of the skull is a cartilaginous box, as in Sharks, enclosing the brain and supporting the cartilaginous jaws and gill arches. In higher Fishes this box is overlaid with bony plates and partly ossified. In Frogs the skull is mainly bony, althongh a good deal of the cartilage remains inside the bones. In higher Vertebrates the cartilage never makes an entire box, and early disappears.

The cervical vertebrce, or bones of the neck, are peculiar in having an orifice on each side of the centrum for the passage of an artery. The first, called atlas, because it supports the head, has no centrum, and turns on the second, called axis, around a blunt process, called the odontoid. The centra are usually wider than deep, and the neural spines very short, except on the last one. The number of cervical vertebræ ranges from 1 in the Frog to 25 in the Swan.

The dorsal vertebrce are such as bear ribs, which, uniting with the breast-bone, or sternum, form a bony arch over the heart and lungs, called the thorax. The sternum may be wanting, as in Fishes and Snakes, or greatly developed, as in Birds. When present, the first vertebra whose ribs are connected with it is the first dorsal. The neural spines of the dorsal series are generally long, pointing backward.

The lumbar vertebrce are the massive vertebræ lying in the loins between the dorsals and the hip-bones.

The sacral vertebra lie between the hip-bones, and are 
generally consolidated into óne complex bone, called sa crim.

The caudal vertebrce are placed behind the sacrum, and form the tail. They diminish in size, losing processes and neural arch, till finally nothing is left but the centrum. They number from 3 or 4 in Man to 270 in the Shark.

Besides the lower jaw, hyoid, and ribs, Vertebrates have other appendages to the spinal column-two pairs of limbs. ${ }^{78}$ The fore limb is divided into the pectoral arch (or shoulder girdle), the arm, and the hand. The arch is fastened to the ribs and vertebræ by powerful muscles, and consists of three bones, the scapula, or shoulder-blade, the coracoid, and the clavicle, or collar-bone. The scapula and coracoid are generally united in Mam. mals, the latter forming a process of the former; and the clavicles are frequently wanting, as in the hoofed animals. The humerus, radius, and ulna are the bones of the arm, the first articulating by ball-and-socket joint with the scapula, and by a hinge-joint with the radius and ulna. The humerus and radius are always present, but the ulua may be absent. The bones of the hand are divided into those of the carpus, or wrist; the metacarpus, or palm: and the phalanges, or finger's. The fingers, or "digits," range in number from 1 to 5 .

The hind limb is composed of the pelvic arch (or hipbones), the leg, and the foot. These parts correspond closely with the skeleton of the fore limb. Like the shoulder, the pelvic arch, or os innominatum, consists of three bones-ilium, ischium, and pubis. The three are distinct in Amphibians, Reptiles, and in the young of higher animals; but in adult Birds and Mammals they become united together, and are also (except in Whales) solidly attached to the sacrum. The two pelvic arches and the sacrum thus soldered into one make the petvis. The leg-bones consist of the fernur, or thigh; the tibia, or 
shin-bone; and the fibula, or splint-bone. The rounded head of the femur fits into a cavity (acetabulum) in the pelvic arch, while the lower end articulates with the tibia, and sometimes (as in Birds) with the fibula also. An extra bone, the patella, or knee-pan, is hung in a tendon in front of the joint between the femur and tibia of the higher animals. The foot is made up of the tarsus, or ankle; the metatarsus, or lower instep; and the phalanges, or toes. The toes number from 1 in the Horse to 5 in Man.

Certain parts of the skeleton, as of the skull, are firmly joined together by zigzag edges or by overlapping; in either case the joint is called a suture. But the great majority of the bones are intended to move one upon another. The vertebræ are locked together by their processes, and also by a tough fibrous substance between the centra, so that a slight motion only is allowed. The limbs furnish the best examples of movable articulations, as the ball-and-socket joint at the shoulder, and the hinge-joint at the elbow. The bones are held together by ligaments, and, to prevent friction, the extremities are covered with cartilage, which is constantly lubricated with an unctuous fluid called synovia.

\section{CHEMICAL COMPOSITION OF BONES.}

\begin{tabular}{|c|c|c|c|c|}
\hline \multirow[b]{2}{*}{$\begin{array}{l}\text { Phosphate of Lime, with trace of } \\
\text { Fluoride } \text { Lime..................... }\end{array}$} & Con. & TORTOIвF. & HAWK. & Man. \\
\hline & 57.29 & 52.66 & 64.39 & 59.63 \\
\hline 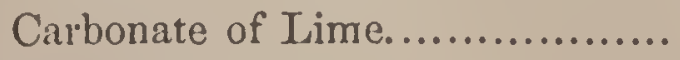 & 4.90 & 12.53 & 7.03 & 7.33 \\
\hline Phosphate of Magnesia.............. & 2.40 & 0.82 & 0.94 & 1.32 \\
\hline $\begin{array}{l}\text { Sulphate, Carbonate, and Chlorate } \\
\text { of Soda....................................... }\end{array}$ & 1.10 & 0.90 & 0.92 & 0.69 \\
\hline Glutin and Chondrin.................. & 32.31 & 31.75 & 25.73 & 29.70 \\
\hline \multirow[t]{2}{*}{ 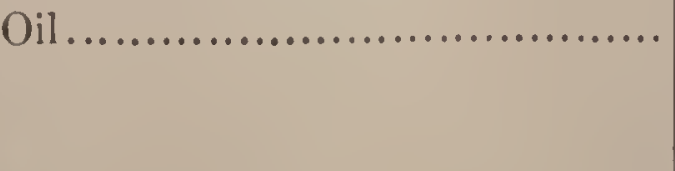 } & 2.00 & 1.34 & 0.99 & 1.33 \\
\hline & 100.00 & 100.00 & 100.00 & 100.00 \\
\hline
\end{tabular}




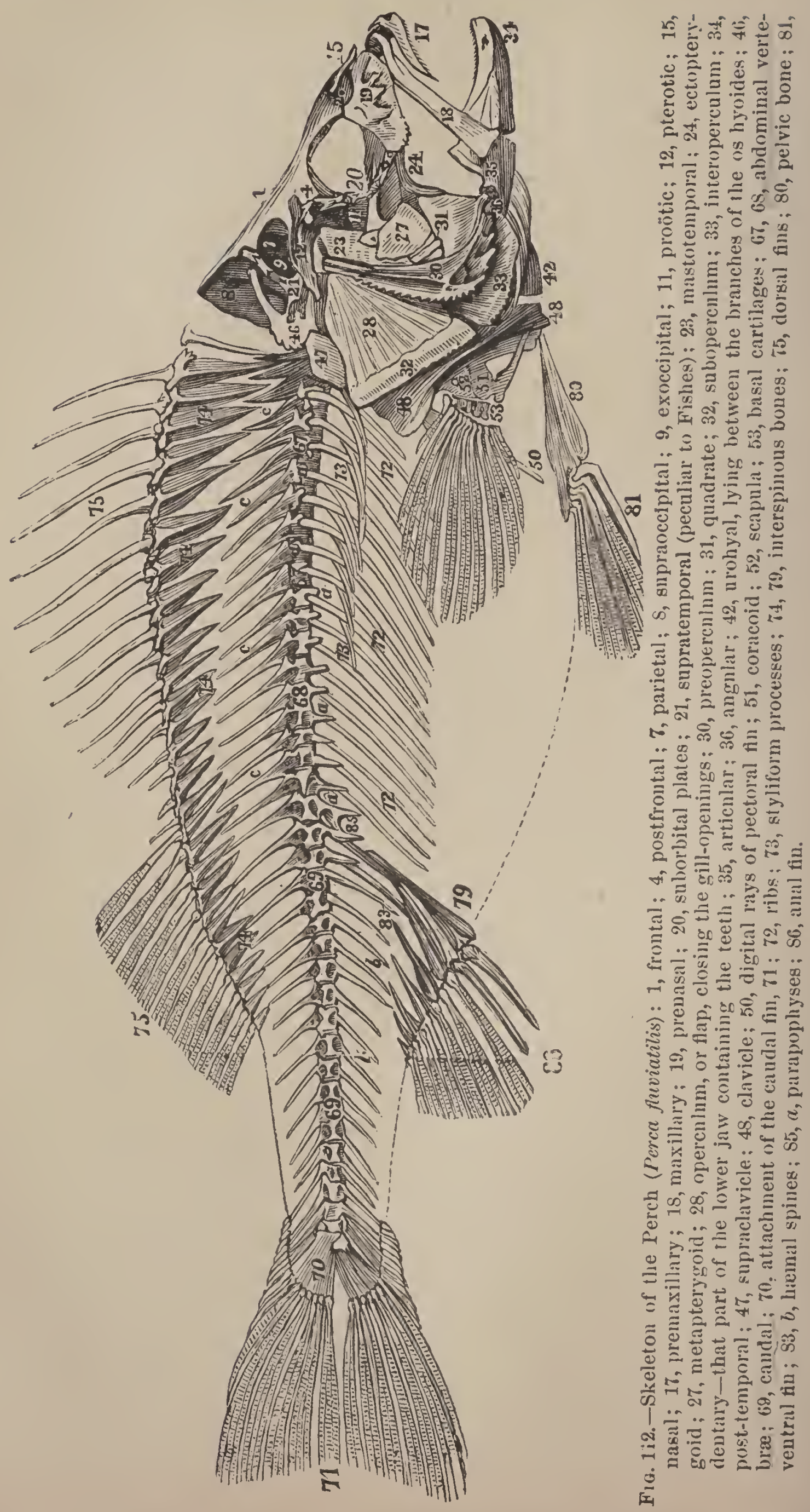



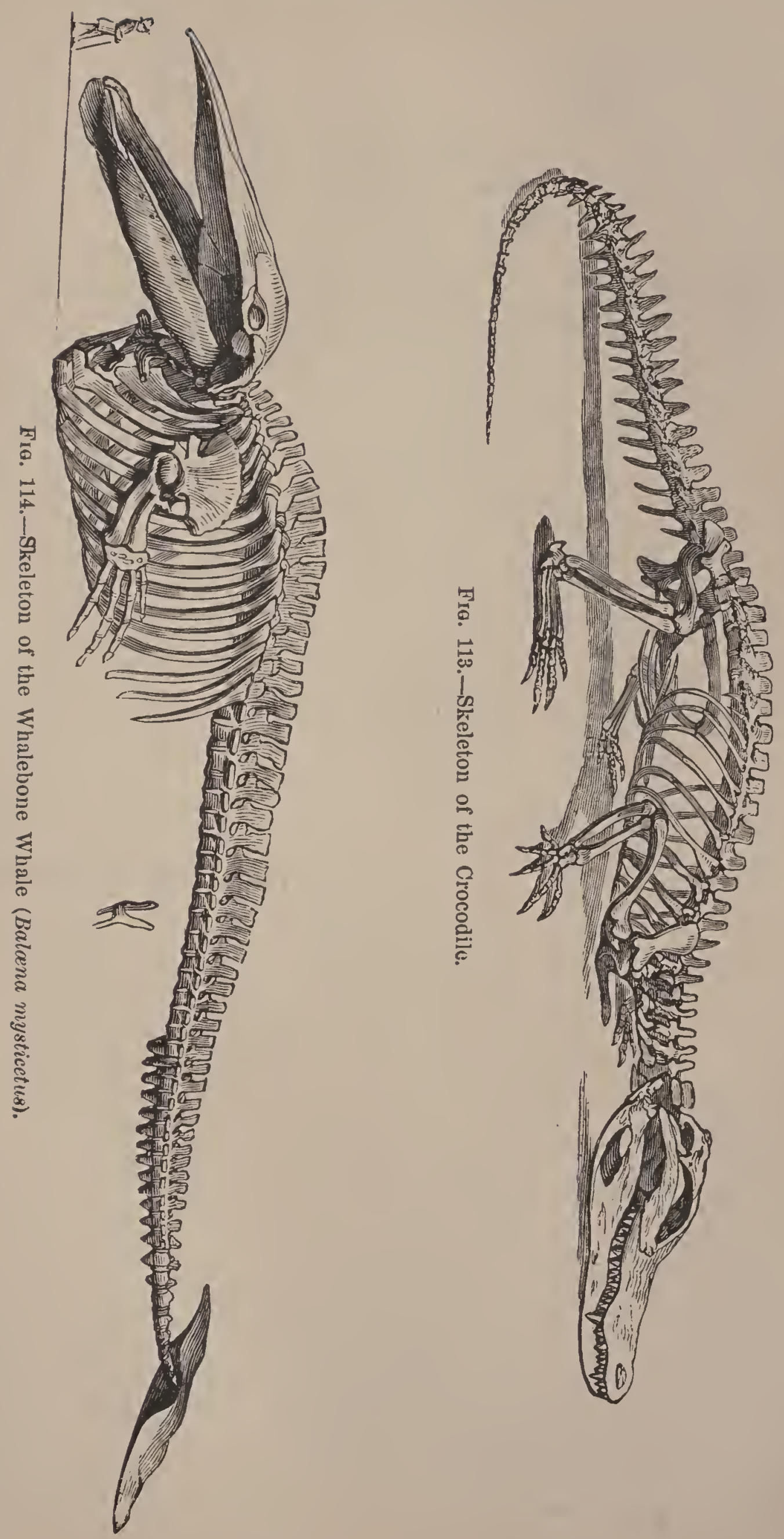


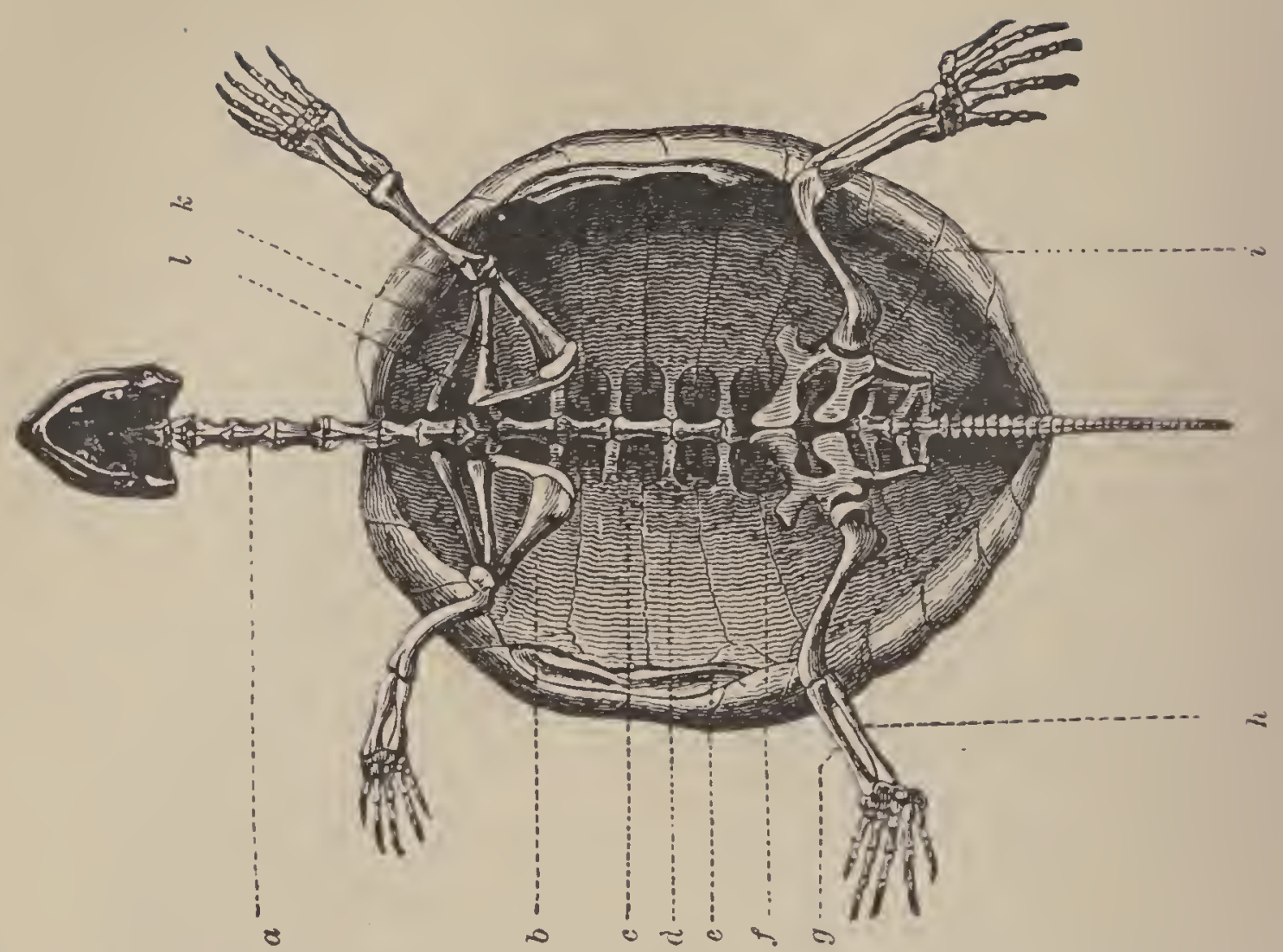

Fra. 115. -Skeleton of the Tortoise (plastron removed): $\alpha$, cervical vertebræ; $c$, dorsal vertebre; $d$, ribs; $e$, marginal bones of the carapace; $l$, scapula; $k$, precoracoid ; $b$, coracoid ; $f$, pelvis ; $i$, femur; $g$, tibia ; $h$, tibula.

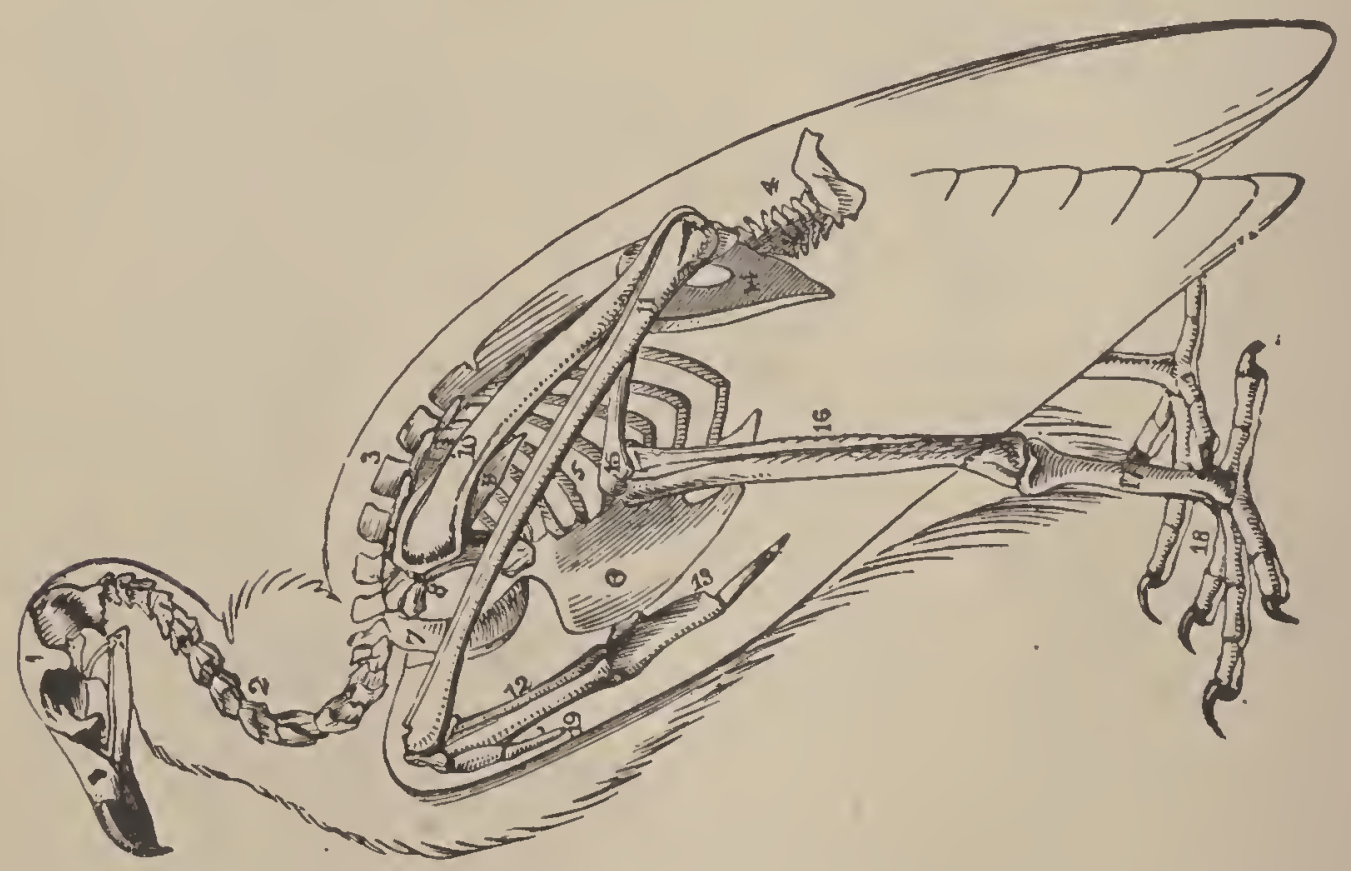

Fig. 116. -Skeleton of a Vulture: 1, craninm-the parts of which are separable only in the chick; 2, cervical vertebre; 3 , dorsal; 4 , coccygeal, or candal; the lumbar and sacral are consolidated; 5 , ribs; 6 , sternnm, or breast-bone, extraordinarily developed; 7, furculum, clavicle, or "wish-bone:" S, coracoid; 9, scapula ; 10 , humerns: 11 , nlna, with rudimentary radius; 12 , metacarpals; 13 , phalanges of the great digit of the wing: 19 , thumb; 14 , pelvis; 15 , femur: 16 , tibia-tarsns and fibula, or crus; 17 , tarso-metatarsus; 18 , internal digit, or toe, formed of three phalanges; the middle toe has fonr phalanges; the outer, five; and the back toe, or thumb, two. 


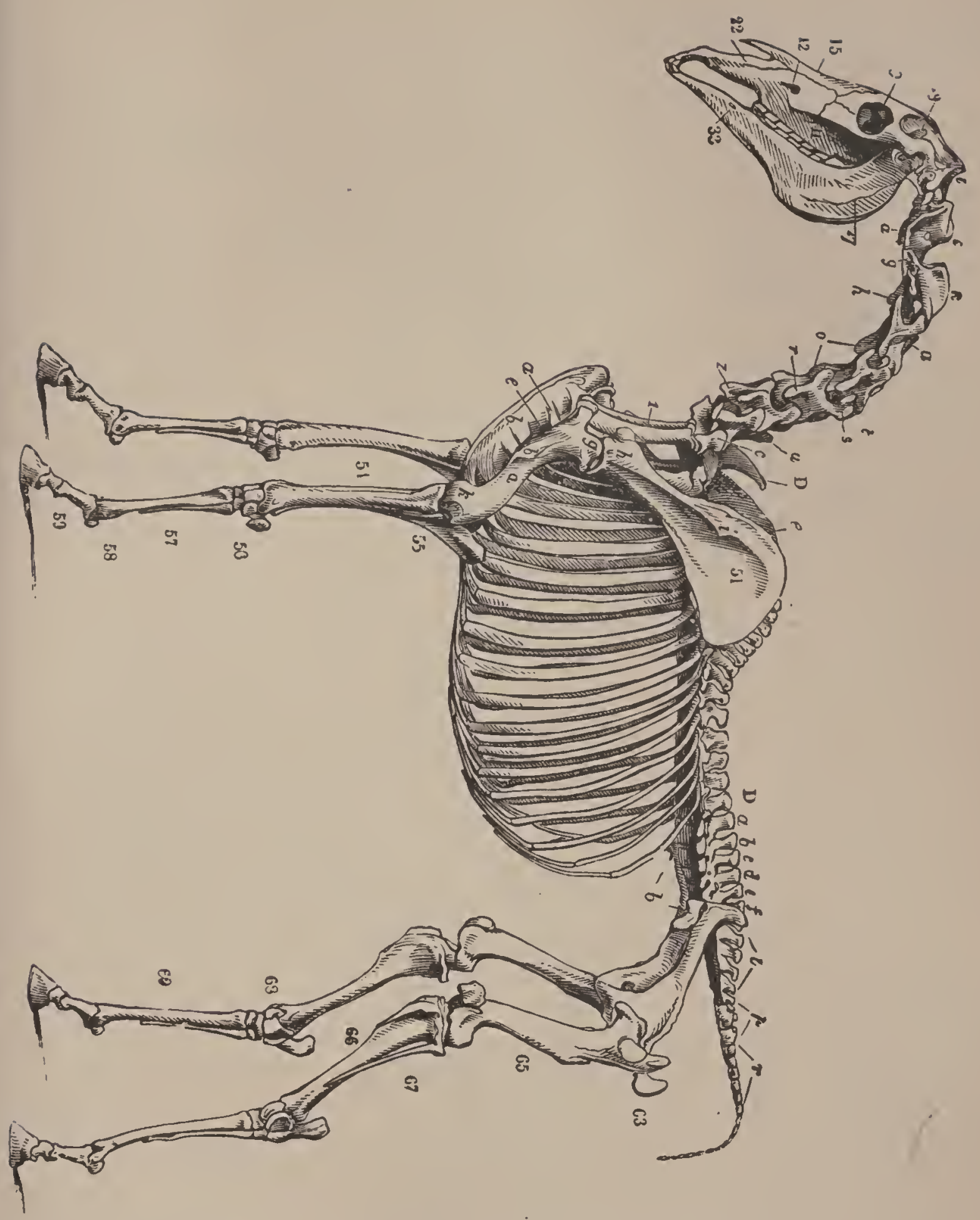

Fig. 117.-Skeleton of the Horse (Equus caballus): 22, premaxillary: 12, foramen in the maxillary; 15 , nasal ; 9 , orbit ; 19 , coronoid process of lower jaw ; 17 , surtace of implantation for the masseter muscle; there are seven cervical vertebræ, nineteen dorsal, D-D; five lumbar, $a-e$; five sacral, $f-l$; and seventeen caudal, $p-r$; 51 , scapnla, or shoulder-hlade; $i$, spine, or crest $; h$, coracoid process (acromion wanting); 1 , first pair of ribs (clavicle wanting, as in all Uvgnlates); $e$, sternum : $a$, shaft of hnmerus; $b$, deltoid ridge $: g$, head fitting in the glenoid cavity of the scapula-near it is a great tuberosity for the attachment of a powerful muscle $k$, condyles: 54 , radius, to which is firmly anchylosed a rudimentary ulna, 55 , the olecranon; 56 , the seven bones of the carpus, or wrist; 57 , larve metacarpal, or "cannon-bone," with two "splint-bones;" 5S, fetlock-joint; 59, phalanges of the developed digit, corresponding to the third finger in Man: 62, pelvis: 63 , the great trochanter, or prominence on the femur, $65 ; 66$, tibia; 67 , rudimentary Eluula; 68 , hock, or heel, falsely called knee; 69 , metatarsals. 


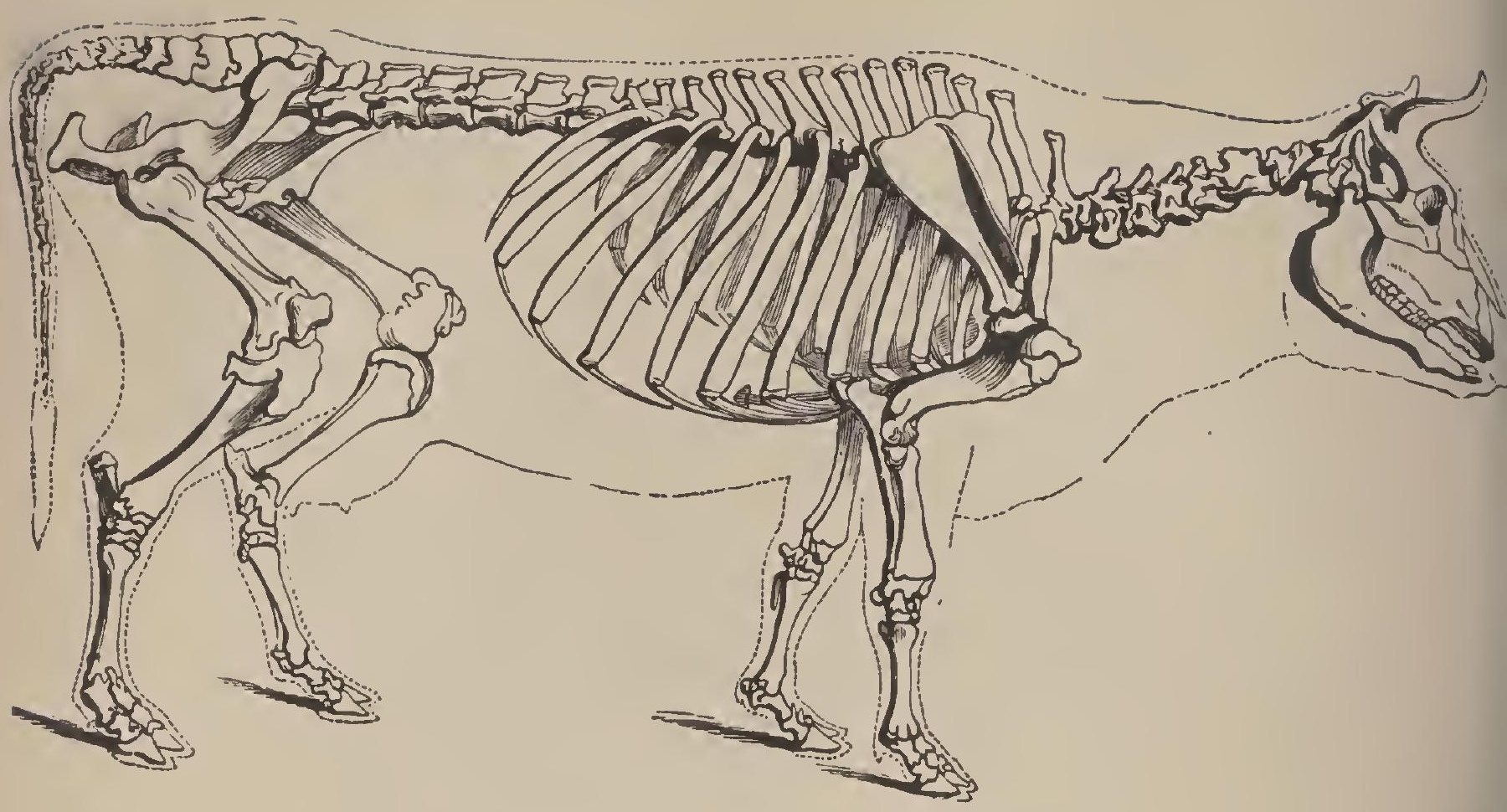

FIG. 118.-Skeleton of the Cow (Bos taurus).

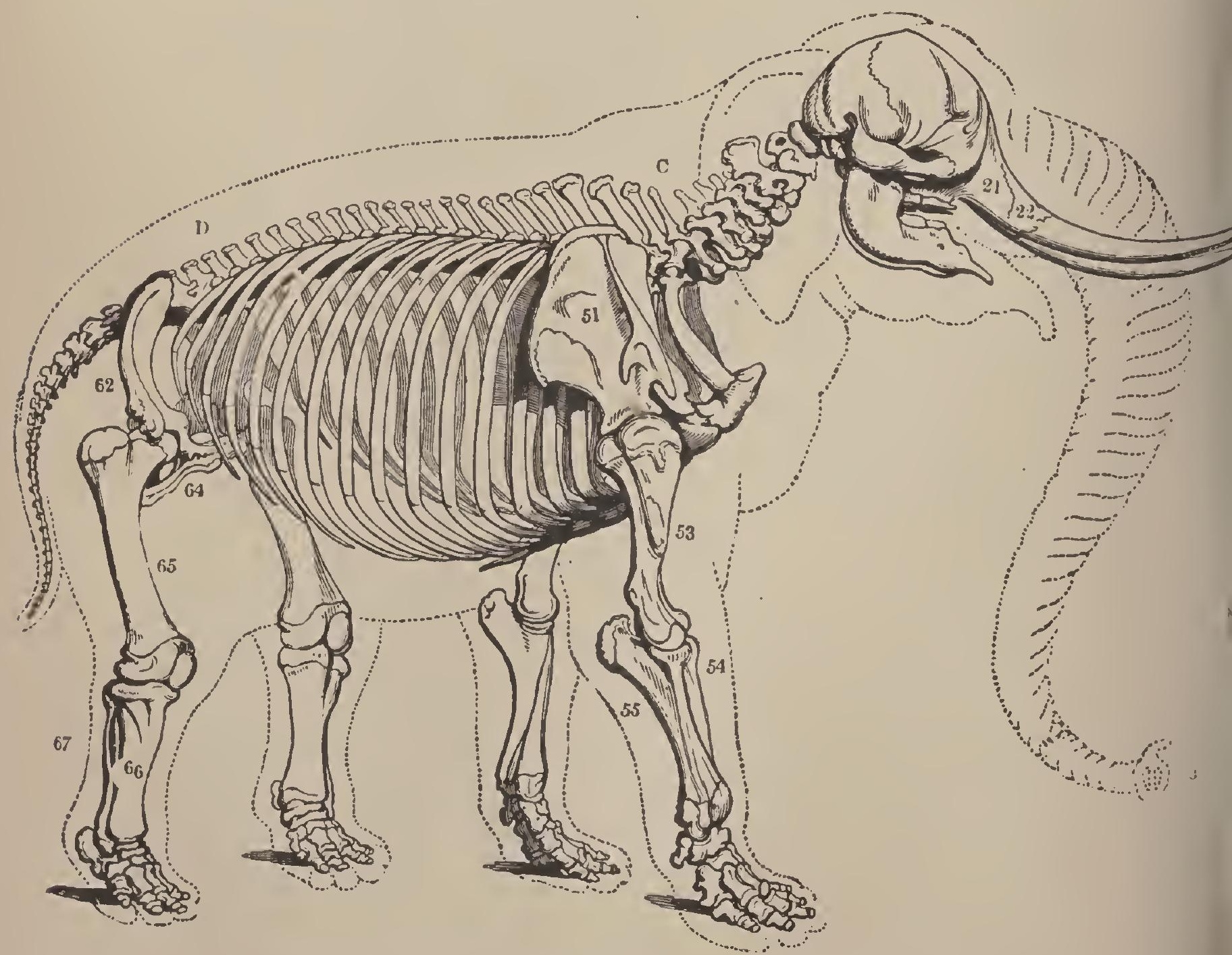

Fra. 119.-Skeleton of an Elephant (Elephas Indicus). 


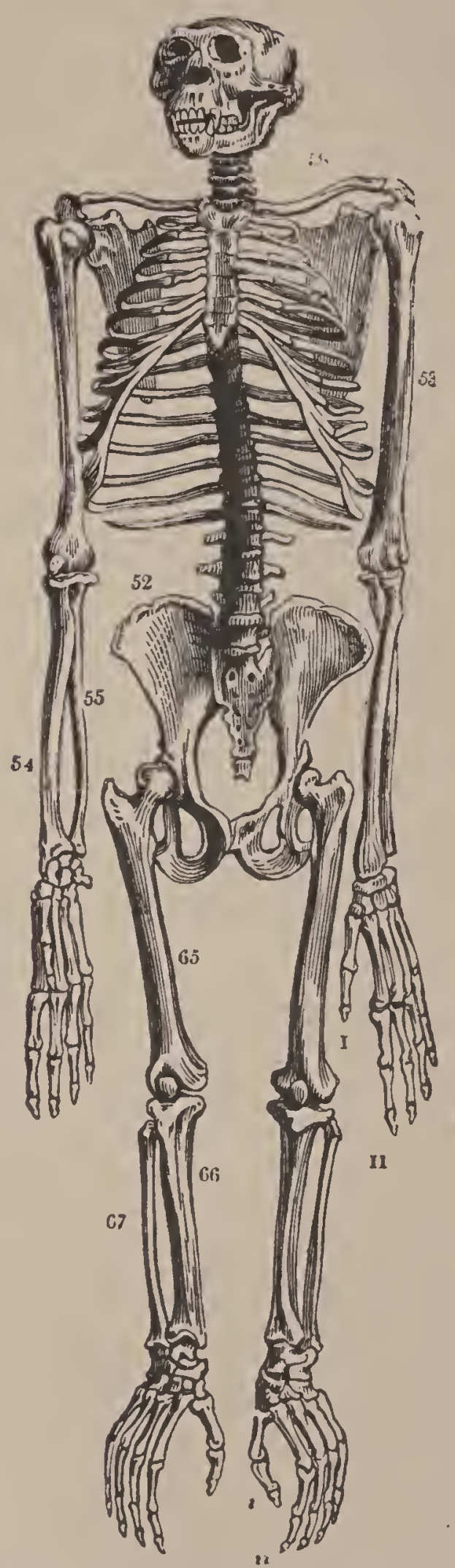

FIG. 120.-Skeleton of the Chimpanzee (Troglodytes Niger). 


\section{CHAP'TER XVII.*}

\section{H OW A I M A L S M O E.}

1. THE power of animal motion is vested in protoplasm, cilia, and muscles. The power of contractility is one of the ultimate physiological properties of protoplasm, like sensibility and the power of assimilation. Protoplasmic animals, like the Amøeba and Rhizopoda, move by the contractility of their protoplasm, as also may the germs of higher animals upon the yolk of the egg. Protoplasm may be extended into projections called pseudopodia, by whose contraction the animal may move (Fig. 185).

Infusoria, and nearly all higher animals, possess cilic (Fig. 188). These are short microscopic threads of protoplasm (Fig. 2,b) which have the power of bending into a sickle-shape and straightening out. As they bend much faster than they straighten, and as they all work together, they can cause motion of the animal, or may serve to produce currents in the water, the animal remaining at rest. They are seen on the outside of Infusoria, and of very many embryos of higher animals, serving as paddles for locomotion; they fringe the gills of the Oyster, creating currents for respiration; and they line the passage to our lungs to expel the mucus. Flagella (Figs. 187, 189) are a sort of long cilia, which are thrown into several curves when active, resembling a whip-lash, whence their name. Both cilia and flagella seem to be wanting in Arthropods.

The cause of ciliary motion is unknown. Their onesided contraction is their property, as the straight contraction of the muscle-fibre belongs to it. No structure can, however, be seen in then with the microscope. No 
nerves go to them, yet they work in concert, waves of motion passing over a surface covered with cilia, as over a field of grain moved by the wind.

1. Muscle.-Muscular tissue is the great motor agent, and exists in all animals from the Coral to Man. ${ }^{79}$ The power of contractility, which in the Amœba is diffused throughont the body, is here confined to bundles of highly elastic fibres, called muscles. When a inuscle contracts, it tends

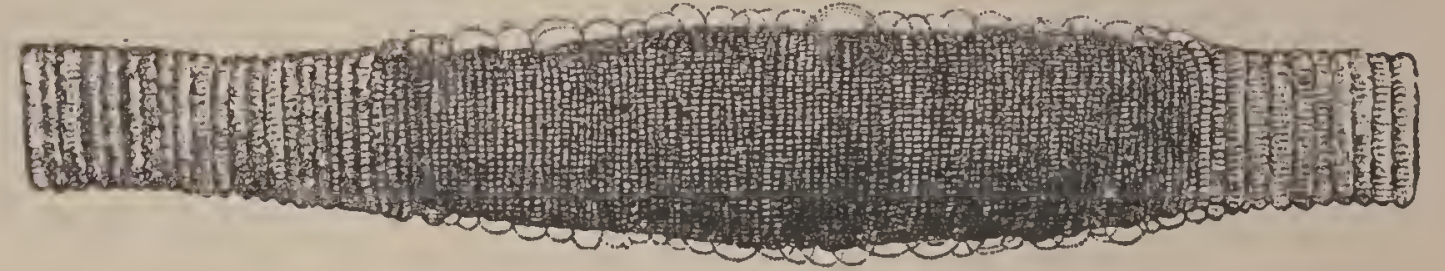

FsG. 121.-A Contractiug Muscle.

to bring its two ends together, thus shortening itself, at the same time increasing in thickness. This shrinking property is excited by external stimulants, such as electricity, acids, alkalies, sudden heat or cold, and even a sharp blow; but the ordinary canse of contraction is an influence from the brain conveyed by a nerve. The property, however, is independent of the nervous system, for the muscle may be directly stimnlated. The amount of force with which a muscle contracts depends on the number of its fibres; and the amount of shortening, on their length.

As a rule, muscles are white in cold-blooded animals, and red in the warm-blooded. They are white in all the Invertebrates, Fishes, Batrachians, and Reptiles, except Salmon, Sturgeon, and Shark; and red in Birds and Mammals, except in the breast of the common fowl, and the like. ${ }^{\text {so }}$

It is also a rule, with some exceptions, that

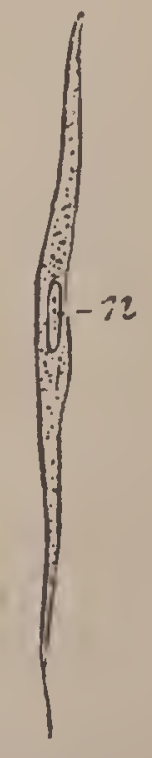

Fia. 122. - Unstriped Muscular Fibre, much enlarged; $n$, nucleus. the voluntary muscles of Vertebrates, and all the muscles 
of the Lobster, Spider, and Insect tribes, are striated; while the involuntary muscles of Vertebrates, and all the muscles of Radiates, Worms, and Mollusks, are smooth. All muscles attached to internal bones, or to a jointed external skeleton, are striated. The voluntary muscles of Vertebrates are generally solid, and the involuntary surround cavities. ${ }^{81}$

This leads to another classification of muscles: into those which are attached to solid parts within the body; those which are attached to the skin or its modifications; and those having no attachments, being complete in themselves. The last are hollow or circular muscles, enclosing a cavity or space, which they reduce by contraction. Examples of such are seen in the heart, blood-vessels, stomach, iris of the eye, and around the mouth. In the lower Invertebrates, the muscular system is a net-work of longitudinal, transverse, and oblique fibres intimately blended with the skin, and not divisible into separate muscles. As in the walls of the human stomach, the fibres are usually in three distinct layers. This arrangement is exhibited by soft-bodied animals, like the Sea-anemone, the Snail, and the Earth-worm. Four thousand muscles have been counted in a Caterpillar. There are also "skin-muscles" in the higher animals, as those by which the Horse produces a twitching of the skin to shake off insects, and those by which the hairs of the head and the feathers of Birds are made to stand on end. Invertebrates whose skin is hardened into. a shell or crust have muscles attached to the inside of such a skeleton. Thus, the Oyster has a mass of parallel fibres connecting its two valves; while in the Lobster and Bee fibres go from ring to ring, both longitudinally and spirally. The muscles of all Invertebrates are straight parallel fibres, not in bundles, but distinct, and usually flat, thin, and soft.

The great majority of the muscles of Vertebrates are attached to the bones, and such are voluntary. The fibres, 
which are coarsest in Fishes (most of all in the Rays), and finest in Birds, are bound into bundles by connective tissue; and the muscles thus made up are arranged in layers around the skeleton. Sometimes their extremities are attached to the bones (or rather to the periosteum) directly; but generally by means of white inelastic cords, called tendons. In Fishes, the chief masses of muscle are disposed along the sides of the body, apparently in longitudinal bands, reaching from head to tail, but really in a series of vertical flakes, one for each vertebra. In proportion as limbs are developed, we find the muscles concentrated about the shoulders and hips, as in quadrupeds. The bones of the limbs are used as levers in locomotion, the fulcrum being the end of a bone with which the moving one is articulated. Thus, in raising the arm, the humerus is a lever working upon the scapula as a fulcrum. The most important muscles are called extensors and flexors. The latter are such as bring a bone into an angle with its fulcrum-as in bending the arm-while the former straighten the limb. Abductors draw a limb away from the middle line of the body, or a finger or toe away from the axis of the limb, while adductors bring them back.

2. Locomotion.-All animals have the power of voluntary motion, and all, at one time or another, have the means of moving themselves from place to place. Some are free in the embryo-life, and fixed when adult, as the Sponge, Coral, Crinoid, and Oyster. There may be no regular well-defined means of progression, as in the Amœba, which extemporizes arms to creep over the surface; or movement may be accomplished by the contraction of the whole body, as in the Jelly-fish, which, pulsating about fifteen times in a minute, propels itself through the water. So the Worms and Snakes swim by the undulations of the body.

But, as a rule, animals are provided with special organs 
for locomotion. These become reduced in number, and progressively perfected, as we advance in the scale of rank. Thus, the Infusorian is covered with thousands of hair-like cilia; the Star-fish has hundreds of soft, unjointed, tubular suckers; the Centipede has from 30 to 40 jointed hollow legs; the Lobster, 10 ; the Spider, 8 ; and the Insect, 6 ; the Quadruped has 4 solid limbs for locomotion; and Man, only 2.

(1) Locomotion in Water.-As only the lower forms of life are aquatic, and as the weight of the body is partly sustained by the element, we must expect to find the organs of progression simple and feeble. The Infusoria swim with great rapidity by the incessant vibrations of the delicate filaments, or cilia, on their bodies. The common Squid on our coast admits water into the interior of the body, and then suddenly forces it ont through a funnel, and thus moves backward, or forward, or around, according as the funnel is turned-towards the head, or tail, or to one side. The Lobster has a fin at the end of its tail, and propels itself backward by a quick downward and forward stroke of the abdomen.

But Fishes, whose bodies offer the least resistance to progression through water, are the most perfect swimmers. Thus, the Salmon can go twenty miles an hour, and even

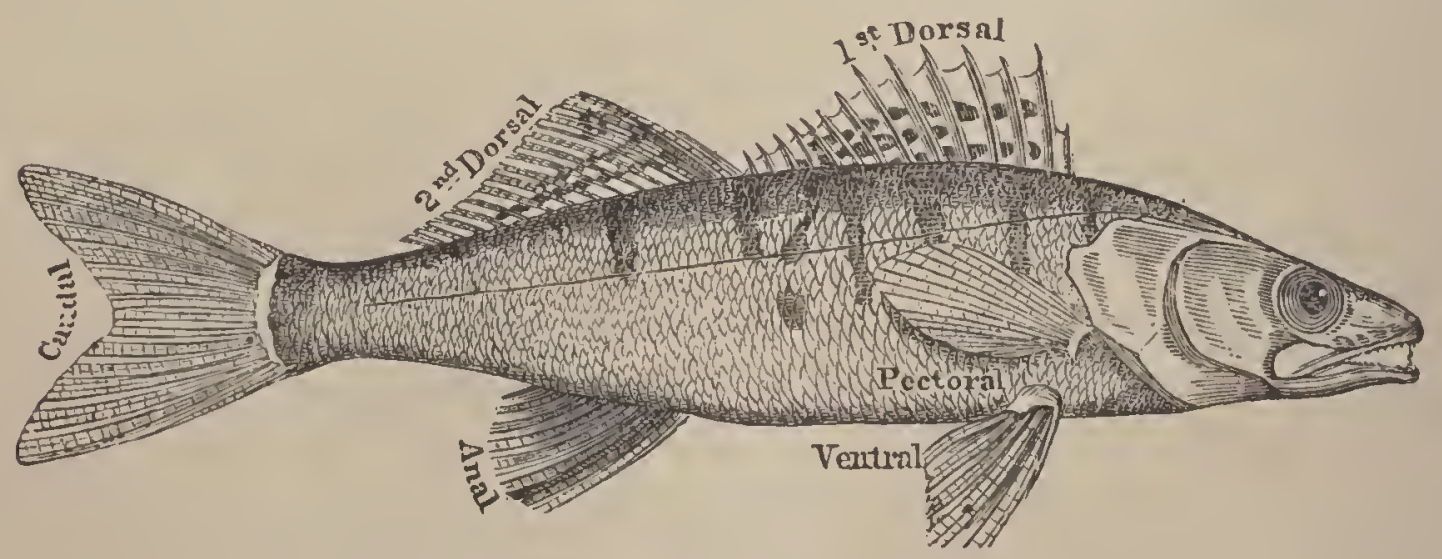

Frg. 123. -The Fins of a Firh (Pike-perch).

ascend cataracts. They have fins of two kinds: those set obliquely to the body, and in pairs; and those which are 
vertical, and single. The former, called pectoral and ventral fins, represent the fore and hind limbs of Quadrupeds. The vertical fins, which are only expansions of the skin, vary in number; but in most Fishes there are at least three: the caudal, or tail-fin; the dorsal, or back-fin; and the anal, situated on the abdomen, near the tail. The chief locomotive agent is the tail, which sculls like a stern-oar; the other fins are mainly used to balance and raise the body. When the two lobes of the tail are equal, and the vertebral column stops near its base, as in the Tront, it is said to be homocercal. If the vertebræ extend into the upper lobe, making it longer than the lower one, as in the Shark, the tail is called heterocercal. The latter is the more effective for varying the course; the Shark, e. g., will accompany and gambol around a ship in full sail

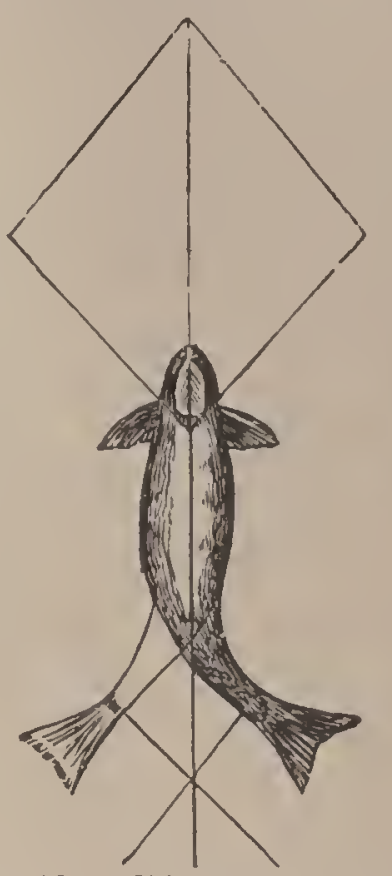

FIG. 124. -Diagram illustrating the locomotion of it Fish. The tail describes the arc of an ellipse; the resultaut of the two im. pulses is the straight line in front. across the Atlantic. The Whale swims by striking the water up and down, instead of laterally, with a fin-like horizontal tail. Many air-breathing animals swim with facility on the surface, as the Water-birds, having webbed toes, and most of the Reptiles and Quadrupeds.

(2) Locomotion in Air.-The power of flight requires a special modification of structure and an extraordinary muscular effort, for air is 800 times lighter than water. Nevertheless, the velocity attainable by certain Birds is greater than that of any Fish or Quadruped; the Hawk being able to go at the rate of 150 miles an hour. The bodies of Insects and Birds are made as light as possible by the distribution of air-sacs or air-cavities. ${ }^{82}$

The wings of Insects are generally four in number; 
sometimes only two, as in the Fly. They are moved by muscles lying inside the thorax. They are simple expansions of the skin, or crust, being composed of two delicate films of the epidermis stretched upon a net-work of tubes. There are three main varieties: thin and transparent, as in the Dragon-fly; opaque, and covered with minute colored scales, which are in reality flattened hairs, as in the Butterfly; and hard and opaque, as the first pair (called elytra) of the Beetle.

The wings of Birds, on the other hand, are modified fore-limbs, consisting of three sets of feathers (called primary, secondary, and tertiary), inserted on the hand, forearm, and arm. The muscles which give the downward stroke of the wing are fastened to the breast-bone; and their power, in proportion to the weight of the Bird, is very great. Yet the Insect is even superior in vigor and velocity of flight. ${ }^{83}$ In ascending, the Bird slightly rotates the wing, striking downward and a little backward; while the tail acts as a rudder. A short, rounded, concave wing, as in the common Fowl, is not so well fitted for high and prolonged flight as the long, broad, pointed, and flat wing

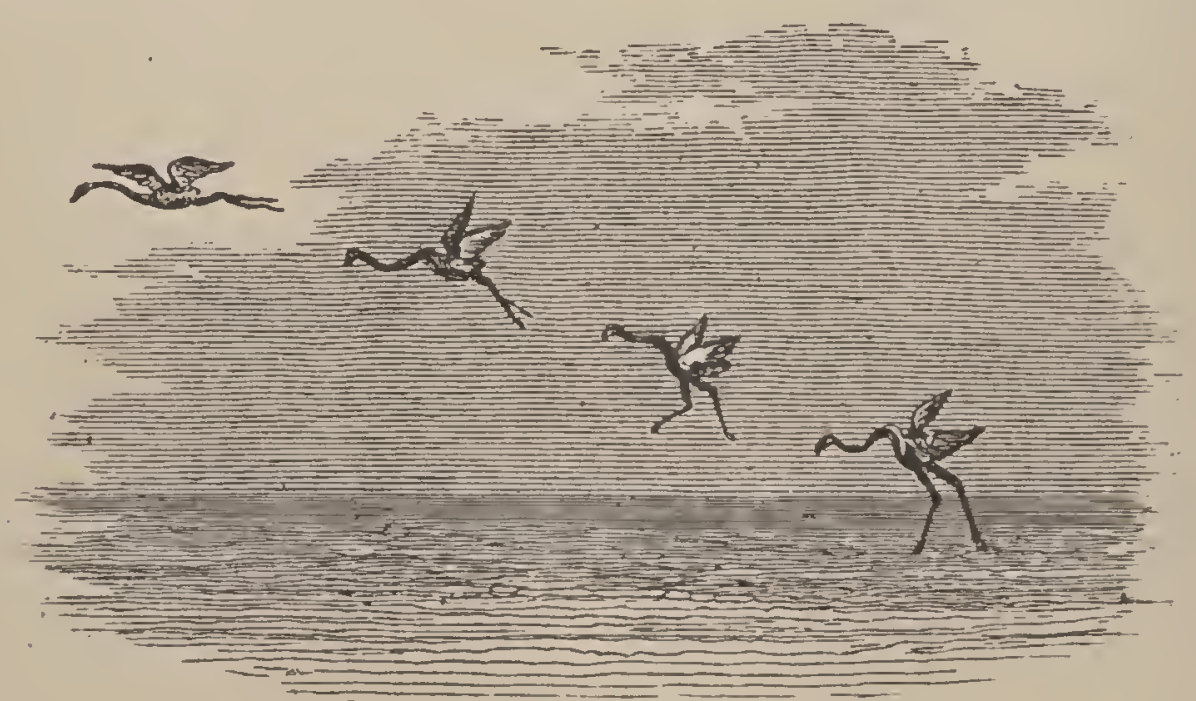

Fra. 125.-Flamingoes taking Wing.

of the Eagle. The wing is folded by means of an elastic skin and muscle connecting the shoulder and wrist. Besides Insects and Birds, a few other animals have the power 
of flight, as Bats, by means of long-webbed fingers; Flying Fishes, by large pectoral fins. Flying Reptiles, Flying Squirrels, and the like, have a membrane stretched on the long ribs, or connecting the fore and hind limbs, which they use as a parachute, enabling them to take very long leaps.

(3) Locomotion on Solids. - This requires less muscular effort than swimming or flying. The more unyielding the basis of support, the greater the amount of force left to move the animal along. The simplest method is the suctorial, the animal attaching itself to some fixed object, and then, by contraction, dragging the body onward. But the higher and more common method is by the use of bones, or other hard parts, as levers.

The Star-fish creeps by the working of hundreds of tubular suckers, which are extended by being filled with

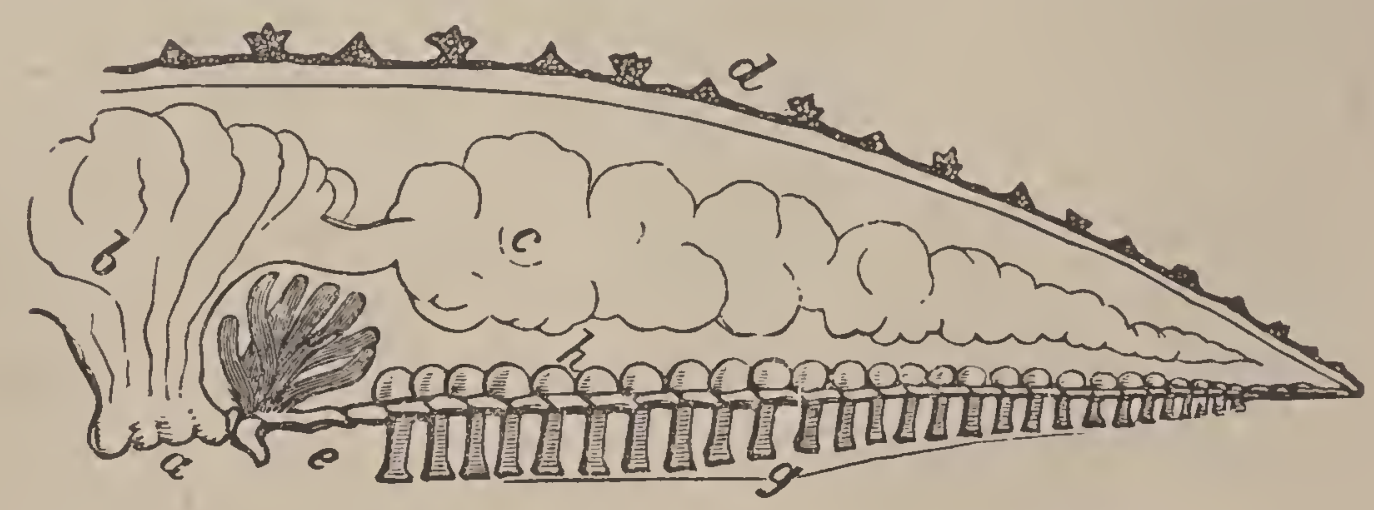

Fig. 126.-Diagrammatic section of Star-fish: $a$, mouth; $b$, stomach ; $c$, hepatic cxcum; $d$, dorsal or aboral surface; $e$, ambulacral plates; $f$, ovary ; $g$, tubular feet ; $h$, internal sacs for distending the feet.

fluid forced into them by little sacs. The Clam moves by fixing and contracting a muscular appendage, called a "foot." The Snail has innumerable short muscles on the under side of its body, which, by successive contractions, resembling minute undulations, enable the animal to glide forward apparently without effort. The Leech has a sucker at each end: fixing itself by the one on its tail, and then stretching the body, by contracting the muscular fibres which run around it, the creature fastens its mouth by suction, and draws forward the hinder parts by 
the contraction of longitudinal muscles. The Earth-worm lengthens and shortens itself in the same way as the Leech, but instead of suckers for holding its position, it has numerous minute spines pointing backward; while the Caterpillar has short legs for the same purpose. The legless Serpent moves by means of the scutes, or large scales, on the under side of the body, acted upon by the ribs. In a straight line, locomotion is slow; but by curving the body, laterally or vertically, it can glide or leap with great rapidity.

Most animals have movable jointed limbs, acted upon as levers by numerons muscles. The Centipede has forty-

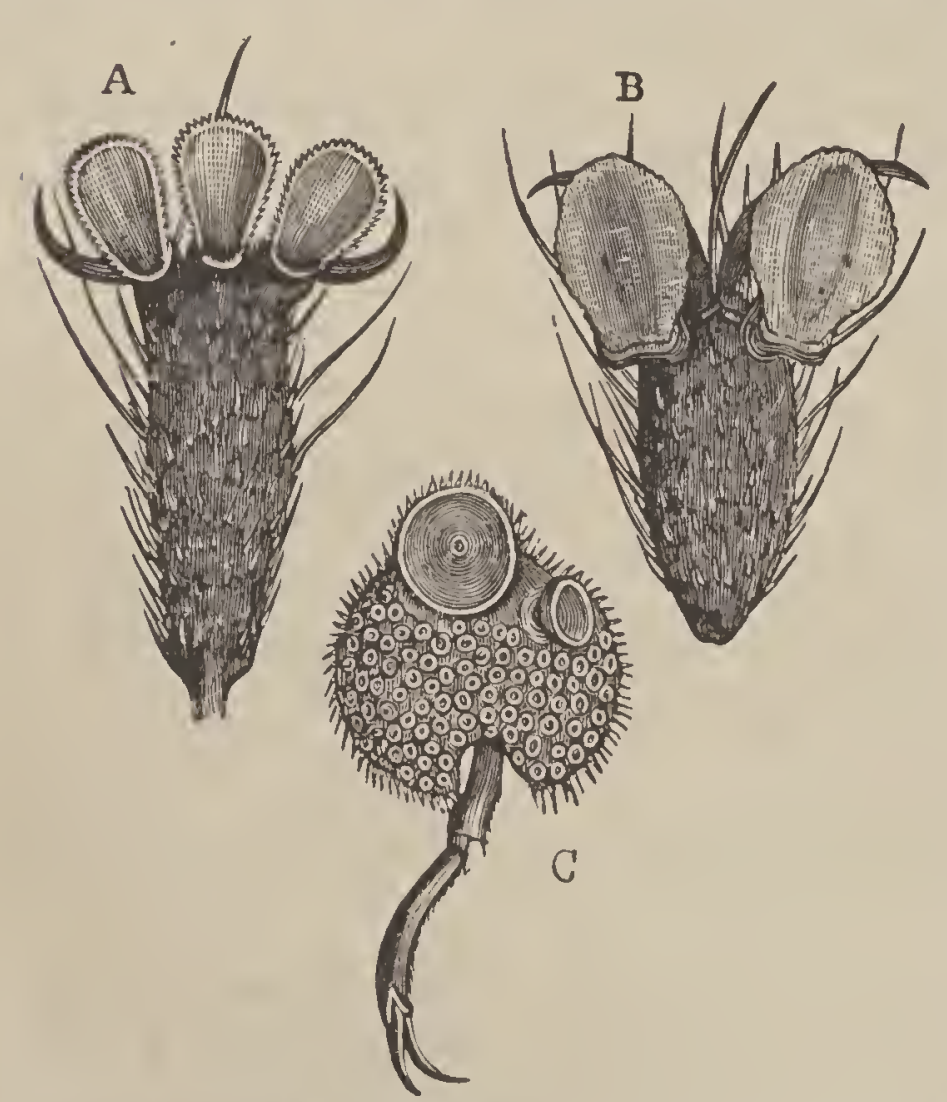
two legs, each with five joints and a claw. The Crab has five pairs of six-jointed legs; but the front pair is modified into pincers for prehension. With the rest, which end in a sharp claw, the Crab moves backward, forward, or sideways. The Spider has eight legs, usually seven-joint. ed, and terminating

Fia. 127. - Feet of Insects: A, Bibio Pebrilis; B, in two claws toothed House-fly (Musca domestica); C, Water-beetle (Dytiscrus).

like a comb, and a third which acts like a thumb. In running, it moves the first right leg, then the fourth left; next, the first left, and then the fourth right; then the third right and second left together; and lastly, the third left and second right together. The front and hind pairs are, therefore, moved like those of a quadruped. The Insect has six 
legs, each of five parts: the coxa; trochanter; femur; tibia, or shank; and tarsus. The last is subdivided usually into five joints and a pair of claws. Such as can walk upside down, as the Fly, have, in addition, two or three pads between the claws. ${ }^{84}$ These pads bear hairs which secrete a sticky fluid, by means of which the Fly adheres to the surface. While the leg-bones of Vertebrates are covered by the muscles which move them, the limbs of Insects are hollow, and the muscles inside. The fore legs are directed forward, and the two hinder pairs backward. In motion, the fore and hind feet on one side, and the middle one on the other, are moved simultaneously, and then the remaining three.

The four-legged animals have essentially the same apparatus and method of motion. The Crocodile has an awkward gait, owing to the fact that the limbs are short, and placed far apart, so that the muscles act at a mechanical disadvantage. The Tortoise is proverbially slow, for a similar reason. Both swim better than they walk. Lizards are light and agile, but progression is aided by a wriggling of the body.

The locomotive organs of the mammalian quadrupeds are much more lighly organized. The bones are more compact; the vertebral column is arched, and yet elastic, between the shoulder and hip, and the limbs are placed vertically underneath the body. The bones of the fore limb are nearly in a line; but those of the hind limb, which is mainly used to project the body forward, are more or less inclined to one another, the angle being most marked in animals of great speed, as the Horse. Some walk on hoofs, as the Ox (Ungulate); some on the toes, as the Cat (Digitigrade); others on the sole, touching the ground with the heel, as the Bear (Plantigrade). In the Pinnigrade Seal, half of the fore limb is buried under the skin, and the hind limbs are turned backward to form a fin with the tail. The normal number of toes is five; but 

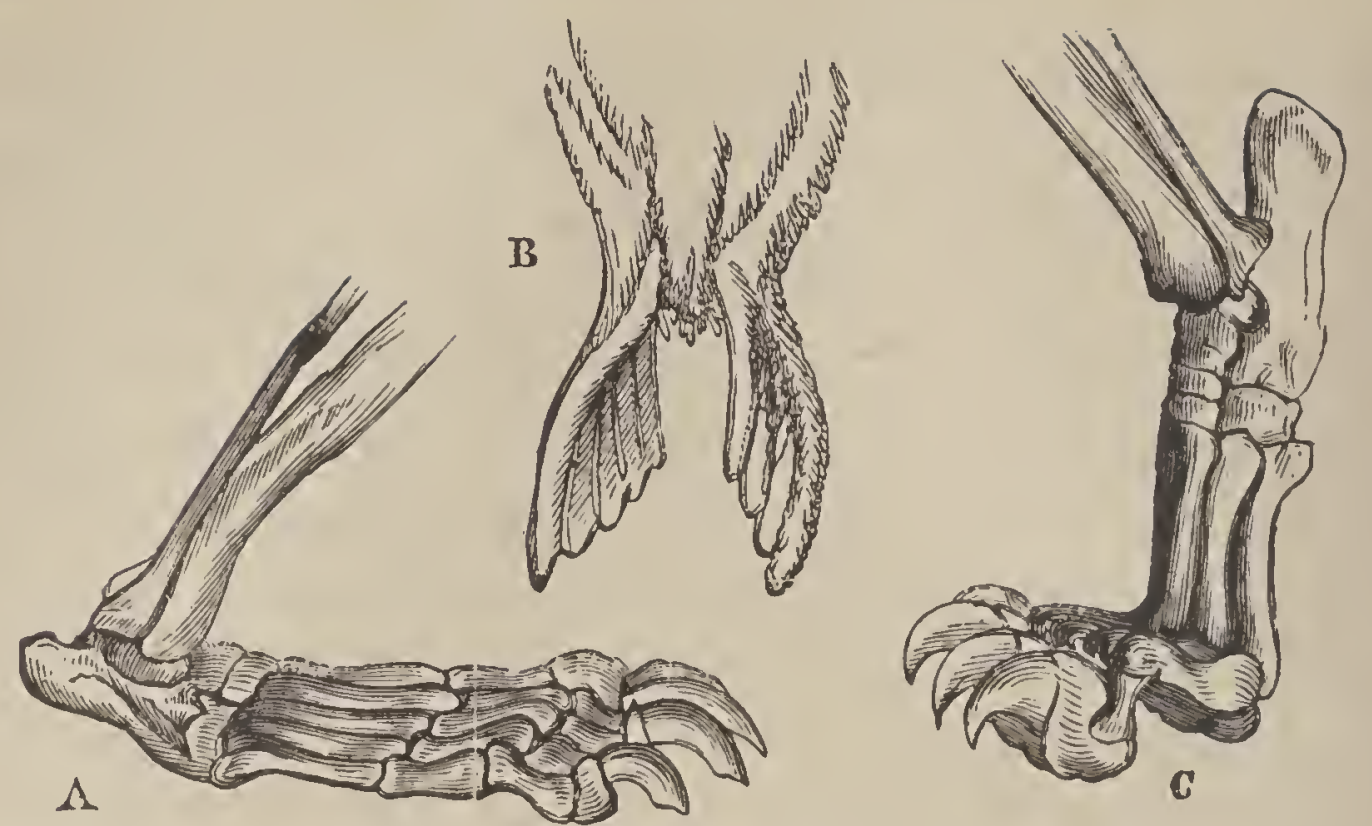

Fra. 129. - Feet of Caruivores : A, Plantigrade (Bear): B, Pinuigrade (Seal): C, Digitigrade (Lion).

some may be wanting, so that we have one-toed animals (as Horse), two-toed (as Ox), three-toed (as Rhinoceros), four-toed (as Hippopotamus), and five-toed (as the Elephant). The Horse steps on what corresponds to the nail of the middle finger; and its swiftness is conditioned on the solidity of the extremities of the limbs. Horses of the greatest speed have the shoulder-joints directed at a considerable angle with the arm.
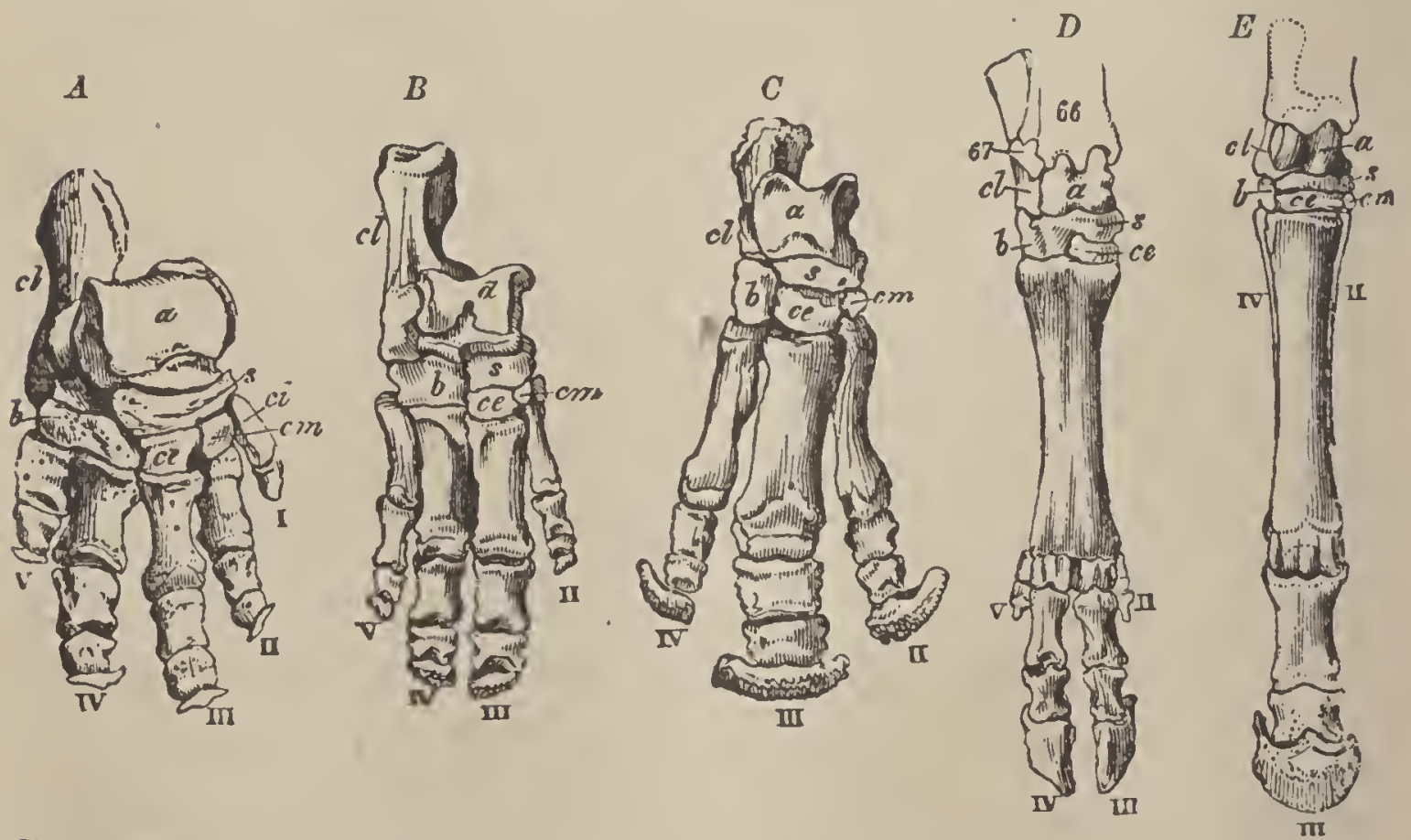

Fre. 129. - Feet of Honfed Mammals: $A$, Elephant: $B$, Hippopotnmus; $C$, Rhinoceros: $D, O x ; l$, Horse. $a$, astragalus ; $c l$, calcaneum, or heel $: s$, vaviculare ; $b$, cuboides: $c e, c i, c m$, cuneiform bones; the numbers indicate the digits in use. 
The order in which the legs of Quadrupeds succeed each other determines the various modes of progression, called the walk, trot, gallop, and leap. Many, as the Horse, have all these movements; while some only leap, as the Frog and Kangaroo. In leaping animals, the hind limbs are extraordinarily developed. In many Mammals, like the Squirrel, Cat, and Dog, the fore legs are used for prehension as well as locomotion. Monkeys use all four,

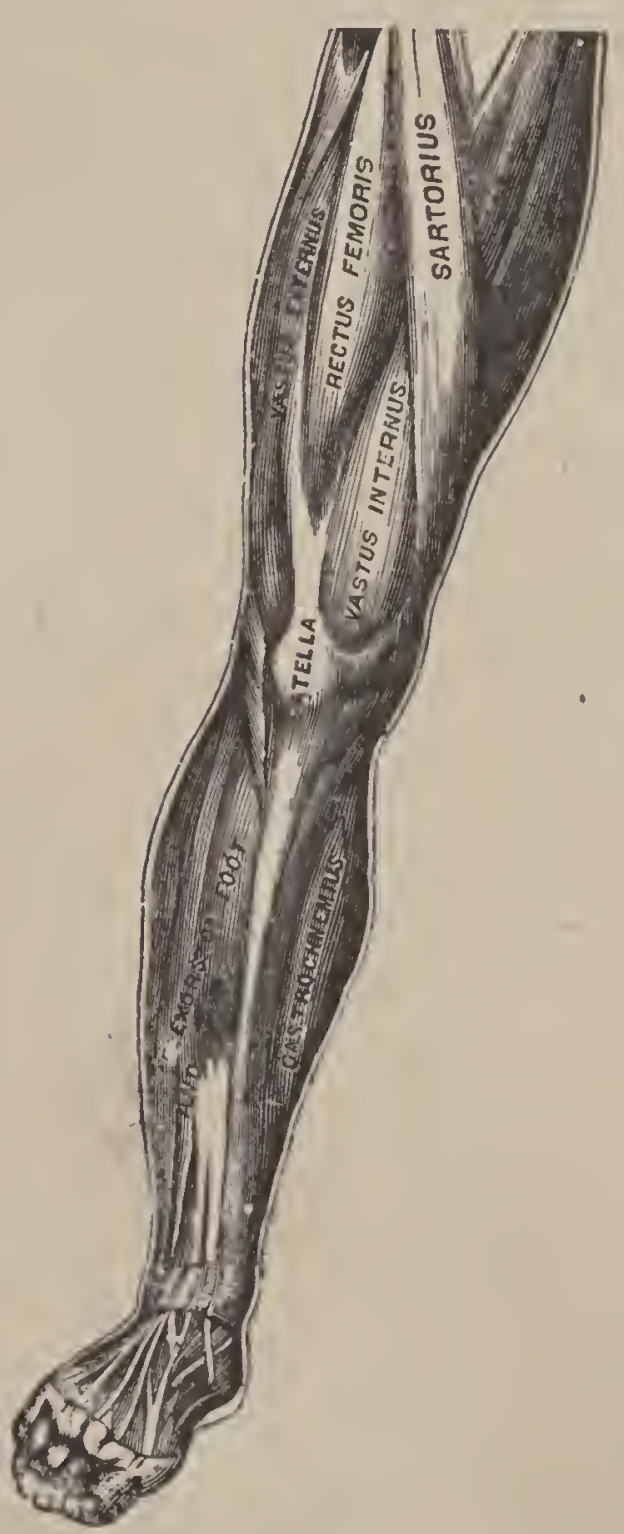

Fia. 130.-Murcles of the Human Leg: sarforius, or "tailor's muscle," the longest muscle in the body, flexes the leg upon the thigh; rectus femoris and vastus externus and internus extend the leg, maintaining an erect posture; gastrocneminus, or "calf," used chiefly in walking, for raising the heel. Another layer underlies these superficial muscles.

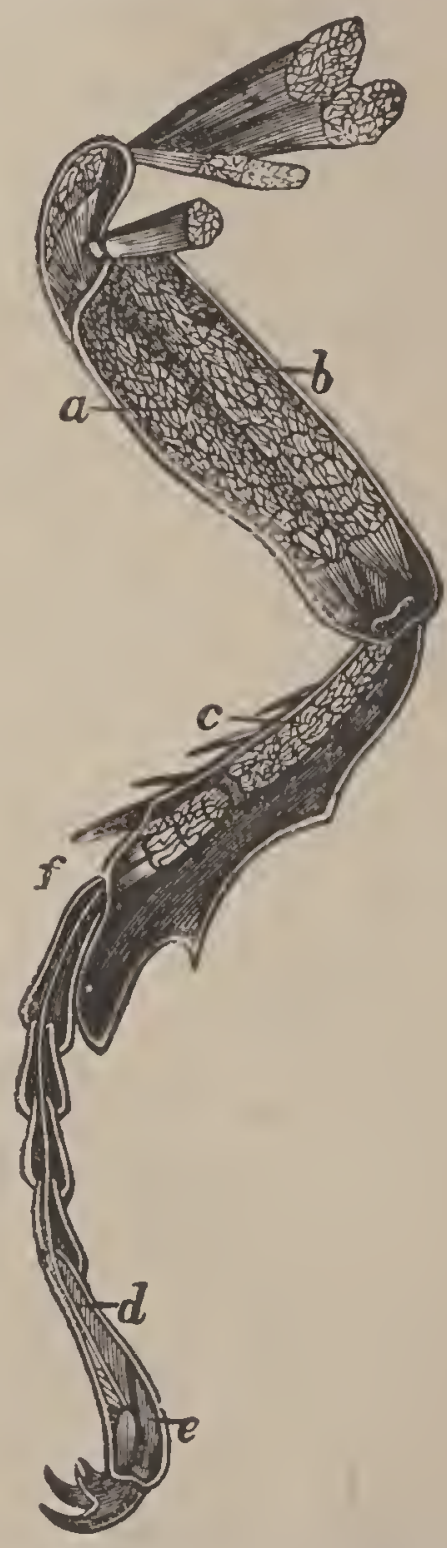

Fi木. 131. - Miscles of an Insect's Leg (Melolontha vulgaris): $a$, flexor, and $b$, extensor, of tibia ; $c$, flexor of foot ; $d$, accessory muscle; $e$, extensor of claw; $f$, extensor of tarsus. The joints are restricted to movements in one plane: and therefore the mus. cles are simply flexors and extensors. All the muscles are within the skele. ton. 
and also the tail, for locomotion and prehension, keeping a horizontal attitude; while the Apes, half erect, as if they were half-quadruped, half-biped, go shambling along, touching the ground with the knuckles of one hand and then of the other. In descending the scale, from the most anthropoid Ape to the true Quadruped, we find the centre of gravity placed increasingly higher up-that is, farther forward. Birds and Men are the only true bipeds; the former standing on their toes, the latter on the soles of the feet. Terrestrial Birds walk and run; while Birds of flight usually hop. The Ostrich can for a time outrun the Arabian Horse; and the speed of the Cassowary ex. ceeds that of the swiftest Greyhound.

\section{CHAPTER XVIII.*}

THE NERVOUS SYSTEM.

Nervous Matter exists in the form of cells and fibres. In the cellular state it is grayish, and accumn-

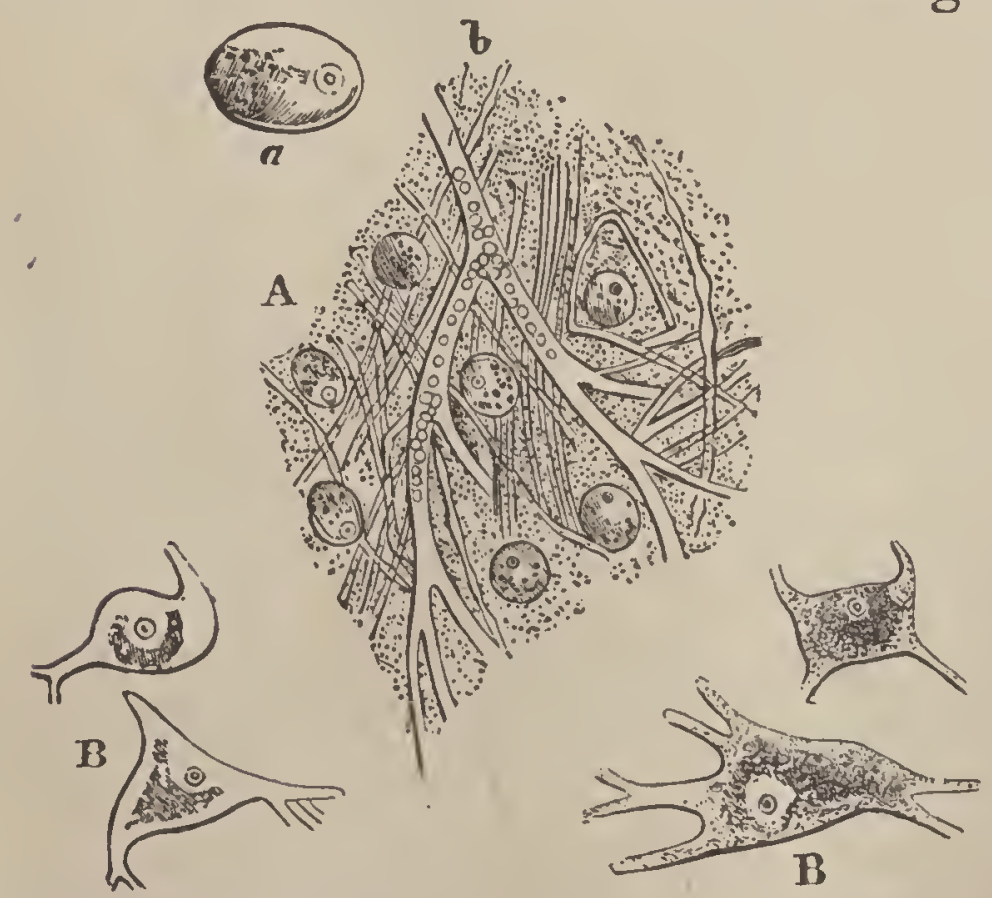
lated in masses, called ganglia, or centres, which alone originate nervous force; the fibres are generally white, and arranged in bundles, called nerves, which serve only as conductors. Most nerves contain two

Fra. 132. - Nerve-cells from Human Brain: A, associ- kinds of fibres, like
ated with nerve-tubes and blood-vessels; B, multipolar nucleated cells. Highly magnified. in structure, but * See Appendix. 
each having its distinct office: one carries impressions received from the external world to the gray centres, and hence is called an afferent, or sensory, nerve; the other conducts an influence generated in the centre to the muscles, in obedience to which they contract, and hence it is called an efferent, or motor, nerve. Thus, when the finger is pricked with a pin, afferent nerve-fibres convey the Fra. 133.-Nervons System of Starimpression to the centre - the spinal cord, which immediately

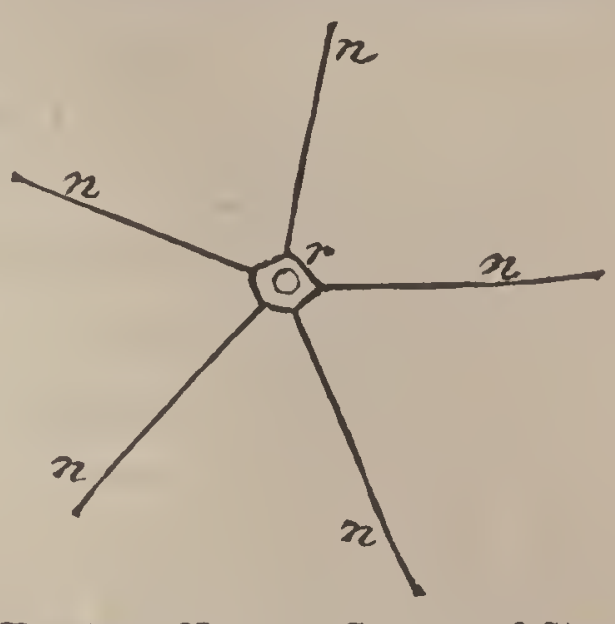
fish: Diagram $-r$, nervous ring around mouth; $n$, radial nerves to each arm, ending in the eye. transmits an order by efferent fibres to the muscles of the hand to contract. If the former are cut, sensation is lost, but voluntary motion remains; if the latter are cut, the animal loses all control over the muscles, although sensibility is perfect; if both are cut, the animal is said to be

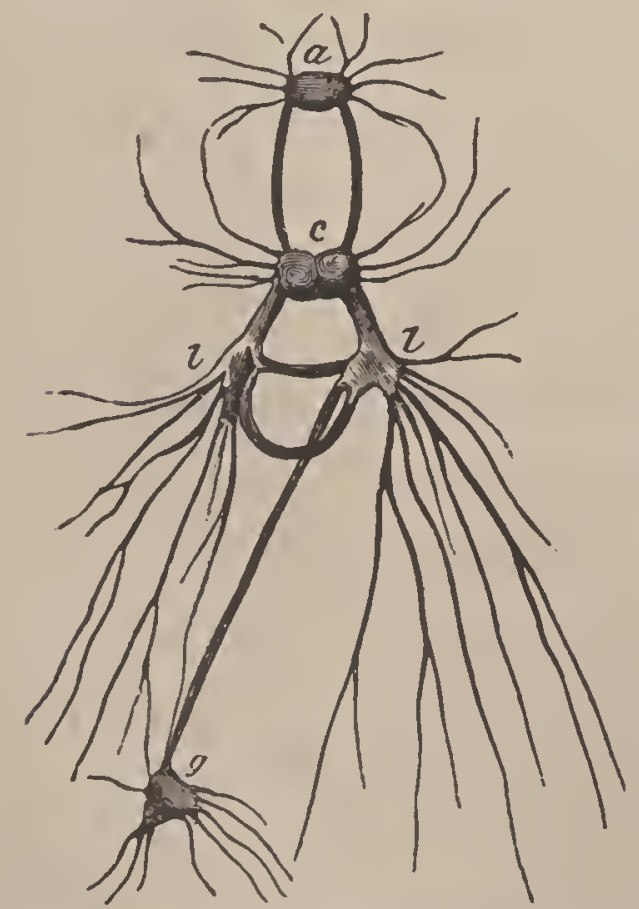

Fra. 134. - Nervons System of a $i a): a$, anterior ganglion; $c$, cephalic; $l$, lateral ; $g$, abdominal. Mollusk (the Gasteropod Aplys-

paralyzed. The nerve-fibres are connected with nerve-cells in the central organs, and at the onter ends are connected with the muscular fibres, or with various sensory end-organs in the skin or other parts of the body. The nature of nerve-force is not known. As to the velocity of a nervous impulse, we know it is far less than that of electricity or light, and that it is more rapid in warm-blooded than in cold-blooded animals, being faster in Man than in the Frog. In the latter it averages about 85 feet per second, in the former over 100 feet. 
The very lowest animals, like the Amceba and Infuso。 ria, have no nerves, although their protoplasm has a gen-

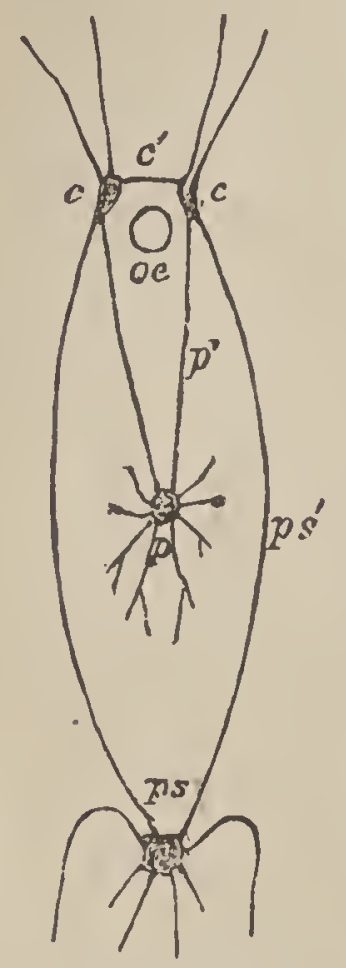

Fra. 135.-Nervous System of Clam: $c$, cerebral ganglion ; $p$, pedal ganglia ; $p s$, parietosplauchnic ganglia; $c^{\prime}$, cerebral commissure $; p^{\prime}$, commissure from cerebral to pedal ganglia ; $p s^{\prime}$, commissure from cerebral to parietosplanchnic ganglia; oe, œsophagus.

eral sensibility. The Hydra has certain cells which are, perhaps, partly nervous and partly muscular in function. The Jelly-fish has a nervous system, consisting of a net-work of threads and ganglia scattered all over its disk. We should look for a definite system of ganglia and nerves only in those animals which possess a definite muscular structure, and show definitely co-ordinated muscular movements. In the Star-fish we detect the first clear specimen of such a system. It consists of a ring around the month, made of five ganglia of equal size, with radiating nerves. The Mollusks are distinguished by an irregularly scattered nervous system. The Clam has three main pairs of connected ganglia - one near the mouth, one in the foot, and the third in the posterior region, near the siphons. In the Snail, these are united into a ring around the gullet, and there are other ganglia scattered through the body. The same is true of the Cuttle- Fra. 136. - Nervons fish, where the brain is partly enclosed in a cartilaginons box (Fig. 151).

In the simpler worms there is but a sin-

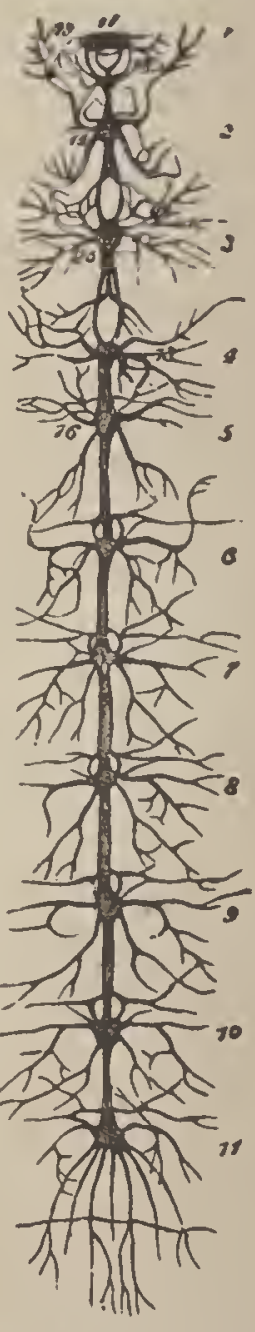
System of a Caterpillar (Sphinx ligustri): the first is the cephalic, or hend, gauglion. gle ganglion or a single pair. The Earth-worm has a pair of brain-ganglia lying above the gullet, and connected by 
two cords with a ventral chain of ganglia-one pair, apparently a single ganglion, for each segment. In the lower Arthropods, such as Crustacea, Centipedes, and larval Insects, the arrangement is substantially the same. In higher Insects and Crustacea, many of the ganglia are fused together in the head and thorax, indicating a concentration of organs for sensation and locomotion.

In Vertebrates, the nervons system is more highly developed, more complex, and more concentrated than in the lower forms. In fact, there are some parts, as the brain, to which we find nothing homologous in the Invertebrates; and while the actions of the latter are mainly, if not wholly, automatic, those of backboned animals are largely voluntary. Its position, moreover, is peculiar, the great mass of the nervous matter being accumulated on the dorsal side, and enclosed by the neural arches of the skeleton.

The brain and spinal cord lie in the cavity of the skull and spinal column, wrapped in three membranes. Each consists of gray and white nervous matter; but in the brain the gray is on the outside, and the white within; while

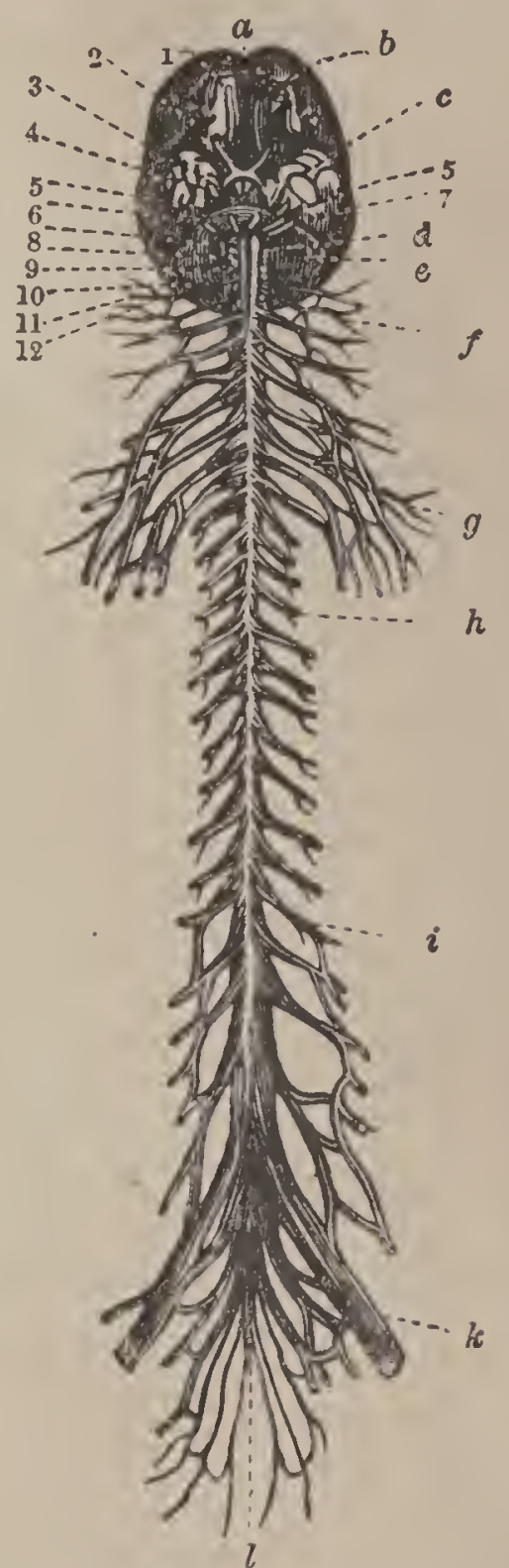

Fra. 137.-Human Brain aud Spinal Cord, about one tenth natural size; $a$, great longitudinal fissure; $b$, anterior lobe; $c$, middle lobe; $d$, medulla oblongata ; $e$, cerebellum ; $f$, first spiual nerve ; $g$, brachial plexus of nerves supplying the arms; $h$, dorsal nerves; $i$, lumbar nerves; $k$, sacral plexus of nerves for the limbs; $l$, cauda equina: the figures indicate the twelve pairs of clanial nerves, of which 1 is olfactory, 2 optic, and 8 auditory. the white of the spinal cord is external, and the gray internal. Both are double, a deep fissure running from the 
forehead backward, dividing the brain into two hemispheres, and the spinal cord resembling two columns welded together; even the nerves come forth in pairs to the right and left. The brain is the organ of sensation and voluntary motion; the spinal cord is the organ of involuntary life and motion. The brain, above the medulla oblongata, may be removed, and yet the animal, though it cannot feel, will live for a time, showing that it is not absolutely essential to life; in fact, the brain does nothing in apoplexy and deep sleep. All of the cord, except that part containing the centres for respiration and circulation, may also be destroyed, without causing immediate death.

The Brain is that part of the nervous system contained in the skull. ${ }^{85}$ It increases in size and complexity as we pass from the Fishes, by the Amphibians, Reptiles, and Birds, to Mammals. Thus, the body of the Cod is 5000 times heavier than its brain-in fact, the brain weighs less than the spinal cord; while in Man, the brain, compared with the body, is as 1 to 36 , and is 40 times heavier than the spinal cord. The brains of the Cat weigh only $1 \mathrm{oz}$; of the Dog, 6 oz. $5 \frac{1}{2} \mathrm{dr}$.; and of the Horse, 22 oz. $15 \mathrm{dr}$. The only animals whose brains outweigh Man's are the Elephant and Whale--the maximum weight of the Elephant's being $10 \mathrm{lbs}$., and of the Whale's 5 lbs.; while the human does not exceed 4 lbs. Yet the human brain is heavier in proportion to the body. But quality must be considered as well as quantity, else the Donkey will outrank the Horse, and the Canary-bird, Man; for their brains are relatively heavier.

The main parts of the brain are the cerebrum, cerebellum, and medulla oblongata.

The cerebrum is a mass of white fibrous matter covered by a layer of gray cellular matter. In the lower Vertebrates, the exterior is smooth; but in most of the Mammals it is convoluted, or folded, to increase the amount of 
the gray surface. The convolutions multiply and deepen as we ascend the scale of size and intelligence, being very complex in the Elephant and Whale, Monkey and Man. As a rule, they are proportioned to the intelligence of the animal; yet the brains of the Dog and Horse are smoother than those of the Sheep and Donkey. Evidently the quality of the gray matter must be taken into account. Save in the bony Fishes, the cerebrum is the largest portion of the brain; in Man it is over eight times heavier than the cerebellum.

The cerebellum, or "little brain," lies behind the cerebrum, and, like it, presents an external gray layer, with a white interior. In Mammals, it is likewise finely convoluted, consisting of gray and white laminæ, and is divided into two lobes,

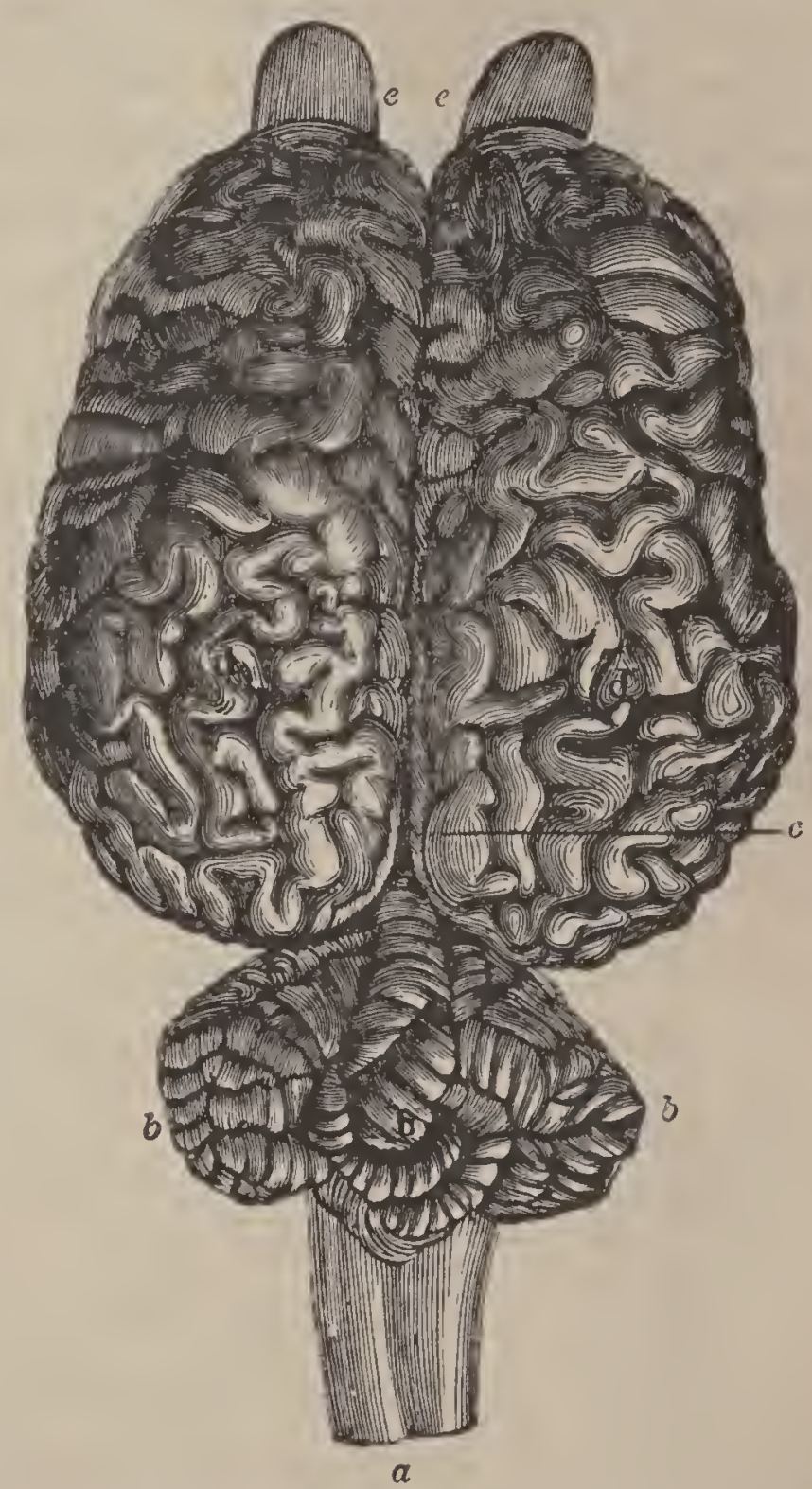

Frg. 138. - Brain or the Horse-upper view, one fourth natural size: $a$, medulla oblongata $b$, lateral and middle lobes of cerebellum; $c$, interlobular fissure; $d$, cerebral hemispheres; $e$, olfactory lobes.

or hemispheres. In the rest of the Vertebrates, the cerebellum is nearly or quite smooth; and in the lowest Fishes it is merely a thin plate of nervous matter. In many Vertebrates, however, it is larger, compared with the cerebrum, than in Man, since in Man the cerebrum is extraordinarily developed. 
The medulla oblongata is the connecting link between the cerebrum and cerebellum and the spinal cord. In structure, it resembles the spinal cord-the white matter being external and the gray internal. The former lies beneath or behind the brain, pdssing through the foramen magnum of the skull, and merging imperceptibly into the cord. The latter is a continuous tract of gray matter enclosed within strands of white fibres. It usually ends in the lumbar region of the vertebral column, but in Fishes it reaches to the end of the tail. In Fishes, Amphibians, and Reptiles, the cord outweighs the brain: in Birds and Mammals, the brain is heavier than the cord. In Man, the cord weighs about an ounce and a half.

Besides these parts, there are also the olfactory and the optic lobes, which give rise respectively to the nerves of smell and sight.

The parts of the brain are always in pairs; but in rela-

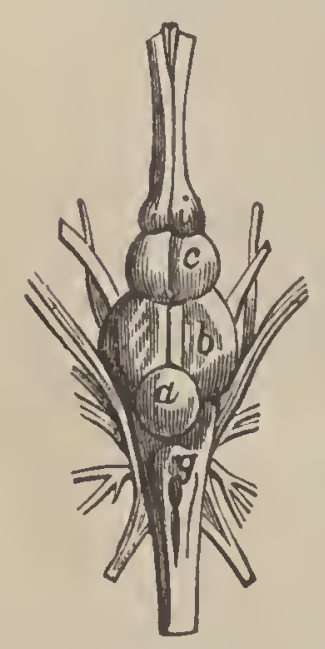

Fig. 139.-Brain of the Perch, upper view : $a$, cerebel$\operatorname{lnm} ; \quad b$, optic lobes; $c$, cerebrum; $i$, olfactory lobes ; $g$, medulla oblongata. tive development and position they differ widely in the several classes of Vertebrates. In Fishes and Reptiles, they are arranged in a horizontal line; in Birds and Mammals, the axis of the spinal cord bends to nearly a right angle in passing through the brain, so that the lobes no longer lie in a straight line. In Man, the fore-brain is so developed that it cov. ers all the other lobes. In looking down upon the brain of a Perch, we see in front a pair of olfactory lobes (which send forth the nerves of smell), behind

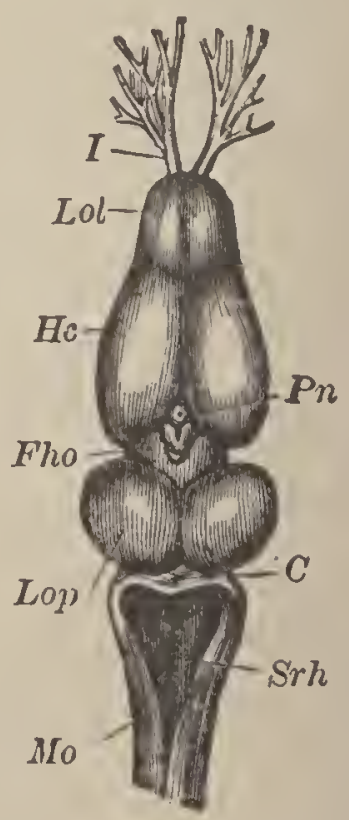

Fig. 140, - Brain of the Frog, upper view, $\times 4$ : $I$, olfactory nerves; $L o l$, olfactory lobes; $H c$, cerebral hemispheres; $P n$, pineal gland; Fho and Srh, third and fourth ventricles; $L \curvearrowleft p$, optic lobes; $C$, cerebellum: $\mathbf{M o}$, medulla oblungata. 
them the small cerebral hemispheres, then the large optic lobes (near which originate the nerves of sight $t$ ), and, last of all, the cerebellum. Not till we reach Man and the Apes do we find the cerebrum so highly developed as to overlap both the olfactory lobes in front and the cerebellum behind.

Functions of the Brain.-The cerebrum is the seat of intelligence and will. It has no direct communication with the outside world, receiving its consciousness of external objects and events throngh the spinal cord and the nerves of special sense. ${ }^{86}$

The cerebellum seems to preside over the co-ordination of the muscular movements. When removed, the animal

A
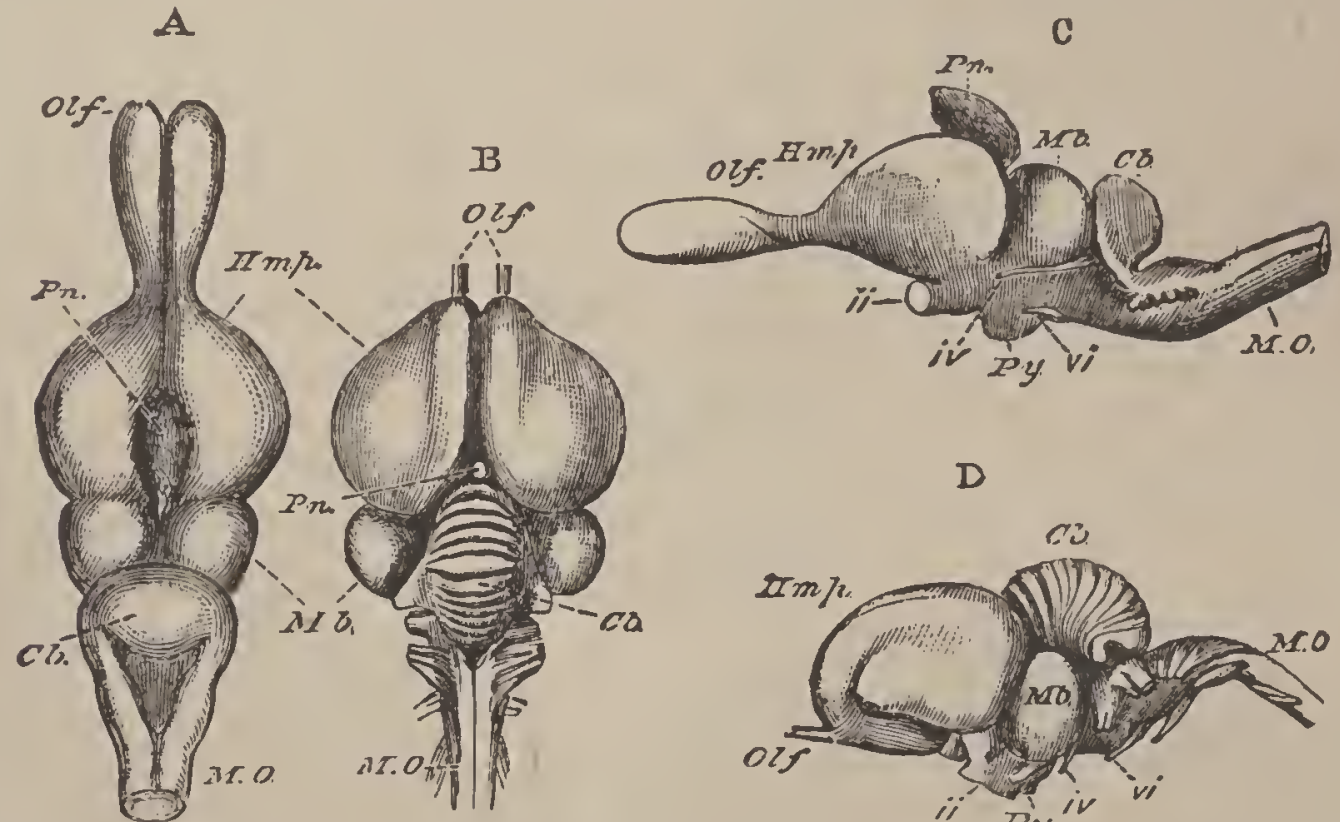

Frn. 141.-A, C, npper and side views of the Brain of a Lizard; B, D, upper and side views of the Brain of a Turkey: Olf, olfactory lobes; Hmp, cerebral hemispheres; $P n$; pineal gland; $M 1 b$, optic lobes of the middle brain ; $C b$, cerebellum ; $M O$, medulla oblongata; $i i$, optic nerves; $i v$ and $v i$, nerves for the muscles of the eye: $P y$, pituitary body.

desires to execute the mandates of the will, but cannot; its motions are irregular, and it acts as if intoxicated. It is usually largest in animals capable of the most complicated movements; being larger in the Ape than in the Lion, in the Lion than in the $\mathrm{Ox}$, in Birds than in Rep. tiles. The cerebellum of the Frog is, however, smaller than that of Fishes (Figs. 139, 140). The olfactory and op. tic lobes receive the messages from their respective nerves. 
The medulla oblongata is not only the medium of communication between the brain and the spinal cord, but it

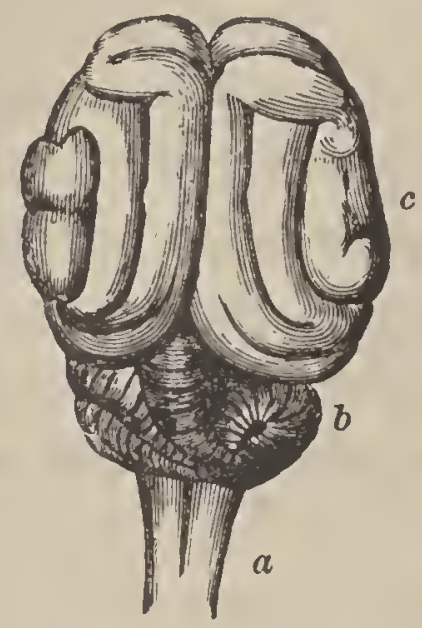

Frg. 142.-Brain of the Cat (Felis domestica) : $a$, medulla oblongata; $b$, cerebellum; cerebrum.

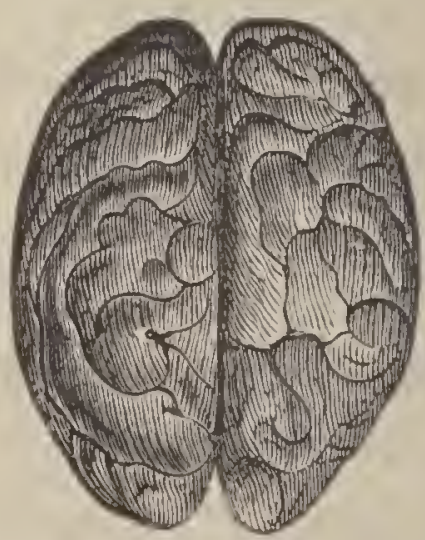

Frg. 143. - Brain of the Orang-utan, upper surface; one third uatural size.

is itself a nervous centre: the brain above and the cord below may be removed without death to the animal, but the destruction of the medulla is fatal. Of the twelve pairs of nerves issuing from the contents of the skull (encephaton), ten come from the medulla oblongata. Among these are the nerves of hearing

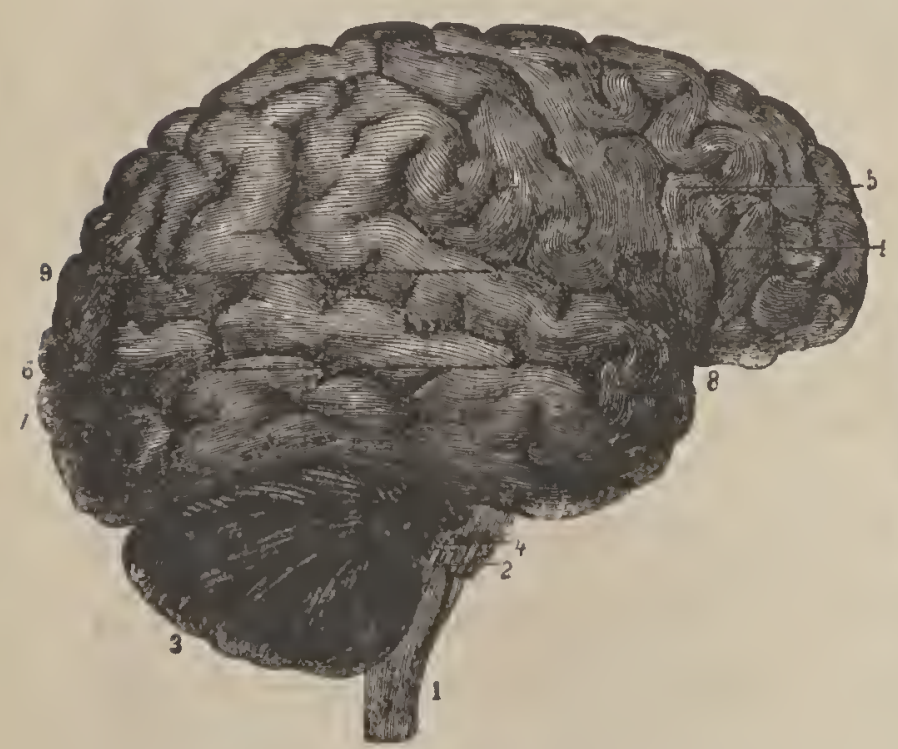

Frg. 144.-Human Brain, side view: 1, medulla oblongata : 3 , cerebellum ; 5 , frontal convolutious of cerebrum.

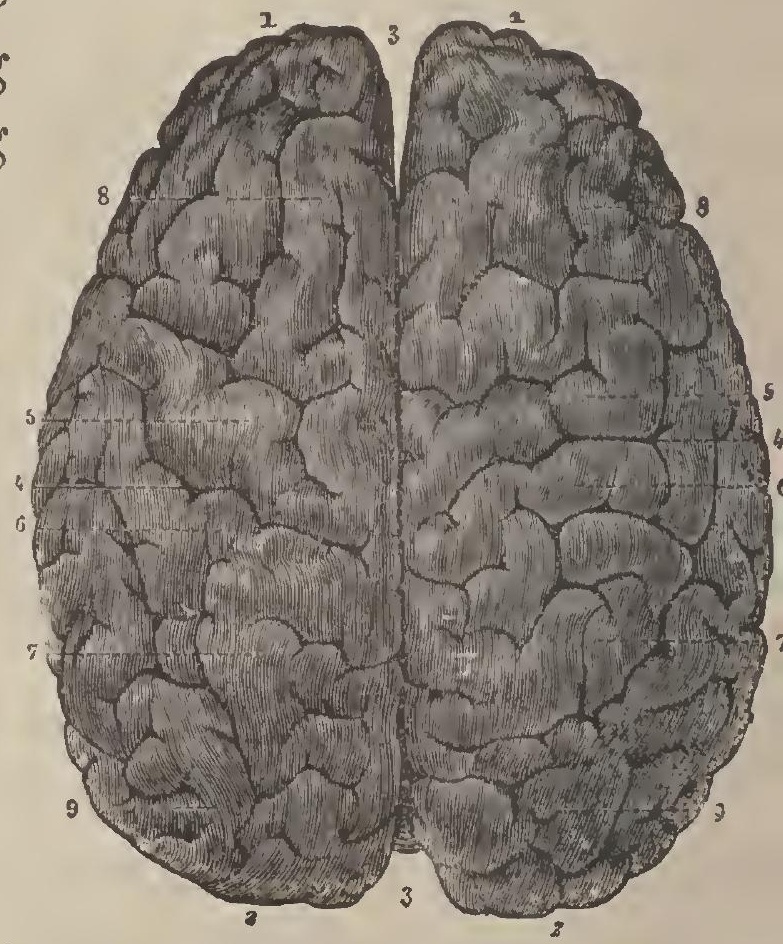

Frg. 145. - Human Brain, upper view, one fonrth natural size: 1 , anteriol lobes; 2 , posterior; 3 , great median fissure.

and taste, and those that control the lungs and heart. Respiration ceases immediately when the medulla is injured. 
The spinal cord is a centre for originating involuntary actions, and is also a conductor-propagating through its central gray matter the impressions received by the nerves to the brain, and taking back through its fibrous part the impulses of the brain. In Man, thirty-one pair's of nerves arise from the cord to supply the whole body, except the head. Each nerve has an anterior and a posterior root. The fibres of the former go to the muscles, and hence carry the impulses which cause muscular contraction (hence called motor fibres); those of the posterior root conrey sensations from the exterior to the central organs (sensory). The fibres leading from the brain to the cord cross one another in the medulla oblongata, so that if the right cerebral hemisphere be diseased,

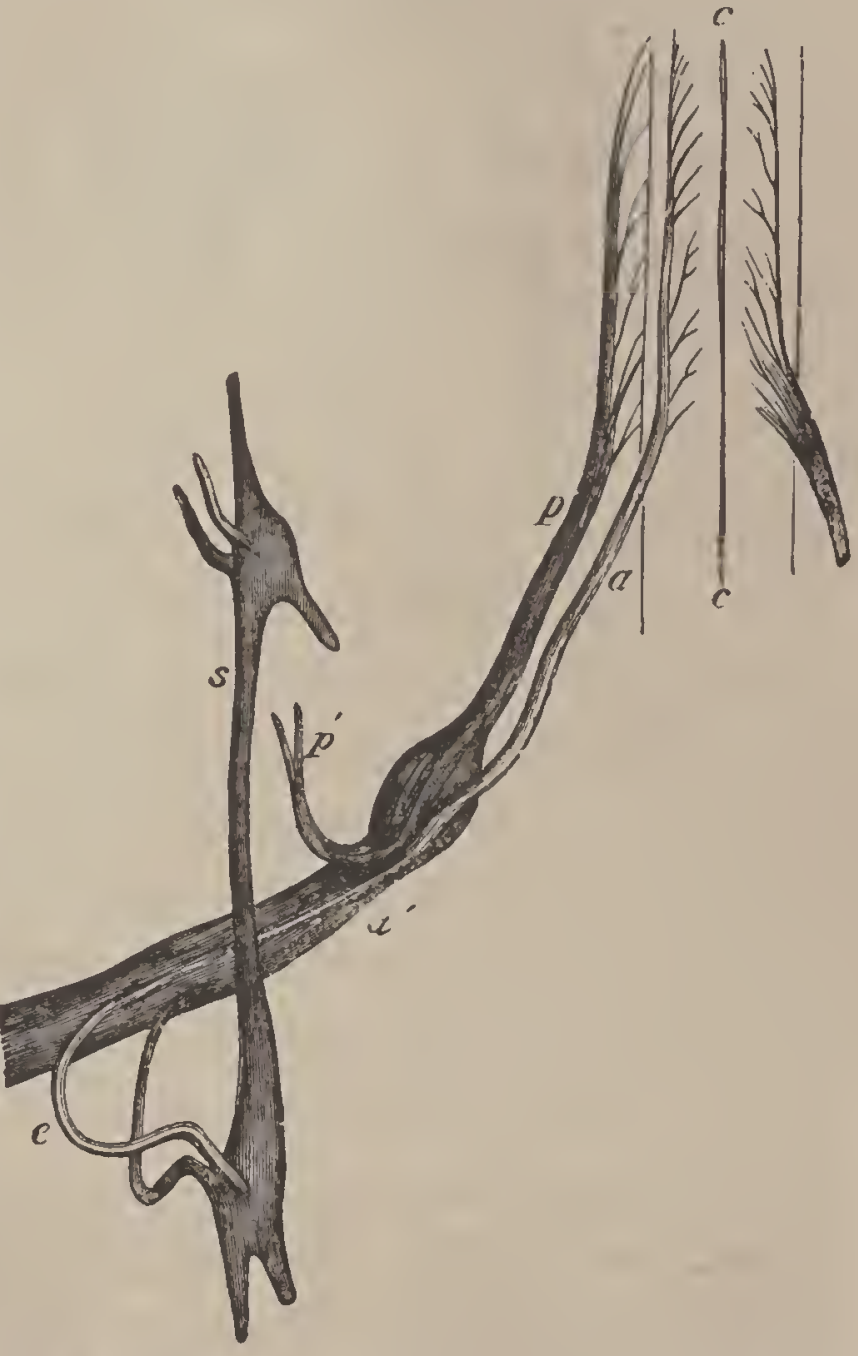

Fig. 146.-Relation of the Sympathetic and Spinal Nerves : $c$, fissure of spinal cord; $a$, anterior root of a dorsal spinal nerve; $p$, posterior root, with its ganglion; $a^{\prime}$, anterior branch; $p^{\prime}$, posterior branch; $s$, sympathetic; $e$, its double junction by white and gray tilaments. the left side of the body loses the power of voluntary motion.

The sympathetic nervous system is a double chain of ganglia, lying along the sides of the vertebral column in the ventral cavity. From these ganglia nerves are given off, which, instead of going to the skin and muscles, like the spinal nerves, form net-works about those internal organs over which the will has no control, as the heart, stomach, 
and intestines. Their apparent office is to stimulate these organs to constant activity, but is little understood.

\section{The Senses.}

Sensation is the consciousness of impressions on the sensory nerves. These impressions produce some change in the brain; but what that change is, is a darkness on which no hypothesis throws light. Obviously, we feel only the condition of our nervous system, not the objects which excite that condition. ${ }^{37}$

All animals possess a general sensibility diffused over the greater part of the body. ${ }^{88}$ This sensibility, like assimilation and contractility, is one of the primary physiological properties of protoplasm. But, besides this (save in the very lowest forms), they are endowed with special nerves for receiving the impressions of light, sound, etc. These nerves of sense, as they are called, although structurally alike, transnit different sensations: thus, the Ear caunot recognize light, and the Eye cannot distinguish sounds. In the Vertebrates, the organs of sight, hearing, and smell are situated in pairs on each side of the head; that of taste, in the mucous membrane covering the tongue; while the sense of touch and that of temperature are diffused over the skin, including the mucous membrane of the mouth, throat, and nose. Sight and hearing are stimulated, each by one agent only; while tonch, taste, and smell may be excited by various substances. The agents awakening sight, hearing, touch, and the sense of temperature are physical; those causing taste and smell are chemical. Animals differ widely in the numbers and keenness of their senses. But there is no sense in any one which does not exist in some other.

Touch is the simplest and the most general sense; no animal is withont it, at least in the form of general sensibility. It is likewise the most positive and certain of the senses. In the Sea-anemone, Snail, and Insect, it is most acute in 
the "feelers" (tentacles, horns, and antennæ); $;^{80}$ in the Oyster, the edge of the mantle is most sensitive; in Fishes, the lips; in Snakes, the tongue; in Birds, the beak and under side of the toes; in Quadrupeds, the lips and tongue; and in Monkeys and Man, the lips and the tips of the tongue and fingers. In the most sensitive parts of Birds and Mammals, the true skin is raised up into multitudes of minute elevations, called

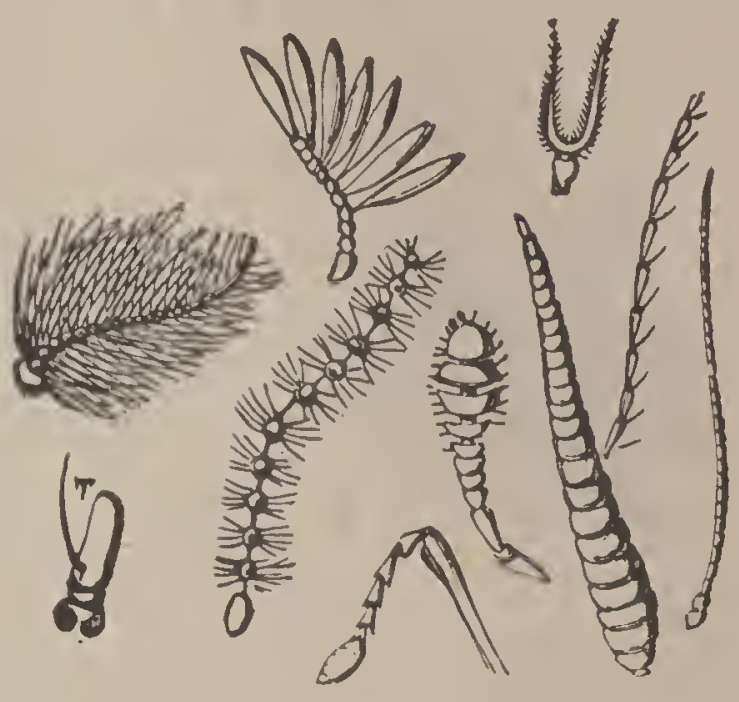

Fra. 147.-Antennæ of Various Insects. papilloe, containing loops of capillaries and nerve-filaments. At the ends of the latter are the essential organs of touch, the tactile corpuscles and the touch-cells. There is a correspondence between the delicacy of touch and the development of intelligence. The Cat and Dog are more sagacions than hoofed animals. The Elephant and Parrot are remarkably intelligent, and are as celebrated for their tactual power.

Taste is more refined than touch, since it gives a knowledge of properties which cannot be felt. It is always placed at the entrance to the digestive canal, as its chief purpose is to guide animals in their choice of food. Special

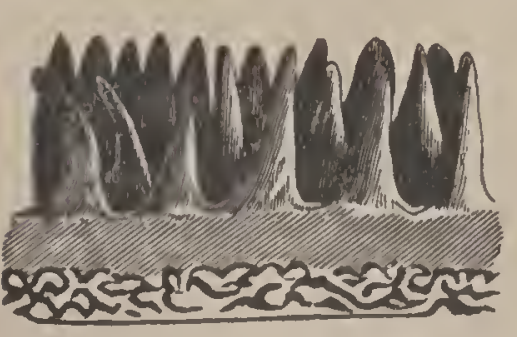

Fig. 14s.-Papillæ of 11 uman Palm, $\times 35$, the cuticle beilig removed. organs of taste have been detected in only a few of the Invertebrates, thongh all seem to exercise a faculty in selecting their food. Even in Fishes, Amphibians, Reptiles, and Birds this sense is very obtuse, for they bolt their food. But the higher Vertebrates have it well developed. It is confined to the tongue, and is most delicate at the root. ${ }^{00}$ A state of solution and an actual contact of the fluid are necessary conditions.

Smell is the perception of odors, $i$. e., certain substances 
in the gaseous state. Many Invertebrates have this sense: Snails, e.g., seem to be guided to their food by its scent, and Flies soon find a piece of meat. In the latter the organ is probably located on the antennæ. In Verte-

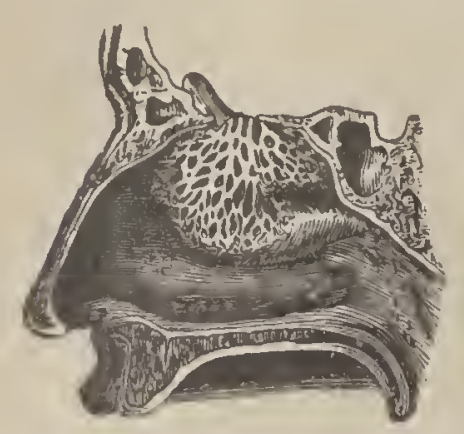

FrG. 149.-Olfactory Nerves on the wall of the nasal cavity. brates, it is placed at the entrance to the respiratory tube, in the upper region of the nose. There the olfactory nerves, which issue from the olfactory lobe of the brain, and pass through the ethmoid bone, or roof of the nasal cavity, are distributed over a moist mucous membrane. The odorous substance, in a gaseous or finely divided state, is dissolved in the mucus covering this membrane. In Fishes and Reptiles generally, this organ is feebly developed; Sharks, however, gather from a great distance around a carcass. In the Porpoises and Whales it is nearly or entirely wanting. Among Birds, Waders have the largest olfactory nerves. It is most acnte in the carnicorous Quadrupeds, and in some wild herbivores, as the Deer. In Man it is less delicate, but has a wider range than in any brute.

Hearing is the perception of sound. The simplest form of the organ is a sac filled with fluid, in which float the soft and delicate ends of the auditory nerve. The vibratious of the fluid are usually strengthened by the presence of minute hard granules, called otoliths. Most Invertebrates have no more complicated apparatus than this; and it is probable that they can distinguish one noise from another, but neither pitch nor intensity. The organ is generally double, but not always located in the

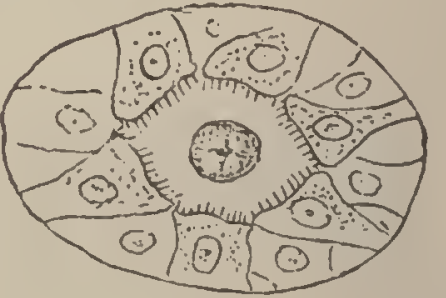

Fis. 150.-Eal of a Mollusk (Cyclas), greatly enlarged, showing the otolith in the centre of a cavity which is filled with fluid, and whose walls are lined by ciliated cells. head. In the Clam, it is found at the base of the foot; some Grasshoppers have it in the fore-legs; and in 
many Insects it is on the wing. Lobsters and Crabs have the auditory sacs at the base of the antennæ. ${ }^{91}$

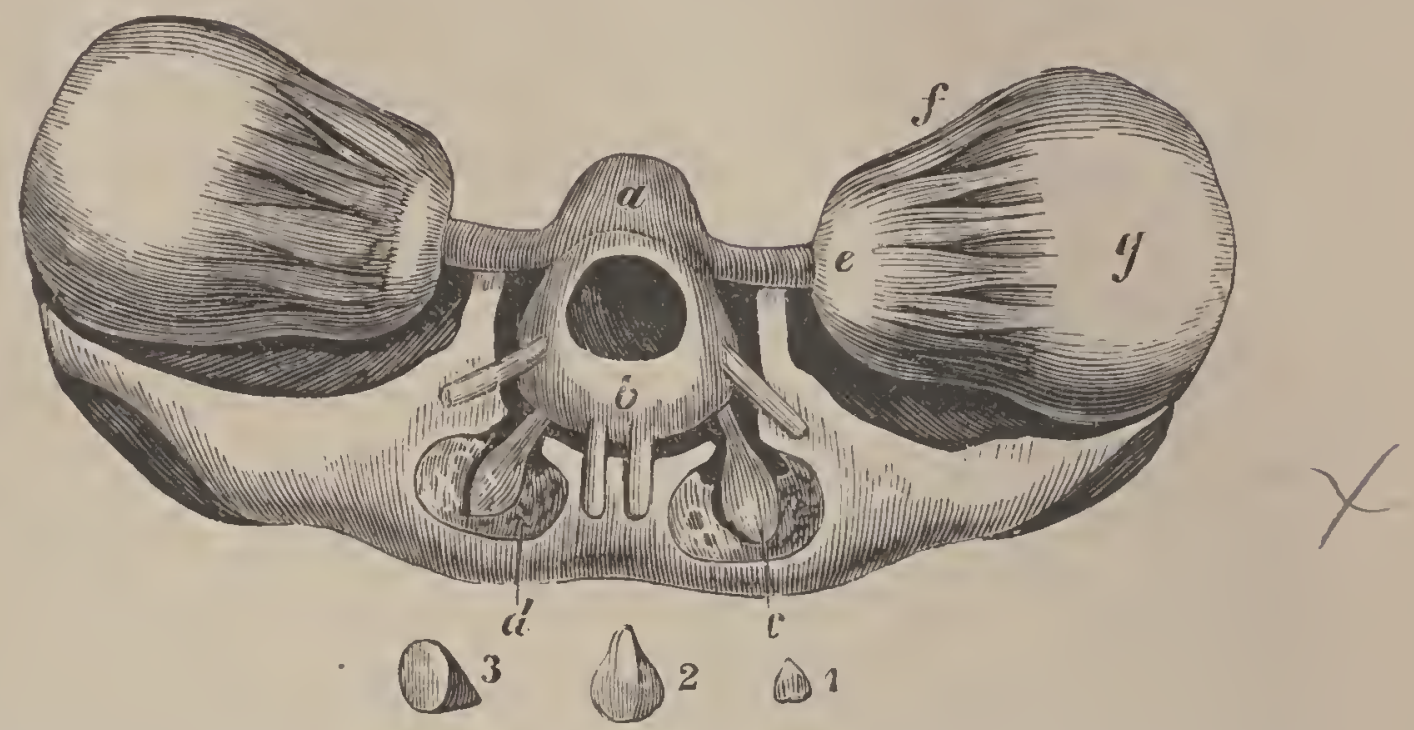

Fig. 151.-Brain and Auditory Apparilus of the Cuttle-fish: $a, b$, brain ; $c$, auditory apparatus ; $d$, the cavity in which it is lodged ; $e, f, g$, eyes; $1,2,3$, otoliths.

A complex organ of hearing, located in the head, exists in all Vertebrates, save the very lowest Fishes. As complete in Man, it consists of the following parts: 1st. The external ear (which is peculiar to Mammals) ; the auditory canal, about an inch long, lined with hairs and a waxy secretion, and closed at the bottom by a membrane, called tympanum, or "drum of the ear." 2 d. The middle ear, containing three little bones (the smallest in the body), malleus, incus, and stapes, alticulated together. The cavity communicates with the external air by means of the Eustachian tube, which opens at the back part of the mouth. $3 \mathrm{~d}$.

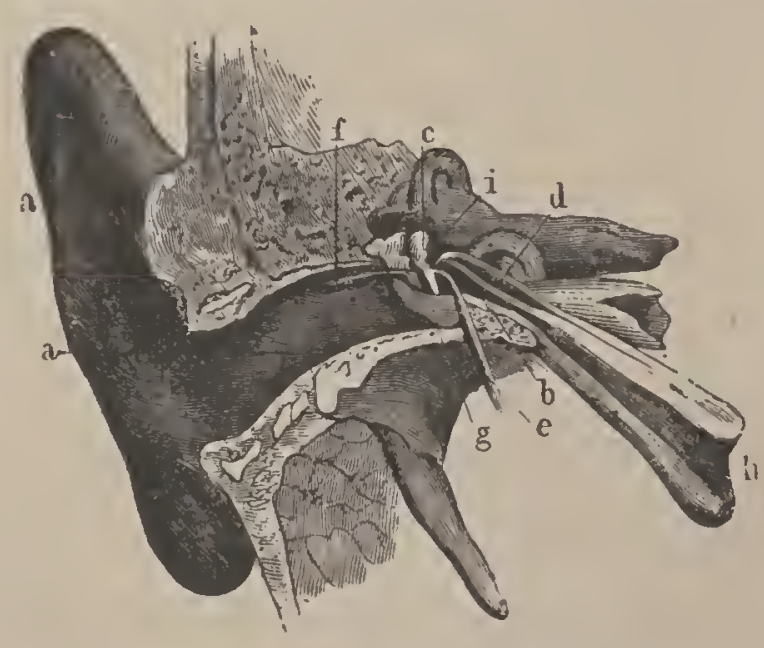

Fig. 152.-Section of Human Ear: $\alpha$, external ear, with anditory canal : $b$, tympanic cavity containing the three bones; $c$, hammer, and its three muscles, $d, e, f ; g$, tympanic membrane, or head of the drum; $h$, Eustachian tube leadung to the pharynx; $i$, labyrinth, with semicircular canals and cochlea visible.

The internal ear, or labyrinth, an irregular cavity in the solid part of the temporal bone, and sevarated from the 
middle ear by a bony partition, which is perforated by two sinall holes. The labyrinth consists of the vestibule, or entrance; the semicircular canals, or tubes; and the cochlea, or spiral canal. While the other parts are full of air, the labyrinth is filled with a liquid, and in this are the ends of the anditory nerve. The vibrations of the air, collected by the external ear, are concentrated upon the tympanum, and thence transmitted throngh the chain of little bones to the fluid in the labyrinth.

The essential organ of hearing is the labyrinth, which is, substantially, a bag filled with fluid and nerve-filitments. Fishes generally have but little more. In Amplibians and Reptiles there are added a tympanum, a single bone, connecting this with the internal ear, the cochlea, and the Eustachian tube; the tympanum being external. Birds have, besides, an auditory passage, opening on a level with the surface of the head, and surrounded by a circle of feathers. Mammals only have an external ear..$^{22}$

Sight is the perception of light. ${ }^{93}$ In all animals it depends upon the peculiar sensitiveness of the optic organ to the luminous vibrations. In Vertebrates the optic nerve comes from the middle mass of the brain, in Invertebrates it is derived from a ganglion. Many animals are utterly destitute of visual organs, as the Protozoa, and the lower Radiates and Mollusks, besides intestinal Worms and the blind Fishes and many cave-animals. Around the margin of the Jelly-fish are colored spots, supposed to be rudimentary eyes; but, as a lens is wanting, there is no image; so that the creature can merely distinguish light from darkness and color without form. Such an eye is nothing but a collection of pigment granules on the expansion of a nervous thread, and the perception of light is the sensation of warmth, the pigment absorbing the rays and converting them into heat. 
Going higher, we find a lens introduced forming a distinct image. The Snail, for example, has two simple eyes, called ocelli, mounted on the tip of its long tentacles, consisting of a globular lens, ${ }^{94}$ with a transparent skin (cornea) in front, and a

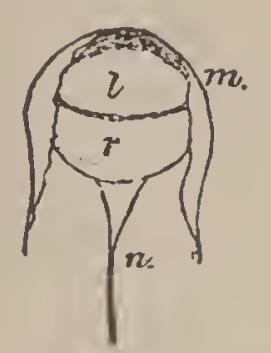

c o l o l e d meinblane (c horo id) and a nelvous netFro. 153, - liye of work (letiPecten, much enlarged: $m$, mouth; $l$, lens ; $r$, retina and choroid; $n$, nerve.

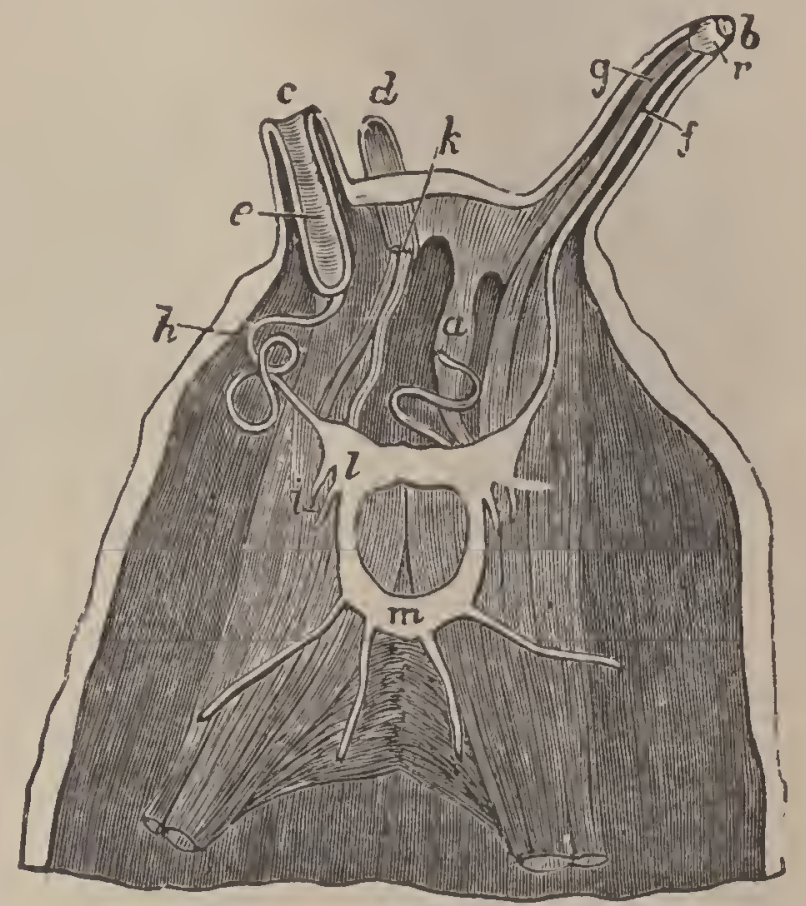

such eyes in the edge of its mantle (Fig. 153). Sucls organs are the only eyes possessed by Myriapods, Spiders, Scorpions, and Caterpillars. Adult In-

Fic. 154. - Head of a snail birected, sluwing structure of tentacles: $a$, right inferior tentacle retracted within the body; $b$, right $s u$ perior tentacle fully protinded; $c$, left superior tentacle partially inverted; $d$, left inferior tentacle; $f$, optic nerve; $g$, retractor muscle; $h$, optic nerve in loose folds; $i$, retractor nuscle of head; $k$, nerve and muscle of left inferior tentacle; $l, m$, wervous collar.

sects usually have three ocelli on the top of the head. But the proper visnal organs of Lobsters, Crabs, and Insects are two compound eyes, perched on pedestals, or fixed on the sides of the head. They consist of an immense number of ocelli pressed together so that they take an angular form-foursided in Crustacea, six-sided in Insects. They form two rounded protuberances variously colored - white, yellow, red, Fra 155.-Hend of the Bee, green, purple, brown, or black. Under the microscope, the surface is seen to

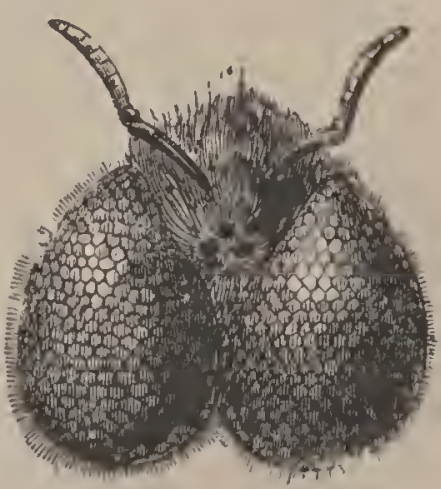
showing compoundeves, the three ocelli, or stem. mata, and the antennæ. Magnified. be divided into a host of facets, ${ }^{95}$ each being an ocellus complete in itself. Each cornea is convex on one side, 
and either convex or flat on the other, so that it produces

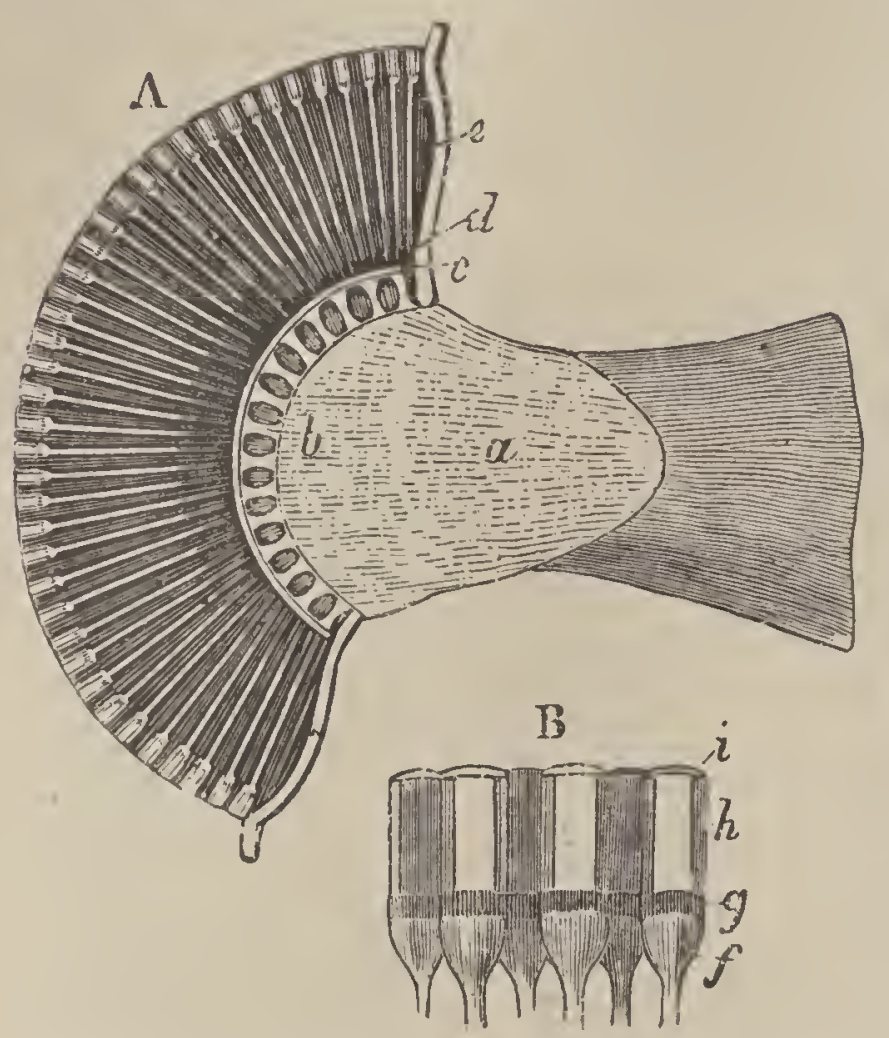
a focus like a lens. Behind the cornea, or lens, is the pigment, having a minute aperture or "pupil." Next is a conical tube-one for each facet - with sides and bottom lined with pigment. These tubes converge to the optic ganglion, the fibres of which pass through the tubes to the colnea. ${ }^{96}$ Vision

Fig. 156. - Eye of a Beetle (Melolontha): A, section; by such a collupound
$a$, optic ganglion; $b$, secondary nerves; $c$, retina; $a$, prgment layer; e, proper optic nerves; $B$, group eye is not a mosaic; of ocelli; $f$, bulb of optic nerve; $g$, layer of pigment; $h$, vitreous humor; $i$, cornea. Minguified. but each ocellus gives a complete inage, although a different perspective from its neighbor. The multiplied images are reduced to one melltal stereoscopic pict$11 \mathrm{l}^{\circ}$, on the principle of single vision in ourselves.

The eyes of the Cuttle-fish are the largest and the most perfect among Invertebrates. They resemble the eyes of ligher animals in having a crystalline lens

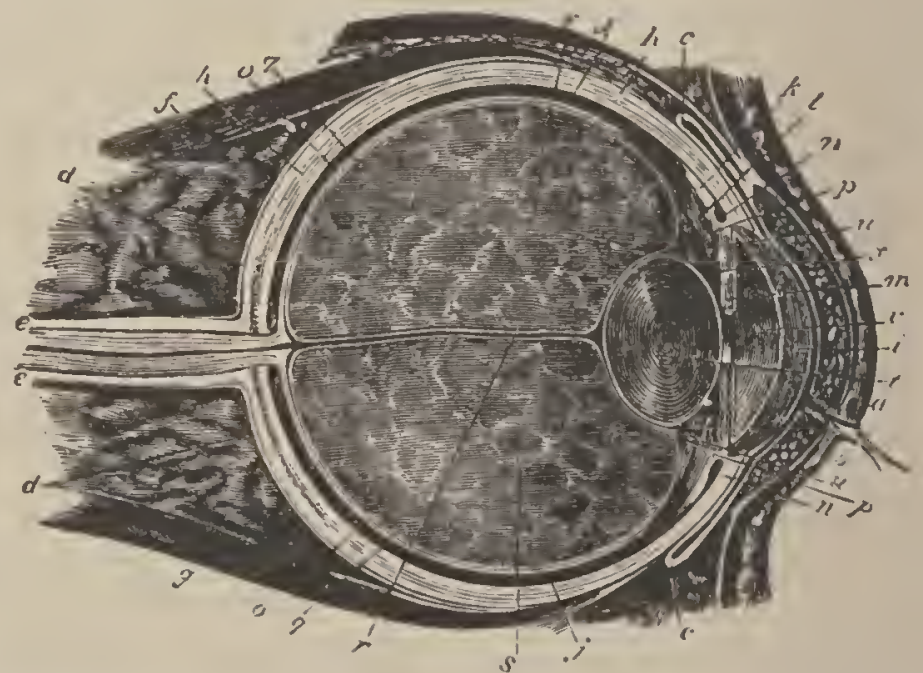

Fig. 157.-Section of Human Eye: $a$ and $b$, npper and lower lid: $c$, conjunctiva, or mucous membraue, lining the inner surface; $d$, external membrane; $e$, sheath of optic nerve: $f, g$, muscles for rolling the eye np or down; $h$, sclerotic; $i$, transparent cornea $;$, chorrid ; $k, l$, ciliary muscle for adjusting the eye for distance; $m$, iris and pupil; $n$, canal; $o$, retina ; $s$, vitreous humor; $t$, crystalline lens ; $v$, anterior clamber ; $x$, posterior chamber.

with a chamber in front (open, however, to the sea- 
water), and a chamber behind it filled with "vitreous humor."

The eye of Vertebrates is formed by the infolding of the skin to create a lens, and an outgrowth of the brain to make a sensitive layer; both enclosed in a white spherical case (sclerotic) made of tough tissue, with a transparent front, called the cornea. This case is kept in shape by two fluids-the thin aqueous humor filling the cavity just behind the cornea, and the ielly-like vitreous humor occupying the larger posterior chamber. Between the two humors lies the doubleconvex crystalline lens. On the front face of the lens is a contractile circular curtain (iris), with a hole in the centre (pupil); and lining the sclerotic coat is the choroid membrane, covered with
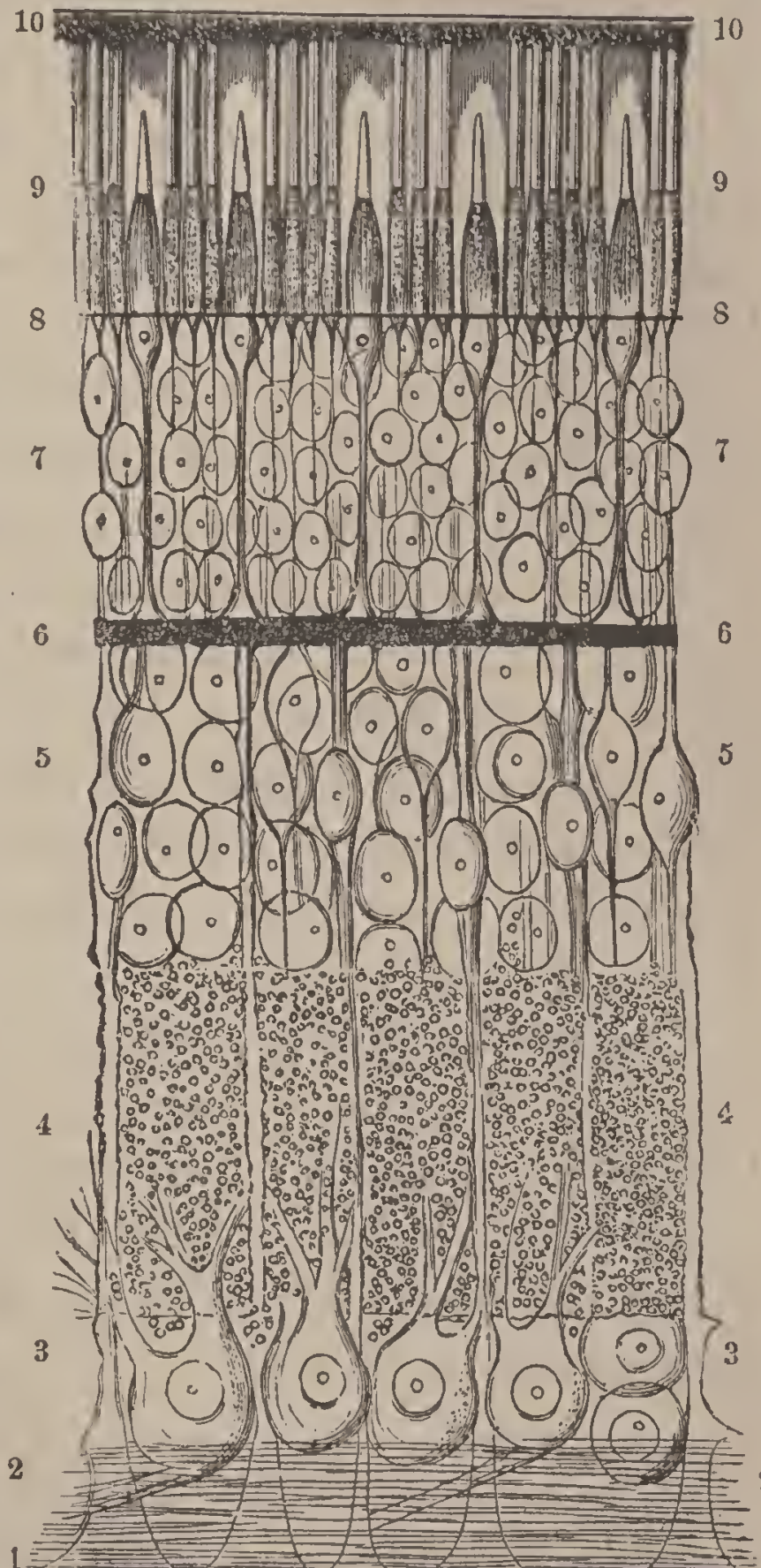
dark pigment. The optic nerve, entering at the back of the eye Fra. 158.-Section of the IIuman Retina, $\times 400: 1$, internal limiting membrane; 2 , optic-nervefibres; 3 , ganglion cells; 4 , internal molecular layer; 5 , internal granules; 6 , external molecular layer; 7 , externalgranules; 8, externallimiting membrane; 9, layer of rods and cones; 10 , pigment layer.

through the sclerotic and choroid coats, expands into the transparent retina, which consists of several layers - 
fibrous, cellular, and granular. The most sensitive part is the surface lying next to the black piginent. And here is a peculiarity of the vertebrate eye: the nerve-fibres, entering from behind, turn back and look towards the bottom of the eye, so that vision is directed backward; while invertebrate vision is directly forward. In Vertebrates only, the optic nerves cross each other (decussate) in passing from the brain to the eyes; so that the right side of the brain, e.g., receives the impressions of objects on the left side of the body. ${ }^{97}$

Generally, the eyes of Vertebrates are on opposite sides of the head; but in the Flat-fishes both are on the same side. Usually, both eyes see the same object at once; but in most Fishes the eyes are set so far back, the fields of vision are distinct. The cornea may be flat, and the lens globular, as in Fishes; or the cornea very convex, and the lens flattened, as in Owls. Purely aquatic animals have neither eyelids nor tears, but nearly all others (especially Birds) have three lids..$^{93}$ The pupil is usually round; but it may be rhomb-shaped, as in Frogs; vertically oval, as in Crocodiles and Cats: or transversely oval, as in Geese, Doves, Horses, and Ruminants. Many Quadrupeds, as the Cat, have a membrane (tapetum) lining the bottom of the eyeball, with a brilliant metallic lustre, usually green or pearly: it is this which makes the eyes of such animals luminous in the dark.

\section{Instinct and Intelligence.}

The simplest form of nervous excitement is mere sensation. Above this we have sensation awakening consciousness, out of which come those voluntary activities grouped together under the name of Instinct; and, finally, Intelligence.

The lowest forms of life are completely under law, for their movements seem to be due solely to their organiza- 
tion. They are automatons, or creatures of necessity. In the higher animals certain actions are automatic, as breathing, the beating of the heart, the contractions of the iris, and all the first movements of an infant. ${ }^{90}$ But, generally, the actions of animals are not the result of mere bodily organization.

The inferior orders are under the control of Instinct, i. e., an apparently untaught ability to perform actions which are useful to the animal. ${ }^{100}$ They seem to be born with a measure of knowledge and skill (as Man is said to have innate ideas), acquired neither by reason nor experiment. For what could have led Bees to imagine that by feeding a worker-larva with royal jelly, instead of beebread, it would turn out a queen, instead of a neuter? In this case, neither the habit nor the experience conld be inherited, for the worker-bees are sterile. We can only guess that the discovery has been communicated by the survivors of an older swarm. Uniformity is another characteristic feature of instinct. Different individuals of the same species execute precisely the same movements under like circumstances. The career of one Bee is the career of any other. We do not find one clever and another stupid. Honey-combs are built now as they were before the Christian era. The creatures of pure instinct appear to be tied down, by the constitution of their nervous system, to one line of action, from which they cannot spontaneously depart. The actions vary only as the structure changes. ${ }^{101}$ There is a wonderful fitness in what they do, but there is no intentional adaptation of means to ends.

All animals, from the Star-fish to Man, are guided more or less by instinct; but the best examples are furnished by the insect-world, especially by the social Hymenopters (Ants, Bees, and Wasps). The Butterfly carefully provides for its young, which it is destined never to see; many Insects feed on particular species of plants, which 
they select with wonderful sagacity; and Monkeys avoid poisonous berries; Bees and Squirrels store up food for the future; Bees, Wasps, and Spiders construct with marvellous precision; and the subterranean chamber's of Ants and the dikes of the Beaver show engineering skill; while Salmon go from the ocean up the rivers to spawn; and Birds of the temperate zones migrate with great regularity:

But in the midst of this antomatism there are the glimmerings of intelligence and free-will. We see some evidence of choice and of designed adaptation. Pure instinct should be infallible. Yet we notice mistakes that remind us of mental aberrations. Bees are not so economical as has been generally supposed. A mathematician can make five cells with less wax than the Bee uses for four; while the Humble-bee uses three times as much material as the Hive bee. An exact hexagonal cell does not exist in nature. Flies lay egge on the carrion-plant because it happens to have the odor of putrid meat. The domesticated Beaver will build a dam across its apartment. Birds frequently make mistakes in the construction and location of their nests. In fact, the process of cheating animals relies on the imperfection of instinct. Nor are the actions of the brute creation always perfectly uniform; and, so far as animals conform to circumstances, they act from intelligence, not instinct. There is proof that some animals profit by experience. Birds do learn to make their nests; and the older ones build the best. Trappers know well that young animals are more easily caught than old ones. Birds brought up from the egg, in cages, do not make the characteristic nests of their species; nor do they have the same song peculiar to their species, if they have not heard it. Chimney-swallows certainly built their nests differently in America three hundred years ago. A Bee can make cells of another shape, 
for it sometimes does; its actions, therefore, being elective and conditional, are in a measure the result of calculation.

The mistakes and variations of instinct are indications that animals have something more - a limited range of that principle of Intelligence so luminous in Man. No precise line can be drawn between instinctive and intelligent acts; all we can say is, there is more freedom of choice in the latter than the former'; and that some aniinals are most instinctive, others most intelligent. Thus, we speak of the instinct of the Ant, Bee, and Beaver, and the intelligence of the Elephant, Dog, and Monkey. Instinct loses its peculiar character as intelligence becomes developed. Ascending from the Worm and Oyster to the Bee, we see the movements become more complex in character and more special in their objects; but instinct is supreme. Still ascending, we observe a gradual fadingaway of the instincts, till they become subordinate to higher faculties-will and reason. We can predict with considerable certainty the actions of animals guided by pure instinct; but in proportion as they possess the power of adapting means to ends, the more variable their actions. Thus, the architecture of Birds is not so uniform as that of Insects. ${ }^{103}$

We must credit brutes with a certain amount of observation and imitation, curiosity and cunning, memory and reason. Animals have been seen to pause, deliberate, or experiment, and resolve. The Elephant and Horse, Dog and Monkey, particularly, participate in the rational nature of Man, up to a certain point. Thinking begins wherever there is an intentional adaptation of means to ends; for that involves the comparison and combination of ideas. Animals interchange ideas: the whine of a Dog at the door on a cold night certainly implies that lie wants to be let in. Bees and Ants, it is well known, confer by 
passing their antenuæ. All the higher animals, too, have similal emotions-as joy, fear, love, and anger.

While instinct culminates in Insects, the highest development of intelligence is presented in Mall. ${ }^{103}$ In Man only does instinct cease to be the controlling power. He stands alone in having the whole of his organization conformed to the demands of his brain; and his intelligent acts are characterized by the capacity for unlimited progress. The brates can be improved by domestication; but, left to themselves, they soon relapse into their original wildness. Civilized Man also goes back to savagery; yet Man (though not all Men) has the ambition to exalt his mental and moral nature. He has a soul, or conscious relation to the Infinite, which leads him to aspire after a lofty ideal. Only he can form abstract ideas. And, finally, he is a completely self-determining agent, with a prominent will and conscience-the highest attribute of the animal creation. In all this, Man differ's profoundly from the lower forms of life.

\section{The Voices of Animals.}

Most aquatic animals are mute. Some Crabs make noises by rubbing their fore-legs against their carapace; and many Fishes produce noises in various ways, mostly by means of the swim-bladder. Insects are the Invertebrates which make the most noise. Their organs are usually external, while those of Vertebrates are internal. Insects of rapid flight generally make the most noise. In some the noise is produced by friction (stridulation); in others, by the passage of air through the spiracles (humming). The shrill notes of Crickets and Grasshoppers are produced by rubbing the wings against each other, or against the thighs; but the Cicada, or Harrest-fly, has a special apparatus - a tense membrane on the abdomen, acted upon by muscles. The buzzing of Flies and humming of Bees 
are caused, in part, by the vibrations of the wings; but the true voice of these Insects comes from the spiracles of the thorax.

Snakes and Lizards have no vocal cords, and can only hiss. Frogs croak ${ }^{104}$ and Crocodiles roar, and the huge Tortoise of the Galapagos Islinds ntters a hoarse, bellowing noise.

The vocal apparatus in Birds is situated at the lower end of the trachea, where it divides into the two bronchi. $^{105}$ It consists mainly of a bony drum, with a crossbone, having a vertical membrane attached to its upper edge. The membrane is put in motion by currents of air passing on either side of it. Five pair's of muscles (in the Songsters) adjust the length of the windpipe to the pitch of the glottis. The various notes are produced by differences in the blast of air, as well as by changes in the tension of the membrane. The range of notes is commonly within an octave. Birds of the same family have a similar voice. All the Parrots have a harsh ntterance; Geese and Ducks quack; Crows, Magpies, and Jays caw; while the Warblers differ in the quality, rather than the kind, of note. ${ }^{108}$ The Parrot and Mocking-bird use the tongue in imitating human sounds. Some species possess great compass of voice. The Bell - bird can be heard nearly three miles; and Livingstone said he could distinguish the voices of the Ostrich and the Lion only by knowing that the former roars by day, and the latter by night.

The vocal organ of Marnmals, unlike that of Birds, is in the upper part of the larynx. It consists of four cartilages, of which the largest (the thyroid) produces the prominence in the human throat known as "Adam's apple," and two elastic bands, called "vocal cords," just below the glottis, or upper opening of the windpipe. The various tones are determined by the tension of these cords, which is effected by the raising or lowering of the thyroid 
cartilage, to which one end of the cords is attached. The will cannot influence the contraction of the vocalizing muscles, except in the very act of vocalization. The vo-

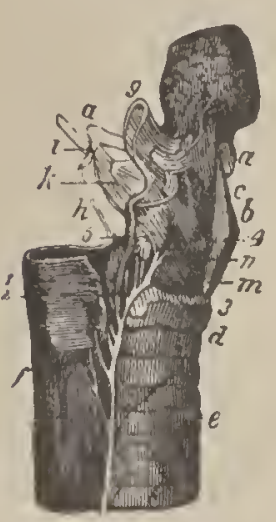

Fig. 159.-Humau Larynx, seen in profile; $a$, half of the hyoid bone; $e$, trachea: $f$, œsophngus ; $g$, epiglottis.

cal sounds produced by Mammals may be distinguished into the ordinary voice, the cry, and the song. The second is the sound made by brutes. The Whale, Porpoise, Armadillo, Ant-eater, Porcupine, and Giraffe are generally silent. The Bat's voice is probably the shrillest sound audible to human ears. There is little modulation in brute utterance. The Opossum purrs, the Sloth and Kangaroo moan, the Hog grunts or squeals, the Tapir whistles, the Stag bellows, and the Elephant gives a hoarse trumpet sound from its trunk and a deep groan from its throat. All Sheep have a guttural voice; all the Cows low, from the Bison to the Musk-ox; all the Horses and Donkeys neigh; all the Cats miau, from the domestic animal to the Lion; all the Bears growl; and all the Canine familyFox, Wolf, and Dog-bark and howl. The Howlingmonkeys and Gorillas have a large cavity, or sac, in the throat for resollance, enabling them to utter a powerful voice; and one of the Gibbon-apes has the remarkable power of emitting a complete octave of musical notes. The human voice, taking the male and female together, has a range of nearly four octaves. Man's power of speech, or the utterance of articulate sounds, is due to his intellectual development rather than to any structural difference between him and the Apes. Song is produced by the rocal cords, speech by the mouth. 


\section{CHAPTER XIX.}

\section{REPRODUCTION.}

IT is a fundamental truth that every living organism has had its origin in some pre-existing organism. The doctrine of "spontaneous generation," or the supposed origination of organized structures out of inorganic particles, or ont of dead organic matter, has not yet been sustained by facts.

Reproduction is of two kinds-sexual and asexual. All animals, probably, have the first method, while a very great number of the lower forms of life have the latter also.

Of asexual reproduction there are two kinds - Self-division and Budding.

Self-division, the simplest mode possible, is a natural breaking-up of the body into distinct surviving parts. This process is sometimes extraordinarily rapid, the increase of one animalcule (Paramœcium) be-

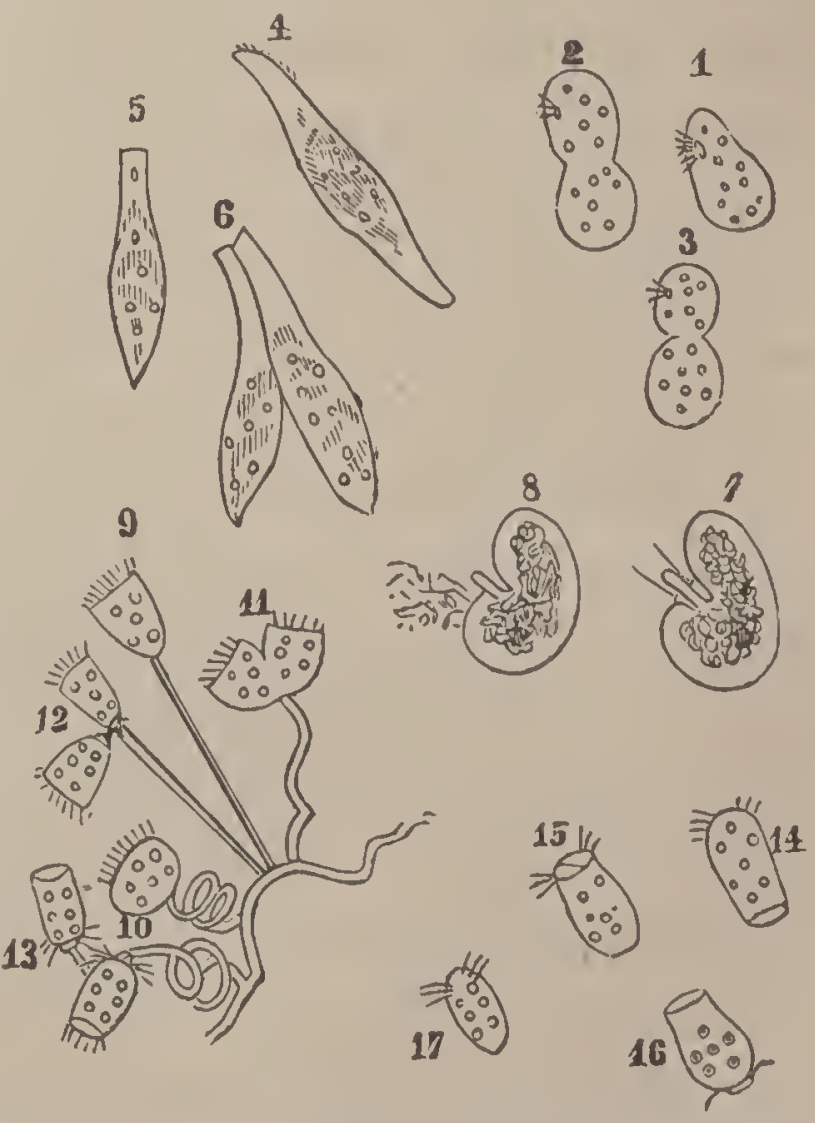
ing computed at 268 Fra. 160.-Reproduction of Infusoria (Vorticelmillions in a month. It may be either transverse or longitudinal. Of the first sort, Figs. 1,2, and 3 (Fig. 160) are examples; of the latter, 
Figs. 4, 6, 9-13. This form of reproduction is, naturally, confined to animals whose tissues and organs are simple, and so can easily bear division, or whose parts are so arranged as to be easily separable without serious injury. The process is most common in Protozoa, Worms, and Polyps.

Budding is separated by no sharp line from Self-division. While in the latter a part of the organs of the parent go to the offspring, in the former one or more cells of the original animal begin to develop and multiply so as to grow into a new animal like the parent. The process in animals is quite akin to the same operation in plants. The buds may remain permanently attached to the parent-stock, thus making a colony, as in Corals and Bryozor (continuous budding), or they may be detached at some stage of growth (discontinuous budding). This separation may occur when the bud is grown up, as in Hydra (Fig. 191), or as in Plant-lice, Daphnias (Fig. 255), and among other animals the buds may be internal, and detached when entirely undeveloped and externally resembling an egg. They differ, however, entirely from a true egg in developing directly, without fertilization.

Sexual Reproduction requires cells of two kinds, usually from different animals. These are the germ-cell or egg, and the sperm-cell. The embryo is developed from the union of the two cells. ${ }^{107}$

The egg consists essentially of three parts, the germinal vesicle, the yolk, and the vitelline membrane, which surrounds both the first. It is ordinarily globular in shape. Of the three parts, the primary one is the germinal vesicle-a particle of protoplasm. The yolk serves as food for this, and the membrane protects both. When a great mass of yolk is present, it is divisible into two parts-formative and food yolk. The latter is of a more oily nature than the former, and is usually not segmented with the 
egg. The structure of the hen's egg is more complicated. The outside shell consists of earthy matter (lime) deposited in a net-work of animal matter. It is minutely porous, to allow the passage of vapor and air to and fro. Lining the shell is a double membrane (membrana putaminis) resembling delicate tissue-paper. At the larger end, it separates to enclose a bubble of air for the use of the chick. Next comes the albumen, or" "white," in spirally arranged layers, within which floats the yolk. The yolk is

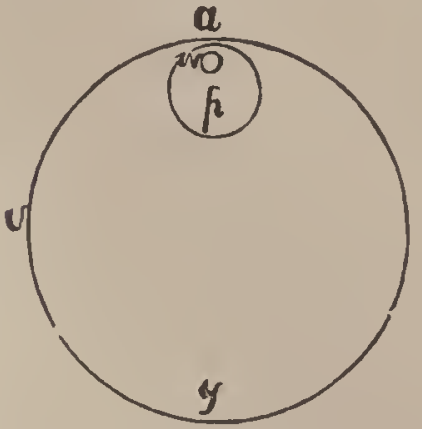

Fit. 161. - Theoretical Ergg, or Cell : $v$, vitelline membrane; $y$, oleaginous pole ; $a$, albuminous pole; $p$, Purkinjenn, or germinal, vesicle; $w$, Wagnerian, or germinal, dot. prevented from moving towards either end of the egg by two twisted cords of albumen, called chalazoe; yet is allowed to rise towards one side, the yolk being lighter than the albumen. The yolk is composed of oily granules (about $\frac{1}{2} \frac{1}{50}$ of an inch in diameter), and is enclosed in a sac, called the vitelline membrane, and disposed in concentric layers, like a set of vases placed one within the other. That part of the yolk which extends from the centre to a white

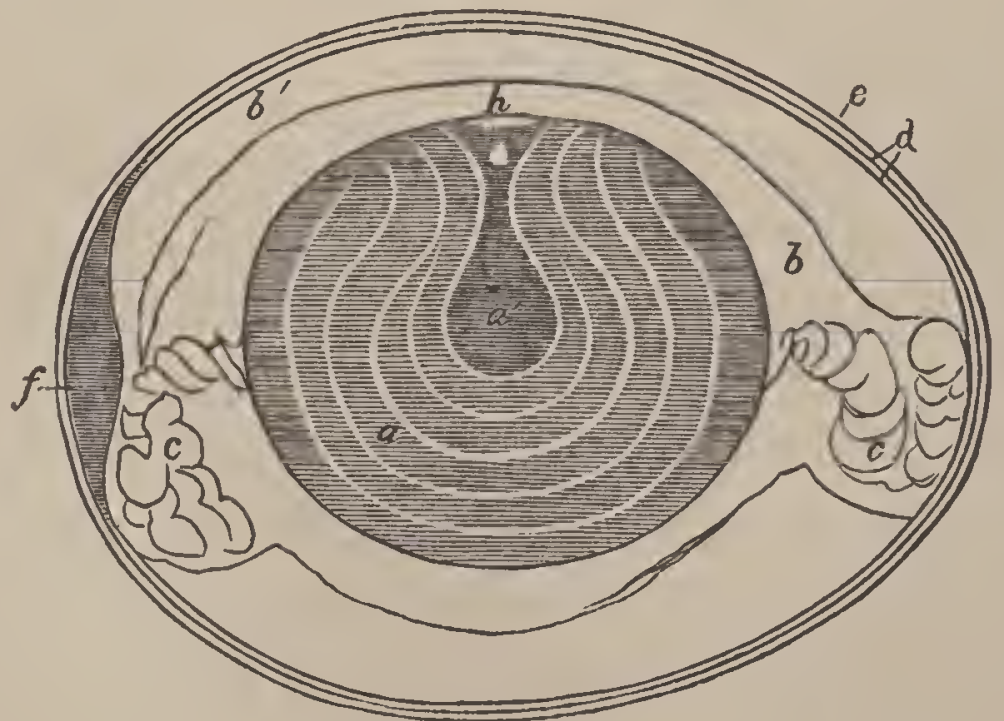

Fra.162. - Longitndinal Section of Hen's Egg before incubation : $\alpha$, yolk, showing concentric inyers; $a^{\prime}$, its semi-fluid centre, consisting of a white granniar snbstance - the whole yolk is enclosed in the vitelline membrane: $b$, inner dense part of the albumen ; $b^{\prime}$, onter, thinner part; $c$, the chalazæ, or albumen, twisted by the revolutions of the yolk; $d$, double shell-membrane, split at the large end to form the chamber, $f ; e$, the shell; $h$, the white spot, or cicatricula. 
spot (cicatricula) on the outside cannot be hardened, even with the most prolonged boiling. The cicatricula, or en!bryo-spot-the part for which all the rest was made-is a thin disk of cellular structure, in which the new life first appear's. This was originally a simple cell, but development has gone some way before the egg is laid. It is always on that side which naturally turns uppermost, for the yolk can turn upon its axis; it is, therefore, always nearest to the external air and to the Hen's bodytwo conditions necessary for its development. There is another reason for this polarity of the egg: the lighter and most delicate part of the yolk is collected in its upper part, while the heavy, oily portion remains beneath.

In most eggs the shell and albumen are wanting. When the albumen is present, it is commonly covered by a membrane only. In Sharks, the envelope is horny; and in Crocodiles it is calcareous, as in Birds.

The egg of the Sponge has no true vitelline membrane, and is not unlike an ordinary amœboid cell. An egg is,

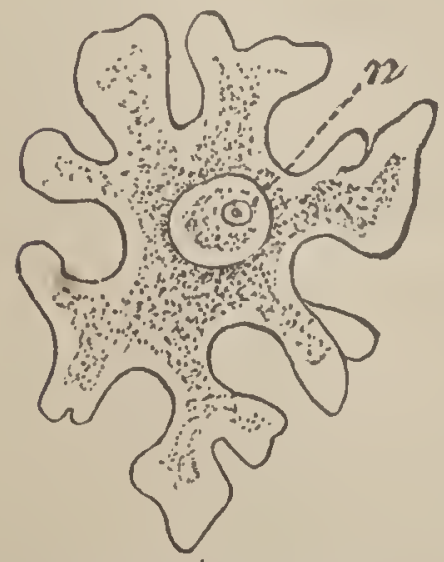

Fig. 163.-Egg of Sponge $n$, nucleus. Magnified. in fact, little more than a very large cell, of which the germinal vesicle is the nucleus.

The size of an egg depends mainly upon the quantity of yolk it contains; and to this is proportioned the grade of derelopment which the embryo attains when it leaves the egg. ${ }^{108}$ In the eggs of the Star-fishes, Worms, Insects, Mollusks (except the Cuttle-fishes), many Amphibians, and Mammals, the yolk is very minute and formative, $i . e$, it is converted into the parts of the future embryo. In the eggs of Lobsters, Crabs, Spiders, Cephalopods, Fishes, Reptiles, and Birds, the yolk is large and colored, and consists of two parts - the formative, or 
germ-yolk, immediately surrounding the germinal vesicle; and the nutritive, or food-yolk, constituting the greater part of the mass, by which the young animal in the egglife is nourished. In the latter case, the young come forth more mature than where the food-yolk is wanting.

As to form, eggs are oval or elliptical, as in Birds and Crocodiles; spherical, as in Turtles and Wasps; cylindrical, as in Bees and Flies; or shaped like a hand-barrow, with tendrils on the corners, as in the Shark. The eggs

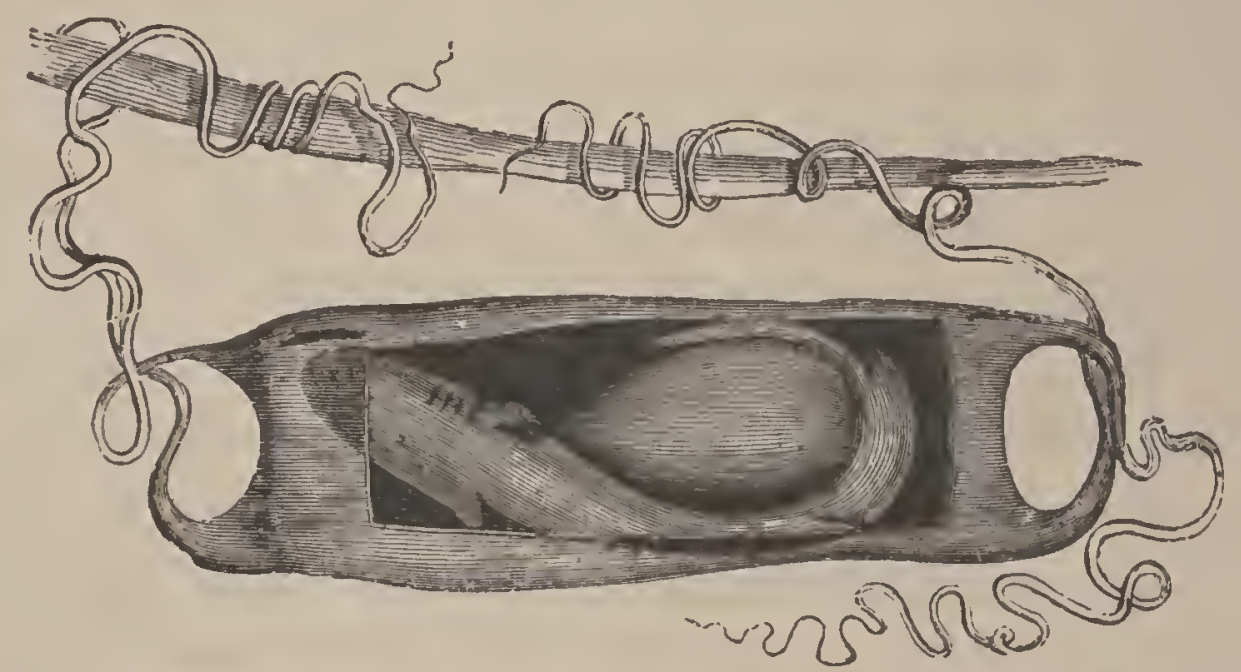

Fia. 164.-Egg of a Shark (the external gills of the embryo are not represented).

of some very low forms are sculptured or covered with hairs or prickles.

The number of eggs varies greatly in different animals, as it is in proportion to the risks during development. Thus, the eggs of aquatic tribes, being unprotected by the parent, and being largely consumed by many animals, are multiplied to prevent extinction. The spawn of a single Cod contains millions of eggs ; that of the Oyster, 6,000,000. A Queen-bee, during the five years of her existence, lays about a million eggs.

Eggs are laid one by one, as by Birds; or in clusters, as by Frogs, Fishes, and inost Invertebrates. The spawn of the Sea-snails consists of vast numbers of eggs adhering together in masses, or in sacs, forming long strings.

As a rule, the higher the rank, the more care animals 
take of their eggs and their young, and the higher the temperature needed for egg-development. In the majority of cases, eggs are left to themselves. The fresh-water Mussel (Unio) carries them within its gills, and the Lobster under its tail. The eggs of many Spiders are enveloped in a silken cocoon, which the mother guards with jealous care. Insects, as Flies and Moths, deposit their eggs where the larva, as soon as born, can procure its own food. Most Fishes allow their spawn, or roe, to float in the water; but a few build a kind of flat nest in the sand or mud, hovering over the eggs until they are hatched; while the Acará of the Amazons carries them in its moith. The Amphibians, generally, envelop their eggs in a gelatinous mass, which they leave to the elements; but the female of the Surinam Toad carries hers on her back, where they are placed by the male. The great Amazon Turtles lay their eggs in holes two feet deep, in the sand; while the Alligators simply cover theirs with a few leaves and sticks. Nearly all Birds build nests, those of the Perchers being most elaborate, as their chicks are dependent for a time on the parent. ${ }^{100}$ The young of Marsupials, as the Kangaroo, which are born in an extremely immature state, are nourished in a pouch ontside of the body. But the embryo of all other Mammals is developed within the parent to a more perfect condition, by means of a special organ, the placenta. It is a general law, that animals receiving in the embryo state the longest and most constant parental care ultimately attain the highest grade of development.

The Protozoa, which have no true eggs, have a sort of reproduction called conjugation. In this process two individuals unite into one mass, surround themselves with a case, in which they divide into several parts, each portion becoming a new individual.

The sperm-cells differ from the egg in being very small, 
usually motile, and in that a large number are usually produced from a single primary reproductive cell of the animal, while the egg represents the entire primary cell. The union of the sperm-cell with the germinal vesicle (fertilization) is the first step in development, and without it the egg will not develop. But the nature of the process is unknown.

\section{CHAPTER XX.*}

DEVELOPMENT.

Development is the evolution of a germ into a complete organism. The study of the changes within the egg constitutes the science of Embryology; the transformations after the egg-life are called metamorphoses, and include growth and repair.

The process of development is a passage from the general to the special, from the simple to the complex, from the homogeneous to the heterogeneous, by a series of differentiations. It brings out first the profounder distinctions, and afterwards those more external. That is, the most essential parts appear first. And not only does development tend to make the several organs of an individual more distinct from one another, but also the individual itself more distinguished from other individuals and from the medium in which it lives. With advancing development, the animal, as a rule, acquires a more specific, definite form, and increases in weight and locomotive power. Life is a tendency to individuality.

The first step in development, after fertilization, is the segmentation of the egg, by a process of self-division. In the simplest form, the whole yolk divides into two parts; these again divide, making four, eight, sixteen, etc., parts, * See Appendix. 
until the whole yolk is subdivided into very small portions (cells) surrounding a central cavity. This stage is known as the "mulberry-mass," or blastula (Fig. 165, c).

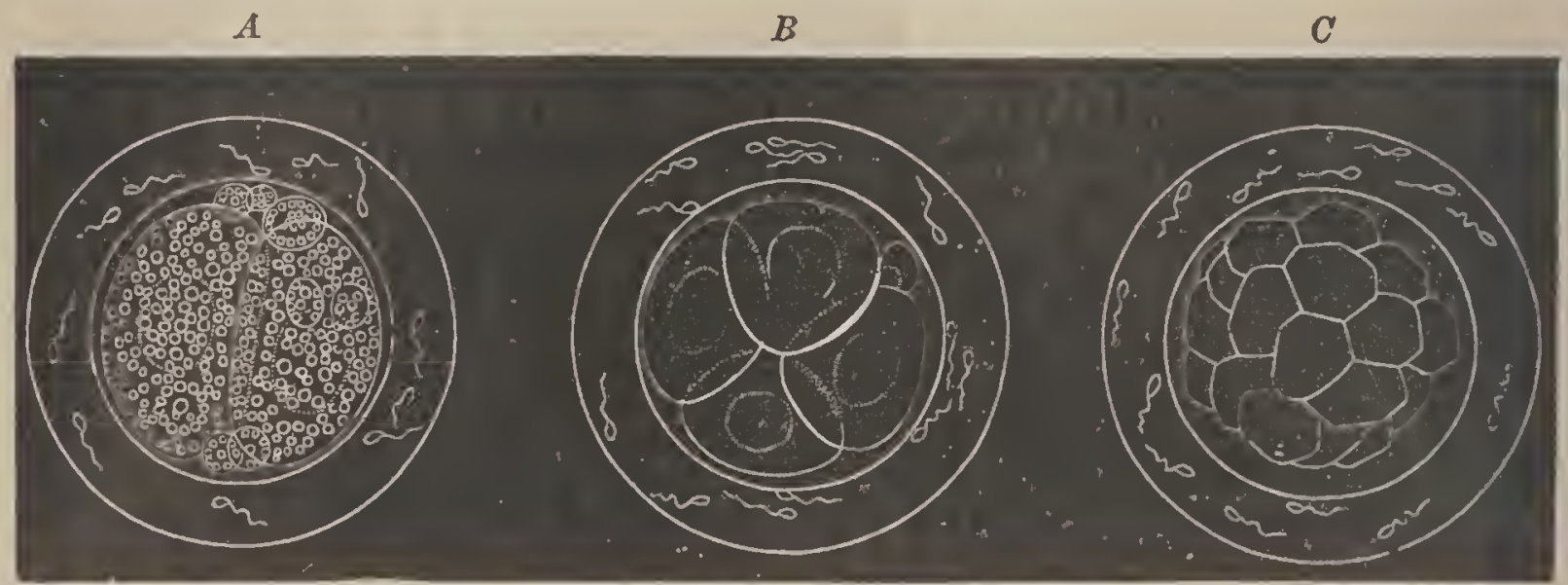

Fra. 165. - First Stages in Segmentation of a Mammalian Egg: $A$, first division into halves, with spermatozoa around it; $B$ and $C$, progressive subdivision, ultimatcly transforming the vitellus, or yolk, into a "mulberry mass" of globules, or embryo-cells.

If the yolk is larger, relatively to the germinal vesicle, the process of division may go on more slowly in one of the two parts of the egg, first formed; or in very large eggs, like those of Birds and Cuttle-fishes, only a sinall part of the yolk subdivides.

In some form, the process of segmentation is found in the eggs of all animals, as is also the following stage.

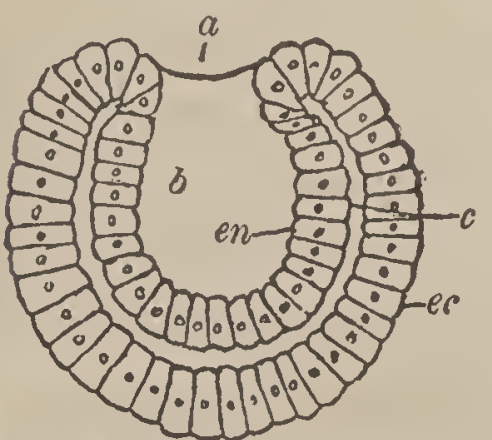
This step is the differentiation of the single layer of cells into two parts, one for the body-wall, the other for the wall of the digestive tract. In the typical examples, this is accomplished by one part of the wall of Frg. 166. -Diagram of Gastru- the blastula turning in, so far as to la of a Worm (Sagitta): $a_{\text {r }}$ convert the blastula into a sort of
primitive mouth; $b$, primi- convert

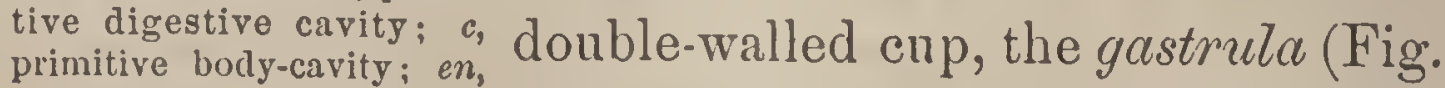
endoderm; ec, ectoderm. 166). One half of the wall of the blastula is now the outer wall of the germ, the other half that of the digestive cavity; the original blastula-cavity is now the body-cavity, and the new cavity formed by the infolding is the stomach, and its opening is both mouth 
and vent (Figs. 165, 166). Some adult animals are little more than such a sac. Hydra (Fig. 191), for instance, is little different from a gastrula with tentacles, and one of its relatives wants even these additions.

Ordinarily, however, development goes much further. From the two original layers arises, in various ways, a third between them, making the three primitive germ-layersepiblast, mesoblast, and hypoblast. This new layer is necessarily in the primitive body-cavity, which it may fill up; or usnally a new body-cavity is formed, in different ways in different groups. In by far the great majority of animals the digestive tract gets a new opening, which usually becomes the mouth; and the old mouth may close, or serve only the functions of the vent. From this point the development of each group must be traced in detail.

Development of a Hen's Egg. - After the segmentation the germinal disk divides into two layers, between which a third is soon formed. The upper layer (epiblast) gives
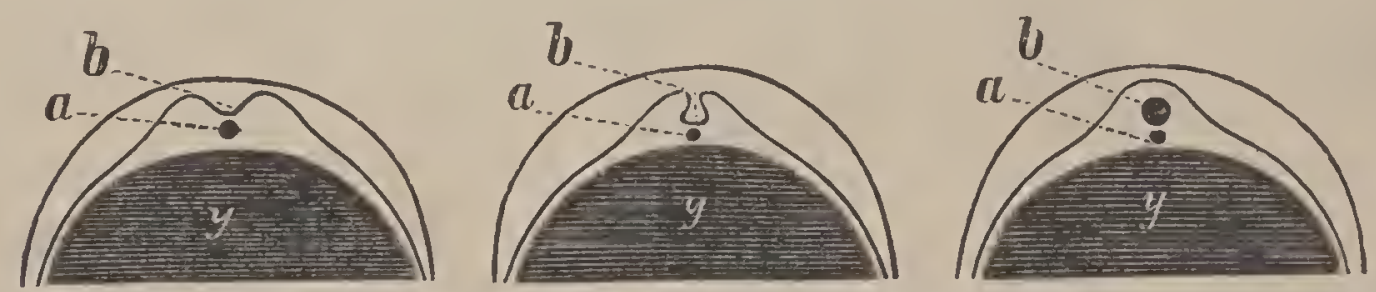

Fic. 167.-Transverse Vertical Sections of an Egg, showing progressive stages of development: $a$, notochord; $b$, medullary furrow, becoming a closed canal in the last.

rise to the cuticle, brain, spinal cord, retina, crystalline lens, and internal ear. From the lower layer (hypoblast) is formed the epithelium of the digestive canal. From the middle layer (mesoblast) come all the other organsmuscles, nerves, bones, etc. The mesoblast thickens so as to form two parallel ridges running lengthwise of the germ, and leaving a groove between them (medullary furrow and ridges). ${ }^{110}$ The ridges gradually rise, carrying with them the epiblast, incline towards each other. and at last unite along the back. So that we have a 
tube of epiblast surrounded by mesoblast, which is itself covered by epiblast. This tube becomes the brain and spinal cord, whose central canal, enlarging into the ven. tricles of the brain, tells the story of its original formation. Beneath the furrow, a delicate cartilaginous thread appears (called notochord)--the predecessor of the backbone. Meanwhile the mesoblast has divided into two layers, except in the middle of the animal, beneath the spinal cord, and in the head. One of these layers remains attached to the epiblast, and with it forms the body-wall; the other bends rapidly down ward, carrying the hypoblast with it, and forms the wall of the intestine. The space thus left between the layers of the mesoblast is the body. cavity. At the same time, the margin of the germ extends farther and farther over the yolk, till it completely encloses it. So that now we see two cavities - a small one, containing the nervous system; and a larger one below, for the digestive organs. Presently, numerous rows
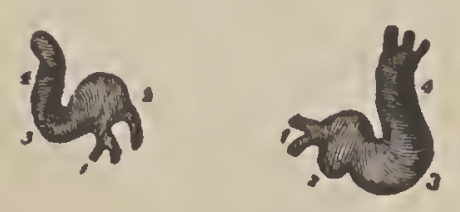
of corpuscles are seen on the middle layer, which are subsequently enclosed, forming a net-work of capillaries,

Pig. 168.-Rudimentary Hearts, humau: 1, venons trunks; 2 , auricle; 3 , ventricle; 4 , bulbus arteriosus.

called the vascular area.

A dark spot indicates the situation of the heart, which is the first distinctly bounded cavity of the circulatory system. It is a short tube lying lengthwise just behind the head, with a feeble pulsation, causing the blood to flow backward and forward. The tube is gradually bent together, until it forms a double cavity, resembling the heart of a Fish. On the fourth day of incubation, partitions begin to grow, dividing the cavities into the right and left auricles and ventricles. The septum between the auricles is the last to be finished, being closed the moment respiration begins. 
The embryo lies with its face, or ventral surface, towards the yolk, the head and tail curving towards each

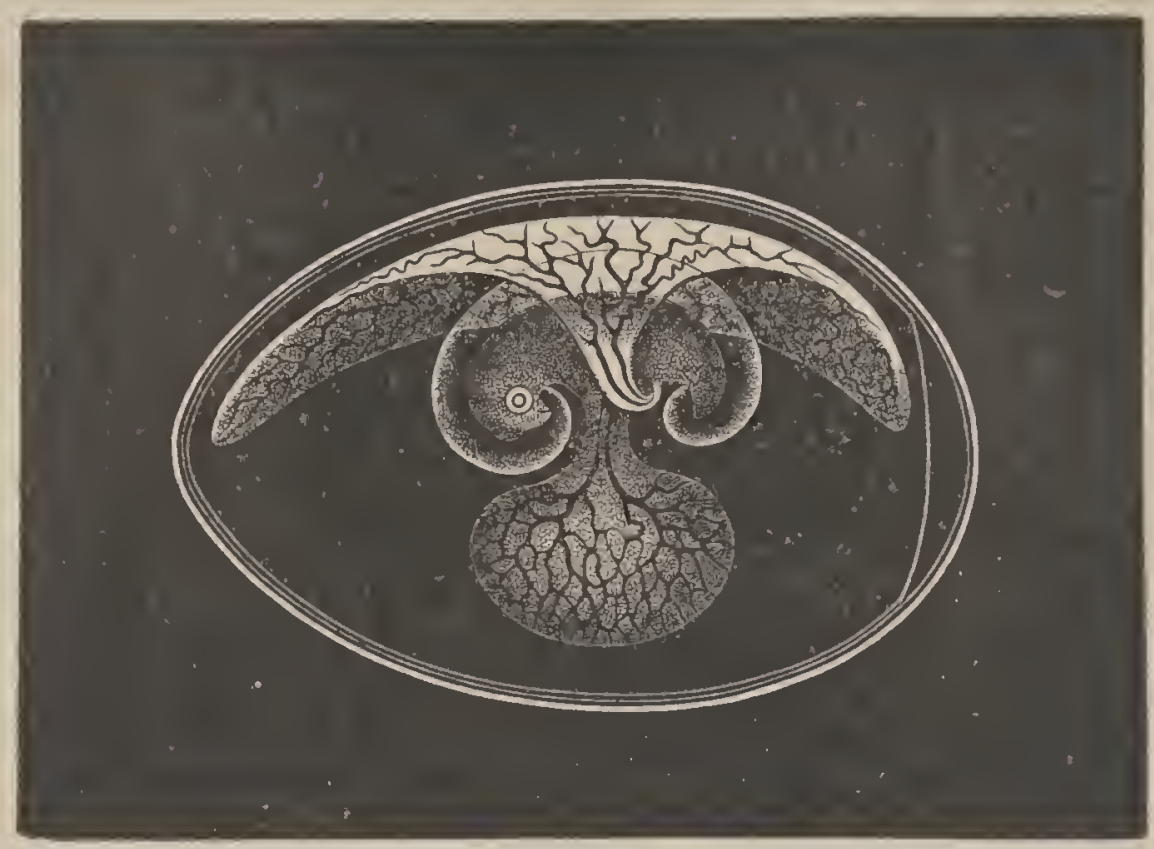

Fig. 170.-Hen's Egg, more highly developed. The embryo is enveloped by the amnion, and has the umbilical vessel, or remuant of the yolk, hanging from its under surface; while the allantois turns upward, and spreads out over the interual surface of the shell-membrane. (Froin Dalton's "Physiology.")

other. Around the embryo on all sides the epiblast and upper layer of the mesoblast rise like a hood over the

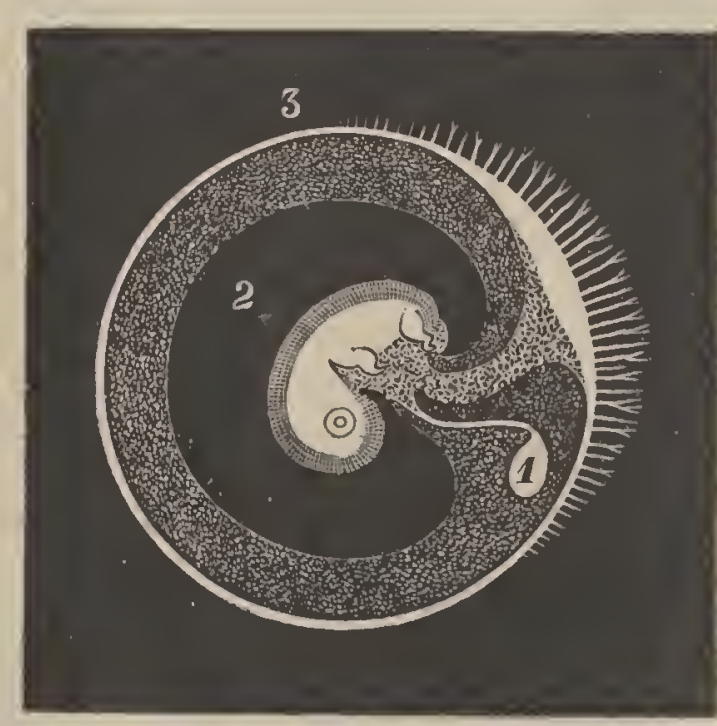

FIG. 171. - Mammalian Embryo, with allantois fully formed: 1 , umbilical vesicle, containiug the last of the yolk; 2 , amnion; 3, allantois, on which the fringes of the placenta are developing. (From Dalton's "Physiology.")

whole inner surface of the shell, so that it partly surrounds both embryo and inner layer of the amnion (amnion prop-

back of the embryo till they form a closed sac, called the amnion. It is filled with a thin liquid, which serves to protect the embryo. Meanwhile, another important organ is forming on the other side. From the hinder portion of the alimentary canal an outgrowth is formed which extends beyond the wall of the embryo proper into the cavity of the amnion and spreads out over the . 
er). This is the allantois. It is full of blood-vessels, and it serves as the respiratory organ until the chick picks the shell and breathes by its lungs. ${ }^{112}$ The chorion is the outermost part of the allantois, and the placenta of Mammals is the shaggy, vascular edge of the chorion.

The alimentary canal is at first a straight tube closed at both ends, the middle being connected with the yolk-bag. As it grows faster than the body, it is thrown into a spiral coil; and at several points it dilates, to form the crop, stomach, gizzard, etc. The mouth is developed from an infolding of the skin. The liver is an ontgrowth from the digestive tube, at first a cluster of cells, then of follicles, and finally a true gland. The lungs are developed on the third day as a minute bud from the upper part of the alimentary canal, or pharynx. As they grow in size, they pass from a smooth to a cellular condition.

The skeleton at the beginning consists, like the notochord, of a cellular material, which gradually turns to cartilage. Then ininute canals containing blood-vessels arise, and earthy matter (chiefly phosphate of lime) is deposited between the cells. The primary bone thus formed is compact: true osseous tissue, with canaliculi, laminæ, and Haversian canals, is the result of subsequent absorption. ${ }^{113}$ Certain bones, as those of the face and cranium, are not preceded by cartilage, but by connective tissue: these are called membrane bones. Ossification, or bone-making, begins at numerous distinct points, called centres; and, theoretically, every centre stands for a bone, so that there are as many bones in a skeleton as centres of ossification. But the actual number in the adult animal is much smaller, as many of the centres coalesce. ${ }^{114}$ The development of the backbone is not from the head or from the tail, but from a central point midway between: there the first vertebræ appear, and from thence they multiply forward and backward. 
The limbs appear as buds on the sides of the body; these lengthen and expand so as to resemble paddlesthe wings and legs looking precisely alike; and, finally, they are divided each into three segments, the last one subdividing into digits. The feathers are developed from the outside cells of the epidermis: first, a horny cone is formed, which elongates and spreads out into a vane, and this splits up into barbs and barbules.

The muscle-fibres are formed either by the growth in length of a single cell, or by the coalescence of a row of cells: the cell-wall thus produces a long tube-the sarcolemma of a fibre-and the granular contents arrange themselves into linear series, to make fibrillæ.

Nervous tissue is derived from the multiplication and union of embryo-cells. The white fibres at first resemble the gray. The brain and spinal marrow are developed from the epiblastic lining of the medullary furrow. Soon the brain, by two constrictions, divides into fore-brain, mid-brain, and lind-brain. The fore-brain throws out two lateral hemispheres (cerebrum), and from these protrude forward the two olfactory lobes. From the middle-brain grow the optic lobes; and the hind-brain is separated into cerebellum and medulla oblongata. The essential parts of the eye, retina and crystalline lens, are developed, the former as a cup-like outgrowth from the fore-brain, the latter as an ingrowth of the epidermis. An infolding of the epidermis gives rise to the essential parts of the inner ear, and from the same layer come the olfactory rods of the nose and the taste-buds of the tongue. So that the central nervous system and the essential parts of most of the sense-organs have a common origin.

Modes of Development.-The structure and embryology of a Hen's egg exhibit many facts which are common to all animals. But every grand division of the Animal Kingdom has its characteristic method of developing. 
Protozoans differ from all higher forms in having no true eggs.

The egg of the Hydroid, after segmentation, becomes a hollow, pear-shaped body, covered with cilia. Soon one end is indented; then the indentation deepens until it reaches the interior and forms the mouth. The animal fastens itself by the other end, and the tentacles appear as buds. In the Sea-anemone, the stomach is turned in, and the partitions appear in pairs.

In the Oyster, the egg segments into two unequal parts, one of which gives rise to the digestive tract and its derivatives, while from the smaller part originate the skin, gills, and shell. It is soon covered with cilia, by whose help it swims about.

The embryo of an Insect shows from the first a right and left side; but the first indication that it is an Articulate is the development of a series of indentations dividing the body into successive rings, or joints. Next, we observe that the back lies near the centre of the egg, the rentral side looking outward; i.e., the embryo is doubled upon itself backward. And, finally, the appearance of three pairs of legs proves that it will be an Insect, rather than a Worm, Crustacean, or Spider.

The Vertebrate embryo lies with its stomach towards the yolk, reversing the position of the Articulate; but the grand characteristic is the medullary groove, which does not exist in the egg of any Invertebrate. This feature is connected with another, the setting apart of two distinct regions - the nervous and nutritive. There are three modifications of Vertebrate development: that of Fishes and Amphibians, that of True Reptiles and Birds, and that of Mammals. The amnion and allantois are wanting in the first group; while the placenta (which is the allantois vitally connected with the parent) is peculiar to Mammals. In Mammals, the whole yolk is segmented; in 
Birds, segmentation is confined to the small white speck seen in opening the shell.

At the outset, all animals, from the Sponge to Man, are structurally alike. All moreover, undergo segmentation, and most have one form or other of the gastrula stage. But while Vertebrates and Invertebrates can travel together on the same road up to this point, here they diverge-never to meet again. For every grand group early shows that it has a peculiar type of construction. Every egg is from the first impressed with the power of developing in one direction only, and never does it lose its fundamental characters. The germ of the Bee is divided into segments, showing that it belongs to the Articulates; the germ of the Lion has the medullary furrow-the mark of the coming Vertebrate. The blastodermic layer of the Vertebrate egg rolls up into two tubes -one to hold the viscera, the other to contain the nervous cord; while that of the Invertebrate egg forms only one such tubular division. The features which determine the subkingdom to which an animal belongs are first developed, then the characters revealing its class.

There are differences also in grade of development as well as type. For a time there is no essential difference between a Fish and a Maminal: they have the same nervous, circulatory, and digestive systems. There are many such cases, in which the embryo of an animal represents the permanent adult condition of some lower form. In other words, the higher species, in the course of their development, offer likenesses, or analogies, to finished lower species. The human germ, at first, cannot be distinguished from that of any other animal: for aught we can see, it may turn out a Frog or a Philosopher. The appearance of a medullary furrow excludes it at once from all Invertebrates. It afterwards has, for a time, structures found in the lower classes and orders of Vertebrates as permanent 
organs. For a time, indeed, the human embryo so closely resembles that of the lower forms as to be indistinguishable from them; but certain structures belonging to those forms are kept long after the embryo is clearly human. ${ }^{115}$ All the members of a group do not reach the same degree of perfection, some remaining in what corresponds to the immature stages of the higher animals. Such may be called permanently embryonic forms.

Sometimes an embryo develops an organ in a rudimentary condition, which is lost or useless in the adult. Thus, the Greenland Whale, when grown up, has not a tooth in its head, while in the embryo life it has teeth in both jaws; unborn Calves have canines and upper incisors; and the female Dugong has tusks which never cut the gum. The "splint-bones" in the Horse's foot are unfinished metatarsals.

Animals differ widely in the degree of development reached at ovulation and at birth. The eggs of Frogs are laid when they can hardly be said to have become fully formed as eggs. The eggs of Birds are laid when segmentation is complete, while the eggs of Mammals are retained by the parent till after the egg-stage is passed. ${ }^{116}$ Ruminants and terrestrial Birds are born with the power of sight and locomotion. Most Carnivores, Rodents, and perching Birds come into the world blind and helpless; while the human infant is dependent for a much longer time.

\section{Metamorphosis.}

Few animals come forth from the egg in perfect condition. The vast majority pass through a great variety of forms before reaching maturity. These metamorphoses (which are merely periods of growth) are not peculiar to Insects, though more apparent in them. Man himself is developed on the same general principles as the Butterfly, but the transformations are concealed from view. The 
Coral, when, hatched, has six pairs of partitions; afterwards, the spaces are divided by six more pairs; then twelve intermediate pairs are introduced; next, twentyfour, and so on. The embryonic Star - fish has a long body, with six arms on a side, in one end of which the young Star-fish is developed. Soon the twelve-armed body is absorbed, and the young animal is of age. Worms are continually growing by the addition of new segments. Nearly all Insects undergo complete metamorphosis, i.e., exhibit four distinct stages of existence-egg, larva, pupa, and imago. The worm-like larva ${ }^{117}$ may be called a locomotive-egg. It has little resemblance to the parent in structure or habits, eating and growing rapidly. Then it enters the pupa state, wrapping itself in a cocoon, or case, and remaining apparently dead till new organs are developed; when it escapes a perfect winged Insect,

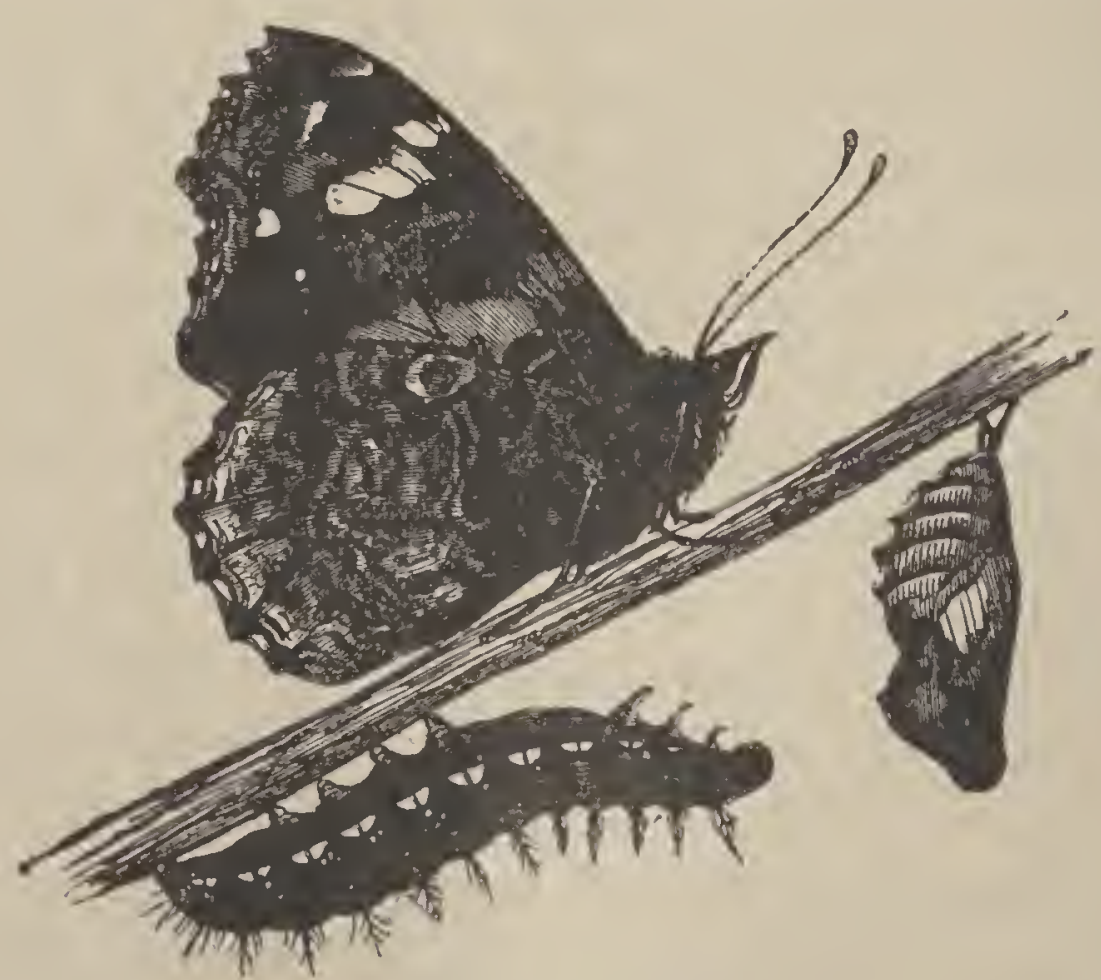

Fia. 172.-Butterfy in the Imago, Pupa, and Larva States.

or imago. ${ }^{118}$ Wings never exist externally in the larva; and some Insects which undergo no apparent metamorphosis, as Lice, are wingless. The Grasshopper develops from the young larva to the winged adult without chang. 
ing its mode of life. In the development of the common Crab, so different is the ontward form of the newly

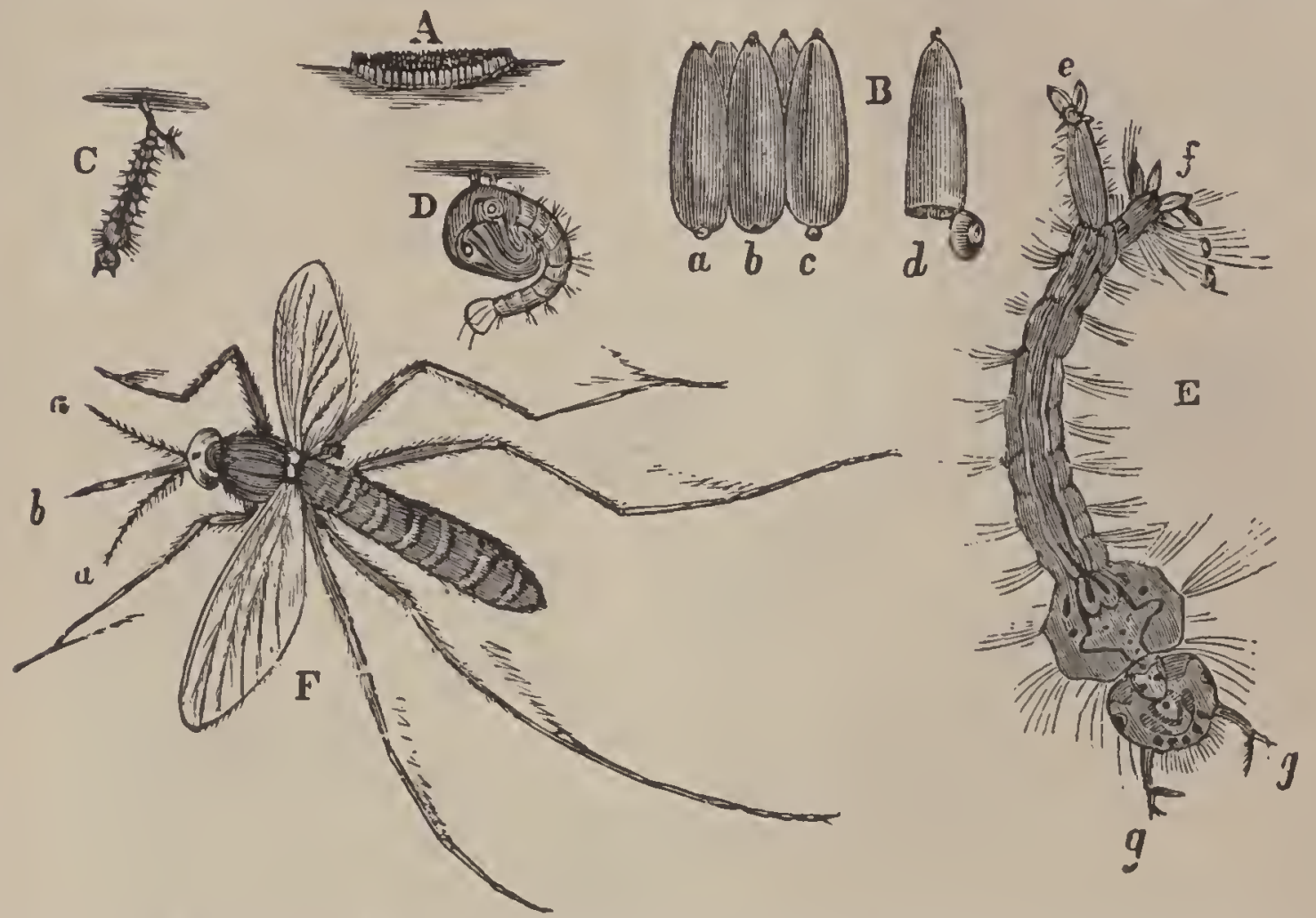

Fig. 173.-Metamorphosis of the Mosquito (Culex pipiens): A, bont of eggs; B, some of the eggs highly magnified; $d$, with lid open for the escape of the larvn, $C ; D$, pupa; E, larva magnified, showing respiratory tube, $e$, anal fins, $f$, antennæ, $g$; $\mathrm{F}$, imago; $a$, antennæ; $b$, beak.

hatched embryo from that of the adult, that the former has been described as a distinct species.

The most remarkable example of metamorphosis among Vertebrates is furnished by the Amphibians. A Tadpole - the larva of the Frog-has a tail, but no legs; gills, instead of lungs; a heart precisely like that of the Fish; a horny beak for eating vegetable food, and a spiral intestine to digest it. As it matures, the hinder legs show themselves, then the front pair; the beak falls off; the tail and gills waste away; lungs are formed; the digestive apparatus is changed to suit an animal diet; the heart is altered to the Reptilian type by the addition of another auricle; in fact, skin, muscles, nerves, bones, and bloodvessels vanish, being absorbed atom by atom, and a new set is substituted. Moulting, or the periodical renewal of epidermal parts, as the shell of the Lobster, the skin of 
the Toad, the scales of Snakes, the feathers of Birds, and the hair of Mammals, may be termed a metamorphosis.

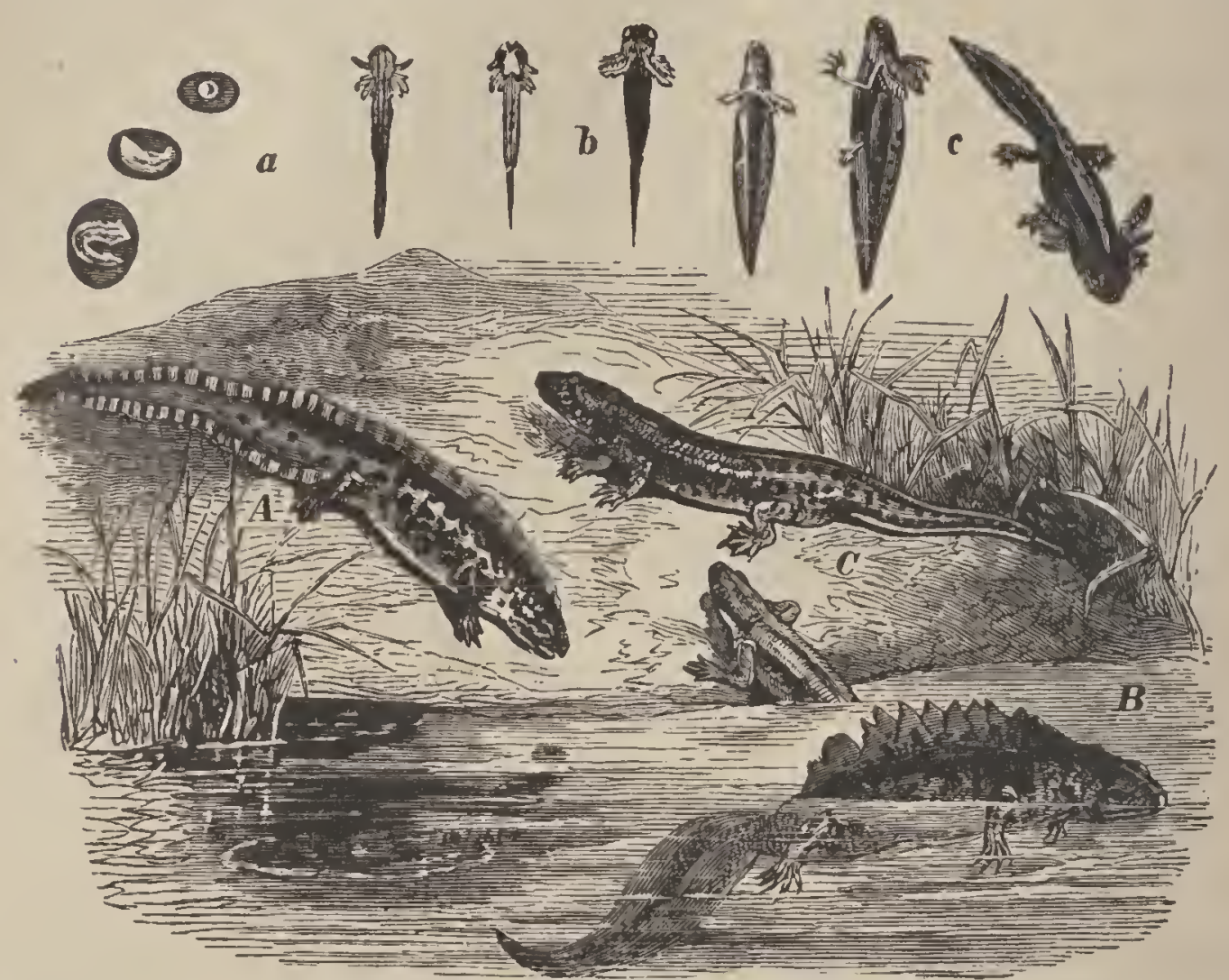

Fig. 174.-Metamorphosis of the Newt.

The change from milk-teetl to a permanent set is another example.

An animal rises in organization as development advances. Thus, a Caterpillar's life has nothing nobler about it than the ability to eat, while the Butterfly expends the power garnered up by the larva in a gay and busy life. But there are seeming reversals of this law. Some mature animals appear lower in the scale than their young. The larval Cirripede has a pair of magnificent compound eyes and complex antennæ; when adult, the antennæ are gone, and the eyes are reduced to a single, simple, minute eye-spot. So the germs of the sedentary Sponge and Oyster are free and active. The adult animal, however, is always superior in alone possessing the power of reproduction. Such a process is known as retrograde metamorphosis.

There are certain larval forms so characteristic of the 
great groups of the animal kingdom as to demand notice. Most Worms leave the egg as a larva, called the trocha sphere (Fig. 175), an oval larva, having mouth and anus, and a circle of cilia anterior to the mouth. This larval stage is common to Worms with the most diverse adult forms and habits. It is also found in all the great groups of Mollusks. Clams, Snails, and Cuttle-fish all have the stage represented in their history. The Mol-

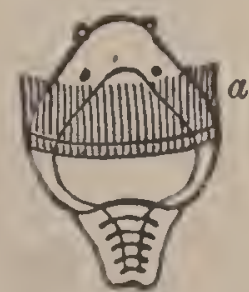

FIG. 175. -Trochosphere of Worm (Phyllodoce) : $a$, circle of cilia. lusks usually pass through a later stage called the veliger
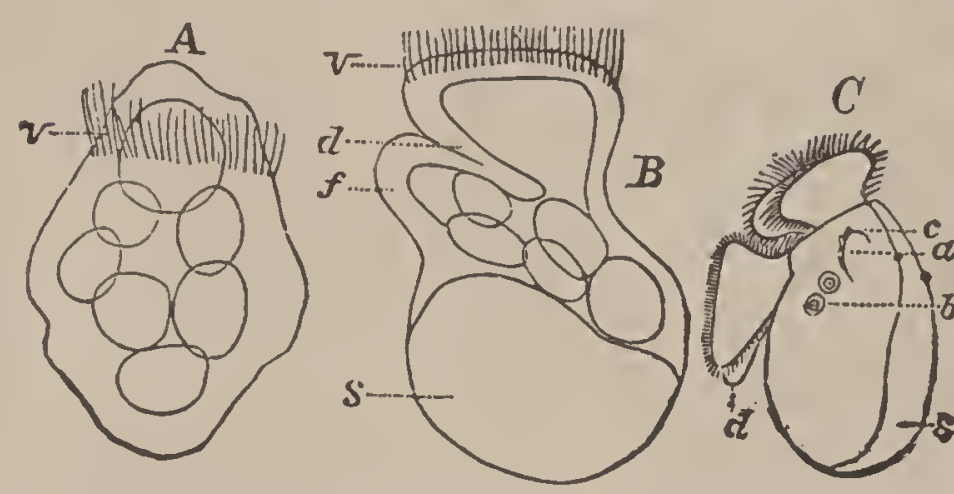

Fig. 176.-Larval Gasteropoda: $A, B$, Trochus ; $C$, Tergipes: $\mathrm{A}$, trochosphere ; $v$, velum; $\mathrm{B}$, veliger $d$, mouth ; $f$, foot; $s$, shell; $\mathrm{C}$, veliger $; d$, foot; $c$, tenta-

$c l e ; b$, ear. Magnified. exhibit so gieat a range of form in the adult state, all pass through a stage in which they are substantially alike. Forms as different in appearance as Barnacles, Entomostracans, and Prawns hatch out as $N$ cuplii, little oval animals, with a straight intestine, three pairs of legs, and a simple eye. (Fig. 177). See Figs. 253, 254, 255, 256. Fig. 256 represents the Lobster, which does not hatch as a Nauplius, but is not very unlike the Prawn. These larval forms are of great interest, because they disclose the relationships of the adult forms, as the gastrula stage hints at the common relationships of all animals above Protozoa.

\section{Alternate Generation.}

Sometimes a metamorphosis extending over several generations is required to evolve the perfect animal; "in 


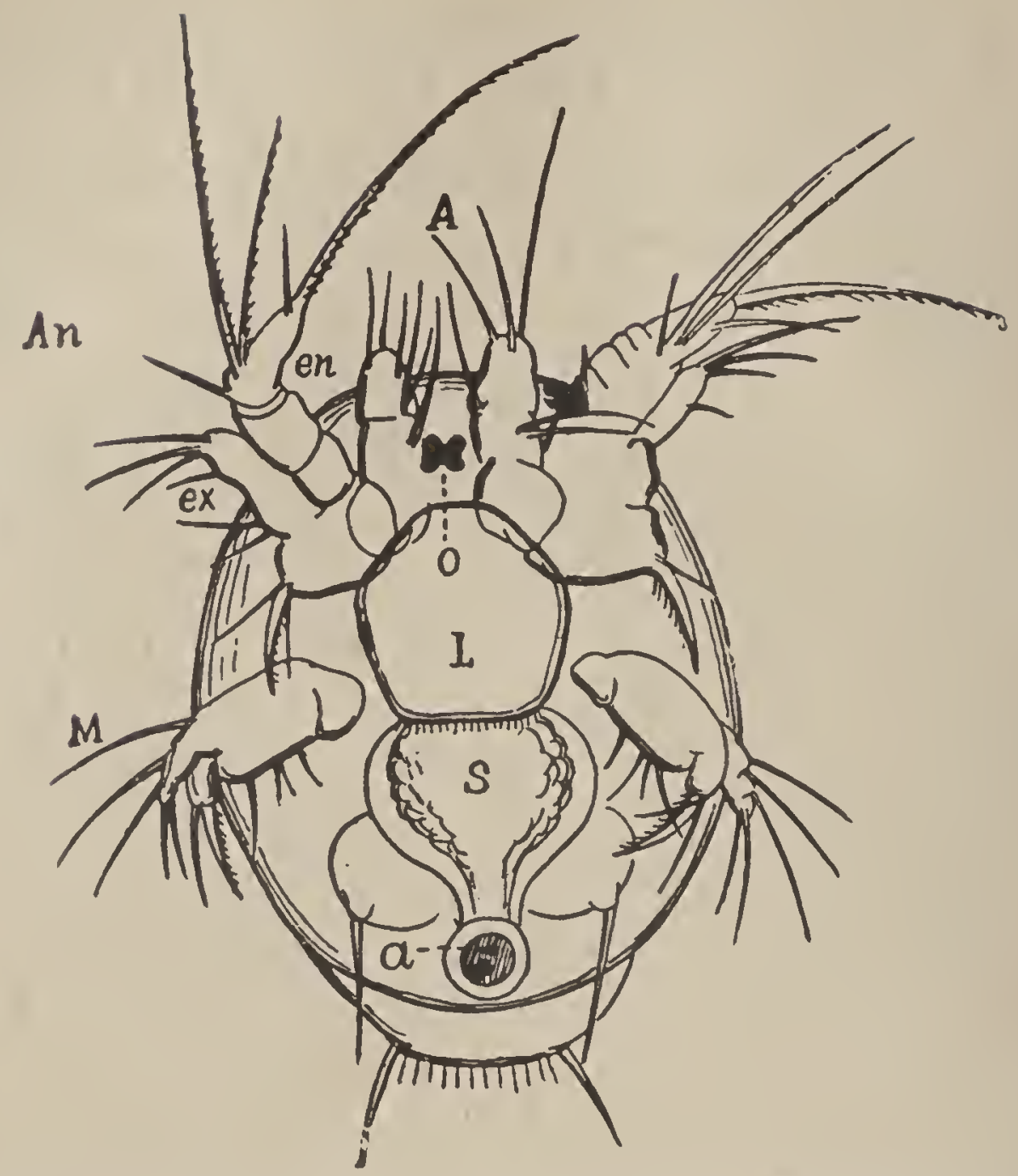

Fra. 177. - Nauplins of Entomostracan (Canthocamptus). See Fig. 255. A, first antenna; $A n$, second antenna; $a$, anus ; $L$, labrum; $O$, ocellus; $S$, stomach. (From Brooks, after Hoek.) Magnifled.

other words, the parent may find no resemblance to himself in any of his progeny, until he comes down to the great-grandson." Thus, the Jelly-fish, or Medusa, lays eggs which are hatched into larvæ resembling Infusorialittle transparent oval bodies covered with cilia, by which they swim about for a time till they find a resting-place. One of them, for example, becoming fixed, develops rapidly; it elongates and spreads at the upper end; a mouth is formed, opening into a digestive cavity; and tentacles multiply till the mouth is surrounded by them. At this stage it resembles a Hydra. Then slight wrinkles appear along the body, which grow deeper and deeper, till the animal looks like "a pine-cone surmounted by a tuft of tentacles;" and then like a pile of saucers (about a dozen 
in number) with scalloped edges. Next, the pile breaks up into separate segments, which are, in fact, so many distinct animals; and each turning over as it is set free, so as to bring the mouth below, develops into an adult Medusa, becoming more and more convex, and furnished with tentacles, circular canals, and other organs exactly like those of the progenitor which laid the original egg (Figs. 178, 195).

Here we see a Medusa producing eggs which develop into stationary forms resembling Hydras. The Hydras

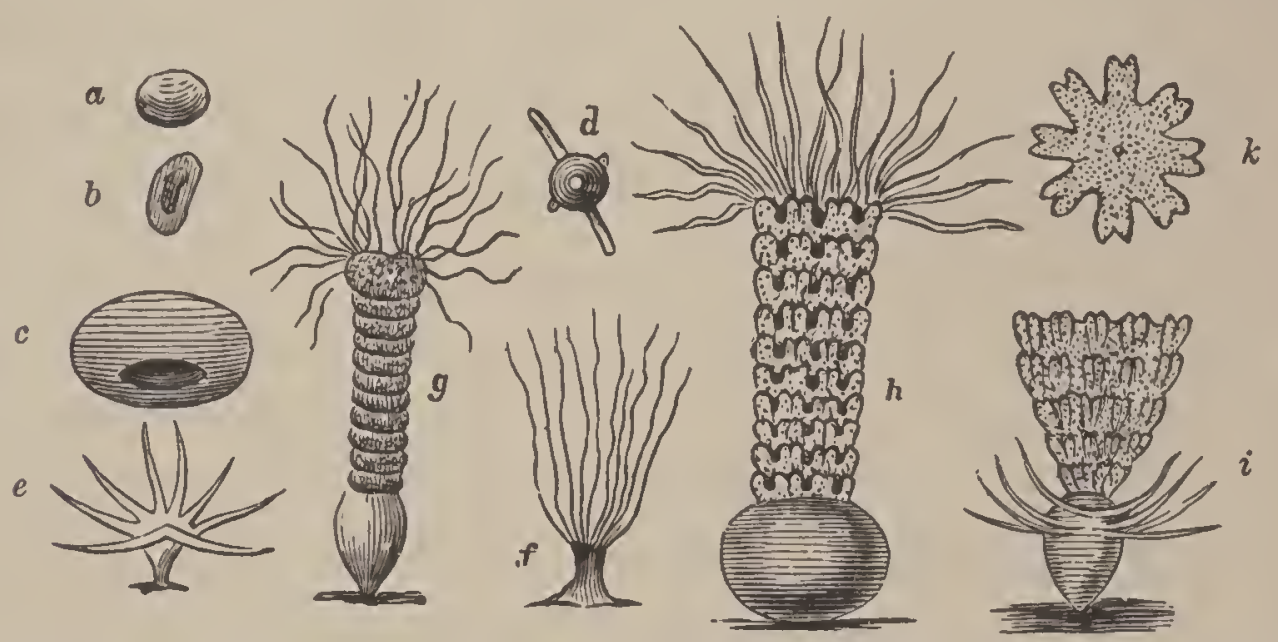

Fig. 178. -Alternate Generation: $a, b, c$, ova of an Acaleph (Chrysaora) ; $d, e, f, \mathrm{Hy}-$ dras; $g, h$, Hydras with constrictions; $i$, Hydra undergoing fission; $k$, one of the separated segments, a free Medusa.

then produce not only Medusæ by budding in the manner described, but also other Hydras like themselves by budding. All these intermediate forms are transient states of the Jelly-fish, but the metamorphoses cannot be said to occur in the same individual. While a Caterpillar becomes a Butterfly, this Hydra-like individual produces a number of Medusæ. Alternate generation is, then, an alternation of asexual and sexual methods of reproduction, one or more generations produced from buds being followed by a single generation produced from eggs. Often, as in the fresh-water Hydra, the two kinds of generations are alike in appearance. The process is as wide-spread as asexual reproduction, being found mostly in Sponges, Cœlenterates, and Worms. It is also found in certain 
Crustacea and Insects. The name is sometimes limited to cases where the two kinds of generations differ in form.

\section{Growth and Repair.}

Growth is increase of bulk, as Development is increase of structure. It occurs whenever the process of repair exceeds that of waste, or when new material is added faster than the tissues are destroyed. There is a specific limit of growth for all animals, although many of the low cold-blooded forms, as the Trout and Anaconda, seem to grow as long as they live. After the body has attained its maturity, $i$. e., has fully developed, the tissues cease to grow; and nutrition is concerned solely in supplying the constant waste, in order to preserve the size and shape of the organs. A child eats to grow and repair; the adult eats only to repair. ${ }^{119}$ Birds develop rapidly, and so spend most of their life full-fledged; while Insects generally, Fishes, Amphibians, Reptiles, and Mammals mature at a comparatively greater age. The perfect Insect rarely changes its size, and takes but little food; eating and growing are almost confined to larval life. The crust of the Sea-urchin, which is never shed, grows by the addition of matter to the margins of the plates. The shell of the Oyster is enlarged by the deposition of new laminæ, each extending beyond the other. At every enlargement, the interior is lined with a new nacreous layer; so that the number of such layers in the oldest part of the shell indicates the number of enlargements. When the shell has reached its full size, new layers are added to the inner surface only, which increases the thickness. It is the margin of the mantle which provides for the increase in length and breadth, while the thickness is derived from the whole surface. The edges of the concentric laminæ are the "lines of growth." The Oyster is full-grown in about five years. The bones of Fishes and Reptiles are 
continually growing; the long bones of higher animals increase in length so long as the ends (epiphyses) are separate from the shaft. The limbs of Man, after birth, grow more rapidly than the trunk.

The power of regenerating lost parts is greatest where the organization is lowest, and while the animal is in the young or larval state. It is really a process of budding. The upper part of the Hydra, if separated, will reproduce the rest of the body; if the lower part is cut off, it will add the rest. Certain Worms may be cut into several pieces, and each part will regain what is needed to complete the mangled organism. The Star-fish can reproduce its arms; the Holothurian, its stomach; the Snail, its tentacles; the Lobster, its claws; the Spider, its legs; the Fish, its fins; and the Lizard, its tail. Nature makes no mistake by putting on a leg where a tail belongs, or joining an immature limb to an adult animal. ${ }^{120}$ In Birds and Mammals, the power is limited to the reproduction of certain tissues, as shown in the healing of wounds. Very rarely an entire human bone, removed by disease or surgery, has been restored. The nails and hair continue to grow in extreme old age.

\section{Likeness and Variation.}

It is a great law of reproduction that all animals tend to resemble their parents. A member of one class never produces a member of another class. The likeness is very accurate as to general structure and form. But it does not descend to every individual feature and trait. In other words, the tendency to repetition is qualified by a tendency to variation. Like produces like, but not exactly. The similarity never amounts to identity. So that we have two opposing tendencies - the hereditary tendency to copy the original stock, and a distinct tendency to deviate from it. 
This is one of the most universal facts in nature. Ev. ery development ends in diversity. All know that no two individuals of a family, human or brute, are absolutely alike. There are always individual differences by which they can be distinguished. Evidently a parent does not project precisely the same line of influences upon each of its offspring.

This variability makes possible an indefinite modification of the forms of life. For the variation extends to the whole being, even to every organ and mental characteristic as well as to form and color. It is very slight from generation to generation; but it can be accumulated by choosing from a large number of individuals those which possess any given variation in a marked degree, and breeding from these. Nature does this by the very gradual process of "natural selection;" Man hastens it, so to speak, by selecting extreme varieties. Hence we have in our day remarkable specimens of Poultry, Cattle, and Dogs, differing widely from the wild races.

Sometimes we notice that children resemble, not theis parents, but their grandparents or remoter ancestors. This tendency to revert to an ancestral type is called atavism. Occasionally, stripes appear on the legs and shoulders of the Horse, in imitation of the aboriginal Horse, which was striped like the Zebra. Sheep have a tendency to revert to dark colors.

The laws governing inheritance are unknown. No one can say why one peculiarity is transmitted from father to son, and not another; or why it appears in one member of the family, and not in all. Among the many causes which tend to modify animals after birth are the quality and quantity of food, amount of temperature and light, pressure of the atmosphere, nature of the soil or water, habits of fellow-animals, etc.

Occasionally animals occur, widely different in struct. 
ure, having a very close external resemblance. Barnacles were long mistaken for Mollusks, Polyzoans for Polyps, and Lamprey-eels for Worms. Such forms are termed homomorphic.

Members of one group often put on the outward appearance of allied species in the same locality: this is called mimicry. "They appear like actors or masqueraders dressed up and painted for amusement, or like swindlers endeavoring to pass themselves off for well-known and respectable members of society." Thus, certain Butterflies on the Amazons have such a strong odor that the Birds let them alone; and Butterflies of another family in the same region have assumed for protection the same form and color of wing, but lack the odor. So we have bee-like Moths, beetle-like Crickets, wasp-like Flies, and ant-like Spiders; harmless and venomous Snakes copying each other, and Orioles departing from their usual gay coloring to imitate the plumage, flight, and voice of quite another style of Birds. The species which are imitated are much more abundant than those which mimic them. There is also a general harmony between the colors of an animal and those of its habitation. We have the white Polar Bear, the sand-colored Camel, and the dusky Twilight-moths. There are Birds and Reptiles so tinted and mottled as exactly to match the rock, or ground, or bark of a tree they frequent; and there are Insects rightly named "Walkingsticks" and "Walking-leaves." These coincidences are not always accidental, but often intentional on the part of nature, for the benefit of the imitating species. Generally, they wear the livery of those they live on, or ape the forms more favored than themselves.

\section{Homology, Analogy, and Correlation.}

The tendency to repetition in the development of ani. mals leads to some remarkable affinities. Parts or organs, 
having a like origin and development, and therefore the same essential structure, whatever their form or function, are said to be homologous; while parts or organs corresponding in use are called analogous. By serial homology is meant the homology existing between successive parts of one animal.

The following are examples of homology: the arms of Man, the fore-legs of a Horse, the paddles of a Whale, the wings of a Bird, the front flippers of a Turtle, and the pectoral fins of a Fish; the proboscis of a Moth, and the jaws of a Beetle; the shell of a Snail, and both valves of a Clam. The wings of the Bird, Flying Squirrel, and Bat are hardly homologous, since the wing of the first is developed from the fore-limb only; that of the Squirrel is an extension of the skin between the fore and lind limbs; while in the Bat the skin stretches between the fingers, and then down the side to the tail. Examples of serial homology: the arms and legs of Man; the upper and lower set of teeth; the parts of the vertebral column, however modified; the scapular and pelvic arches; the humerus and femur; carpus and tarsus; the right and left sides of most Animals; the dorsal and anal fins of Fishes. The legs of a Lobster and Lizard, the wings of a Butterfly and Bird, the gills of a Fish, and the lungs of other Vertebrates, aie analogous. The air-bladder of a Fish is homologous with a lung, and analogons to the air-chambers of the Nantilus.

In the midst of the great variety of form and structure in the animal world, a certain harmony reigns. Not only are different species so related as to suggest a descent from the same ancestor, but the parts of any one organism are so closely connected and mutually dependent that the character of one must receive its stamp from the character of all the rest. Thus, from a single tooth it may be inferred that the animal had a skeleton and spinal cord, 
and that it was a carnivorous, hot-blooded Mammal. Certain structures always co-exist. Animals with two occipital condyles, and non - nucleated blood - corpuscles, suckle

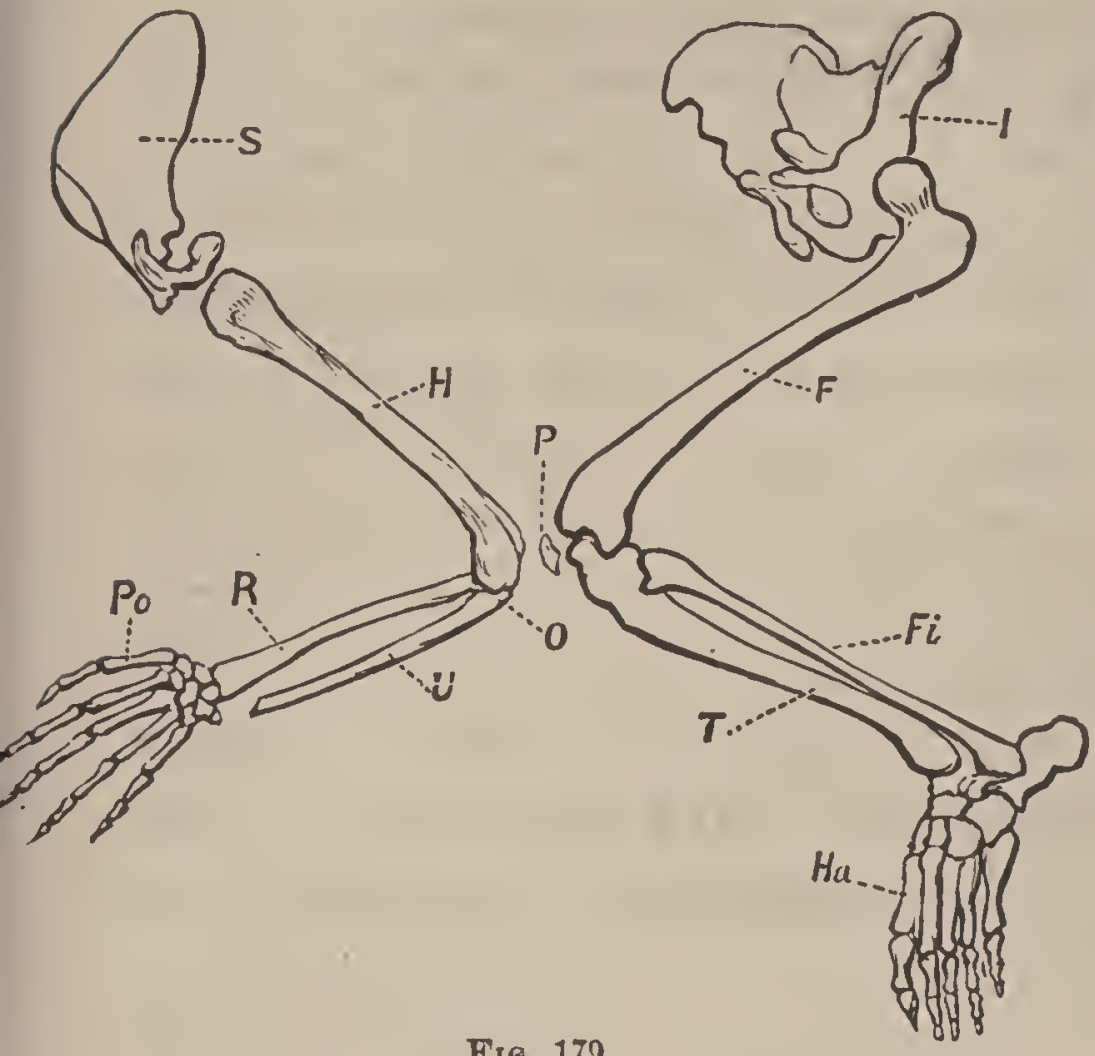

FiG. 179.

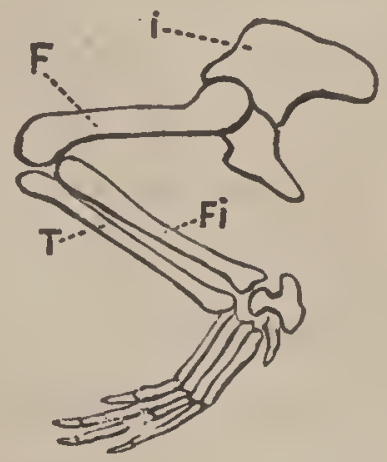

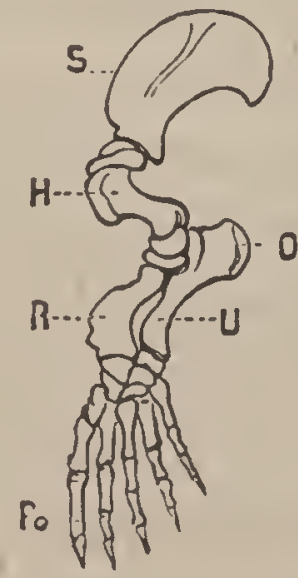

Fig. 181

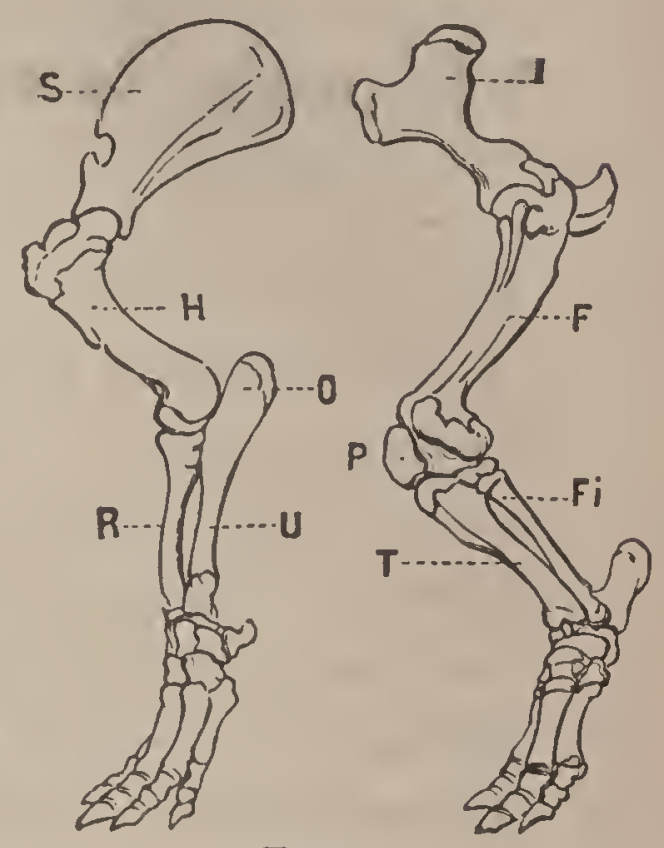

Fig. 18 n.

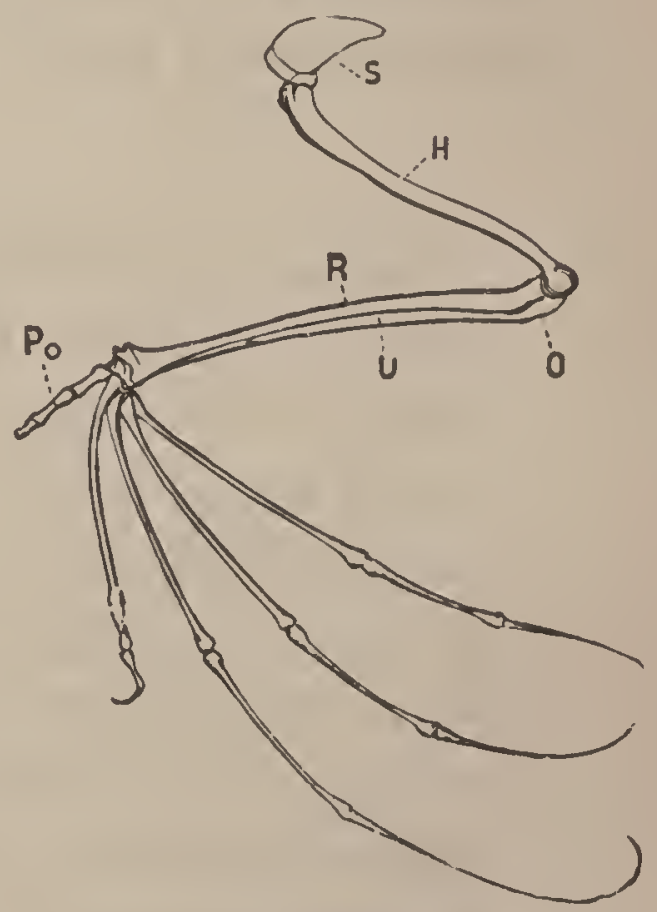

FIG. 1 S2.

HOMOLOGIES OF LIMBS.

Fra. 179.-Arm and Leg of Man, as they are when he gets down on all-fours. Fra. 150.-Fore and Hind Legs of Tapir. Fra. 181.-Fore Leg of Seal and Hind Leg of Alligator. Frg. 182.-Wing of the Bat. S, scapula; I, ilium, or shin-bone of pelvis; $\mathrm{H}$, humerus; $\mathrm{F}$, femur; $\mathrm{O}$, olecranou, or tip of the elbow; $\mathrm{P}$, patella; U, ulna; T, tibia; $R$, radius; Fi, Fibulu; Po, pollex, or thumb; Ha, hallux, or great toe. Compare the fore and hind limbs of the same animal, and the fore or hind limbs of different animals. Nute the directions of the homologous seg. ments. 
their young, $i$. e., they are Mammals. All Ruminant hoofed beasts have horns and cloven-feet. If the hoofs are even, the horns are even, as in the Ox; if odd, as in the Rhinoceros, the horns are odd, $i$. e., single, or two placed one behind the other. Recent creatures with feathers always have beaks. Pigeons with short beaks have small feet; and those with long beaks, large feet. The long limbs of the Hound are associated with a long head. A white spot in the forehead of a Horse generally goes with white feet. Hairless Dogs are deficient in teeth. Long wings usually accompany long tail-feathers. White Cats with blue eyes are usually deaf. A Sheep with numerous horns is likely to have long, coarse wool. Homologous parts tend to vary in the same manner; if one is diseased, another is more likely to sympathize with it than one not homologous. This association of parts is called correlation of growth.

\section{Individuality.}

It seems at first sight very easy to define an individual animal. A single Fish, or Cow, or Snail, or Lobster is plainly an individual; and the half of one such animal is plainly not one. But when we consider animals in colonies, like Corals, it is not so easy to say whether the individual is the colony or the single Polyp. Is the tree the individual, or the bud? If we say the former-the colony - what shall we say to the free buds of a Hydroid colony, living independent lives, and scattered over square miles of ocean? Are they parts of one individual? If we choose the latter as our standard, we are in equal difficnlty; for we must then call an individual the bud of the Portugnese man - of-war, reduced to a mere bladder or feeler, and incapable of leading an independent life. We thus find it necessary to distinguish at least two kinds of individuals - physiological individuals, applying that 
name to any animal form capable of leading an independent life; and morphological individuals, one of which is the total product of an egg. Such an individual may be a single physiological individual, as the Fish; or many united, as the Coral stock; or many separate physiological individuals, as in the Hydroids or Plant-lice. The single members of such a compound morphological individual are called zoöids, or personce, and are found wherever asexual reproduction takes place.

7. Relations of Number, Size, Form, and Rank.

The Animal Kingdom has been likened to a pyramid, the species diminishing in number as they ascend in the scale of complexity. This is not strictly true. The number of living species known is at least 300,000, of which more than nine tenths are Invertebrates. A late enumeration gives the following figures for the number of described species :

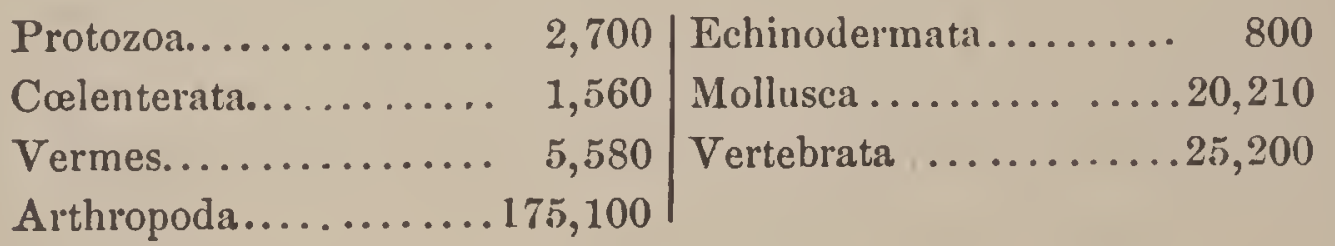

These figures are lower than those usually given. Of Vertebrates, Fishes are most abundant; then follow Birds, Mammals, Reptiles, and Amphibians. There are usually said to be about 200,000 species of Insects.

The largest species usually belong to the higher classes. The aquatic members of a group are generally larger than the terrestrial, the marine than the fresh-water, and the land than the aerial. The extremes of size are an Infusorium, $\frac{1}{16000}$ of an inch in diameter, the smallest animal ever measured, and the Whale, one hundred feet long, the largest animal ever created. The female is sometimes larger than the male, as of the Nautilus, Spider, and Eagle. The higher the class, the more uniform the size. Of all 
groups of animals, Insects and Birds are the most constant in their dimensions.

Every organism has its own special law of growth: a Fish and an Oyster, though born in the same locality, develop into very different forms. Yet a symmetry of plan underlies the structure of all aninals. In the embryo, this symmetry of the two ends, as well as the two sides, is nearly perfect; but it is subsequently interfered with to adapt the animal to its special conditions of life. It is a law that an animal grows equally in those directions in which the incident forces are equal. The Polyp, rooted to the rocks, is subjected to like conditions on all sides, and, therefore, it has no right and left, or fore and hind parts. The lower forms, generally, are more or less geometrical figures: spheroidal, as the Sea-urchin; radiate, as the Star-fish; and spiral, as many Foraminifers. The higher animals are subjected to a greater variety of conditions. Thus, a Fish, always going throngh the water head foremost, must show considerable difference between the head and the hinder end; or a Turtle, moving over the ground with the same surface always down, must have distinct dorsal and ventral sides.

Nevertheless, there is a striking likeness between the two halves or any two organs situated on opposite sides of an axis. And, first, a bilateral symmetry is most common. It is best exhibited by the Articulates and Vertebrates, but nearly all animals can be clearly divided into right and left sides - in other words, they appear to be double. A vertical plane would divide into two equal parts our brain, spinal cord, vertebral column, organs of sight, hearing, and smell; our teeth, jaws, limbs, lungs, etc. In fact, the two halves of every egg are identical. There are many exceptions: the heart and liver of the higher Vertebrates are eccentric; the nervous system of Mollusks is scattered; the hemispheres of the human 
brain are sometimes unequal; the corresponding bones in the right and left arms are not precisely the same length and weight; the Narwhal has an immense tusk on the left side, with none to speak of on the other; the Rattlesnake has but one lung, the second remaining in a rudimentary condition; both eyes of the adult Flounder and Halibut are on the same side; the claws of the Lobster differ; and the valves of the Oyster are unequal. But all these animals and their organs are perfectly symmetrical in the embryo state.

Again, animals exhibit a certain correspondence between the fore and hind parts. ${ }^{121}$ Thus, the two ends of the Centipede repeat each other. Indeed, in some Worms, the eyes are developed in the last segment as well as the first. So a Vertebrate may, theoretically perhaps, be compared to two individuals placed side by side. In the embryo of Quadrupeds, the four limbs are closely alike. But in the adult, the fore and hind limbs differ more than the right and left limbs, because the functions are more dissimilar. An extreme want of symmetry is seen in Birds which combine aerial and land locomotion.

There is also a tendency to a vertical symmetry, or up-and-down arrangement-the part above a horizontal plane being a reversed copy of the part below. A good example is the posterior half of a Cod, while the tail of a Shark shows the want of it. This symmetry decreases as we ascend the scale. In most animals there is considerable difference between the dorsal and ventral surfaces; and in all the nervous system is more symmetrically disposed than the digestive.

Every animal is perfect in its kind and in its place. Yet we recognize a gradation of life. Some animals are manifestly superior to some others. But it is not so easy to say precisely what shall guide us in assorting living 
forms into high and low. Shall we make structure the criterion of rank? Plainly the simple Jelly-fish is beneath complicated Man. An ounce of muscle is worth a pound of protoplasm, and a grain of nervous matter is of more account than a ton of flesh. The intricate and finished build of the Horse elevates him immeasurably above the stupid Snail. The repetition of similar parts, as in the Worm, is a sign of low life. So also a prolonged posterior is a mark of inferiority, as the Lobsters are lower than the Crabs, Snakes than Lizards, Monkeys than Apes. The possession of a head distinct from the region behind it is a sign of power. And in proportion as the fore-limbs are used independently of the hind limbs, the animal ascends the scale: compare the Whale, Horse, Cat, Monkey, and Man.

But shall the Fish, never rising above the "monotony of its daily swim," be allowed to outrank the skilful Bee? Shall the brainless, sightless, almost heartless Amphioxus, a Vertebrate, be allowed to stand nearer to Man than the Ant? What is the possession of a backbone to intelligence? No good reason can be given why we might not be just as intelligent beings if we carried, like the Insect, our hearts in our backs and our spinal cords in our breasts. So far as its activity is concerned, the brain may be as effective if spread out like a map as packed into its present shape. Even animals of the same type, as Vertebrates, cannot be ranked according to complexity. For while Mammals, on the whole, are superior to Birds, Birds to Reptiles, and Reptiles to Fishes, they are not so in every respect. Man himself is not altogether at the head of creation. We carry about in our bodies embryonic structures. That structural affinity and vital dignity are not always parallel may be seen by comparing an Australian and an Englishınan. ${ }^{122}$ 
Function is the test of worth. Not mere work, however; for we must consider its quality and scope. An animal may be said to be more perfect in proportion as its relations to the external world are more varied, precise, and fitting. Complexity of organization, variety, and amount of power are secondary to the degree in which the whole organism is adapted to the circumstances which surround it, and to the work which it has to do. Ascent in the animal scale is not a passage from animals with simple organs to animals with complex organs, but from simple individuals with organs of complex function to complex individuals with organs of simple function: the addition as we ascend being not function, but parts to discharge those functions; and the advantage gained, not another thing done, but the same thing done better. Advance in rank is exhibited, not by the possession of more life (for some animalcules are ten times more lively than the busiest Man), but by the setting apart of more organs for special purposes. The higher the animal, the greater the number of parts combining to perform each function. The power is increased by this division of labor. The most important feature in this specialization is the tendency to concentrate the nervous energy towards the head (cephalization). It increases as we pass from lower to higher animals.

As a rule, fixed species are inferior to the free, water species to land species, fresh-water animals to marine, arctic forms to tropical, and the herbivorous to the carnivorous. Precocity is a sign of inferiority: compare the chicks of the Hen and the Robin, a Colt with a Kitten, the comparatively well-developed Caterpillar with the footless grub of the Bee. Among Invertebrates, the male is frequently inferior, not only in size, but also in grade of organization. Animals having a wide range as to cli- 
mate, altitude, or depth are commonly inferior to those more restricted: Man is a notable exception.

There is some relation between the duration of life and the size, structure, and rank of animals. Vertebrates not only grow to a greater size, but also live longer than Invertebrates. Whales and Elephants are the longest-lived; and Falcons, Ravens, Parrots and Geese, Alligators and Turtles, and Sharks and Pikes, are said to live a century. The life of Quadrupeds generally reaches its limit when the molar teeth are worn down: those of the Sheep last about 15 years; of the Ox, 20; of the Horse, 40 ; of the Elephant, 100. Many inferior species die as soon as they have'laid their eggs, just as herbs perish as soon as they have flowered.

\section{The Struggle for Life.}

Every species of animal is striving to increase in a geometrical ratio. But each lives, if at all, by a struggle at some period of its life. The meekest creatures must fight, or die.

"There is no exception to the rule that every organic being naturally increases at so high a rate that, if not destroyed, the earth would soon be covered by the progeny of a single pair." If the increase of the human race were not checked, there would not be standing-room for the descendants of Adam and Eve. A pair of Elephants, the slowest breeder of all known animals, would become the progenitors, in seven and one half centuries, of $19,000,000$ of Elephants, if death did not interfere. Evidently a vast number of young animals must perish while immature, and a far greater host of eggs fail to mature. A single Cod, laying millions of eggs, if allowed to have its own way, would soon pack the ocean.

Yet, so nicely balanced are the forces of nature, the average number of each kind remains about the same. 
The total extinction of any one species is exceedingly rare. The number of any given species is not determined by the number of eggs produced, but by its surrounding conditions. ${ }^{123}$ Aquatic birds outnumber the land birds, becanse their food never fails, not because they are more prolific. The Fulmar-petrel lays but one egg, yet it is believed to be the most numerous bird in the world.

The main checks to the high rate of increase are: climate (temperature and moisture), acting directly or indirectly by reducing food; and other animals, either rivals requiring the same food and locality, or enemies, for the vast majority of animals are carnivorous. Offspring are continually varying from their parents, for better or worse. If feebly adapted to the conditions of existence, they will finally go to the wall. But those forms having the slightest advantage over others inhabiting the same region, being hardier or stronger, more agile or sagacious, will survive. Should this advantageous variation become hereditary and intensified, the new variety will gradually extirpate or replace other kinds. This is what Mr. Darwin means by Natural Selection, and Herbert Spencer by the Survival of the Fittest. 



\section{PART II.}

\section{SYSTEMATIC ZOOLOGY.}


Facts are stupid things until brought into connection with some general law.-Agassiz.

No man becomes a proficient in any science who does not transcend system, and gather up new truth for himself in the boundless field of research. -Dr. A. P. Peabody.

Never ask a question if you can help it; and never iet a thing go unknown for the lack of asking a question if you can't help it.-BEECHER.

He is a thoroughly good naturalist who knows his own parish thoroughly. -Charles Kingsley. 


\section{CHAPTER XXI.*}

\section{THE CLASSIFICATION OF ANIMALS.}

The Kingdom of Nature is a literal Kingdom. Order and beauty, law and dependence, are seen everywhere. Amidst the great diversity of the forms of life, there is unity; and this suggests that there is one general plan, but carried out in a variety of ways.

Naturalists have ceased to believe that each animal or group is a distinct, circumscribed idea. "Every animal has a something in common with all its fellows: much with many of them; more with a few; and, usually, so much with several, that it differs but little from them." The object of classification is to bring together the like, and to separate the unlike. But how shall this be done? To arrange a library in alphabetical order, or according to size, binding, date, or language, would be unsatisfactory. We must be guided by some internal character. We must decide whether a book is poetry or prose; if poetry, whether dramatic, epic, lyric, or satiric; if prose, whether history, philosophy, theology, philology, science, fiction, or essay. The more we subdivide these groups, the more difficult the analysis.

A classification of animals, founded on external resemblances - as size, color, or adaptation to similar habits of life-would be worthless. It would bring together Fishes and Whales, Birds and Bats, Worms and Eels. Nor should it be based on any one character, as the quality of the blood, structure of the heart, development of the brain, embryo-life, etc.; for no character is of equal value in every tribe. A natural classification must rest on those

* See Appendix. 
prevailing characters which are the most constant. ${ }^{124}$ And such a classification cannot be linear. It is impossible to arrange all animal forms from the Sponge to Man in a single line, like the steps of a ladder, according to rank. Nature passes in so many ways from one type to another, and so multiplied are the relations between animals, that one series is out of the question. There is a number of series, and series within series, sometimes proceeding in parallel lines, but more often divergent. The animals arrange themselves in radiating groups, each group being connected, not with two groups merely, one above and the other below, but with several. Life has been likened to a great tree with countless branches spreading widely from a common trunk, and deriving their origin from a common root; branches bearing all manner of flowers, every fashion of leaves, and all kinds of fruit, and these for every use.

The groups into which we are able to cast the various forms of animal development are very unequal and dissimilar. We must remember that a genus, order, or class is not of equal value throughout the kingdom. Moreover, each division is allied to others in different degrees-the distance between any two being the measure of that affinity. The lines between some are sharp and clear, between others indefinite. Like the islands of an archipelago, some groups merge into one another through connecting reefs, others are sharply separated by unfathomable seas, yet all have one common basis. Links have been found revealing a relationship, near or distant, even between animals whose forms are very unlike. There are Fishes (Dipnor) with some Amphibian characters, and fish-like Amphibians (Axolotl). The extinct Ichthyosaurus was a Lizard with fish-characteristics. Birds seem isolated, but they are closely connected with Reptiles by fossil forms. Even the great gap in the Animal Kingdom-that separating Vertebrates 
and Invertebrates-is partially bridged on the one side by Amphioxus, and on the other by Balanoglossus 'a wormlike animal) and the Tunicates.

We have, then, groups subordinate to groups, and interlocking, but not representing so many successive degrees of organization. For, as already intimated, complication of structure does not rise in continuous gradation from one group to another. Every type starts at a lower point than that at which the preceding class closes; so that the lines overlap. While one class, as a whole, is higher than another, some members of the higher class may be inferior to some members of the lower one. Thus, certain Star-fishes are nobler than certain Mollusks; the Nautilus is above the Worm, and the Bee is more worthy than the lowest Fish. The groups coalesce by their inferior or less specialized members; e.g., the Fishes do not graduate into Amphibians through their highest forms, but the two come closest together low down in the scale. Man appears to be the goal of creation; but even within the Vertebrate series, every step of development, say of the Fish, is away from the goal. The highest Fish is the one farthest from Man.

A number of animals may, therefore, have the same grade of development, but conform to entirely different types. While a fundamental unity underlies the whole Animal Kingdom, suggesting a common starting-point, we recognize several distinct plans of structure. ${ }^{125}$ Animals like the Amœba, with no cellular tissues nor true eggs, form the subkingdom Protozoa. Animals like the Sponge, with independent cells, one excurrent and many incurrent openings, form the subkingdom Porifera. Animals like the Coral, unlike all others, have an alimentary canal but no body - cavity, have no separate nervous and vascular regions, and the parts of the body radiate from a centre. Such form a sublingdom called Coelenterata. Animals like the Star-fish, having also a radiating body, but a closed 
alimentary canal, and a distinct symmetrical nervous sys. tem, constitute the subkingdom Echinodermata. ${ }^{126}$ Animals like the Angle-worm, bilaterally symmetrical, onejointed, or composed of joints following each other from front to rear, with no jointed limbs, constitute the subkingdom Vermes. Animals like the Snail, with a soft, unjointed body, a mantle, a foot, a two or three chambered heart, and a nervous system in the form of a ring around the gullet, constitute the subkingdom Mollusca. Animals like the Bee, with a jointed body and jointed limbs, form the subkingdom Arthropoda. Animals like the Sea-squirts, sack or barrel shaped, with a mantle cavity penetrated by an excurrent and an incurrent opening, with heart and gills, form the subkingdom Tunicata. Animals like the Ox, having a donble nervous system, one (the sympathetic) lying on the upper side of the alimentary canal, the other and main part (spinal) lying along the back, and completely shut off from the other organs by a partition of bone or gristle, known as the "vertebral column," and having limbs, never more than four, always on the side opposite the great nervous cord, constitute the subkingdom Vertebrata.

Comparing these great divisions, we see that the Vertebrates differ from all the others chiefly in having a donble body-cavity and a double nervous system, the latter lying above the alimentary canal; while Invertebrates have one cavity and one nervous system, the latter being placed either below or around the alimentary canal. The Vermes are closely related to all the following subkingdoms of Invertebrates, most nearly to Mollusks and Tunicates, while the latter have affinities with the Vertebrates. The Echinoderms and Colenterates are built on the common type of a star; but they differ from each other in the presence or absence of distinct alimentary, circulatory, and nervous systems. 
But there are types within types. Thus, there are five modifications of the Vertebrate type-Fish, Amphibian, Reptile, Bird, and Mammal; and these are again divided and subdivided, for Mammals, e. g., differ among themselves. So that in the end we have a constellation of groups within groups, founded on peculiar characters of less and less importance, as we descend from the general to the special.

Individuals are the units of the Animal Creation. An animal existence, complete in all its parts, is an individual, whether separate, as Man, or living in a community, as the Coral. ${ }^{127}$

Species is the smallest group of individuals which can be defined by distinct characteristics, and which is separated by a gap from all other like groups. A well-marked subdivision of a species is called a variety. Crosses between species are called hybrids, as the Mule.

Genus is a group of species having the same essential structure. Thus, the closely allied species Cat, Tiger, and Lion belong to one genus.

Family, or Tribe, is a group of genera having a similar form. Thus, the Dogs and Foxes belong to different genera, but betray a family likeness.

Order is a group of families, or genera, related to one another by a common structure. Cats, Dogs, Hyenas, and Bears are linked together by inportant anatomical features; their teeth, stomachs, and claws show carnivorous habits.

Class is a still larger group, comprising all animals which agree simply in a special modification of the type to which they belong. Thus, Fishes, Amphibians, Reptiles, Birds, and Mammals are so many aspects of the Vertebrate type.

Subkingdom is a primary division of the Animal Kingdom, which includes all animals formed upon one of the various types of structure; as Vertebrate. 
The subkingdoms are grouped into two great Series (Protozoa and Metazoa), according to their histological structure and mode of development. ${ }^{128}$

These terms were invented by Linnæus, except Family, Subkingdom, and Series. To Linnæus we are also indebted for a scientific method of naming animals. Thus, a Dog, in Zoology, is called Canis familiaris, which is the union of a generic and a specific name, corresponding to the surname and the Christian name in George Washington, only the specific name comes last. It will be understood that these are abstract terms, expressing simply the relations of resemblance: there is no such thing as genus or species.

Classification is a process of comparison. He is the best naturalist who most readily and correctly recognizes likeness founded on structural characters. As it is easier to detect differences than resemblances, it is much easier to distinguish the class to which an animal belongs than the genus, and the genus than the species. In passing from species to classes, the characters of agreement become fewer and fewer, while the distinctions are more and more manifest; so that animals of the same class are more like than unlike, while members of distinct classes are more unlike than like.

To illustrate the method of zoological analysis by searching for affinities and differences, we will take an example suggested by Professor Agassiz. Suppose we see together a Dog, a Cat, a Bear, a Horse, a Cow, and a Deer. The first feature which strikes us as common to any two of them is the horn in the Cow and the Deer. But how shall we associate either of the others with these? We examine the teeth, and find those of the Dog, the Cat, and the Bear sharp and cutting; while those of the Cow, the Deer, and the Horse have flat surfaces, adapted to grinding and chewing, rather than to cutting and tearing. We 
compare these features of their structure with the habits of these animals, and find that the first are carnivorousthat they seize and tear their prey; while the others are herbivorous, or grazing, animals, living only on vegetable substances, which they chew and grind. We compare, further, the Horse and Cow, and find that the Horse has front teeth both in the upper and the lower jaw, while the Cow has them only in the lower; and going still further, and comparing the internal with the external features, we find this arrangement of the teeth in direct relation to the different structure of the stomach in the two animals-the Cow having a stomach with four pouches, while the Horse has a simple stomach. Comparing the Cow and Deer, we find the digestive apparatus the same in both; but though both have horns, those of the Cow are hollow, and last through life; while those of the Deer are solid, and are shed every year. Looking at the feet, we see that the herbivorous animals are hoofed; the carnivorous, clawed. The Cow and Deer have cloven feet, and are ruminants ; the Horse has a single hoof, and does not chew the cud. The Dog and Cat walk on the tips of their fingers and toes (digitigrade); the Bear treads on the palms and soles (plantigrade). The claws of the Cat are retractile; those of the Dog and Bear are fixed.

In this way we determine the exact place of each animal. The Dog belongs to the kingdom Animalia, subkingdom Vertebrata, class Mammalia, order Carnivora, family Canido, genus Canis, species Familiaris, variety Hound (it may be), and its individual name; perhaps, is "Rover." The Cat differs in belonging to the family Felidoe, genus Felis, species Catus. The Bear belongs to the family Ursidce, genus Ursus, and species F'erox, if the Grizzly is meant. The Horse, Cow, and Deer belong to the order Ungulata; but the Horse is of the family Equida, genus Equus, species Caballus; the Cow is of 
the family Bovidoe, genus Bos, species Taurus; the Deer is of the family Cervidce, genus Cervus, species Virginianus, if the common Deer is meant.

The diagram on the opposite page roughly represents (for the relations of animals cannot be expressed on a plane surface) the relative positions of the subkingdoms and classes according to affinity and rank.*

\section{SERIES I.-PROTOZOA.}

Animals without cellular tissues (the body consisting of a single cell), and with no true eggs. The body which corresponds to the egg does not develop a blastoderm.

\section{Subkingdom I.-ProtozoA.}

This division was proposed by Von Siebold in 1845, to contain that vast cloud of microscopic beings on the verge of the Animal Kingdom which could not be received into the other subkingdoms. Though the division was at first artificial and provisional, the name now has a very definite signification. The classes composing it are not founded on a common type, but are distinguished by the absence rather than the presence of positive characters. Many stand parallel to the Protophyta of the Vegetable World, and no definite line can be drawn between them.

Protozoans agree in being minute, aquatic, and exceedingly simple in structure, their bodies consisting mainly or wholly of the contractile, gelatinous matter called protoplasm, or sarcode - the first homogeneous substance which has the power of controlling chemical and physical forces. They have no cellular organs or tissnes, yet they take and assimilate food, grow, and multiply, which are

* The student should master the distinctions between the great groups, or classes, before proceeding to a minuter classification. "The essential matter, in the first place," says Huxley, "is to be quite clear about the different classes, and to have a distinct knowledge of all the sharply definable modifications of animal structure which are discernible in the Animal Kingdom." 
THE CLASSIFICATION OF ANIMALS.

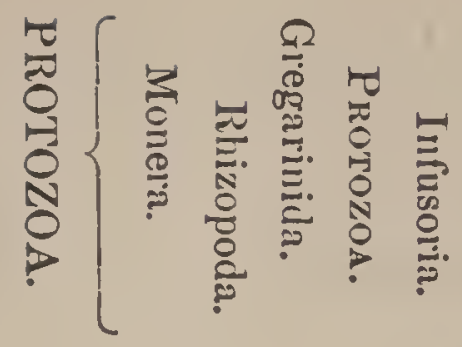
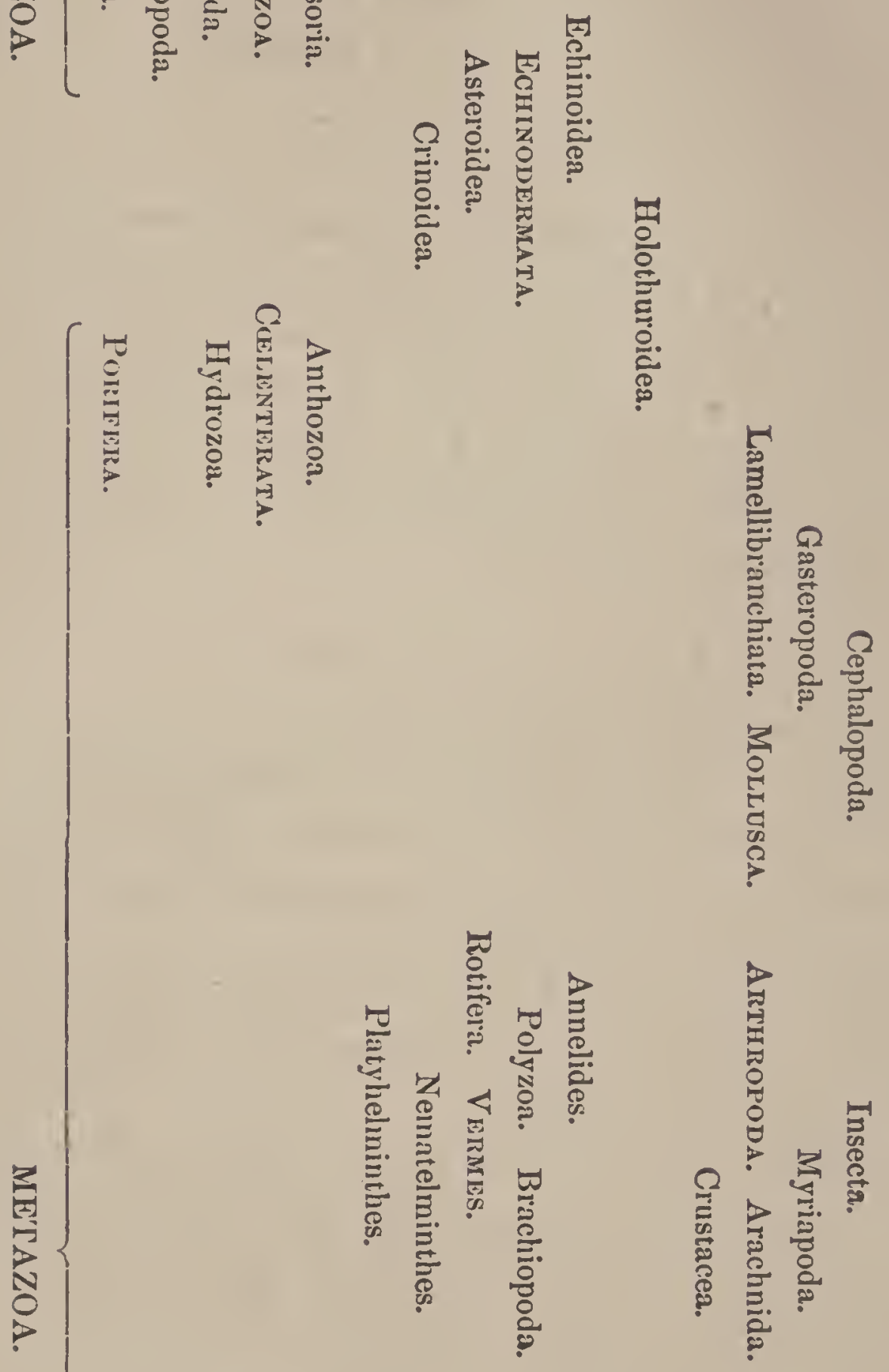

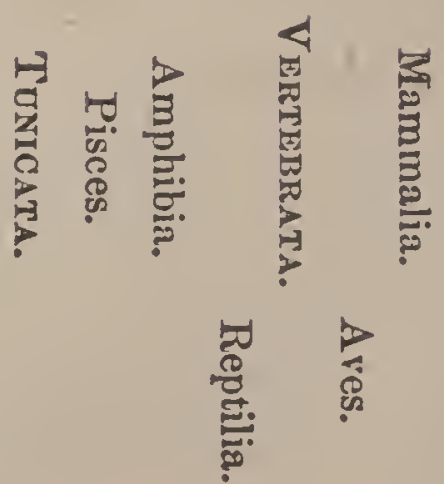


the essential signs of life. The usual methods of reproduction are self-division and budding.

The subkingdom may be divided into four classes: Monera, Rhizopoda, Gregarinida, and Infusoria.

\section{Class I.-Monera.}

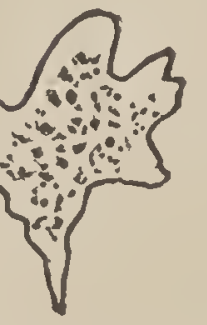

Frg. 183.-Pro. tamoeba primitiva.

These simplest living beings are organless bits of protoplasma, with no distinction of layers, and so far as observed not even a nucleus is present. They are round when at rest, and have psendopodia when active. They are all aquatic, and some are parasitic. Such is Protamœba, Fig. 183.

\section{Class II.-Rhizopoda.}

The Rhizopods are characterized by the power of throwing out at will delicate processes of their bodies, called pseudopodia, or false feet, for prehension or locomotion. They possess no cilia. The representative forms are $A$ moboe, Foraminifera, and Radiolaria.

An Amœba is a naked fresh-water Rhizopod; an indefinite bit of protoplasm, as structureless as a speck of jelly, save that it is made of two rather distinct layers, and has a nucleus and a contractile cavity inside. It thus differs from the Monera. It has no particular form, as it changes continually. It moves by putting forth short, blunt processes, and eats by wrapping

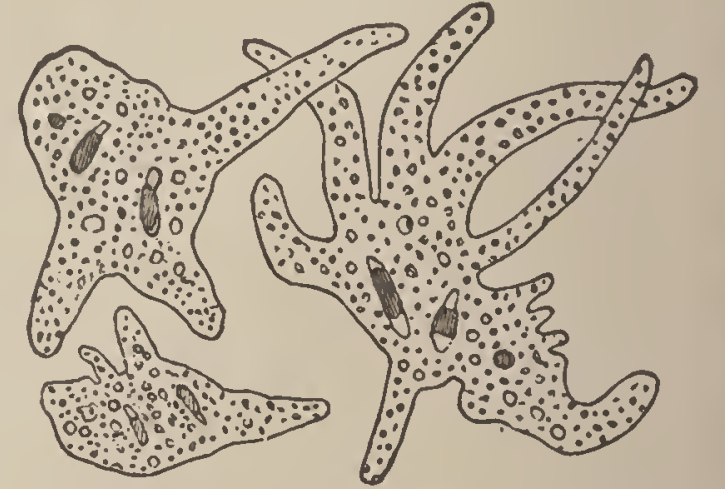

Fig. 134. - A moba princeps, $\times 150$; the same animal in various shapes. its body around the particle of food. The size ranges from $\frac{1}{30}$ to $\frac{1}{2800}$ of an inch in diameter. Specimens can be obtained by scraping the slimy matter from the stems and leaves in stagnant ponds. 
A Foraminifer differs from an Amœba in having an apparently simpler body, the protoplasm being without layers or cavity; its pseudopodia are long and thread-like, and may unite where they tunch each other. It has the property of secreting an envelope, usually of carbonate of
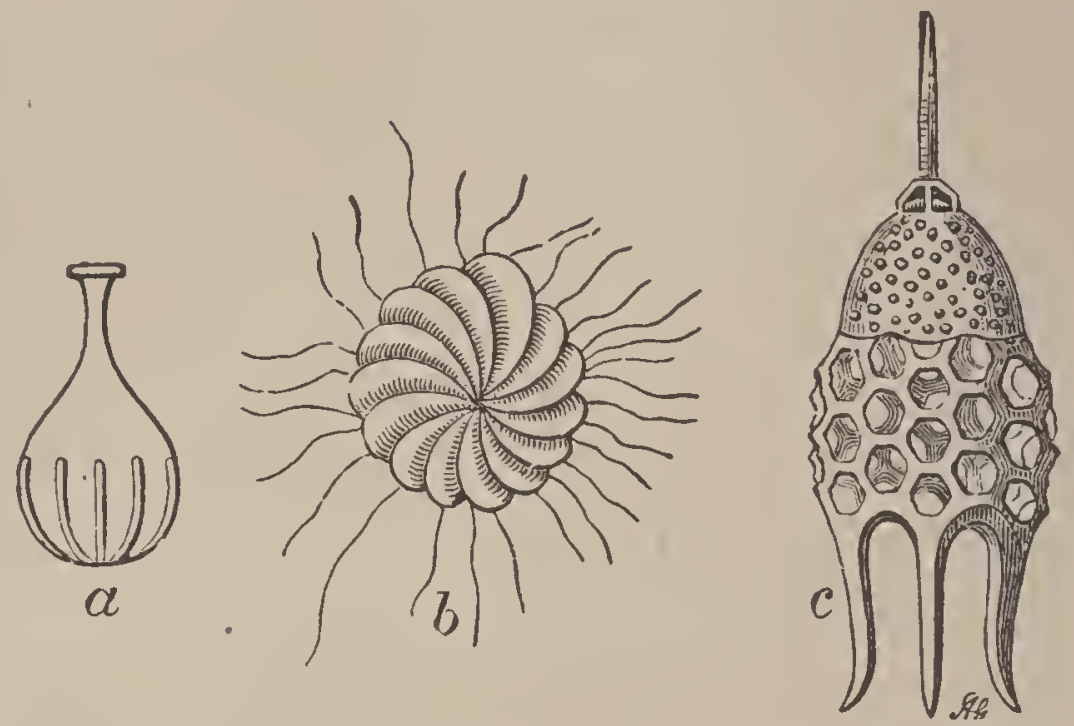

Frg. 185.-Rhizopods: $a$, shell of a monothalamous, or single-chambered, Foraminifer (Lagena striata); $b$, shell of a polythalamous, or many-chambered, Foraminifer (Polystomella crispa), with pseudopodia extended; $c$, shell of a Radiolarian, one of the Polycystives (Podocyrtis Schomburgkii).

lime. The shell thus formed is sometimes of extraordinary complexity and singular beauty. It is generally perforated by innumerable minute orifices (foramina) through which the animal protrudes its myriad of glairy, threadlike arms. The majority are compound, resembling chambered cells, formed by a process of budding, the new cells being added so as to make a straight series, a spiral, or a flat coil. As a rule, the many - chambered species have calcareous, perforated shells; and the one-chambered have an imperforated membranous, porcelaneous, or arenaceous envelope. The former are marine. There are few parts of the ocean where these microscopic shells do not occur, and in astounding numbers. A single ounce of sand from the Antilles was calculated to contain over three millions. The bottom of the ocean, up to about $50^{\circ}$ on each side of the Equator, and at depths not greater than 2400 fathoms, is covered with the skeletons of these ani- 
mals, which are constantly falling upon it (Globigerinaooze). Their remains constitute a great proportion of the so-called sand-banks which block up many harbors. Yet they are the descendants of an ancestry still more prolific; for the Foraminifera are among the most important rockbuilding animals. The chalk-cliffs of England, the buildingstone of Paris, and the blocks in the Pyramids of Egypt are largely composed of extinct Foraminifers. Foraminifera are both marine and fresh-water, chiefly marine.

A Radiolarian differs from a Foraminifer in secreting a siliceous, instead of a calcareous, shell, studded with radiating spines; and the central part of the body is made up of a colony of cells, and surrounded by a strong membrane. They are also more minute, but as widely diffused. They enter largely into the formation of some strata of the earth's crust, and abound especially in the rocks of Barbadoes and at Richmond, Va. The living forms are mostly marine, but some are fresh-water.

\section{Class III.-Gregarinida.}

The Gregarinæ, discovered by Dufour in 1828, are among the simplest animal forms of which we have any knowledge. The only organ is a nuclens, snspended in extremely mobile protoplasin which is covered by a cuticle; and the most conspicnous signs of life are the con-

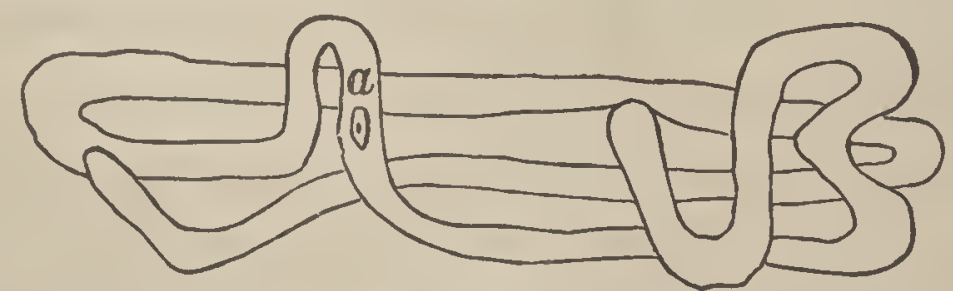

FIG. 186.-Gregarina gigantea, highly magnified: $a$, nucleus.

traction and lengthening of the worm-like body. They feed by absorption, and are all parasites, living in the alimentary canal of higher animals; as in the Cockroach, Earth-worm, and Lobster. The name is derived from the fact that they occur in large numbers crowded together. 


\section{Class IV.-Infusoria.}

This unassorted group of living organisms derived its naine from the fact that they were first discovered in vegetable infusions. Every drop of a stagluant pool is crowded with them. They are all single and microscopic, yet of various sizes, the difference between the smallest and largest being greater than the difference between a Mouse and an Elephant. Some are fixed

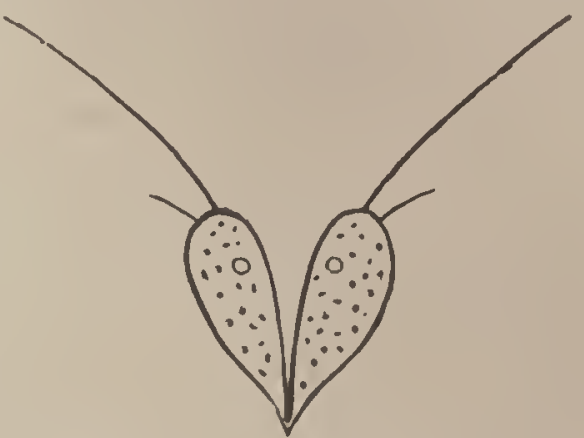

Fia. 187.-A Compound Monad (Uvella), $\times 1000$. (as Vorticella), but the majority are free, and constantly in motion, propelled by countless cilia, as a galley by its

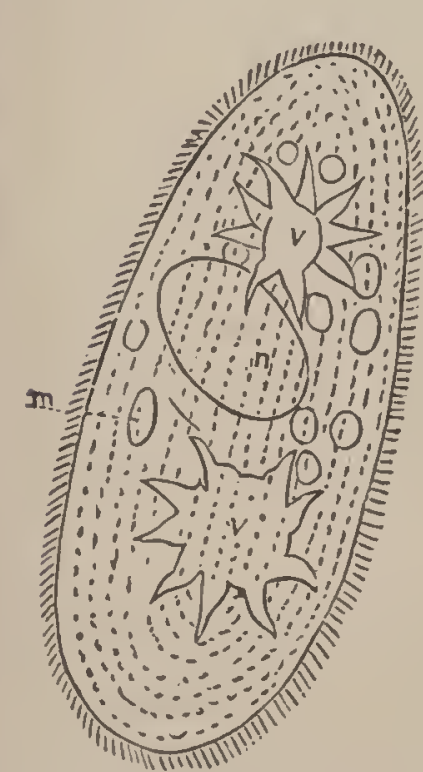

Fig. 18s, - Infusorium (Paramecium anerelia), $\times 300: m$, mouth; $v$, contractile vesicles; $n$, nucleus.

oars. The delicate body consists of two layers of sarcode (there are no cellular tissues, but the whole body is a single cell), covered by a membrane, or skin, having one or two contractile cavities, and a nucleus. Food-granules can often be seen. On one side is a slight depression, or "mouth," leading to a short, funnel-shaped throat. A mouth and a rudimentary digestive cavity are among the distinctive features of these Protozoans. Some have a pigment-speck - the simplest sense organ-and in the stem of Vorticella the first rudiments of muscle may be found. Thiey multiply so rapidly (chiefly by self-division), that a Paramecium, the most common form, may become the parent of 1,364,000 in forty-two days.

There are three main groups: Flagellata, or Monads, provided with one or two flagella, or long, bristle-like cilia; Tentaculifera, with several hollow tentacles; and Ciliata, which are furnished with numerous vibratile cilia. 


\section{SERIES II.-METAZOA.}

The Metazoa include all those animals which reproduce by true eggs and spermatozoa, whose germ develops a blastoderm, and which have cellular tissues. There are seven subkingdoms.

\section{Subkingdom II.-Porifera.}

The position of the Sponges has been much disputed. At first they were thought to be on the border-line between animals and plants, and were assigned by some to the animals and by others to the vegetables. Later, and up to very recent years, they were assigned to the Protozoa. The discovery of their mode of reproduction and development has determined that they belong to the Metazoa.

The Sponges are formed of an aggregate of membraneless amœboid or ciliated cells. They usually have a skeleton, which may be calcareous, horny, or siliceons. They have a central cavity, with numerous incurrent orifices and one excurrent opening. They reproduce by true eggs, as well as by budding and fission.

The cells of the Sponge are relatively independent, whence they have been regarded as colonies of amœboid animals, but by few naturalists are still so considered.

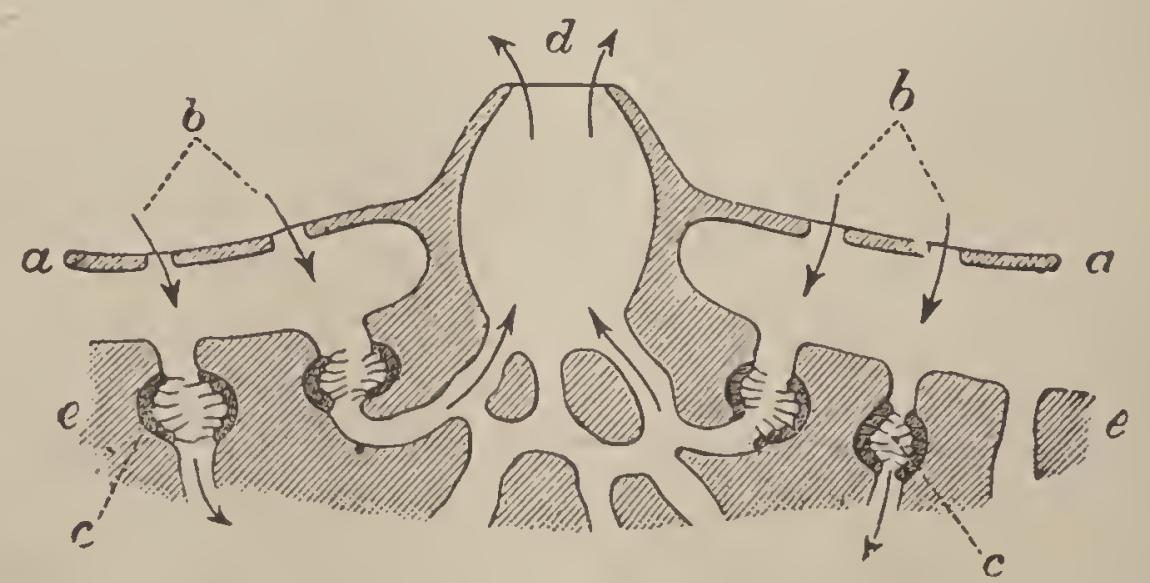

Fra. 159.-Hypothetical Section of a Sponge: $a$, superficial layer; $b$, inhalant pores: $c$, ciliated chambers; $d$, exhaliut aperture, or osculum; $e$, decper substance of the Sponge. 
They develop, however, regularly from the egg, and the cells acquire their independence only at a late date in development. Some of the cells bear cilia, or flagella, and drive the water through numerous channels into the central cavity, whence it is discharged by one opening. Each cell of the Sponge feeds itself from the particles contained in the water circulating through the channels.

The Sponge-individnal contains one exhalant orifice (osculum), with the channels leading into it. An ordinary

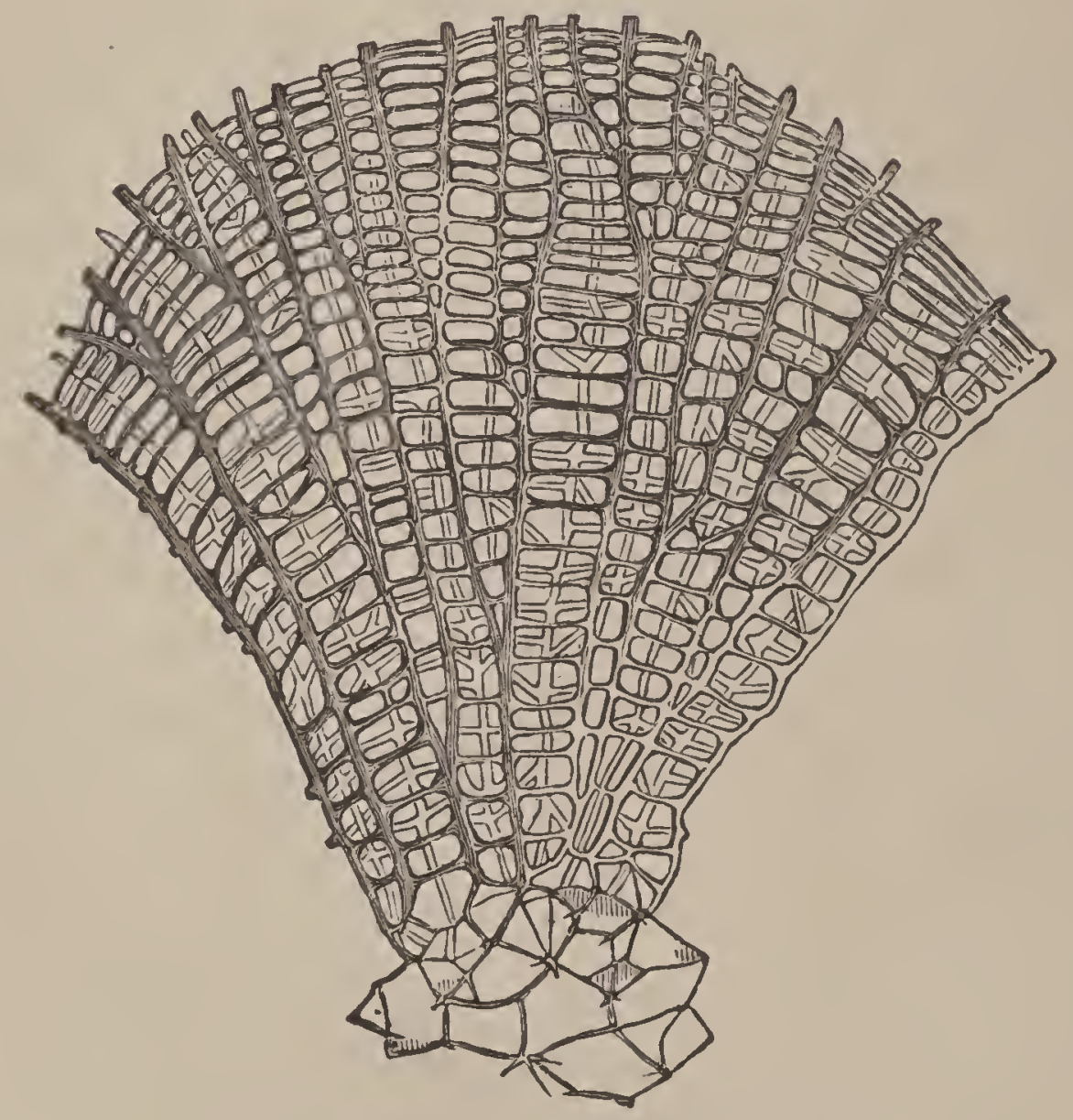

Fig. 190.-Skeleton of a Horny Sponge.

bathing-sponge constitutes a colony of such individuals, which are not definitely marked off from each other. Other Sponges have only one osculum, and such are a single individual.

Some few Sponges have no skeleton. Most have one of horny fibres, strengthened with siliceous spicules. These last are absent in the commercial Sponges, and in them the horny fibres are much tougher than in most Sponges. 
A few Sponges, as the Venus's Flower-basket (Euplectella), have siliceous and others have calcareous skeletons. Excepting a few small fresh-water species (as Spongilla), Sponges are marine. In the former, the cellular part is greenish, containing chlorophyll; in the latter, it is brown, red, or purple. In preparing the Sponge of commerce, this is rotted by exposure, and washed ont. The best fishing-grounds are the eastern end of the Mediterranean and around the Bahama Islands.

\section{Subkingdom III.-CøLenterata.}

These radiate animals are distinguished by having a body cavity, whose walls have, at least, two layers of cellular tissue, an outer (ectoderm) and inner (endoderm), and usually a middle layer (mesoderm), this cavity serving for both digestion and circulation. They have thread-cells, minute sacs containing a fluid, and connected with barbed filaments capable of being thrown ont for stinging purposes. Most are provided with hollow tentacles around the mouth. All are aquatic, and nearly all are marine. There are three classes, represented by the Hydra, Sea-anemone, and Ctenophores. All reproduce by eggs, and the first two also by budding.

\section{Class I.-Hydrozoa.}

These Cœlenterates have no separate digestive sac, so that the body is a simple tube, or cavity, into which the month opens. The nervous system is slightly developed. Such are the fresh-water Hydra and the oceanic Jelly-fish (Acaleph or Medusa).

The body of the Hydra is tubular, soft, and sensitive, of a greenish or brownish color, and seldom over half an inch long. It is found spontaneously attached by one end to submerged plants, while the free end contains the orifice, or month, crowned with tentacles, by which the creature feeds and creeps. The body-wall consists of two cellular layers-ectoderm and endoderm. These surround 
a central cavity with one opening. The animal may be compared to a bag with a two-layered wall, and tentacles around the opening. It buds; and also reproduces by eggs. The buds, when adult, become detached from the parent.

In most of the other Hydroids the colony is permanent, and supported by a horny skeleton. There are two kinds of Polyps in each colony, one for feeding and the other for reproduction.
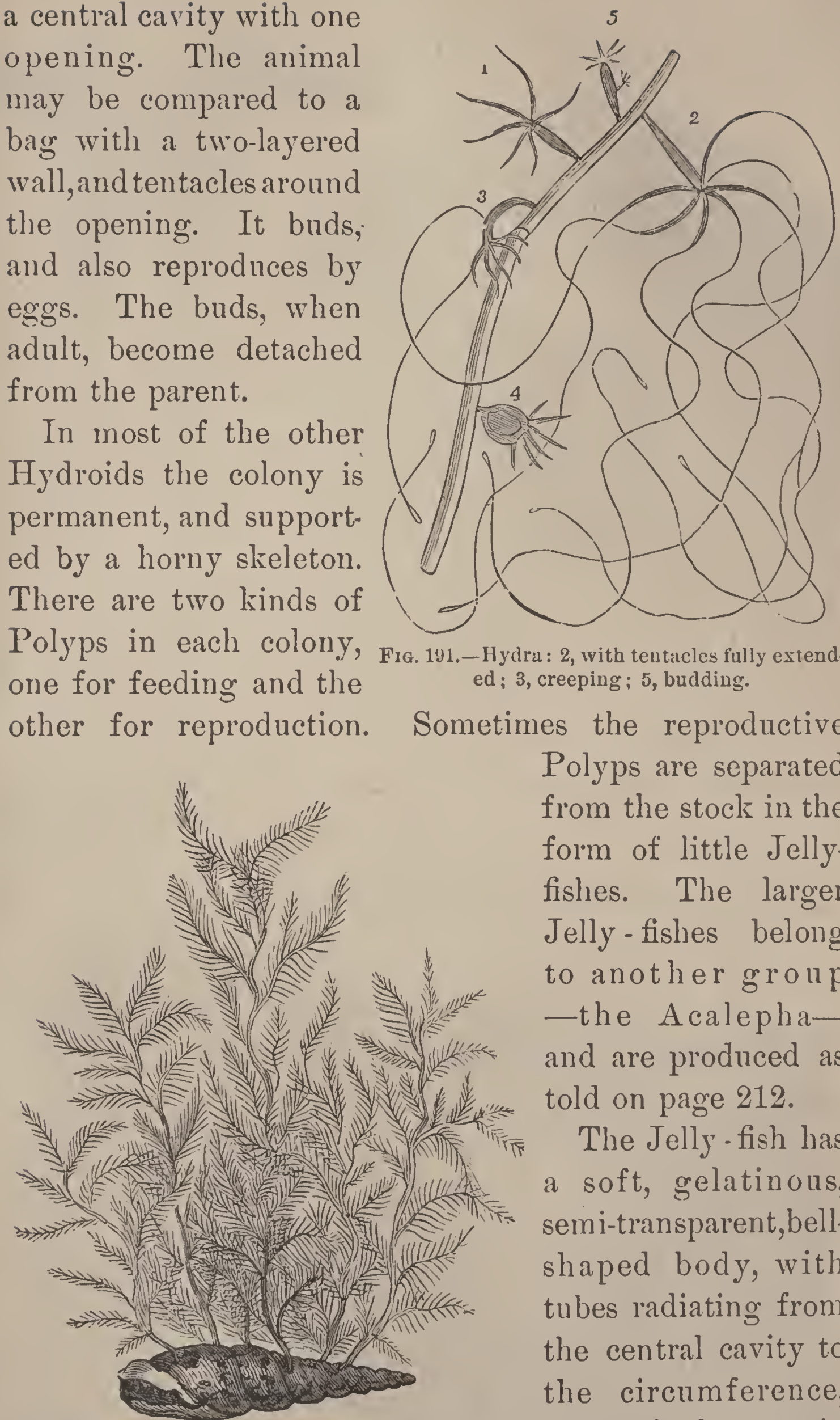

FIG. 191.-Hydra: 2, with teutacles fully extended; 3 , creeping ; 5 , budding.

Sometimes the reproductive Polyps are separated from the stock in the form of little Jellyfishes. The larger Jelly - fishes belong to another group -the Acalephaand are produced as told on page 212.

The Jelly - fish has a soft, gelatinous, semi-transparent,bellshaped body, with tubes radiating from the central cavity to the circumference, Fig. 192,-Hydruid (Sertularia) growing on a Shell. and with the margin 


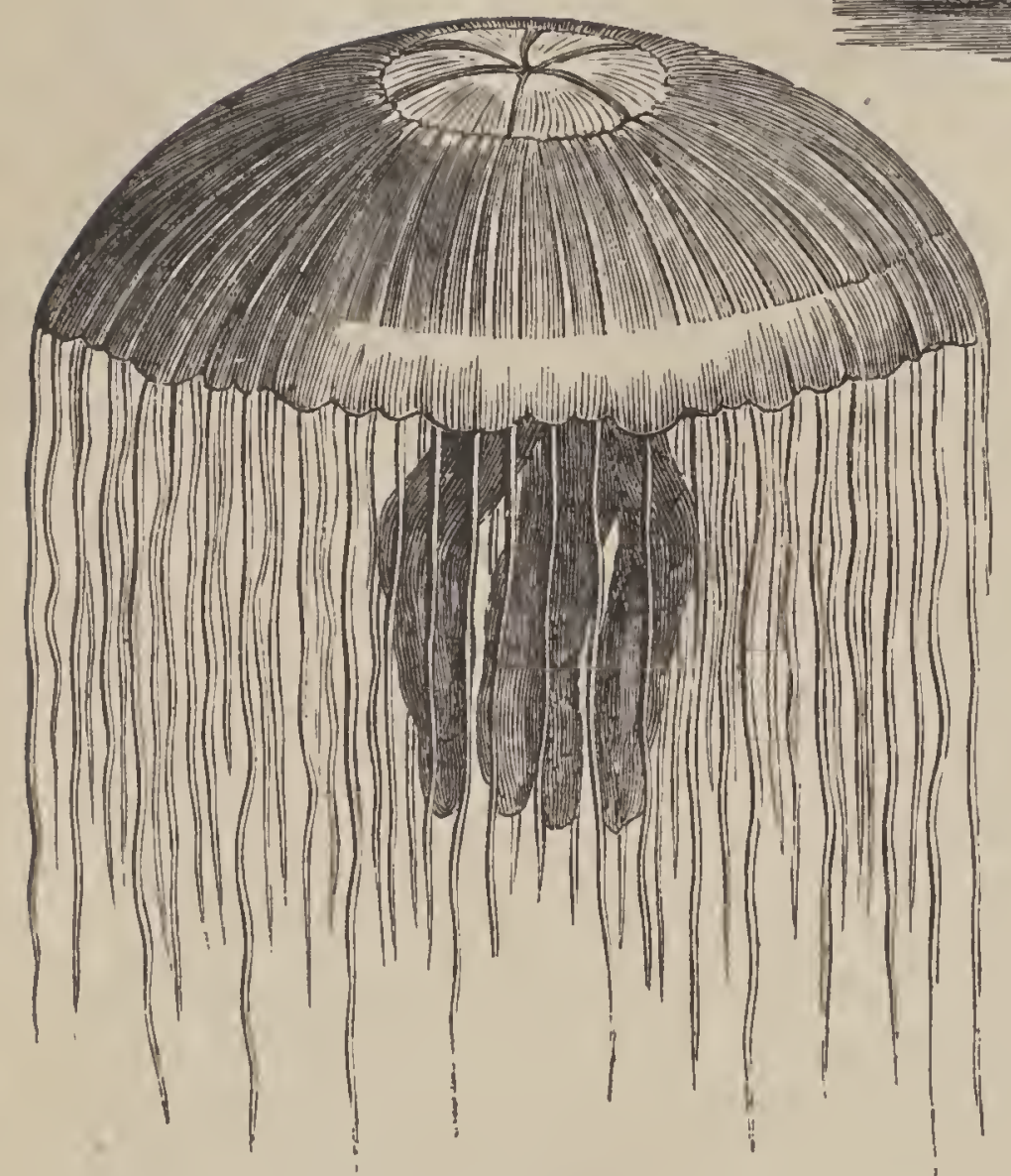

Fra. 193. Jelly-fish (Pelagia noctiluca). Mediterranean.
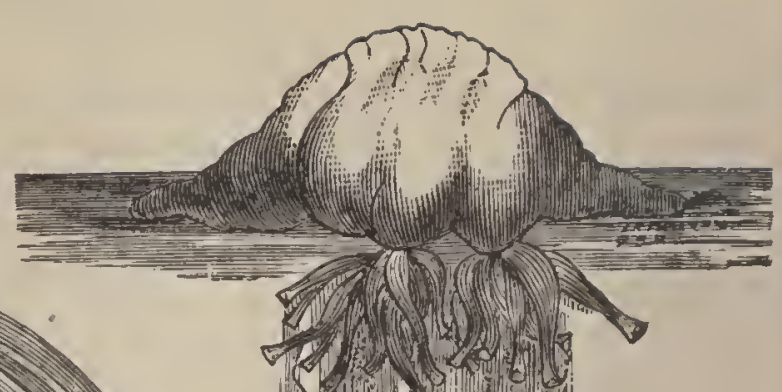

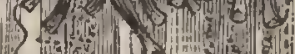

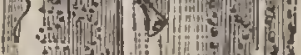

(1)

20

ind

?

गै।

\begin{tabular}{ccc}
4 & $\vdots$ \\
\hdashline & $\vdots$
\end{tabular}

:

(1)

(1)

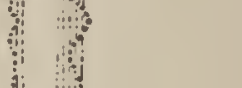

:

:

i:

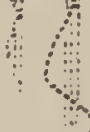

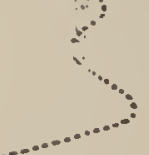

Fig. 194.-Portuguese man. of-war (Physalia), 1/6 nallral size. Tropical Atlautic.

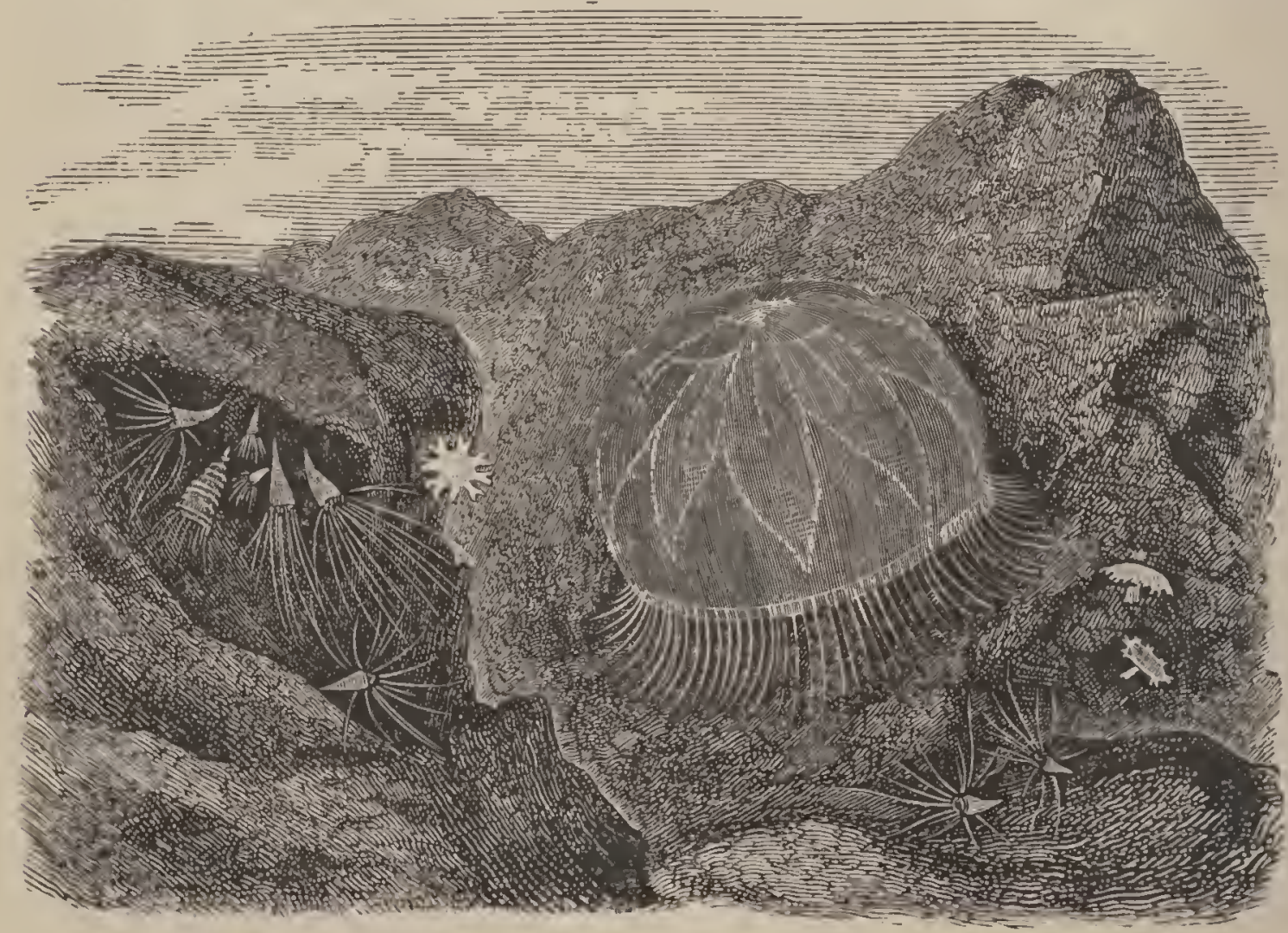

FIG. 195.-Jelly-fish (A urelia aurita), with young 11 various stages. 
fringed with tentacles, which are furnished with stinging thread-cells. The radiating parts are in multiples of four. Around the rim are minute colored spots, the "eye-specks." In fine weather, these "sea-blubbers" are seen floating on the sea, mouth downward, moving about by flapping their sides, like the opening and shutting of an umbrella, with great regularity. They are frequently phosphorescent when disturbed. Some are quite small, resembling little glass bells; the common Aurelia is over a foot in diameter when full-grown; while the Cyanea, the giant among Jelly-fishes, sometimes measures eight feet in diameter, with tentacles one

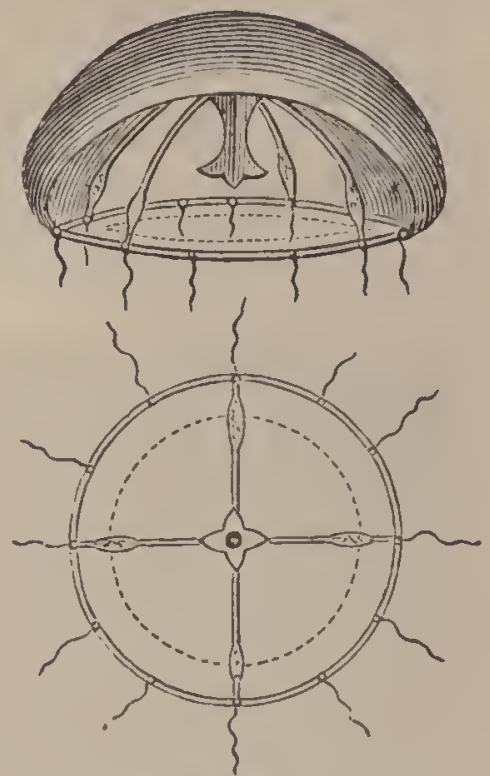

Fia. 196.-A Medusa, seen in profile and from below, showing central polypite, radiating and marginal callals. hundred feet long. The tissues are so watery that, when dried, nothing is left but a film of membrane weighing only a few grains.

There are two representative types: the Lucernaria, the Umbrella-acaleph, having a short pedicel on the back

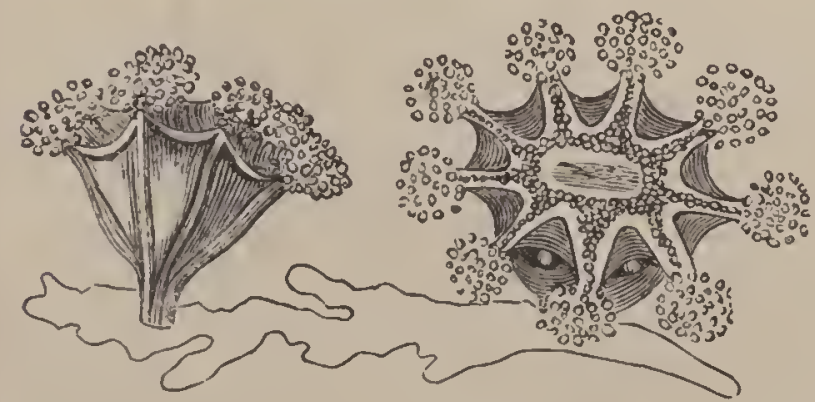

Fia. 197.-Lucernaria auricula attached to a piece of sea-weed; uatural size. The one on the right is abnormal, having a ninth tuft of tentacles. for attaclıment; tentacles disposed in eight groups around the margin, the eight points alternating with the four partitions of the body-cavity and the four corners of the mouth; not less than eight radiating canals, and no membranons veil. The common species on the Atlantic shore, generally found attached to eel-grass, is an inch in diameter, of a green color. Auretia, the ordinary Jelly-fish, is free and oceanic. It differs from the Lncer- 
naria in its usually larger size and solid disk, four radiating canals, which ramify and open into a circular vessel, running around the margin of the disk. ${ }^{12}$

\section{Class II.-Anthozoa.}

These marine animals, which by their gay tentacles convert the bed of the ocean into a flower-garden, or by their

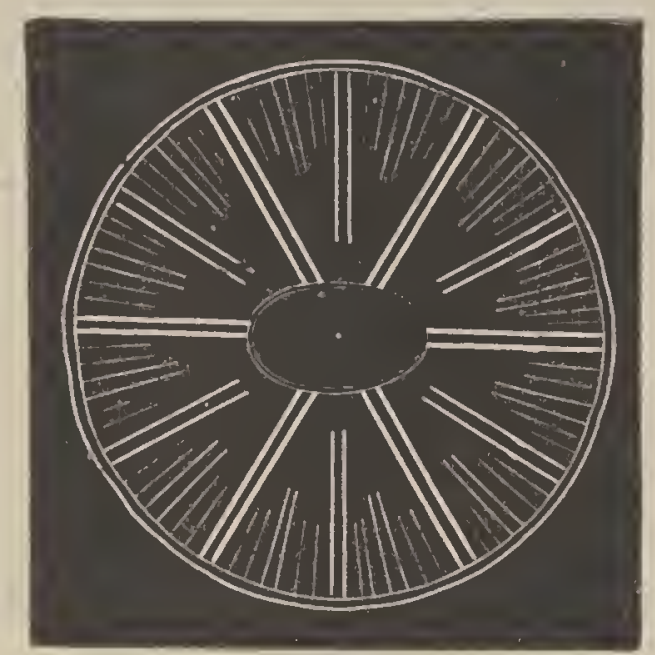

FIG. 198.-Horizuntal Section of Actinia through the stomach, showing septa aud compartments. secretions build up coral-islands, have a body like a cylindrical gelatinous bag. One end, the base, is usually attached; the other has the mouth in the centre, surrounded by numerous hollow tentacles, which are covered with nettling lasso-cells. This upper edge is turned in so as to form a sac within a sac, like the neck of a bottle turned outside in. The inner sac, which is the digestive cavity, does not reach the bottom, but opens into the general body-cavity (Fig. 38). ${ }^{130}$ The space between these two concentric tubes is divided by a series of vertical partitions, some of which extend from the bodywall to the digestive sac, but others fall short of it. Instead, therefore, of the radiating tubes of the Acaleph, there are radiating spaces. No members of this class are microscopic. All are

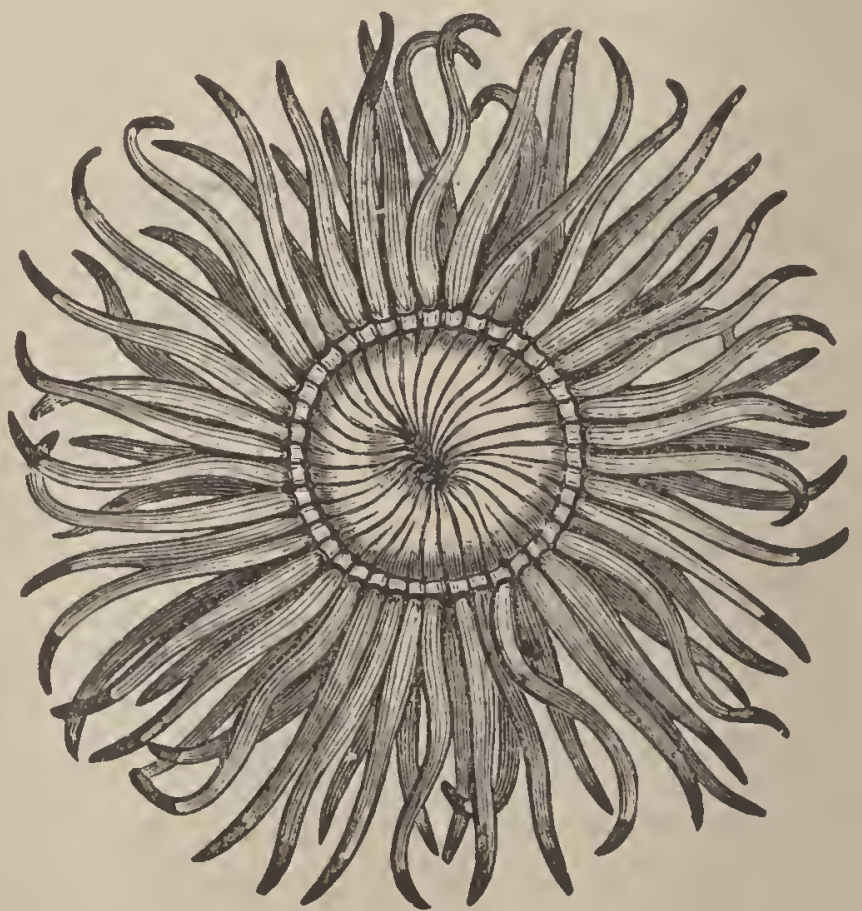

Fro. 199. - Actinia expauded, seen from above, showing mouth. 
long-lived compared with the Hydrozoa, living for several years. One kept in an aquarium in England is now more than sixty years old.

1. Soft-bodied Polyps.-The best-known representative of this group is the Actinia (Metridium), or Sea-anemone. It usually leads a solitary life, though frequently several are found together, some of which have arisen as buds from the others. It is capable of a slow locomotion. Muscular fibres run around the body, and others cross these at right angles. The tentacles, which often number over two hundred, and the partitions, which are in reality donble, are in inultiples of six. At night, or when alarmed, the tentacles are drawn in, and the aperture firmly closed, so that the animal looks like a rounded lump of fleshy substance plastered on the rock. It feeds on crabs and Mollusks. It abounds on every shore, especially of tropical seas. The size varies from one eighth of an inch to a foot in diameter.

2. Coral Polyps. - The majority of Anthozoa secrete a calcareous or horny framework called "coral." With few exceptions, they are fixed and composite, living in colonies formed by a continuous process of budding. Their structures take a variety of shapes: often domelike, but often resembling shrub. hery and cluster's of leaves. The members of a coral community are organically connected; each feeds himself, yet is not independent of the rest. We can speak of the individual Corals, $a, b, c$, but we must write them down $a b c$. The compound mass is "like a living sheet of animal matter, fed and nourished by numerous mouths and as many

stomachs." Life and death go on together, the old

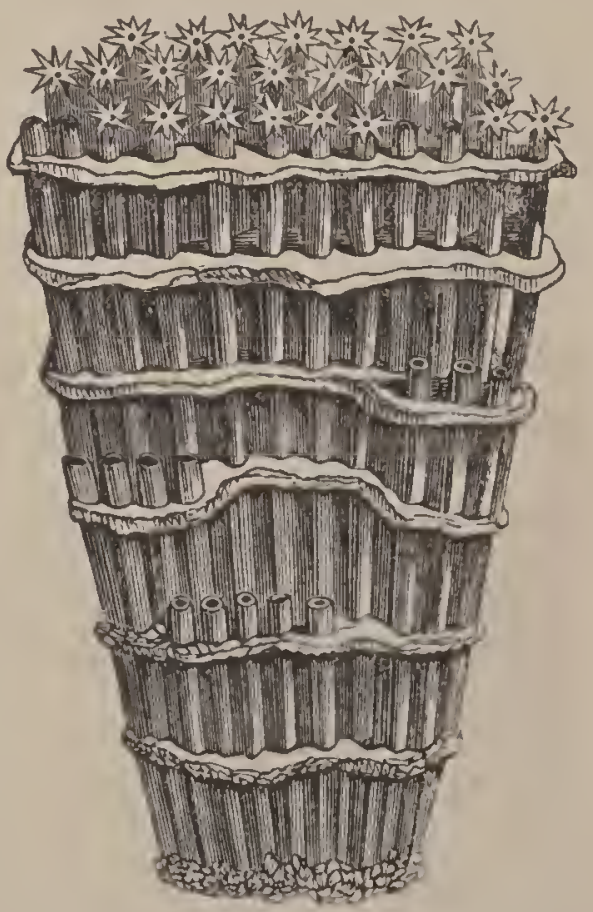

Fig. 200.-Organ-pipe Coral (Tubipora musica). Indian Ocean. 
Polyps dying below as new ones are developed above. The living part of an Astraca is only half an inch thick. 'The growth of the branching Madrepore is about three inches a year. The prevailing color of the Coral Polyps is green; and the usual size varies from that of a pin's head to half an inch, but the Mushroom-coral (which is a single individual) may be a foot in diameter.

Corals are of two kinds: those deposited within the tissues of the animal (sclerodermic), and those secreted by the onter surface at the foot of the Polyp (sclerobasic). The Polyps producing the former are Actinoid, resembling the Actinia in structure. ${ }^{131}$ The skeleton of a single Polyp (called corallite, Fig. 95) is a copy of the animal, except the stomach and tentacles, the earthy matter being secreted within the outer wall and between each pair of partitions. So that a corallite is a short tube with vertical septa radiating towards the centre. ${ }^{132}$ A sclerobasic Coral is a true exoskeleton, and is distinguished by being smootls and solid. The Polyps, having eight fringed tentacles, are situated on the outside of this as a common axis, and are connected together by the fleshy conosarc covering the Coral.

(1) Sclerodermic Corals.-Astrcea is a hemispherical mass covered with large cells. Meandrina, or "Brain-coral," is also globular; but the mouths of the Polyps open into each other, forming furrows. Fungia, or "Mushroomcoral," is disk-shaped, and differs from other kinds in being the secretion of a single gigantic Polyp, and in not being fixed. Madrepora is neatly branched, with pointed extremities, each ending in a small cell abont a line in diameter. Porites, or "Sponge-coral," is also branching, but the ends are blunt, and the surface comparatively smooth. Tubipora, or "Organ - pipe coral," consists of smooth red tubes connected at intervals by cross-plates. The Astrcea, Meandrina, Madrepora, and Porites are the chief reef-forming Corals. They will not live in waters 


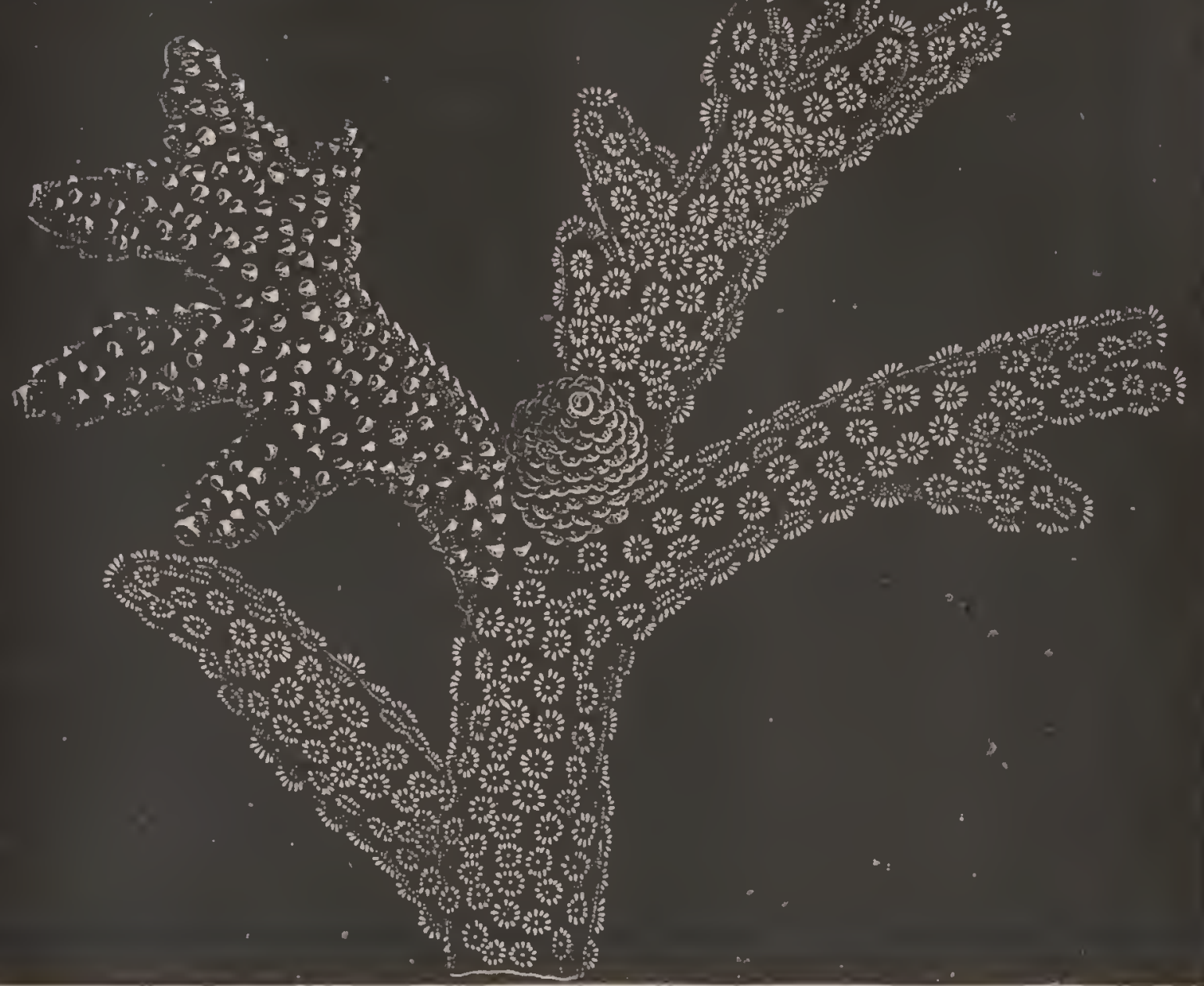

Frf. 201.-Madrepora aspera, living and expanded; natural size. Paciffc.

whose mean temperature in the coldest month is below $68^{\circ}$ Fahr., nor at greater depth than twenty fathoms. The most luxuriant reefs are in the Central and Western $\mathrm{Pa}$ cific and around the West Indies.

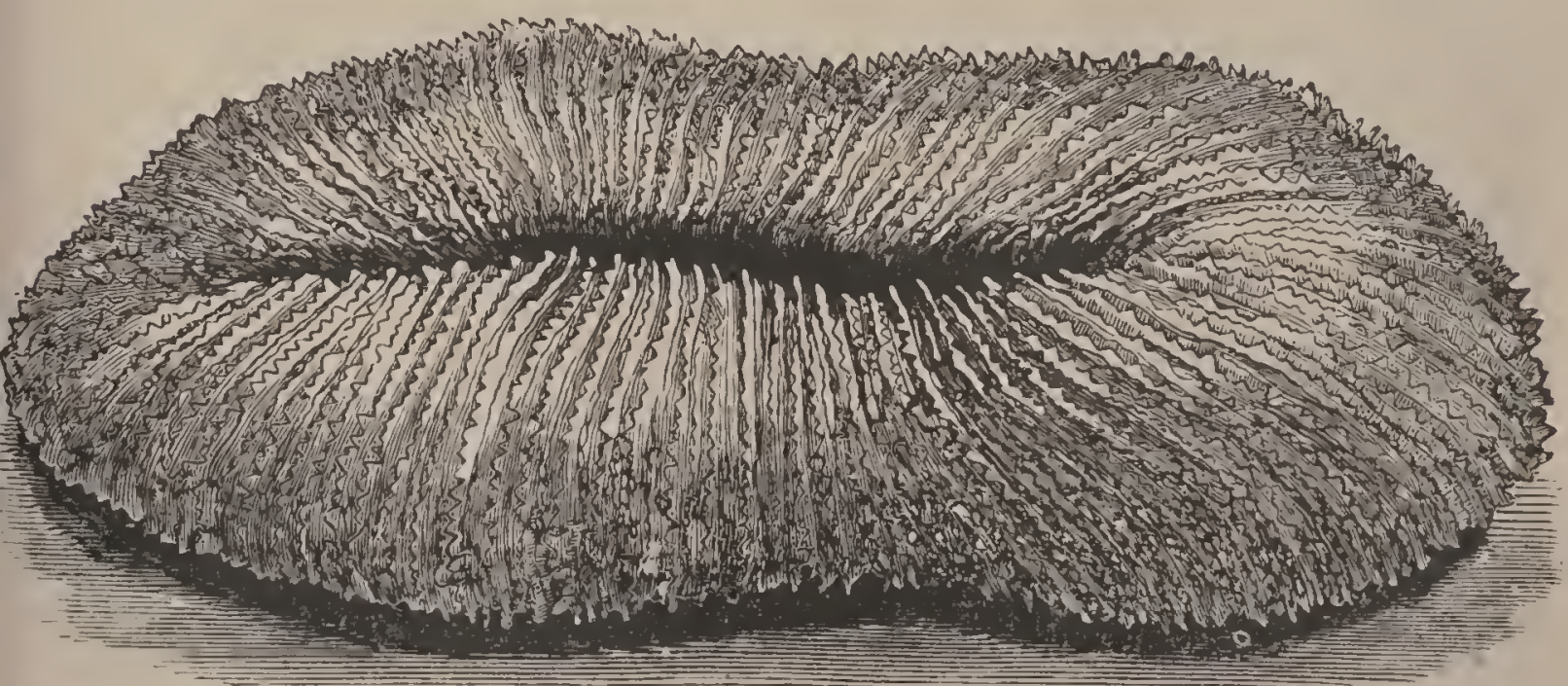

Fig. 202.-Ctenactis echinata, or "Mushroom-coral :" one fourth natural size. Pacific. 


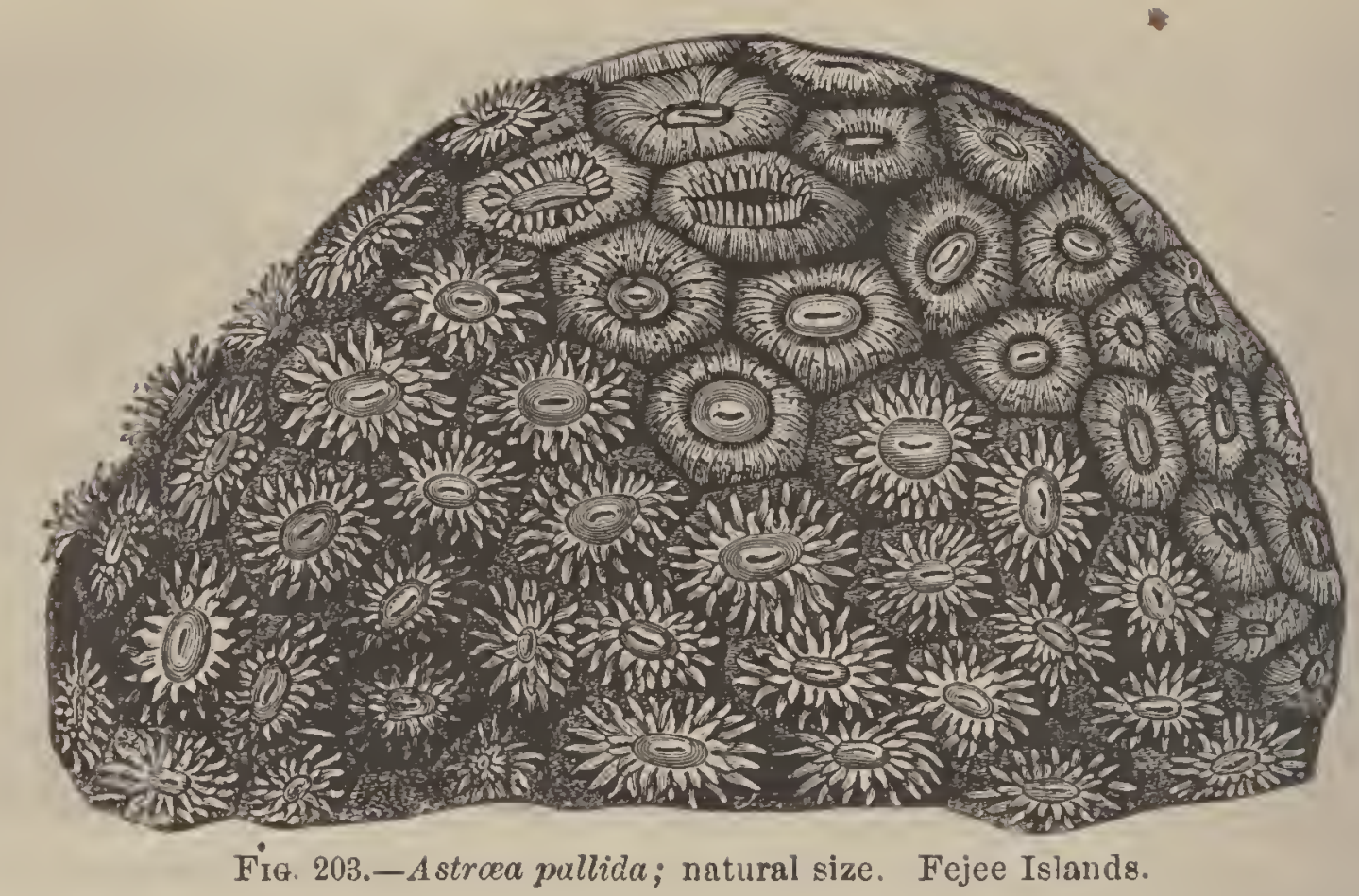

A coral-reef is formed by many Corals growing together. It is to the single Coral-stock as a forest is to a tree.

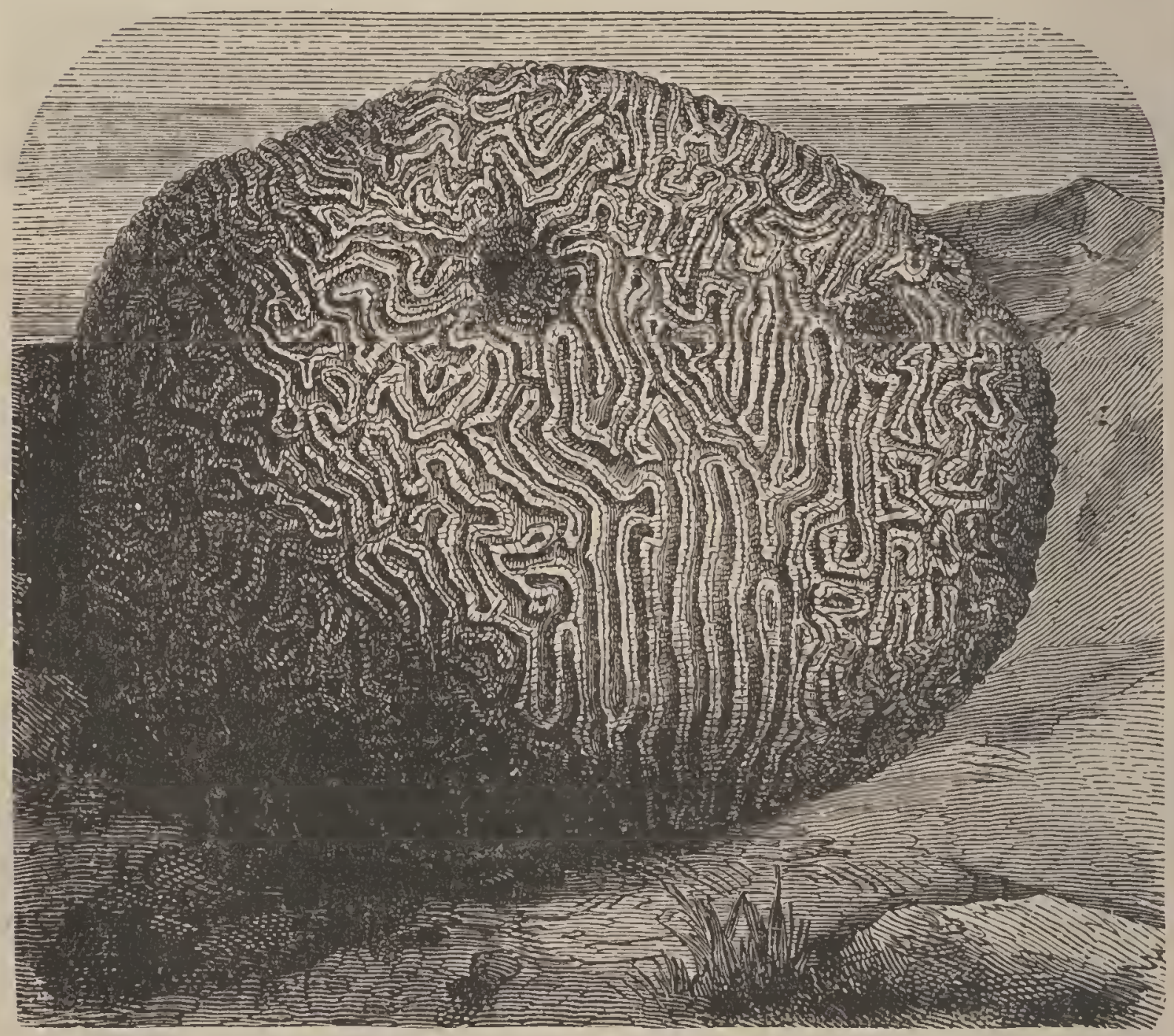

Fra. 204.-Diyloria cerebriformis, or "Brain-coral;" one half natural size. Bermudas. 


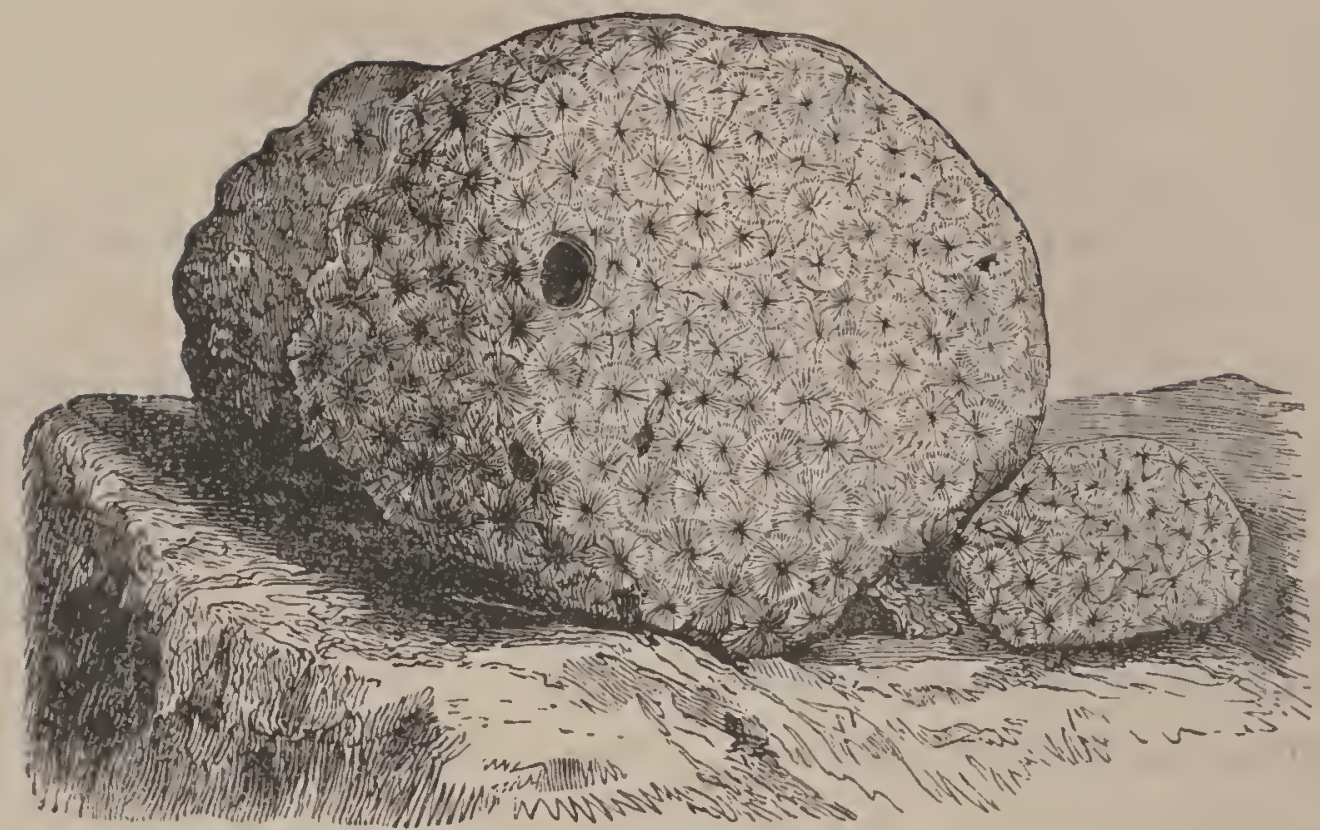

Fig. 205. - Astrcea rotulosa. West Indies.

The main kinds of reefs are fringing, where the reef is close to the shore; barrier, where there is a channel be-

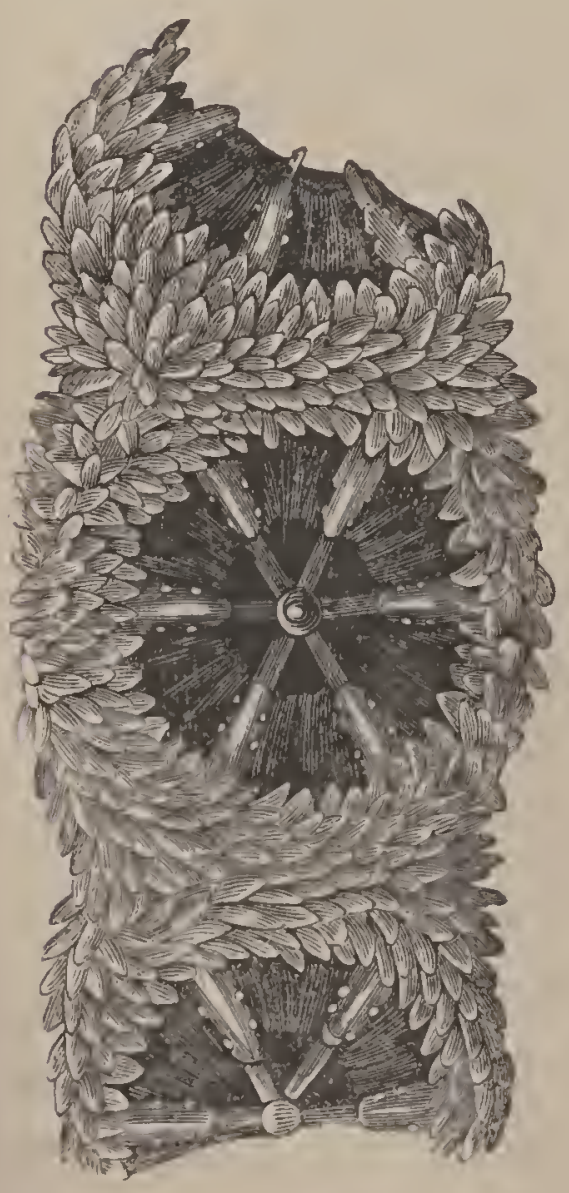

FiG. 206.-Cell of Madrepore Coral, magnified. The cup-like depression at the top of a coral skeleton is called calicle.

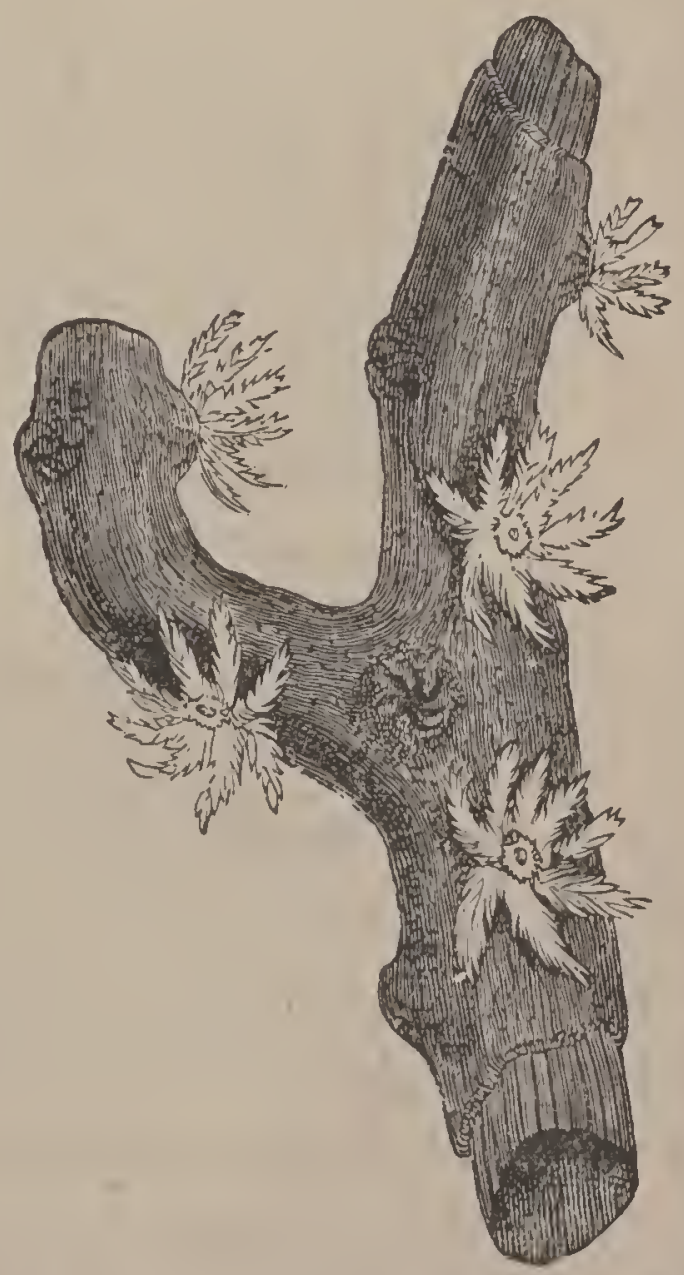

Fra. 207.-Fragment of Red Coral (Coròllium rubrum), showing living cortex and expanded Polyps. Mediterranean. 
tween reef and shore; encircling, where there is a small island inside of a large reef; and coral islands, or atolls, where there is simply a reef with no land inside of it. All reefs begin as fringing-reefs, and are gradually changed into the other forms by the slow sinking of the bottom of the ocean. This sinking must be slower than the upward growth of the reef, else it will be drowned ont. Probably the reef does not grow more than five feet in a thousand years; and, as reefs are often more than two thousand feet thick, they must be very old.

(2) Sclerobasic Corals.-Corallium rubrum, the precious coral of commerce, is shrub-like, about a foot high, solid throughout, taking a high polish, finely grooved on the surface, and of a crimson or rose-red color. In the living

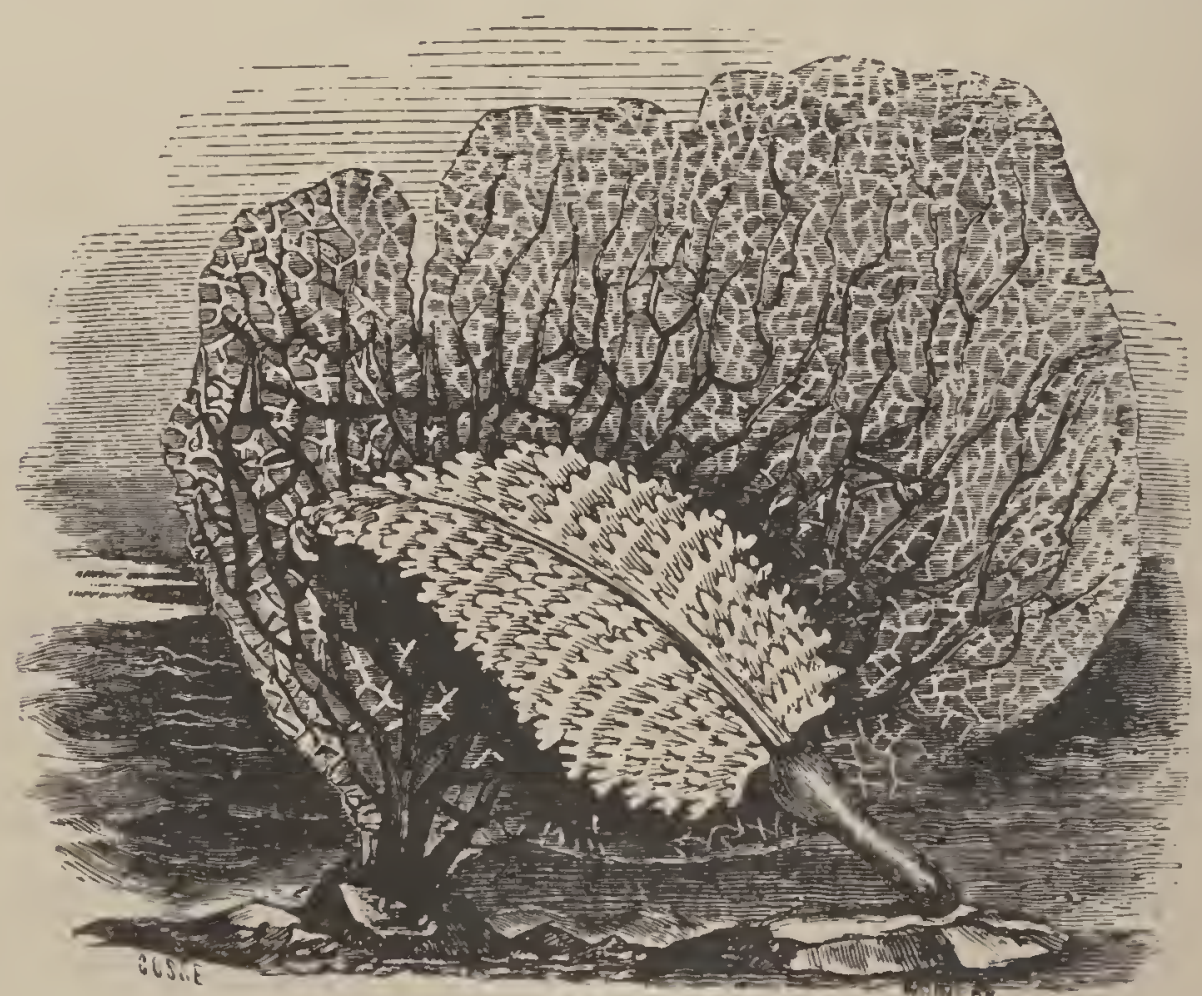

Fig. 208.-Sea-fau (Gorgonia) aud sea-peu (Penratulu).

state the branches are covered with a red conosare studded with Polyps. Grorgonia, or "Sea-fan," differs from all the other representative forms in having a horny axis covered with calcareous spicules. The branches arise in the same vertical plane, and unite into a beautiful network. 
Class III.-Ctenophora.

The Ctenophora (as the Pleurobrachia, Cestum, and Beroë) secrete no hard deposit. They are transparent and gelatinous, swimming on the ocean by means of eight comblike, ciliated bands, which work like paddles. The body is not contractile, as in the Jelly-fishes. They are considered the highest of Coelenterates, having a complex nutritive ap- Fro. 2u9.-A (tenmphure (Pleuparatus and a definite nervous sys- robruchia pilens); natural tem.

\section{Subkingdom IV.-EChinodermata.}

The Echinoderms, as Star-fishes and Sea-urchins, are distinguished by the possession of a distinct nervous system (a ring around the mouth with radiating branches); an ali-

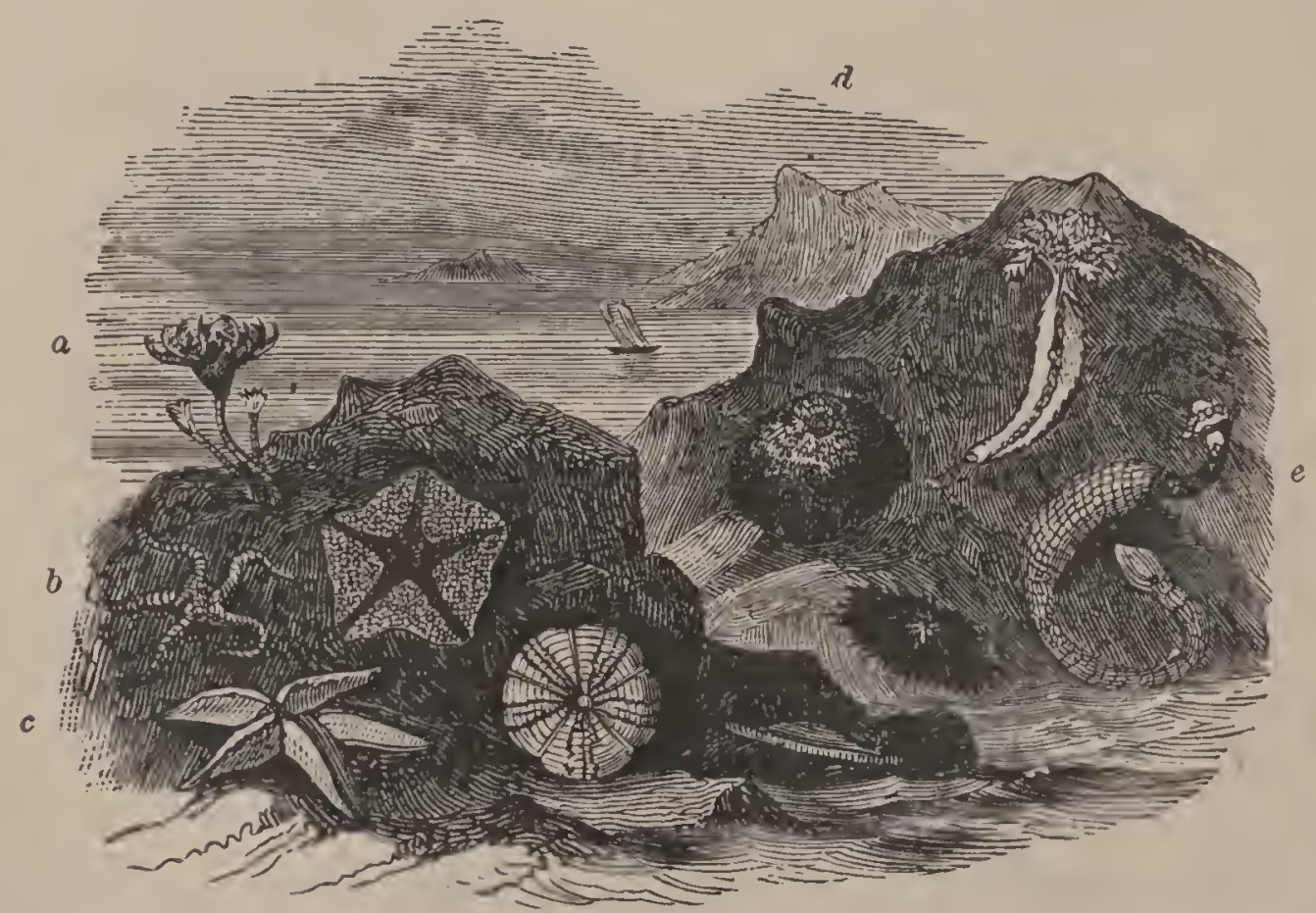

Fra. 210.-Forms of Echinoderms, fiom radiate to annulose type: $a$, Crinoids; $b$, Ophiuraus; $c$, Star-fish ; $d$, Echini ; e, Holothurians.

mentary canaI, completely shut off from the body-cavity, and having both oral and anal apertures; a water-vascular 
system of circular and radiating canals, connected with the outside water by means of the madreporic tubercle, and a symmetrical arrangement of all the parts of the body around a central axis in multiples of five. ${ }^{133}$ There are four principal classes, all exclusively marine and solitary, and all haring the power of secreting more or less calcareons matter.

\section{Class I.-Crinoidea.}

The Crinoids, or "Sea-lilies," are fixed to the sea-bottom by means of a hollow, jointed, Hexible stem. On the top of the stem is the body proper, resembling a bud or expanded flower, containing the digestive apparatus, with the surrounding al'ms, or tentacles. The mouth looks upward. There is a complete skeleton for strength and support, the entire animal-body; arms, and stem-consisting of thousands of stellate pieces connected together by living matter. Crinoids were very abundant in the old geoologic seas, and many limestone strata were formed out of their remains. They are now nearly extinct: dredging in the deep parts of the oceans has brought to light a few living representatives.

\section{Class II.-Asteroidea.}

Ordinary Star-fishes consist of a flat central disk, with five or more arms, or lobes, radiating from it, and containing branches of the viscera. The skeleton is leathery, hardened by small calcareous plates (twelve thousand by calculation), but somewhat flexible. The mouth is below; and the rays are furrowed underneath, and pierced with numerous holes, through which pass the sucker-like tentacles - the organs of locomotion and prehension. The red spots at the ends of the rays are eyes. The usual color of Star-fishes is yellow, orange, or red. They abound on every shore, and are often seen at low tide half buried in the sand, or slowly gliding over the rocks. Cold fresh 


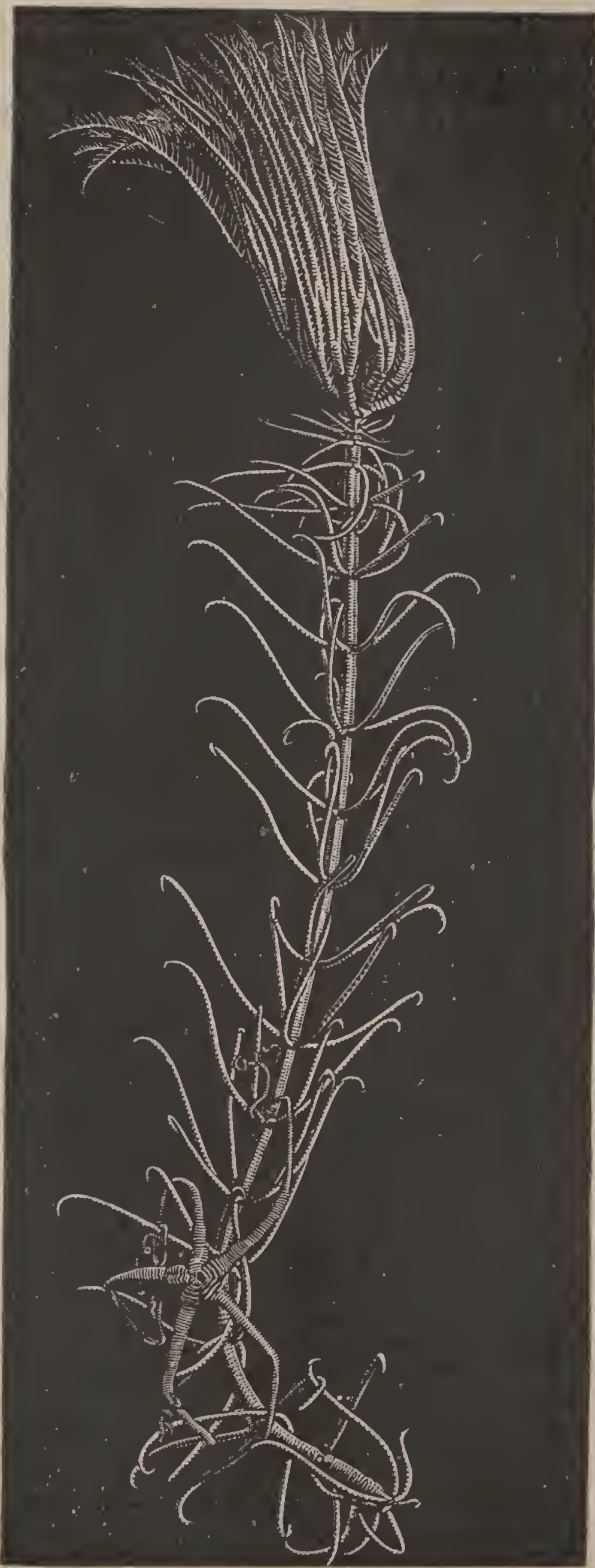

F16. 211. - A living Crinoid (Pentacrinus asteria); one fourth natural size. Wust Indian Seas. 
water is instant death to them. They have the power of reproducing lost parts to a high degree. They are very voracious, and are the worst enemies of the Oyster.

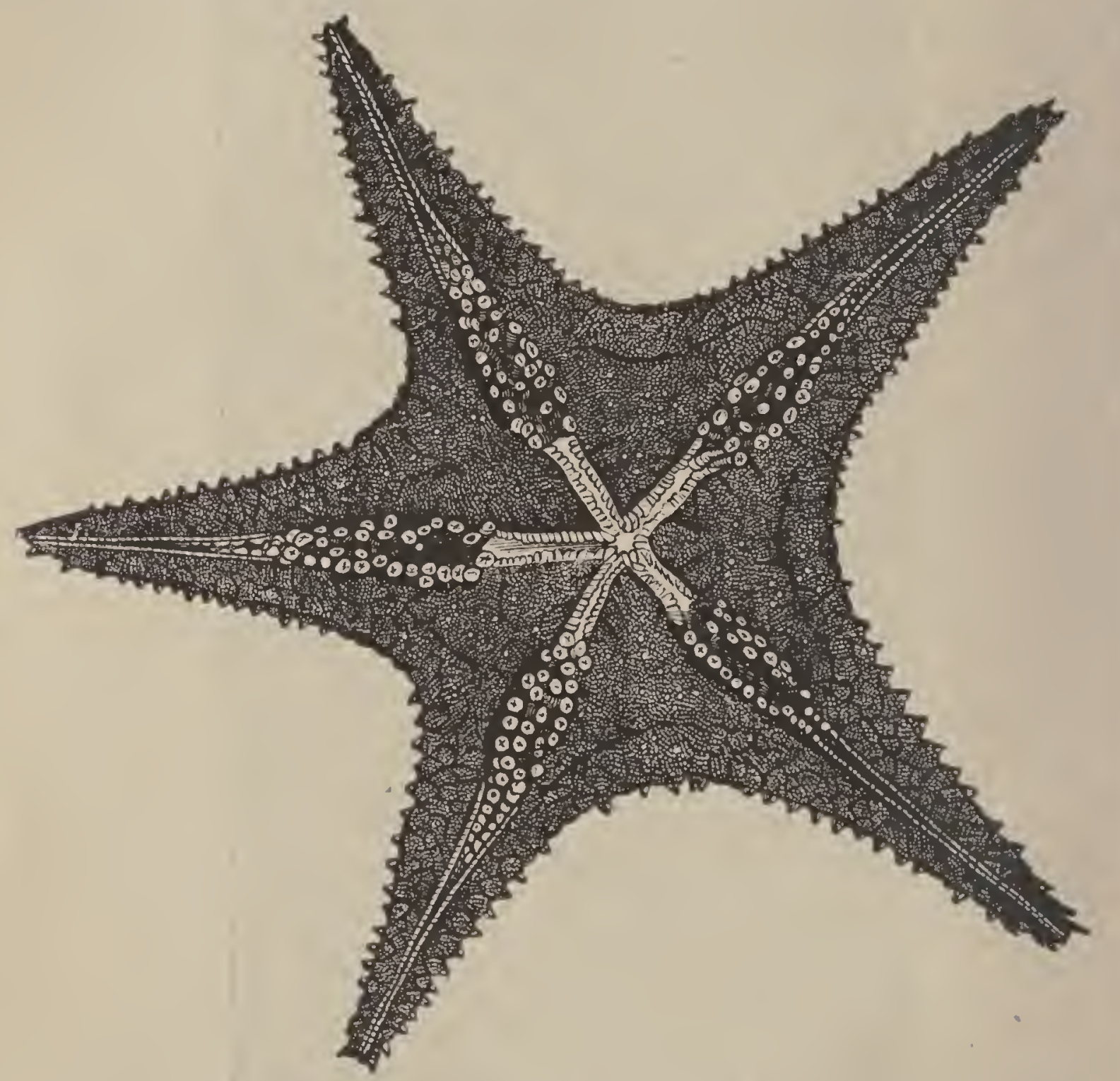

Fra. 212. - Under-surface of Star-fish (Goniaster reticulatus), showing ambulacral grooves aud protrnded suckers.

About one hundred and fifty species are known. These may be divided into two groups: (1) species having four rows of feet, represented by the common five-fingered Asterias; or having two rows of feet, as the many-rayed Solaster, or "Sun-star," and the pentagonal Goniaster; (2) species having long, slender arms, which are not prolongations of the body, and are not provided with suckers, as the Ophiura, or "Brittle-star," and Astrophyton, or "Basket-fish." The last are of inferior rank, and resemble 
inverted, stemless Crinoids. The digestive sac is confined to the disk, and the madreporic tubercle is concealed.

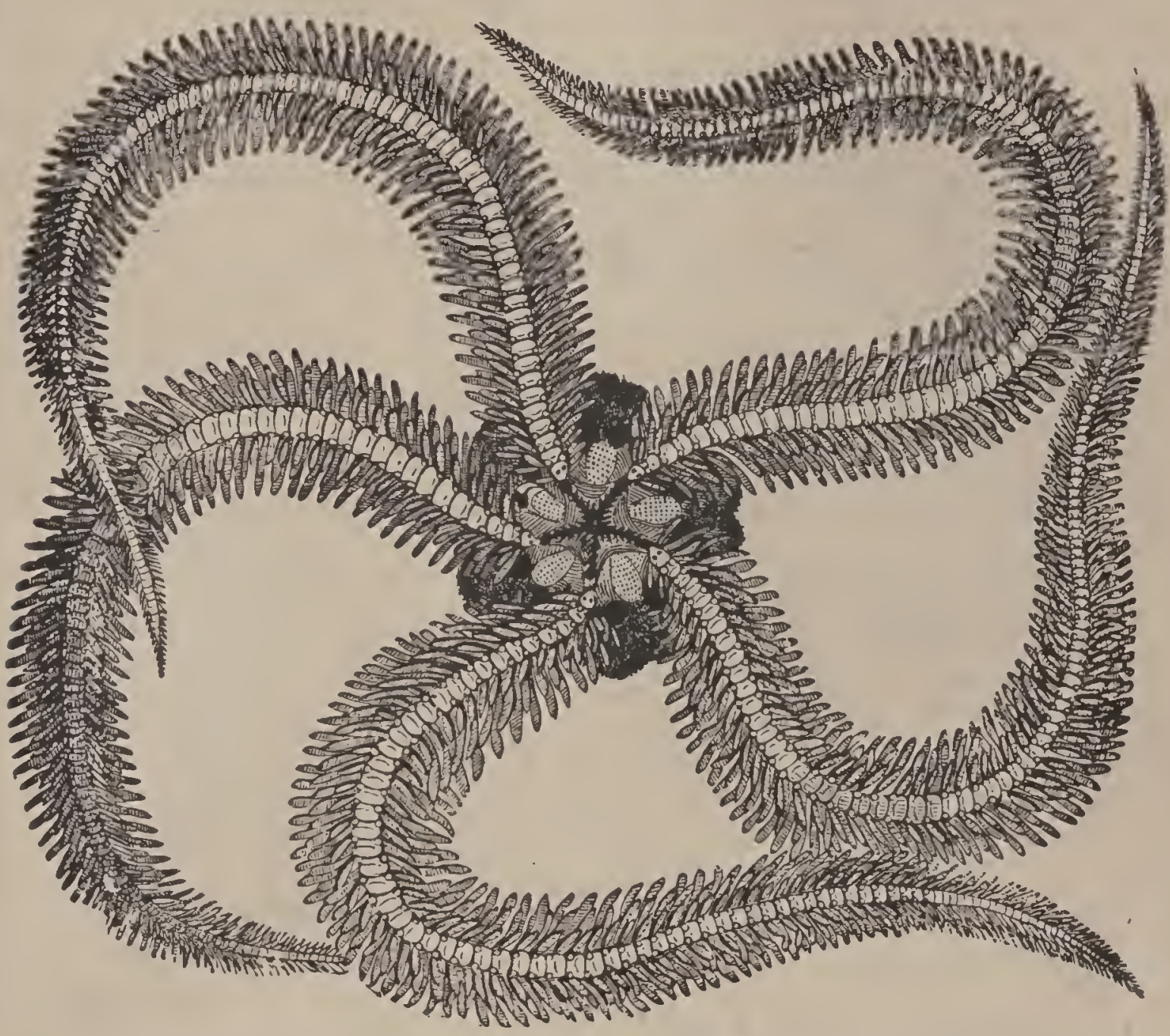

Fig. 213. - Ophiocoma Russei, an Ophiuran; natural size. West Indies.

\section{Class III.-Echinoidea.}

The Sea-urchin is encased in a thin, hollow shell corered with spines, and varying in shape from a sphere to a disk. ${ }^{134}$ The mouth is underneath, and contains a dental apparatus more complicated than that of any other creature. It leads to a digestive tube, which extends spirally to the summit of the body. The spines are for burrowing and locomotion, and are moved by small muscles, each being articulated by ball-and-socket joint to a distinct tubercle. When stripped of its spines, the shell (or "test") is seen to be formed of a multitude of pentagonal plates, fitted together like a rnosaic. ${ }^{135}$ Five double rows of plates, 
passing fiom pole to pole, like the ribs of a melon, alternate with five other double.rows. In one set, called the

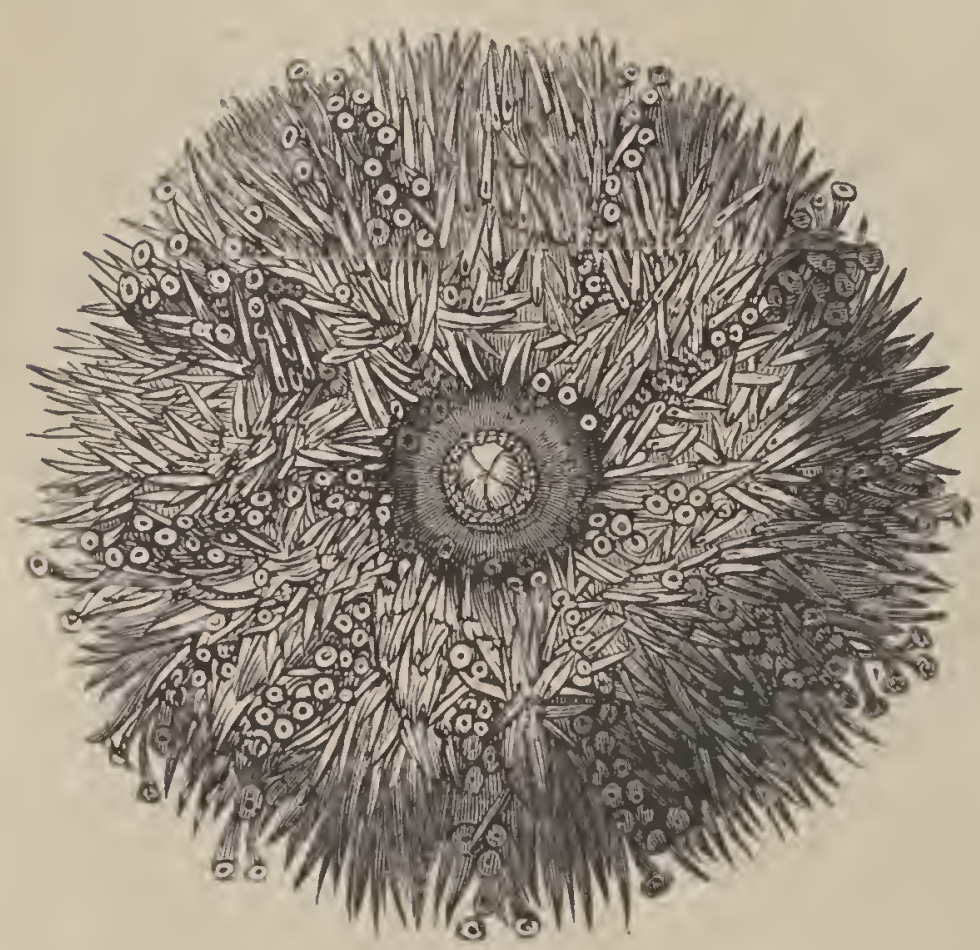

Fig. 214.-Undel-surface of a Ser-urchin (Echinus escrelentus), showing rows of suckers among the syiuts. British seas. ambulacra, the plates are perforated for the protrusion of tubular feet, or suckers, as in the Star-tish. So that altogether there are twenty series of platesten ambulacral, and ten interambulacral. The shell is not cast, but grows by the enlargement of each individual plate, and the addition of new ones around the mouth and the opposite pole. Every part of an Echinus, even sections of the spines, show the principle of radiation. If the upper surface of a Star-fish shonld shriuk so as to bring the points of the arms to meet above the month, we should have a close imitation of a Sea-mechin. Echini live near the shore, in rocky holes or under sea-weed. They are less active than Star-fishes; but, like them, feed on Mollusks, Crabs, and offal. They reproduce by minute red eggrs.

Regular Echini, as the common Cidaris, are nearly globular, and the oral and anal openings are opposite. Irregular Echini, as the Clypeaster, are flat, and the anal orifice is near the margin.

\section{Class IV.-Holothuroidea.}

These worm-like "Sea-slugs," as they are called, have a soft, elongated body, with a tough, contractile skin contain. 
ing calcareous granules. One end, the head, is abruptly terminated, and has a simple aperture for a month, encircled with feathery tentacles. There are usually five longitudinal rows of ambulacral suckers, but only three are used for locomotion, of which one is more developed than the rest. The month opens into a pharynx leading to a long intestinal canal. Holothurians have the singular power of ejecting most of their internal organs, surviving

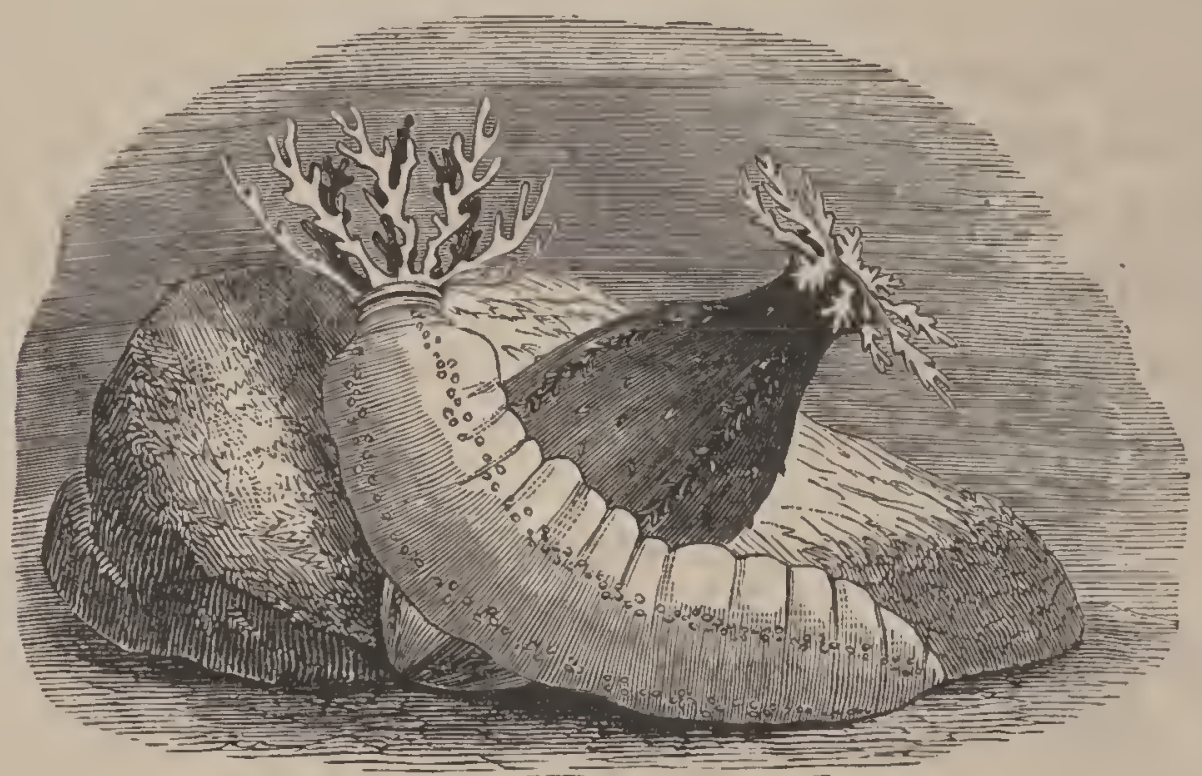

FIG. 215.-Sen-slugs (Holothuria).

for some time the loss of these essential parts, and afterwards reproducing them. They occur on nearly every coast, especially in tropical waters, where they sometimes attain the length of three or four feet. As found on the beach after a storm, or when the tide is out, they are leathery lumps, of a reddish, brownish, or yellowish color. They may be likened to a Sea-urchin devoid of a shell, and long drawn out, with the axis horizontal, instead of vertical.

\section{Subkingdom V.-VERMES.}

The Vermes, ${ }^{136}$ or Worms, form the lowest subkingdom of the bilaterally symmetrical animals. The group includes animals very different in form and rank, and the different classes are widely separated from each other. 
It has also close relations with the other subkingdoms of the bilaterally symmetrical animals. Through the Polyzoa and Brachiopoda, it approaches the Mollusca; through the Annelides, the Arthropoda; and through other forms, the Tunicata, and so the Vertebrata. The subkingdom thus stands in the centre of several subkingdoms, with affinities towards all. Nor are indications of connection with Cœlenterata and Echinodermata wanting.

The Vermes are bilaterally symmetrical animals, with one or many segments, no jointed legs. They usually have a soft . skin, and peculiar excretory organs-the segmental organs.

Many of the Worins are parasitic, and most of the endoparasites belong to this group.

There are numerous classes, of which only the most important are mentioned.

Class I.-Platyhelminthes.

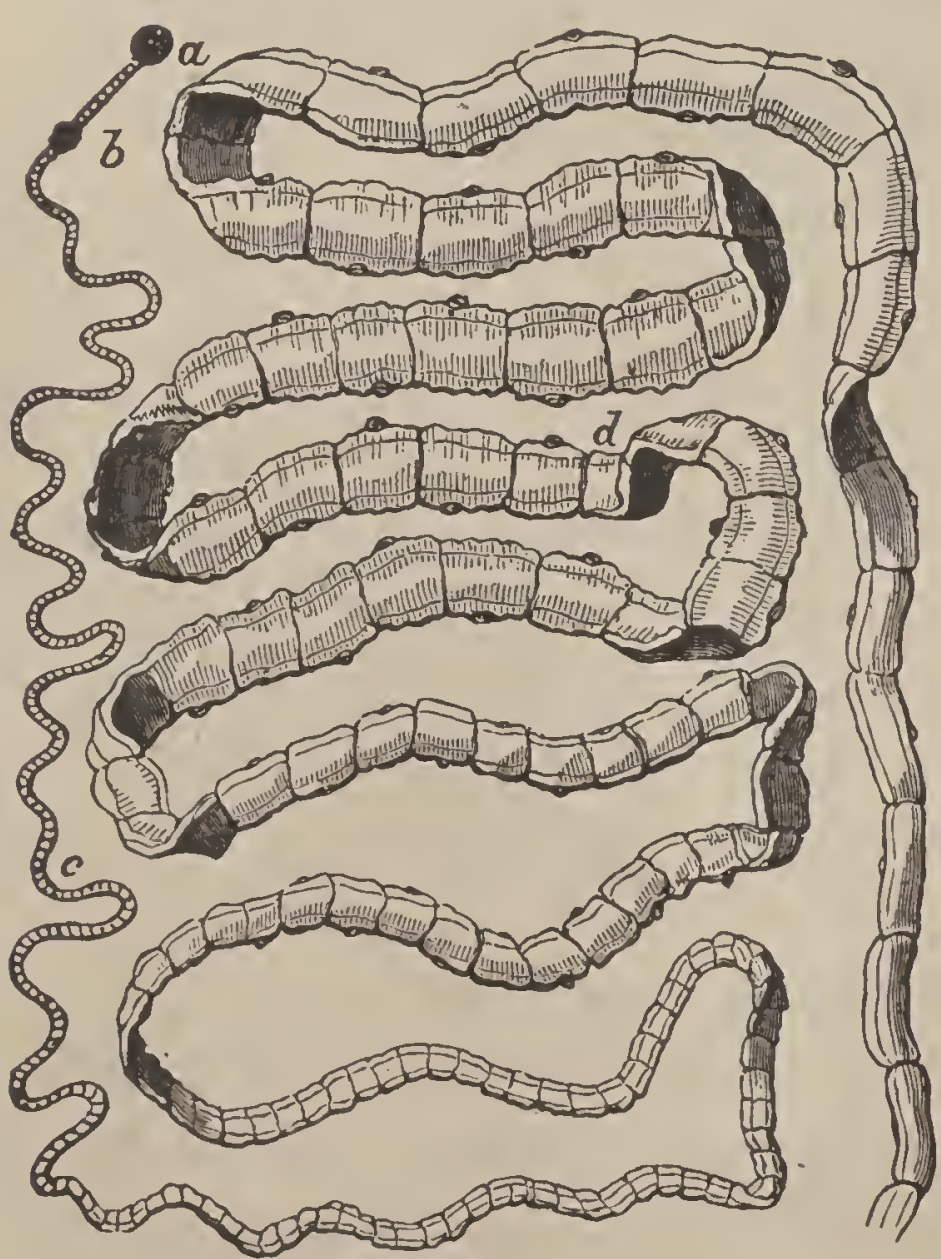

Fra. 216.-Tape-worm (Tania solium): $a$, bead; $b, c$, $d$, segments of the body.

The Flat-worms include some free forms, as the Planaria, common in fresh water, and the Tapeworms and Flukes among the parasites.

The Tape-wolm consists of the socalled head-the proper worm - and the body segments,

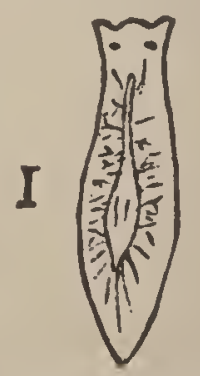

Fio. 217.-Plauarian worm. 
which are really reproductive joints. It develops from the egg in the digestive canal of the Pig, burrows into the cellular tissue of the animal, and there becomes encased. Such pork is called "measly pork." If the pork be eaten by man, in an uncooked condition, this case is dissolved by the gastric juice, and the embryo develops into the Tape-worm, attaching itself to the intestine by its "head," and budding off the reproductive segments. As these become ripe and filled with fertilized eggs, they are detached, and pass off with the excrement.

The disease called "rot," in Sheep, is produced by the Fluke (Distoma), a member of this class.

\section{CLASS II.-Nematelminthes.}

The Round, or Thread, Worms include free forms, as the Vinegar-eel; parasitic forms, as the Pin-worm and Trichina; and forms fiee when adult, and, parasitic when young, as the Hair-worm (Gordius).

The Trichina is usually derived by Man from the flesh of the Pig. It exists in the muscles, enclosed in microscopic cases. If the meat be eaten uncooked or partially cooked, the cases are dissolved, and the Trichinæ become sexually mature in the intestines. The young

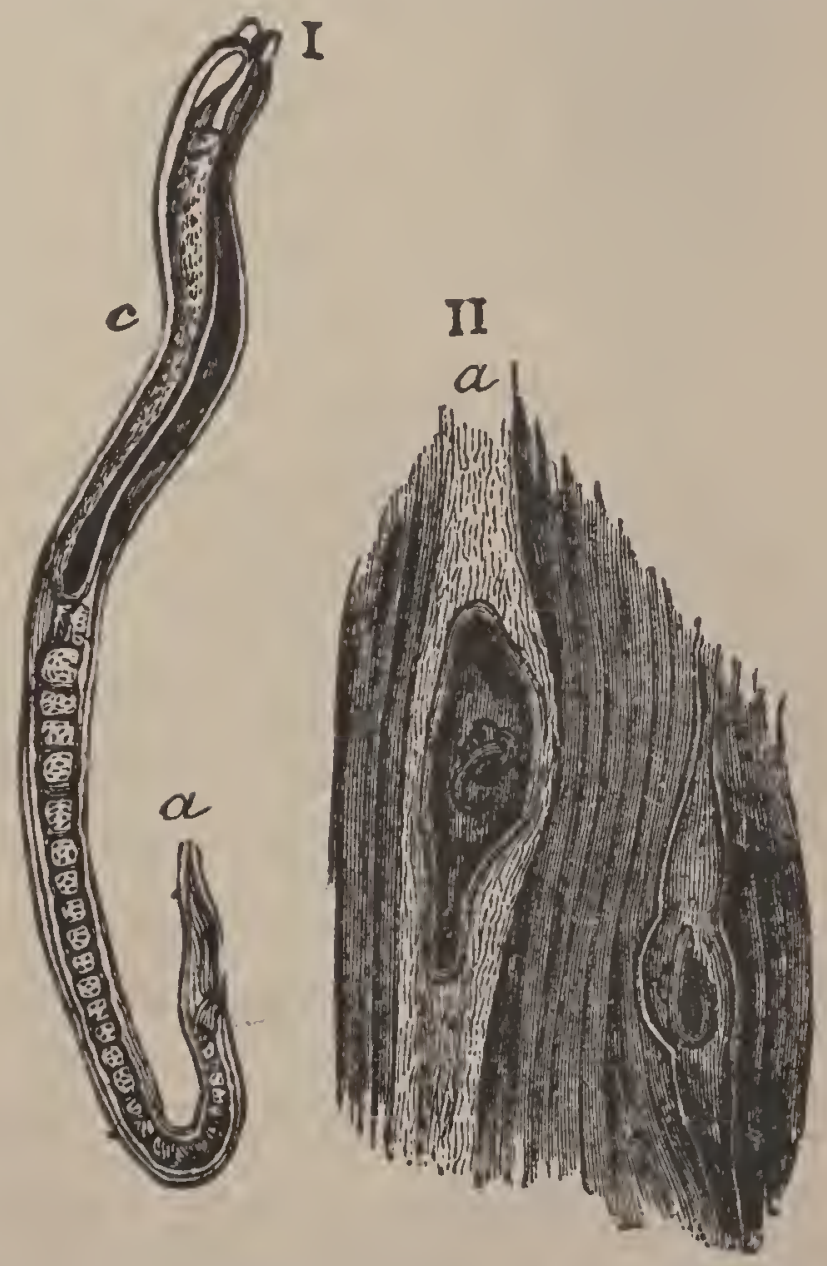
are produced and bur-Fre.218. - Trichina spiralis: I, male: $a$, mouth: row their way into the cle, intestine; II, cups 
muscles, where they become encysted. In burrowing, they cause great pain and fever, and sometimes death. The adult Worm is about $\frac{1}{12}$ inch long.

\section{Class III.-Rotifera.}

The Wheel-animalcules, mostly found in fresh water, are minute Worms of few segments, having on the ante-

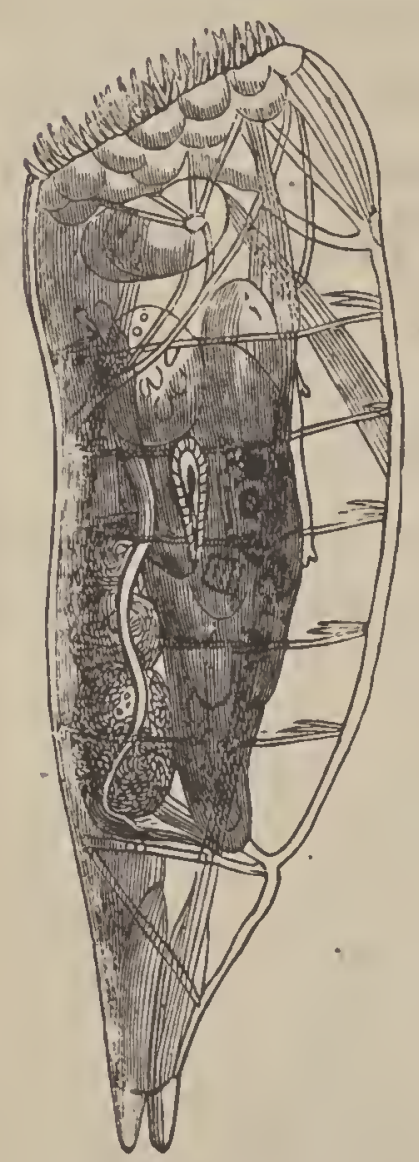
rior end a disk ciliated on the edge, whence their name. They are from $\frac{1}{200}$ to $\frac{1}{36}$ of an inch long. They can bear drying and revivifying, like seeds.

\section{Class IV.-Polyzoa.}

These minute Worms resemble the Polyps in appearance, living in clusters, each individual inhabiting a delicate cell, or tube, and having a simple mouth surrounded with ciliated tentacles. The colony often takes a plant-like form; sometimes spreads, like fairy-chains or lace-work, over other bodies; or covers rocks and sea-weeds in patches with a delicate film. The majority secrete carFro. 219. - Rotifer, or bonate of lime. A Polyzoan shows its su"Wheel-inimalcule" periority to the Coral, which it imitates, magnified.

in possessing a distinct alimentary canal and a well-defined nervous system. The cells of a group never have connection with a common tube, as in Cœlenterates. There are both marine and fresh-water species.

This group and the next following are related to the Mollusca.

\section{Class V.-Brachiopoda.}

These Worms have a bivalve shell, the valves being applied to the dorsal and ventral sides of the body. The valres are unequal, the ventral being usually, larger, and 
fringed "arm," generally coiled up, and supported by a calcareous framework. The animal, having no gills, respires by the arms and the mantle. Brachiopods were once very abundant, over two thousand extinct species having been described; but less than a hundred. species are now living. ${ }^{137}$ They are all marine, and fixed; but of all Worms, they enjoy the greatest range of climate and depth.

\section{Class VI.-Annelides.}

The Annelides include the highest and most specialized Worms. They have many segments, spines or suckers for locomotion, a superosophageal biain, a ventral chain

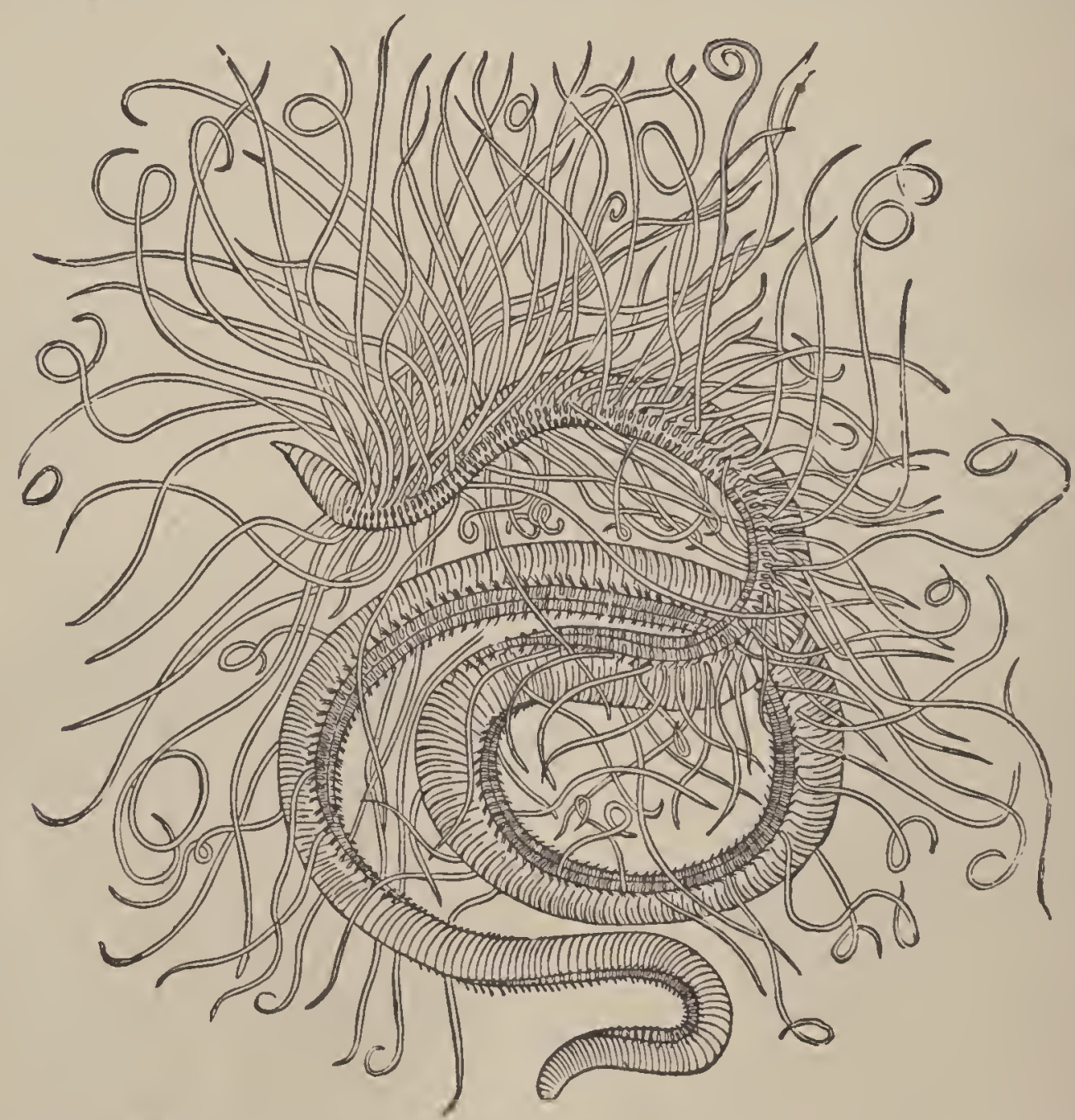

Fro. 223.-Marine Worm (Cirratulus grandis), with extended cirri. Atlantic. of ganglia, and a closed blood-system. There are three main divisions: the flattened Leeches, without definite segments or bristles, and with suckers for locomotion; the 
Earth-worms and their allies, which have few bristles on each segment (Oligochcetce); and the Sea-worms, with numerous bristles, arranged in two clusters on each side of each segment (Polychcetce).

These last are the largest of the Worms, and may have a distinct head, bearing tentacles and eyes. The cesophagus is often turned in, so as to form a proboscis, which bears horny jaws, and can be protruded at the will of the animal (Fig. 17).

\section{Subkingdom VI.-Mollusca.}

A Mollusk is a soft-bodied animal, withont internal skeleton, and without joints, covered with a moist, sensitive, contractile skin, which, like a mantle, loosely envelops the creature. In some cases the skin is naked, but generally it is protected by a calcareous covering (shell). The length of the body is less in proportion to its bulk than in other animals. The lowest class has no distinct head. The nervous system consists of three well-developed pairs of ganglia, which are principally concentrated around the entrance to the alimentary canal, forming a ring around the throat. The other ganglia are, in most cases, scattered irregularly through the body, and in such the body is unsymmetrical. The digestive system is greatly developed, especially the "liver," as in most aquatic animals. Except in the Cephalopods, the muscles are attached to the skin, or shell. There is a heart of two chambers (auricle and ventricle) or three (two auricles and ventricle). As in all Invertebrates, the heart is arterial. In Mollusks, with rare exceptions, we find no repetition of parts along the antero-posterior axis. They are best regarded as Worms of few segments, which are fused together and much developed. The total number of living species probably exceeds twenty thousand. The great majority are water-breathers, and marine; some are 
fluviatile or lacustrine, and a few are terrestrial air-breathers. All bivalves, and nearly all univalves, are aquatic. Each zone of depth in the sea has its particular species.

\section{Class I.-Lamellibranchiata.}

Lamellibranchs are all ordinary bivalves, as the Oyster and Clam. The shells differ from those of Brachiopods

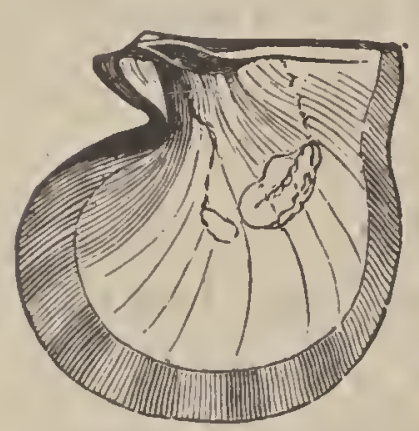

Fig. 224.-1'eirll Oyster (Meleagrina margaritifera): one fourth natural size. Ceylon. in being placed on the right and left sides of the body, so that the linge is on. the back of the animal, and in being unequilateral and equivalved. ${ }^{138}$ The unbo, or beak, is the point from which the growth of the valve commences. Both Brachiopods and Lamellibranchs are headless; but in the latter the mouth points the same way as the umbo, $i$.e., towaids the anterior part. The length of the shell is measured from its anterior to its posterior margin, and its breadth from the dorsal side, where the hinge is, to the opposite, or ventral, edge. The valves are united to the animal by one muscle (as in the Oyster), or two (as in the Clam), and to each other by a hinge. In some species, as some freshwater Mussels, the hinge is simply an elastic ligament, passing on the outside from one valve to the other just behind the beak, so that it is on the stretch when the valves are closed, and another placed between the edges of the valves, so that it is squeezed as they shut, like the spring

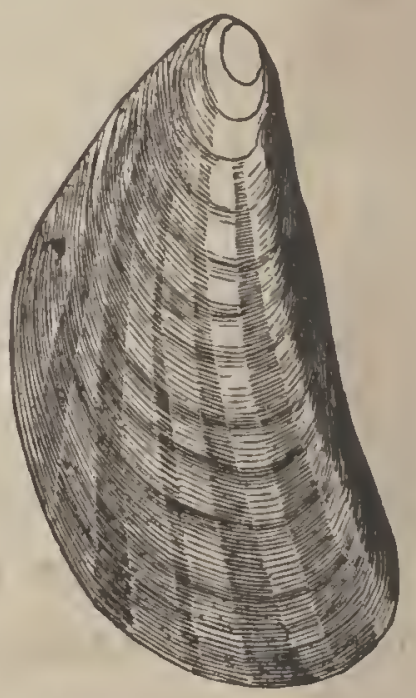

Frg. 225. - Salt - water Mussel (Mytilus pellucidus). Atlautic coasts.

in a watch-case. Such bivalves are said to be edentulons. But in the majority, as the Clam, the valves also articulate by interlocking parts called teeth. The valves are, therefore, opened by the ligaments, and closed by the muscles. 
The margin of the shell on which the ligament and teeth are situated is termed the hinge-line.

Lamellibranchs breathe by four plate-like gills (whence the name), two on each side underneath the mantle (Fig. 78). In the higher forms, the mantle is rolled up into two tubes, or siphons, for the inhalation and exhalation of water. They feed on microscopic organisms filtered from the water. A few are fixed; the Oyster, e.g., habitnally ly-

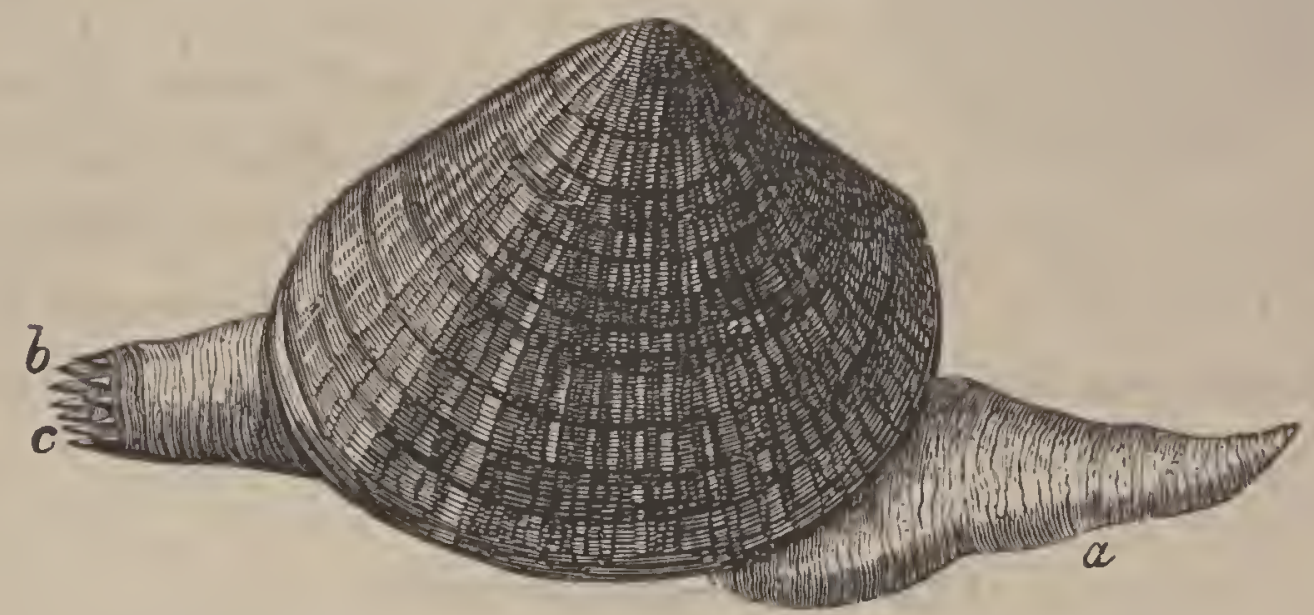

Fra. 226.-Lamellibranch (Mactru) : $a$, foot; $b, c$, siphons.

ing on its left valve, and the Salt-water Mussel hanging to the rocks by a cord of threads called "byssus;" but most have a "foot," by which they creep about. Unlike the Oyster, also, the majority live in an erect position, resting on the edges of their shells. Over four thousand living species are known. These are fresh-water and marine, and range from the shore to a depth of a thousand feet.

The chief characters for distinguishing Lamellibranchs are the muscular impressions, ${ }^{139}$ whether one or two; the presence of a pallial sinus, which indicates the possession of siphons; the structure of the hinge, and the symmetry of the valves. (Fig. 99).

The following are the leading types of structure, as shown by the shells:

1. Monomya: with one adductor muscle; no siphons; foot wanting, or very small; shell unequivalve and eden- 
tulous-as the Oyster (Ostrea), Scallop (Pecten), and Pearl Oyster (Avicula).

2. Heteromya: with two unequal adductor muscles and

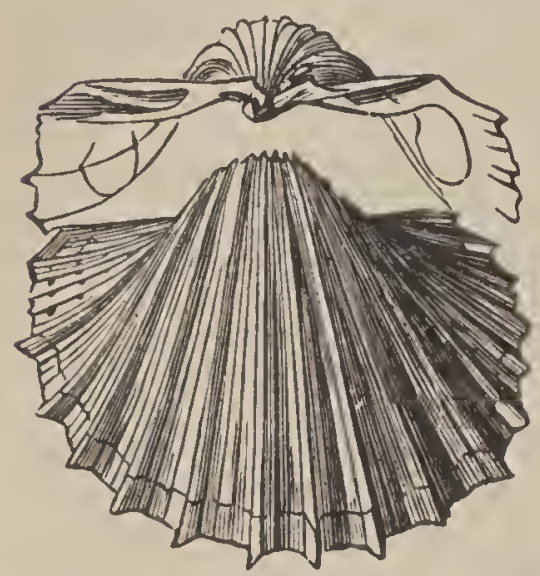

Fig. 227.-Cockle (Cardium costutum); oue third watural size. China seas. no siphons-as the Sea-mussel (Mytilus).

3. Isomya: with two equal adductor muscles. There are two sections of this order: $a$. Those with no siphons, and hence no pallial sinus - as the Fresh - water Mussel (Unio), Cockle (Cardium), and "the giant of the bivalve race" (Tridac$n a)$. b. Those with siphons and pallial sinus-as the common Clam (Mya), Quohog (Venus), and Razor-shell (Solen). ${ }^{140}$

\section{Class II.-Gasteropoda.}

The Snails are, with rare exceptions, all univalves. ${ }^{142}$ 'The body is coiled up in a conical shell, which is usually

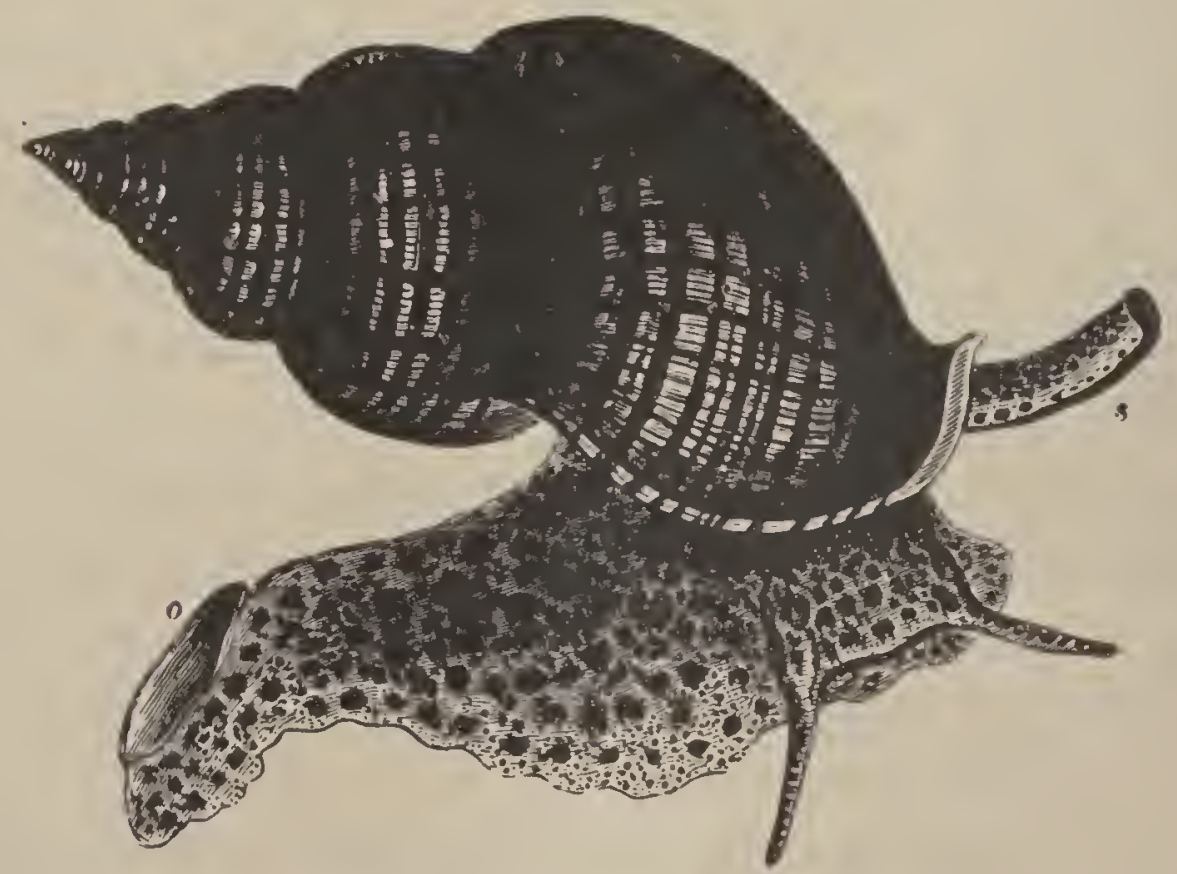

Fra. 228.-Whelk (Buccinum), showing operculum, 0 , and siphon, $\varepsilon_{0}$

spiral, the whorls passing obliquely (and generally from right to left), ${ }^{142}$ around a central axis, or "columella." 
When the columella is hollow (perforated), the end is called the "umbilicus." When the whorls are coiled around the axis in the same plane, we lave a discoidal shell, as the Planorbis. The mouth, or "aperture," of the shell is "entire" in most vegetable-feeding Snails, and notched or produced into a canal for the siphons in the. carnivorous species. The former are generally land and fresh-water forms, and the latter all marine. In some Gasteropods, as the River-snails and must Sea-snails, a horny or calcareous plate (operculum) is secreted on the foot, which closes the aperture when the animal withdraws into its shell. In locomotion, the shell is carried with the apex directed backward.

The body of most Gasteropods is unsymmetrical, the organs not being in pairs, but single, and on one side, instead of central. The mantle is continuous around the body, not bilobed, as in Lamellibranchs. A few, as the common Garden-snail, have a lung; but the vast majority breathe by gills. The head is more or less distinct, and provided with two tentacles, with auditory sacs at their bases; two eyes, which are often on stalks; and a straplike tongue covered with minute teeth. The heart is situated, in the majority, on the right side of the back, and has two cavities. The nervous ganglia are united into an œsophageal ring or collar (Figs. 45, 154). All, except the Pteropods, move by means of a ventral disk or foot.

Gasteropods are now the reigning Mollusks, comprising three fourths of all the living species, and are the types of the subkingdom. They have an extraordinary range in latitnde, altitude, and depth.

Omitting a few rare and aberrant forms, we may separate the class into the following orders:

1. Pteropods.-These are small, marine, floating Mollusks, whose main organs of motion resemble a pair of wings or fins coming out of the neck, whence the com- 
mon name, "Sea-butterflies." Many have a delicate, transparent shell. The head has six appendages, armed

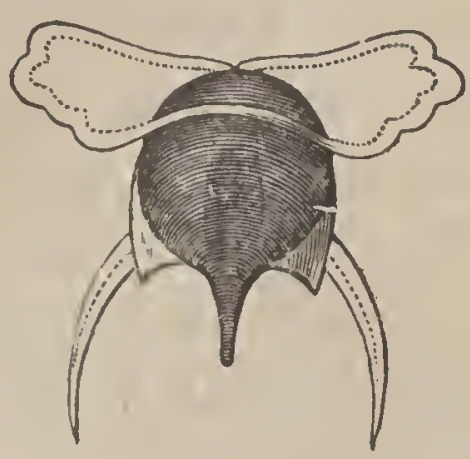

Fig. 229. - A Pteropod (Hyalea tridentata). Atlantic. with several hundred thousand microscopic suckers-a prehensile apparatus unequalled in complication. Pteropods occur in every latitude, but generally in mid-ocean, and in the aretic regions are the food of Whales and Sea-birds.

2. Opisthobranchs.-These low Gasteropods are, for the most part, naked Sea-slugs, a few only having a small shell. The feathery gills are behind the heart (whence the name). They are found in all seas, from the arctic to the torrid, generally on rocky coasts. When disturbed, most of them draw themselves up into a lump of jelly or tough skin.

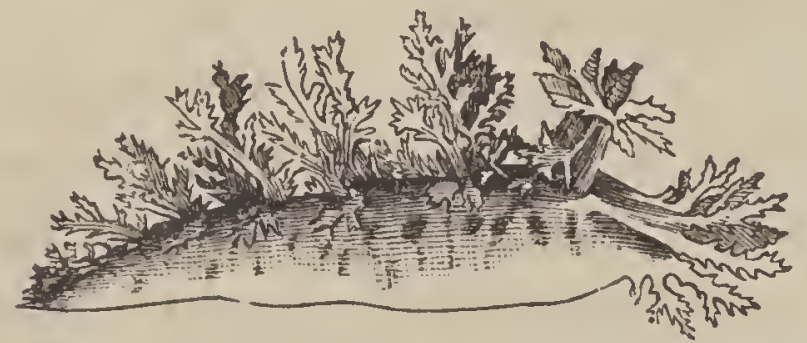

FIG. 230.-A Tritonian (Dendronotus arborescens). British seas.

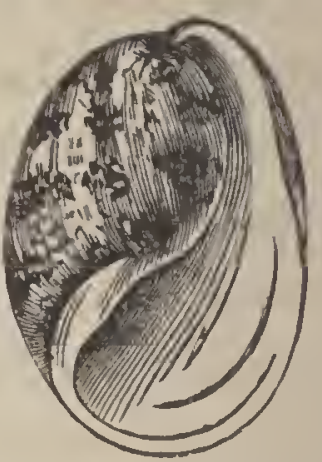

Fia. 231.-Bulla ampulla, or "Bubble-shell:" three fourths natural size. Indian Ocean

Examples: Sea-lemon (Doris), the beautiful Tritonia, the painted Asolis, the Sea-hare (Aplysia), which discharges a purple fluid, and the Bubble-shell (Bulla).

3. Pulmonates.-These air-breathing Gasteropods, represented by the familiar Snail, have the simplest form of lung-a cavity lined with a delicate net-work of bloodvessels, which opens externally on the right side of the neck. This is the mantle-cavity. The entrance is closed by a valve, to shut out the water in the aquatic tribes, and the hot, dry air of summer days in the land species. They are all fond of moisture, and are more or less slimy. Their shells are lighter (being thinner, and containing less 
earthy matter) than those of marine Mollusks, having to be carried on the back without the support of the water.

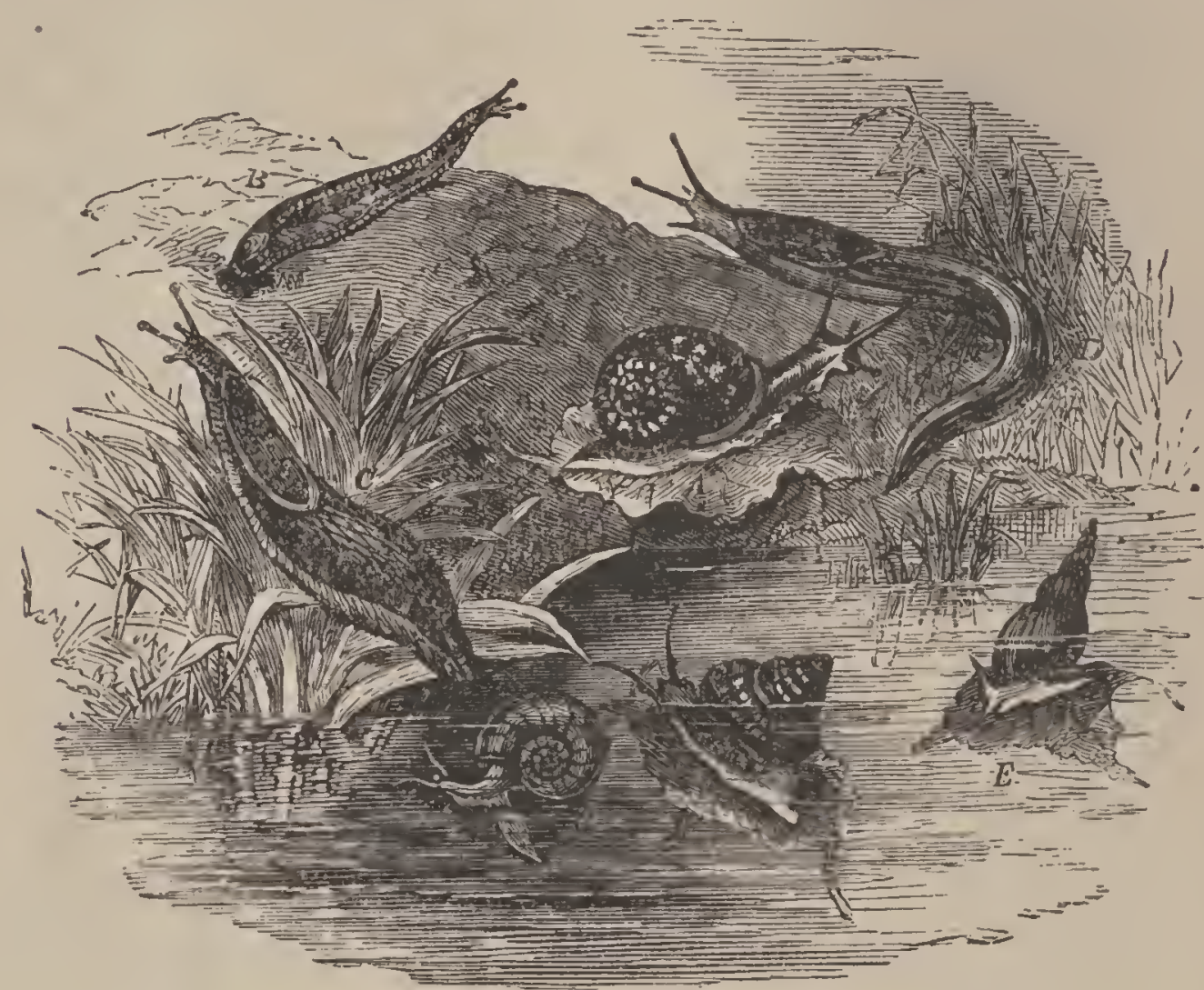

Fro. 232.-A, Land-snail (Helix); $B, C, D$, Slugs (Limax); $E, F, G$, Pond-snails (Limnoea, Paludina, and Planorbis).

Their eggs are laid singly, while the eggs of other orders are laid in chains.

They are found in all zones, but are most numerous where lime and moisture abound. All feed on vegetable matter. A few are naked, as the Slug; some are terrestrial; other's live in fresh water. The Land-snails, represented by the common Helix, the gigantic Bulimus, and the Slug (Limax), are distinguished by their four "horns," the short front pair being the true tentacles, and the long hinder pair being the eye-stumps. They have a saw-like upper jaw for

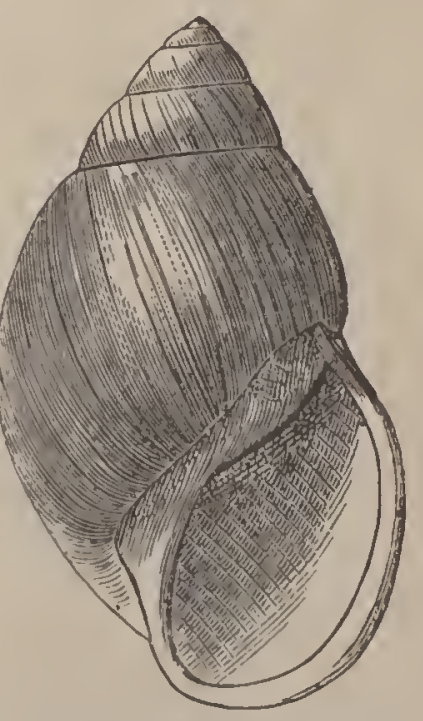

Fıg. 233. - Bulimus oblnngus; one half natural size. Guiana. biting leaves, and a short tongue covered with minute teeth. The Pond-snails, as Limnoea and Planorbis, differ 


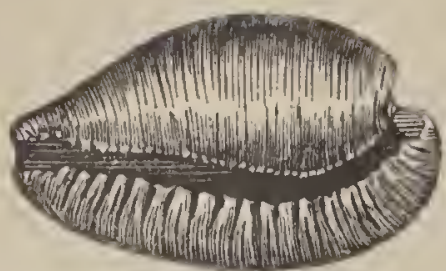

Fig. 234.-Cowry (Cyproea capensis); two thirds natural size. South Africa.

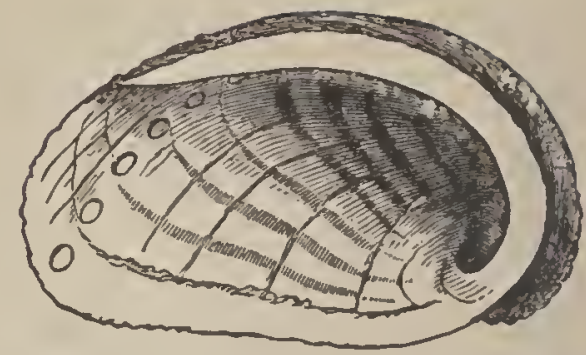

Fia. 235.-Haliotis, or "Pearly Ealshell." Pacific cuasts.

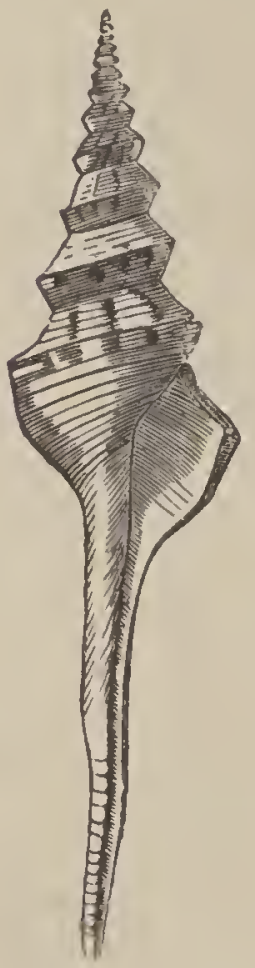

FIG. 236. - Spindleshell (Fusus colus); one half natural size. Ceylon.

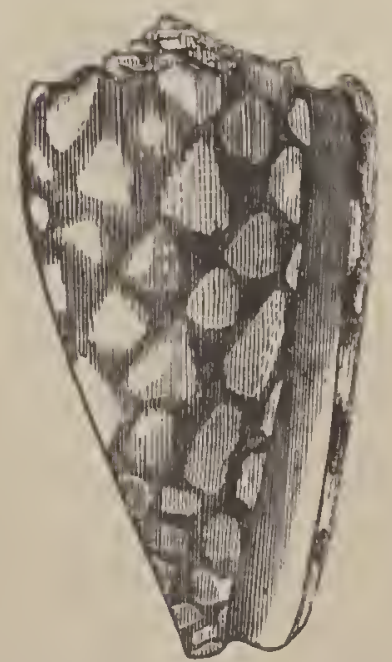

Fig. 239.-Cune-shell (Conus marmoreus); two thirds natural size. China seas.

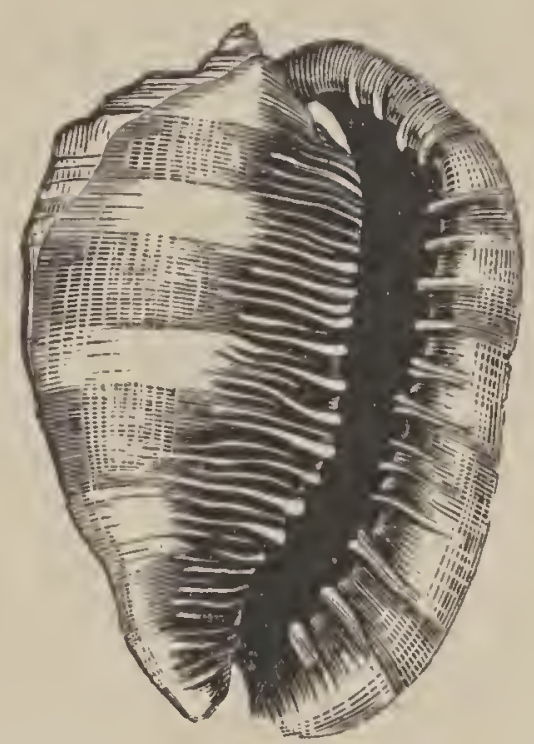

FIG. 237. - Cassis rufa, or "Helmet-shell:" one fourth uatural size. Indian Ocean.

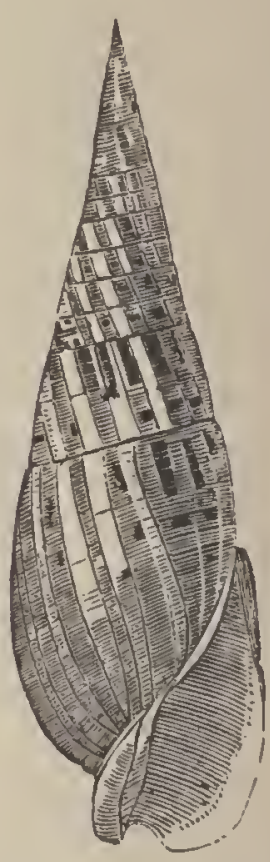

Fig. 23S.-Auger-shell (Terebra maculata): one half natural size. China seas.

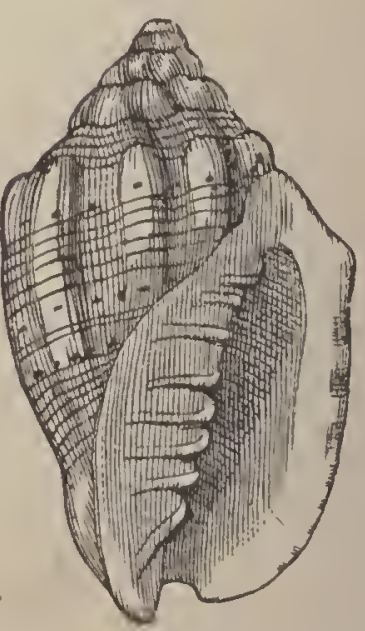

FIG. 240.-Chiton squamosus; one half natural size. West Indies.
Fig. 241.-Volute (Voluta musica); one half natural size. West Indies. 


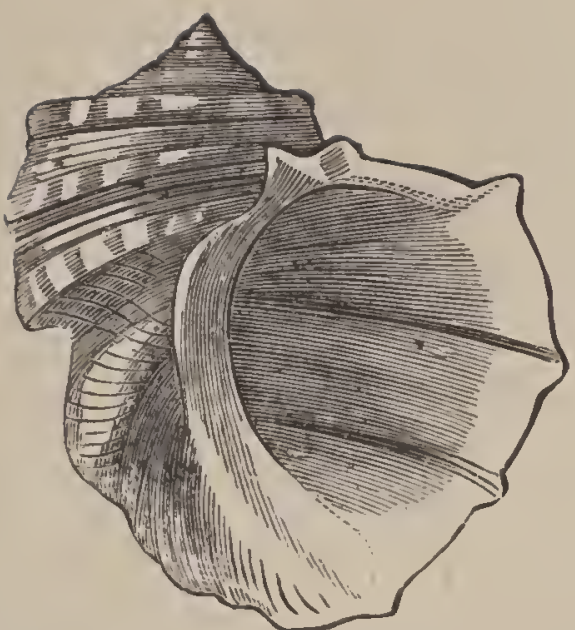

Fig. 242. - Top-shell (Turbo marnoratus); one fourth natural size. China keas.

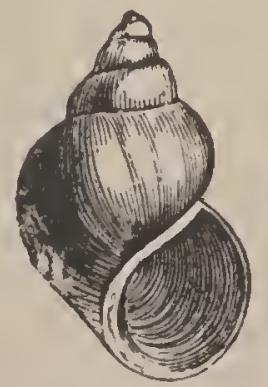

Frc. 244. - Paludina, a Fresh-water Snail.

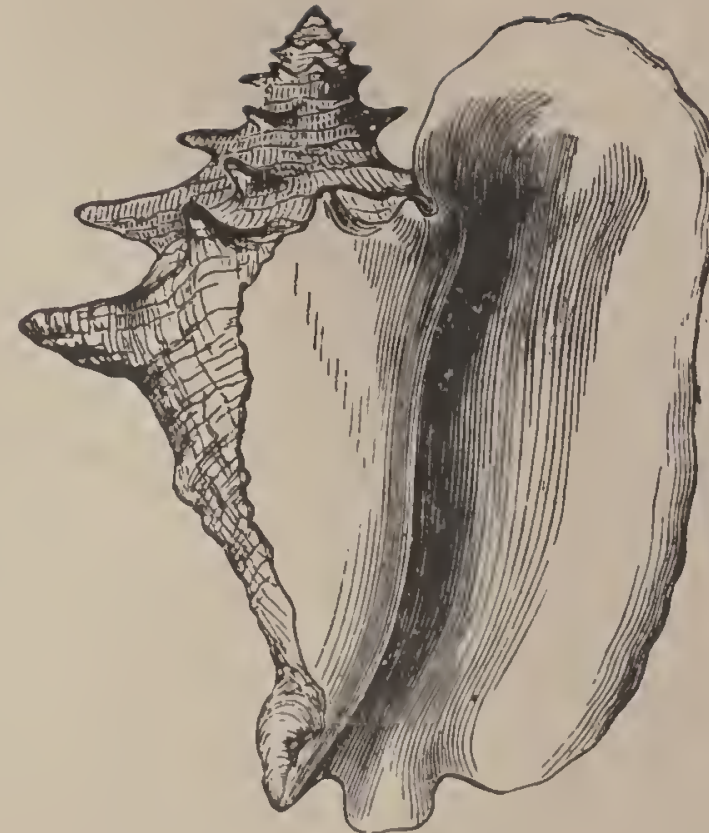

Fra. 243.-Strombus gigas, or "Wingedshell:" one fifth natural size. West Indies.

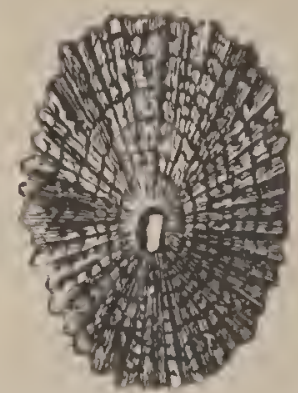

Fig. 245. - Key-hole Limpet (Fissurella listeri). West Indies.

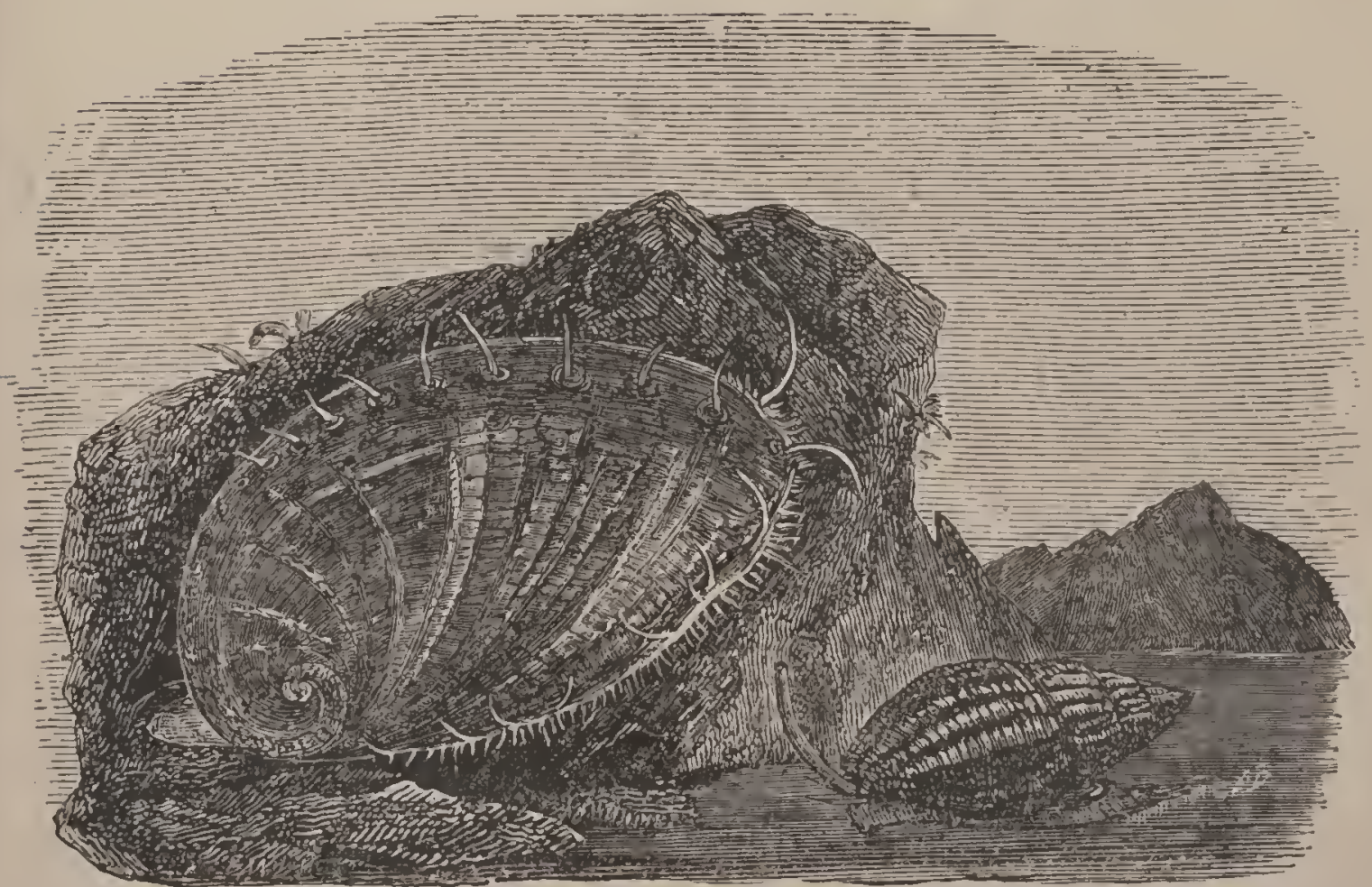

Fra. 246. -Ear-shell (H. tuberculata), and Dog-whell (Nassa reticulata). England. 
in having no eye-stalks, the eyes being at the base of the tentacles. They are obliged to come frequently to the surface of the water to breathe.

4. Prosobranchs. - These are aquatic Gasteropods, breathing by gills situated in front of the heart. They are the most highly organized and the most abundant of the crawling Mollusks. Nearly all are marine, and all have a shell.

Among the lower forms are the singular Chiton, covered with eight shelly plates; Limpet (Patella), well known to every sea-side visitor; and the beantiful Earshell (Haliotis), frequently used for ornaments and inlaidwork.

In the higher Prosobranchs, the gills are comb-shaped and the sexes are distinct. The group includes all the spiral univalve sea-shells, and a few fresh-water shells. Many have the aperture entire, which is closed with an operculum: as the dull-colored Paludina and Melania from fresh water, and the pyramidal Trochus, pearly Turbo, screw-like Turritella, common Periwinkle (Littorina), and globular Natica from the sea. Others, the highest of the race, have the margin of the aperture notched or produced into a canal, and are carnivorous and marine: such are nearly all the sea-shells remarkable for their beautiful forms, enamelled surfaces, and brilliant tints, as the Cowry (Cyprcea), Volute, Olive, Cone, Harp, Whelk (Buccinum), Cameo-shell (Cassis), Rock - shell (Murex), Trumpet-shell (Triton), Spindle-shell (Fusus), and Wingshell (Strombus).

\section{Class III.-Cephalopoda.}

The Cephalopods stand at the head of the subkingdom. The head is set off from the body by a slight constriction, and furnished with a pair of large, staring eyes, a mouth armed with a rasping tongue and a parrot-like beak, and 
eight or more tentacles or arms. The body is symmetrical, and wrapped in a muscular mantle.

The nervous system is more concentrated than in other Invertebrates; the cerebral ganglia are partly enclosed in a cartilaginous cranium. All the five senses are present. The class is entirely marine (breathing by plume-like gills on the sides of the body), and carnivorous. The naked species are found in every sea. Those with chambered shells (as Nautilus, Ammonites, and Orthoceras) were once very abundant: more than two thousand fossil species are known, but only one living representative - the Pearly Nautilus.

1. Tetrabranchs. - This order is characterized by the possession of four gills, forty or more short tentacles, and an external, chambered shell. The partitions, or septa, of the shell are united by a tube called "siphuncle," and the

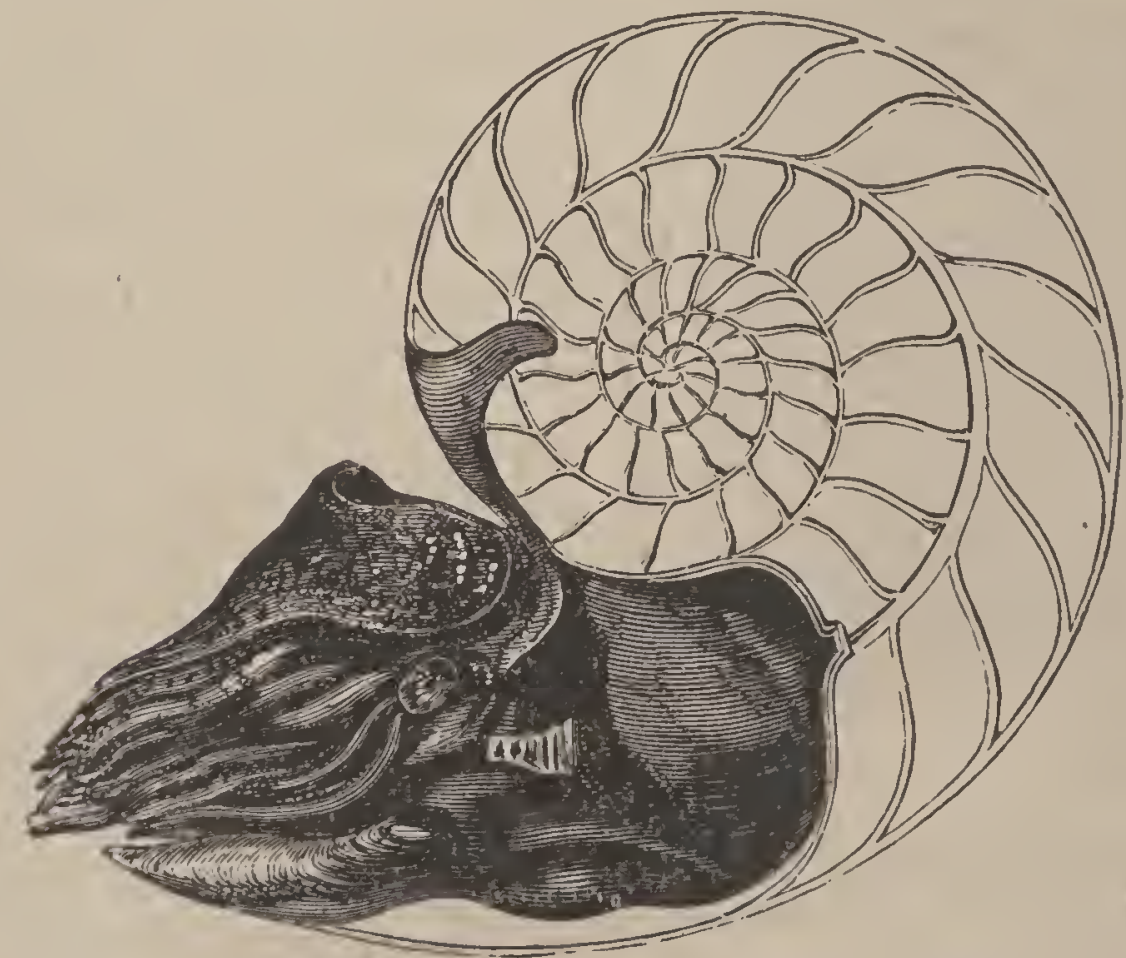

Fro. 247.-Pearly Nautilus, with shell bisected; one half natural sizc. Indian Ocean.

animal lives in the last and largest chamber. ${ }^{143}$ The living Nautilus has a smooth, pearly shell, a head retractile within the mantle or "hood," and calcareous mandibles, well fitted for masticating Crabs, on which it feeds. This 
stragglel of a mighty race dwells in the deep parts of the Indian Ocean, crawling on the bottom; and, while the shell is well known, only a few specimens of the animal have ever been obtained.

2. Dibranchs.-These are the most active of Mollusks, and the tyrants of the lower tribes. Among them are the largest of invertebrate animals. They are naked, having no external shell covering the body, but usually a horny or calcareous part within. They have a distinct

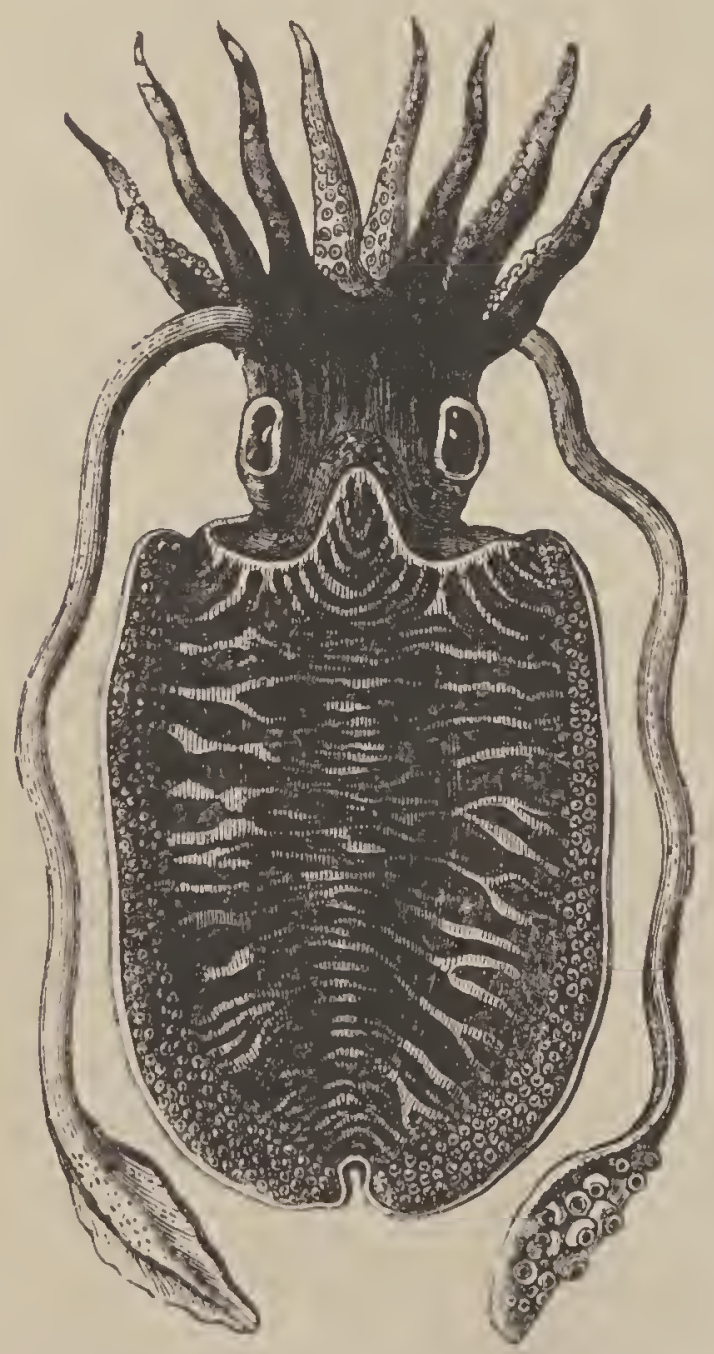

Fic. 24S. -Cuttle-fish (Sexia officinalis): one tifth uatural size. Atlantic coasts. head, prominent eyes, horny mandibles, eight or ten arms furnished with suckers, two gills, a complete tubular funnel, and an ink-bag containing a peculiar fluid (sepia), of intense blackness, with which the water is darkened to facilitate escape. They have the power of changing color, like the Chameleon. They crawl with their arms on the bottom of the sea, head downward, and also swin backward or forward, usually with the back downward, by means of fins, or squirt themselves backward by forcing water forward through their breathing funnels.

The Paper Nantilus ( $A r$ gonauta) and the Poulpe (Octopus) have eight arms. The female Argonant secretes a thin, unchambered shell for carrying its eggs. The Squid (Loligo) and Cuttle-fish (Sepia) have ten arms, the additional pair being much longer than the others. Their eyes are movable, while 
those of the Argonaut and Poulpe are fixed. The Squid, so much used for bait by cod-fishermen, has an internal horny "pen," and the Cuttle has a spongy, calcareous "bone." The extinct Belemnites had a similar structure.

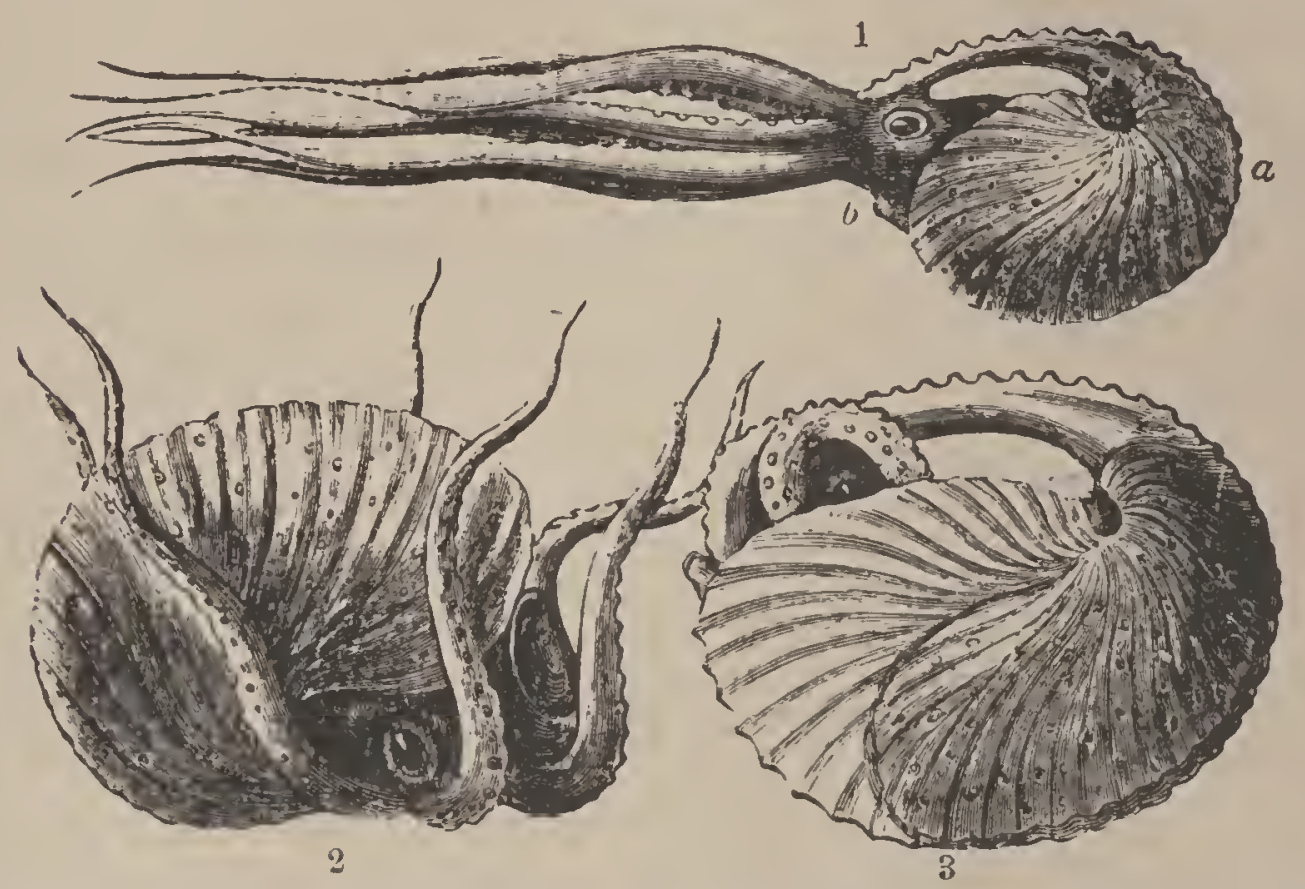

Fig. 249. - Paper Nautilus (Argonauta argo) : 1, swimming towards $a$ by ejecting water from funnel, $b ; 2$, crawling on the bottom; 3 , coiled within its shell, which is one fourth natural size. Mediterranean.

Squid have been found with a body seven feet and arms twenty-four feet long, and parts of others still larger-as much as fifty feet in total length.

\section{Subkingdom VII.-ARTHROPODA.}

This is larger than all the other subkingdoms put together, as it includes the animals with jointed legs, such as Crabs and Insects. These differ widely from the Molluscan type in having numerous segments, and in showing a repetition of similar parts; and from the Worms in having a definite number of segments and jointed legs.

The skeleton is ontside, and consists of articulated segments or rings. The limbs, when present, are likewise jointed and hollow. The jaws move from side to side. The nervons system consists mainly of a double chain of 
ganglia running along the ventral surface of the body under the alimentary canal. The brain is connected to the ventral ganglia by a ring encircling the gullet. The alimentary canal and the circulatory apparatus are nearly straight tubes lying lengthwise-the one through the centre, and the other along the back. The skeleton is composed of a horny substance (chitin), or of this substance with carbonate of lime. All the muscles are striated.

There are four principal classes, of which the first is water-breathing, and the others air-breathing.

\section{Class I.-Crustacea.}

The Crustacea ${ }^{144}$ are water-breathing Arthropoda, usnally with two pairs of antennæ. ${ }^{145}$ Among them are the largest, strongest, and most voracions of the sublingdom, armed with powerful claws and a hard cuirass bris'tling with spines. Although constructed on a common type, Crustaceans exhibit a wonderful diversity of external form: contrast, for example, a Barnacle and a Crab. We will select the Lobster as illustrative of the entire group.

A typical Crustacean consists of twenty segments, of which five belong to the head, eight to the thorax, and seven to the abdomen. ${ }^{146}$ In the Lobster, however, as in all the higher forms, the joints of the head and thorax are welded together into a single crust, called the cephatothorax. On the front of this shield is a pointed process, or rostrum; and attached to the last joint of the abdomen (the so-called "tail") is the sole representative of a tail -the telson. This skeleton is a mixture of chitin and calcareous matter. ${ }^{147}$

On the under-side of the body we find numerous appendages, feeler's, jaws, claws, and legs beneath the cephalo-tho$\mathrm{rax}$, and flat swimmerets under the abdomen. In fact, as a rule, every segment carries a pair of movable append- 
ages. The five segments of the head are compressed into a very small space, yet have the following members: ${ }^{147 a}$ the short and the long antennæ; the mandibles, or jaws, between which the mouth opens; and the two pairs of maxillæ. The thorax carries three pairs of modified limbs, called "foot-jaws," and five pairs of legs. The foremost legs, "the" great claws," are extraordinarily developed, and terminated by strong pincers (chelce). Of the four slender pairs succeeding, two are furnished with claws, and two are pointed. The last pair of swimmerets, together with the telson, form the candal finthe main instrument of locomotion; the others (called "swimmerets") are used by the female for carrying her eggs. The eyes are raised on stalks so as to be movable (since the head is fixed to the thorax), and are compound, made up of about two

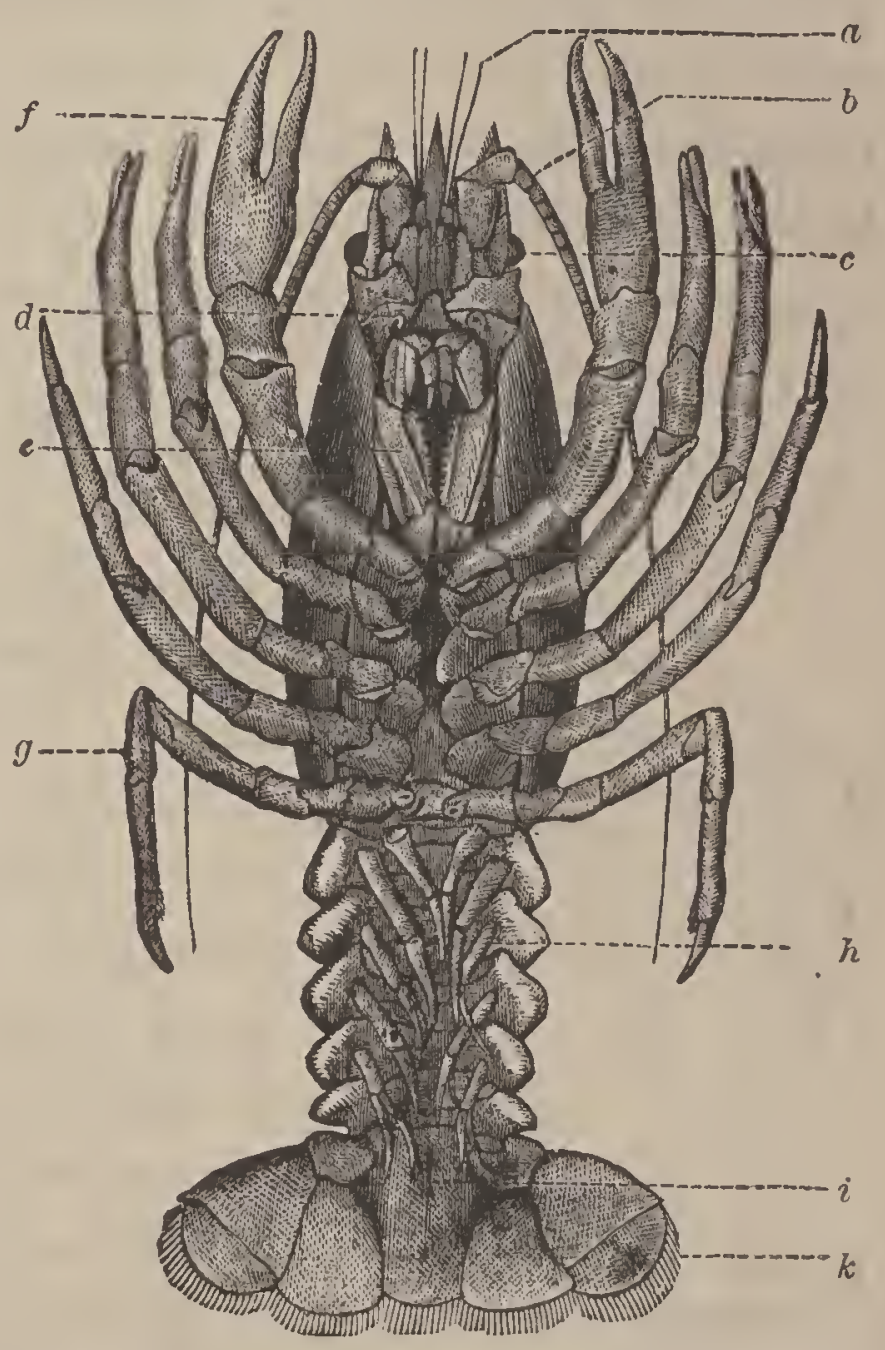

Fig. 250.-Under-side of the Cray-fish, or Freshwater Lobster (A stacus fluviatilis) : a, first pair of antennæ; $b$, second pair ; $c$, eyes; $d$, opening of kidney; $e$, foot-jaws; $f, g$, first and fifth pair of thoracic legs; $h$, swimmerets; $i$, anus; $k$, caudal fin. thousand five hundred square facets. At the base of each small antenna is a minute sac, whose month is guarded by hairs: this is the organ of hearing. The gills, twenty on a side, are situated at the bases of the legs and enclosed in two chambers, into which water is freely admitted, in fact, drawn, by means of a curions attachment to one of the 
maxillæ, which works like the "screw" of a propeller. The heart is a single oval cavity, and drives arterial blood -a milky fluid full of corpuscles. The alimentary canal consists of a short gullet, a gizzard-like stomach, and a straight intestine.

Crustaceans pass through a series of strange metamorphoses before reaching their adult form. They also periodically cast the shell, or moult, every part of the integument being renewed; and another remarkable endowment is the spontaneous rejection of limbs and their complete

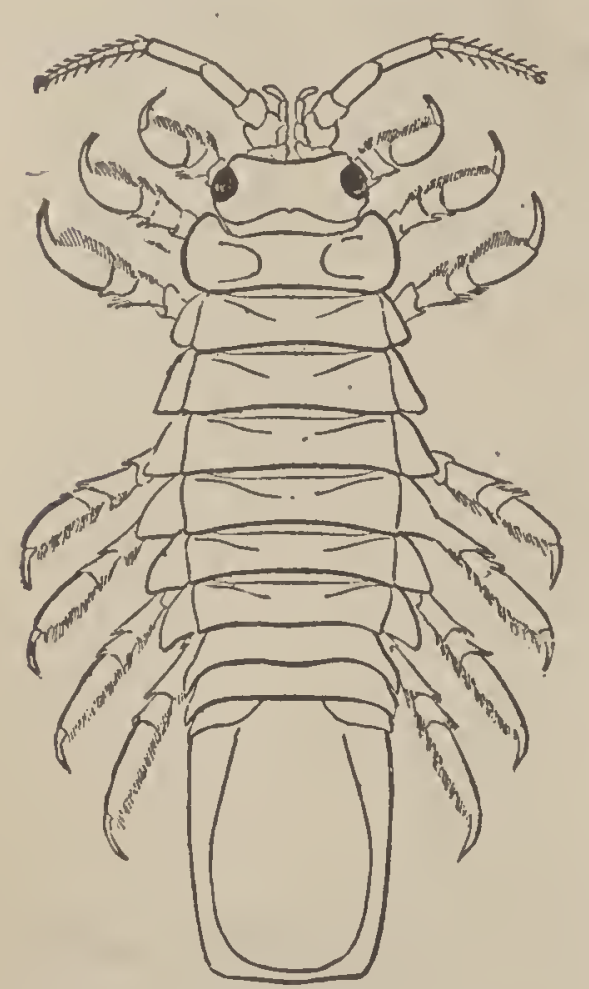

Fig. 251.-1dotea robusta: a Te-

tradecapod. U. S. coast. restoration. Many species are found in fresh water, but the class is essentially marine and carnivorous.

Of the numerous orders of this great class we will mention only four :

1. Cirripeds, distinguished by being fixed, by having a shelly covering, and by their feathery arms (cirri). Such are Barnacles (Lepas)and A corn-shells (Balanus), so common on rocks and timbers by the sea-shore.

2. Entomostracans, which agree in having a horny shell and no abdominal limbs; represented by the little Water-fleas (Cyclops) of our ponds, and

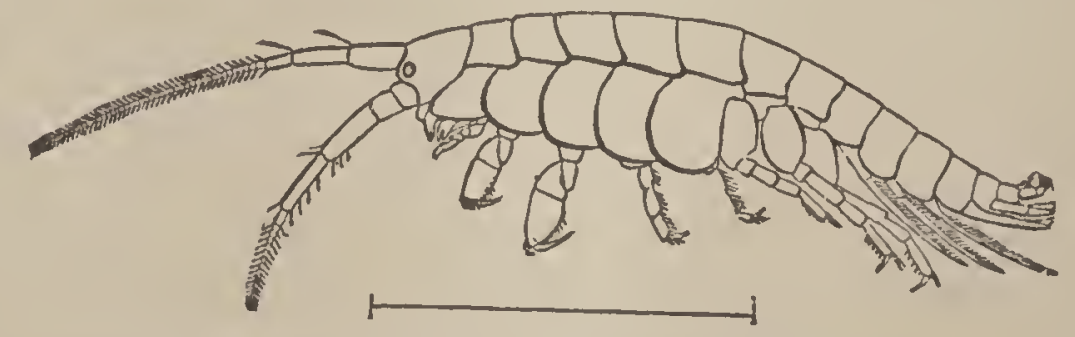

Fra. 252.-Amphithoë maculata: a* Sand-flea.

the Brine-shrimps (Artemia), and many others. The Kingcrabs (Limulus) and the extinct Trilobites were formerly 


\section{ARTHROPUDA.}

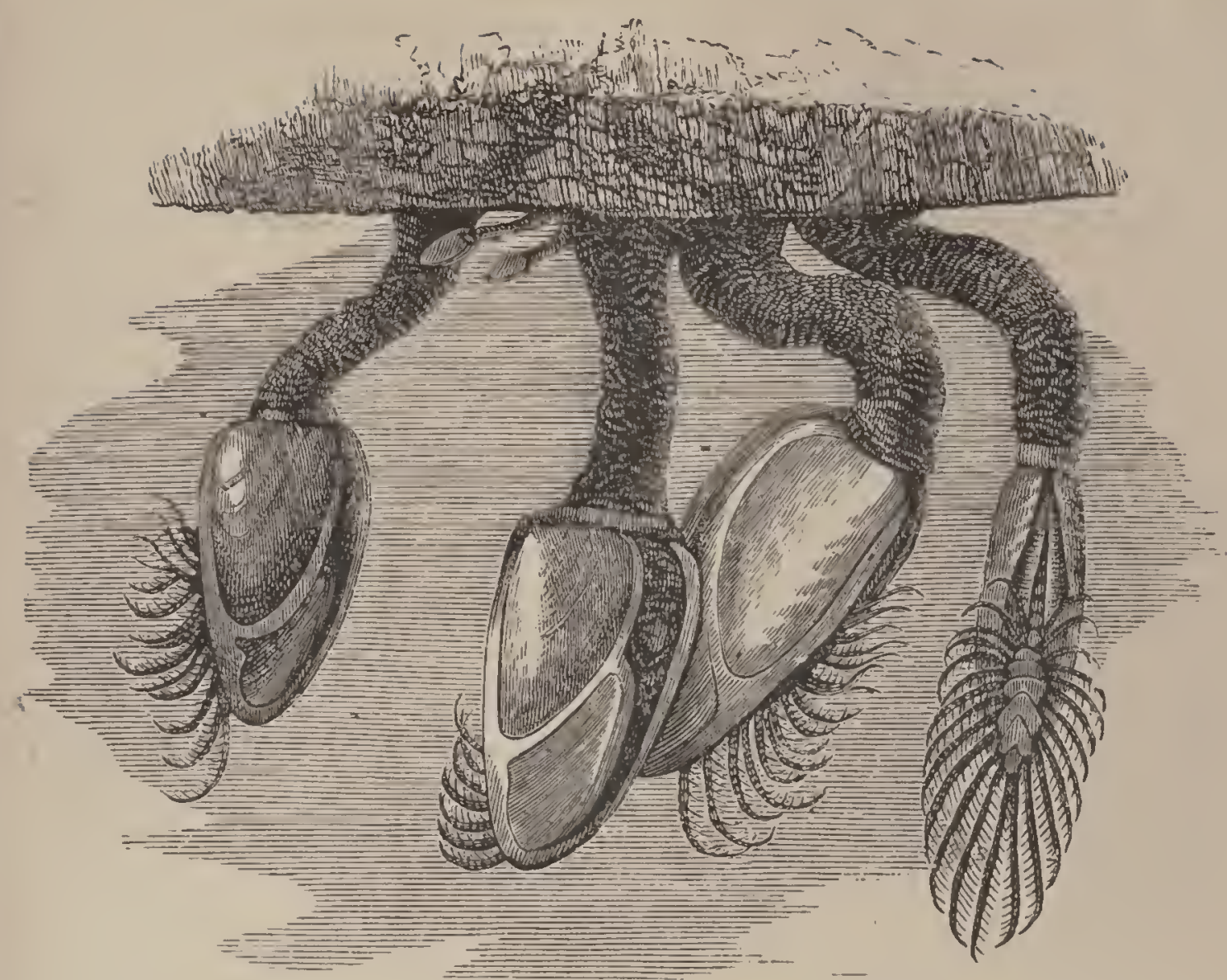

Fig. 253.-Barnacles, or Pedunculate Cirripedes (Lepas anatifera).

united to this class, but now are known to be widely removed from it. The former is by some authors removed from the Crustacea.

3. Tetradecapods, small, fourteen-footed species; as the

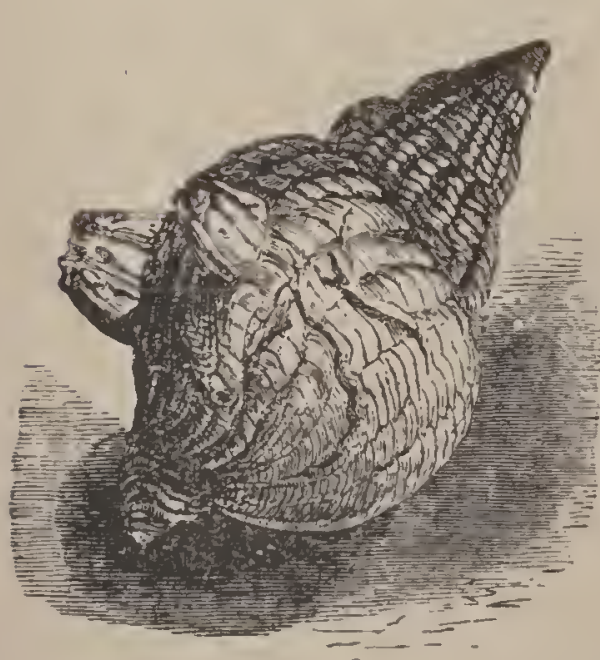

Fig. 254.-Acorn-shells (Balanus) on the Shell of a Whelk (Buccinum).

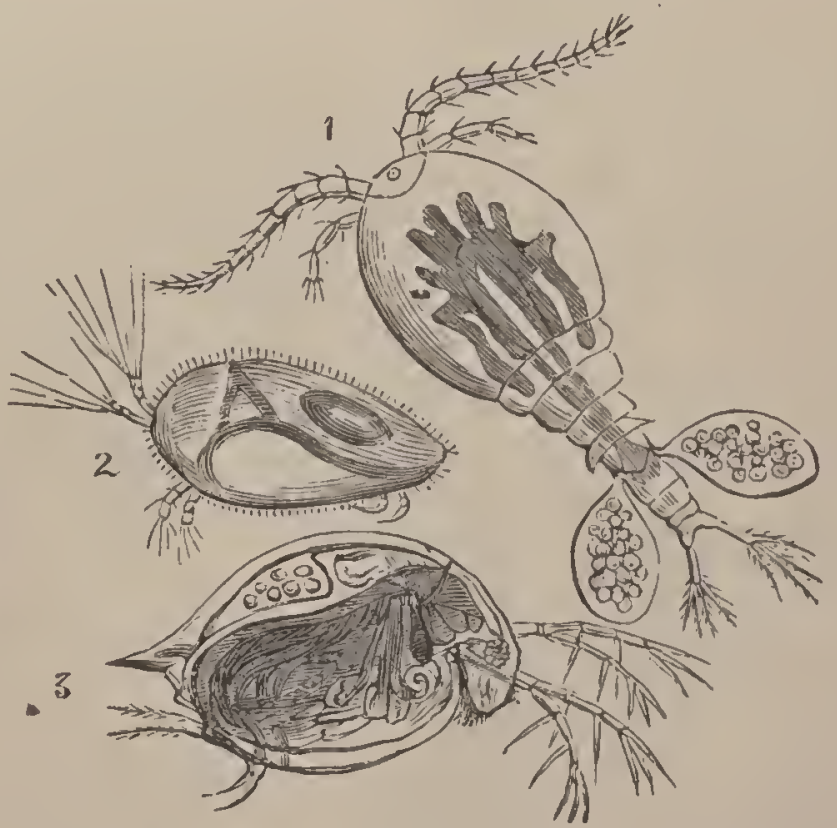

F1a. 255.-Water-Heas: 1, Cyclops communis; 2. Cypris unifasciata; 3, Daphnia pulex. 
Wood-louse, or Sow-bug (Oniscus), so common in damp places, the Slaters (Idotea), and the Sand-fleas (Gammarus), seen by the sea-side.

4. Decapods, having ten legs, as the Shrimp (Crangon),

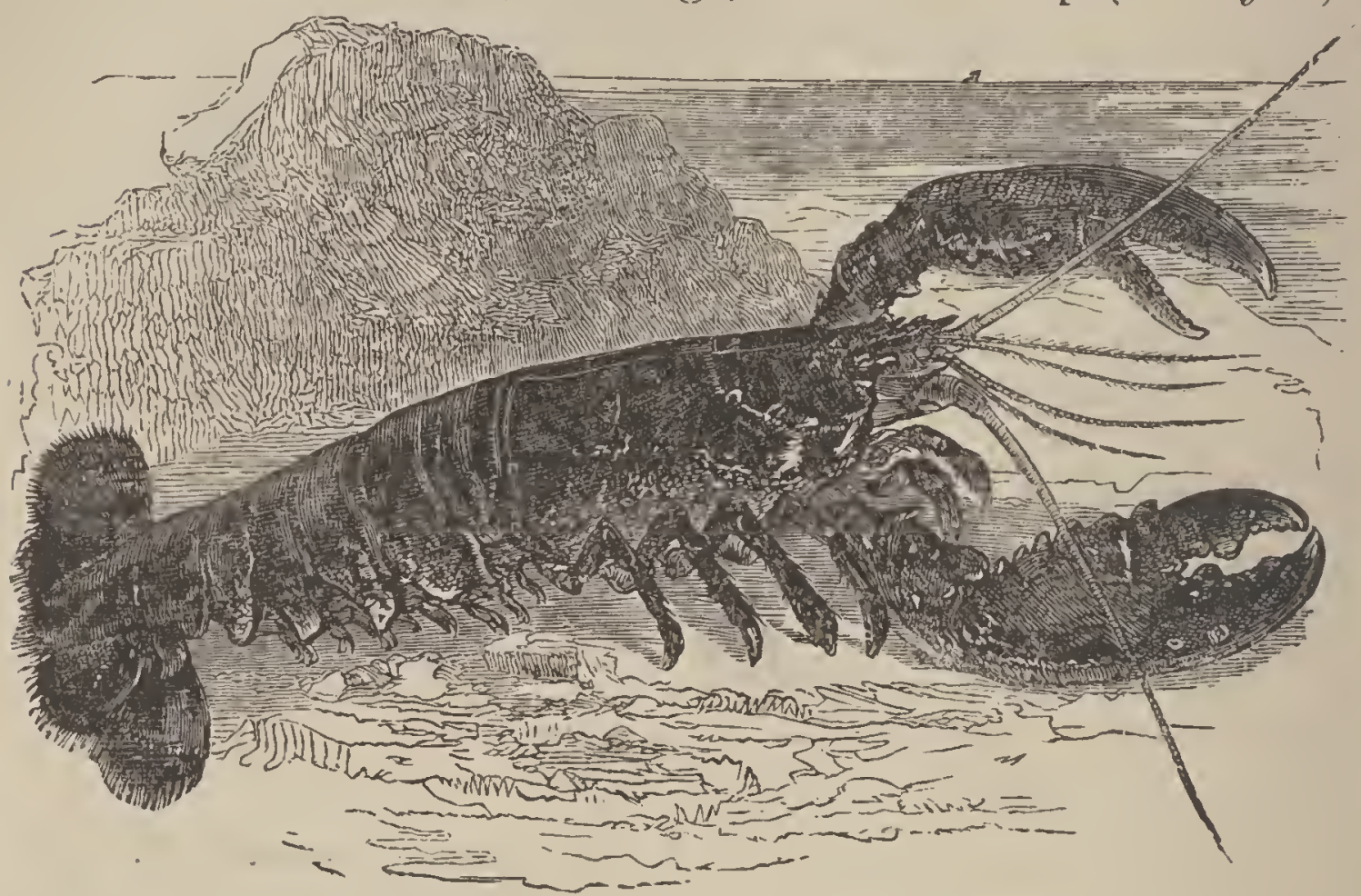

Fia. 256.-Lobster (Homarus vulgaris).

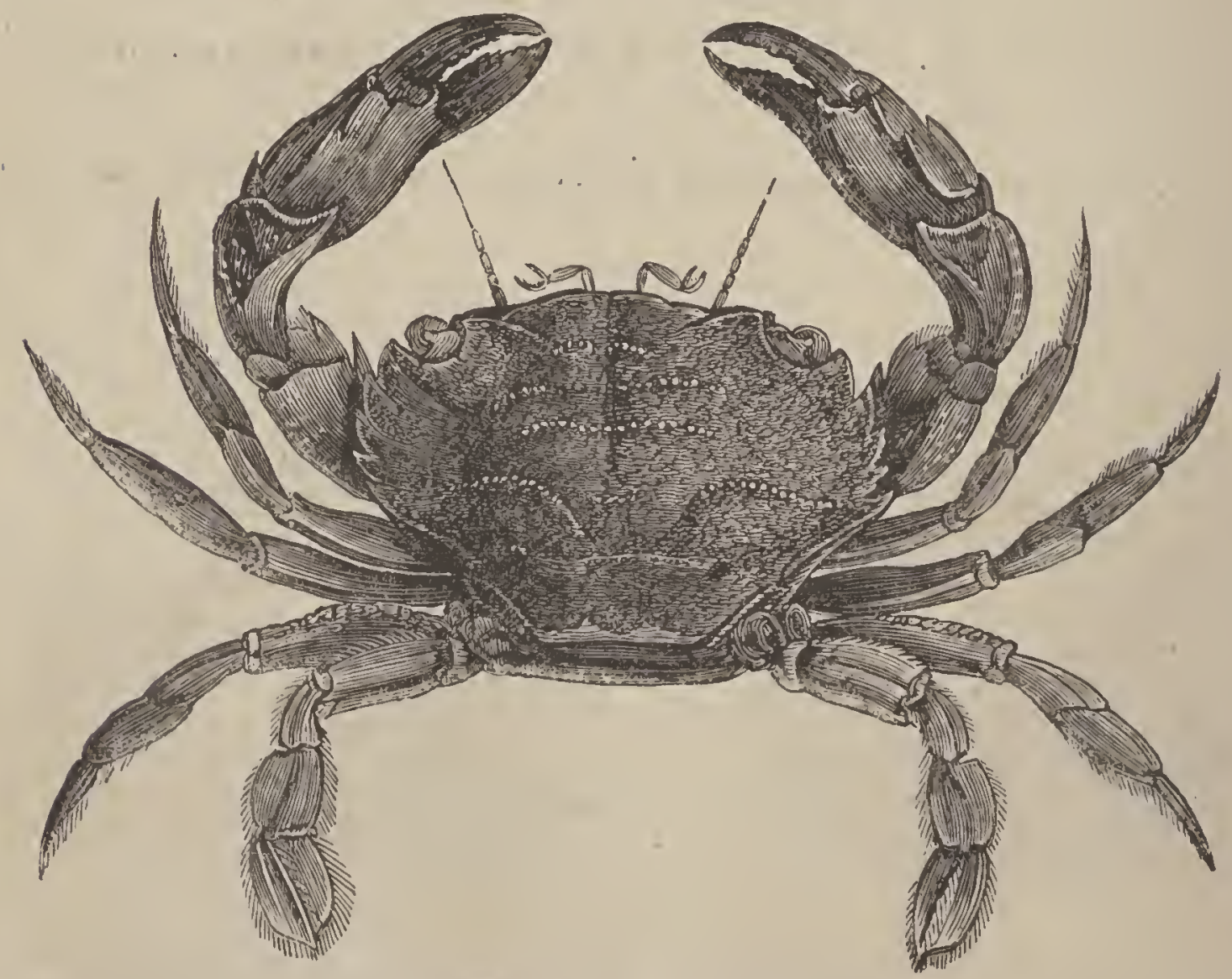

FIG. 257.-Swimming Crab (Platyonychus). 
Cray-fish (Astacus), Lobster (Homarus), and Crab (Cancer). Crabs differ from Lobsters chiefly in being formed for creeping at the bottom of the sea instead of swimming, and in the reduction of the abdomen or "tail" to a rudiment, which folds into a groove under the enormous thorax. They are the highest and largest of living Crustacea: they have been found at Japan measuring fifteen feet between the tips of the claws.

\section{Class. II.-Myriapoda.}

Myriapods differ from Crustaceans and Spiders in having the thorax merged in the abdomen, while the head is free. In other words, the body is divided into similar segments, so that thorax and abdomen are scarcely distinguishable. They resemble Worms in form and in the simplicity of their nervous and circulatory systems; but the skin is stiffened with chitin, and the legs (indefinite in number) are articulated. The legs resemble those of Insects, and the head appendages follow each other in the same order as in Insects-eyes, antennæ, mandibles, maxillæ, and labium. They breathe by tracheæ, and have two antennæ and a variable number of eyes.

There are two orders:

1. Chilognatha, having a cylindrical body, each segment, except the anterior, being furuished with two pairs of legs. They are of slow locomotion, harmless, and vegetarian. The Thousand-legged Worm (Julus) is a common representative.

2. Chilopoda, characterized by having a flattened body composed of about twenty segments, each carrying one pair of legs, of which the hindermost is converted into spines. They have longer antennæ than the preceding, and the mouth is armed with two formidable fangs connected with poisonous glands. They are carnivorous and active. Such is the Centipede (Scolopendra, Fig. 259). 


\section{Class III.-Arachnida.}

The Arachnids are closely related to the Crustaceans, having the body divided into a cephalo-thorax and abdomen. ${ }^{148}$ To the former are attached eight legs of seven joints each; the latter has no locomotive appendages. The head carries two, six, or eight eyes, smooth and sessile ( $i$. e., not faceted and stalked, as in the Lobster), and approaching the eye of the Vertebrates in the completeness and perfection of their apparatus. The anteunæ, if present, are only two, and these are not "feelers," but modified to serve for the prehension of food. ${ }^{149}$ They are all air-breathers, having spiracles which open either into air-sacs or trachex. The young of the ligher forms undergo no metamorphosis after leaving the egg.

Arachuids number nearly five thousand species. The typical forms may be divided into three groups:

1. Acarina, represented by the Mites and Ticks. They have an oval or rounded body, without any marked articulations, the head, thorax, and

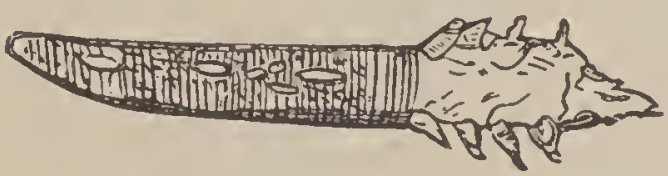
abdomen being apparently merged into one. They have no brain; only a single gan-

Fig. 255. - A Mite (Demodex folliculo rum), one of the lowest Arachnids; glion lodged in the abdomen. a parasite in human hair-sacs; $\times 125$. They breathe by tracheæ. The mouth is formed for suction, and they are generally parasitic. The Mites (Acarus) are among the lowest of Articulates. The body is soft and minute. The Ticks (Ixodes) have a leathery skin, and are sometimes half an inch long. The month is furnished with a beak for piercing the animal it infests.

2. Pedipalpi, or Scorpions, characterized by very large maxillary palpi ending in forceps, and a prolonged, jointed abdomen. The nervous and circulatory systems are more highly organized than those of Spiders; but the 
long, tail-like abdomen and the abnormal jaws place them in a lower rank. The abdomen consists of twelve segments: the anterior half is as large as the thorax, with no well-marked division between; the other part is comparatively slender, and ends in a hooked sting, which is perforated by a tube leading to a poison-sac. The mandibles are transformed into small, nipping claws, and the eyes generally number six. Respiration is carried on by four pairs of pulmonary sacs which open on the under surface

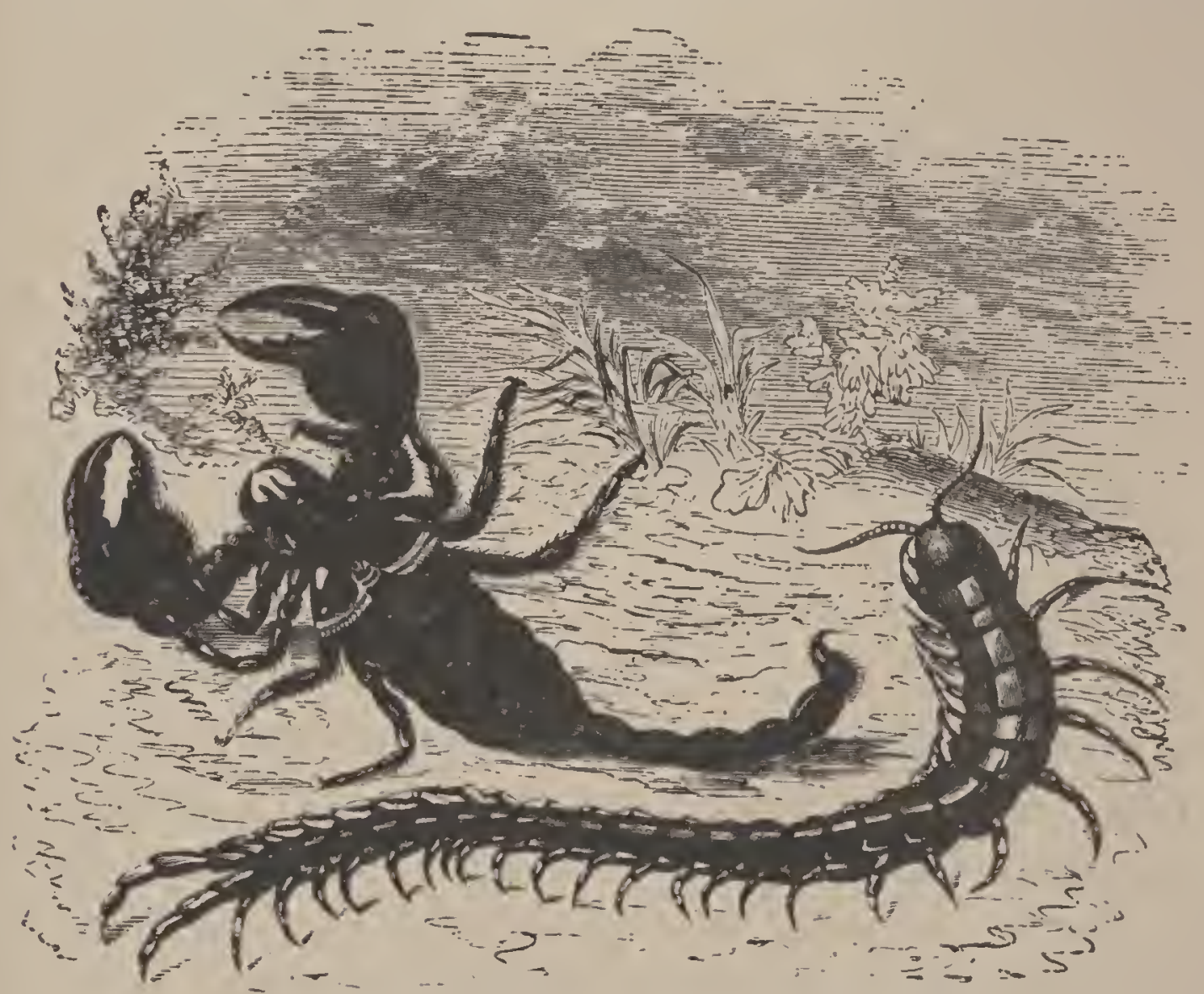

FIG. 259.-Scorpion (under surface) and Centipede.

of the abdomen. The heart is a strong artery, extending along the middle of the back, and divided into eight separate chambers. Scorpions are confined to the warm-temperate and tropical regions, usually lurking in dark, damp places.

The Harvest-men (Phalangium), frequently seen about our houses, belong to this order. They have a short, thick body and extremely long legs, and breathe by tracheæ.

3. Araneina, or Spiders. They are distinguished by 
their soft, unjointed abdomen, separated from the thorax by a narrow constriction, and provided at the posterior end with two or three pairs of appendages, called "spin-

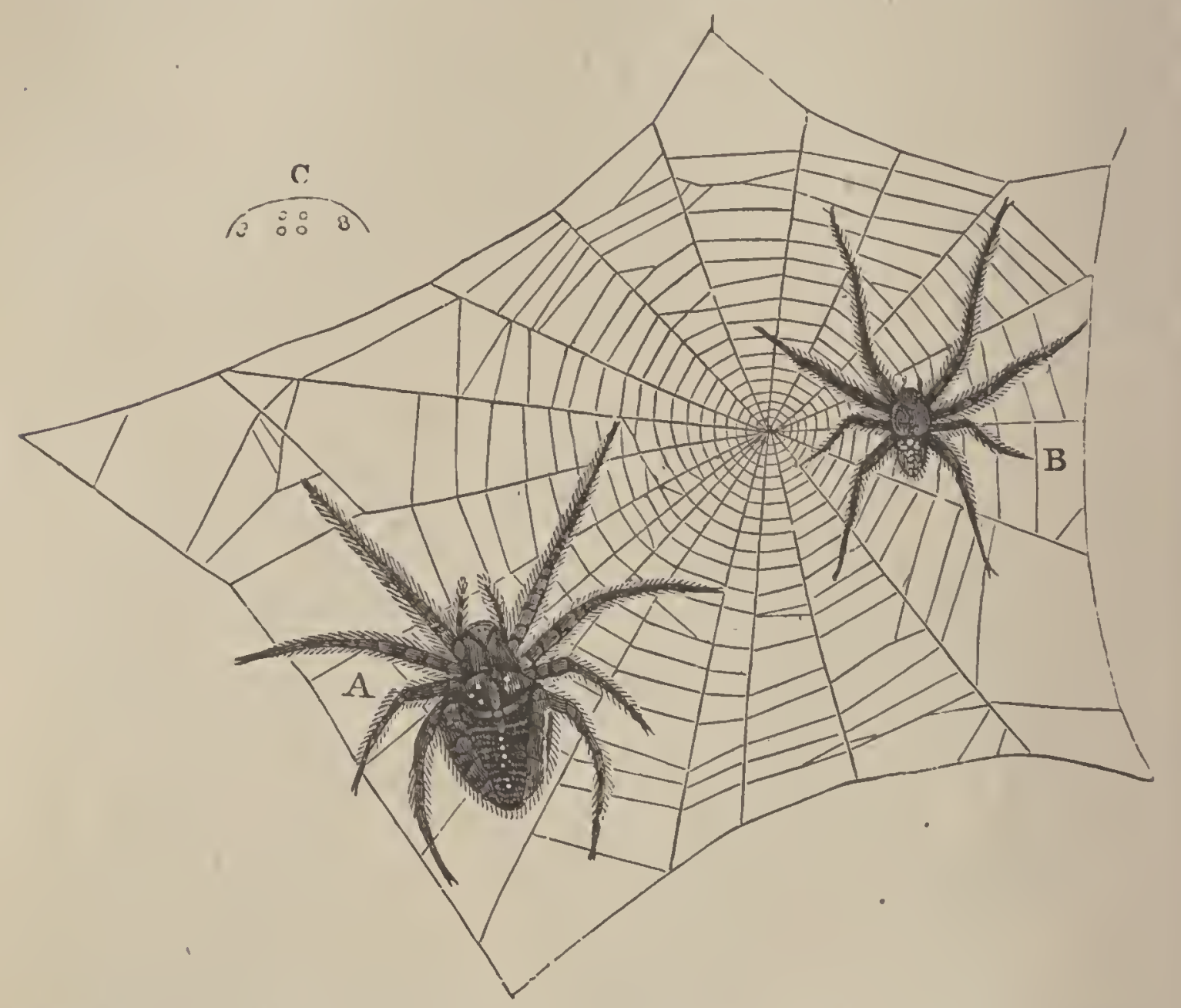

Frg. 260.-A, female Spider ; B, male of same species: C, arrangement of the eyes.

nerets," which are homologons with legs. The office of the spinnerets is to reel out the silk from the silk-glands, the tip being perforated by a myriad of little tubes, through which the silk escapes in excessively fine threads. An ordinary thread, just visible to the naked eye, is the union of a thousand or nore of these delicate streams of silk. ${ }^{150}$ These primary threads are drawn ont and mited by the hind legs.

The mandibles are vertical, and end in a powerful hook, in the end of which opens a duct from a poison-gland in the head. The maxillæ, or "palpi," which in Scorpions are changed to formidable claws, in Spider's resemble the thoracic feet, and are often mistaken for a fifth pair. The brain is of larger size, and the whole nervous system more 
concentrated than in the preceding order. There are generally eight simple eyes, rarely six. They breathe both by trachex and lung-like sacs, from two to four in number, situated under the abdomen. All the species are carnivorous.

The instincts of Spiders are of a high order. They are, perhaps, the most wily of Articulates. They display remarkable skill and industry in the construction of their webs; and some species (called "Mason Spi-

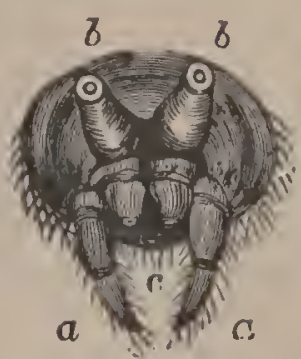

Fig. 261. - Spinnerets of the spider, $b, c ; a$, palpifurm organs. ders") even excavate a subterranean pit, line it with their. silken tapestry, and close the entrance with a lid which moves upon a hinge. ${ }^{151}$

\section{Class IV.-Insecta.}

Insects are distinguished by having head, thorax, and abdomen distinct, three pairs of jointed legs, one pair of antennæ, and generally two pairs of wings. The number of segments in the body never exceeds twenty. The head, apparently one, is formed by the union of fonr segments. The thorax consists of three - the prothorax, mesothorax, and metathorax-each bearing a pair of legs; the wings, if present, are carried by the last two segments. The abdomen is normally composed of ten segments, more or less movable upon one another. The skin is hardened with chitin, and to it, as in all Arthropods, the muscles are attached. The organs of sense are confined to the cephalic division of the body, the motor organs to the thoracic, and the vegetative to the abdominal. All the appendages are hollow.

The antennæ are inserted between or in front of the eyes. There is a great variety of forms, but all are tubular and jointed. They are supposed to be organs of touch, and also seem to be sensitive to sound. The eyes are. usually compound, composed of a large number of hexagomal corneæ, or facets (from fifty in the Ant to inany thou. 
sands in the winged Insects). They are never placed on

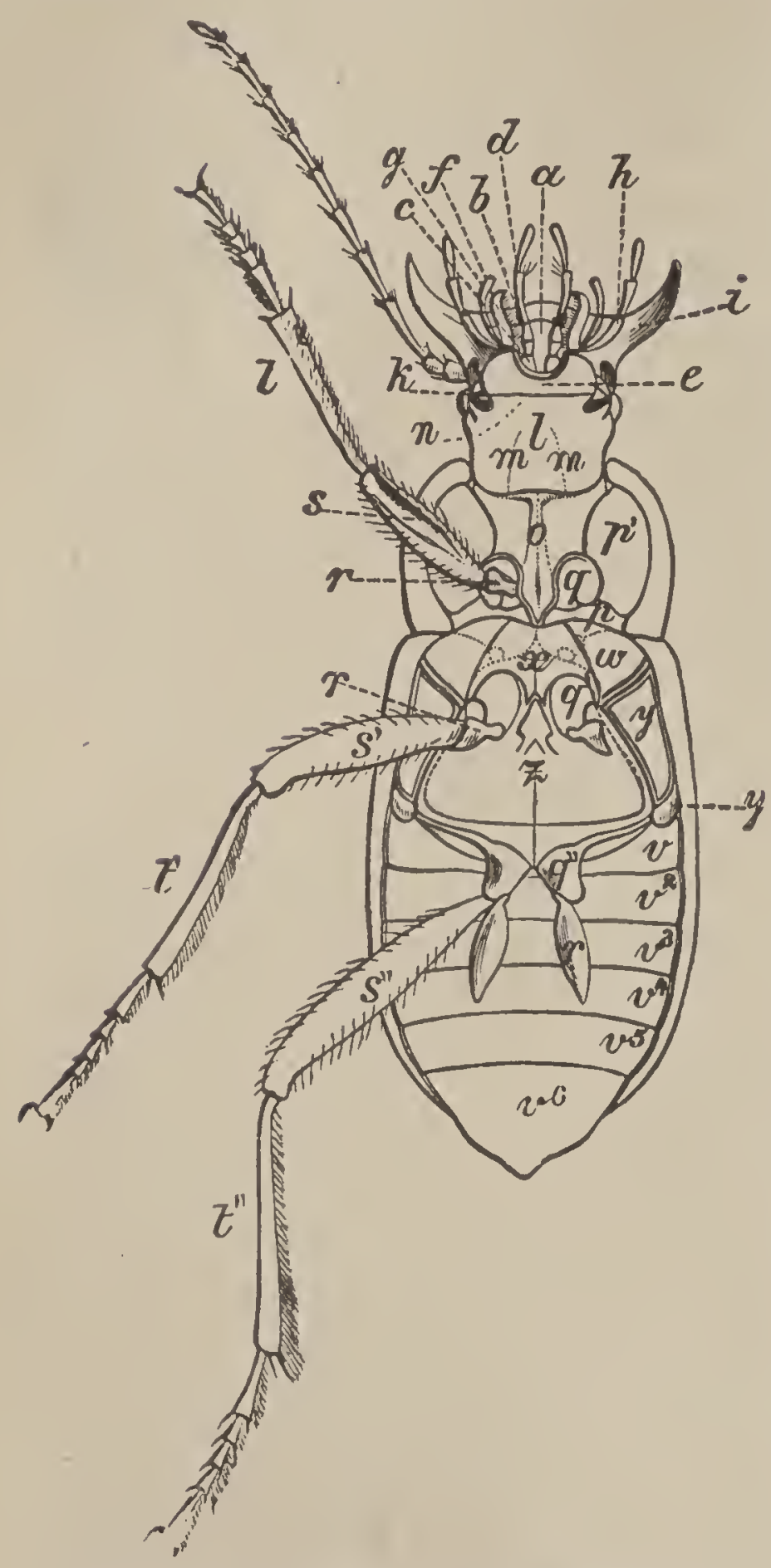

E::c. 262. -Under surface of a Beetle (Harpalus caltginosus): $a$, ligula : $b$, paraglossæ; $c$, supports of labial palpi; $d$, labial palpus; $e$, mentum; $f$, inner lobe of maxilla; $g$, outer lobe; $h$, maxillary palpus: $i$, mandible; $k$, buccal opening: $l$, gula, or throat: $m$, buccal sutures; $n$, gular suture; 0 , prosterunm; $p$, episternum of prothoras ; $p^{\prime}$, epimeron: $q, q^{\prime}, q^{\prime \prime}$, coxæ; $r, r, r$, trochanters; $s_{1}$ $s^{\prime}, s^{\prime \prime}$, femora, or thighs; $t, t^{\prime}, t^{\prime \prime}$, tibæ; $v$, ventral abdumiual segments; $w$, episterna of mesothornx ; $x$, mesosternum; $y$, episterna of metathorax : $y^{\prime}$, epimeron; $z$, metastelnum.

and foot. ${ }^{162}$ Some larvæ have also movable stalks, as the Lobster's. Besides these, there are three simple eyes, called ocelli. 'The mouth may be fitted for biting (masticatory), as in Beetles, or for sucking (suctorial), as in Butterflies. The masticatory type, which is the more complete, and of which the other is but a modification, consists of four horny jaws (mandibles and maxilloe) and an upper and an under lip (labrum and labium). Sensitive palpi (maxillary and labial) are developed from the lower jaw and lower lip. The labium is also prolonged into a ligula, or tongue.

The legs are invariably six in the adult, the fore-legs directed forward and the hinder pairs backward. Each consists of a hip, thigh, shank, "false legs," without 
joints, on the abdomen, upon which they chiefly rely in locomotion. The wings are expansions of the crust, stretched over a net-work of horny tubes. The venation, or arrangement of these tubes (called veins and veinlets), particularly in the fore-wings, is peculiar in each genus. In many Insects, the abdomen of the female ends in a tube which is the sheath of a sting, as in the Bee, or of an ovipositor, or "borer," as in the Ichneumon, by means of which the eggs are deposited in suitable places.

Cephalization is carried to its maximum in this class, and we have animals of the highest instincts under the articulate type. The "brain" is formed of several ganglia massed together, and lies across the upper side of the throat, just behind the mouth. The main cord lies along the ventral side of the body, with a swelling for each segment; besides this, there is a visceral nerve representing, in function, the sympathetic system of Vertebrates. The digestive apparatus consists of a pharynx, gullet (to which a crop is added in the Fly, Butterfly, and Bee tribes), gizzard, stomach, and intestine. There are no absorbent vessels, the chyme simply transuding through the walls of the canal. The blood, usually a colorless liquid, is driven by a chain of liearts along the back, $i$. e., by a pulsating tube divided into valvular sacs, ordinarily eight, which allow the current to flow only towards the head. As it leaves this main pipe, it escapes into the cavities of the body, and thus bathes all the organs. Although the blood does not circulate in a closed system of blood-vessels, as in Vertebrates, yet it always takes one set of channels in going from the heart, and another in returning. Respiration is carried on by tracheæ, a system of tubes opening at the surface by a row of apertures (spiracles), generally nine on each side of the body.

The sexes are distinct, and the larræ are hatched from eggs. As a rule, an Insect, after reaching the adult, or 
imago, state, lives from a few hours to several years, and dies after the process of reproduction. Growth takes place only during larval life, and all metamorphoses occur then. Among the social tribes, as Bees and Ants, the majority (called "workers") do not develop either sex.

Insects (the six-footed Arthropods) comprise nearly one half of the whole Aninal Kingdom, or from one hundred and seventy thousand to two hundred thousand species. They may be grouped into seven principal orders:

Lower series: body usually flattened; prothorax large and Neuroptera, squarish ; mouth-parts usually adapted for biting; met- Orthoptera, amorphosis often incomplete; pupa often active; larra Hemiptera, flattened, often resembling the adult.

Coleoptera.

Higher series: body usually cylindrical; prothorax small; mouth-parts more generally formed for sucking; metamorphosis complete; pupa inactive ; larva usually cylindricul, very unlike the adult.

Diptera, Lepidoptera, Hymenoptera.

1. Neuroptera have a comparatively long, slender body,

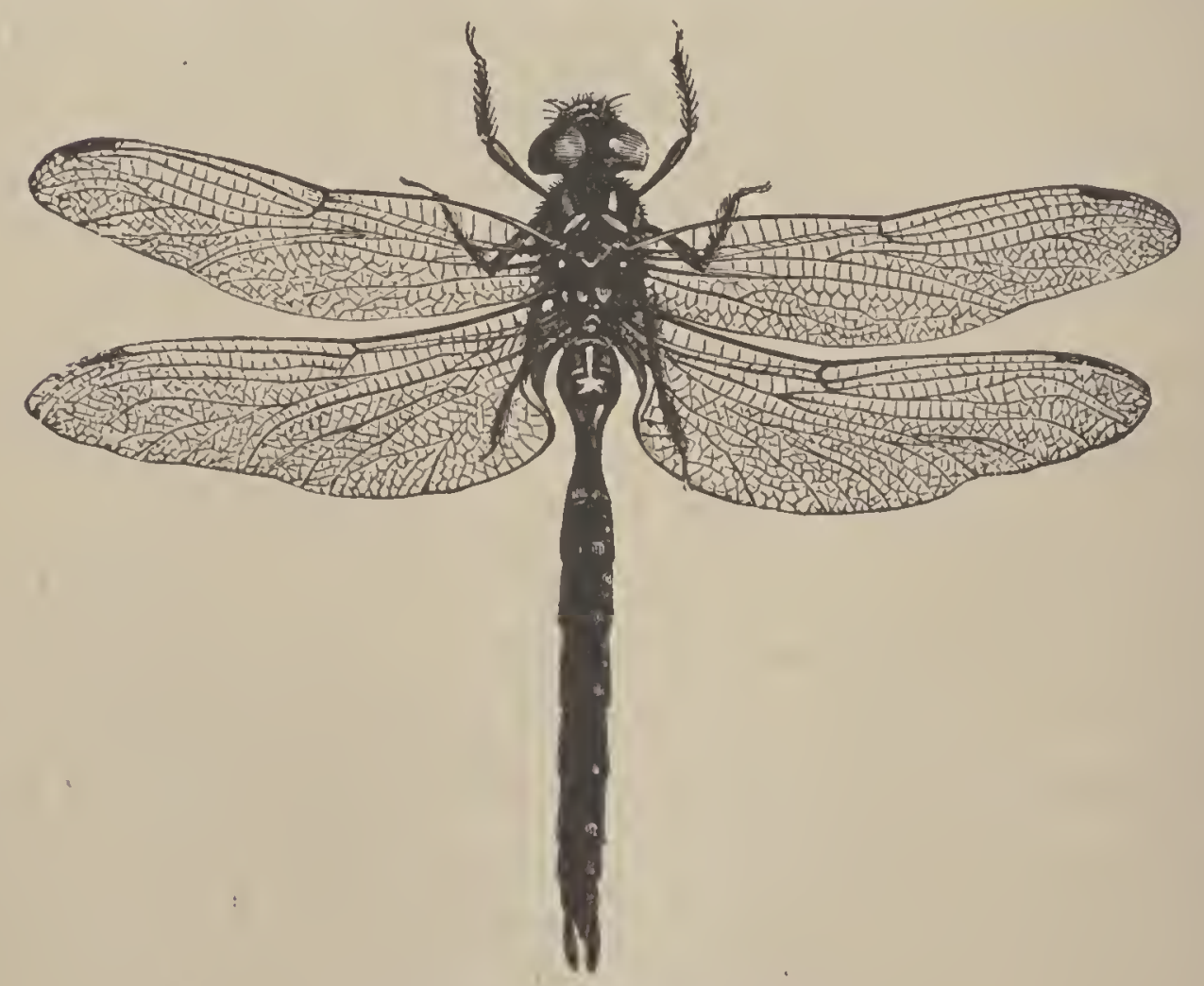

Fra. 263.-Dragon-fly (Libellula).

and four large, transparent wings, nearly equal in size, membranous and lace-like. Such are the brilliant Dragon. 
flies, or Devil's Darning-needles (Libellula), well known by the enormous head and thorax, large, prominent eyes (each furnished with twenty-eight thousand polished lenses), and Scorpion-like abdomen; the delicate and short-lived May-flies (Ephemera); Caddis-flies (Phryga$n e a)$, whose larvæ live in a tubular case made of minute stones, shells, or bits of wood; the Horned Corydalis (Corydalus), of which the male lias formidable mandibles twice as long as the head; and the White Ants (Termes) of the tropics.

2. Orthoptera have four wings: the front pair somewhat thickened, narrow, and overlapping along the back; the hind pair broad, net-veined, and folding up like a fan

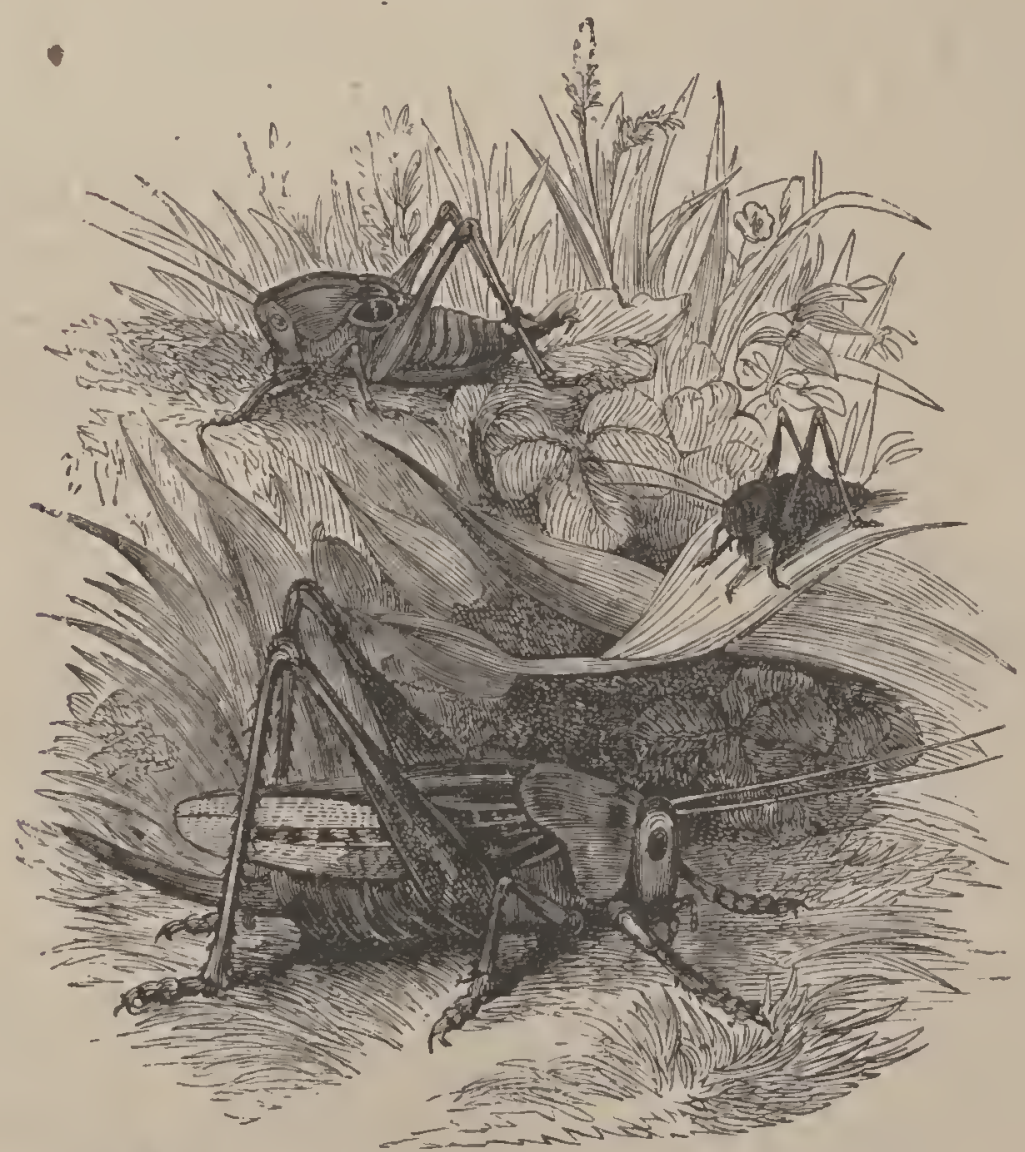

Fra. 264.-Metimurphosis of a Cricket (Grylltrs).

upon the abdomen. The hind legs are usually large, and fitted for leaping, all the species being terrestrial, although some fly as well as leap. The eyes are small, the mouth remarkably developed for cutting and grinding. The lar- 


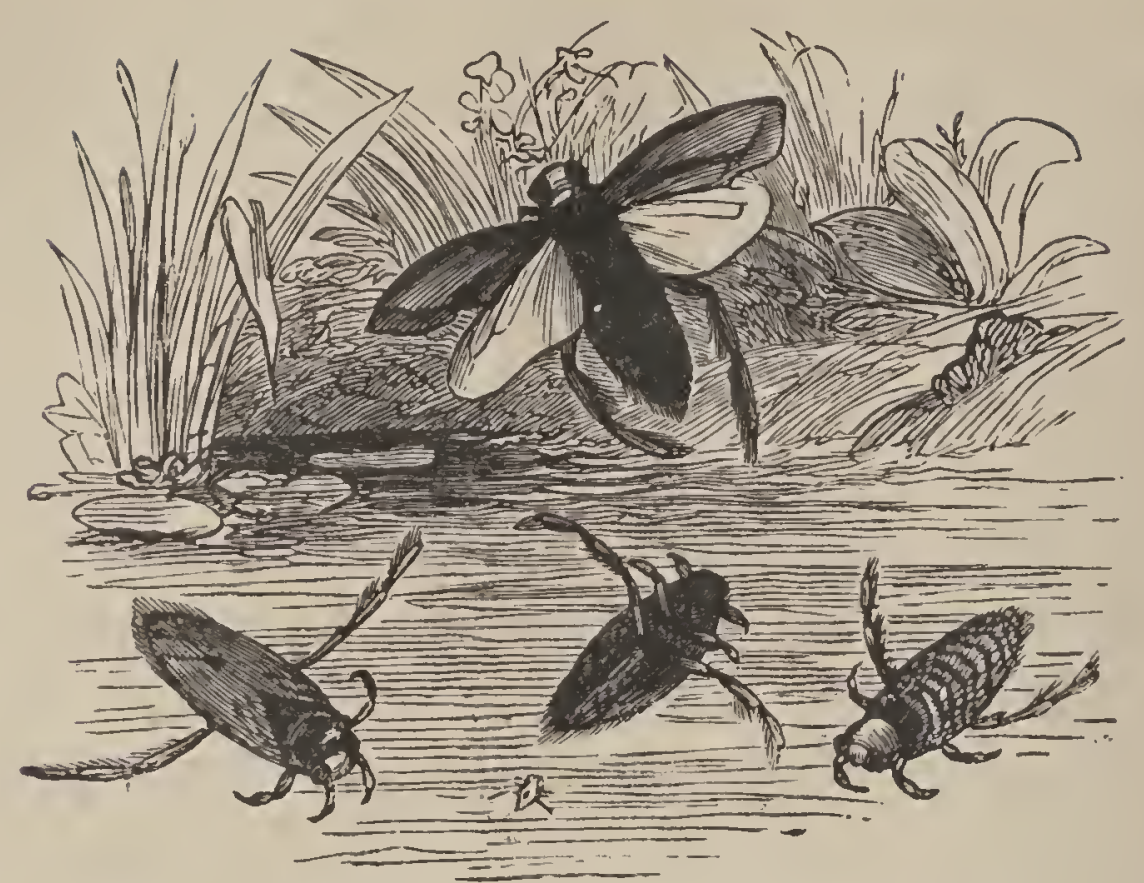

Fig. 265.-Metamorphosis of an Hemipter, Water-boatman (Notonecta).

væ and pupæ are active, and resemble the imago. They are nearly all vegetarian. Each family produces characteristic sounds (stridulation). The representative forms

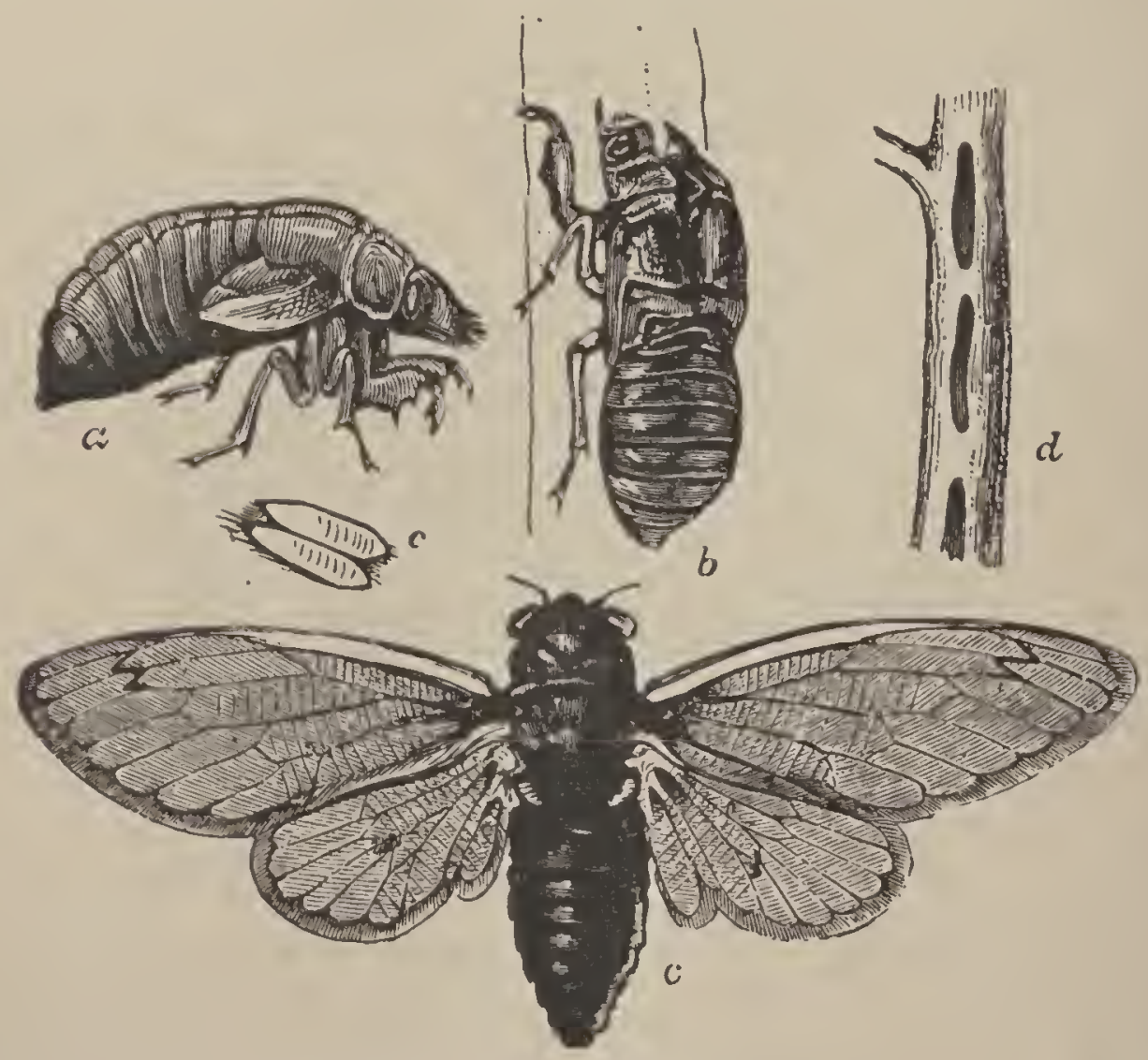

Fia. 266. -Seventeen-year Cicada (Cicada septendecim): $a$, pupa; $b$, the same, after the imago, $c$, has escaped through a rent in the back; $d$, holes in a twig, where the eggs, $e$, are inserted. 
are Crickets (Gryllus), Locusts (Locusta), Grasshopper's (Acrydium), Walking-sticks (Phasma), and Cockroaches (Blatta).

3. Hemiptera, or "Bugs," are chiefly characterized by a suctorial mouth, which is produced into a long, hard, beak, in which mandibles and maxillæ are modified into bristles and enclosed by the labium. The four wings are irregularly and sparsely veined, sometimes wanting. The body is flat above, and the legs slender. The larva differs from the imago in wanting wings. In some species the fore-wings are opaque at the base and transparent at the apex, whence the name of the order. Some feed on the juices of animals, others on plants. Here belong the wingless Bed-bug (Cimex) and Louse (Pediculus), the Squash-bug (Coreus), Water-boatman (Notonecta), Seventeen-year Locust (Cicada), Cochineal (Coccus), and Plantlouse (Aphis).

4. Coleoptera, or "Beetles." This is the largest of the order's, the species numbering about ninety thousand. They are easily recognized by the elytra, or thickened,
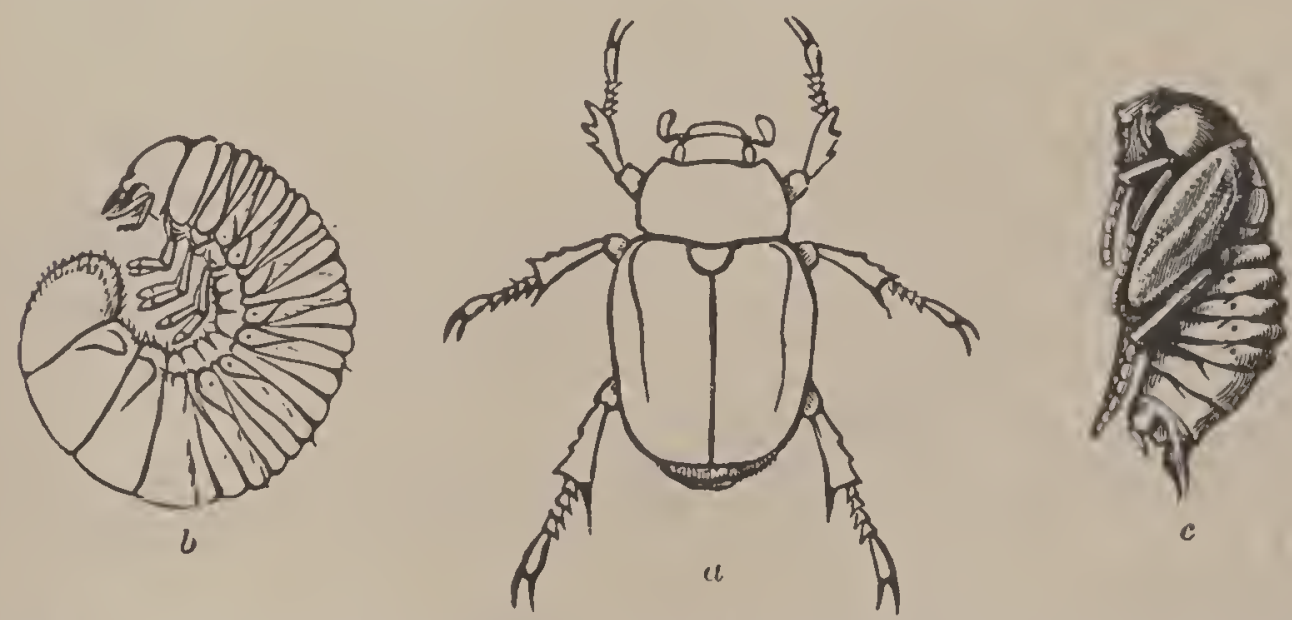

Fig. 267. $-a$, imago, and $b$, larva, of the Goldsmith Beetle (Cotalpa lanigera); $c$, pupa of june-bug (Lachnosterna fusca).

horny fore-wings, which are not used for flight, but serve to cover the hind pair. When in repose, these elytra are always united by a straight edge along the whole length. The hind wings, when not in use, are folded transversely. 
The mandibles are well developed, and the integument generally is hard. The legs are strong, for the Beetles are among the most powerful running Insects. The larvæ are worm-like, and the pupa is motionless. The highest tribes are carnivorous. The most prominent forms

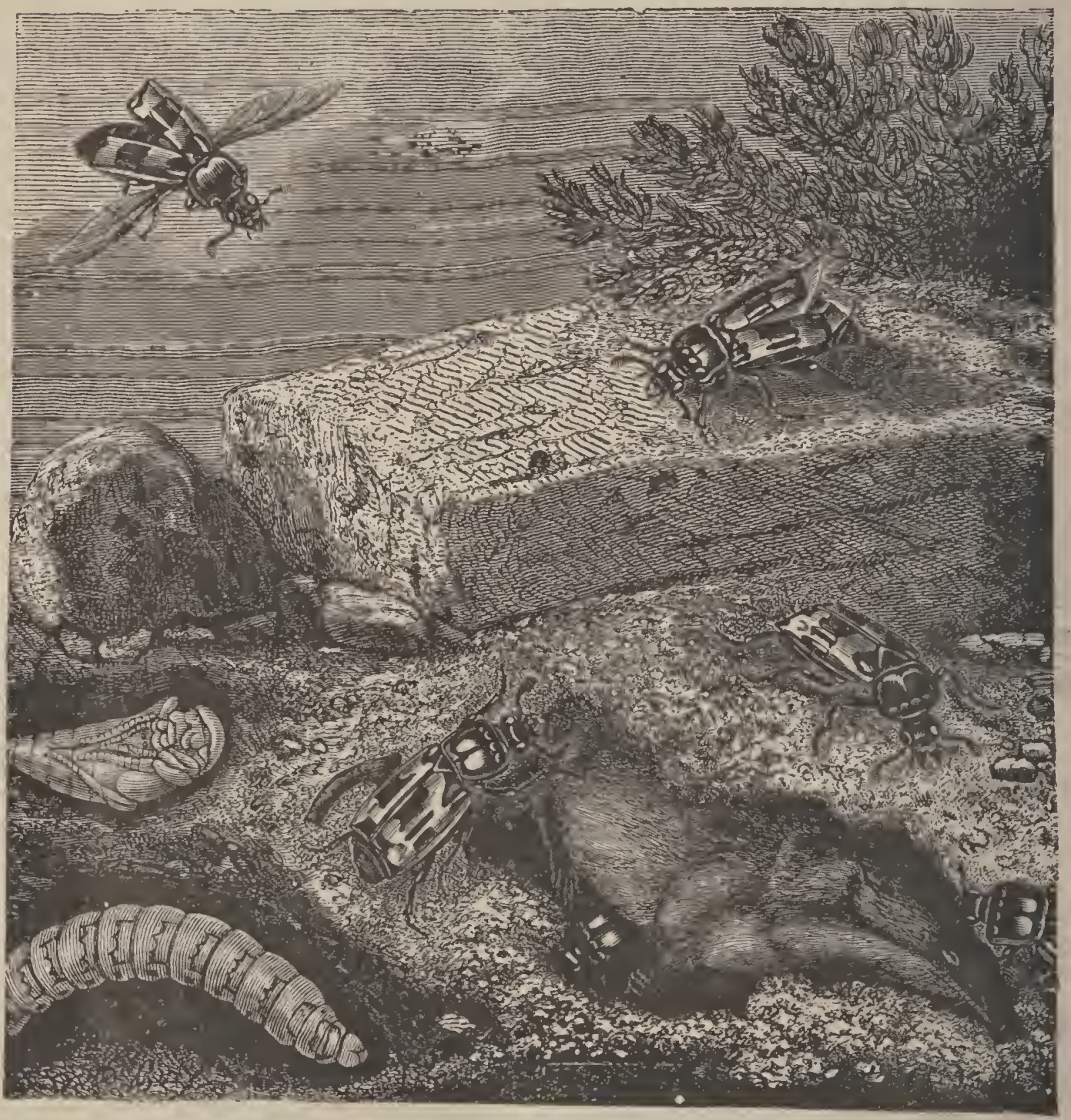

Fir. 265.-Dexton Beetles (Necrophorus vesprillo), with latva and nymph. They are burying a mouse, prepuratory to laying their eggs in it. ,

are the savage but beautiful Tiger Beetles (Cicindela); the cornmon Gronnd Beetles (Carabus), whose hind wings are often absent; the Diving Beetles (Dytiscus), with boat-shaped body, and hind legs changed into oars; the Carrion Beetles (Silpha), distinguished by their black, flat 
bodies and club-shaped antennæ; the Goliath Beetles (Scurabous), the giants of the order'; the Snapping-bugs (Elater); the Lightning-bugs (Pyrophorus); the spotted Lady-birds (Coccinella); the showy, Long-horned Beetles

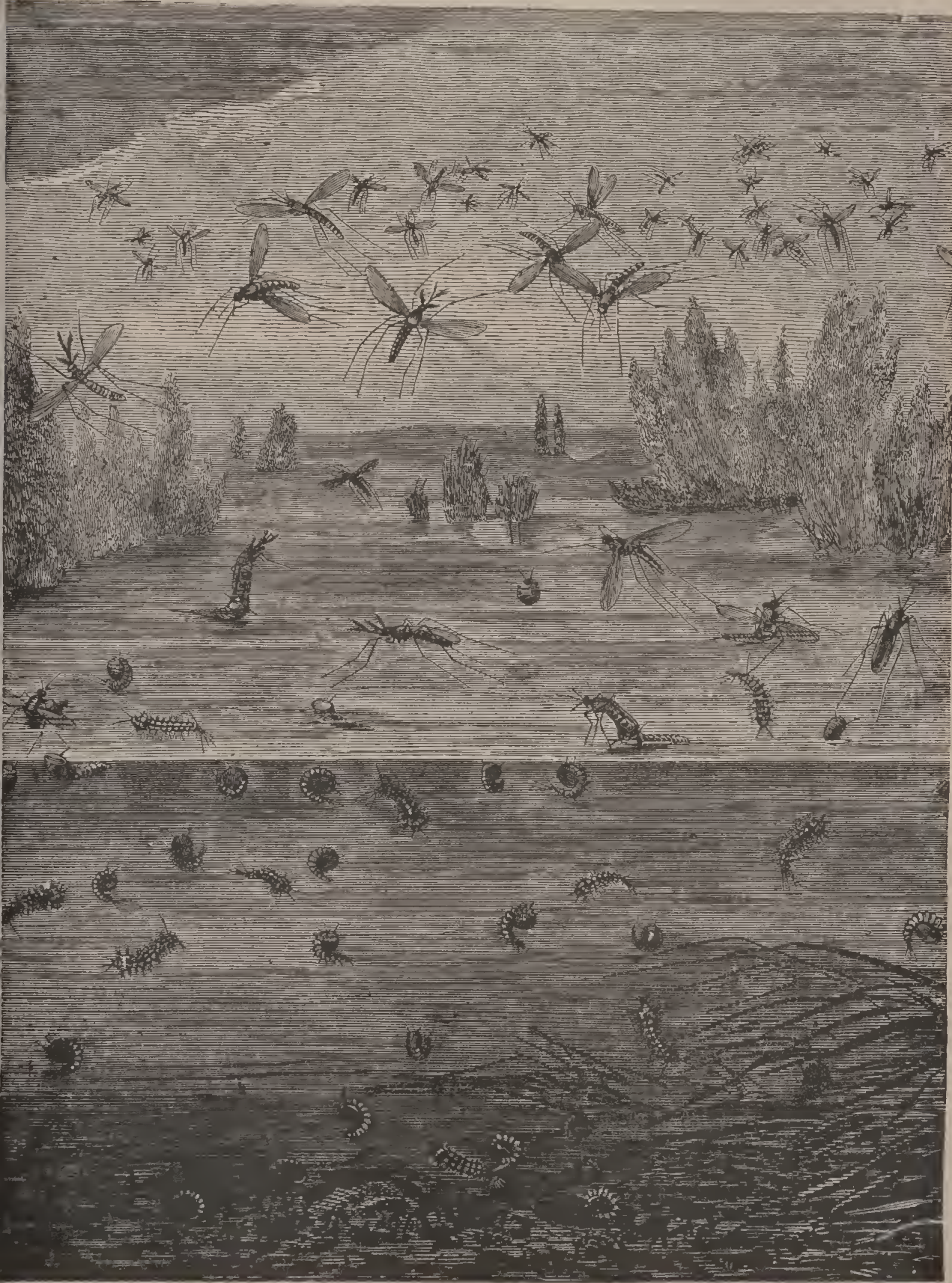

FIG. 269.-Metamorphosis of the Mosquito (Culex pipiens). 
(Cerambycidce); and the destructive Weevils (Curculio. nidce), with pointed snouts.

5. Diptera, or "Flies," are characterized by the rudimentary state of the hinder pair of wings. Although having, therefore, but one available pair, they are gifted with the power of very rapid flight. While a Bee moves its wings one hundred and ninety times a second, and a Butterfly nine times, the House-fly makes three hundred and thirty strokes. A few species are wingless. The eyes are large, with numerous facets. In some forms, as the Housefly, all the mouth-parts, except the labium, are rudimentary; and the labinim has an expanded tip, by means of

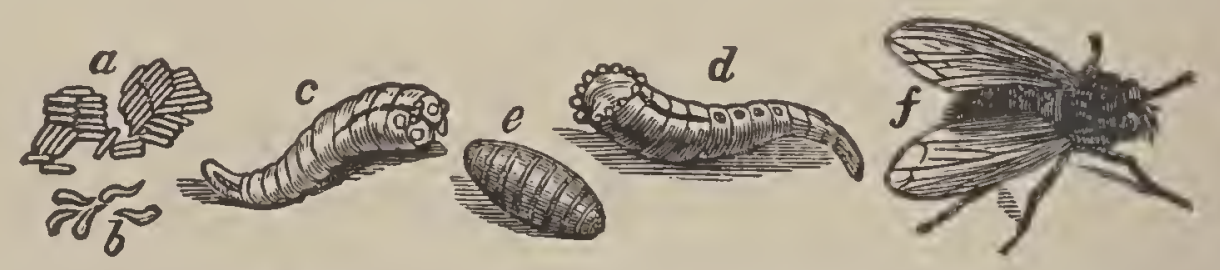

Fig. 270. -Metamorphosis of the Flesh-fly (Sarcophaga carnaria): $a$, eggs; $b$, young maggots just hatched; $c, d$, full-grown maggots; $e$, pupa; $f$, imago.

which the fly licks up its food. In other forms, as the Mosquito, the other mouth-parts are present as bristles or lancets, fitted for piercing; the thorax is globular, and the legs slender. The larvæ are footless grubs. The Diptera number about twenty-four thousand. Among them are the Mosquitoes (Culex); Hessian-fly (Cecidomyia), so destructive to wheat; Daddy-long-legs (Tipula), resembling a gigantic Mosquito; the wingless Flea (Pulex); besides the immense families represented by the Honse-fly (Mus-

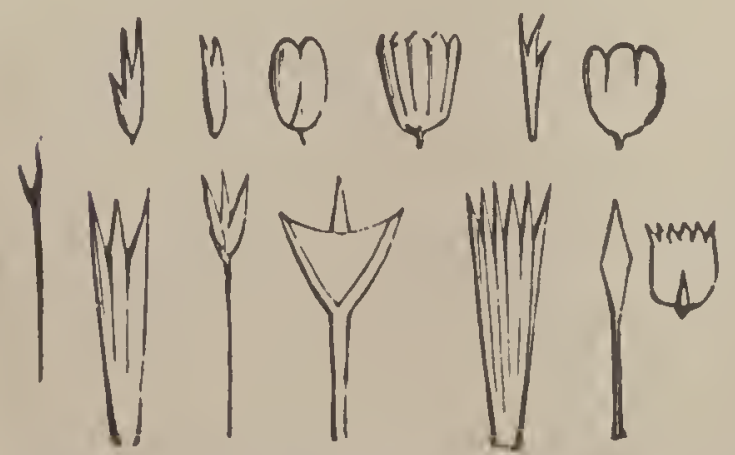

Fig. 271. -Scales from the Wings of various Lepidoptera. $c a$ ) and Bot-fly (CEstrus).

6. Lepidoptera, or "Butterflies" and "Moths," are known chiefly by their four large wings, which are thickly covered on both sides by minute, overlapping scales. The scales are of different 
colors, and are often arranged in patterns of exquisite beauty. They are in reality modified hairs, and every family has its particular form of scale. The head is small, and the body cylindrical. The legs are of but little use for locomotion. All the mouth parts are nearly obsolete except the maxillæ, which are fashioned into a "proboscis" for pumping up the nectar of flowers.

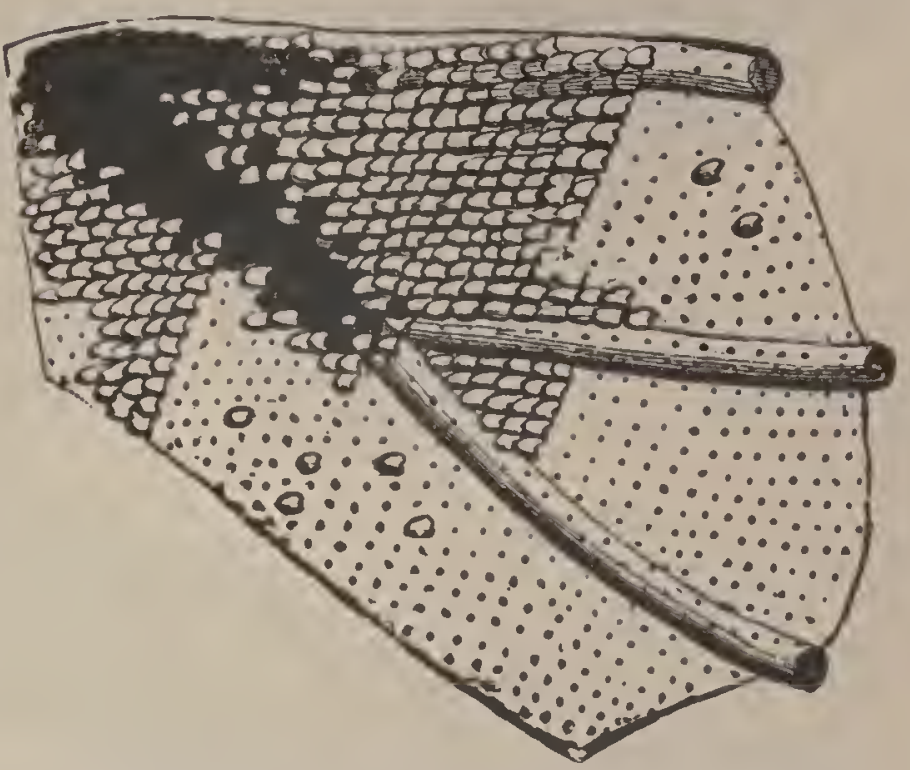

Fia. 272. - Part of the Wing of a Moth (Saturnia), magnified to show the allangement of scales. The larvæ, called "caterpillars," have a worm-like form, and from one to five pairs of abdominal legs, in addition to the three on the thorax. The mouth is formed for mas-

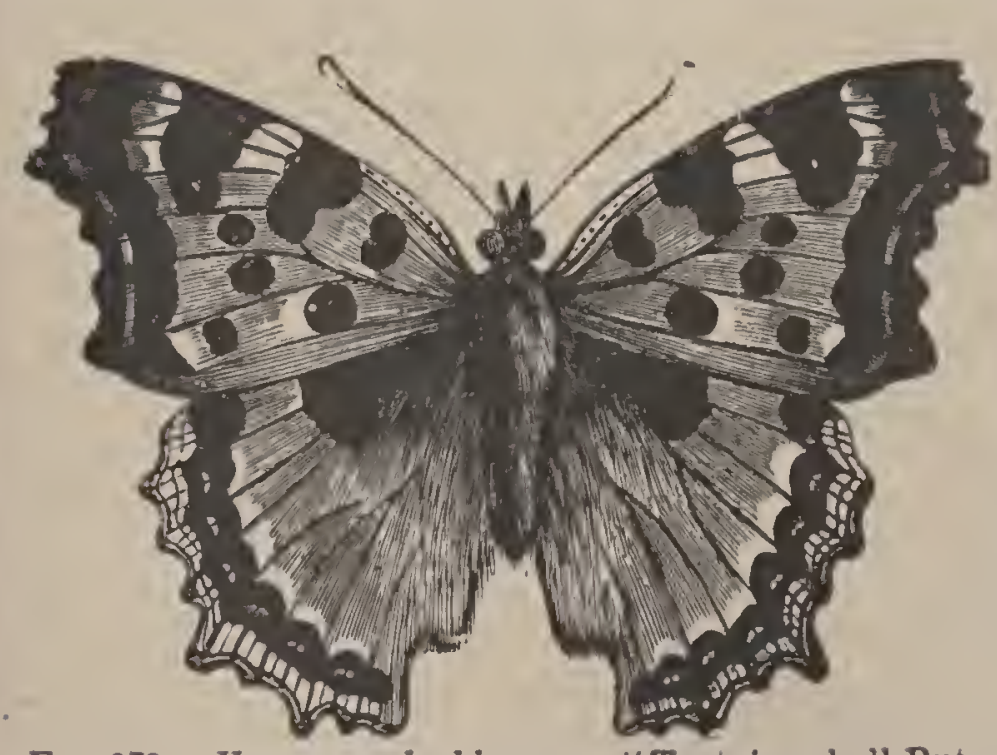

Fra. 273.-Vanessa polychloros, or "Tortoise-shell Butterfly." tication, and (except in the larvæ of Butterflies) the lip has a spinneret connected with silkglands.

There are three groups: the gay Butterflies, having knobbed or hooked antennæ, and flying in the day only; the dull-colored Sphinges, with antennæ thickened in the middle, and flying at twilight; and the nocturnal Moths, which generally prefer the night, and whose antennæ are thread-like and often feathery. Generally, when at rest, the Butterflies keep their wings raised rertically, while 


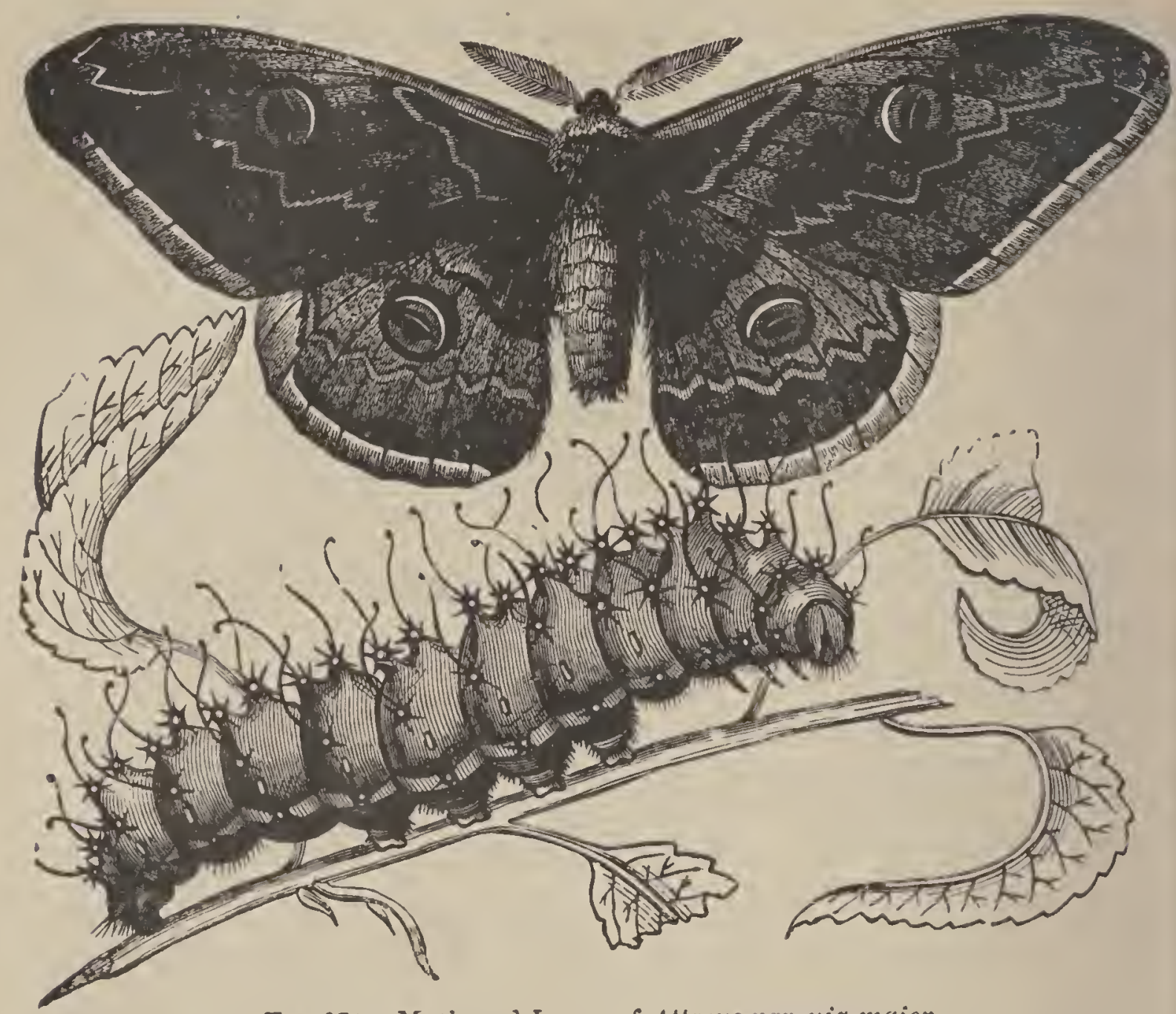

Fig. 274.-Moth and Larva of Attacus pavmia-major.

the others hold theirs horizontally. The pupa of the former is unprotected, and is nsually suspended by a bit of silk : $:^{153}$ the prpa of the Moths is enclosed in a cocoon.

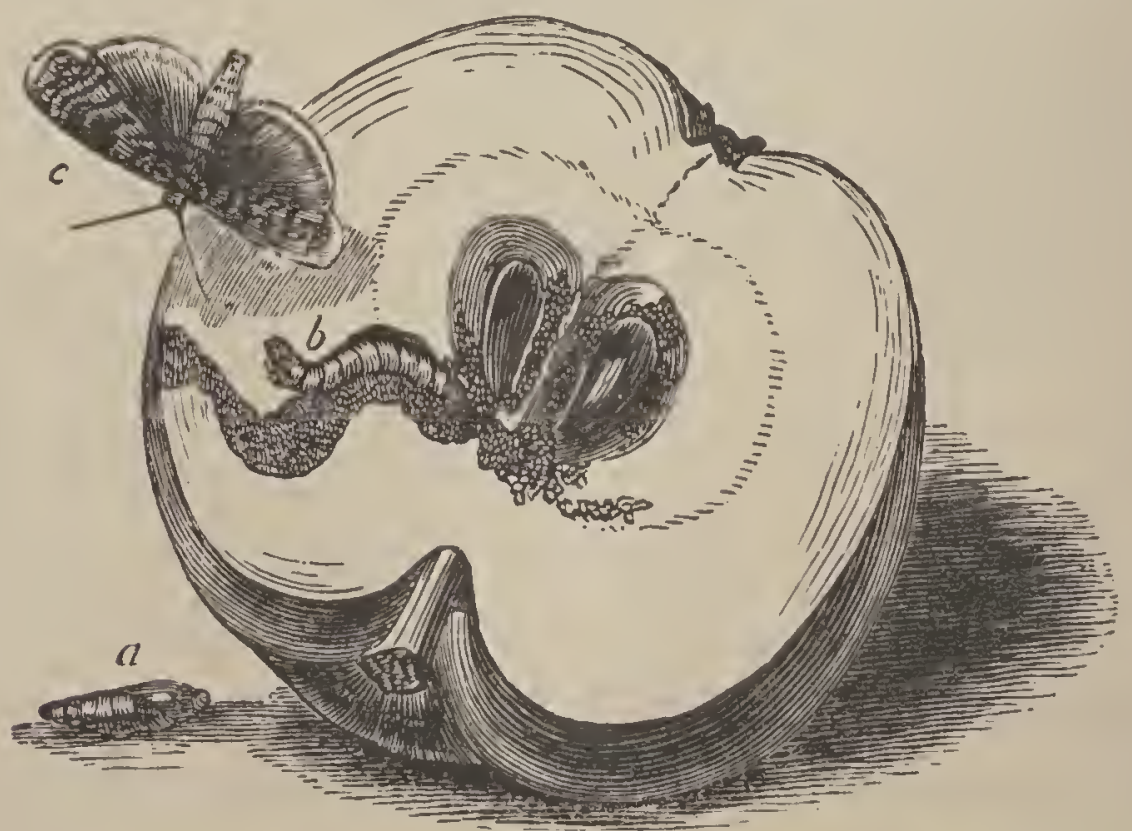

F'16. 275.-Fruit-moth (Carpocapsa pomonella): $b$, latva ; $a$, chrysalis ; $c$, imago. 
From twenty-two thousand to twenty-four thousand Lepidopterous species have been identified. Some of the most common Butterflies are the swallow-tail Papilio, the white Pieris, the sulphuryellow Colias; the Argynnis, with silver spots on the under side of the hind wings; the Vanessa, with notched wings. The Sphinges exhibit little variety. They have narrow, powerful wings, and are sometimes mistaken for Hurmmingbirds. The "potato-worm" is the caterpillar of a Sphinx. The most conspicuons Mothis are the large and beantiful Attacus, distinguished by a triangular, transparent spot in the centre of the wing; the white Bombyx, or " silk-

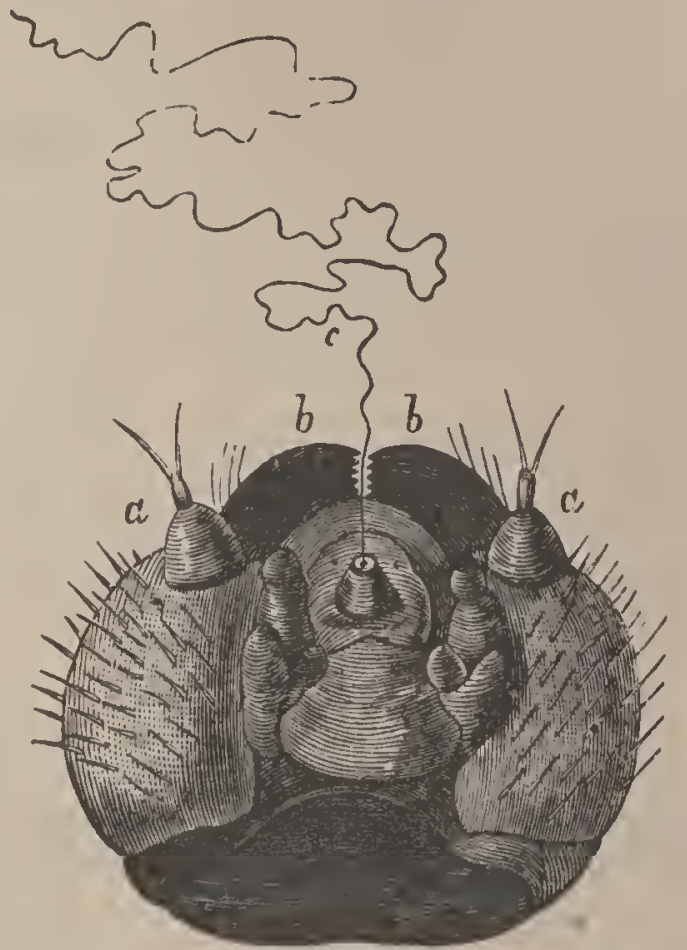

Frg. 276. - Head uf a Caterpillar, from beneath: $a$, antennæ; $b$, horuy jaws: $c$, thread of silk from the conical fusulus, on either side of which are rudimentary palpi. Magnified. worm ;" the reddish-brown Clisiocampa, whose larva, "the American Tent-caterpillar," spreads its web in many an apple and cherry tree; the pale, delicate Geometrids; and the small but destructive Tineids, represented by the Clothes-moth.

7. Hymenoptera, comprising at least twenty-five thousand species, include the highest, most social, and, we may add (if we except the Silk-worm), the most useful, of Insects. They have a large head, with compound eyes and three ocelli, mouth fitted both for biting and lapping, legs formed for locomotion as well as support, and four wings equally transparent, and interlocking by small hooks during flight. The females are usually prorided with a sting, or borer. The larvæ are footless, helpless grubs, and generally nurtured in cells, or nests. Such are 
the Honey-bees (Apis), Humble-bees (Bombus), Wasps (Vespa), Ants (Formica), Ichneumon-flies, and Gall-flies. Those living in societies exhibit three castes: females, or "queens ;" males, or "drones," and neuters, or sexless "workers." There is but one queen in a hive, and she is treated with the greatest distinction, even when dead. She dwells in a large, pear-shaped cell, opening downward. She lays three kinds of eggs: from the first come forth workers, the second produces males, and the last females. The drones, of which there are about eight hundred in an ordinary hive, are marked by their great size, their large eyes meeting on the top of the head, and

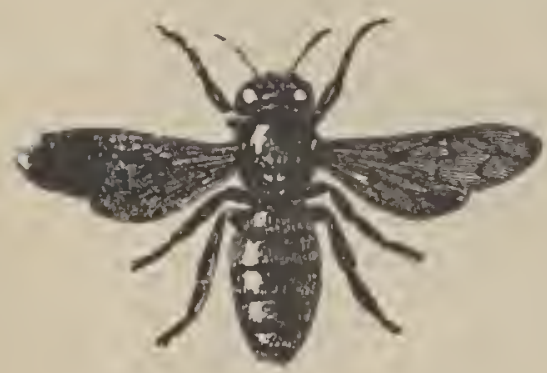

a

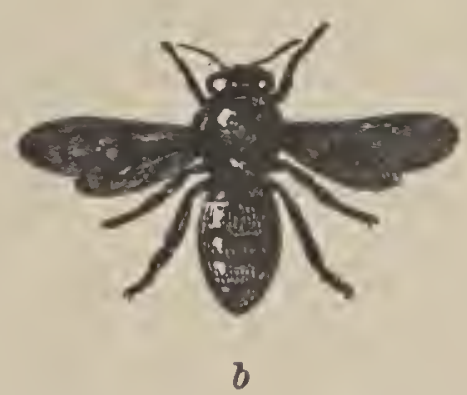

$b$

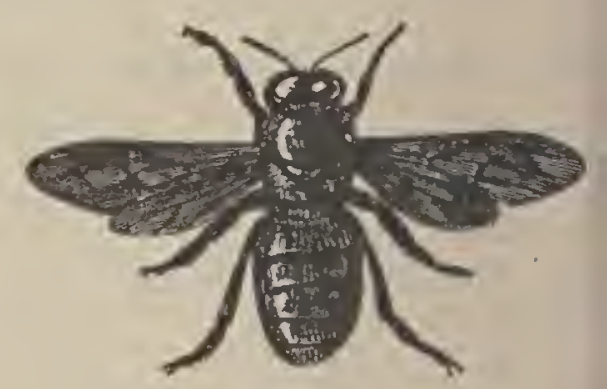

c

Frg. 277. -Honey-bee (Apis mellifica) : $a$, female ; $b$, worker ; $c$, male.

by being stingless. The workers, which number twenty to, one drone, are small and active, and provided with stings, and hollow pits in the thighs, called "baskets," in which they carry pollen. Their honey is nectar elaborated in the crop by an unknown process; while the wax is secreted from the sides of the abdomen and mixed with saliva. There is a subdivision of extra labor: thus there are wax-workers, masons, and nurses. Ants (except the Saüba) have but two classes of workers. While Ants live in hollow trees or subterranean chambers (called formicarium), Honey-bees and Wasps construct hexagonal cells. The comb of the Bee is hung vertically, that of the Wasp is horizontal. 


\section{Subkingdom VIII.-VERTEBrata.}

This grand division includes the most perfect animals, or such as have the most varied functions and the most numerous and complex organs. Besides the unnumbered host of extinct forms, there are abont twenty-five thousand living species, widely differing among themselves in shape and habits, yet closely allied in the grand features of their organization, the general type being endlessly modified.

The fundamental distinctive character of Vertebrates is the separation of the main mass of the nervous system from the general cavity of the body. A transverse section of the body exhibits two cavities, or tubes - the dorsal, containing the cerebro-spinal nervous system; the ventral, inclosing the alimentary canal, heart, lungs, and a double chain of ganglia, or sympathetic system. This ventral, or hæmal, cavity corresponds to the whole body of an Invertebrate; while the dorsal, or neural, is added.

Vertebrates are also distinguished by an internal, jointed skeleton,
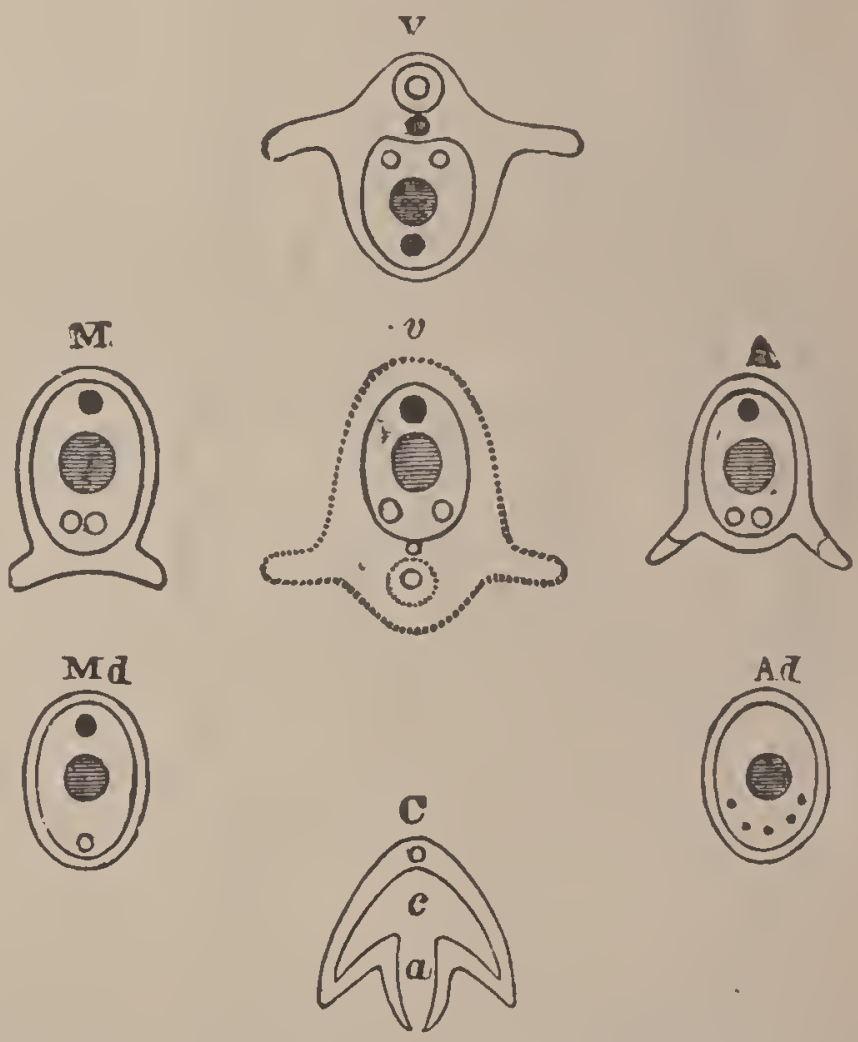

Fig. 278. -Ideal Plans of the Subkingdoms. $T$, transverse section of vertebrate type; $v$, the same, inverted. $\boldsymbol{M}$, transverse section of molluscous type; and $M d$, of mollnscoid. $A$ and $A d$, transverse sections of articulate type, high and low. $C$, longitudinal section of cœleuterate type; $a$, alimentary canal; $c$, body-cavity. In the other figures, the alimentary canal is shaded, the heart is black, and the nervous cords are open rings.

endowed with vitality, and capable of growth and repair. During embryo-life it is represented by the notochord; but in the higher forms this is afterwards replaced 


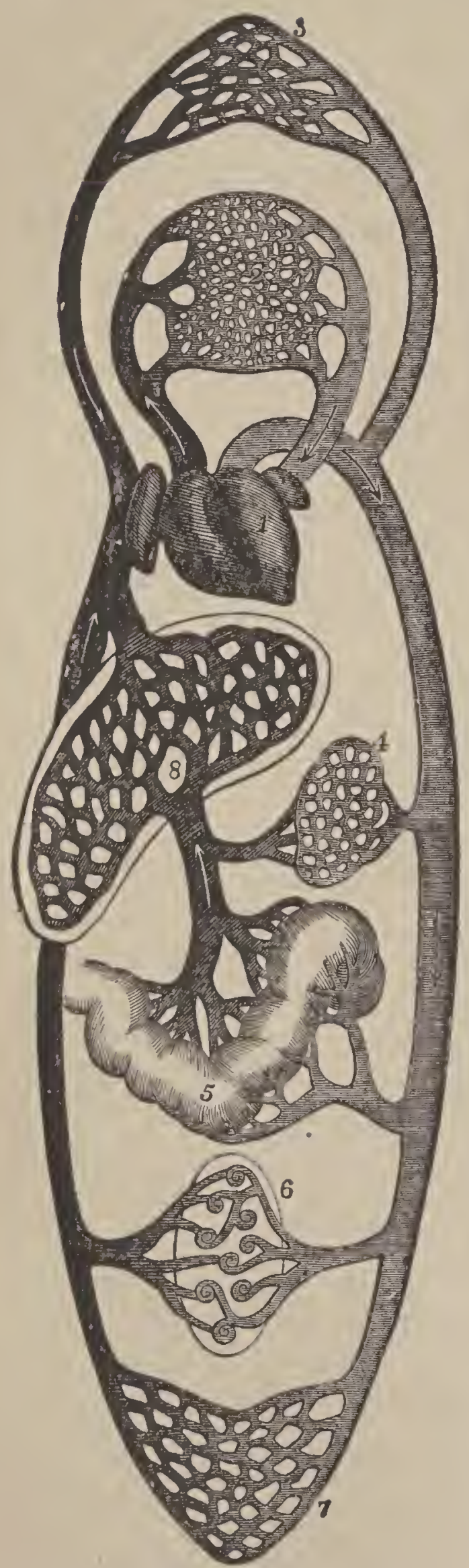

Fic. 279,-Diagram of Circulation in the higher Vertebrates: 1, heart: 2, lungs; 3 , head and upper extremities: 4 , spleen; 5 , intestiue ; 6 , kiduey : 7 , lower extremities; 8, liver. (From Daltou's "Physiology.") by a more highly developed vertebral column of cartilage or bone. The column and cranium are never absent in the craniota; other parts may be wanting, as the ribs in Frogs, limbs in Snakes, etc. ${ }^{154}$ The limbs are never more than four, and are always articulated to the hæmal side of the body, while the legs of Invertebrates are developed from the neural side. The muscles moving the limbs are attached to the endoskeleton.

The circulation of the blood is complete, the arteries being joined to the veins by capillaries, so that the blood never escapes into the visceral cavity as in the Invertebrates. All have a portal vein, carrying blood through the liver; all have lacteals and lymphatics. The blood is red, and contains both kinds of corpuscles. ${ }^{165}$ The teeth are developed from the dermis, never from the cuticle, as in Mollusks and Articulates; the jaws move vertically, and are never modified limbs. The liver and kidneys are always present. The respiratory organs are either gills or lungs, 
or both. Vertebrates are the only animals which breathe through the mouth.

The nervous system has two marked divisions: the cerebro-spinal, presiding over the functions of animal life (sensation and locomotion); and the sympathetic, which partially controls the organic functions (digestion, respiration, and circulation). In no case does the gullet pass through the nervous system, as in Invertebrates, and the mouth opens on the side opposite to the brain. Probably none of the five seuses is ever altogether absent. The form of the brain is modified by the relative development of the various lobes. In the lower Vertebrates, the cerebral hemispheres are small - in certain Fishes they are actually smaller than the optic lobes-in the higher, they nearly or quite overlap both olfactories and cerebellum. The brain may be smooth, as in most of the cold-blooded animals, or richly convoluted, as in Man.

There is no skull in Amphioxus. In the Marsipobranchii and Elasmobranchii it is cartilaginous. In other fishes it is cartilage overlaid with bone. In Amphibians and Reptiles, it is mingled bone and cartilage. In Birds and Mammals, mainly or wholly bony. The human skull contains fewer bones than the skull of most animals, excepting Birds. The skull of all Vertebrates is divisible into two regions: the cranium, or brain-case, and the face. The size of the cranial capacity, compared with the area of the face, is generally the ratio of intelligence. In the lower order's, the facial part is enormonsly predominant, the eye-orbits are directed ontward, and the occipital condyles are nearly on a line with the axis of the body. In the higher orders, the face becomes subordinate to the cranium, the sensual to the mental, the eyes look forward, and the condyles approach the base of the cranium. Compare the "snouty" skull of the Crocodile and the almost rertical profile of civilized Man. A straight line drawn from the middle of the ear to the base of the nose, and 
another from the forehead to the most prominent part of the upper jaw, will include what is called the facial angle, which roughly gives the relation between the two regions, and therefore the rank of the animal..$^{156}$ In the cold-blooded Vertebrates the brains do not fill the cranium; while in Birds and Mammals a cast of the cranial cavity well exhibits the general features of the cerebral surface. ${ }^{157}$

All higher Vertebrates are single and free. Mammals bring forth their young alive, having directly nourished them from the mother before birth (viviparous). In almost all the others the nourishment is laid up in the egg, which is laid before hatching (oviparous), or is retained in the mother until hatched (ovoviviparous), as in some Reptiles and Fishes.

Of the sublingdom Vertebrata or Chordata there are three great divisions, Urochordata, Acrania, and Craniota. The first division includes the Tunicates, and the second the Vertebrates withont skulls-e.g., the Amphioxus. ${ }^{157 \mathrm{a}}$

The Craniota are divided into five great classes: Fishes, Amphibians, Reptiles, Birds, and Mammals. The first three are "cold-blooded," the other two are "warmblooded." Fishes and Amphibians have gills during the whole or a part of their lives, while the rest never have gills. Fishes and Amphibians in embryo have neither amnion nor allantois, while the other three are provided with both.

There are three provinces of skull-bearing Vertebrates.

Fishes and Amphibians agree in having gills, in wanting amnion and allantois, and in possessing nucleated red blood-corpuscles (Ichthyopsida).

Birds and Reptiles agree in having no gills, but both amnion and allantois, in the articulation of the skull with the spine by a single condyle, in the development of the skin into feathers or scales, and in circulating oval, nucleated, red corpuscles (Sauropsida).

Manmals differ from Birds and Reptiles in having two occipital condyles, and their red blood-corpuscles are not nucleated ${ }^{168}$ (Mammalia). 


\section{Division I.-Urochordata.}

Class I.-Tunicata.

The Tunicates form a small and singular group of animals having relations with the worms on the one hand and with the Vertebrates on the other. The most common forms (the solitary Ascidians) are enclosed in a leathery, elastic bag, one end of which is fastened to the rocks, while the other has two orifices, for the inlet and exit of a current of water for nutrition and respiration. They are withont head, feet, arms, or shell. Indeed, few animals seem more

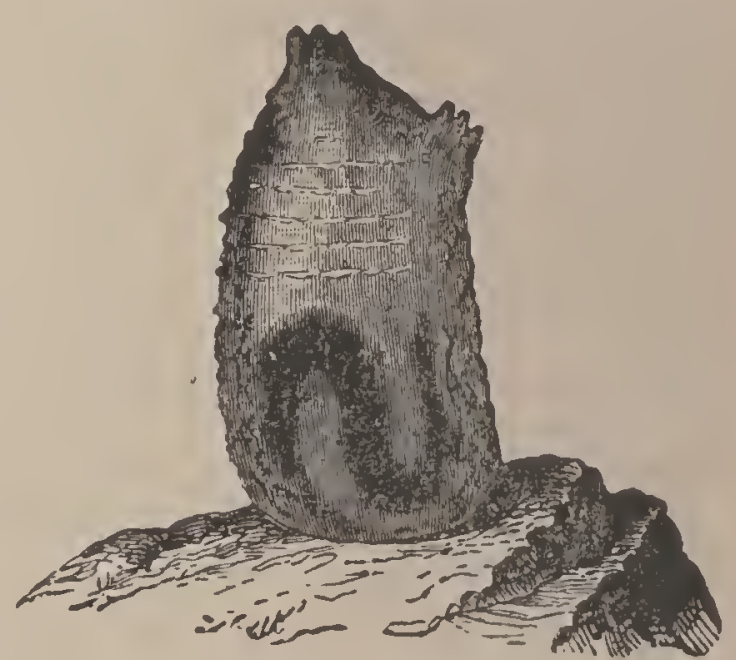

FIG. 2S0. -An Ascidian. helpless and apathetic than these apparently shapeless beings. The tubular heart exhibits the curious phenomenon of reversing its action at brief intervals, so that the blood

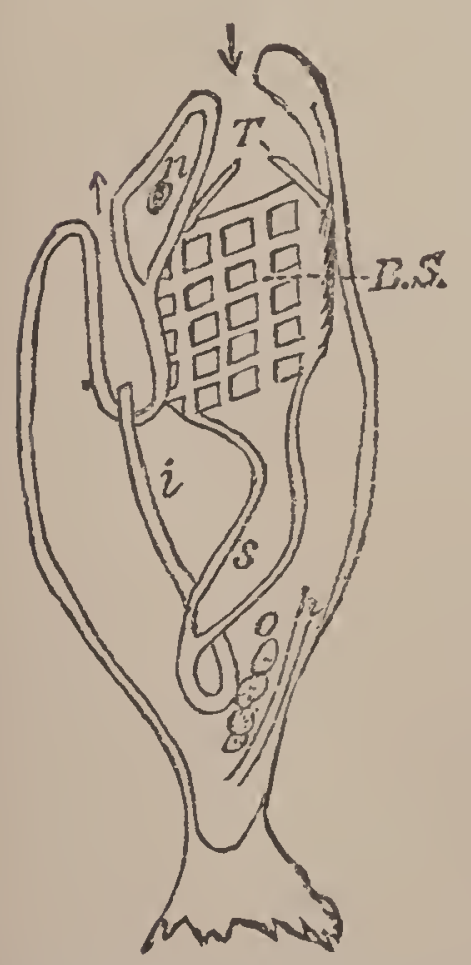

Frg.2S1.-Diagram of simple Ascidian: $B, S$, branchial sac; $n$, nervous intestine ; 0 , reproductive orgau; $h$, heart. same vessels. Another peculiarity is the presence of cellulose in the skin. The water is drawn by cilia into a branchial sac, an enlargement of the first part of the intestine, whence it escapes throngh openings in the sides, to the excurrent orifice, while the particles of food drawn in with the water are retained and passed into the intestine. The larva is active, swimming by means of a long tail. It looks like a tadpole, and has a notochord and a nerrous system closely resembling those of a Vertebrate. Afterwards it attaches itself by the head, the tail is absorbed, and the nervons system is reduced to a single small ganglion. Thus oscillates backward and forward in the 
the animal, whose larval structure is that of a Vertebrate, degenerates in its adult stage into an Invertebrate.

\section{Division II.-Acrania.}

Vertebrates without a skull.

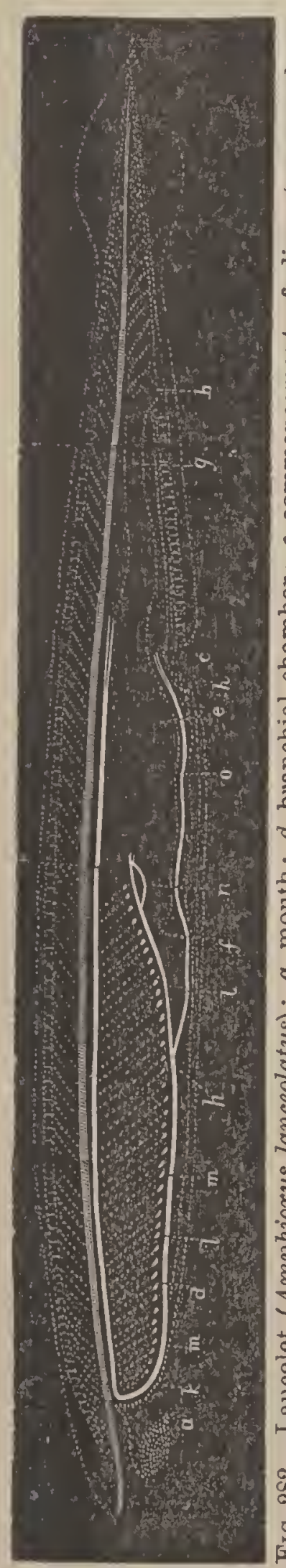

Class.-Pharyngobranchii.

The Acrania are represented by the singular animal Amphioxus or Lancelet. It is about two inches long, semi-transparent, without skull, limbs, brain, heart, or red blood-corpuscles. It has for a skeleton a notochord only. It breathes by very numerous gill arches, withont fringes, and the water is drawn in by cilia, which line the gill slits. The embryo develops into a gastrula closely resembling that of the Invertebrates. The animal lives in the sandy bottom of shallow parts of the ocean, and has been found in the Mediterranean Sea, in the Indian Ocean, and on the east coast of North and Sonth America.

Drvision III.-Craniota. .

Vertebrates with a distinct skull.

\section{Class I.-Pisces.}

Fishes are the lowest of Vertebrates. They fall far behind the rest in strength, intelligence, and sensibility. The eyes, though large, are almost immovable, bathed by no tears, and protected by no lids. Dwelling in the realm of silence, ears are little 
needed, and such as they have are without external parts, the sound being obliged to pass through the cranium. Taste and smell are blunted, and touch is nearly confined to the lips.

The class yields to no other in the number and variety of its forms. It includes nearly one half of all the vertebrated species. So great is the range of variation, it is difficult to frame a definition which will characterize all the finny tribes. It may be said, however, that Fishes are the only backboned animals having median fins (as dorsal and anal) supported by fin-rays, and whose limbs (pectoral and ventral fins) do not exhibit that threefold division (as thigh, leg, and foot) found in all other Vertebrates. ${ }^{159}$

The form of Fishes is admirably adapted to the element in which they live and move. Indeed, Nature nowhere presents in one class such elegance of proportions with such variety of form and beanty of color. The head is

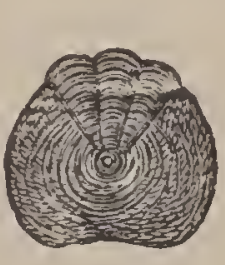

$A$

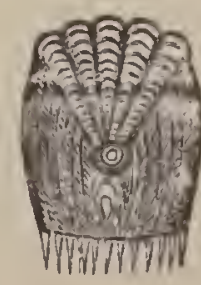

$B$

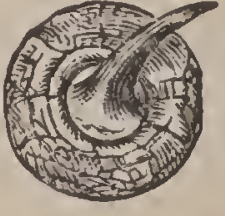

$C$

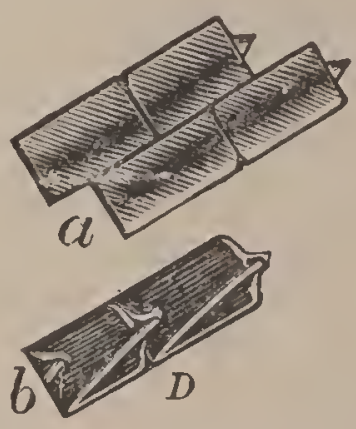

O. D

Fig. 283.-Scales of Fishes: $A$, cycloid scale (Salmon); $B$, ctenoid scale (Perch); $C$, placoicl scale $(R a y) ; D$, ganoid scales (Anblypterus) - a, upper surface ; $b$, under surface, showing articulating processes.

disproportionately large, but pointed to meet the resistance of the water. The neck is wanting, the head being a prolongation of the trunk. The viscera are closely packed near the head, and the long, tapering trunk is left free for the development of muscles which are to more the tail-the instrument of locomotion. The biconcave vertebræ, with intervening cavities filled with elastic gelatin, are designed for rapid and versatile movements. The body is either naked, as in the Lamprey, or covered with 
polished, overlapping scales, as in the Perch. Rarely, as in the Sturgeon, it is defended by bony plates, or by minute, hard spines, as in the Shark. Scales with smooth, circular outline are called cycloid; those with notched or spiny margins are ctenoid. Enameled scales are ganoid, and those with a sharp spine, like those of the Shark, are placoid.

The vertical fins (dorsal, anal, and caudal) are peculiar to Fishes. The dorsal vary in number, from one, as in the Herring, to three, as in the Cod; and the first dorsal may be soft, as in the Tront, or spiny, as in the Perch.

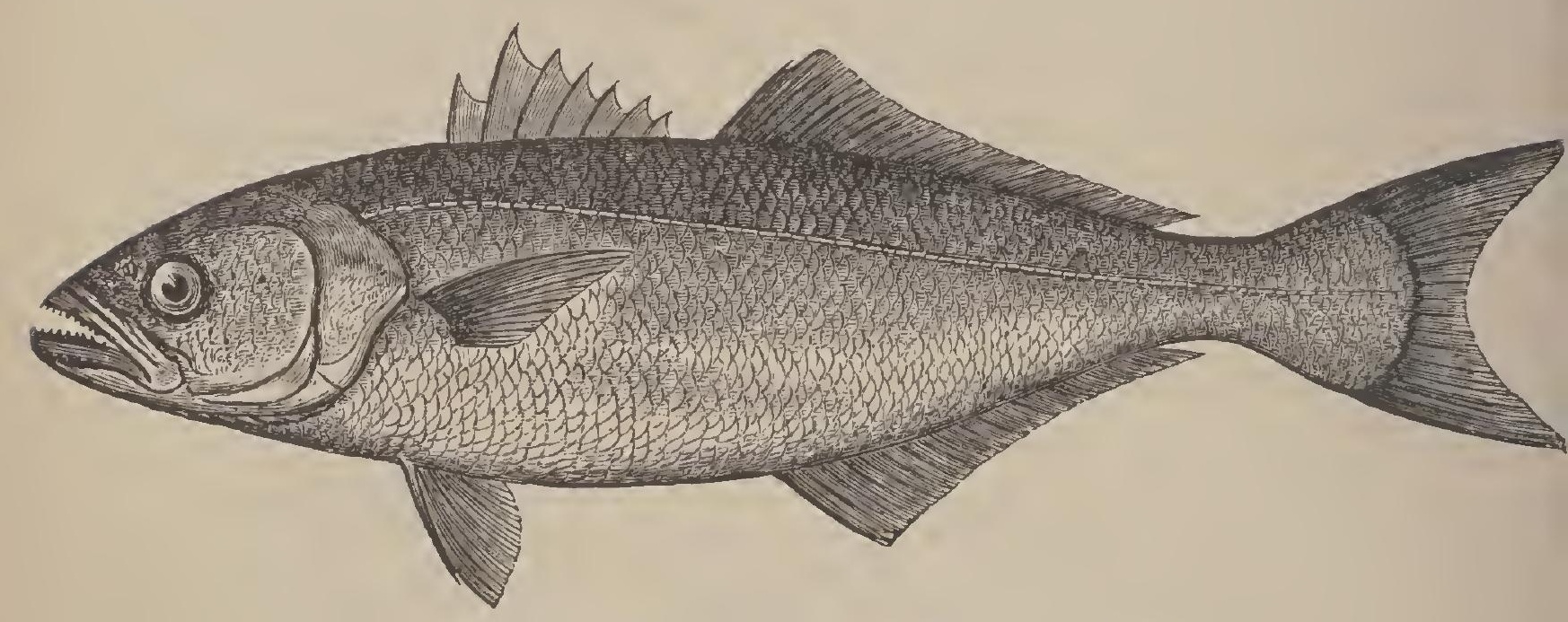

Fi6. 2S4.-Blue-fish (Temnodon saltator). All seas.

If the dorsals are cut off, the Fish reels to and fro. The caudal may be homocercal, as in ordinary species; or heterocercal, as in Sharks. In ancient heterocercal Fishes, the tail was frequently vertebrated. The pectoral and ventral fins stand for the fore and hind limbs of other Vertebrates. As the specific gravity of the body is greater than that of the water, most Fishes are provided with an air-bladder, which is an outgrowth from the œsophagus. This is absent in such as grovel at the bottom, as the Rays, and in those, like the Sharks, endowed with compensating muscular power.

Fishes have no prehensile organ besides the month. Both jaws are movable. The teeth are numerous, and 
may be recurved spines, as in the Pike; flat and triangular, with serrated edges, in the Shark; or flat and tessellated in the Ray. They feed principally on animal matter. The digestive tract is relatively shorter than in other Vertebrates. ${ }^{160}$ The blood is red, and the heart has rarely more than two cavities, an auricle and a ventricle, both on the venous side. Ordinary Fishes have four gills, which are covered by the operculum, and the water escapes from an opening behind this. In Sharks there is no operculum,

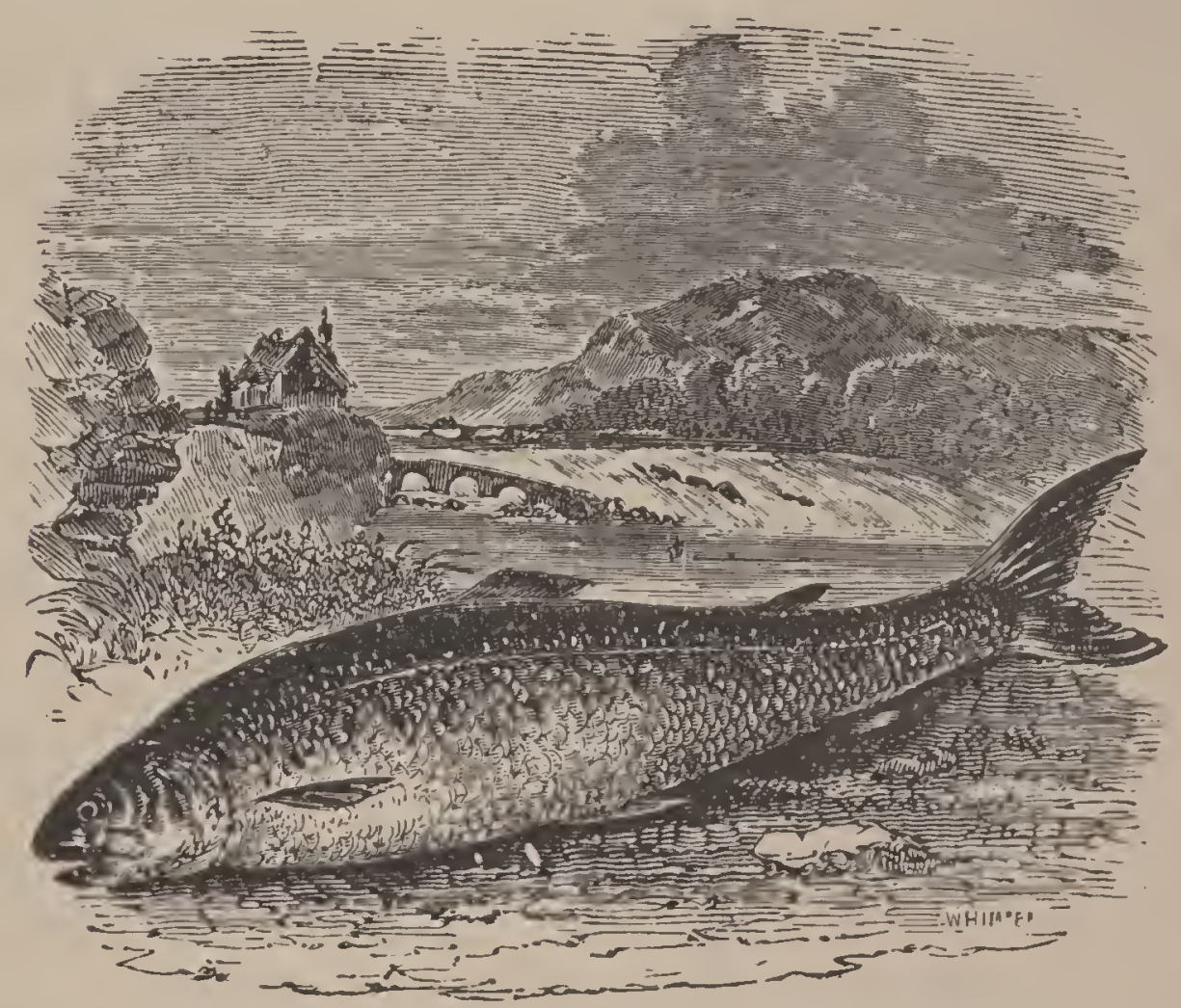

Fig. 255.-Silmou (Salmo salar). Both hemispheres.

and each gill opens separately. The brain consists of several ganglia placed one behind the other, and occupies but a small part of the cranial cavity. Its average weight to the rest of the body may be as low as 1 to 3000 . The eggs of bony Fishes are naked and multitudinous, sometimes numbering millions in a single spawn; those of the Sharks are few, and protected by a horny shell (Fig. 164).

There are about thirteen thousand species of Fishes, of which over two thirds are Teleostei. There are two sub. classes of Pisces. 
SuBClass I.-Marsipobranchii.

The Lampreys and Hag-fish have a persistent noto-

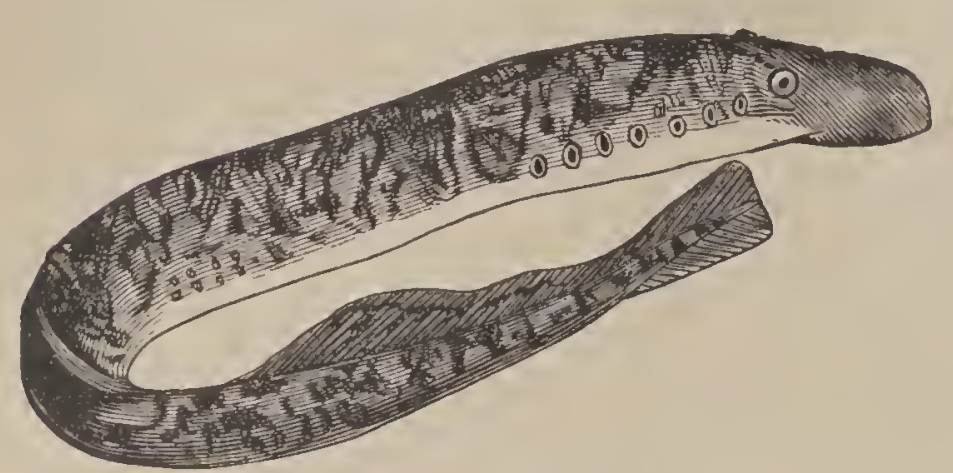

Fig. 2s6.-Lamprey (Petromyzon Americanus). Atlantic. chord, a cartilaginous skull, no lower jaw, a round, suctorial mouth, horny teeth, one nasal-organ, no scales, limbs, or gillarches. The gills are pouch-like (whence the name of the class), and open separately. They are found both in salt and fresh water.

\section{Subclass II.-Pisces Proper.}

The true Fishes have two nasal organs, and well-developed jaws and gill-arches. There are four orders:

1. Elasmobranchii, having a.cartilaginous skeleton, and a skin naked or with placoid scales. The gill-openings are uncovered; and the mouth is generally under the head. The ventral fins are placed far back; the pectorals are large, in the Rays enormously developed; and the tail is heterocercal. Such are the Sharks, Rays, and Chimæra.

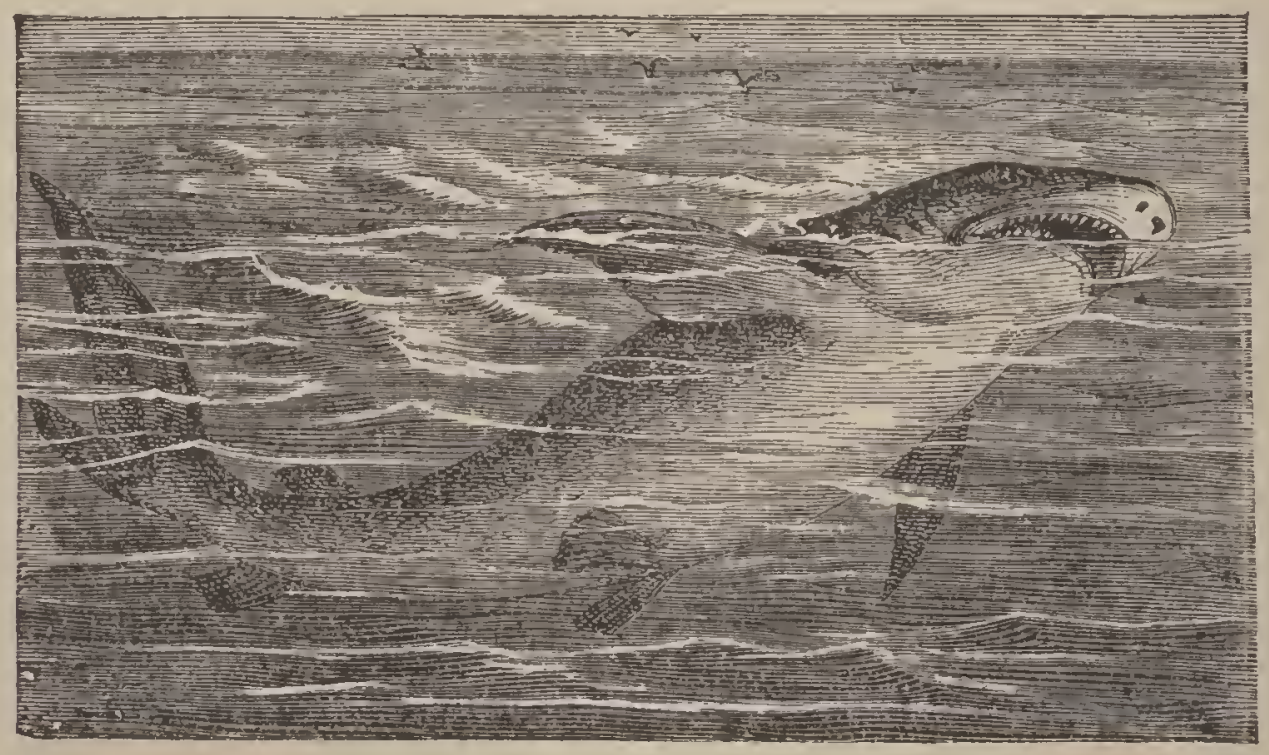

FIG. 2St.-Shark (Carcharias vulgaris). Atlantic. 
They are all marine. The largest Shark found, and therefore the largest Fish, measured forty feet in length.

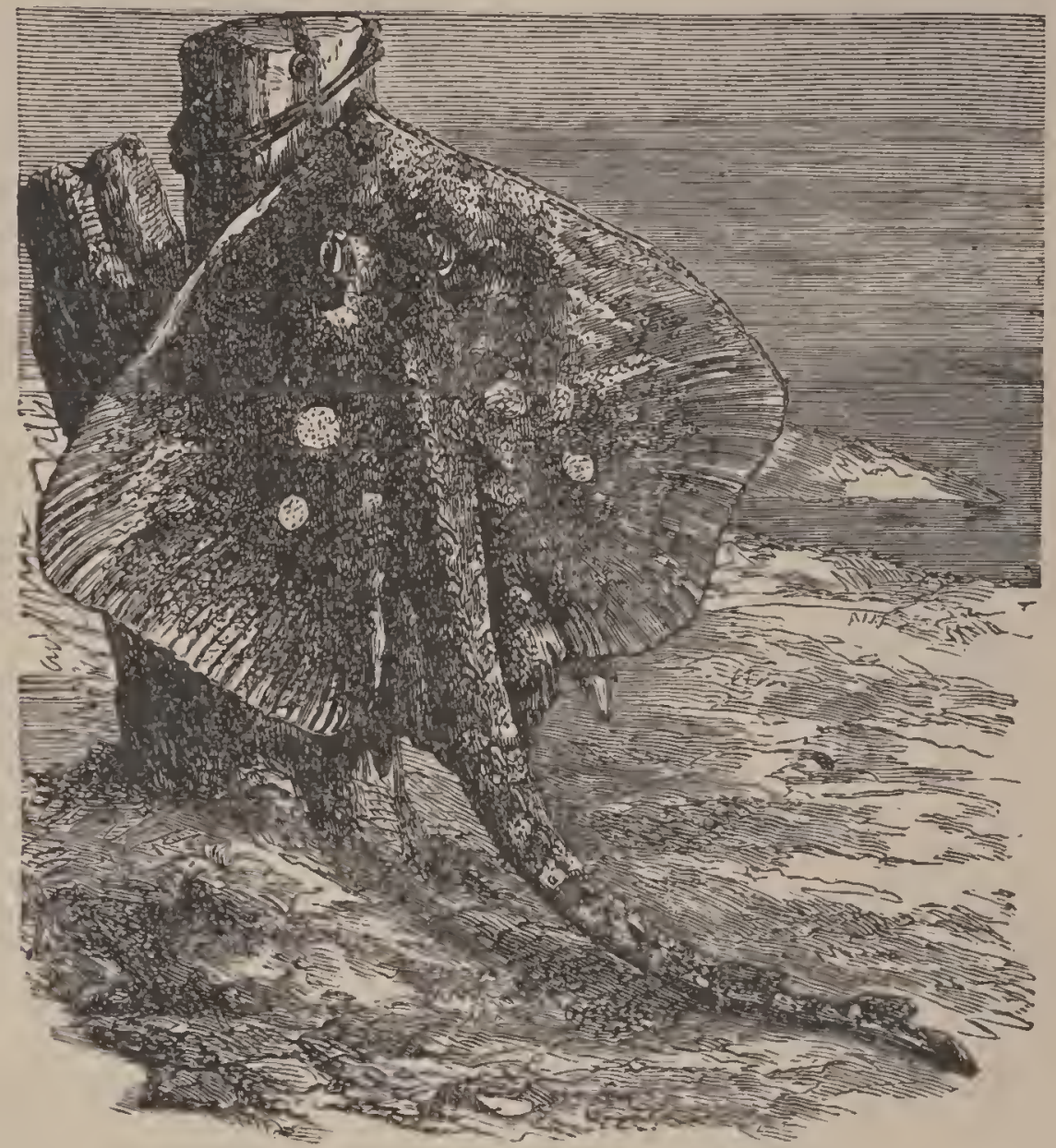

FIG. 2SS.-Thurnback (Raia clavata). European seas.

2. Ganoidei, distinguished by their enameled scales or bony plates. The endoskeleton is usually not completely ossified; the ventral fins are placed far back; and the tail is generally heterocercal. The gills are like those of the bony Fishes, and the air-bladder has a duct, and may aid in respiration. This was one of the largest orders in old geological history. The few modern representatives, as the Sturgeon, Gar-pike, Mud (or Dog) Fish, and Polypterus, are essentially fresh-water.

3. Teleostei, including all the common Fishes having a bony endoskeleton

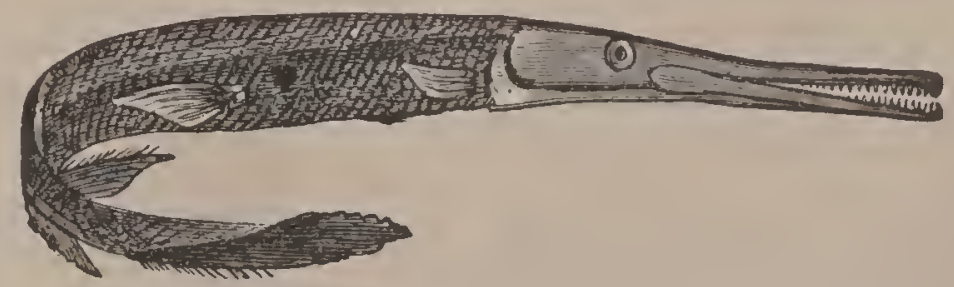

FIa. 2S9.-Gar-pike (Lepidosteus osseus). Lake Ontario 


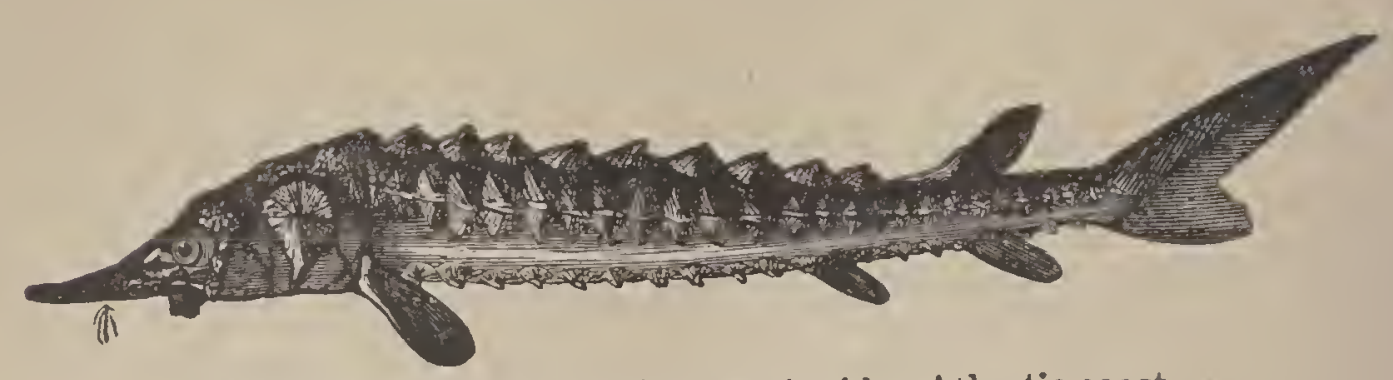

FIG. 290.-Sturgeon (Acipenser sturio). Atlantic coast.

and a scaly exoskeleton. The skull is extremely complicated; the upper and lower jaws are complete, and the

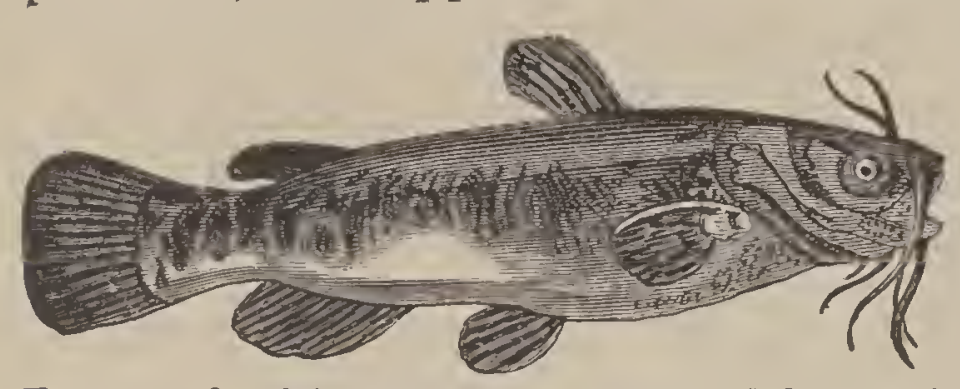

Frg. 291.-Cat-fish, or Horued Pout (Pimelodus catus). American rivers.

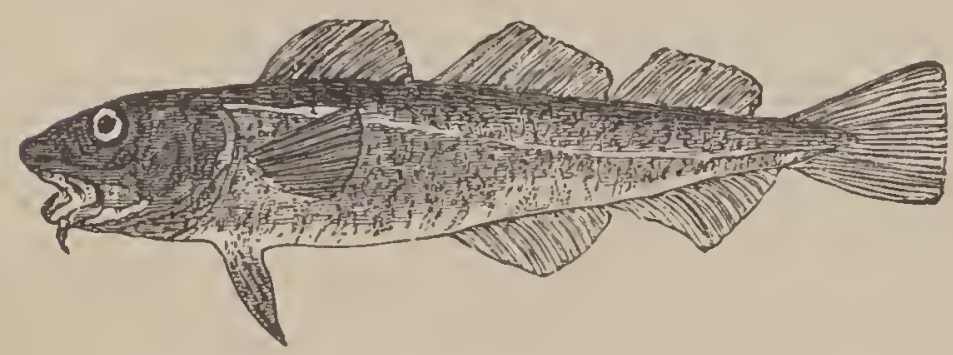

Fig. 292.-Cod (Gadus morrhua). Atlantic coast. gills are comb-like or tufted. The tail is homocercal; the other fins are variable in number and pusition. In the soft - fiuned Fishes, the ventrals are absent, as in the Eels; or attached to the abdomen, as in the Salmons, Herrings, Pikes, and Carps; or placed nuder the throat, as in the Cod, Haddock, and Flounder. In the spiny-finned Fishes, the ventrals are generally under or in front of the pectorals, and the scales ctenoid, as in the Perches, Mullets, and Mackerels.

4. Dipnoi. 'These Fishes connect the class with the Amphibia. They have an eel-like body, covered with cycloid scales; an embryonic notochord for a back-bone;

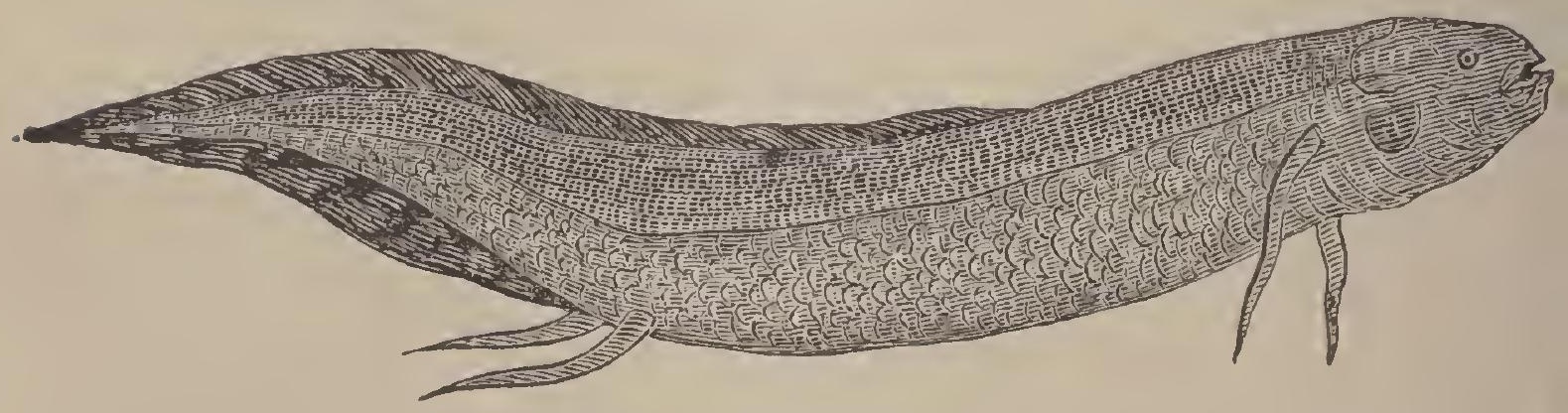

FIG. 293.-Protopterus annectens; one fourth natural size. African rivers. 
long, ribbon-like pectoral and ventral fins, set far apart; two auricles, and one ventricle; and, besides gills, a cellular air-bladder, which is used as a lung.

The representatives are Ceratodus from Australia, Pro topterus from Africa, and Lepidosiren from Brazil.

\section{Class II.-Amphibia.}

These cold-blooded Vertebrates are distinguished by having gills when young, and true lungs when adult. They lave no fin-rays, and the limbs, when present, have the same divisions as those of higher animals. The skin is soft, and generally naked, and the skeleton is ossified. The skull is flat, and articulates with the spinal column by two condyles. There is no distinct neck; and the ribs are usually small or wanting. The heart consists of two auricles and one ventricle. All undergo metamorphosis upon leaving the egg, passing through the "tadpole" state (Fig. 174). They commence as water-breathing larvæ, when they resemble Fishes in their respiration, circulation, and locomotion. In the lowest forms, the gills are retained through life; but all other's have, when mature, lungs only, the gills disappearing. The cuticle is frequently shed, the mode varying with the habits of the species. ${ }^{161}$ The common Frog, the type of this class, stands intermediate between the two extremes of the vertebrate series; no fundamental part is excessively developed.

There are about seven hundred living species, grouped in four orders:

1. Proteida have a naked skin, a tail, and two or four limbs. Some retain their gills through life, as the

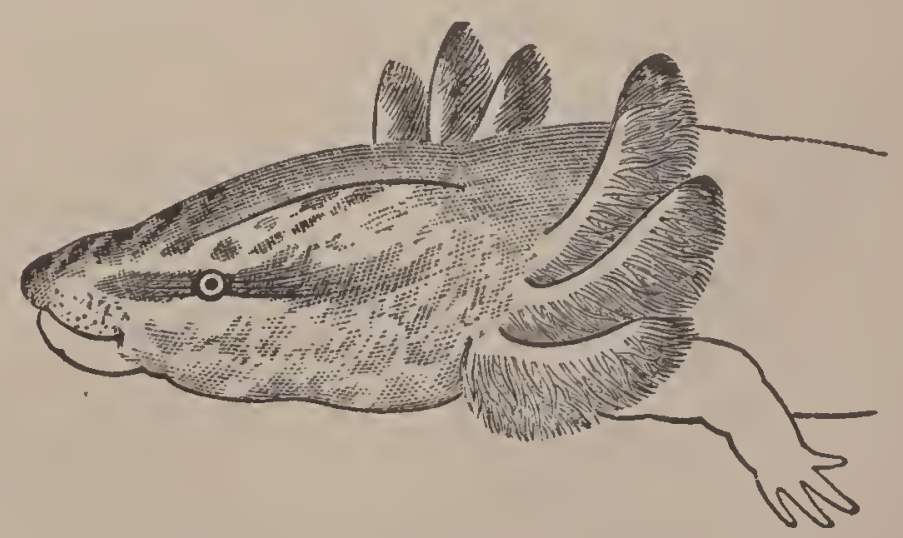

Frg. 294.-Head and Gills of Necturus. Cayuga Lake. 
Proteus of Austria, and Necturus of the eastern United States.

2. Urodela, as the aquatic Newts and land Salamander's, always have four limbs, but the gills rarely persist in the adult stage. ${ }^{162}$

3 Caccilia have neither tail nor limbs, a snake-like form,

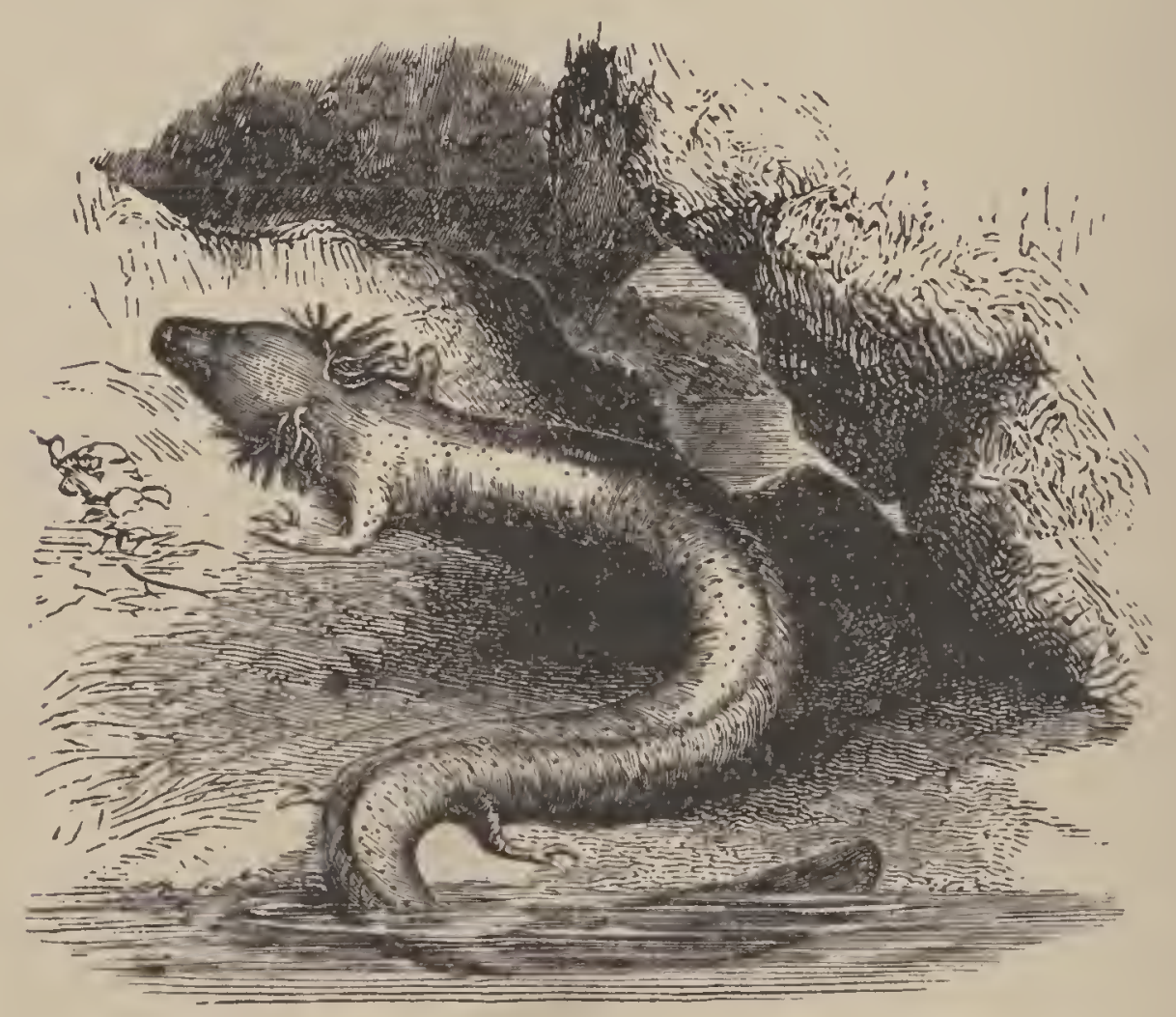

Frg. 295.-Proteus anguinus. Europe.

minute scales in the skin, and well-developed ribs. They are confined to the tropics.

4. Anura include all the well-known tailless Am-

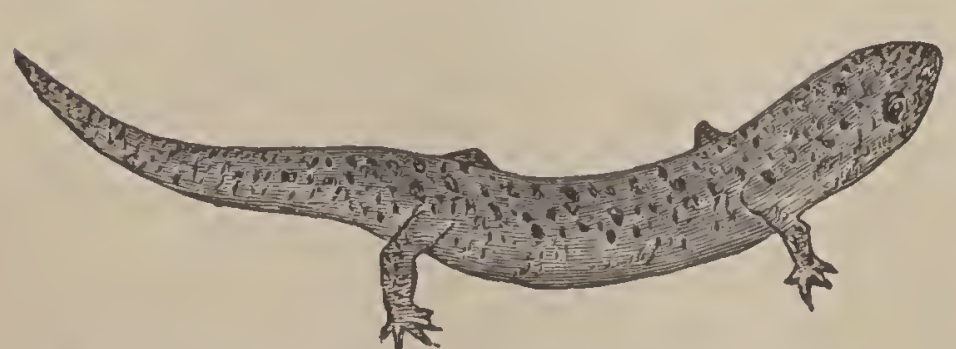

Fig. 296. - Red Salamander (Pseudotriton ruber). United States. phibians, as Frogs and Toads. They have a moist, naked skin, ten vertebræ, and no ribs. As they breathe by swallowing the air, they can be suffocated by holding the mouth open. They have 
four limbs-the hinder the longer, and the first developed. They have four fingers and five toes. The tongue is long, and, fixed by its anterior end, it can be rapidly thrown out as an organ of prehension. ${ }^{163}$ The eggs are laid in the water enveloped in a glairy mass; and the tadpoles

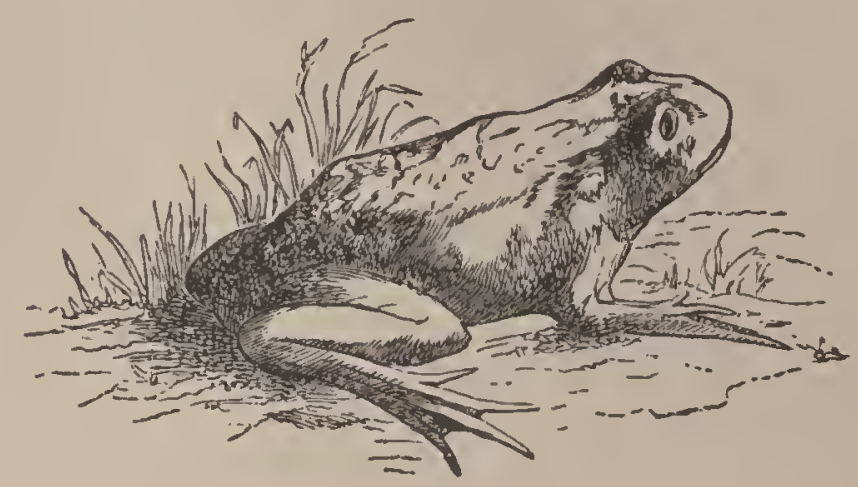

Fia. 297.-Frog (Rana). resemble the Urodelans, till both gills and tail are absorbed. Frogs (Rana) have teeth in the upper jaw, and webbed feet; Toads (Bufo) are higher in rank, and have neither teeth nor fully webbed feet. The former have been known to live sixteen years, and the latter thirty-six.

\section{Class III.-Reptilia.}

These air-breathing, cold-blooded Vertebrates are distinguished from all Fishes and Amphibians by never having gills, and from Birds by being covered with horny scales or bony plates. The skeleton is never cartilaginous; and the skull has one occipital condyle. The vertebræ are ordinarily concave in front; and the ribs are well developed. With few exceptions, all are carnivorous; and teeth are always present, except in the Turtles, where a horny sheath covers the jarvs. The teeth are never fastened in sockets, except in Crocodiles. The jaws are usually very wide. The heart has three chambers, save in Crocodiles, where the ventricle is partitioned. But in all cases a mixture of arterial and renous blood is circulated. The lungs are large, and coarsely cellular. The limbs, when present, are provided with three or more fingers as well as toes.

There are about three thousand species of living Reptiles, and of these there are four main groups: the first 
two have horny scales, the others have bony plates combined with scales.

1. Ophidia, or Snakes, are characterized by the absence of visible limbs $;^{164}$ by the great number of vertebræ, amounting to over four hundred in the great Serpents; by a corresponding number of ribs, but no sternum; and no true eyelids, the eyes being covered with a transparent

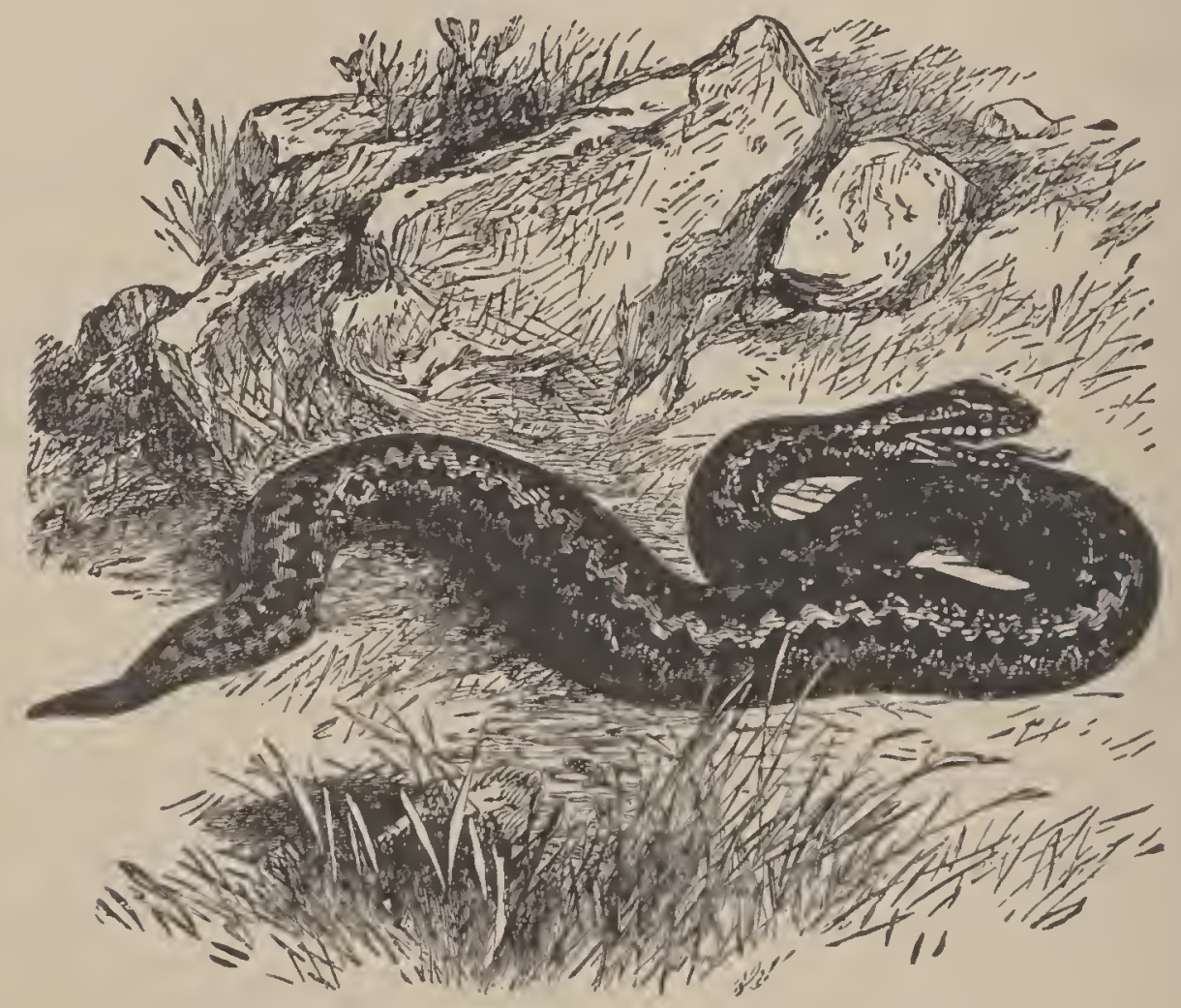

Fig. 29S.-Adder, or Viper (Pelias berus). England.

skin. The tongue differs from that of nearly all other Reptiles in being bifid and extensile. The mouth is very dilatable. The skin is frequently shed, and always by reversing it. Snakes make their way on land or in water with equal facility.

As a rule, the venomous Snakes, as Vipers and Rattlesnakes, are distinguished by a triangular head covered with small scales; a constriction behind the head; two or more fangs, and few teeth; small eyes, with vertical pupil; and short, thick tail. In the harmless Snakes, the head gradually hlends with the neck, and is covered with plates; the teeth are comparatively numerous in both jaws; the pu- 


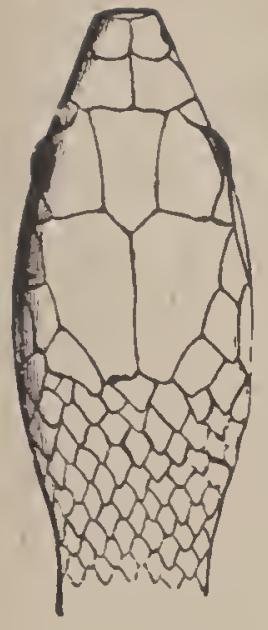

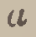

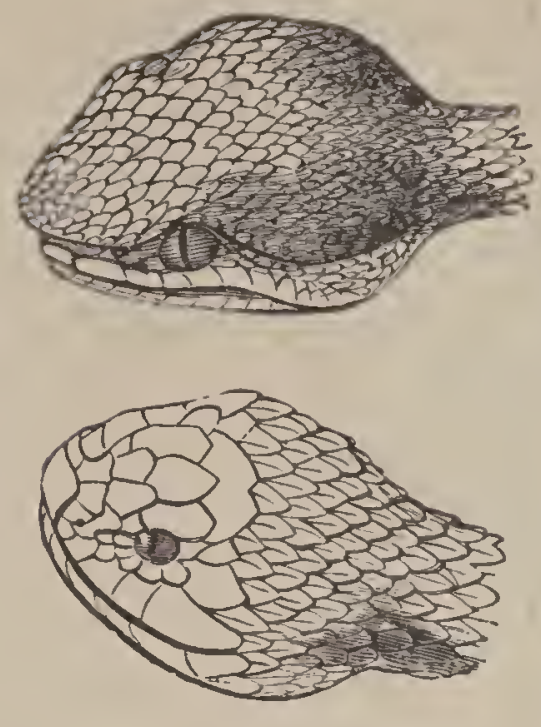

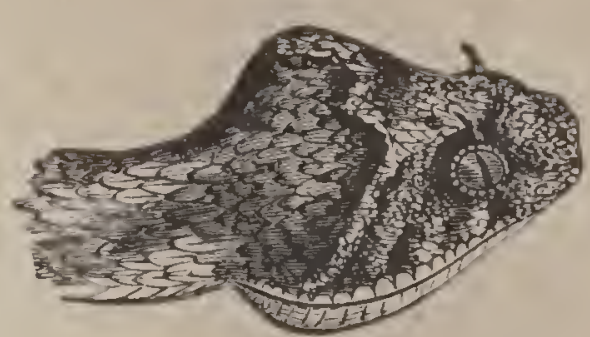

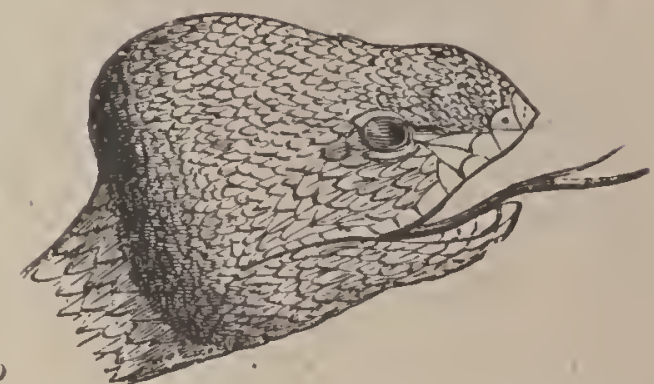

F1G. 299. —a, Head of a Harmless Sunke (upper view); $b$, heads of various Venomous Snakes.

pil is round, and the tail tapering. This rule, however, has many exceptions.

2. Lacertilia, or Lizards, may be likened to Snakes provided with four limbs, each having five digits. ${ }^{105}$ The body is covered with horny scales. All have teeth, which are simple in structure; and the halves of the lower jaw are firmly united in front, while those of Snakes are

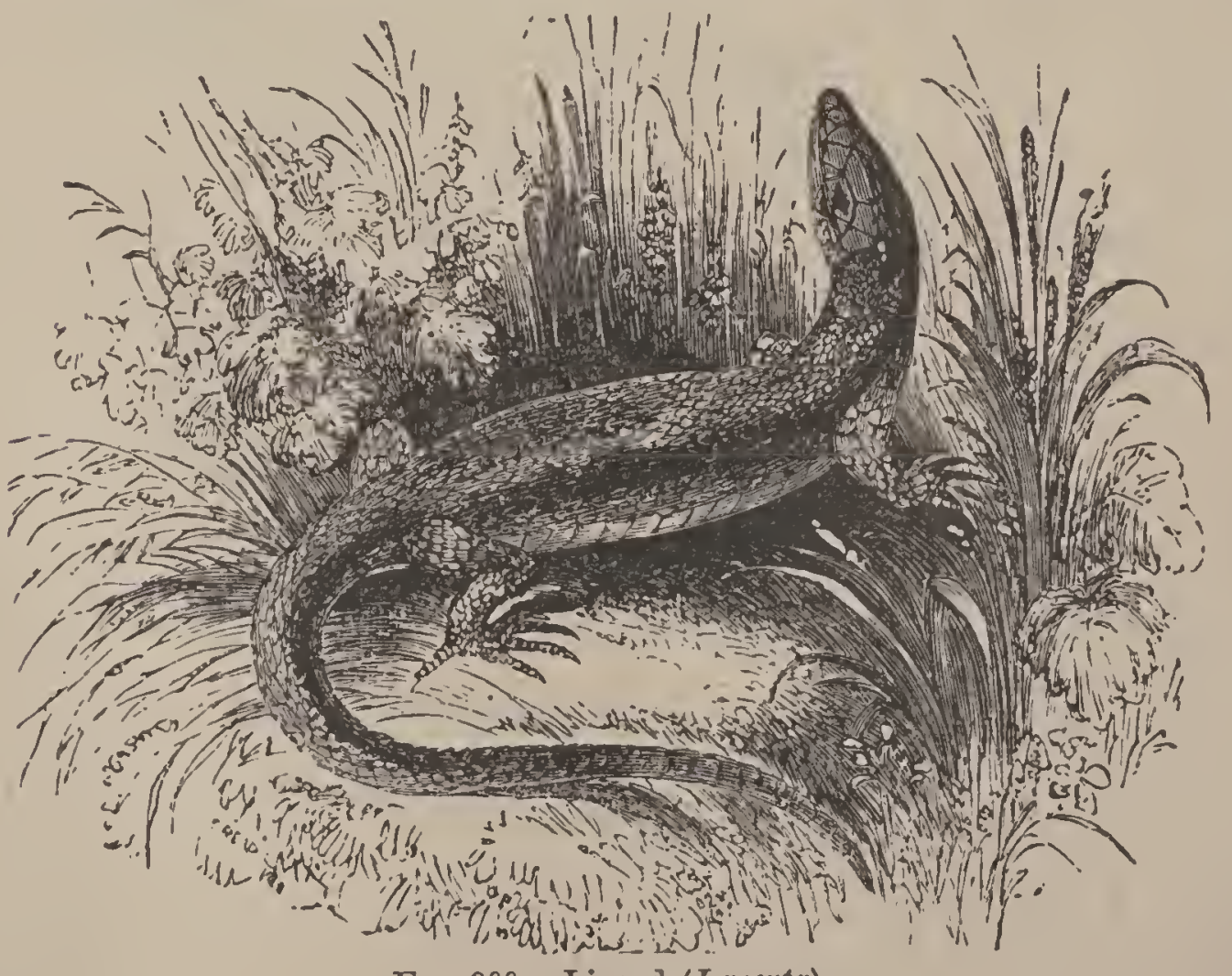

FIG. 300.-Lizard (Lacerta). 
loosely tied together by ligaments. Nearly all have a breast-bone, and the eyes (save in the Gecko) are furnished with movable lids. In the common Lizards and Chameleon, the tongue is extensile. The tail is usually long, and in some cases each caudal vertebra has a divis' ion in the middle, so that the tail, when grasped, breaks off at one of these divisions. The Chameleon has a prehensile tail. The Iguana is distinguished by a dewlap on the throat and a crest on the back. Except some of the Monitors of the Old World, all the Lizards are terrestrial.

3. Chelonia, or Tortoises and Turtles, are of anomalous structure. The skeleton is external, so as to include not only all the viscera, but also the whole muscular system, which is attached internally; and even the limbs are

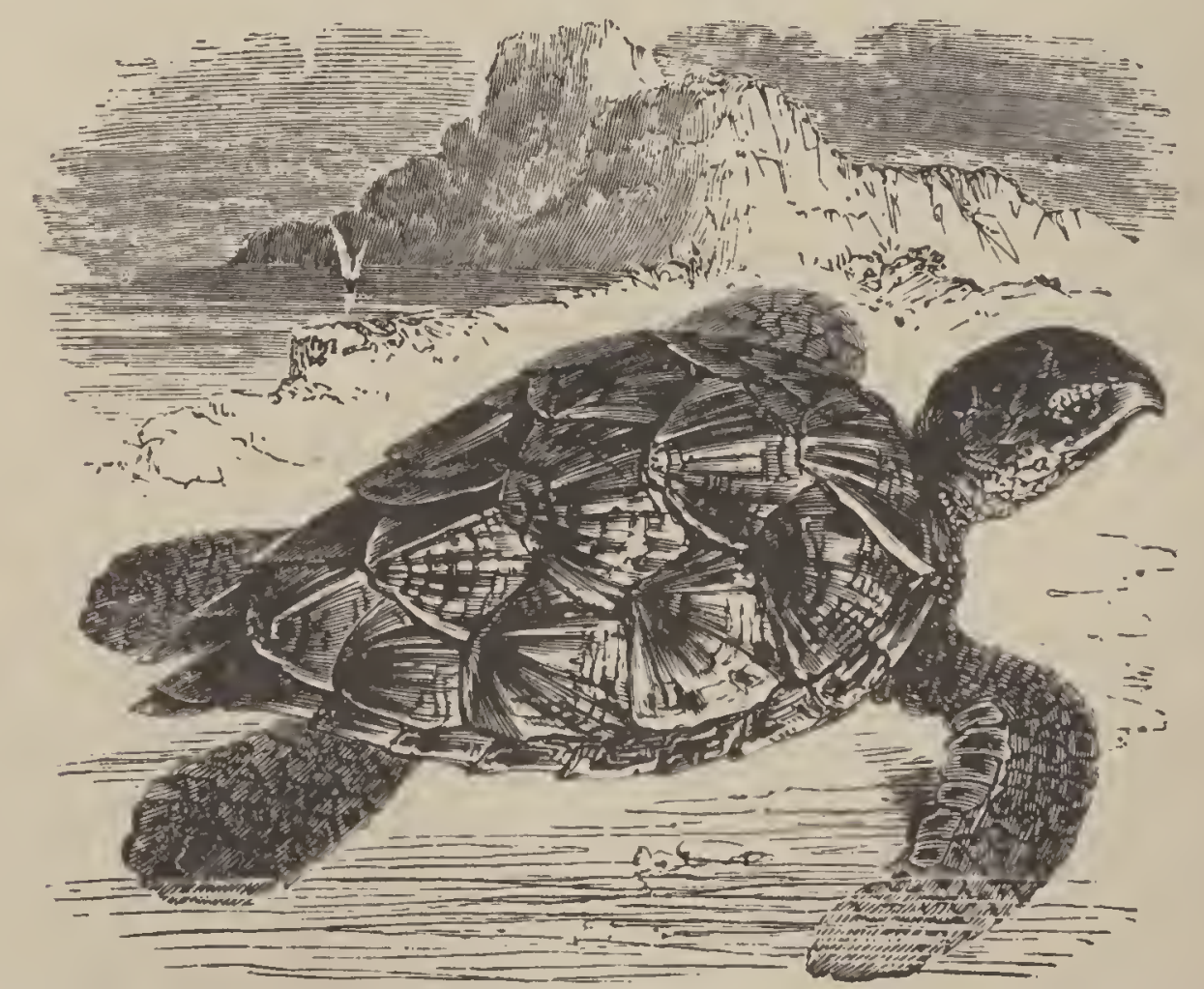

FIG. 301.-Hawk's-bill Turtle (Eretmochelys imbricata). Tropical Atlantic.

inside, instead of outside, the thorax. The exoskeleton unites with the endoskeleton, forming the carapace, or case, in which the body is enclosed. The exoskeleton consists of horny plates, known as "tortoise-shell" (in the soft Tortoises, Trionyx, this is wanting), and of dermal 
bones, united to the expanded spines of the vertebræ and to the ribs, making the walls of the carapace. The ventral pieces form the plastron, or sternum. ${ }^{166}$ All are toothless. There are always four stout legs; and the order furnishes the only examples of Vertebrates lower than

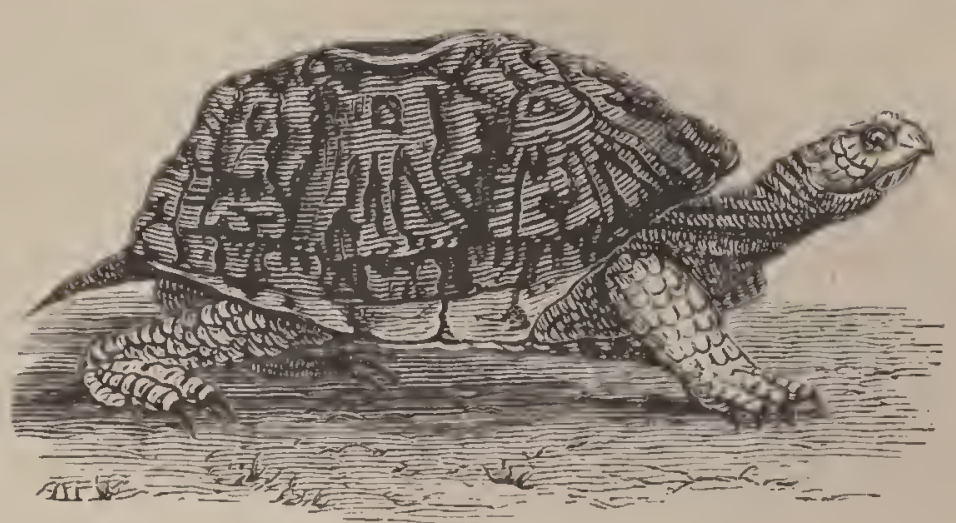
FIG. 302, - Box-tortoise (Cistudo Virginea). United States.

Birds that really walk, for Lizards and Crocodiles wriggle, and drag the body along. There are no teeth, but a horny beak. The eggs are covered with a calcareous shell.

The Sea-turtles, as the edible Green Turtle and the Hawk's-bill Turtle, which furnish the "tortoise-shell" of commerce, have the limbs converted into paddles. The fresh-water forms, represented by the Snapping Turtle (Chelydra), are amphibious, and have palmated feet. Land Tortoises (Testudo) have short, clumsy limbs, fitted for slow motion on the land; the plastron is very broad, and the carapace is arched (while it is flattened in the aquatic species), and head, legs, and tail can be drawn within it. The land and marine species are vegetable-feeders; the others, carnivorous.

4. Crocoditia, the highest and largest of Reptiles, have also two exoskeletons-one of horny scales (epidermal), and another of bony plates (dermal). The bones of the skull are firmly united, and furnished with numerous teeth, implanted in distinct sockets. The lower jaw extends back of the cranium. The heart has four cavities, but the pulmonary artery and aorta communicate with each other, so that there is a mixture of venous and arterial blood. They have external ear-openings, closed by a flap of the 
skin, and eyes with movable lids; a muscular gizzard; a lung, compressed tail; and four legs, with feet more or less webbed, and having five toes in front and four behind. The existing species are confined to tropical river's, and are carnivorous. The eggs are covered with a hard shell.

There are three representative forms: the Gavial of the Ganges, remarkable for its loug snout and uniform teeth; the Crocodiles, mainly of the Old World, whose teeth are unequal, and the lower canines fit into a notch in the edge of the upper jaw, so that it is visible when the mouth is

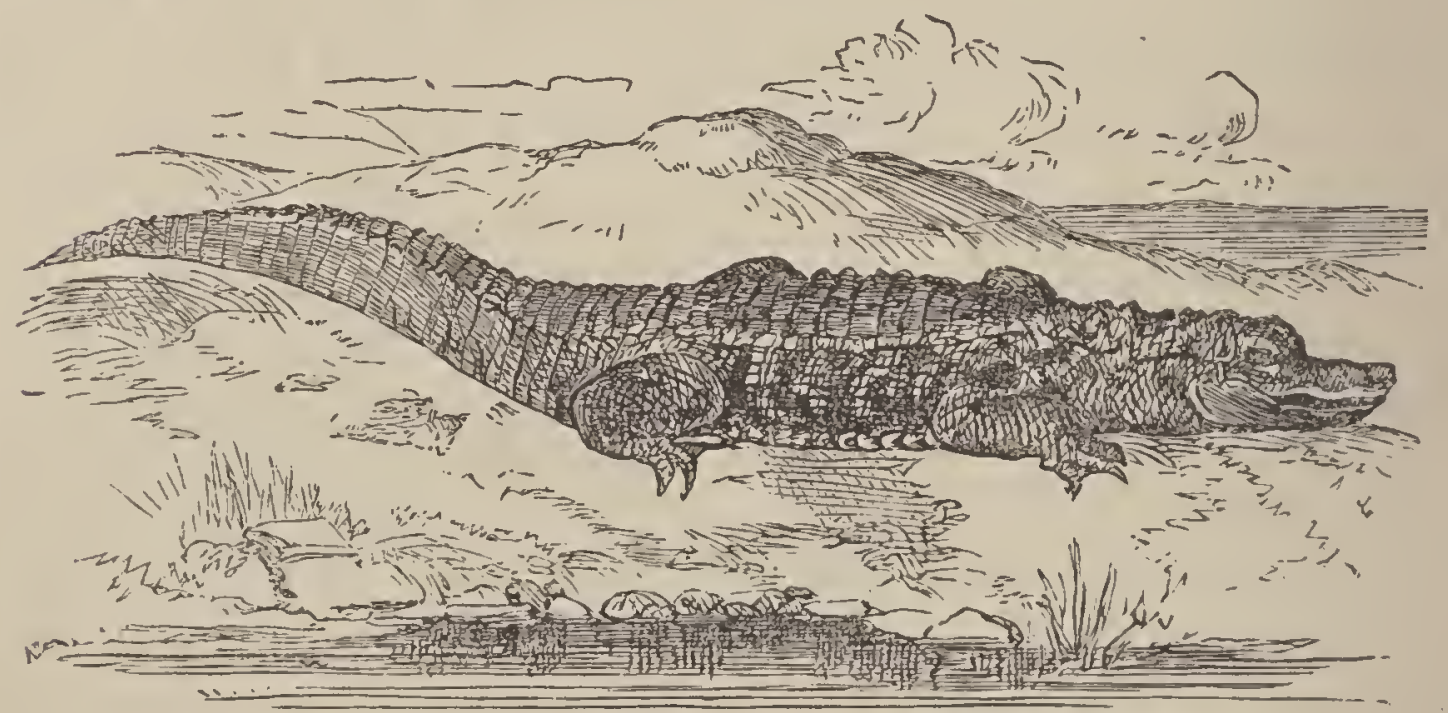

Frg. 303.-Alligator (A. Mississippiensis). Southern States.

closed; and the Alligators of the New World, whose canines, in shutting the mouth, are concealed in a pit in the upper jaw. The toes of the Gavials and Crocodiles are webbed to the tip; those of the Alligators are not more than half-webbed.

In the medirval ages of geological history, the class of Reptiles was far more abundantly represented than now. Among the many forms which geologists have unearthed are numerous gigantic Saurians, which cannot be classified with any of the four living order's. Such are the Ichthyosaurus, Plesiosaurus, Pterodactyle, Megalosaurus, and Iguanodon. 


\section{Class IV. - Aves.}

Birds form the most clearly defined class in the whole Animal Kingdom. The Eagle and Hummer, the Ostrich and Duck, widely as they seem to be separated by size, form, and habits, still exhibit one common type of structure. On the whole, Birds are more closely allied to Reptiles than to Mammals. In number, they approach the Fishes, ornithologists having determined eight thousand species, or more.

A Bird is an air-breathing, egg-laying, warm-blooded, feathered Vertebrate, with two limbs (legs) for perching, walking, or swimming, and two limbs (wings) for flying or swimming. Organized for flight, it is gifted with a light skeleton, very contractile muscular fibre, and a respiratory function of the highest development.

The skeleton is more compact than those of Reptiles and Mammals, at the same time that it is lighter, and the bones are harder and whiter. It contains fewer bones than nsual, many parts being anchylosed together, as the skull-bones, the dorsal vertebræ, and bones of the tarsus and metatarsus. The lumbar vertebra are united to the ilia. The neck is remarkably long (containing from nine to twenty-four vertebræ) and flexible, enabling the head to be a most perfect prehensile organ. The ribs are generally jointed in the middle, as well as with the backbone and sternum. The last, where the muscles of flight originate, is highly developed. The skull articulates with the spinal column by a single condyle, and with the lower jaw, not directly, as in Mammals, but through the intervention of a separate bone, as in Reptiles.

All Birds always have four limbs, while every other vertebrate class shows exceptions. The fore-limbs are fitted for flight. They ordinariiy consist of nine separate bones, and from the hand, fore-arm, and humerus are de- 
veloped the primary, secondary, and tertiary feathers of the wing. The hind-limbs are formed for progressionwalking, hopping, running, paddling, and also for perching and grasping. The modifications are more numerous and important than those of the bill, wing, or tail. There are twenty bones ordinarily, of which the tibia is the principal; but the most characteristic is the tarso-metatarsus,

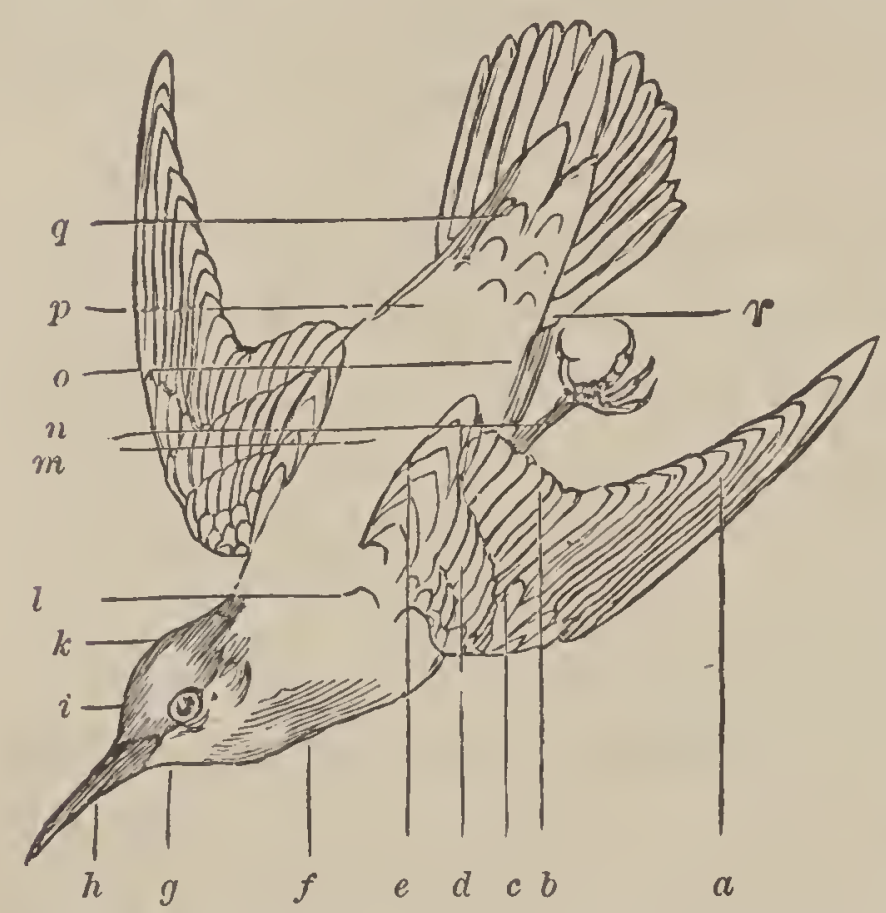

Fig. 304.-Principal Parts of a Bird: $\alpha$, primnries; $b$, secondaries; $c$, spurious wing ; $d$,wing-coverts : $e$, tertiaries; $f$, throat, or jugulum ; $g$, chin ; $h$, bill; the meetiug-liue between the two mandibles is the commissure; the ridge on the upper mandible is called cnlmen; that of the lower, gonys; the space between the base of the $11 \mathrm{pper}$ mandible and the eye is the lore $i$, forehead; $k$, crown; $l$, scapular feathers; $m$, back; $n$, metatarsus, often called tarsus or tarso-metatarsus; $o$, abdomen; $p$, rump ; $q$, upper tail-coverts ; $r$, lower tail-coverts.

which is a fusion of the lower part of the tarsus with the metatarsus. The rest of the tarsus is fused with the tibia. The thigh is so short that the knee is never seen outside of the plumage; the first joint visible is the heel. ${ }^{167}$ Most Birds have four toes (the external or "little" toe is always wanting); many have three, the hallux, or "big" toe, being absent; while the Ostrich has but two, answering to the third and fourth. The normal number of phalanges, reckoning from the hallux, is $2,3,4,5$. The toes always end in claws.

Birds have neither lips nor teeth, epiglottis nor diaphragm. The teeth are wanting, because a heavy masticating apparatus in the head would be unsuitable for flight. The beak, crop, and gizzard vary with the food. It is a peculiarity of all Birds, though not confined to 
them, that the generative products and the refuse of digestion are all discharged through one common ontlet.

The sole organs of prehension are the beak and feet. The circulation is double, as in Mammals, starting from a four-chambered heart. Respiration is more complete than in other Vertebrates. The lnngs are fixed, and communicate with air-sacs in varions parts of the body, as along the vertebral column, and also with the interior of many bones, as the humerus and femur, which are usually hollow and marrowless. ${ }^{188}$ Both brain and cord are much larger relatively than in Reptiles; the cranium is larger in proportion to the face; and the parts of the brain are not situated in one plane, one behind the other. The cerebrum is round and smooth, and the cerebellum singlelobed. The ears resemble those of Crocodiles; but the eyes are well developed, and protected by three lids. They are placed on the sides of the head, and the pupil is always round. The sexes generally differ greatly in plumage, in some cases more widely than two distinct species, but the coloration of either sex of any one species is very constant.

There are two subclasses. ${ }^{180}$

\section{Subclass I.-Ratitæ (Cursores).}

This small and singular group is characterized by having no keel on the breastbone, rudimentary wings, feather's with disconnected barbs, and stout legs. The African Ostrich has two toes, the Cassowary three, and the Apteryx four.

Its representatives are the Ostrich (Struthio) of Africa and Arabia, South American Ostrich (Rhea), Cassowary (Casuarius) of the East Indian Archipelago and Australia, Emu (Dromceus) of Australia, and Apteryx, or Kiwikiwi, of New Zealand. Besides these, there are extinct gigantic forms from Madagascar (Epyornis) and from 


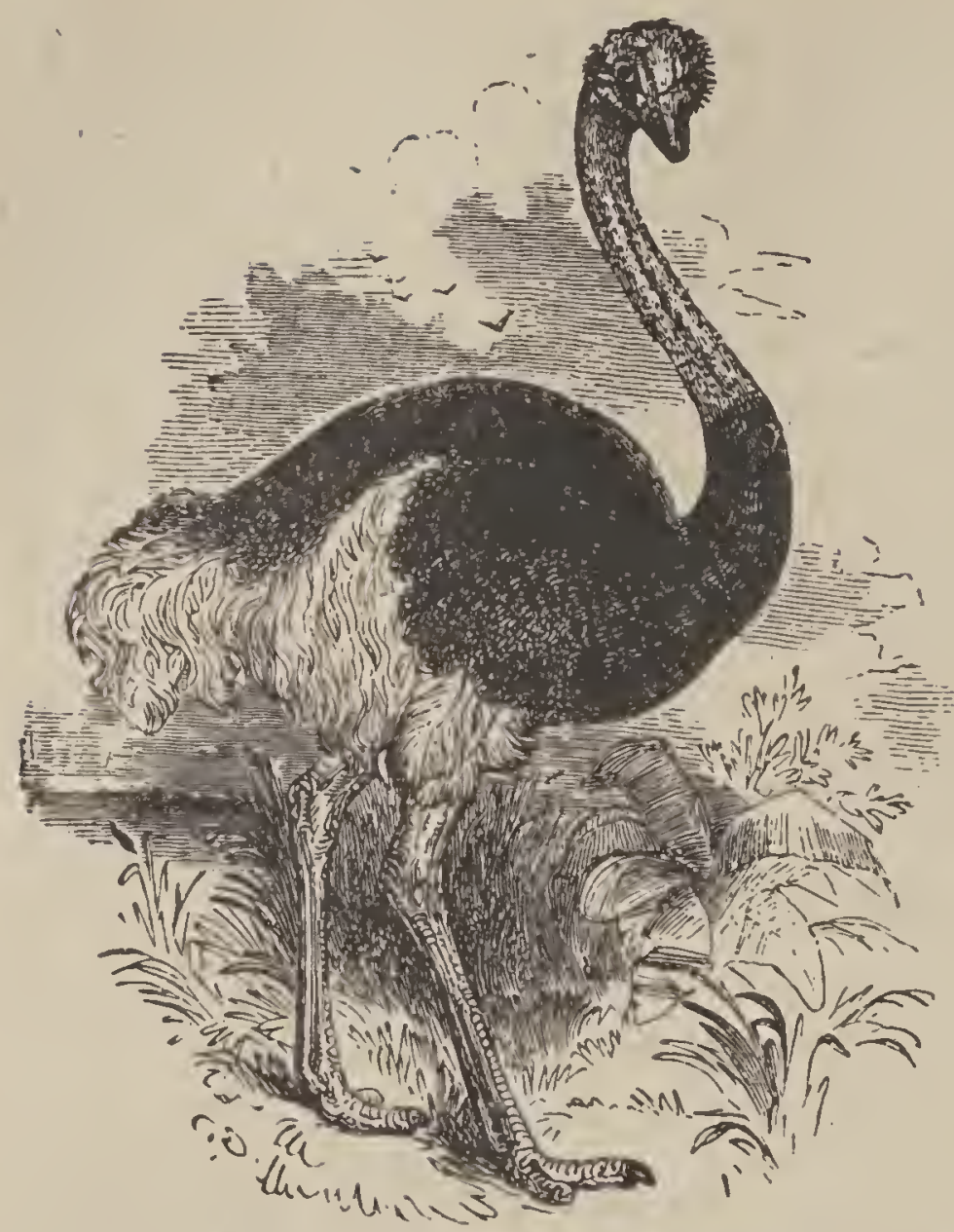

FIG.305.-African Ostrich (Struthio camelus).

oped functional wings.

New Zealand ( $D i$ nornis, or Moa). This singular geographical distribution, like that of the Dipnoi and Marsupials, shows that the group was once widely spread over the earth, but is now greatly restricted in area.

Subclass II.

Carinatæ.

Birds with a keeled sternum, and with devel-

A. Aquatro Birds. - Specially organized for swimming; the body flattened, and covered with water-proof clothing-feathers and down; the legs short (the knees being wholly withdrawn within the skin of the body), and set far apart and far back; the feet webbed, and hind-toe elevated or absent. The legs are always feathered to the heel at least. They are the only Birds whose neck is sometimes longer than the legs.

1. Pygopodes, or Divers. These lowest of the feathered

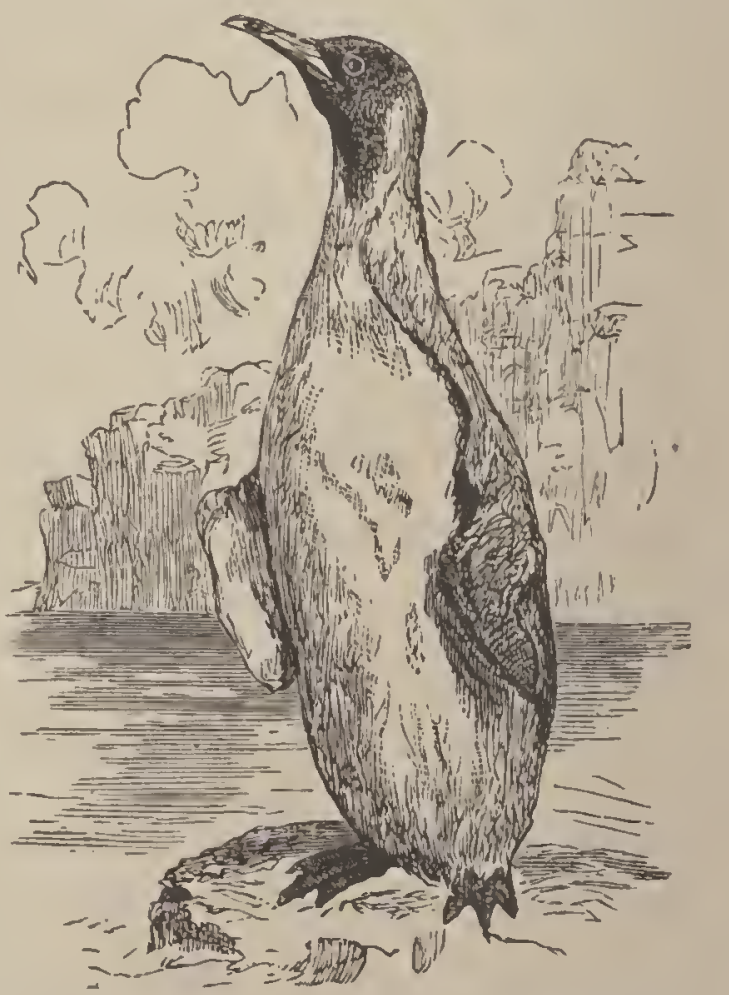

Fis. 306.-Penguin (Aptenodytes Pennanm tii). Falkland Islands. 


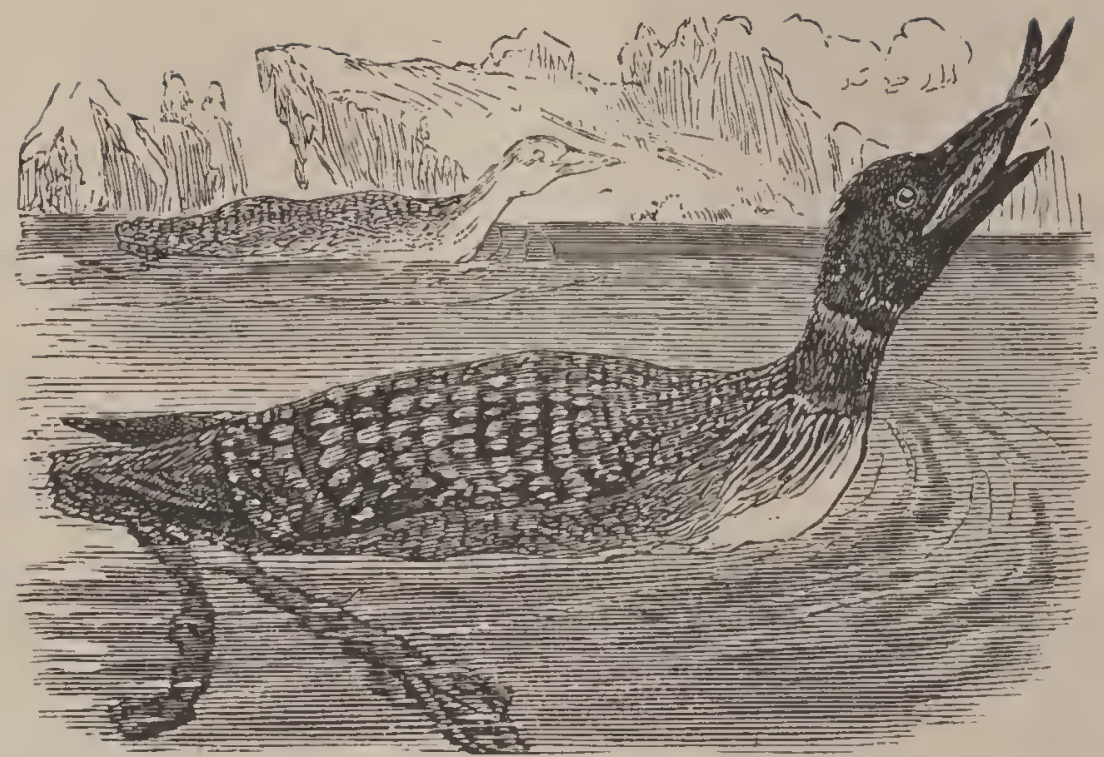

FIG. 307.-Loon (Colymbus torquatus). North America.

tribe have very short wings and tail, and the legs are placed so far back that they are obliged, when on land, to stand nearly bolt upright. They are better fitted for diving than for flight, or even swimming. They belong to the high latitudes, living on Fishes mainly, and are represented by the Penguins, Auks, Loons, and Grebes.

2. Longipennes, or Gulls.-Distinguished by their long,

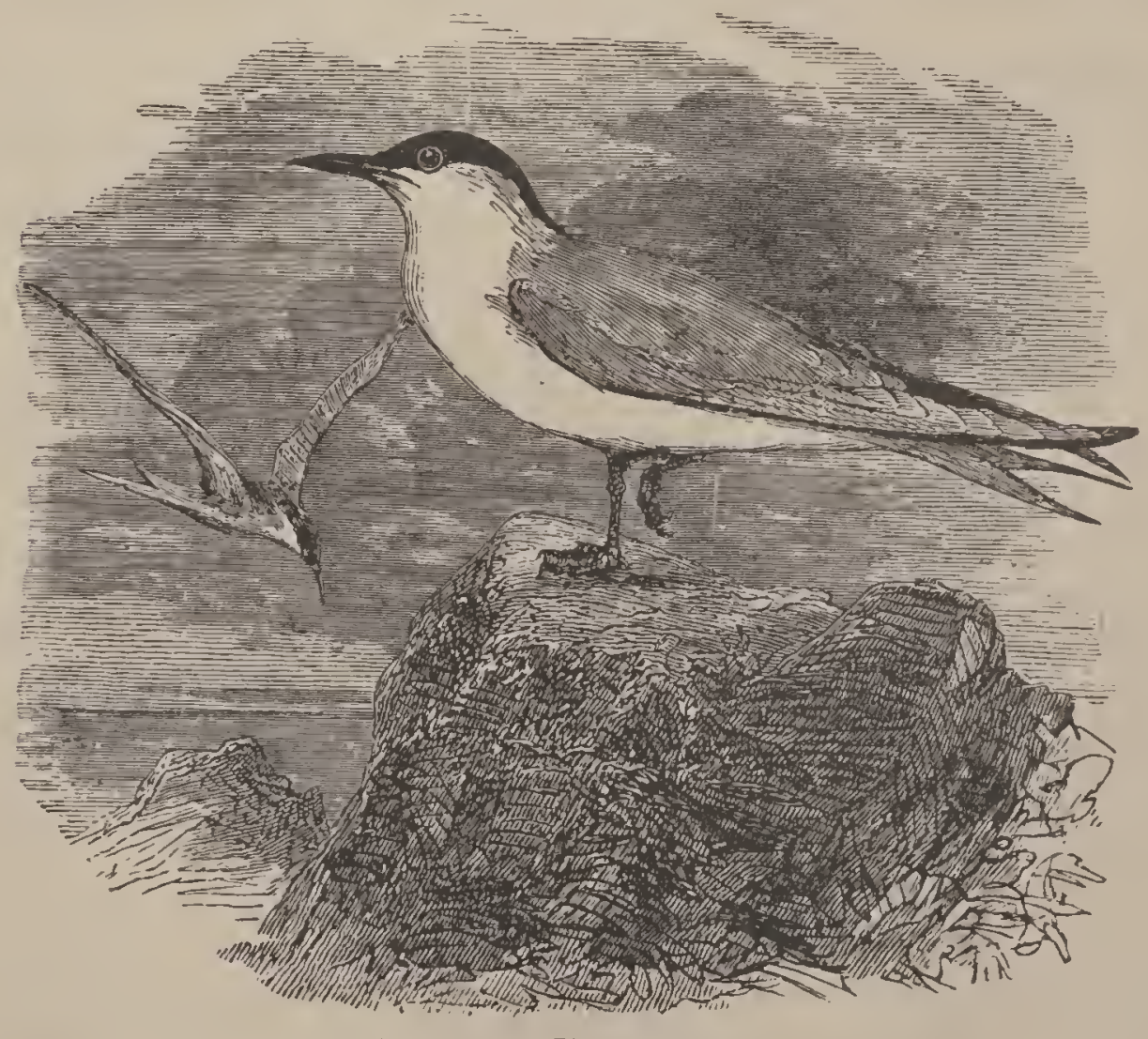

Fia. 308.-Tern (Sterna). 


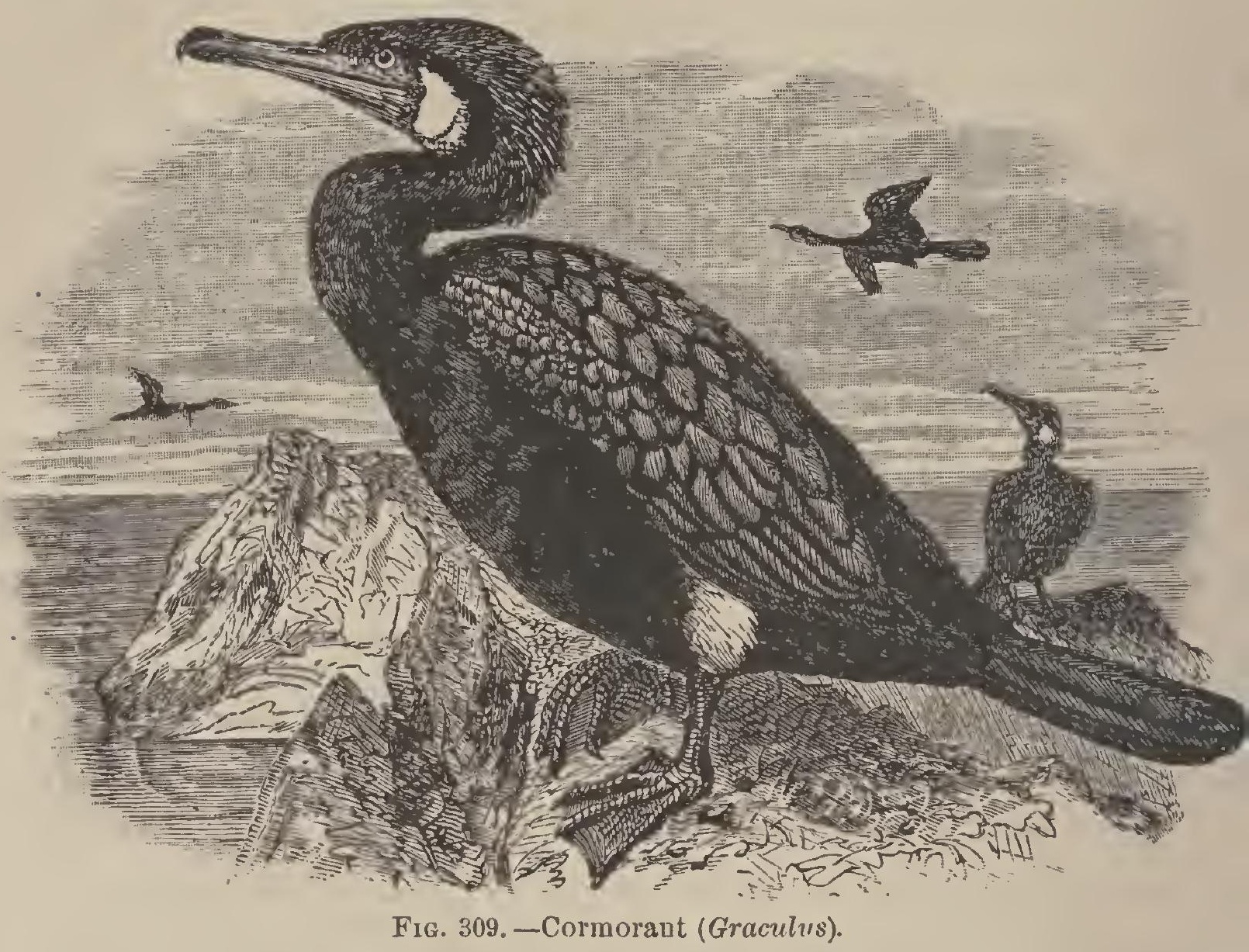

pointed wings, usually long tail, and by great powers of flight. They are all carnivorous. Such are the Gulls and Terns, which frequent the sea-coast, lakes, and rivers; and

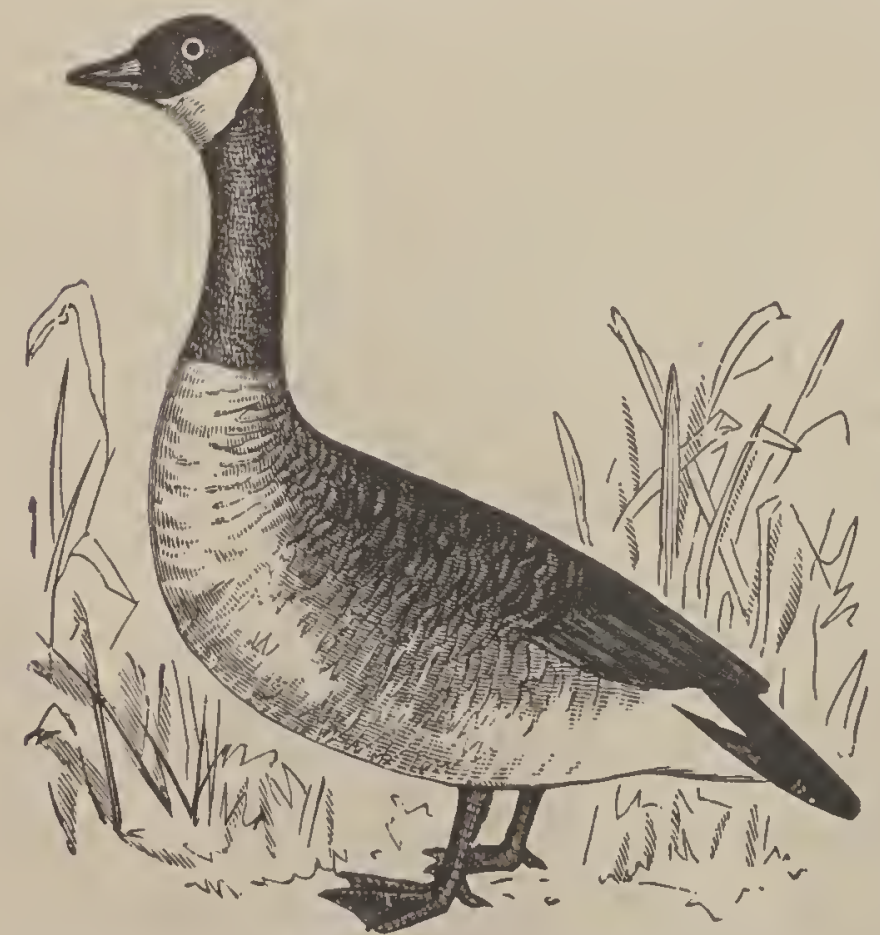

FrG. 310.-Wild Guose (Bernicla Canadensis). United States. the Albatrosses and $\mathrm{Pe}$ trels (the largest and smallest of web-footed Birds), which are oceanic.

3. Totipalmates, or Cormorants. - Characterized by a long bill, generally hooked; wings rather long; and toes long, and all four joined together by broad webs. Throat generally naked, and furnished with a sac. The majority are large sea-birds, 
and feed on Fishes, Mollusks, and Insects. Examples are the Cormorants, Pelicans, and Gannets.

4. Lamellirostres, or Ducks, have a heavy body, moderate wings, short tail, flattened bill, covered by a soft skin,

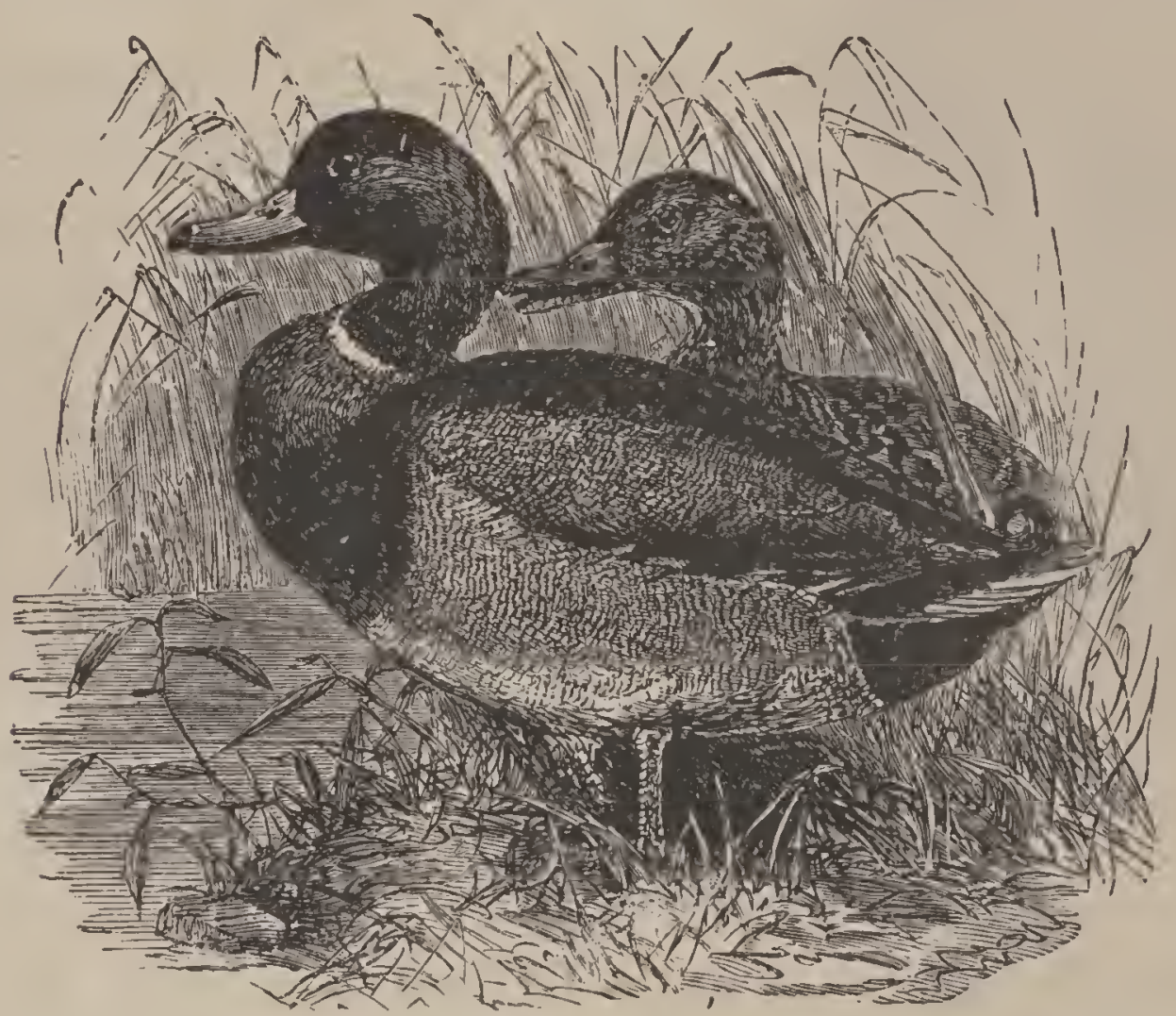

Frg. 311. -Wild Duck (Anas boschas). North America.

with ridges along the edges. Diet more commonly regetarian than animal. The majority inhabit fresh wateras the Ducks, Geese, Swans, and Flamingoes.

$B$. Terrestrial Birds. - This group exhibits great diversity of structure; but all agree in being especially terrestrial in habit, spending most of the time on the ground, not on trees or the water, although many of them fly and swim well. Fra. 312.-Sandpiper (Tringa hypoleacea). Englaud.

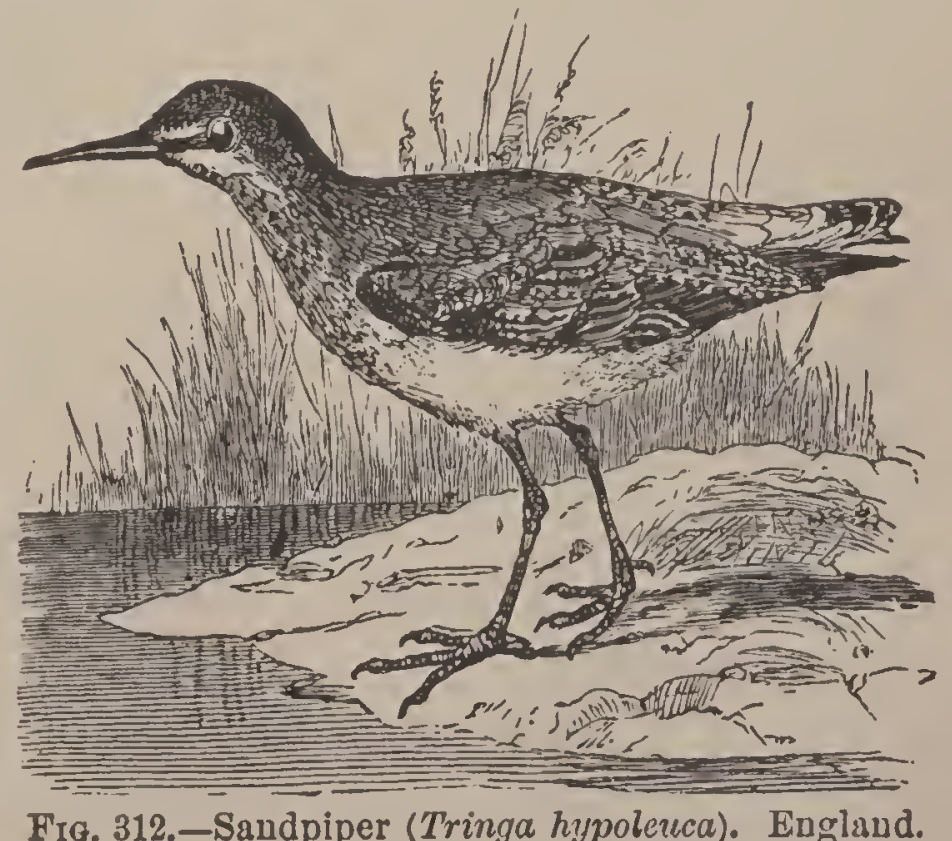


The legs are long or strong, and the knee is free from the

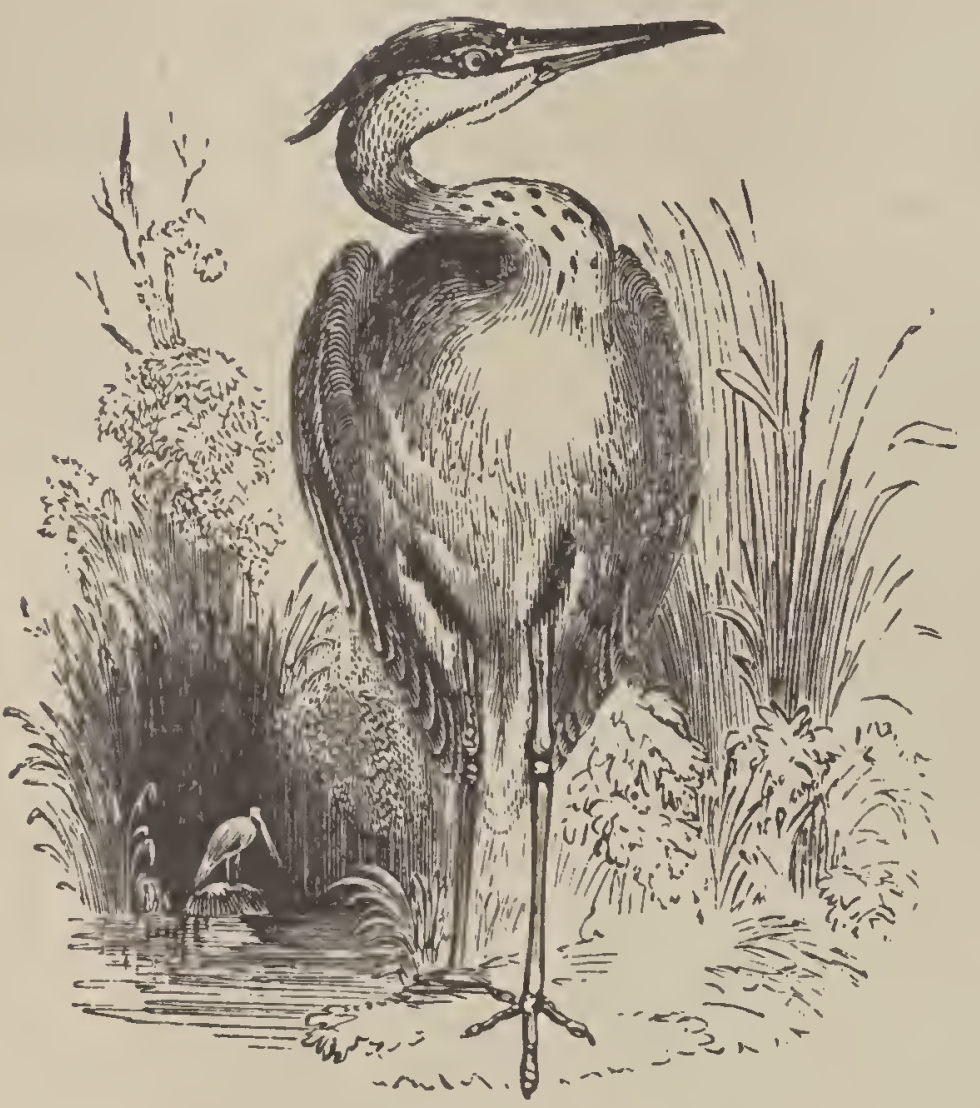

Fig. 313.-Heron (Ardea). body. The hind toe, when present, is small and elevated.

5. Grallatores, or $\mathrm{W}$ a ders.-These are readily distinguished by their long and bare legs. Generally, also, the toes, neck, and bill are of proportionate length, and the tail short. They feed on small animals, and, with a few exceptions, frequent the banks of rivers. In flying, their legs are stretched ont behind, while in most other Birds they are folded under the body. Such are the Rails, Cranes, Herons, Storks, Ibises, Stilts, Snipes, Sandpipers, and Plover's.

6. Rasores, or Scratchers. - As a rule, this order, so valuable to Man, is characterized by a short, arched bill; short and concave wings, unfitted for protracted flight; stout

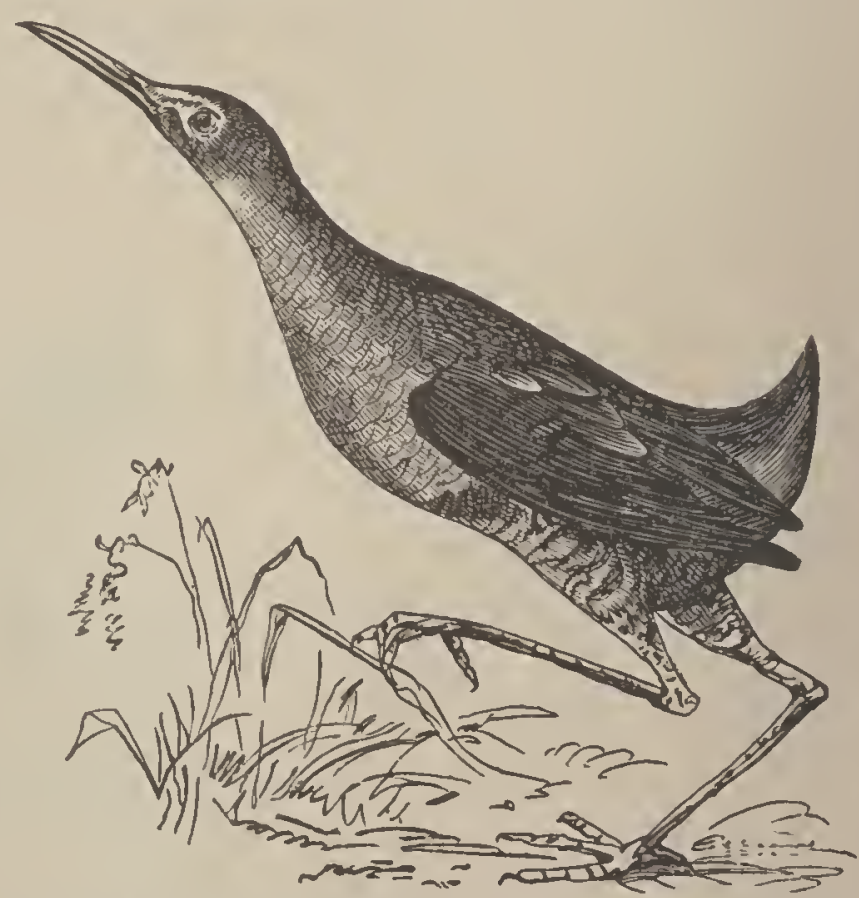

Fig. 314.-Rail, or Marsh Hen (Rallus elegans). United States. legs, of medium length; and four toes, the three in front 
being united by a short web, and terminating in blunt claws. The legs are usually feathered to the heel, sometimes (as in Grouse) to the toes. The feather's of the body are large and coarse. The males generally have gay plumage, and some appendage to the head. The nostrils are covered by a scale or valve. Their main food is grain. Such are the

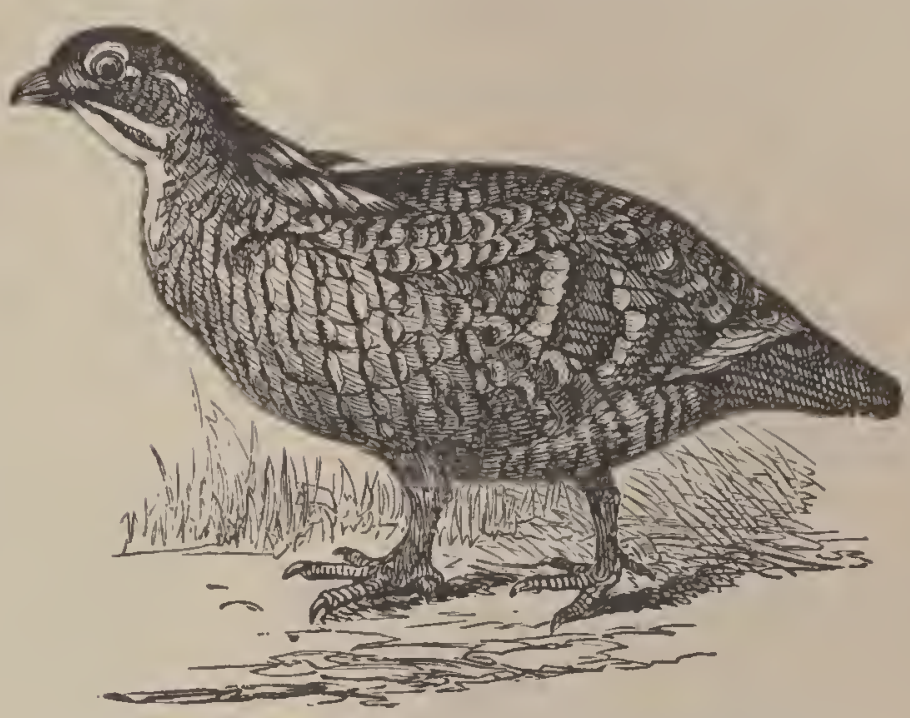

Fis. 315.-Prairie-chicken (Cupidonia cupido). Western prairies.

Grouse, Partridges, Turkeys, Pheasants, Poultry, and $\mathrm{Cu}$ rassows.

C. Ä̈rial Birds.- This highest and largest group in-

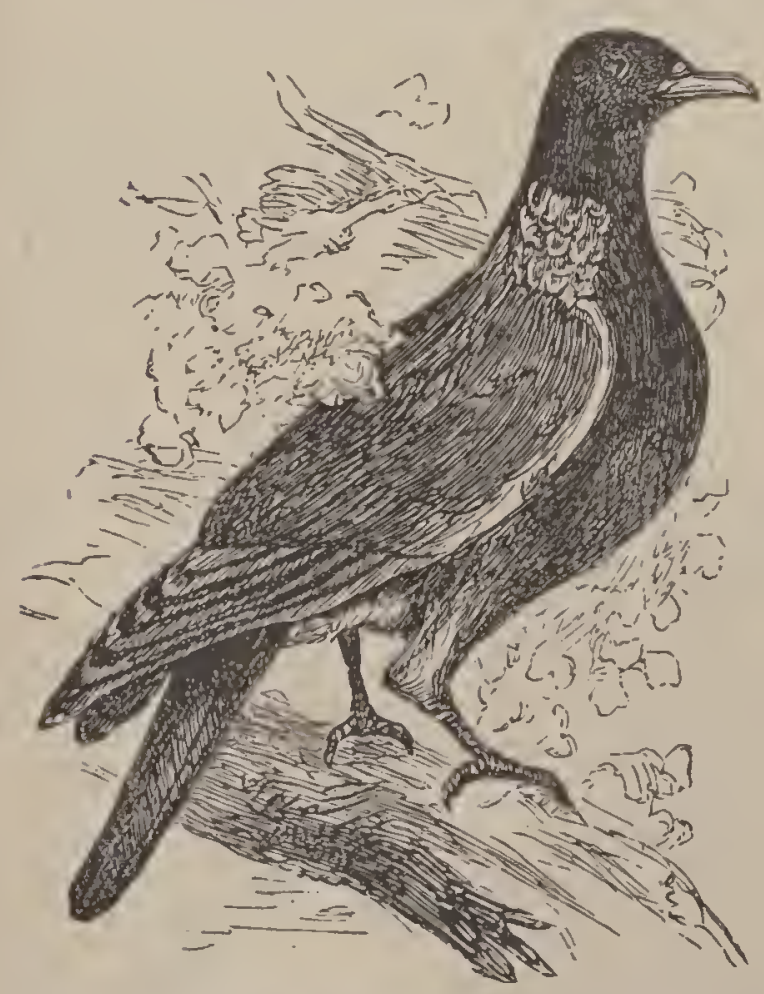

Fig. 316. -Ring-dove (Columba palumbus). England. cludes all those Birds whose toes are fitted for grasping or perching, the hind toe being on a level with the rest. The knee is free from the body, and the leg is generally feathered to the heel. The wings are adapted for rapid or long flight ; and they hop, rather than walk, on the ground. ${ }^{170}$ They always live in pairs; and the young are hatched helpless.

7. Columbce, or Pigeons and Doves, stand intermediate between the terrestrial and perching Birds, as the Flamingoes, and link the aquatic and terrestrial. They differ from the typical Rasores in 
having wings for prolonged flight, and slender legs, fitted rather for an arboreal life, with toes not united, and the hind toe on a level with the rest.

8. Raptores, or Birds of Prey, differ from all other

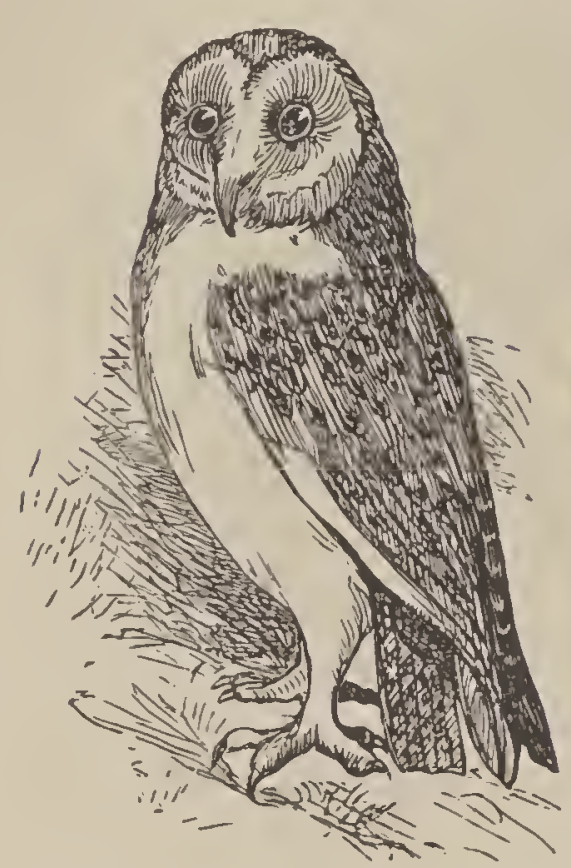

FIG. 317.-Bard-owl (Strix flammea). Both hemispheres.
Birds, except Parrots, in having a strongly hooked bill and a waxy membrane (cere) at the base of the upler mandible; and from Parrots,

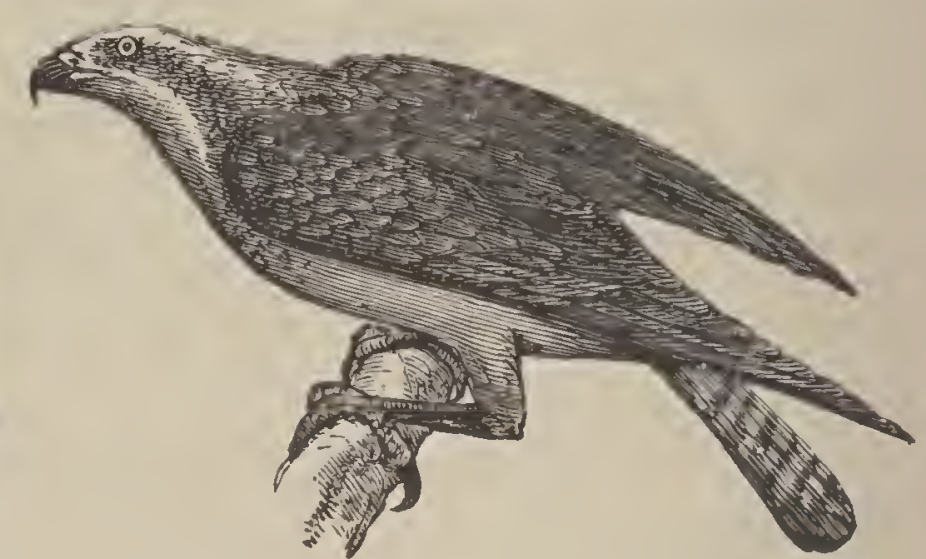

FIG. 31S. - Fish-hawk (Pandion Carolinensis). United States.

in having three toes in front and one behind. The toes are armed with long, strong, crooked talons; the legs are robust; and the wings are of considerable size, adapted

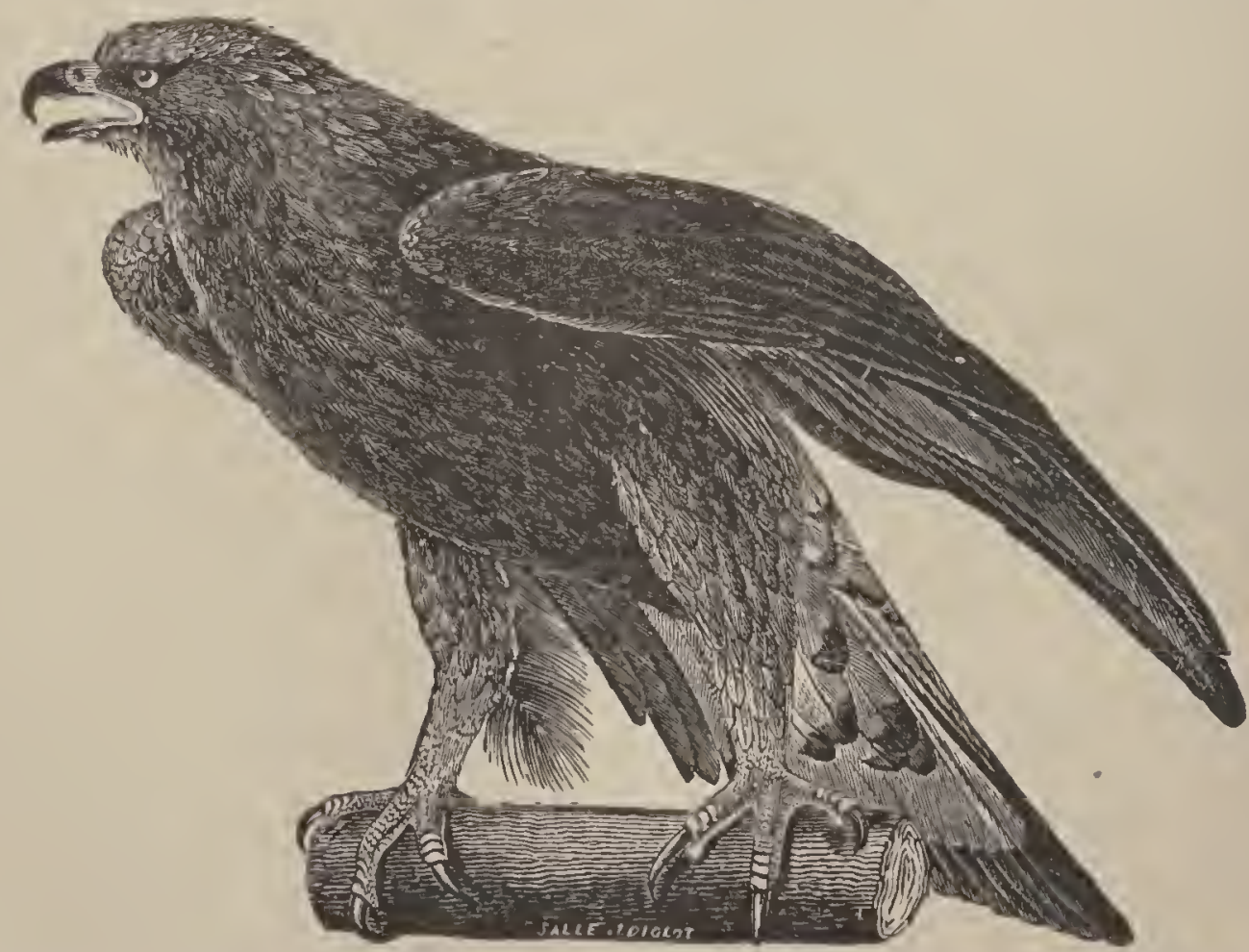

Fia. 319.-Golden Eagle (Aquila chrysaetos). North America and Europe. 
for rapid and powerful flight. The bill is stout and sharp, and usually toothed. All are carnivorous. The female is larger than the male, except the Condor. There are two
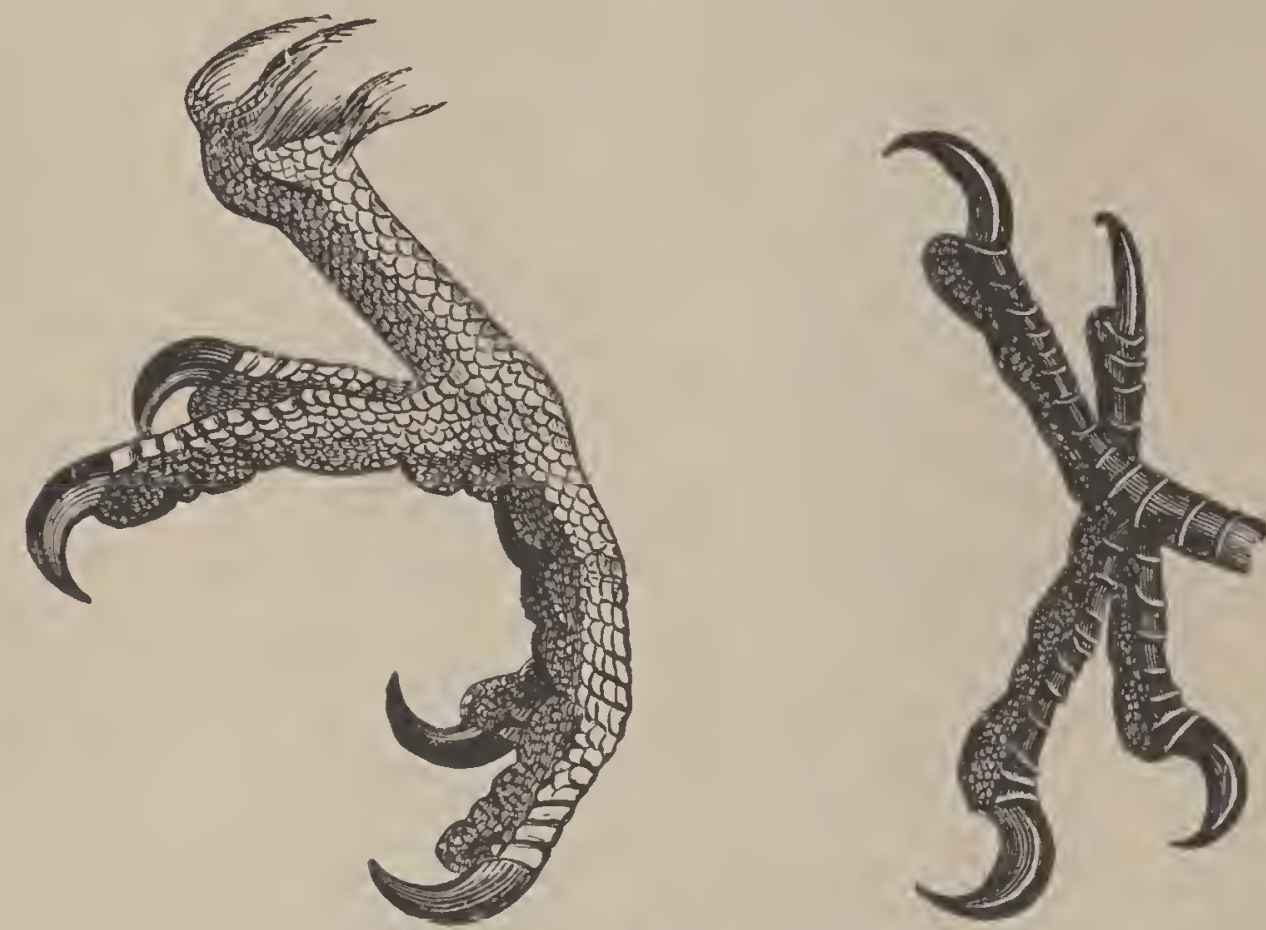

Fra. 320.-Foot of Parrot and Woodpecker.

sections: the Diurnal, whose eyes are on the sides of the head, wings pointed, and metatarsus and toes covered over with scales, as the Vultures, Kites, Hawks, Falcons, and Eagles; the Nocturnal, whose large eyes are directed forward, and surrounded by radiating feathers, metatarsus feathered, and plumage soft, as the Owls.

9. Picarice.-This polymorphic group has hardly any peculiarities in common. ${ }^{171}$ The toes are usually paired, two in front and two behind.

There are three divisions of the order: Cypseli, or Swifts, Goat-suckers, and Humming-birds; Cuculi, or Cuckoos, Kingfishers, Trogons, Toucans, Hornbills, and Iloopoes; and Pici, or Woodpeckers. These Birds are not musical, and only ordinary fliers. They feed on Insects or fruit. The majority make nests in the hollows of old trees; but the Cuckoos lay in the nests of other Birds. In climbing, the Woodpeckers are assisted by their stiff tail. 


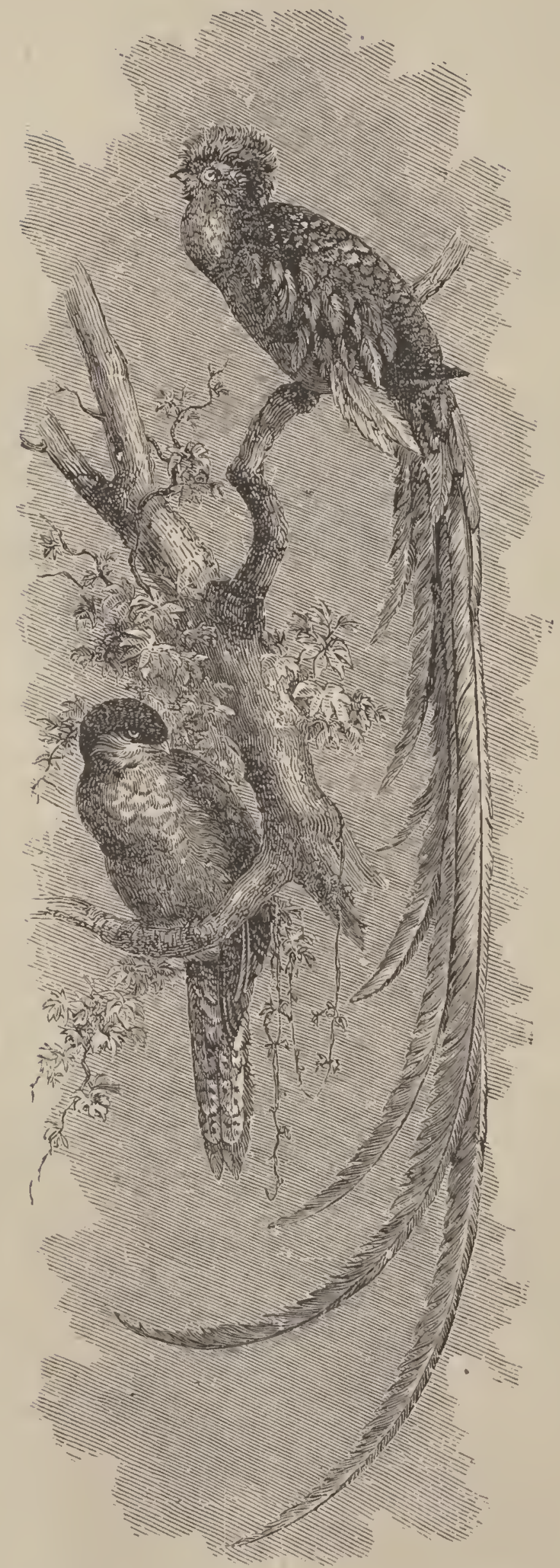

ITI. 321.-Trogon elegans. Central America. 
10. Psittaci, or Parrots.-These birds have a strong, arched upper biil, with a cere at the base, a fleshy, thick,

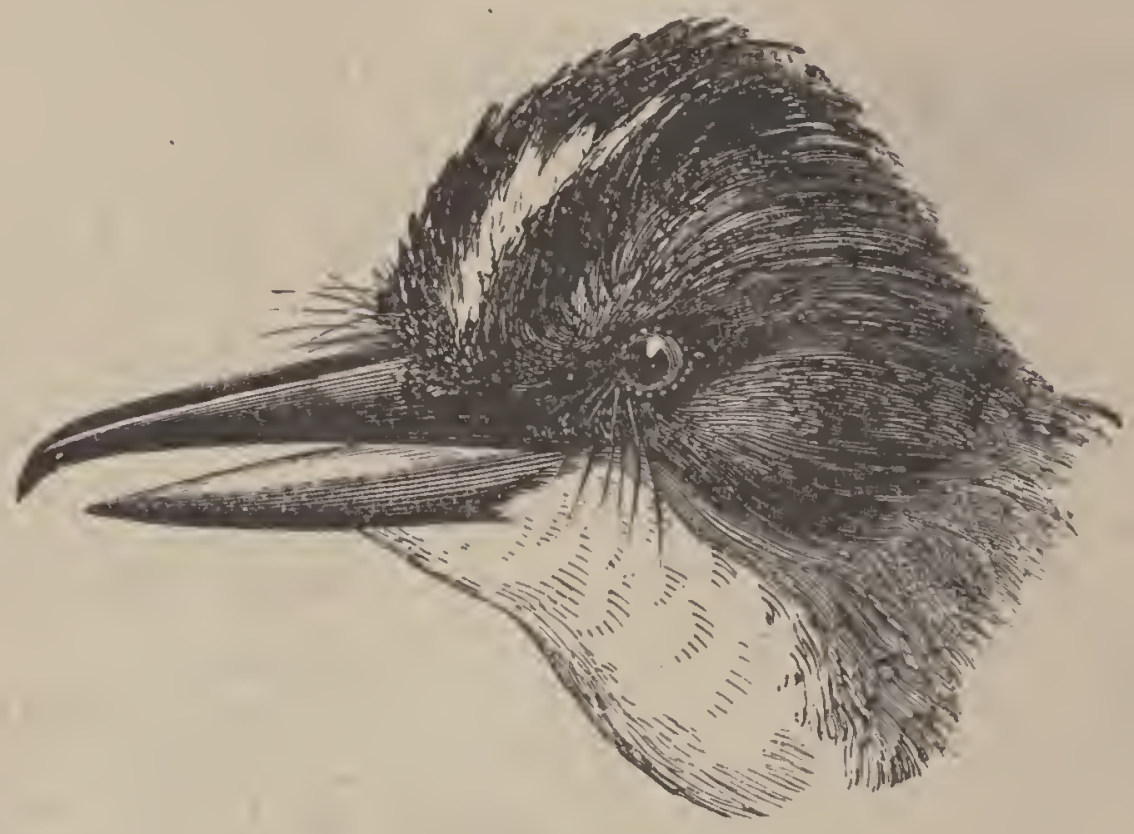

FIG. 322.-Head of a Fly-catcher (Tryrannus).

and movable tongue, and paired toes. They have, usually, brilliant plumage. They live in trees and feed on fruits. Such are the Parrots, Paroquets, and Cockatoos.

11. Insessores, or Perchers.-This order is the most nu-

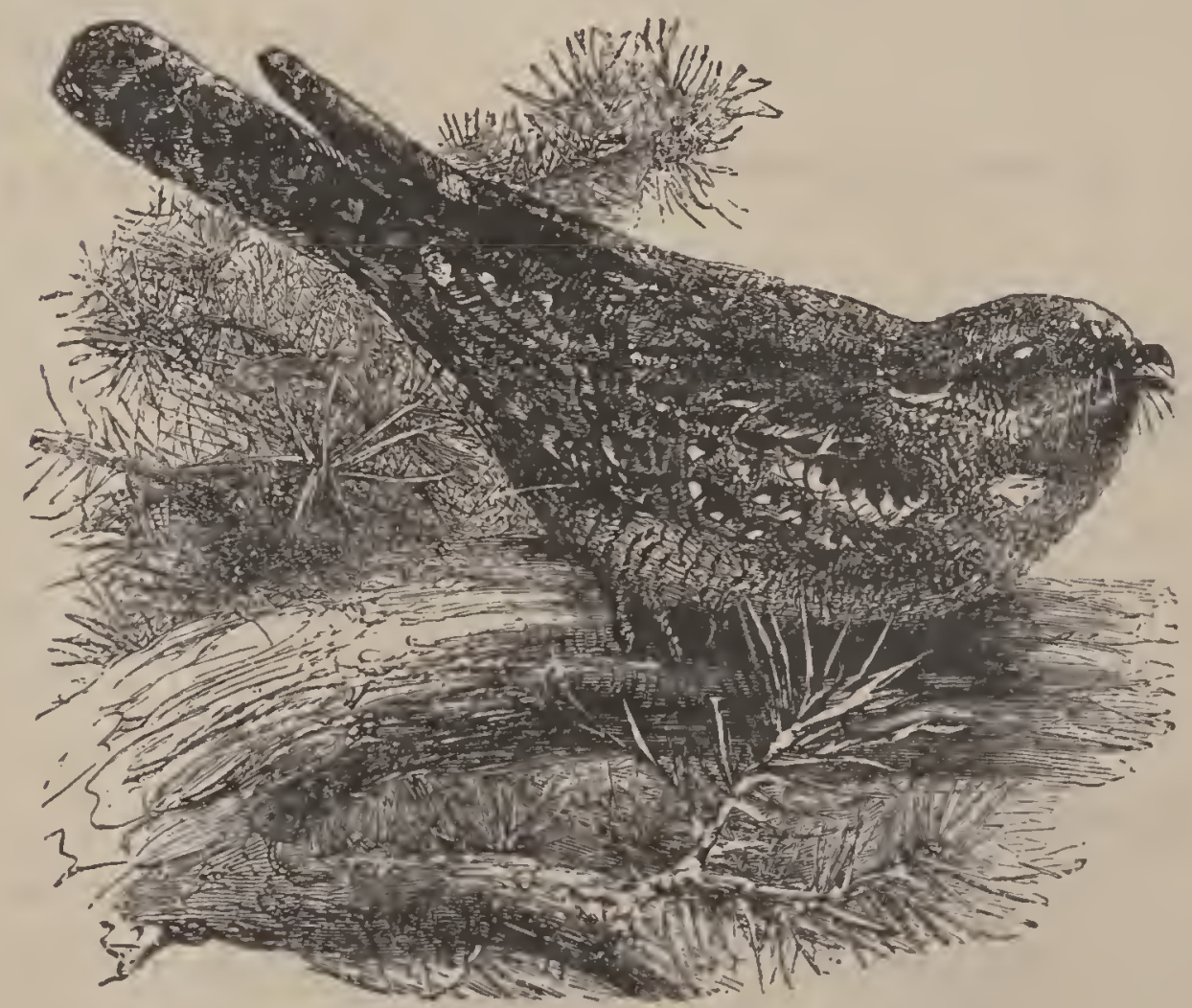

FIG. 323.-Goat-sucker (Caprimulgus). 
merous and varied in the whole class. It comprehends all those tribes which live habitually among trees, excepting

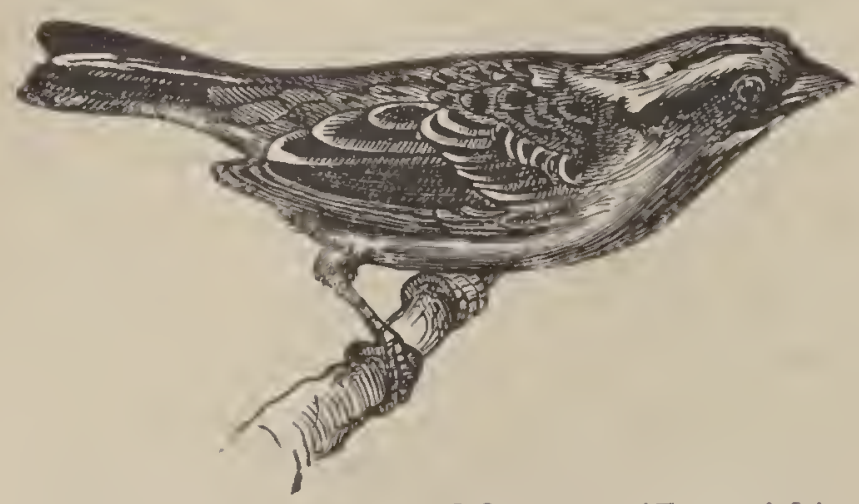

Fra. 324.-White-throated Sparrow (Zonotrichia albicollis). United States.

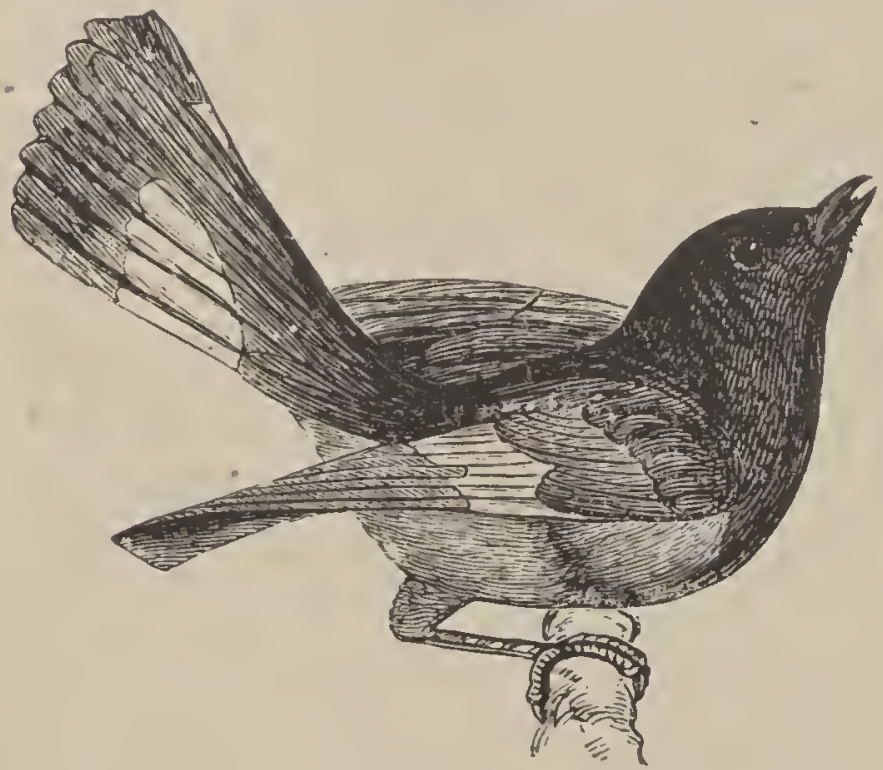

FIx. 325.-Redstart (Setophaga ruticilla). United States.

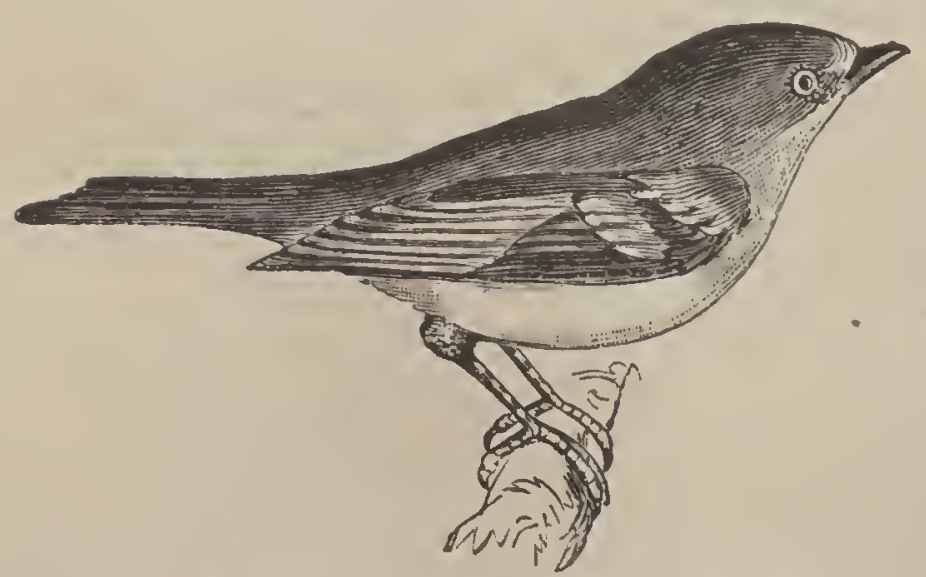

Fig. 326. White-eyed Vireo (Vireo Noveboracensis). United States.

the Rapacious and Climbing Birds, and whose toes - three in front, and one behind-are eminently fitted for perching only. The legs are slender, and seldom used for locomotion.

They are divisible into two sections: a. Clamatores, with nothing in common but a harsh voice. In most, the tarsus is enveloped in a row of plates, which meet behind in a groove, and the bill broad, and bent down abruptly at the tip. The typical representatives are the Tyrant Fly-catchers. b. Oscines, or Songsters, all of whom have a vocal apparatus, though not all sing. The anterior face of the tarsus is one continuous plate, or divided transversely into large scales; and the plates on the sides meet be- 
hind in a ridge. The toes, always three in front and one behind, are on the same level. The eggs are usu-

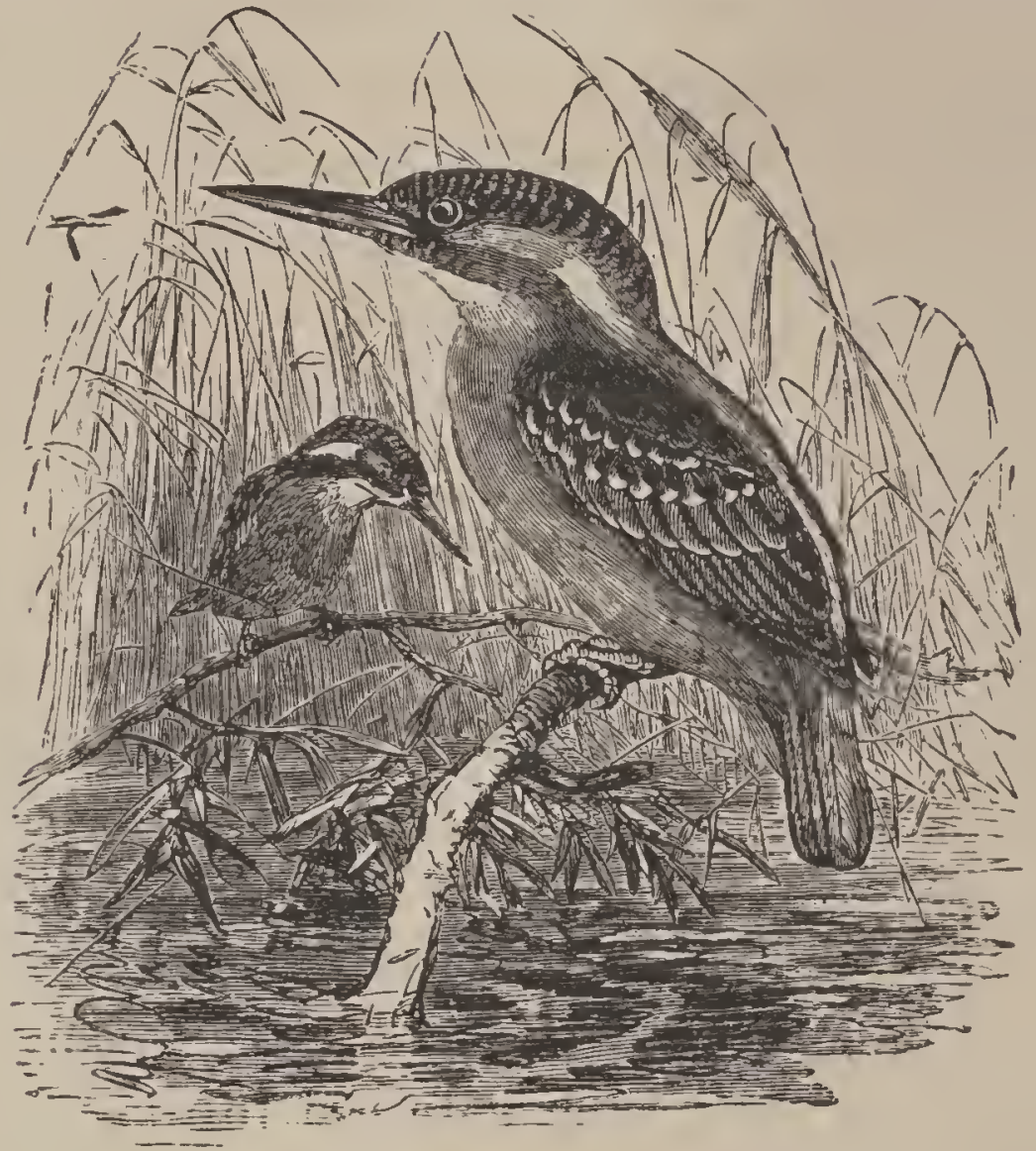

Fra. 327. -Kingfisher (Ceryle).

ally colored. Here belong the Ravens, Crows, Jays, Birdsof - Paradise, Blackbirds, Orioles, Larks, Sparrows, Tan-

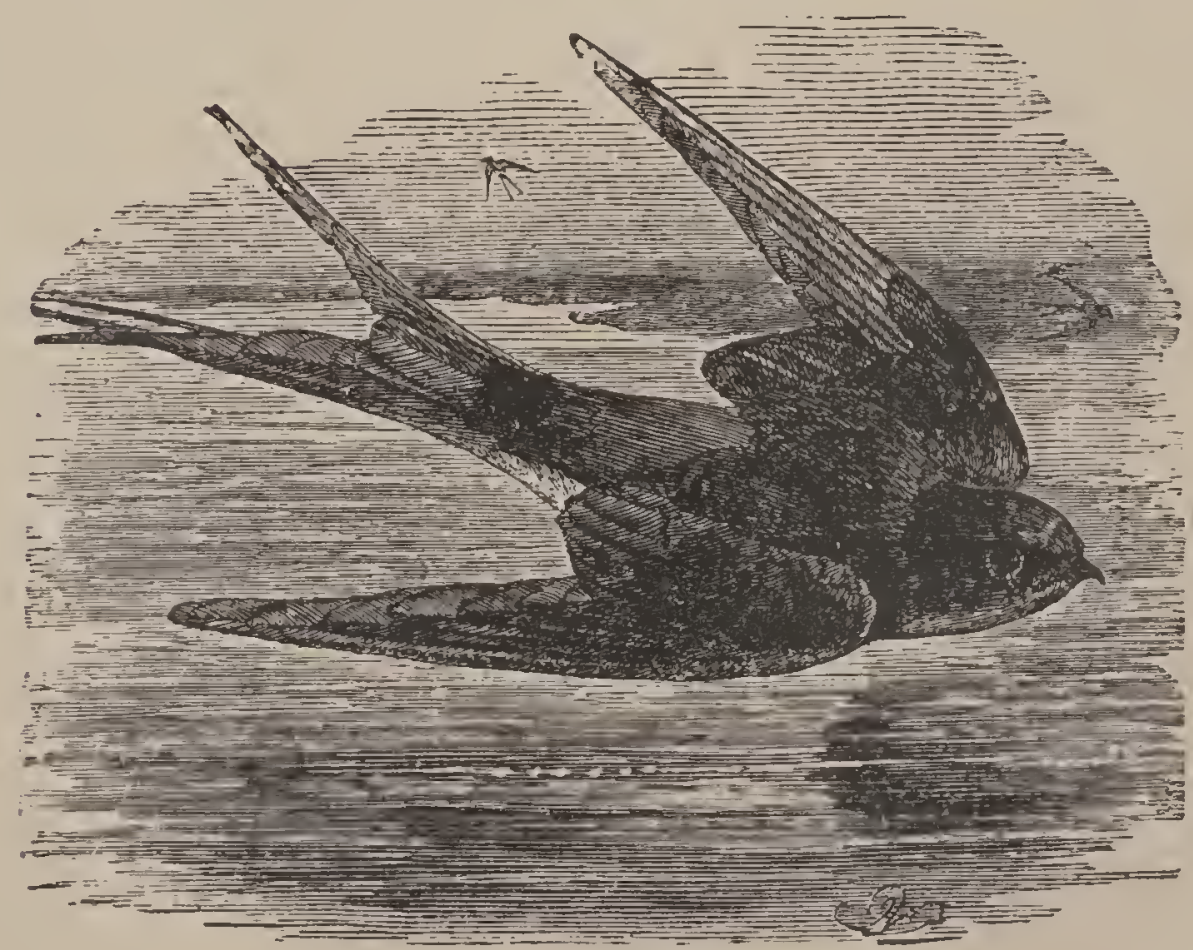

FIG. 328.-Swallow (Hirundo). 
agers, Wax-wings, Swallows, Wrens, Warblers, Thrushes, etc.

\section{Crass V.-Mammalia.}

Mammals are distinguished from all other Vertebrates by any one of the following characters: they suckle their young; the thorax and abdomen are separated by a perfect diaphragm; the red corpuscles of the blood have no nucleus, and are therefore double-concave; and either a part or the whole of the body is hairy at some time in the life of the animal. ${ }^{172}$

They are all warm-blooded Vertebrates, breathing only by lungs, which are suspended freely in the thoracic cavity; the heart is four-chambered, and the circulation is double, as in Birds; the aorta is single, and bends over the left bronchial tube; the large veins are furnished with valves; the red corpuscles differ from those of all other Vertebrates in having no nucleus and in being circular (except in the Camel); the entrance to the windpipe is always guarded by an epiglottis; the cerebrum is more highly developed than in any other class, containing a greater amount of gray matter and (in the higher orders) more convolutions; the cerebellum has lateral lobes, a mammalian peculiarity, and there is a corpus callosum and a pons varolii; the cranial bones are united by sutures, and they are fewer than in cold-blooded Vertebrates; the skull has two occipital condyles, a feature shared by the Amphibians; the lower jaw consists of two pieces only (often united), and articulates directly with the cranium; with four exceptions there are always seven cervical vertebræe $;^{173}$ the dorsal vertebræ, and therefore the ribs, vary from ten to twenty-four; the lumbar vertebræ number from two to nine; the sacral from three to nine, and the caudal from two to forty-six ; the articulating surfaces of the vertebræ are generally flat; the fore-limbs are never wanting, and the hind-limbs only in 
a few aquatic forms; excepting the Whales, each digit carries a nail, claw, or hoof; the teeth (always present, save

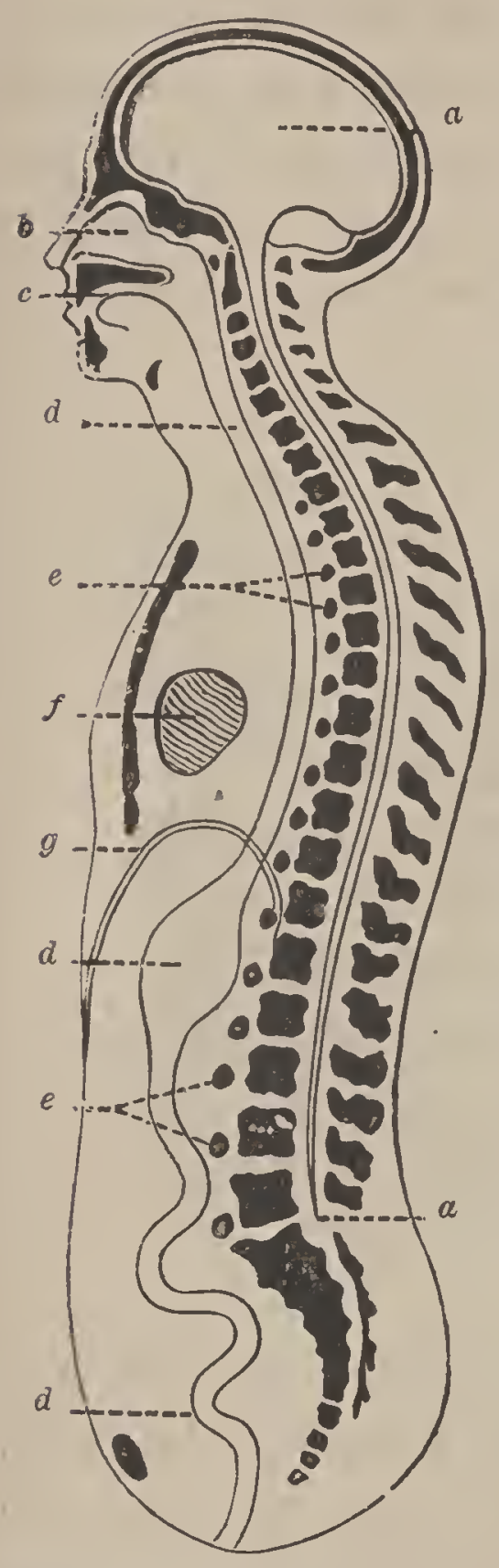

FiG. 329.-Longitudinal Section of Human Body (theoretical): $a$, cerebro-spinal nervous system ; $b$, cavity of nose; $c$, cavity of mouth; $d$, alimentary canal ; $e$, chain of sympathetic ganglia ; $f$, heart; $g$, diaphragm. in certain low tribes) are planted in sockets; the mouth is closed by flexible lips; an"external ear is rarely absent $;{ }^{174}$ the eyes are always present, though rudimentary in some burrowing animals; they are viviparons; and, finally, and perhaps above all, while in all other animals the embryo is developed from the nourishment laid up in the egg itself, in Mammals it draws its support, almost from the beginning, directly from the parent, and, after birth, it is sustained for a time by the milk secreted by the mammary glands. From the first, therefore, till it can care for itself, the young. Mammal is in vital connection with the parent.

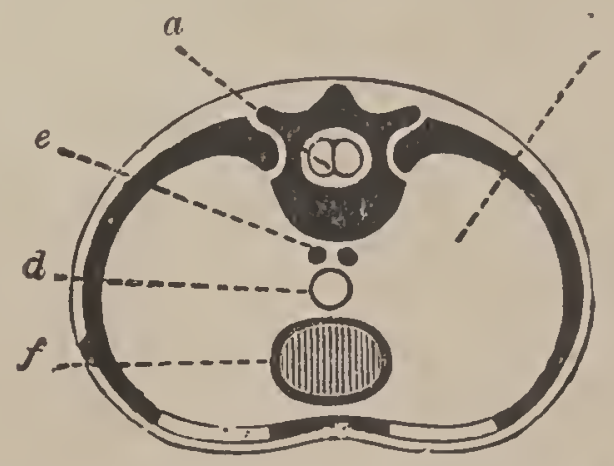

Frg. 330. - Transverse Section of Human Body (theoretical): $a$, cerebro-spinal nervous axis contained in neural tube; e, chain of sympathetic ganglia; $d$, alimentary canal; $f$, heart : $h$, hæmal tube.

\section{Subchass I.-Ornithodelphia.}

These Mammals have but one outlet for the intestine, urinary and reproductive organs, as in Birds. They are implacental. There is but one order. 
1. Monotremata. - This order includes two singular forms, the Duck-mole (Omithorhynchus) and Spiny Anteater (Echidna), both confined to the Australian continent and New Guinea. The former has a covering of fur, a bili like that of a Duck, and webbed feet. The latter is covered with spines, has a long, toothless snont, like the Ant-eater's, and the feet are not webbed. Both bur-

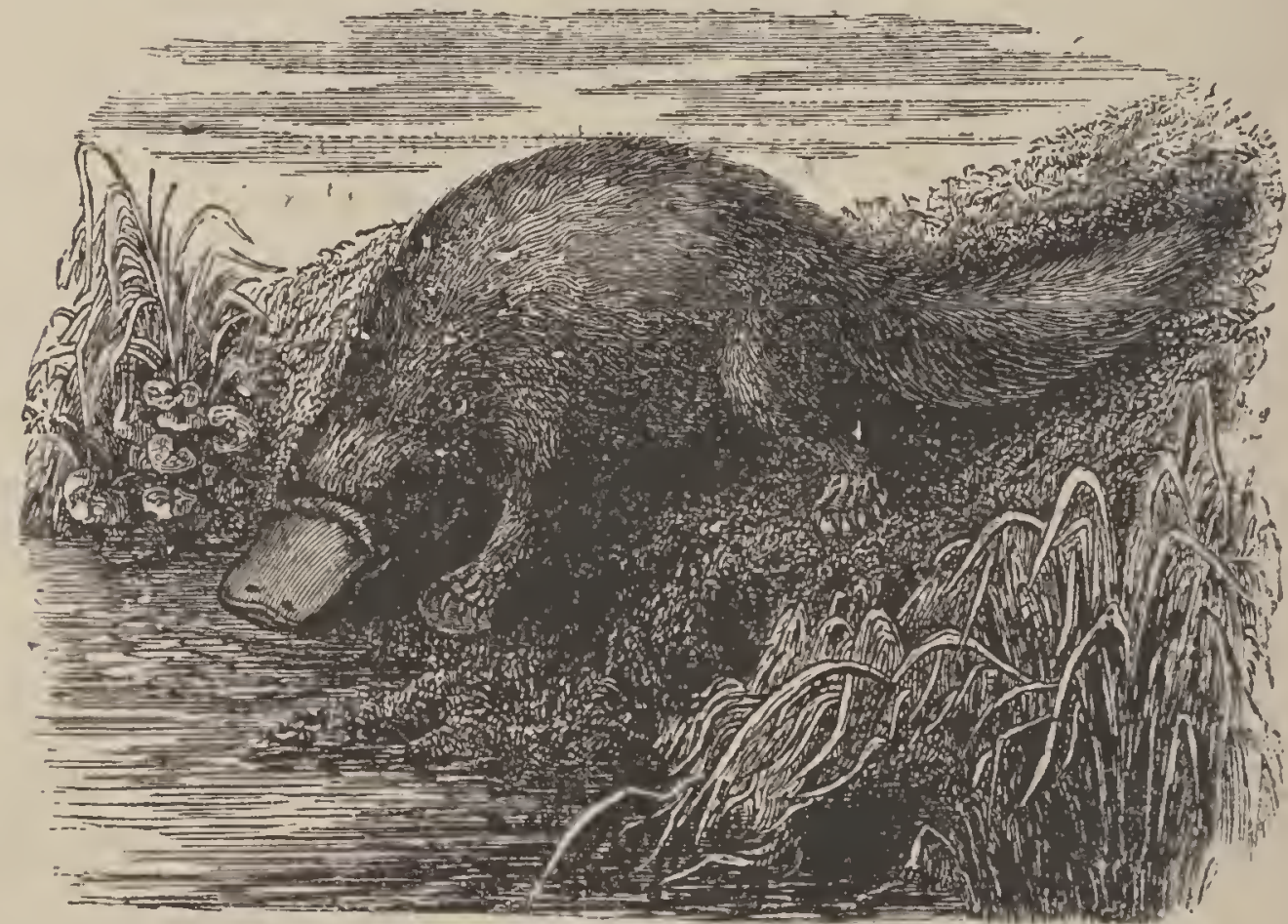

FIG. 331,-Ornithorhynchus.

row, and feed upon Insects. The brain is smooth in the Ornithorhynchus, and folded in the Echidna. In both, the cerebral hemispheres are loosely united by transverse fibres, and do not cover the cerebellum and olfactory lobes. ${ }^{175}$ Both lay eggs which resemble those of Birds and from which the young are hatched.

\section{Subclass II.-Didelphia.}

In these implacental Mammals the uterus is divided into two parts.

2. Marsupiatia are distinguished by the fact that the young, always born premature, are transferred by the mother to a pouch on the abdomen, where they are attached to the nipples, and the milk is forced into their 
mouths by special muscles. ${ }^{176}$ They have "marsupial bones" projecting from the pelvis, which may serve to support the pouch; but as the Monotremes have the same bones, but no pouch, they doubtless have some other function. These bones are peculiar to animals having no placenta, namely, to Monotremes and Marsupials. The brains of Marsupials resemble those of the Monotremes, except that the cerebrum of the Kangaroo covers the olfactory lobes. All have the four kinds of teeth, and all are covered with fur, never with spines or scales. Except the Opossums of America, all are restricted to Australia and

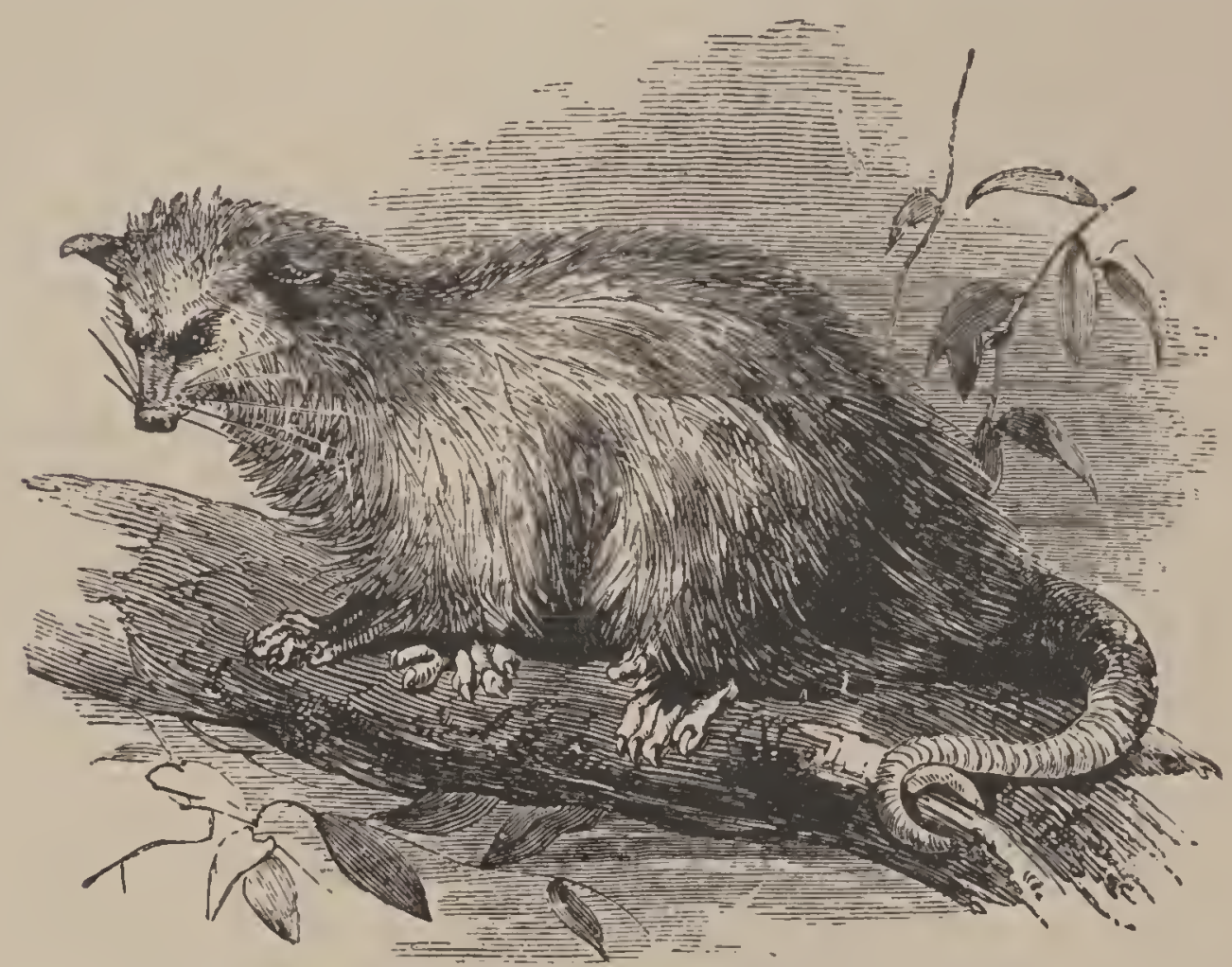

FıG. 332.-Virginian Opossum (Didelphys Virginiana).

adjacent islands. The Marsupials are almost the only Mammals of A ustralia, a few species of Rodents and Bats being the only placental Mammals. The Marsupials have here developed into forms corresponding in their habits to the orders of placental Mammals in the rest of the world. The Kangaroos take the place of the large herbivores - the Ungulates. The Thylacinus and Dasyurus are the marsupial carnivora. Other forms are squirrellike in shape and habits, and stili others are insectivorons. 
SubCLAss III.-Monodelphia or Placental Mammals.

In these Mammals the young are connected with the mother by means of a vascular structure, the placenta, by which they are nourished. They are born in a relatively perfect condition.

3. Edentata.-This strange order contains very diverse forms, as the leaf-eating Sloths and the insectivorous Anteaters and Armadillos of South America, and the Pangolin and Orycteropus of the Old World. The gigantic fos-

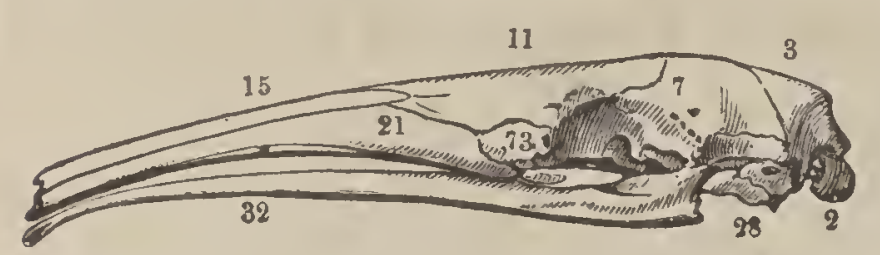

Fig. 333.-Skull of the Great Ant-eater (Myrmecophaga jubata): 15, nasal; 11 , frontal; 7 , parietal; 3 , superoccipital ; 2, occipital condyles; $2 S$, tympanic ; 73 , lachrymal; 32 , lower mandible. Teeth wanting. sils, Megatherium and Glyptodon, belong to this group. The Sloths and Ant-eaters are covered with coarse hair; the Armadillos and Pangolins, with an armor of plates or scales. The Ant-eaters and Pangolins are strictly edentate, or toothless; the rest have molars, wanting, however, enamel and roots. In general, it may be said that the order includes all quadrupeds having separate, clawed toes and no incisors. The Sloths are arboreal; the

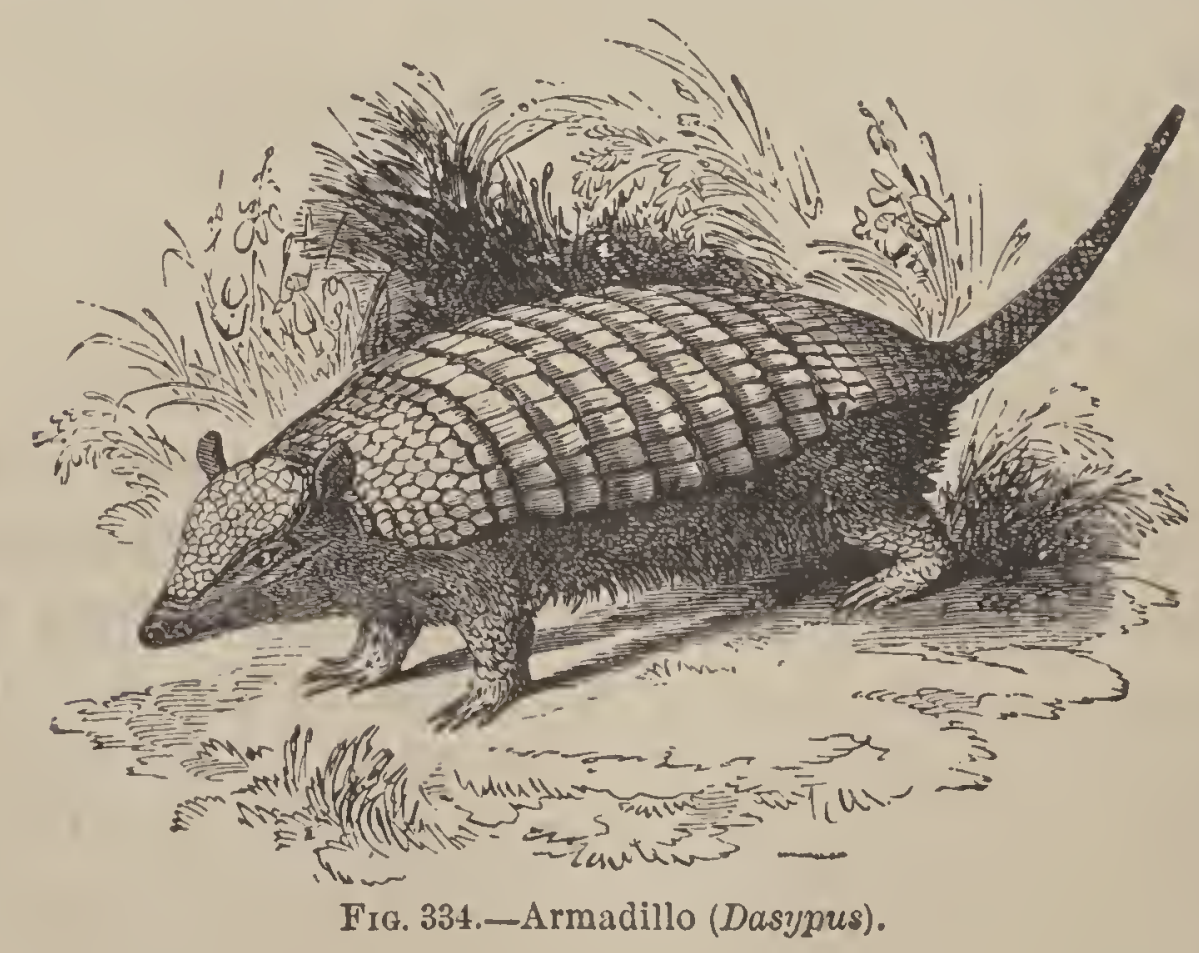


others burrow. 'The brain is generally smooth; but that of the Ant-eater is conroluted, and has a large corpus callosum; but in all the cerebellum and part of the olfactory lobes are exposed.

4. Rodentia, or Gnawers, are characterized by two long, curved incisors in each jaw, enameled in front, and perpetually growing; they are specially formed for nibbling.

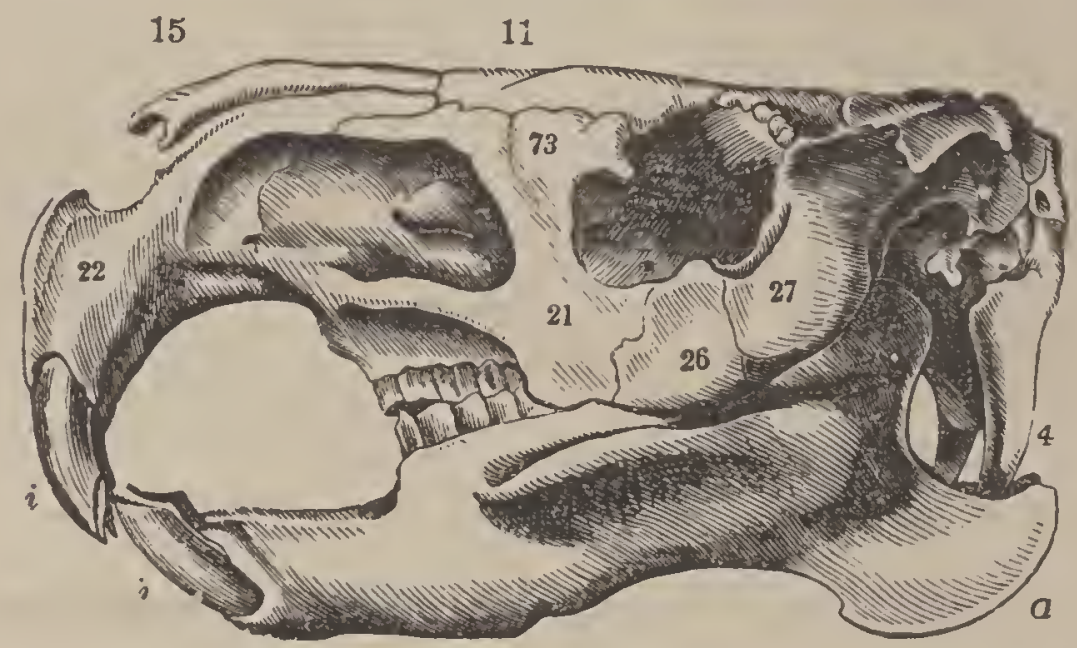

Fra. 335.-Skull of a Rodent (Capybara): 22, premaxillary ; 21, maxillary; 26, molar ; 27 , squamosal; 73 , lachrymal; 15 , nasal; 11 , frontal; 4, occipital processes, unusually developed; $i$, incisors; $a$, angle of lower jaw.

Separated from them by a wide space (for canines are wanting), are the flat molars, admirably fitted for grinding. The lower jaw has longitudinal condyles, which work freely backward and forward in longitudinal furrows. Nearly all have clavicles; and the toes are clawed. The cerebrum is nearly or quite smooth, and covers but a small part of the cerebellum. All are vegetarian.

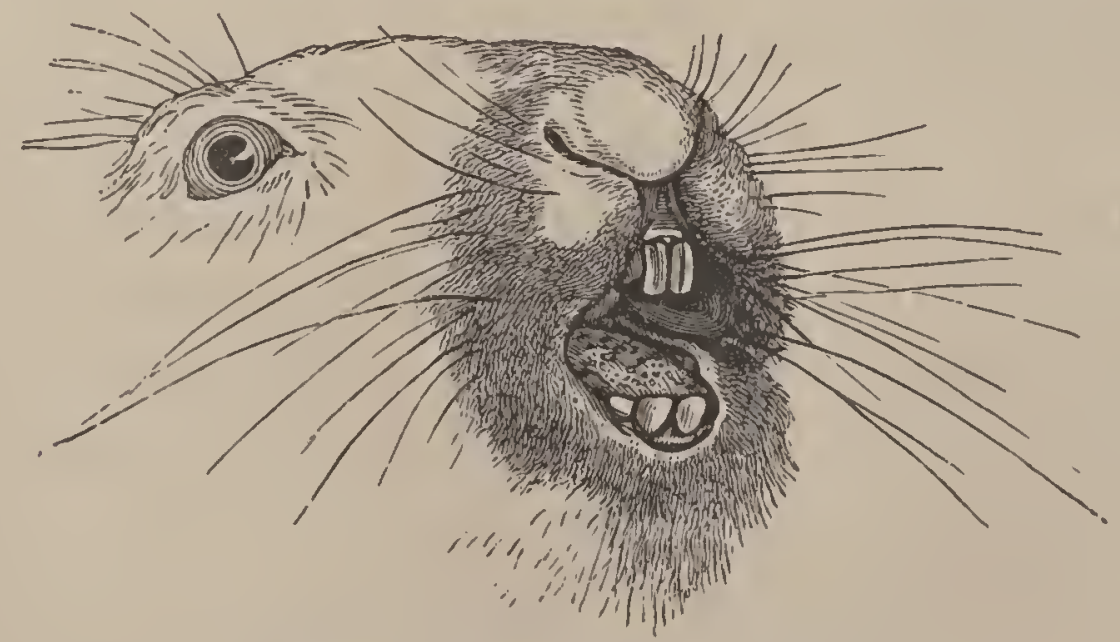

Fıc. 336.-Incisor 'Teeth of the Hare. 
More than one half of all known Mammals are Rodents. They range from the equator to the poles, over every continent, over mountains and plains, deserts and woods. The

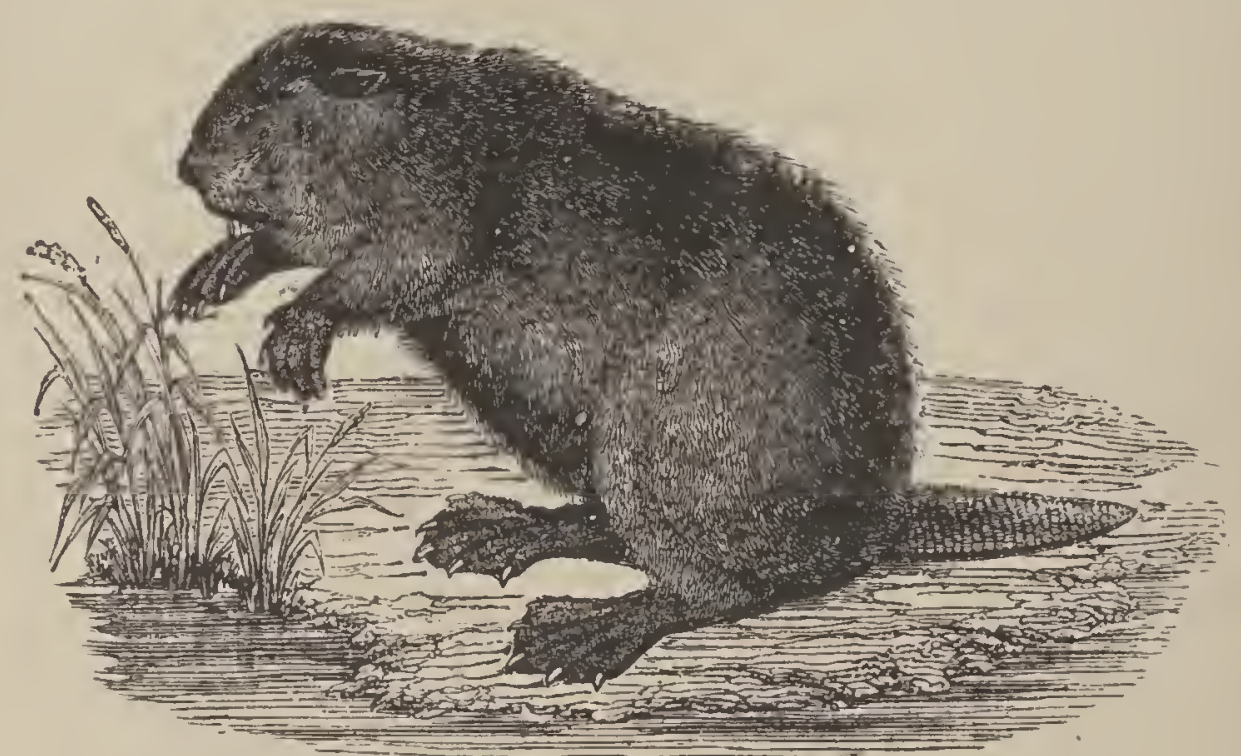

Fit, 337.-Beaver (Castor Canadensis). North America.

more important representatives are the Porcupines, Capybaras, Guinea-pigs, Hares, Mice, Rats, Squirrels, and Beavers. The Capybara and Beaver are the giants of the race.

5. Insectivora are diminutive, insect-eating animals, some, as the Shrew, being the smallest of Mammals.

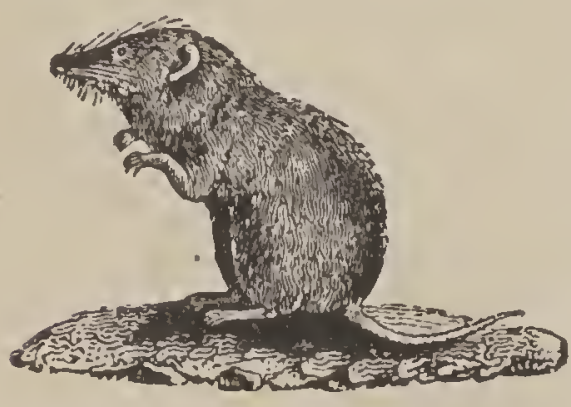

Fig. 33S.-Shrew Mouse (Sorex). They have small, smooth brains, which, as in the preceding orders, leave uncovered the cerebellum and olfactory lobes. The molar teeth bristle with sharp, pointed cusps, and are associated with canines and incisors. They have a long muzzle, short legs, and clavicles. The feet are formed for walking or grasping, and are plantigrade, five-toed, and clawed. The Shrew, Hedgehog, and Mole are examples.

6. Cheiroptera, or Bats, repeat the chief characters of the Insectivores; but some (as the Flying-fox) are fruiteaters, and have corresponding modifications of the teeth. They are distinguished by their very long fore-limbs, 
which are adapted for flight, the fingers being immense. ly lengthened, and united by a membranons web. The toes, and one or two of the fingers, are armed with hooked

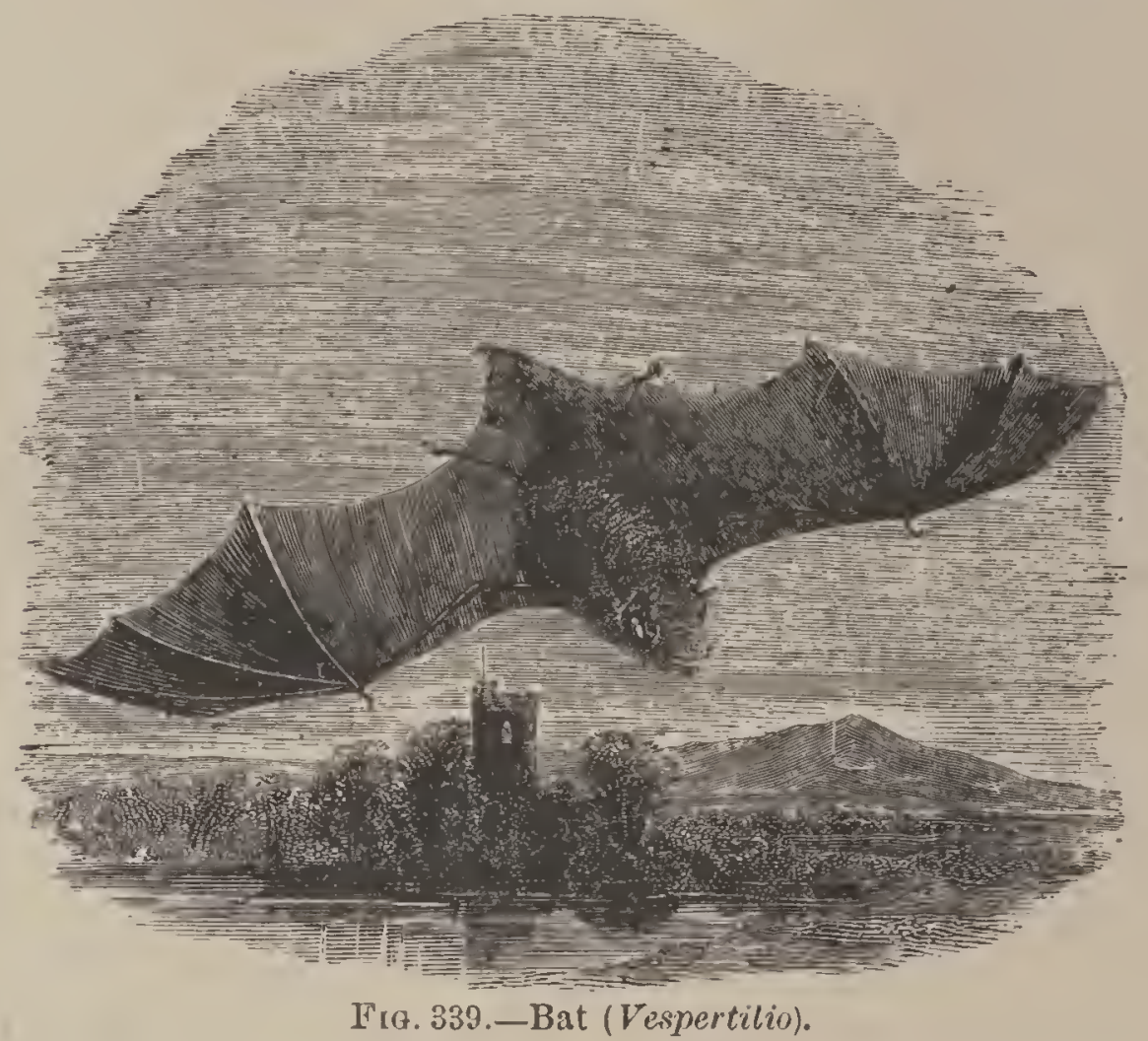

nails. The clavicles are remarkably long, and the sternum is of great strength; but the whole skeleton is ex.. tremely light, though not filled with air, as in Birds. The eyes are small, the ears large, and the sense of touch is very acute. The favorite attitude of a Bat when at rest is that of suspension by the claws, with head downward. They are all nocturnal.

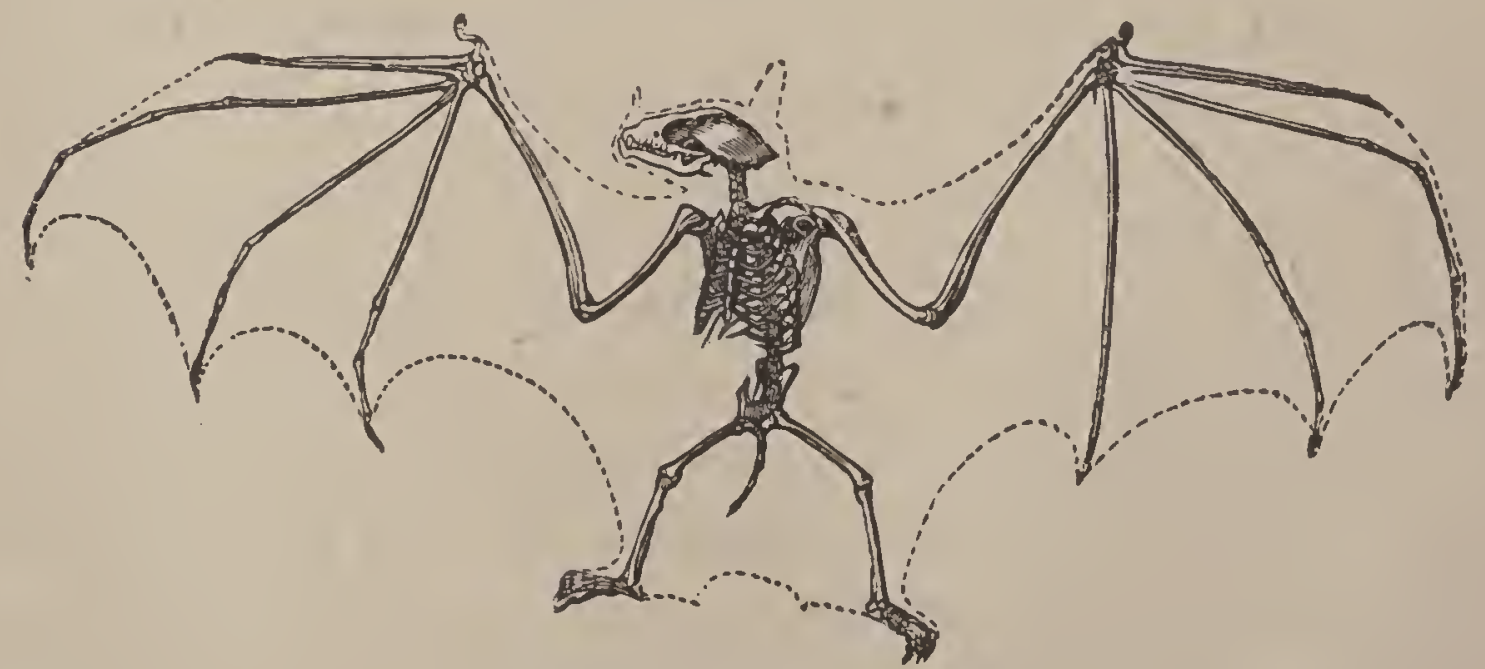

Fi6. 340.-Skeleton of a Bat. 
7. Cetacea, or Whales, have the form and life of Fishes, yet they possess a higher organization than the preceding orders. They have a broad brain, with many and deep foldings; the foramen magnum of the skull is entirely posterior; the whole head is disproportionately large, and the jaws greatly prolonged. The body is covered with a thick, smooth skin, with a layer of fat ("blubber") under-

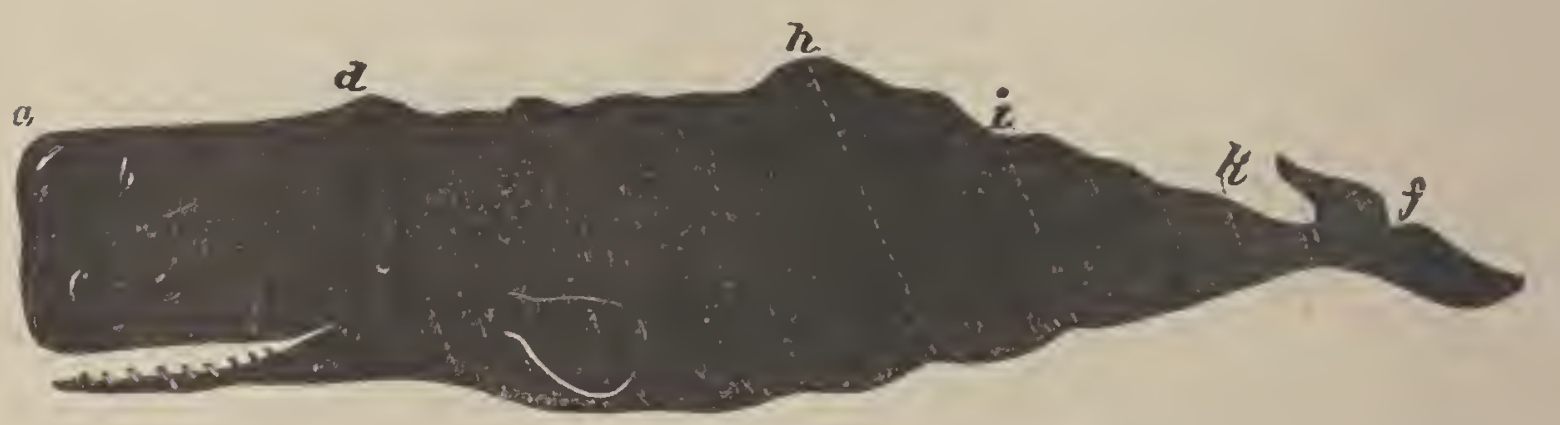

Fig. 341.-Outline of the Sperm-whale (Physeter) : $a$, blow-hole ; $b$, the case containing spermaceti ; $c$, junk; $d$, bunch of the neck-between it and the corner of the mouth is the eye; $h$, hump; $i$, ridge; $k$, the small $f$, tail, or flukes. Between the dotted lines are the spiral strips of blubber. Maximum length, sixty feet. South Atlantic.

neath; there are no clavicles; the hind-limbs are wanting, and the front pair changed to paddles; the tail expands into a powerful, horizontal fin; neck and external ears are wanting; the eyes small, with only two lids; the nostrils ("blow-holes")-double in the Whale, single in the Porpoise-are on the top of the head. All are carnivorous, and essentially marine, a few Dolphins only being found in the great rivers. In the Whalebone Whales, the teeth are absorbed, and disappear before birth, and their place is supplied by hoiny "baleen" plates. "The Whale feeds by putting this gigantic strainer into operation, as it swims through the shoals of minute Mollusks, Crustaceans, and Fishes, which are constantly found at the surface of the sea. Opening its capacious month, and allowing the sea-water, with its multitudinous tenants, to fill the oral cavity, the Whale shuts the lower jaw upon the baleen plates, and, straining out the water through them, swallows the prey stranded upon its vast tongue." In the 


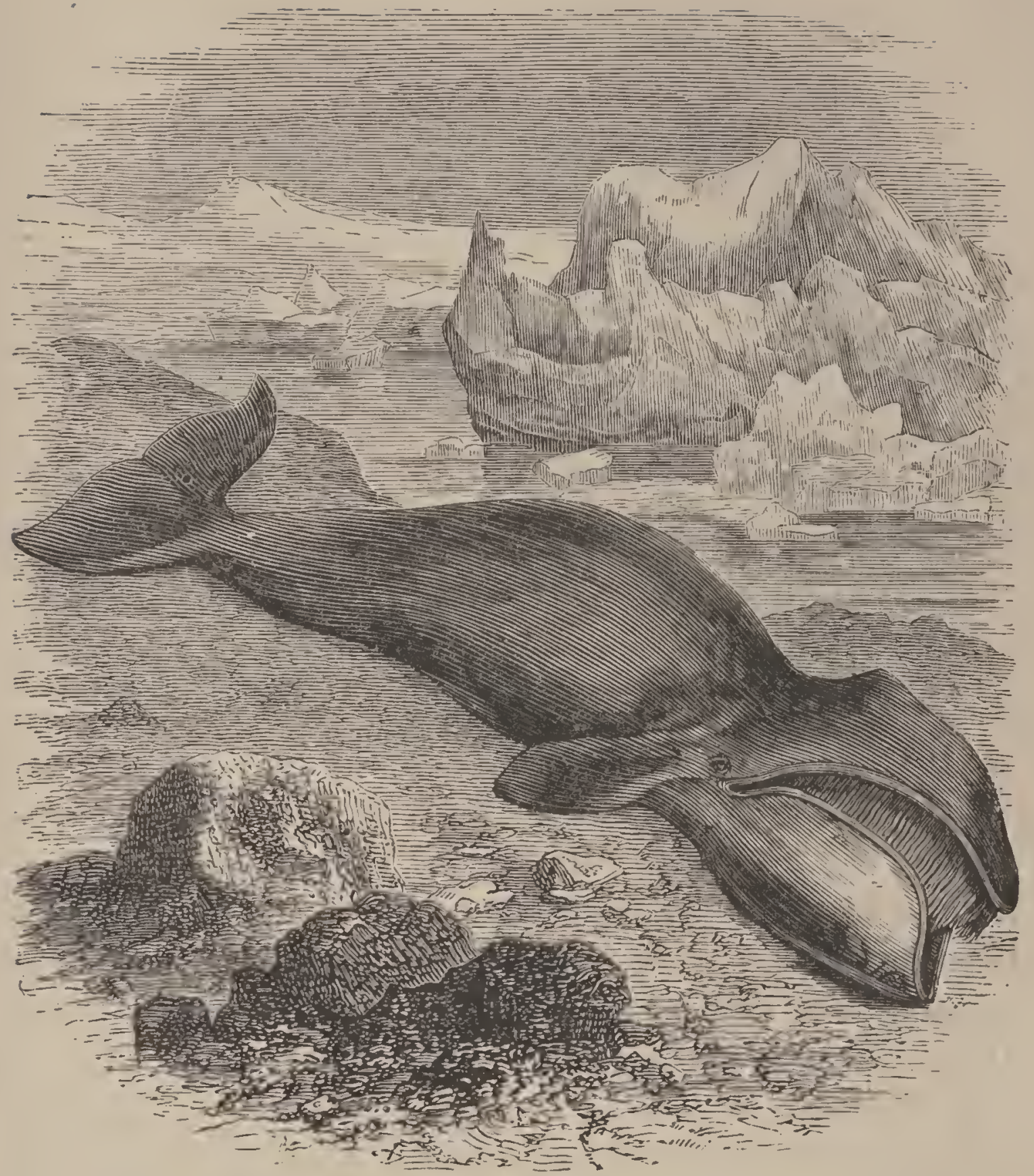

Fig. 342.-Greenland Whale (Baloena mysticetus). North Atlantic.

other Cetaceans teeth are developed, especially in Dolphins and Porpoises; but the Sperm Whale has them only in the lower jaw, and the Narwhal can show but a single tusk. The Dolphins are the only Mammals having no organ of smell.

8. Sirenia resemble the Cetaceans in shape, but are closely allied to the hoofed animals in organization. They have the limbs of the Whales, and are aquatic; but they are herbivorous, and frequent great rivers and estuaries. They have two sets of teeth, the Cetaceans having but 


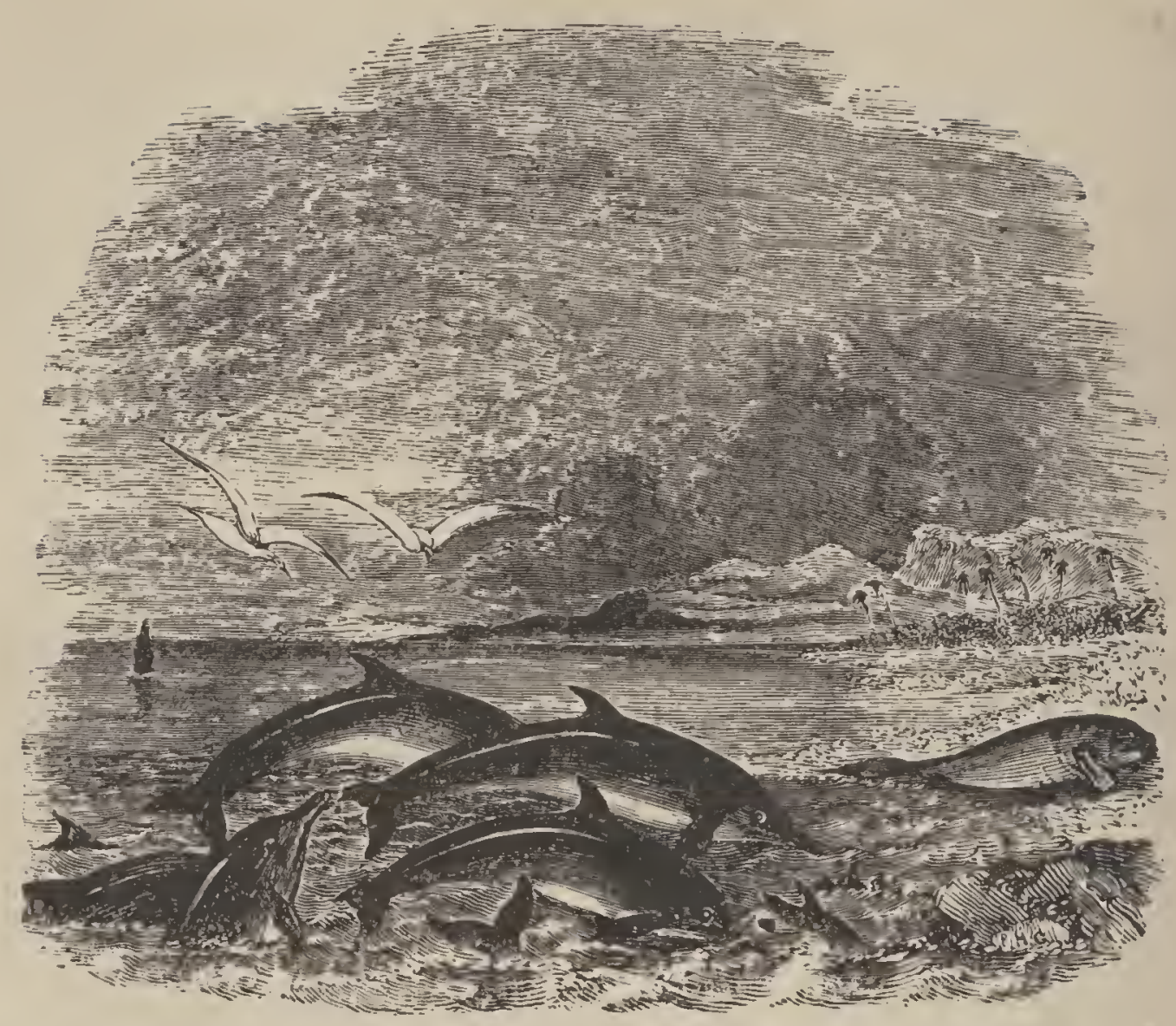

Fı. 343.-Troop of Dolphins, with Mauatee in the distance.

one. They have a narrow brain; bristles scantily covering the body; and nostrils placed on the snout, which is large and fleshy. The living representatives are the Manatee, of both sides of the tropical Atlantic Ocean, and the Dugong, of the East Indies.

9. Proboscidia. - This race of giants, now nearly extinct, is characterized by two upper incisors in the form of tusks, mainly composed of dentine (ivory). In the extinct Dinotherium the tusks projected from the lower jaw; and in the Mastodon, from both jaws. Canines are wanting. The molar's are few and large, with transverse ridges (Ele- phant) or tubercles (Mastodon). The cerebrum is large and convoluted, but does not cover the cerebellum. The skull is enormous, the size arising in great measure from the development of air-cavities between the inner and outer plates. The nose is prolonged into a flexible trunk, which is a strong and delicate organ of prehension. There are four massive limbs, each with fire toes incased in 
broad, shallow hoofs, and also with a thick, tegumentary pad. The knee is below and free from the body, as in Monkeys and Men. Clavicles are wanting. The body of the Elephant is nearly naked; but the Mammoth, an extinct species, had a covering of long woolly hair. Elephants live in large herds, and subsist on foliage and grass. There are but two living species: the Asiatic, with long head, concave forehead, small ears, and short tusks; and the African, with round head, convex forehead, large ear's, and long tusks. ${ }^{177}$

10. Ungulata, or Hoofed Quadrupeds.-This large order, comprehending many animals most useful to Man, is distinguished by four well-developed limbs, each furnished with not more than four complete toes, and each toe incased in a hoof. The leg, therefore, has no prehensile power; it is only for support and locomotion. Clavicles are wanting; and the radius and ulna are so united as to prevent rotation. There are always two sets of teeth, $i . e$, milk-teeth are succeeded by a permanent set. The grinders have broad crowns. As a rule, all are herbivorous. The brain is always convoluted, but the cerebellum is largely uncovered.

Ungulates are divided into the odd and even toed. $a$. The Odd-toed, as the three-toed Rhinoceros and Tapir, ${ }^{178}$ and the one-toed Horse. ${ }^{179}$ The first is distinguished by its very thick skin, the absence of canines, and one or two horns on the nose. The Tapir has the four kinds of teeth, and a short proboscis. The dental formula of the Horse is-

$$
i \frac{3-3}{3-3}, c \frac{1-1}{1-1}, p m \frac{3-3}{3-3}, m \frac{3-3}{3-3}=40 \text {. }
$$

The canines are often wanting in the mare. The Horse walks on the third finger and toe. The metacarpals and metatarsals are greatly elongated, so that the wrist and heel are raised to the middle of the leg. $b$. The Even-toed 
Ungulates - Hog, Hippopotamus, and Ruminants - have two or four toes. The Hog and Hippopotamus have the

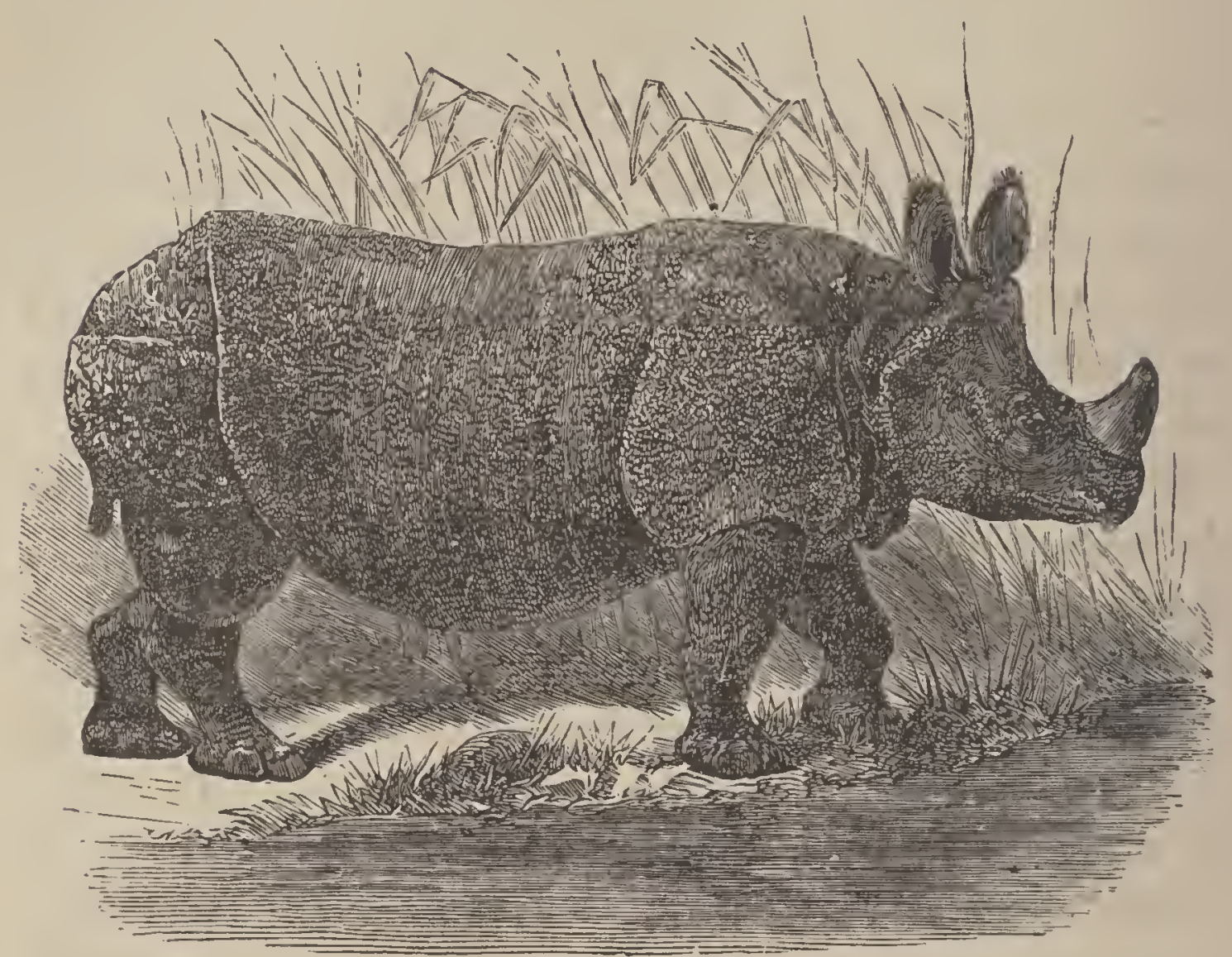

Fıg. 344.-Iudian Rhiuoceros (R. unicornis).

four kinds of teeth, and, in the wild state, are vegetarian. The Ruminants have two toes on each foot, enveloped in hoofs which face each other by a flat side, so that they appear to be a single hoof split or "cloven." Usually there are also two supplementary hoofs behind, but they do not ordinarily touch the ground. All chew the cud, and have a complicated stomach. They have incisors in the lower jaw only, and these are apparently eight; but the two outer ones are canines. ${ }^{180}$ The molars are flat, typical grinders. The dental formula of the $\mathrm{Ox}$ is-

$$
i \frac{0-0}{3-3}, c \frac{0-0}{1-1}, p m \frac{3-3}{3-3}, m \frac{3-3}{3-3}=32 \text {. }
$$

With few exceptions, as the Camel, all Ruminants have horns, which are always in pairs. Those of the Deer are solid, bony, and deciduous; those of the Giraffe and An- 


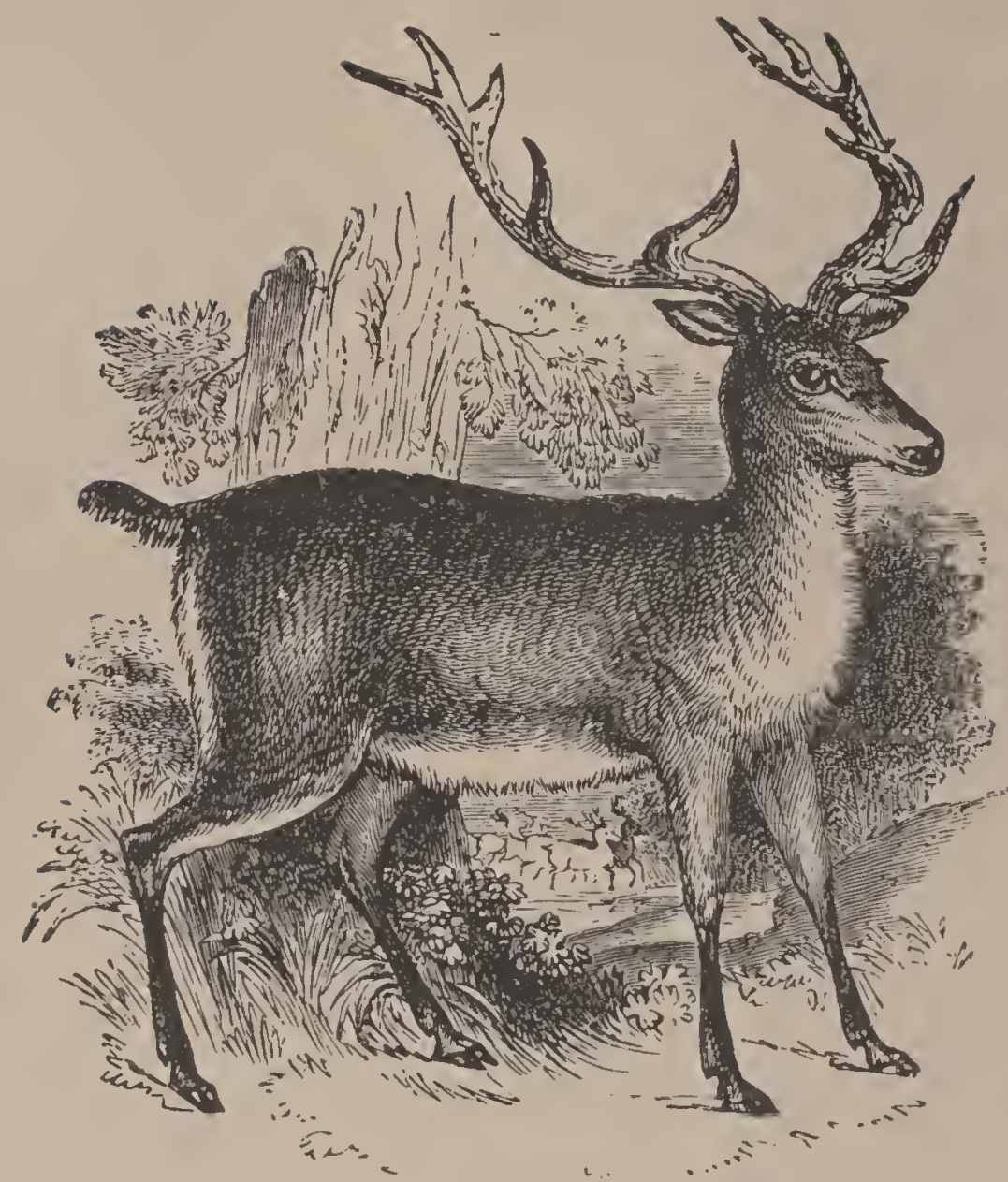

FIG. 345._Stag, or Red Deer (Cervus claphus). Europe.

telope are solid, horny, and permanent; in the Goat, Sheep, and $\mathrm{Ox}$ they are hollow, horny, and permanent.

i1. Carnivora, or Beasts of Prey, may be recognized by their four long, curved, acute, canine teeth, the gap between the incisors and canines in the upper jaw for the reception of the lower canine, and molars graduating from a tuberculate to a trenchant form, in proportion as the diet deviates from a miscellaneous kind to one strictly of flesh. The incisor's, except in the Pinnigrades, number Fra. 346.-Raccoon (Procyon lotor). United States. 
six in each jaw. There are always two sets. The skull is comparatively small, the jaws are shorter and

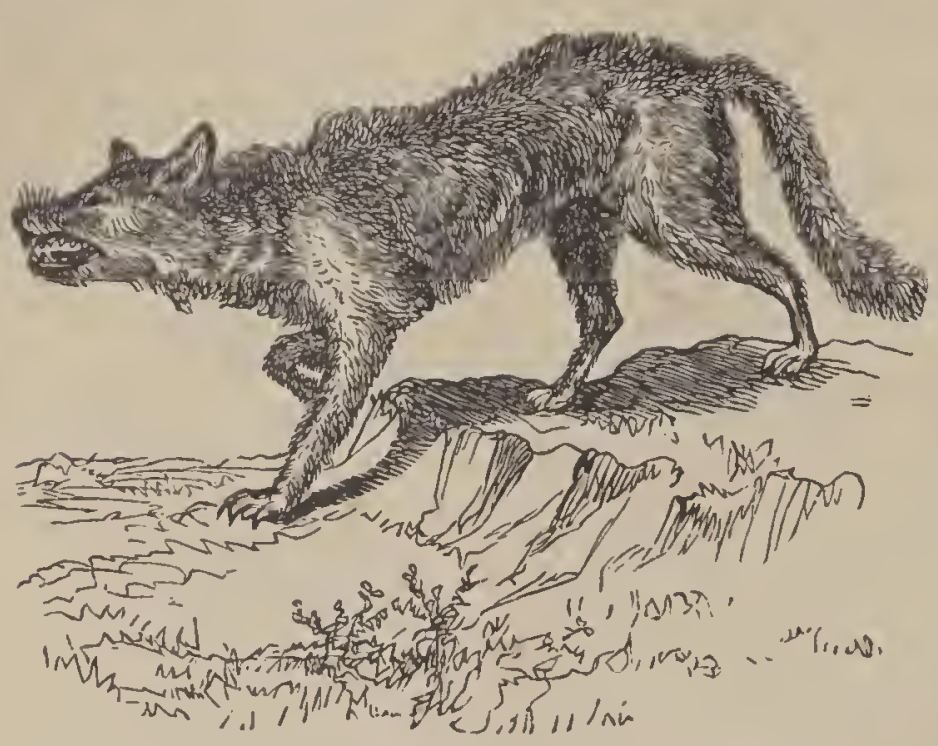

Fig. 347._Wolf (Lupus occidentalis). United States.

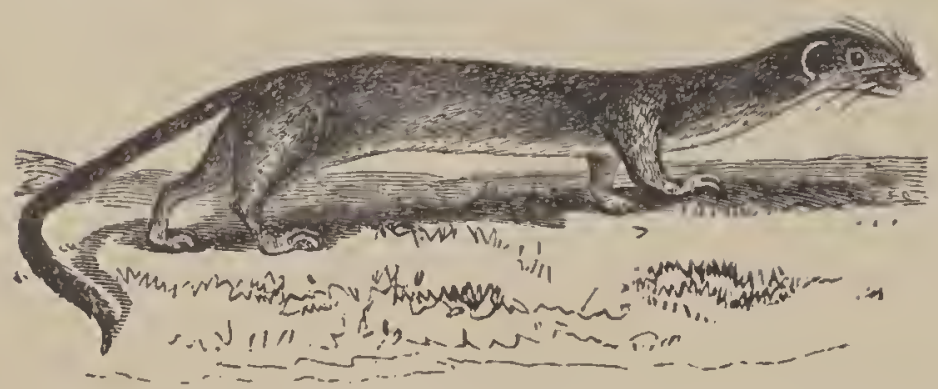

Fig. 34S.-Ermine-weasel (Putorius Noveboracensis). United States.

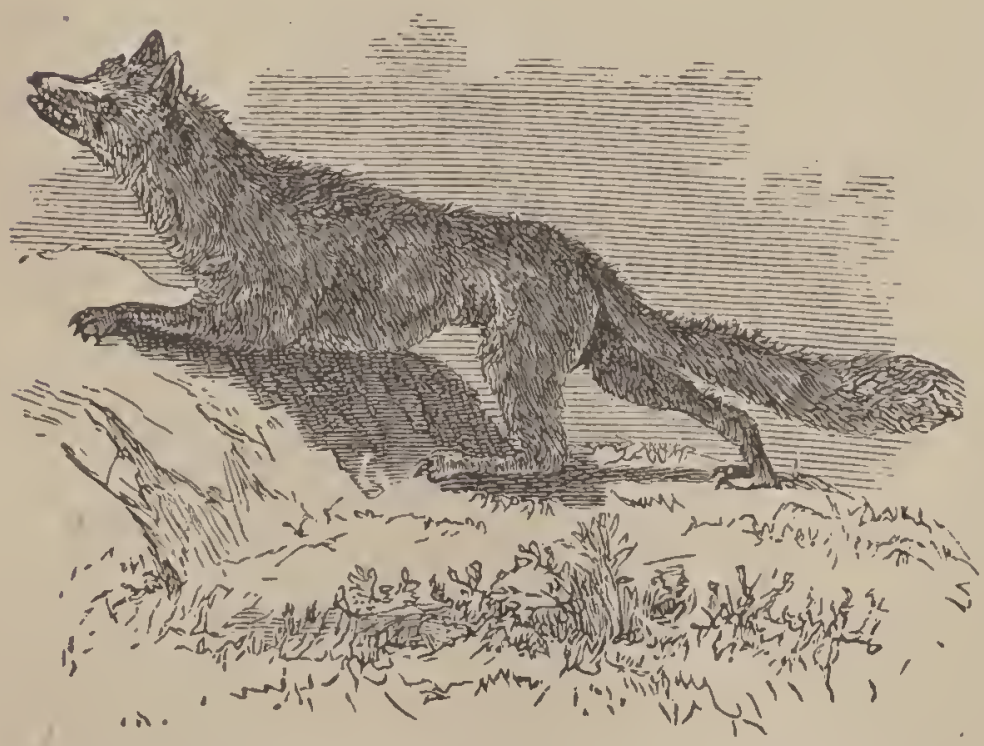

FIG. 349.-Red Fox (Vulpes fulvus). United States. deeper than in Ungulates, and there are numerousbony ridges on the inside and outside of the craniumthe high occipital crest being specially characteristic. The cerebral hemispheres are joined by a large colpus callosum, but the cerebellum is never completely covered. Both pairs of limbs are well developed, the front being prehensile; but the clavicles are rudimentary. Thehumerus and femur are mainly enclosed in the body. The digits, never less than four, always have sharp and pointed claws. ${ }^{181}$ The body is covered with abundant hair.

Carnivores are divided according to the modifications of the limbs : a. Pinnigrades, having short feet expanded 
into webbed paddles for swimming, the hinder ones being bound in with the skin of the tail. Snch are the Seals, Walrus, and Eared Seals, or Sea-lions. 6. Plantigrades, in which the whole, or nearly the whole, of the hind-foot forms a sole, and rests on the ground. The claws are not retractile; the ears are small, and tail short. Bears, Badgers, and Raccoons are well-known examples. c. Digitigrades keep the heel raised above the ground, walking on the toes. The majority have long tails. Such are the Weasels, Otters, Civets, Hyenas, Foxes, Jackals, Wolves, Dogs, Cats, Panthers, Leopards, Tigers, and Lions. The

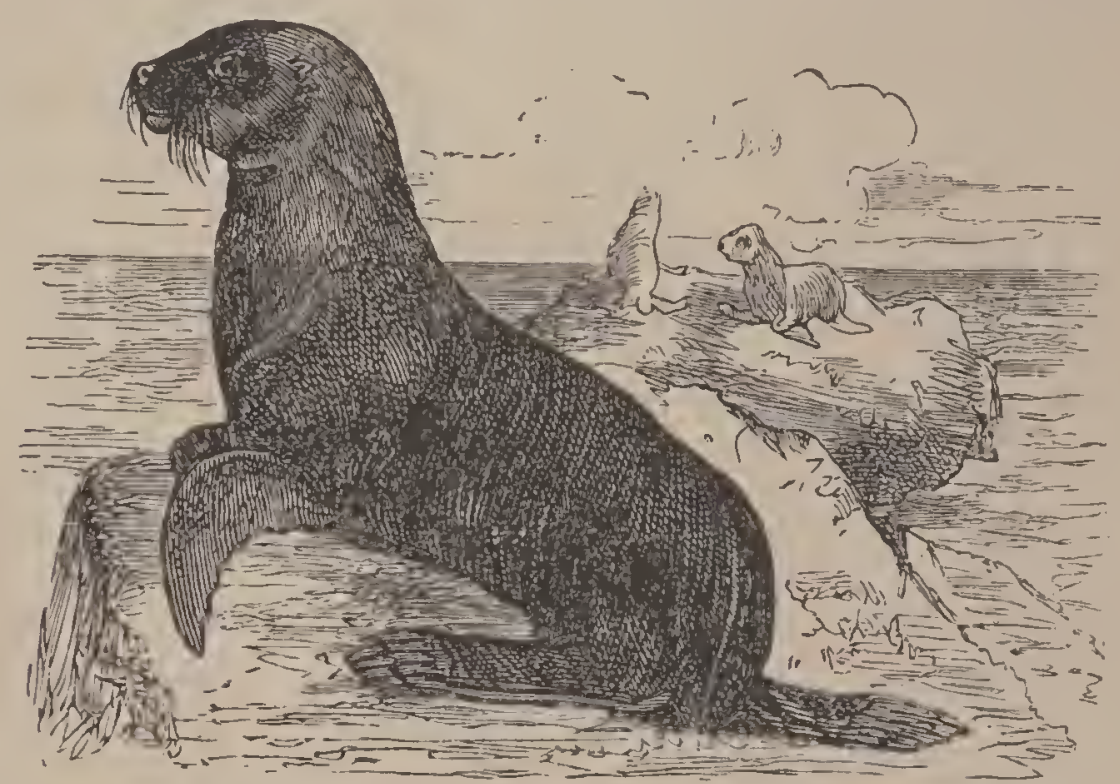

FIG. 350.-Southern Sea-lion (Otaria jubata). Antarctic Ocean.

last five differ from all others in having retractile claws, and the radius rotating freely on the ulna. The Cats have thirty teeth; the Dogs, forty-two, or twelve more molars. In the former, the tongue is prickly; in the latter, smooth.

12. Prosimii or Lemurs. These singular mammals, sometimes included in the next order, have affinities with Rodents, Insectivora, and Primates. They are covered with soft fur, have usually a long tail, pointed ears, foxlike muzzle, and curved nostrils. They walk on all fours, and the thumb and great toe are generally opposable to the digits. The second toe has a long, pointed claw in- 
stead of a nail. The cerebrum is relatively small, and flattened, and does not cover the cerebellum and olfactory

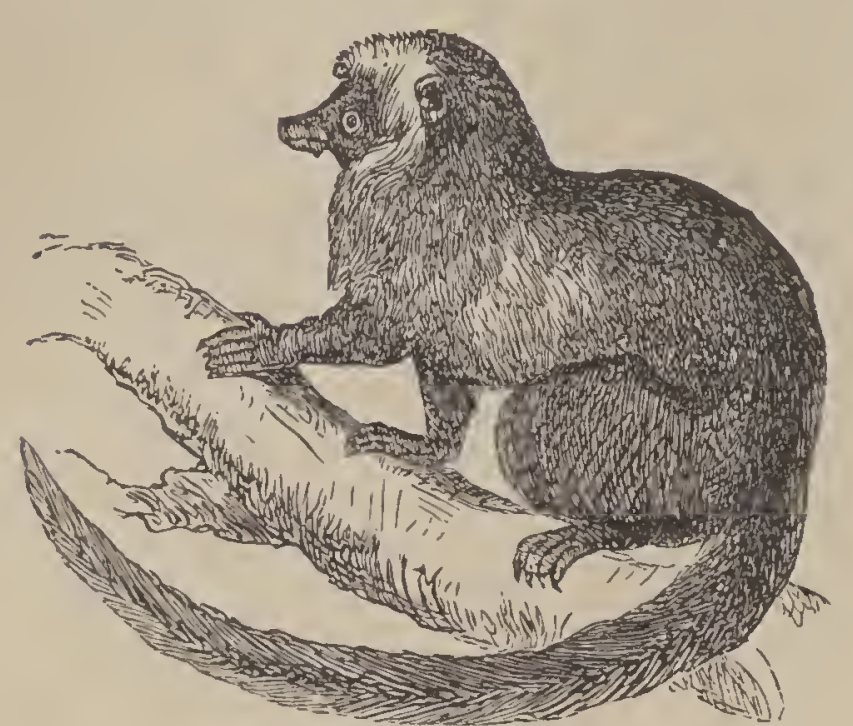

Fig. 351.-Lemur (L. ruber). Madayascar. lobes. ${ }^{182}$ They are found mainly in Madagascar.

13. Primates, the head of the kingdom, are characterized by the possession of two hands and two feet. The thigh is free from the body, and all the digits are furnished with nails, the first on the foot enlarged to a "great toe." Throughout the order, the hand is eminently or wholly prehensile, and the foot, however prehensile it may be, is always locomotive. ${ }^{193}$ The clavicles are perfect. The eyes are situated in a complete bony cavity, and look forward. There are two sets of teeth, all enamelled; and the incisors number four in each jaw. They are divided into Monkeys and Apes, and Man.

The Monkeys of tropical America have, generally, a long, prehensile tail; the nostrils are placed far apart, so that the nose is wide and flat; the thumbs and great toes are fitted for grasping, but are not opposable to the other digits; and they have four molars more than the Apes or Man - that is, thirty-six teeth in all. In the Apes of the Old World the tail is never prehensile; and is sometimes wanting; the nostrils are close together; both thumbs and great toes are opposable; and the teeth, though numbering the same as Man's, are uneven (the incisors being prominent, and the canines large), and the series is interrupted by a gap on one side or other of the canines. Their average size is much greater than that of the Monkeys, and they are not so strictly arboreal. In both Monkeys and Apes, the cerebrum covers the cere- 
VER'TEBRATA.

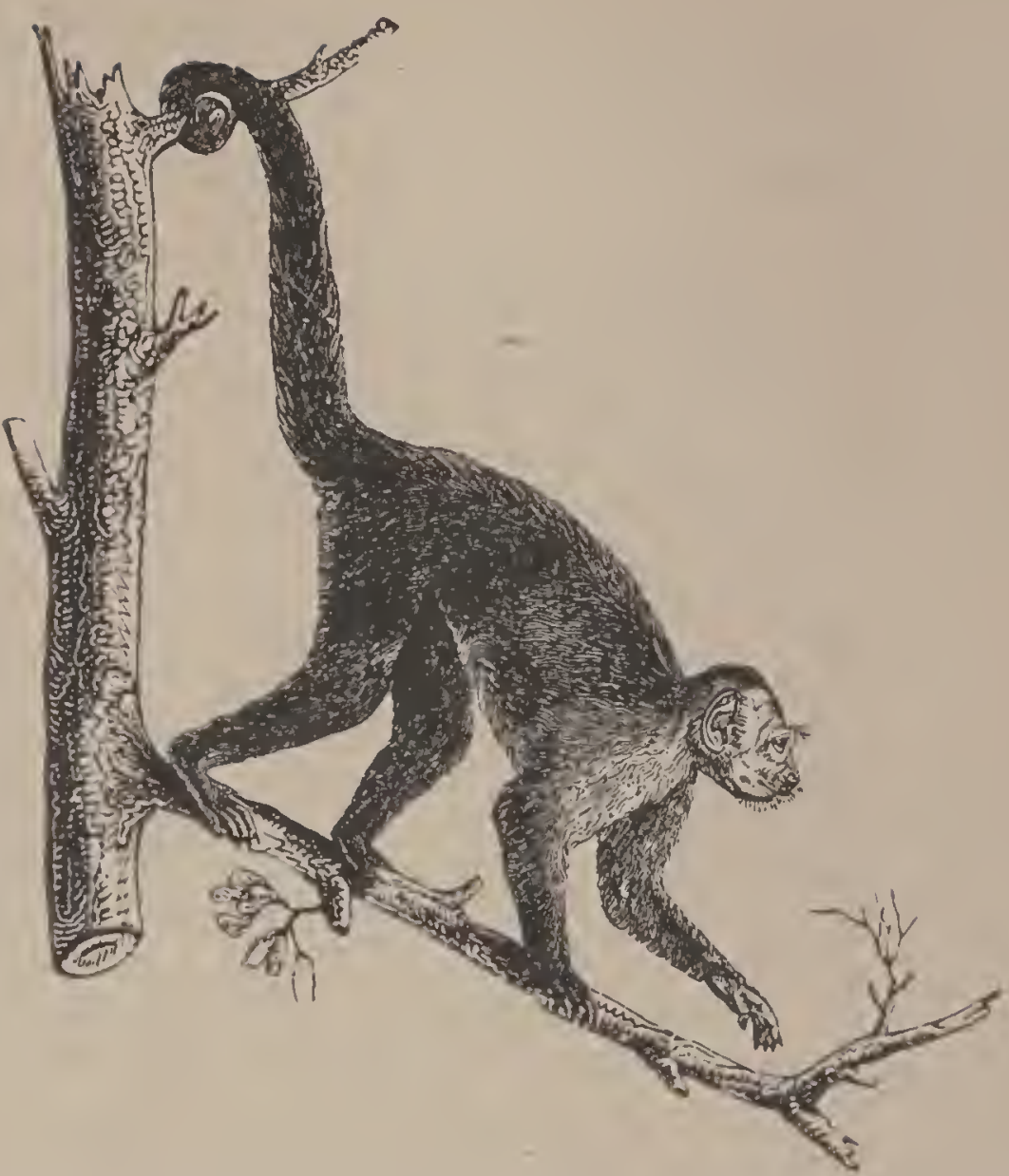

Fir. 352.-White-throated Sapajou (Cebus hypolencus). Central America.

bellum. ${ }^{184}$ While in the Monkeys the skull is rounded and smooth, that of the Apes, especially those coming nearest to Man-the anthropoid, or long-armed, Apes, as Gorilla, Chimpanzee, Orang, and Gibbon-is characterized by strong crests. Monkeys take a horizontal position;

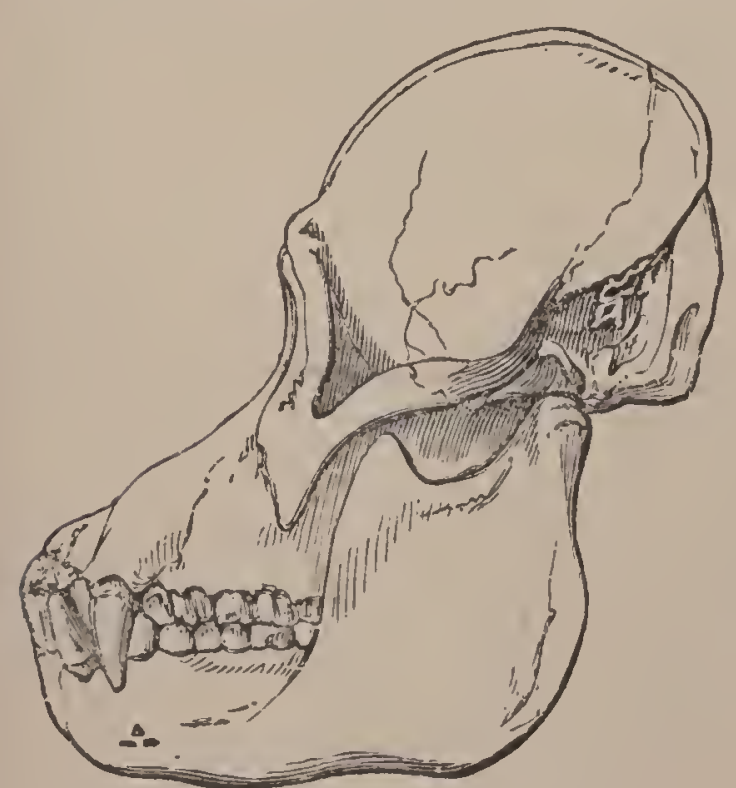

Fra. 353.-Skull of Orang-utan (Simia satyrus). but the Apes assume a semierect attitude, the legs being shorter than the arms. In

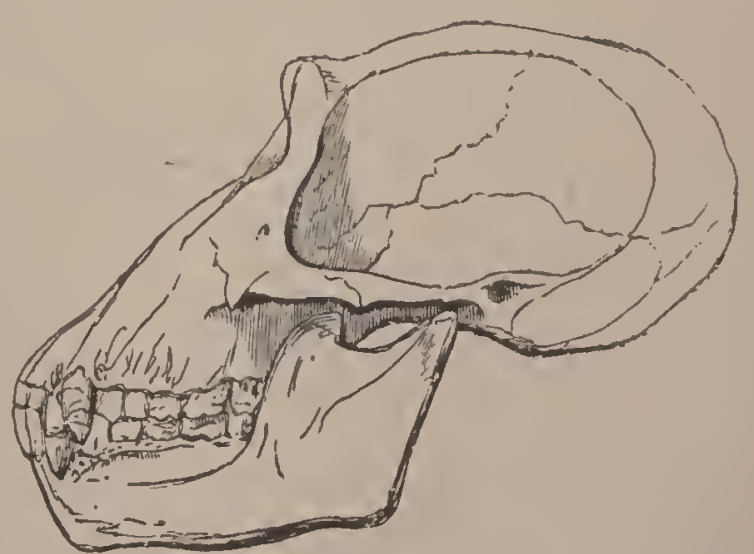

Fig. 354.-Skull of Chimpanzee (Troglo. dytes Niger). 
all the Primates but Man, the body is clothed with hair, which is generally longest on the back. Several Monkeys and Apes have a beard, as the Howler and Orang.

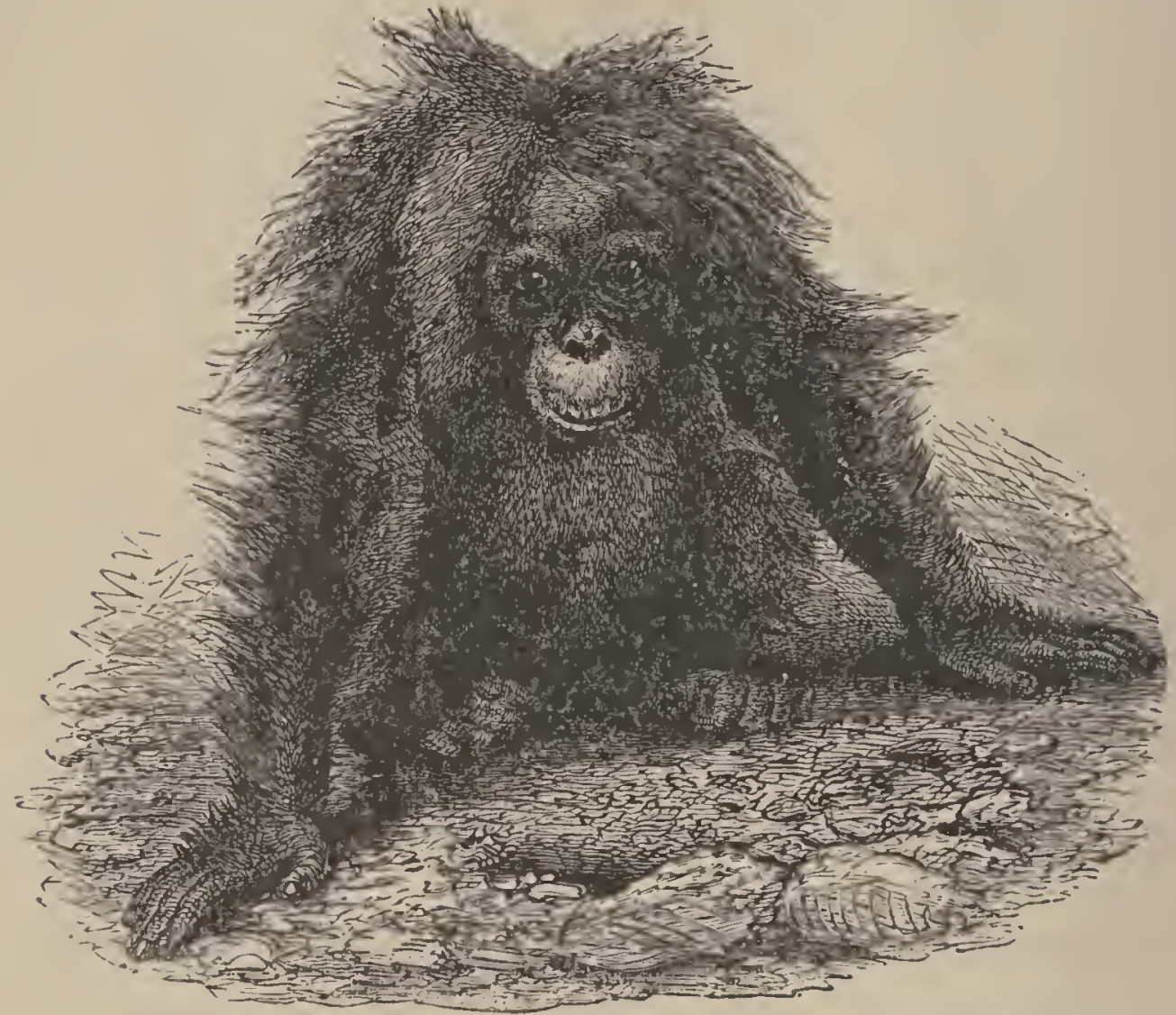

Fig. 355.-Female Orang-utan (from photograph). Borneo.

The Orang is the least human of all the anthropoid

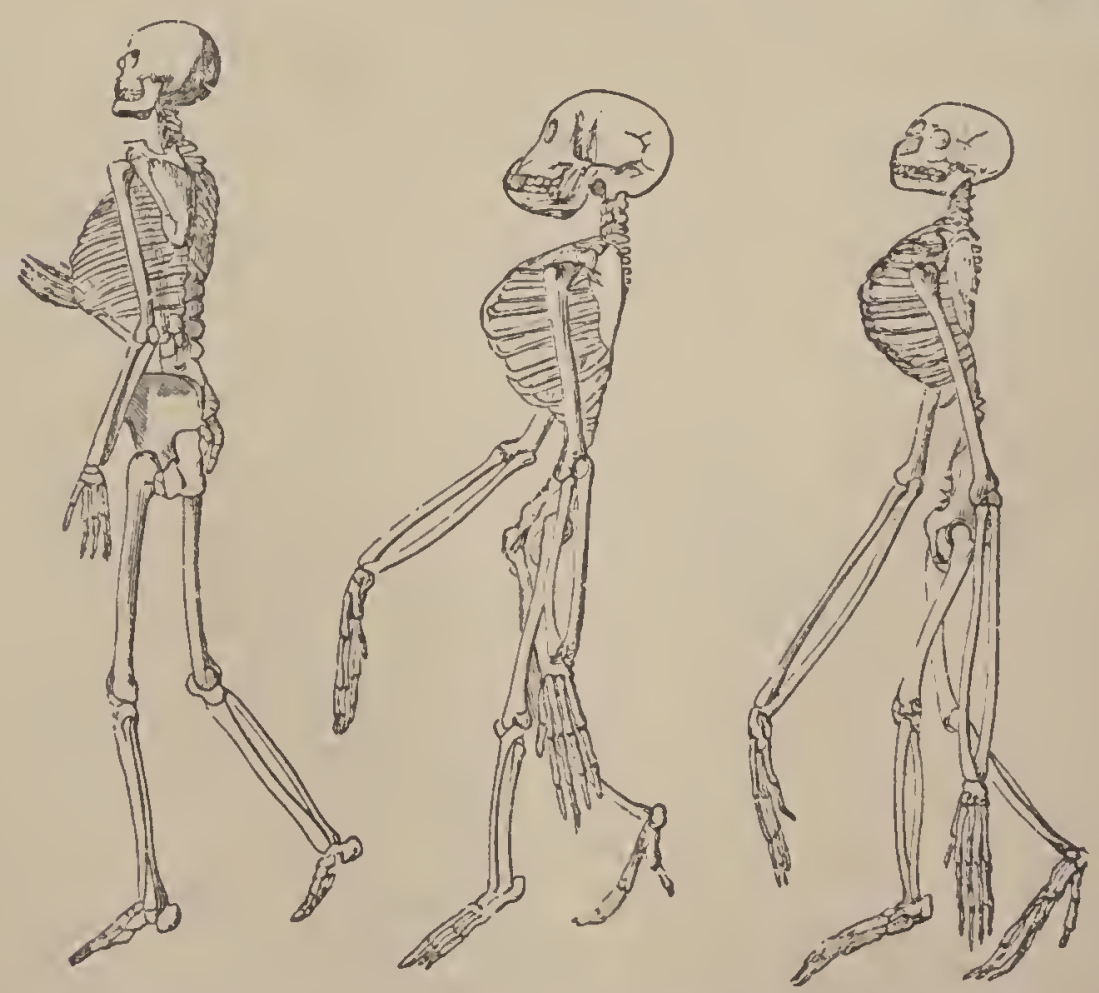

Fra. 356.-Skeletons of Man, Chimpauzee, and Oraug. 
Apes as regards the skeleton, but comes nearest to Man in the form of the brain. The Chimpanzee approaches Man more closely in the character of its cranium and teeth, and the proportional size of the arms. The Gorilla is most Man-like in bulk (sometimes reaching the height of five feet six inches), in the proportions of the leg to the body and of the foot to the hand, in the size of the heel, the form of the pelvis and shoulder-blade, and volume of brain. ${ }^{185}$

Man differs from the Apes in being an erect biped. In him, the vertebrate type, which began in the horizontal Fish, finally became vertical. No other animal habitually stands erect; in no other are the fore-limbs used exclusively for head-purposes, and the hind pair solely for locomotion.

His limbs are naturally parallel to the axis of his body, not perpendicular. They have a near equality of length, but the arms are always somewhat shorter than the legs. In all the great Apes the arms reach below the knee, and the legs of the Chimpanzee and Gorilla are relatively shorter than Man's.

Man only has a finished hand, most perfect as an organ of touch, and most versatile. Both hand and foot are relatively shorter than in the Apes. The foot is planti-
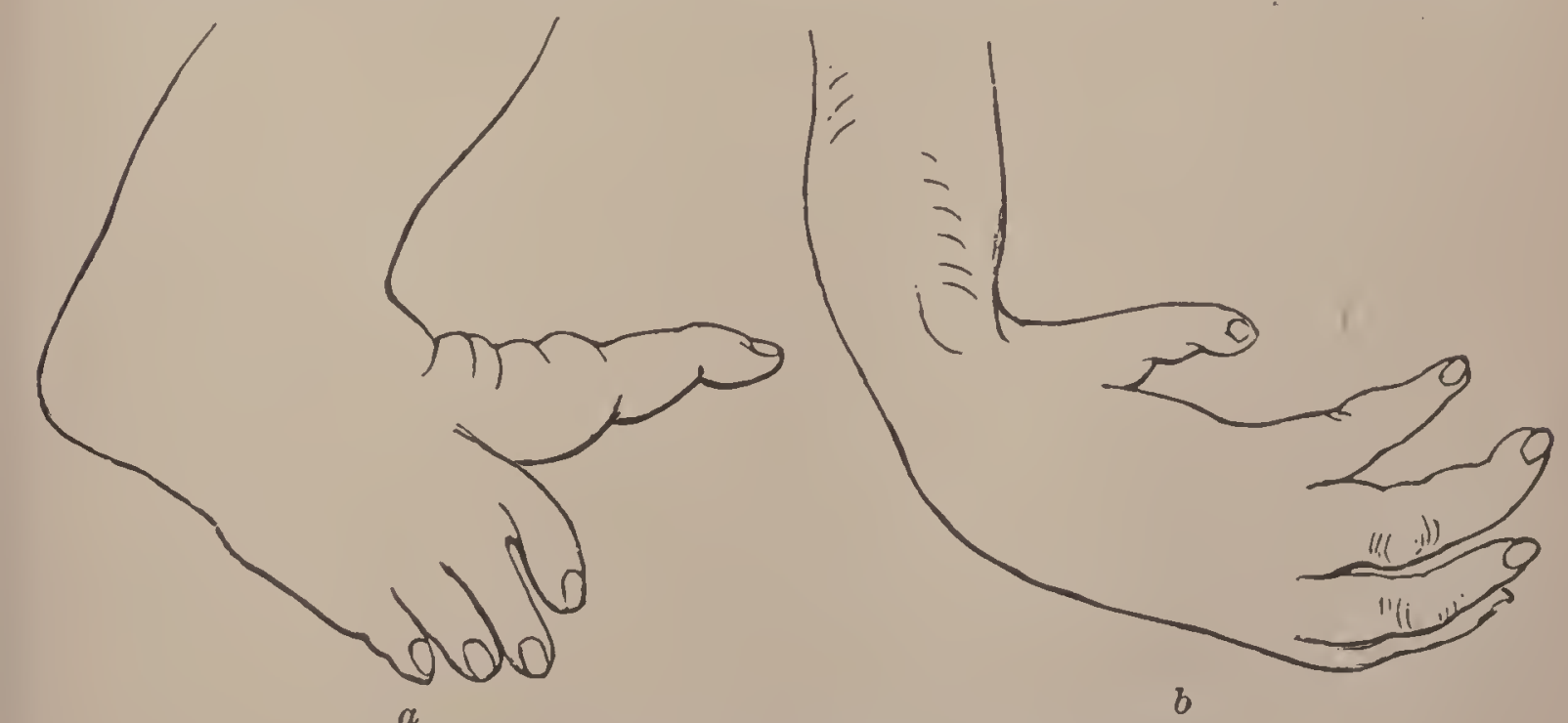

FrG. 357.-Foot (a) and Hand (b) of the Gorilla. 
grade; the leg bears vertically upon it; the heel and great toe are longer than in other Primates; and the great toe is not opposable, but is used only as a fulcrum in locomotion. The Gorilla has both an inferior hand and inferior foot. The hand is clumsier, and with a shorter thumb than Man's; and the foot is prehensile, and is not applied flat to the ground. ${ }^{186}$

The scapular and pelvic bones are extremely broad, and the neck of the femur remarkably long. Man is also singular in the double curve of the spine: the Baboon comes nearest to Man in this respect.

The human skull has a smooth, rounded outline, elevated in front, and devoid of crests. The cranium greatly predominates over the face, being four to one ${ }^{187}$ and no other animal (except the Siamang Gibbon) has a chin.

Man stands alone in the peculiarity of his dentition: his teeth are vertical, of nearly uniform height, and close together. In every other animal the incisors and canines are more or less inclined, the canines project, and there are vacant spaces. ${ }^{188}$

Man has a longer lobule to his ear than any Ape, and no muzzle. The bridge of his nose is decidedly convex; in the Apes generally it is flat.

Man has been called the only naked terrestrial Mammal. His hair is most abundant on the scalp; never on the back, as in the Apes.

Man has a more pliable constitution than the Apes, as shown by his world-wide distribution. The animals nearest him soon perish when removed from their native places.

Though Man is excelled by some animals in the acuteness of some senses, there is no other animal in which all the senses are capable of equal development. He only has the power of expressing his thoughts by articulate speech, and the power of forming abstract ideas.

Man differs from the Apes in the absolute size of 


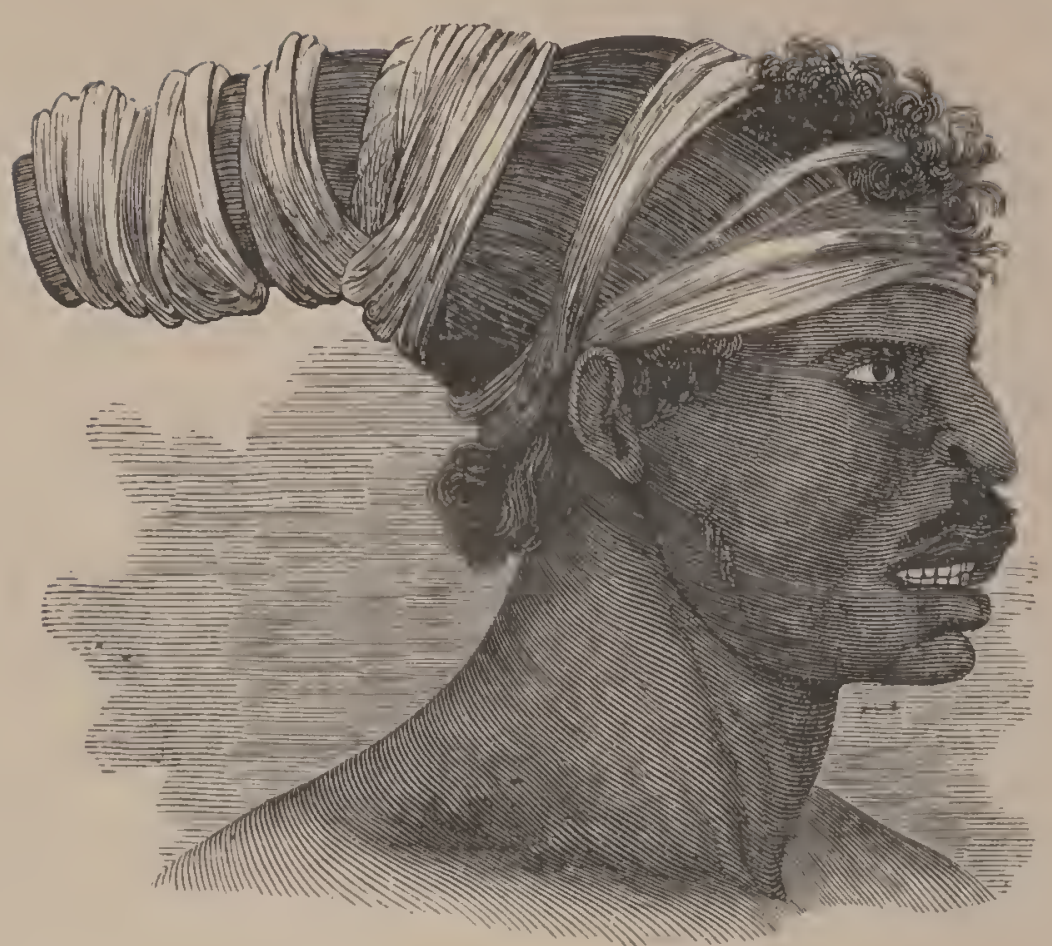

Frg. 35S.-Australian Savage.

brain, and in the greater complexity and less symmetrical disposition of its convolutions. The cerebrum is larger in proportion to the cerebellum (being as $8 \frac{1}{2}$ to 1 ), and the former not only covers the latter, but projects beyond it. The brain of the Gorilla scarcely amounts to one third in volume or one half in weight of that of Man.

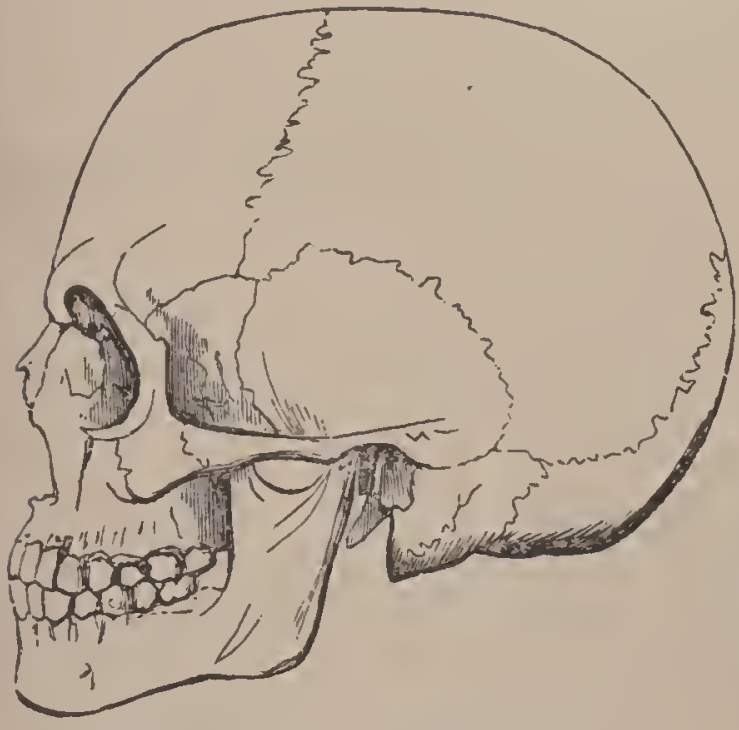

Fig. 359._Skull of European.

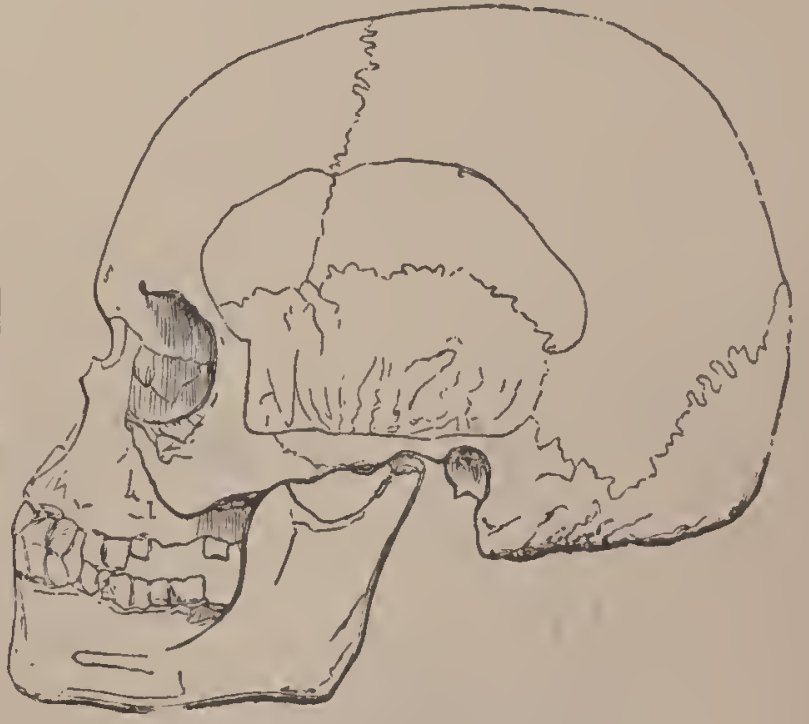

FIf. 360.-Slkull of Negro.

Yet, so far as cerebral structure goes, Man differs less from the Apes than they do frorn the Monkeys and Lemurs. The great gulf between Man and the brute is not physical, but psychical. ${ }^{188}$ 

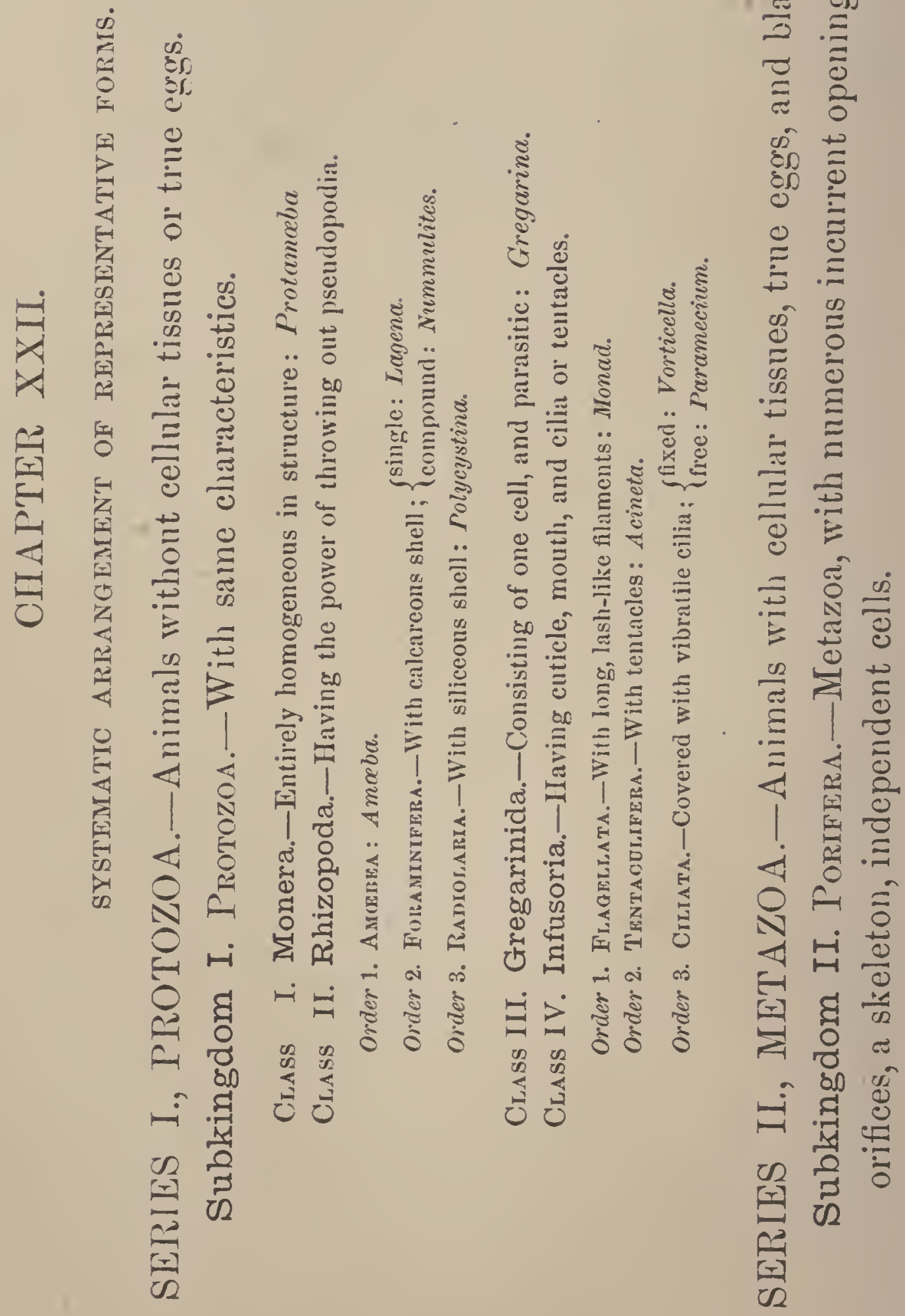
ARRANGEMENT OF REPRESENTATIVE FORMS. 363

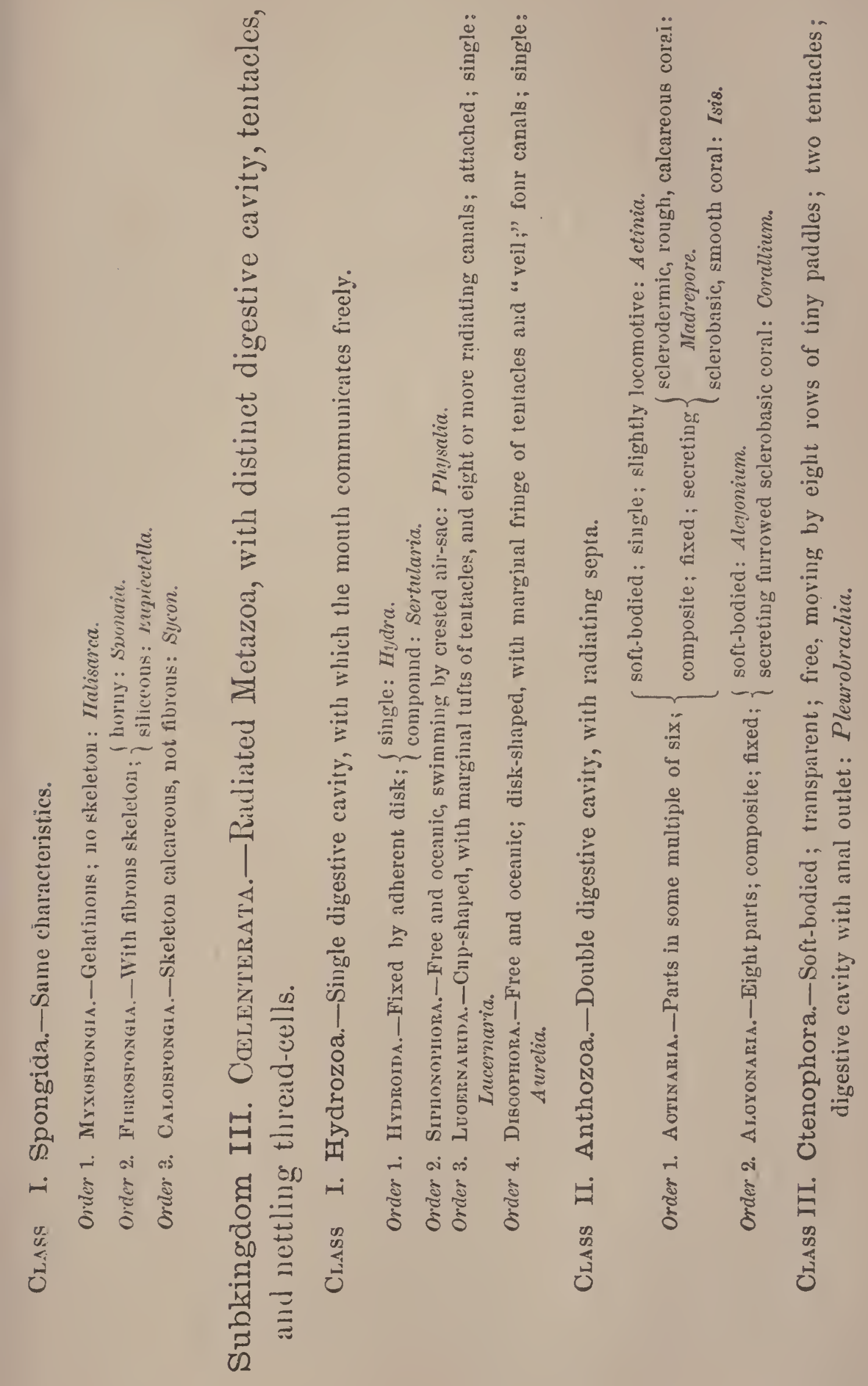




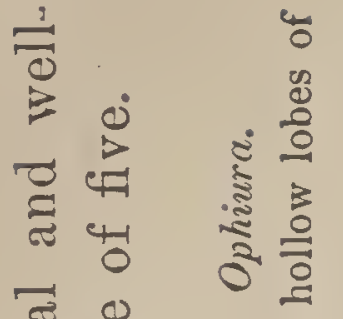

䒿 $\frac{0}{2}$ :ै

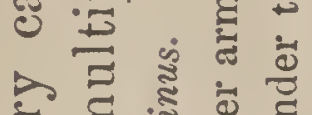

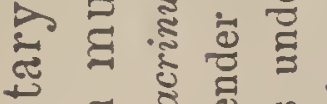

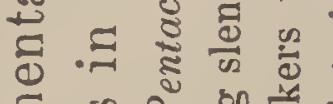

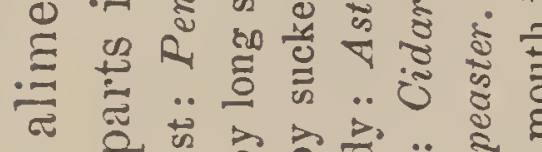

艹 $\cdots$ है

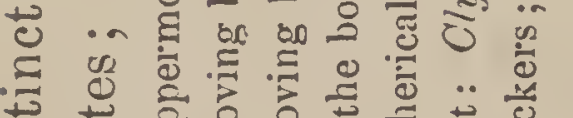

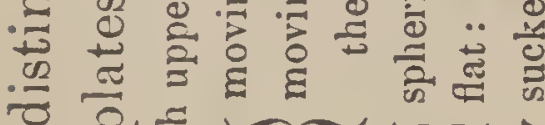

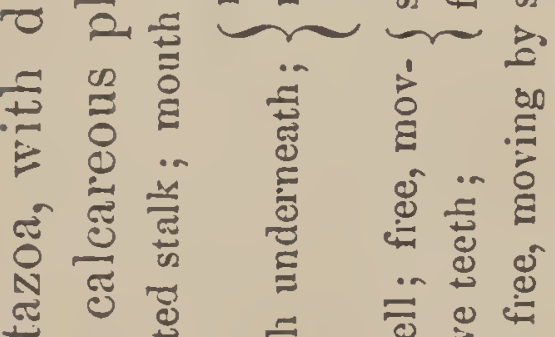

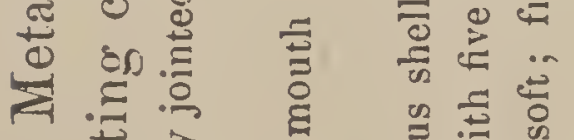

- $\overrightarrow{0}$

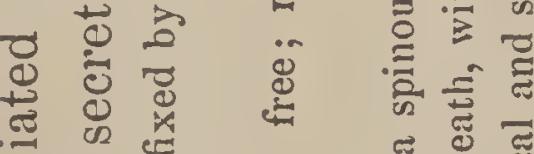

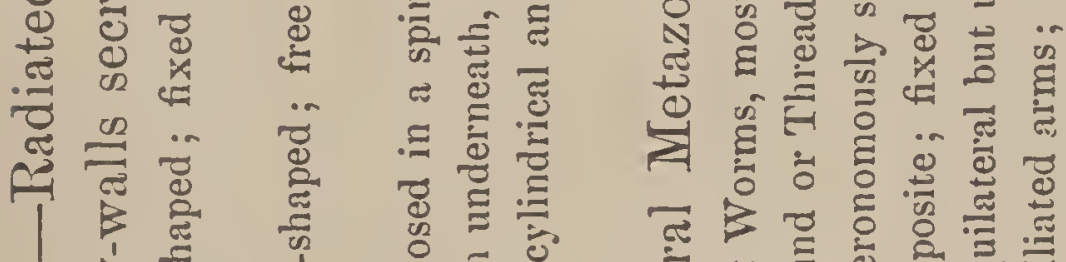

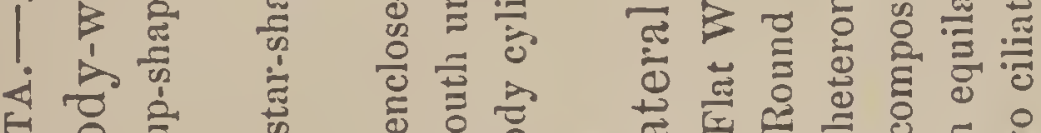

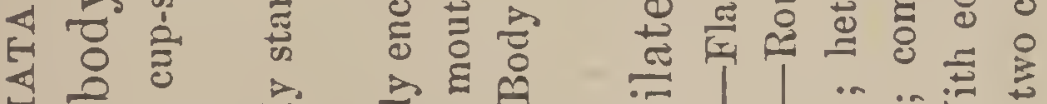

…

ज्ञ

焉

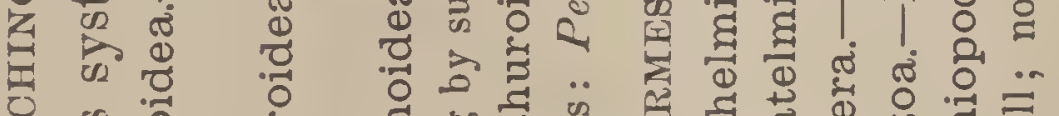

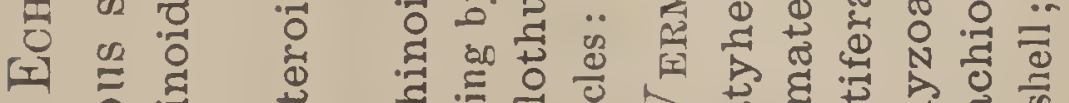

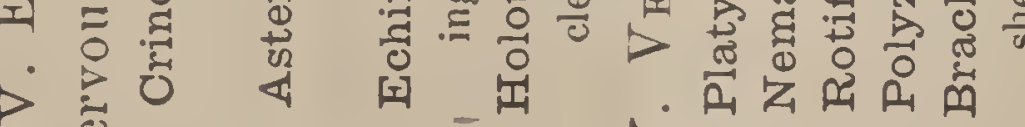

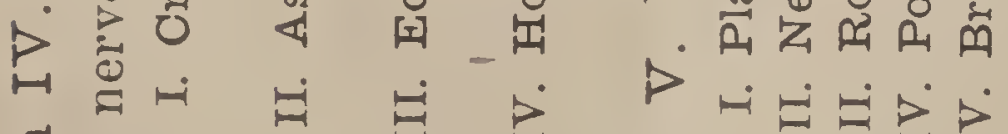

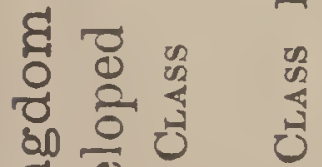

(०)

웡

(3)

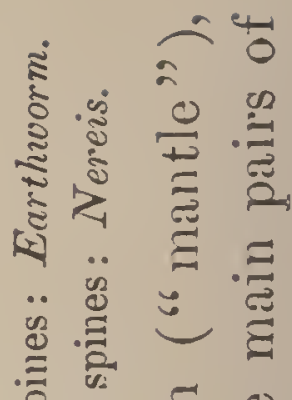

क्ष

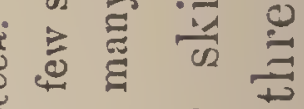

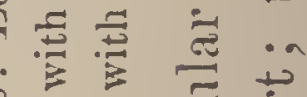

设

离 $\stackrel{0}{\Xi}$

志

离

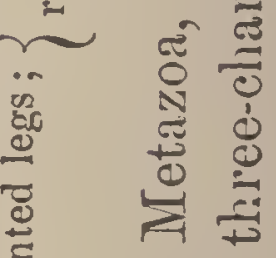

๑ ठ

$\stackrel{0}{\cong} \quad$.

宊 $\frac{\bar{c}}{\bar{\varepsilon}}$

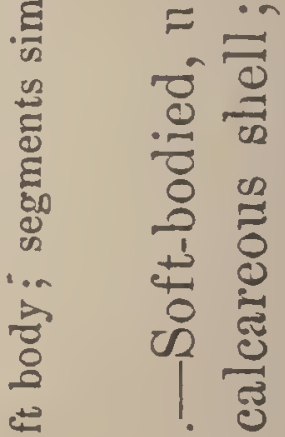

范

के

:

\&

$\dot{P} \stackrel{\circ}{\circ}$

क द्व

क 00

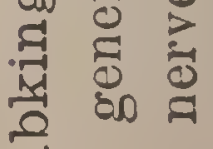

क 


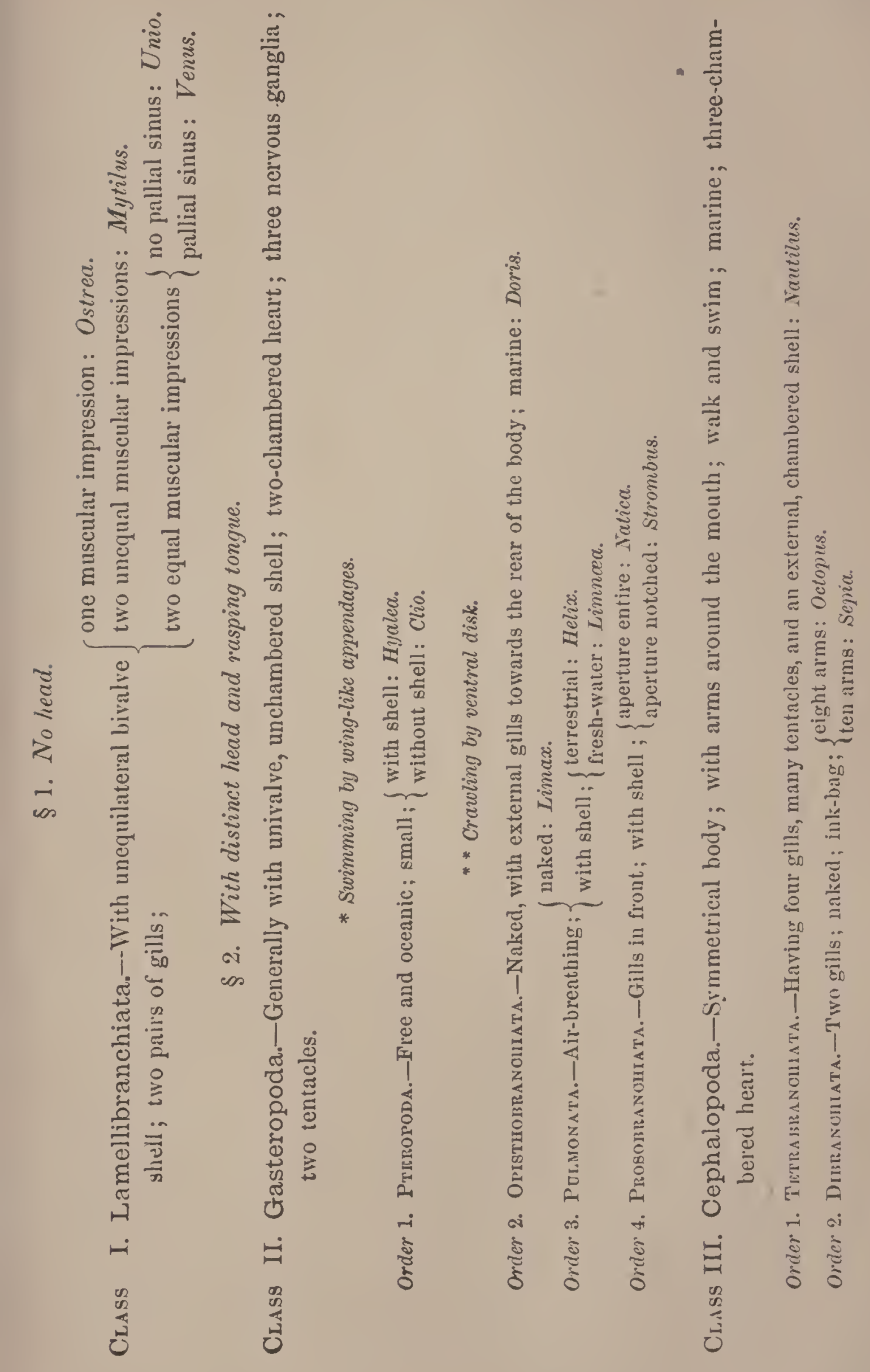




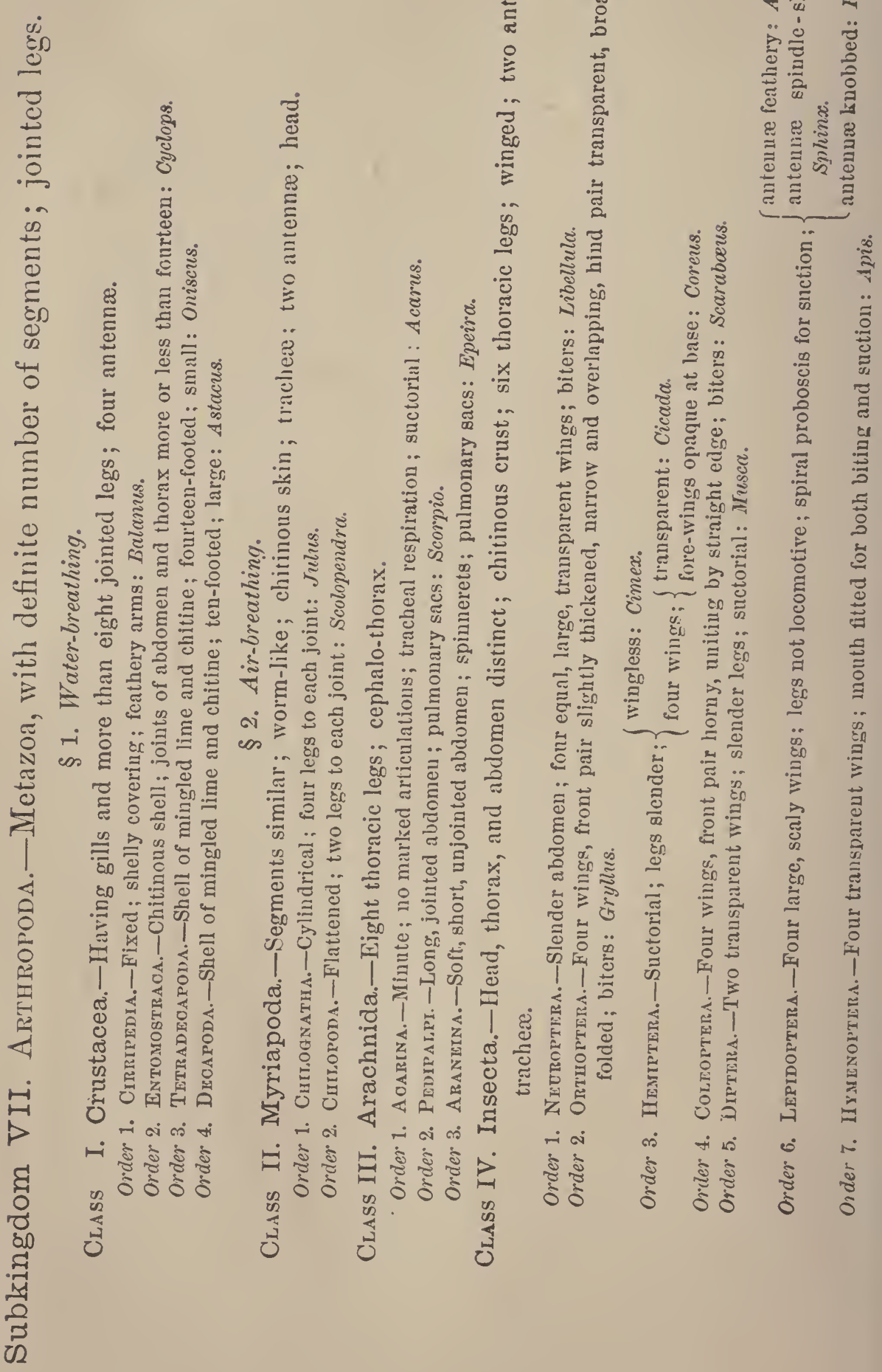




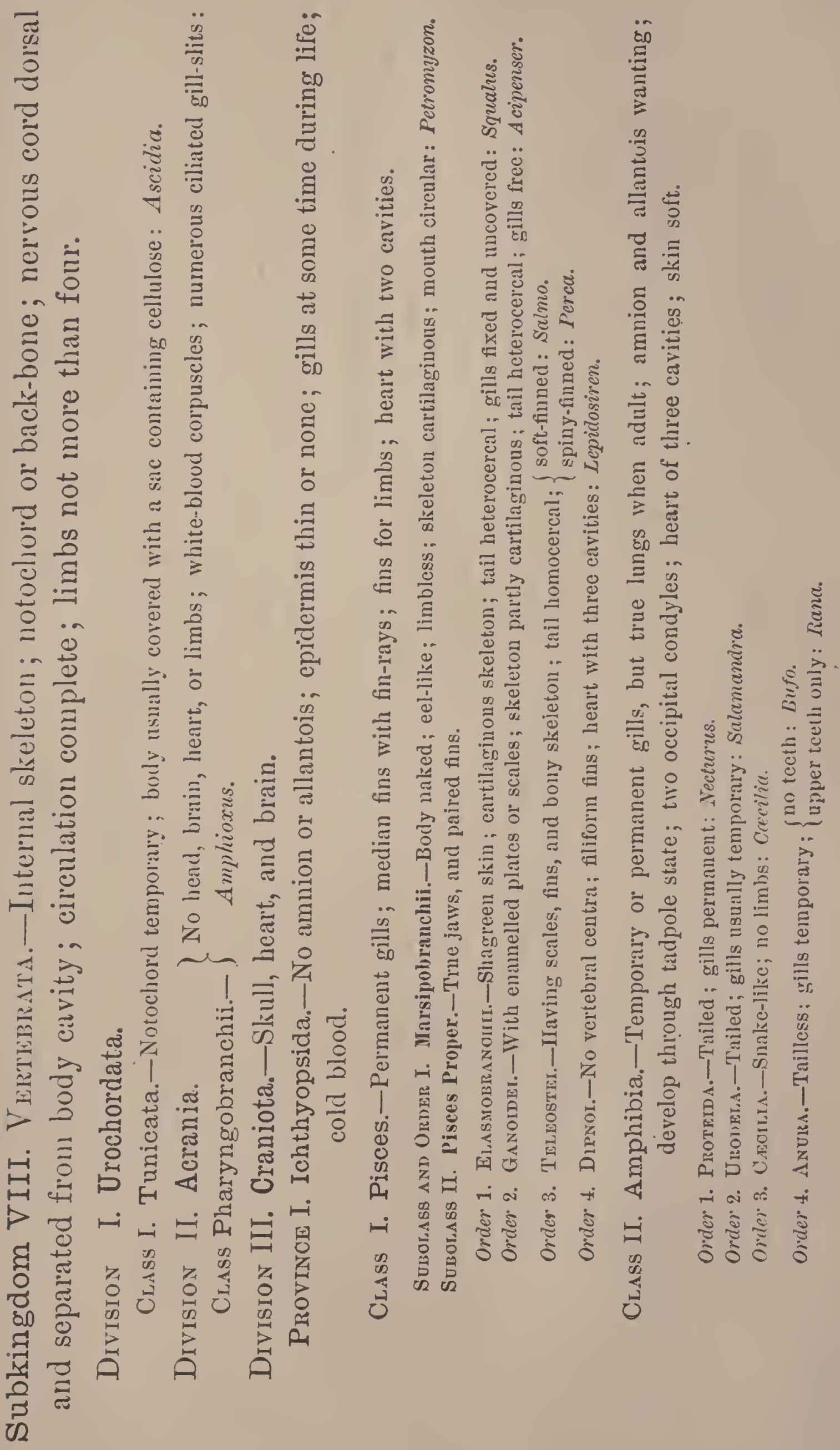




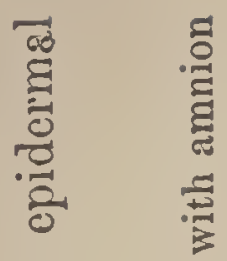

○

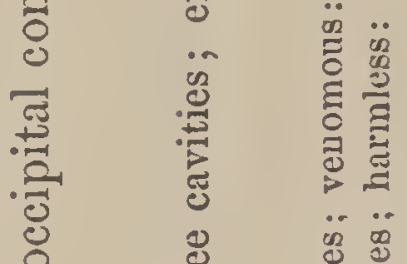

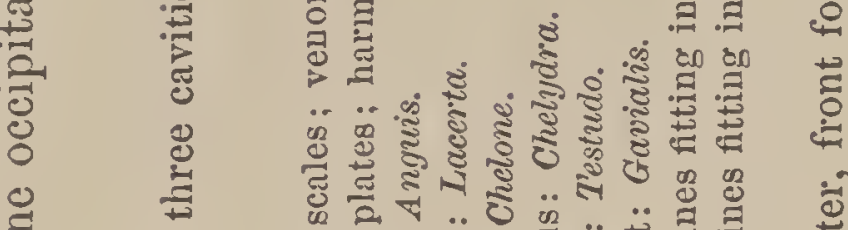

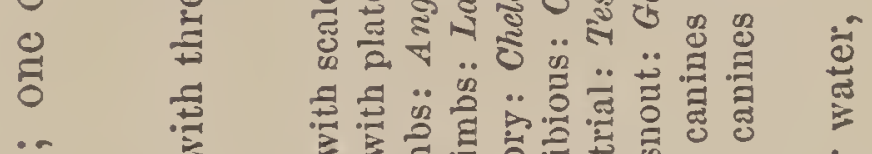

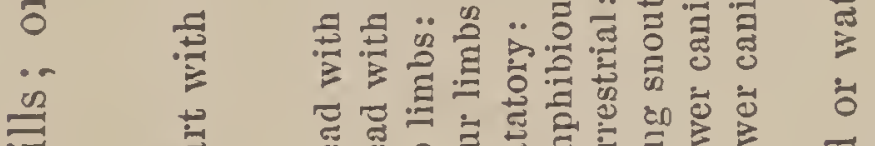

हु

$\stackrel{ }{g}$

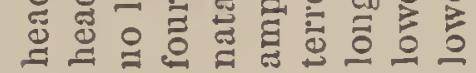

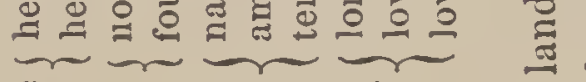

ô

跑

究

క్రి

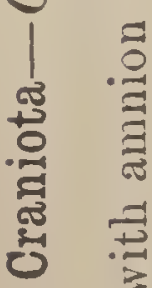

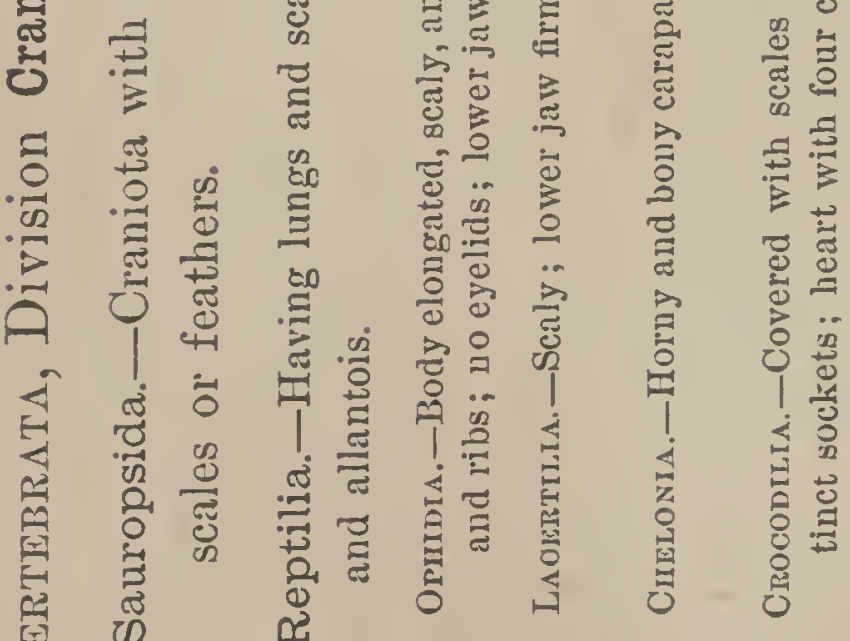

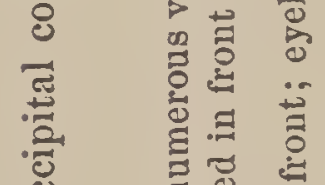

"ृ.

$\stackrel{\Xi}{0}$

要

的挡

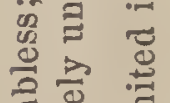

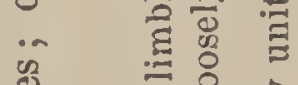

$\frac{\mathscr{0}}{\mathrm{E}}$

혈을

迋

:

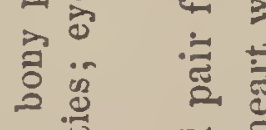

สี

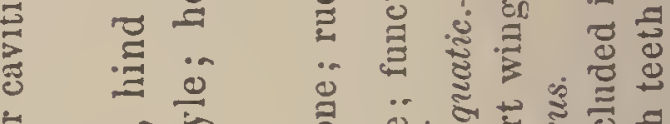

:

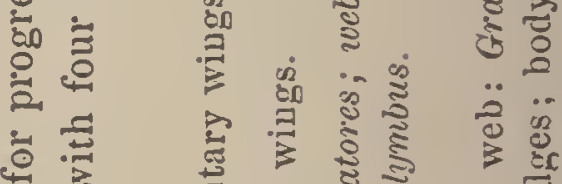

:

范

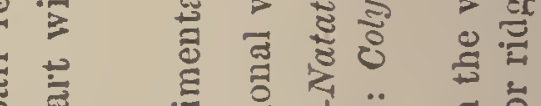

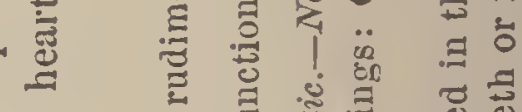

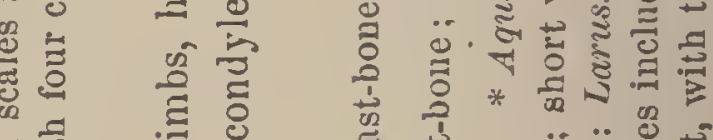

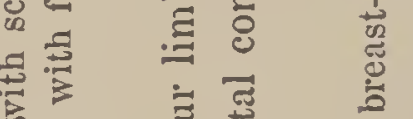

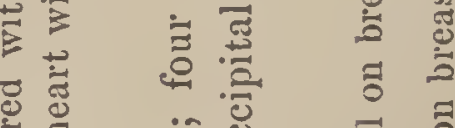

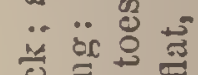

है

更苛

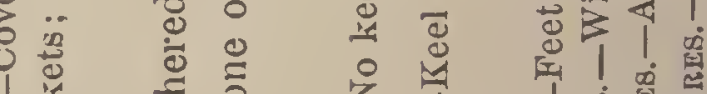

范苛

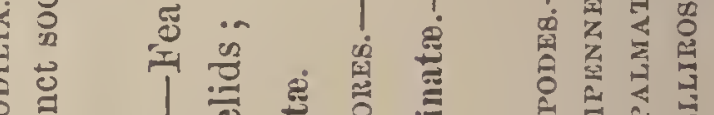

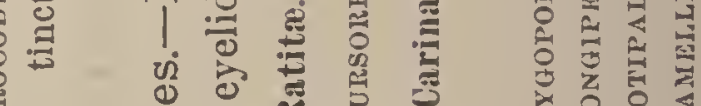

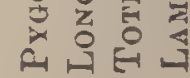

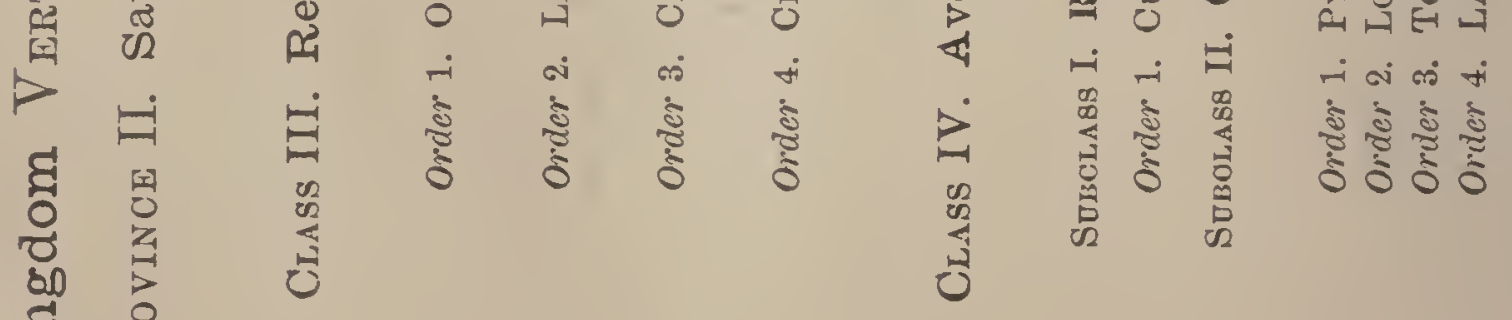




\section{9}

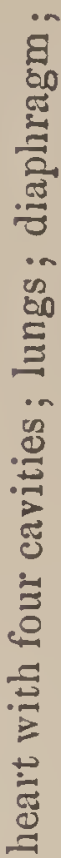

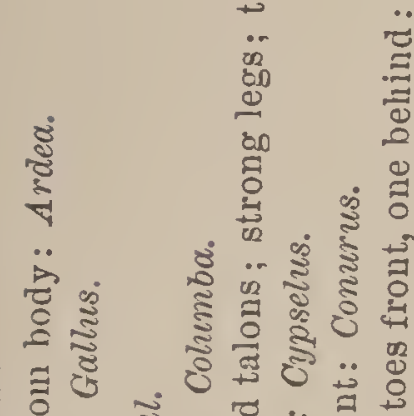

.క.

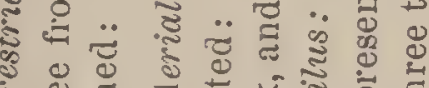

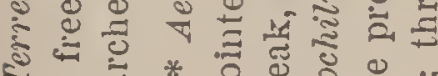

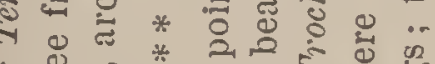
*

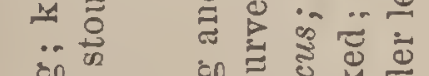

ô 음 …

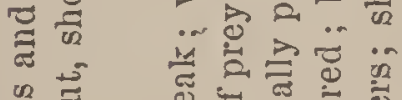

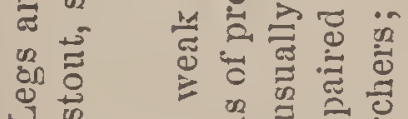

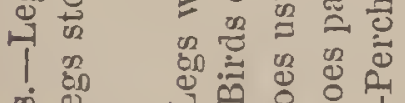
की 


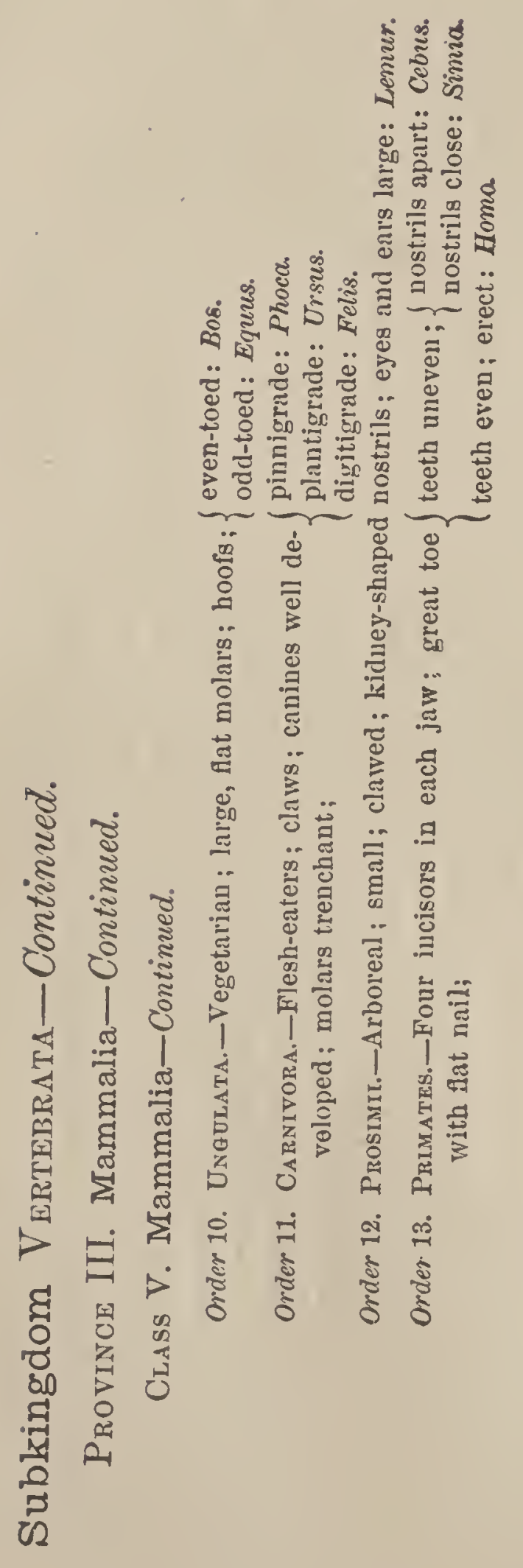




\section{CHAPTER XXIII.}

\section{THE DISTRIBUTION OF ANIMALS.}

LIFE is everywhere. In the air above, the earth beneath, and the waters under the earth, we are surrounded with life. Nature lives: every pore is bursting with life; every death is only a new birth, every grave a cradle. The air swarms with Birds, Insects, and invisible animalcules. The waters are peopled with innumerable forms, from the Protozoan, millions of which would not weigh a grain, to the Whale, so large that it seems an island as it sleeps upon the waves. The bed of the sea is alive with Crabs, Molluscs, Polyps, Star-fishes, and Foraminifera. Life everywhere - on the earth, in the earth, crawling, creeping, burrowing, boring, leaping, running.

Nor does the vast procession end here. The earth we tread is largely formed of the débris of life. The quarry of limestone, the flints which struck the fire of the old Revolutionary muskets, are the remains of countless skeletons. The major part of the Alps, the Rocky Mountains, and the chalk cliffs of England are the monumental relics of by-gone generations. From the ruins of this liring architecture we build our Parthenons and Pyramids, our St. Peters and Louvres. So generation follows generation. But we have not yet exhausted the surrey. Life cradles within life. The bodies of animals are little worlds having their own fauna and flora. In the fluids and tissues, in the eye, liver, stomach, brain, and' muscles, parasites are found; and these parasites often have their parasites liv. ing on them. 
"Great fleas have little fleas and smaller fleas to bite 'em; And these again have other fleas, and so ad infinitum."

Thus the ocean of life is inexhaustible. It spreads in every direction, into time past and present, flowing everywhere, eagerly surging into every nook and corner of creation. On the mountain-top, in the abysses of the Atlantic, in the deepest crevice of the earth's crust, we find traces of animal life. Nature is prodigal of space, but economical in filling it. ${ }^{190}$

Animals are distributed over the globe according to definite laws, and with remarkable regularity.

Each of the three great provinces, Earth, Air, and Water, as also every continent, contains representatives of all the classes; but the various classes are unequally represented. Every great climatal region contains some species not found elsewhere, to the exclusion of some other forms. Every grand division of the globe, whether of land or sea, each zone of climate and altitude, has its own fauna. And, in spite of the many causes tending to disperse animals beyond their natural limits, each country preserves its peculiar zoological physiognomy.

The space occupied by the different groups of animals is often inversely as the size of the individuals. Compare the Coral and Elephant.

The fauna now occupying a separate area is closely allied to the fauna which existed in former geologic times. Thus, Australia has always been the home of Marsupials, and South America of Edentates.

It is a general rule that groups of distinct species are circumscribed within definite, and often narrow, limits. Man is the only cosmopolitan; yet even he comprises ser. eral marked races, whose distribution corresponds with the great zoological regions. The natives of Australia are as grotesque as the animals. Certain brutes likewise have a great range: thus, the Puma ranges from Canada to Pata- 
gonia; the Musk - rat, from the Arctic Ocean to Florida; the Ermine, from Behring's Straits to the Himalayas; and the Hippopotamus, from the Nile and Niger to the Orange River. ${ }^{192}$

Frequently, species of the same genus, living side by side, are widely different, while there is a close resem-1 blance between forms which are antipodes. The Mud-eel of South Carolina and Menobranchus of the Northern States have their relatives in Japan and Austria. The American Tapir has its mate in Sumatra; the Llama is related to the Camel, and the Opossum to the Kangaroo.

The chief causes modifying distribution are temperature, topography, ocean and wind currents, humidity and light. To these may be added the fact that animals are ever intruding on each other's spheres of existence. High mountain-ranges, wide deserts, and cold currents in the ocean are impassable barriers to the migration of most species. Thus, river-fish on opposite sides of the Andes differ widely, and the cold Peruvian current prevents the growth of coral at the Galapagos Islands. So a broad river, like the Amazons, or a deep, narrow channel in the sea, is an effectual barrier to some tribes. Thus, Borneo belongs to the Indian region, while Celebes, though but a few miles distant, is Australian in its life. The faunæ of North America, on the east coast, west coast, and the open plains between, are very different.

Animals dwelling at high elevations resemble those of colder latitudes. The same species of Insects are found on Mount Washington, and in Labrador and Greenland.

The range does not depend upon the powers of locomotion. The Oyster extends from Halifax to Charleston, and the Snapping - turtle from Canada to the equator; while many Quadrupeds and Birds have narrow hab. itats.

The distribution of any group is qualified by the nature 
of the food. Carnivores have a wider range than herbivores.

Life diminishes as we depart from the equator north or south, and likewise as we descend or ascend from the level of the sea.

The zones of geography have been divided by zoologists into narrower provinces. Five vertical regions in the sea have been recognized: the Littoral, extending between tide-marks; the Laminarian, from low water to fifteen fathoms; the Coralline, from fifteen to twenty fathoms; the deep-sea Coral, from fifty to one hundred fathoms; and the Bathybian, from one hundred fathoms down; but since life has been found to extend to great depths in the ocean-as great as three thousand fathoms -these divisions are of little importance. Every marine species has its own limits of depth. It would be quite as difficult, said Agassiz, for a Fish or a Mollusk to cross from the coast of Europe to the coast of America as for a Reindeer to pass from the arctic to the antarctic regions across the torrid zone. Marine animals congregate mainly along the coasts of continents and on soundings. The meeting - place of two maritime currents of different temperatures, as on the Banks of Newfoundland, favors the development of a great diversity of Fishes.

Every great province of the ocean contains some representatives of all the subkingdoms. Deep-sea life is diversified, though comparatively sparse. Examples of all the five invertebrate divisions were found in the Bay of Biscay, at the depth of two thousand four hundred and thirty-five fathoms. ${ }^{192}$

Distribution in the sea is influenced by the temperature and composition of the water and the character of the bottom. The depth acts indirectly by modifying the temperature. Northern animals approach nearer to the equator in the sea than on the land, on account of cold 
currents. The heavy aquatic Mammals, as Whales, Walruses, Seals, and Porpoises, are mainly polar.

The land consists of the following somewhat distinct areas : the Neotropic, comprising South America, the West Indies, and most of Mexico; the Nearctic, including the rest of America; the Palæarctic, composed of the eastern continent north of the Tropic of Cancer, and the Himalayas; the Ethiopian, or Africa south of the Tropic of Cancer; the Oriental, or India, the southern part of China, the Malay Peninsula, and the islands as far east as Java, Borneo, and the Philippine Islands; and the Australian, or the eastern half of the Malay Islands and Australia. These are Mr. Wallace's regions. Other writers unite the northern parts of both hemispheres into one region, and the Oriental with the Ethiopian regions.

Life in the polar regions is characterized by great uniformity, the species being few in number, though the number of individuals is immense. The same animals inhabit the arctic portions of the three continents; while the antarctic ends of the continents, Australia, Cape of Good Hope, and Cape Horn, exhibit strong contrasts. Those three continental peninsulas are, zoologically, separate worlds. In fact, the whole southern hemisphere is peculiar. Its fauna is antique. Australia possesses a strange mixture of the old and new. South America, with newer Mammals, has older Reptiles; while Africa has a rich vertebrate life, with a striking uniformity in its distribution. Groups, old geologically and now nearly extinct, are apt to have a peculiar distribution; as the Edentata in South America, Africa, and India ; the Marsupials in Australia and America; the Ratitæ in South America, Africa, Australia, and New Zealand.

In the tropics, diversity is the law. Life is more varied and crowded than elsewhere, and attains its highest development. 
The New-world fauna is old-fashioned, and inferior in rank and size, compared with that of the eastern continents.

As a rule, the more isolated a region the greater the variety. Oceanic islands have comparatively few species, b but a large proportion of endemic or peculiar forms. Batrachians are absent, and there are no indigenous terrestrial Mammals. The productions are related to those of the nearest continent. When an island, as Britain, is separated from the mainland by a shallow channel, the mammalian life is the same on both sides.

Protozoans, Cœlenterates, and Echinoderms are limited to the waters, and nearly all are marine. Sponges are mostly obtained from the Grecian Archipelago and Bahamas, but species not commercially valuable abound in all seas. Coral-reefs abound throughout the Indian Ocean and Polynesia, east coast of Africa, Red Sea, and Persian Gulf, West Indies, and around Florida; and Corals which do not form reefs are much more widely distributed, being found as far north as Long Island Sound and England. Crinoids have been found, usually in deep sea, in very widely separated parts of the world-off the coast of Norway, Scotland, and Portugal, and near the East and West Indies. The other Echinoderms abound in almost every sea: the Star-fishes chiefly along the shore, the Seaurchins in the Laminarian zone, and the Sea-slugs around coral-reefs. Worms are found in all parts of the world, in sea, fresh water, and earth. They are most plentiful in the muddy or sandy bottoms of shallow seas. Living Brachiopods, though few in number, occur in tropical, temperate, and arctic seas, and from the shore to great depths. Polyzoa have both salt and fresh water forms, and Annelids include land forms, as the Earth-worm and some Leeches.

Mollusks have a world -wide distribution over land and 
sea. The land forms are restricted by climate and food, the marine by shallows or depths, by cold currents, by a sandy, gravelly, or mud bottom. The Bivalves are also found on every coast and in every climate, as well as in rivers and lakes, but do not flourish at the depth of much more than two hundred fathoms. The fresh-water Mussels are more numerous in the United States than in Europe, and west of the Alleghanies than east. The seashells along the Pacific coast of America are unlike those of the Atlantic, and are arranged in five distinct groups: Alentian, Californian, Panamic, Peruvian, and Magellanic. On the Atlantic coast, Cape Cod and Cape Hatteras separate distinct provinces. Of land Snails, Helix has an almost universal range, but is characteristic of North America, as Butimus is of South America, and Achatina of Africa. The Old World and Ainerica have no species in common, except a few in the extreme north.

The limits of Insects are determined by temperature and vegetation, by oceans and mountains. There is an insect-fauna for each continent, and zone, and altitude. The Insects near the snow-line on the sides of mountains in the temperate region are similar to those in polar lands. The Insects on our Pacific slope resemble those of Europe, while those near the Atlantic coast are more like those of Asia. Not half a dozen Insects live in the sea.

The distribution of Fishes is bounded by narrower limits than that of other animals. A few tribes may be called cosmopolitan, as the Sharks and Herrings; but the species are local. Size does not appear to bear any relation to latitude. The marine forms are three times as numerous as the fresh-water. The migratory Fishes of the northern hemisphere pass to a more sonthern region in the spring, while Birds migrate in the autumn.

Living Reptiles form but a fragment of the immense number which prevailed in the Middle Ages of Geology. 
Being less under the influence of Man, they have not been forced from their original habitats. None are arctic. America is the most favored spot for Frogs and Salamanders, and India for Snakes. Australia has no Batrachians, and two thirds of its Snakes are venomous. In the United

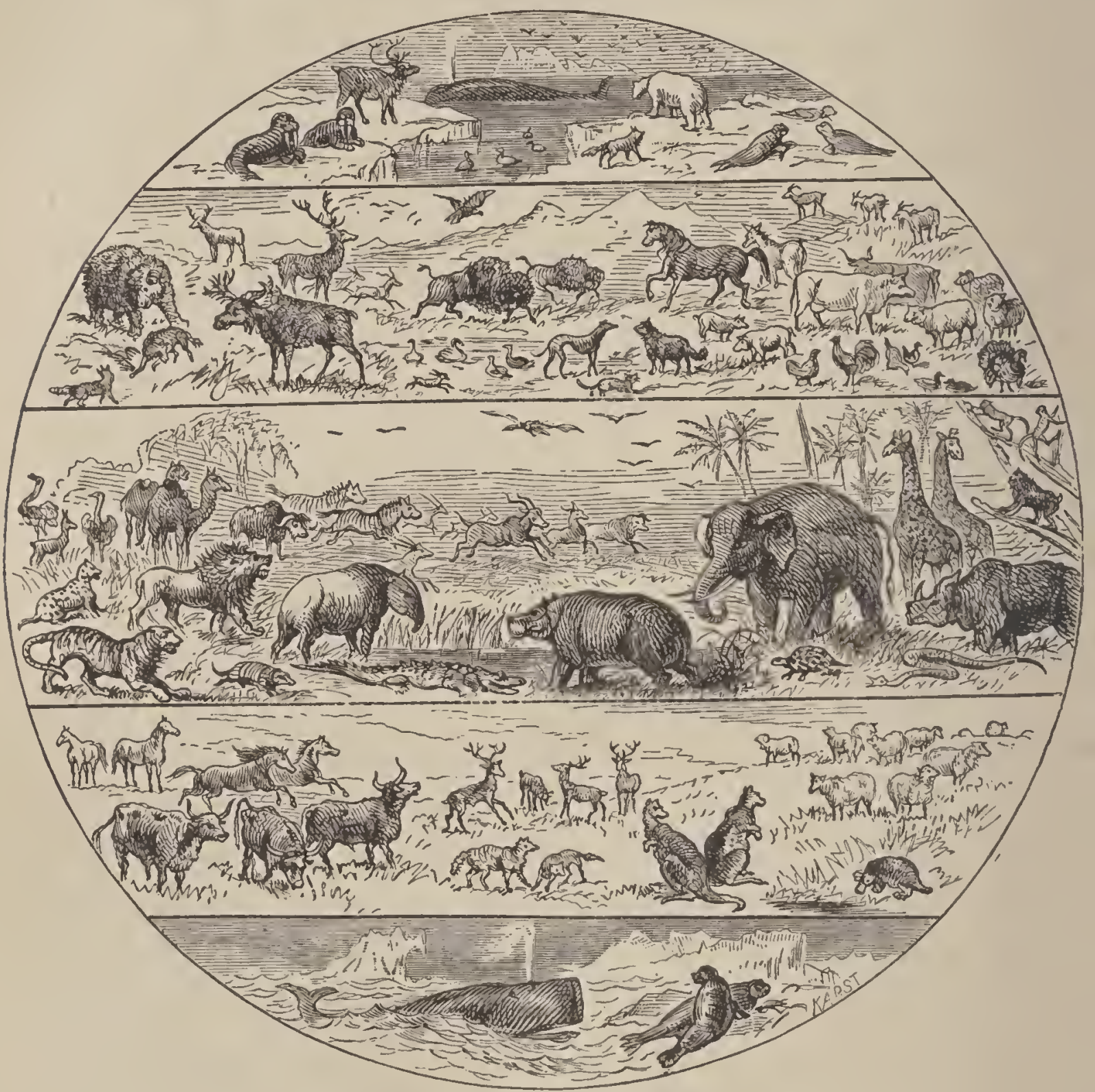

Fig. 361.-Zones of Animal Life.

States, only twenty-two out of one hundred and seventysix are venomons. Frogs, Snakes, and Lizards occur at elevations of over fifteen thousand feet. Crocodiles, and most Lizards and Turtles, are tropical.

Swimming Birds, which constitute about one fourteenth of the entire class, form one half of the whole number in 
Greenland. As we approach the tropics, the variety and number of land Birds increase. Those of the torrid zone are noted for their brilliant plumage, and the temperate forms for their more sober hues, but sweeter voices. India and South America are the richest regions. Hummers, Tanagers, Orioles, and Toucans are restricted to the New World. Parrots are found in every continent except Europe; and Woodpeckers occur everywhere, save in Australia.

The vast majority of Mammals are terrestrial; but Cetaceans and Seals belong to the sea, Otters and Beavers delight in lakes and rivers, and Moles are subterranean. As of Birds, the aquatic species abound in the polar regions. Marsupials inhabit two widely separated areas - America and Australia. In the latter continent they constitute two thirds of the fauna, while all placental Mammals, except Bats and a few Rats and Squirrels, are wanting. Excepting a few species in South Africa and South Asia, Edentates are confined to tropical Sonth America. The equine family is indigenous to South and East Africa and Southern Asia. In North America, Rodents form about one half the number of Mammals; there are but three species in Madagascar. Ruminants are sparingly represented in America. Carnivores flourish in every zone and continent. The prehensile-tailed Monkeys are strictly South American; while the anthropoid Apes belong to the west coast of Africa, and to Borneo and Sumatra. Both Monkeys and Apes are most abundant near the equator; in fact, their range is limited by the distribution of palms. 



\section{NOTES.}

I The complete and elaborate natural history of a single species or limited group is called a Monograph, as Darwin's "Monograph of the Cirripedia." A Memoir is not so formal or exhaustive, giving mainly original investigations of a special subject, as Owen's "Memoir on the Gorilla."

${ }^{2}$ Before the time of Linnæus, the Lady-bug, $e_{.} g$. , was called "the Coccinella with red coleopters having seven black spots." He called it Cuccinella septem-punctata.

3 Mandino (1315) and Berenger (1518), of Bologna, and Vesalius, of Brussels (1550), were the first anatomists. Circulation of the blood discovered by Harvey, 1616. The lacteals discovered by Aseltius, 1622, and the lym. phatics by Rudbek, 1650. Willis made the first minute anatomy of the brain and nerves, 166t. The red blood-corpuscles were discovered by Leeuwenhoek and Malpighi, 1675. Infusoria first observed by Leeuwenhoek, 1675 ; the name given by Müller, 1786. Swammerdam was the founder of Entomology, 1675. Comparative anatomy was first cultivated by Perrault, Pecquet, Duverney, and Méry, of the Academy of Paris, the latter part of the seventeenth century. Malpighi, the founder of structural anatomy, was the first to demonstrate the structure of the lungs and skin, 1690. About the same time, Ray and Willoughby first classified Fishes on structural grounds. Foraminifers were seen by Beccarius one hundred and fifty years ago; but their true structure was not demonstrated till 1835, by Dujardin. Yeyssonel published the first elaborate treatise on Corals, 1727. Haller was the first to distinguish between contractility and sensibility, 1757. White blood-corpuscles discovered by Hewson in 1775. Spallanzani was the first to demonstrate the true nature of the digestive process, 1780. Cuvier and Geoffroy, in 1797, proposed the first natural classification of animals. Before that, all Invertebrates were divided into Insects and Worms. Lamarck was the first to study Mollusks, 1800; before him, attention was confined to the shell. He separated Spiders from Insects in 1812. 'The law of correlation enunciated by Cuvier, 1826. Von Baer was the founder of Embryology, establishing the doctrine omnia ex ovo, 1827 ; but the first researches in Reproduction were made by Fabricius about 1600, and by Harvey in 1651. Wolff, in the last century, was the pioneer in observing the phenomena of Development. Sars first observed alternate generation, 1833. Duméril is considered the father of Herpetology, and Owen of Odontology. Schleiden and Schwann published their celebrated researches in cell-structure, 18t1; but Bichat, who died 1802, was the founder of Histology. Protoplasm was discovered by Dujardin in 1835, and called Sarcode. 
${ }^{4}$ This twofold division is arbitrary. No essential distinction, founded on the nature of the elements concerned, or the laws of their comoination, can be made; and so many so-called organic substances, as urea, ammonia, alcohol, tartaric and oxalic acids, alizarine, and glucose, have been prepared by inorganic methods, that the boundary-line is daily becoming fainter, and may in time vanish altogether. We would here utter our protest against the introduction of any more terms like inoryanic, invertebrate, acephalous, etc., which express no qualities.

${ }^{5}$ Even the works of nearly all animals proceed in curves.

${ }^{6}$ London Quarterly Review, January, 1869, p. 142. It is true of any great primary group of animals, as of a tree, that it is much more easy to define the summit than the base.

"De Bary on "Myxomycete ;" Darwin on "Insectivorous Plants."

8 "There are certain phenomena, even among the ligher plants, connected with the habits of climbing plants and with the functions of fertilization, which it is very difficult to explain without admitting some low form of a general harmonizing and regulating function, comparable to such an obscure manifestation of reflex nervous action as we have in Sponges and in other animals in which a distinct nervous system is absent."-Prof. Wrville Thosson's Introductory Leeture at Edinburgh.

9 "If nature had endowed us with microscopic powers of vision, and the integuments of plants had been rendered perfectly transparent to our eyes, the vegetable world would present a very different aspect from the apparent immobility and repose in which it is now manifested to our senses." - HowBoL.DT's Cosmos, i., 341.

${ }^{10}$ See Gray's "Structural Botany," Sixth ed., Introduction; also Rolleston's "Forms of Animal Life," Introduction.

11 "Life has been called the vital force, and it has been suggested that it may be found to belong to the same category as the convertible forces, heat and light. Life seems, however, to be more a property of matter in a certain state of combination than a force. It does no work, in the ordinary sense." -Prof. Wryille Thomson.

12 There was a time in our history when a single membrane discharged all the functions of life-digesting, respiring, secreting. The separation of a heart, lung, stomach, liver, etc., for special duty was an after-considera. tion.

${ }^{13}$ The vegetable cell usually consists of a cell-wall surrounding the primordial utricle or protoplasmic sac. In animal cells the former, though often present, is usually not easily seen. As a general fact, animal cells are smaller than vegetable cells.

${ }^{14}$ Cells are not the sources of life, as once thought, but are the products of protoplasm. "They are no more the producers of vital phenomena than the shells scattered in orderly lines along the sea-beach are the instruments by which the gravitation-force of the moon acts upon the ocean. Like these, the cells mark only where the vital tides liave been and how they have acted."-Prof. HoxLEY.

${ }^{15}$ Many of the bones of the skull are preceded by membrane-hence called membrane-bones. 
${ }^{26}$ In the heart, the muscular fibres are striated, yet involuntary; but the sarcolemma is wanting.

${ }^{17}$ Other names are medullary sheath and white substance of Schwann.

${ }^{18}$ We may, however, infer that the animal functions are not absolutely essential to the vegetative, from the facts that plants digest without muscles or nerres, and that nutrition takes place in the embryo long before the nerves have been developed.

19 This is not strictly true, for the Elm and Oak, the Trout and Alligator, do reach a maximum size.

${ }^{20}$ Scorpions and Spiders properly feed upon the juices of their victims after lacerating them with their jaws, but fragments of Insects have been found in their stomachs.

${ }^{21}$ The real tongue forms the floor of the mouth, and is found as a distinct part in a few Insects, as the Crickets.

${ }^{22}$ In the Marsipobranchii, it is circular or oval.

${ }^{23}$ The mouth of the Whale is exceptional, the walls not being dilatable. The act of sucking is characteristic of all young Mammals, hence the need of lips.

24 'The Ant-eater has two callous ridges in the mouth, against which the insects are crushed by the action of the tongue.

${ }^{25}$ 'The baleen plates do not represent teeth; for in the embryo of the Whale we find minute calcareous teeth in both jaws, which never cut the gum. The whalebone is a peculiar development of hair in the palate, and under the microscope it is seen to be made up of fibres which are hollow tubes.

${ }^{26}$ The "tusks" of the Elephant are prolonged incisors ; those of the Walrus, Wild Boar, and Narwhal are canines.

${ }^{27}$ "I was one day talking with Prof. Owen in the Hunterian Museum, when a gentleman approached, with a request to be informed respecting the nature of a curious fossil which had been dug up by one of his workmen. As he drew the fossil from a small bag, and was about to hand it for examination, Owen quietly remarked, "That is the third molar of the underjaw of an extinct species of rhinoceros." "-Lewes's Studies in Animal Life.

${ }_{29}$ This gap or interspace, so characteristic of the inferior Mammals, is called diastema. It is wanting in the extinct Anoplotherium, is hardly perceptible in one of the Lemurs, and is not found in Man.

${ }_{29}$ In the Spermaceti-whale, the teeth are fixed to the gum.

so The Iguma among Reptiles, and Fishes with pavement-teeth, approach the Mammals in this respect.

${ }^{31}$ This movement is called peristaltic or vermicular, and characterizes all the successive movements of the alinentary canal.

¿2 Fishes 'and Amphibians have no saliva, but a short gullet. Birds are aided by a sudden upward jerk of the head.

${ }^{33}$ Fishes and Reptiles have no pharynx proper, the nostrils and glottis opening in to the mouth.

${ }^{34}$ This movement of the pharynx and cesophagus is wholly involnntary. Liquids are swallowed in exactly the same way as solids. 
95 The few animals in which the digestive cavity is wanting are called agastric, and agree in having a very simple structure. Such are some Entozoa (as Tape-worm) and unicellular Protozoa (as Gregarina). They absorb the juices, already prepared, by the physical process of endosmose. There are other minute organisms which seem to be able to extract the necessary elements, C H O N, from the medium in which they live.

${ }^{36}$ The cavity of a Sponge is perhaps homologous with the digestive cavity, but is not functionally such. Each cell lining it does its own digestion, taking the food from the water circulating in the cavity.

37 "Nothing is more curious and entertaining than to watch the neatness and accuracy with which this process is performed. One may see the rejected bits of food passing rapidly along the lines upon which these pedicellariæ occur in greatest number, as if they were so many little roads for the conveying away of the refuse matter's; nor do the forks cease from their labor till the surface of the animal is completely clean and free from any foreign substance."-AGassiz's Sea-side Studies.

${ }^{38}$ In the larva of the Bee, the anal orifice is wanting.

${ }^{39}$ The length of the canal in Insects is not so indicative of the habits as in Mammals. Thus, it is nearly as long and more complicated in the carnivorous Beetles than in the honey-sipping Butterflies.

40 The object of this is unknown. It does not occur in the Oyster.

${ }^{41}$ In the Nautilus, this is preceded by a capacious crop.

${ }^{42}$ In the Shark, this is impossible, owing to a great number of fringes in the gullet hanging down towards the stomach.

43 At the beginning of the large intestine in the Lizards (and in many Vertebrates above them, especially the vegetarian orders), there is a blind sac, called coecum.

44 The Crocodile is said to swallow stones sometimes, like Birds, to aid the gastric mill.

45 In the crop of the common Fowl, regetable food is detained sixteen hours, or twice as long as animal food. The Dormonse, among Mammals, has an approach to a crop.

46 In Invertebrates, the gizzard, when present, is situated between the crop and the true stomach; in Birds, it comes after the stomach.

47 The Tape-worm has no digestive apparatus, but absorbs the already digested food of its host. This is no exception to the rule. The chemical jreparation of the food has preceded its absorption.

48 We find the most abundant saliva in those Mammals that feed on herbs and grain, but its action on starch is extremely feeble.

49 The acid in the gastric juice has an important function in killing or preventing the growth of bacteria which are taken in with the food. The gastric juice also dissolves the albuminous walls of fat cells, thus permitting the contained fats to escape. The drops of fat fuse together into larger masses, which are later broken up into droplets or emulsified by the pancreatic juice.

${ }^{50}$ It is probable that the digestive part of the alimentary canal in all animals manifests a similar mechanical movement. It is most remarkable in the gizzard of a fowl, which corresponds to the pyloric end of the human stomacl. This muscular organ, supplying the want of a masticatory appa- 
ratus in the head, is powerful enough to pulverize not only grain, but even pieces of glass and metal. This is done by two hard muscles moving obliquely upon each other, aided by gravel purposely swallowed by the bird. The grinding may be heard by mcans of the stetloscope.

${ }^{51}$ Chyle is opaque in carnivores; more or less transparent in all other Vertebrates, as in Birds, since the food does not contain fatty matter.

${ }^{51^{a}}$ In Fishes, the villi are few or wanting. In Man, they number about 10,000 to the square inch.

${ }^{52}$ Except, perliaps, the tendons, ligaments, epidermis, etc.

53 The phenomenon produced by these properties conjointly, capillary attraction and diffusion, is called endosmosis.

54 The blood is colorless also in the muscular part of Fishes. That of Birds is of the dcepest red. The coloring matter of the red blood in worms is not in the corpuscles, but in the plasma.

${ }^{55}$ Coagulation may be artificially arrested by common salt. Arterial blood coagulates more rapidly than venous. The disposition of the red corpuscles in chains, or rouleux, does not occur within the blood-vessels. The cause has not been discovered.

56 The corpuscles of Invertcbrates are usually colorless, even when the blood is tinged.

${ }^{57}$ Exccpt during the foetal life. The corpuscles of the Camel are nonnucleated, as in other Mammals. If the transparent fluid from a boil be examined with a microscope, it will be seen to be almost composed of colorless corpuscles, showing their use in r'epairing injuries.

${ }_{58}$ There are no valves in the veins of Fishes, Reptiles, and Whales, and few in Birds.

${ }^{59}$ Capillaries are wanting in the epidermis, nails, hair, teeth, and cartilages. Hence, the epidermis, for example, when worn out by use, is not removed by the blood, like other tissues, but is shed.

${ }^{60} \mathrm{~A}$ part of the blood, lowever, in going from the capillaries to the heart, is turned aside and made to pass through the liver and kidneys for purification. This is callcd the portal circulation, and exists in all Vertebrates, except that in Birds and Mammals it is confined to the liver.

${ }^{61}$ Two in the higher Mammals, thrce in the lower Mammals, Birds, and Reptiles. They are called vence cave.

62 Tricuspid in Mammals, triangular in Birds.

${ }^{63}$ The pulse of a Hen is 140 ; of a Cat, 110 to 120 ; of a $\mathrm{Dog}, 90$ to 100 ; and of an $0 x, 25$ to 42.

${ }^{64}$ The bivalvc Brachiopods breathe by delicate arms about the mouth, and by the "mantle."

${ }_{65}$ The air-bladder, found in most Fishes, is anotlıcr rudiment of a lung, although it is used, not for respiration, but for altering the specific gravity of the Fish. In the Gar-pike of our Northern lakes, it very closely rescmbles a lung, having a cellular structure, a tracheal tube, and a glottis. It is here functional. The gills represent lungs only in function; they are totally distinct parts of the organism.

${ }^{66}$ In the human lungs they number $600,000,000$, each about $\frac{1}{100}$ of an inch in diameter, with an aggregate area of 132 square feet. The thicliness 
of the membranc between the blood and the air is $\frac{1}{2400}$ of an inch. The lungs of Carnivores are more highly developed than those of Herbivores. In the Manatee, they are not eonfined to the thorax, but extend down nearly to the tail.

${ }^{67}$ Croeodiles are the only Reptiles whose nostrils open in the throat behind the palatc, instead of directly into the mouth-cavity. This enables tlic Croeodile to drown its vietim without drowning itself; for, by keeping its snout above water, it can breathe whilc its mouth is wide open.

${ }^{68}$ A rudimentary diaphragm is secn in the Crocodile and Ostrich.

${ }^{69}$ The poison-glands of venomous Serpents and the sill-vessels of Caterpillars are eonsidered to be modified salivary glands. Birds, Snakes, and Cartilaginous Fishes have 110 urinary bladder.

${ }^{70}$ Since the weight of a full-grown animal remains nearly uniform, it must lose as much as it receives; that is, the excretions, including the solid residuum ejected from the intestinal canal, equal the food and drink.

71 Other names for derm are, cutis, corium, enderon, and true stin; and for epidermis, cuticle, ecderon, and scarf-skin. The derm is often so intimatcly blended with the museles that its existenee as a distinct layer is not easily made out. Even in Infusoria, we find the covering double, an outside cuticula lined by a soft cortical layer; and in Jelly-fishes, naturalists distinguish an ectoderm, endoder'm, and mesoder'm.

${ }^{72}$ Papillæe are scarcely visible in the skin of Reptiles and Birds.

${ }^{73}$ The animal basis of this structure is chitin, a peeuliar horn-like substance found in the hard parts of all the articulated animals.

74 The shell is always an epidermal structure, even when apparently internal. The horny "pen" of the squid, the "bone" of the Cuttle-fish, and the calcareous spot on the batk of the Slug are only eoncealed under a fold of the mantle. So the shell of the common Unio, or fresh-water clam, is covered with a brownish or greenish membrane, which is the outer layer of the epidermis. Where the mantle covers the lips of a shcll, as in most of the large seasnails, or where its folds cover the whole exterior, as in the polished Cowry, the epidermis is wanting, or covered up by an additional layer.

75 The pearls of eommerce, found in the mantle of some Mollusks, are similar in structure to the shell; but what is the innermost layer in the shell is placed on the outside in the peurl, and is much finer and more compact. The pearl is formed around some nucleus, as an organic particle, or grain of sand.

${ }^{76}$ When the eentrum is concave on both sides, as in Fishes, it is said to be ampliccelous; when concave in front and convex behind, as in Crocodiles, it is called proccelous; when coneave behind and convex in front, as in the neck-vertebræe of the $O x$, it is opisthoccelous. In the last two cases, the vertebra unite by ball-and-socket joints.

77 Whether the skull represents any definite number of vertebræ is still under diseussion. We cannot speak of "cranial vertebræ" in the same sense as "eervical vertebrx." The most that can be said is that in a general way the sliull is homologous to part of the vertebral column.

${ }^{7}$ A few have but one pair, the Whale and Siren wanting the hind pair; while some luave none at all, as the Snakes and lowest fishes. In land animals, the posterior limbs are generally most developed: in aquatic animals, 
the anterior. Dr. Wyman contends that the limbs are tegumentary organs, and attached to the vertebral column in the same sense that the teetl are attached to the jaws. Other theories are that they originate from gill-arches (Gegenbaur) or that they are remains of a once continuous lateral fin (Thacher).

${ }^{79}$ The first trace of muscular tissue is found in the stem of Vorticella-an Infusorian. In Hydra we find neuro-muscular cells, and the Jelly-fishes have muscular tissue.

${ }^{80}$ The muscles of some Invertebrates, as Spiders, are yellow.

81 The muscles of the heart and gullet are striped. In the lower animals these distinctions of voluntary and involuntary, striated and smooth, solid and hollow, muscles can seldom be made.

82 The skeleton of the Carrion-crow, for example, weiglıs, when dry, only twenty-three grains.

${ }^{83}$ The Dragon-fly can outstrip the Swallow; nay, it can do in the air more than ally bird-it can fly backward and sidelong, to right or left, as well as forward, and alter its course on the instant without turning. It makes twenty-eight beats per second with its wings, while the Bee makes one hundred and ninety, and the House-fly three hundred and thirty. The swiftest Racehorse can double the rate of the Salmon. So that Insect, Bird, Quadruped, and Fish would be the order according to velocity of movement.

${ }^{84}$ The theory that flies adhere by atmospheric pressure is now abandoned.

85 More precisely, the term brain, or brains, applies only to the cerebrum, while the total contents of the cranium are called encephalon.

${ }^{86}$ The exact functions of the cerebrum are not yet clearly understood. If we remove it from Fishes, or even Birds, their voluntary movements are little affected, while the Amphioxus, the lowest of Fishes, has no brain at all, but its life is regulated by the spinal cord. Such mutilated animals, however, make no intelligent efforts. The substance of the cerebrum, as also the cerebellum, is insensible, and may be cut away without pain to the animal; and when both are thus removed, the animal still retains sensation, but not consciousness.

${ }^{87}$ It is very difficult to define sensation, or sensibility. The power is possessed by animals which have neither nervous system nor consciousness. These low manifestations of sensibility are called irritability-the power by which an animal is capable of definitely responding to a stimulus from without. The response is not called out by the direct action of the stimulus, but is determined mainly by the internal structure and condition of the animal.

85 Purts destitute of blood-vessels, as hair, teeth, nails, cartilage, etc., are not sensitive. The impressibility of the nerves is proportioned to the activity of circulation. According to the recent investigations of Dr. Bowditch, the channels of motor and sensitive impressions lie in the lateral, and not in the anterior and posterior columns of the spinal cord.

89 "Tentacles" and "liorns" are more or less retractile, while antennæ are not, but all are hollow. Antennæ alone are jointed.

${ }^{90}$ In Man, the soft palate and tonsils also have the power of tasting.

${ }^{91}$ No organ of hearing has been discovered with certainty in the Padiates and Spiders. The "ear" of many lower animals is probably an organ for perceiving the animal's position rather than sound-an "equilibrium orgall." 
${ }_{92}$ It is wanting in the aquatic Mammals. Crocodiles have the first representative of an outside ear in the form of two folds of skin.

${ }_{93}$ This, like the definition of smell and hearing, is loose language. There is no such thing as sound till the vibrations strike the tympanum, nor even then, for it is the work of the brain, not of the auditory nerve. Sound is the sensation produced by the wave-movement of the air. If thus defined in terms of sensation, light is nothing; without eyes the world would be wrapped in darkness. Some Protozoa have a pigment spot as an eye.

${ }_{94}$ In Invertebrates and aquatic Vertebrates, the crystalline lens is globular; or, in other words, it is round in short-sighted animals, and flattish in the long-sighted. The lens of the Invertebrate is not exactly the same as the lens of the Vertebrate eye, though it performs the same function; it is really a part of the cornea.

${ }_{95}$ The Ant has fifty in each eye, the House-fly four thousand, the Dragonfly twenty-eight thousand.

96 The pigment, therefore, while apparently in front of the retina, is really behind it, as in Vertebrates. The layer beneath the cornea, serving as an "iris," is wanting in nocturnal Insects, since they need every lay of light. The optic nerve alone is insensible to the strongest light.

${ }^{97}$ It should be noticed that this corresponds with another peculiar fact already mentioned, that either hemisphere of the brain controls the muscles on the opposite side of the body. In Invertebrates, the motor apparatus is governed on its own side.

${ }^{98}$ Sharks have eyelids, while Suakes have none. The third eyelid (called nictıtating membrane) is rudimentary in many Marnmals.

${ }^{99}$ An infant would doubtless learn to walk if brouglit up by a wild beast, since it was made to walk. Just as an Infusorium moves its cilia, not because it has any object, but because it can move them. New-born puppies, deprived of brains, have suckled; and decapitated Centipedes run rapidly. Such physical instincts exist without mind, and may be termed "blind impulses."

100 We say "apparently," because it may be a fixed habit, first learned by experience, transmitted from generation to generation. A duckling may go to the water, and a hound may follow game in some sense, as Sir John Herschel takes to astronomy, inheriting a taste from his father. Breeders take advantage of this power of inheritance.

${ }_{101}$ We may divide the apparently voluntary actions of animals into tliree classes. First, organic, in which consciousness plays no part, and which are due wholly to the animal machine. Second, instinctive, in which conscious. ness may be present, but which are not controlled by intelligence. Third, assuciative, in which the animals act under conscious combination of distinct, single ideas, or past inpressions. To these we may add rational acts, in which the mental process takes place under the laws of thought.

102 "Thus, while the human organism may be likened to a keyed instrument, from which any music it is capable of producing can be called forth at the will of the performer, we may compare a Bee, or any other Insect, to a barrel-organ, which plays with the greatest exactness a certain number of tunes that are set upon it, but can do nothing else."-Carpenter's Mental Physiology, p. 61. This constancy may be largely due to the uniformity of conditions under which Insects live. 
${ }^{103} \mathrm{~W}$ e may say, as a rule, that the proportion of instinct and intelligence in an animal corresponds to the relative development of the spinal cord and cerebrum. As a rulc, also, the addition of the power to reason comes in with the addition of a cerebrum, and is proportioned to its development. Bctween the lowest Vertebrate and Man, therefore, we observe successive types of intelligence. Intelligence, howerer, is not according to the size of the brain (else Whales and Elcphants would be wisest), but rather to the amount of gray matter in it. A loncy-comb and an Oriole's nest are constructed with more care and art thin the liut of the savage. It is true, this is no test of the capability of the animal in any other direction; but when they are fashioned to suit circumstances, there is proof of intelligence in one direction.

${ }^{104}$ An exception to the general rule that the smaller animals have more acute voices.

105 It is wanting in a few, as the Storks.

106 The Nightingale and Crow have rocal organs similarly constructed, yet one sings and the other croaks.

107 These cells are detached portions of the parental organisms. Generally, these two kinds of cells are produced by separite sexes; but in some cases, as the Snail, they originatc in the same individual. Such an animal, in which the two sexcs are combined, is called an hermaphrodite.

108 The egrgs of Mammals are of nearly uniform size; those of Birds; Insects, and most other animals are proportioned to the size and habits of the adult. Thus, the egg of the Apyornis, the gicat cxtinct bird of Madagascar, has the capacity of fifty thousand Humming-birds' egrgs.

109 As a gcueral rule, when botl sexes are of gay and conspicuous colors, the nest is such as to conceal the sitting Bird; while, whenever there is a striking contrast of colors, the male being gay and the female dull, the ncst is open. Such as form no nest are many of the Waders, Swimmers, Scratcher's, and Goatsuckers.

110 This lies at first transversely to the long axis of the egg. As the chick develops, it turus upon its side.

111 The blood appears before the true blood-vessels, in intercellular spaces.

It is at first colorless, or yellowish.

112 Exactly as the blood in the copillaries of the lungs is aërated by the external air.

113 Thus, the liollow wing-bone was at first solid, then a marrow-bone, and finally a thin-walled pneumatic bone. The solid bones of Penguins arc examples of arrested development.

114 The thigh-bone ossifies from five centres. The bone eventually unites to one piece.

115 Muscle is mainly fibrine and myosin, while nerve is neurin.

116 lior this reason, Manmmals are called viviparous; but, strictly spenking, they are as oriparous as Birds. The process of reproduction is the same, whether the egg is hatched within the parent or without. The eggs of Birds contain whatever is wanted for the developnent of the embryo, except lieat, which must come from without. Mammals, having no food-yolk, obtain their nutrition from the blood of the parent, and alter birtl from milk.

117 The larve of Buttertlies and Moths are called caterpillars; those of 
Beetles, grubs ; those of Flies, maggots; those of Mosquitoes, wigglers. The terms larva, pupa, and imago arc relative only; for, while the grub and caterpillar are quite different from the pupa, the bee-state is reached by a vcry gradual change of form, so that it is difficult to say where the pupa cnds and the imago begins. In fact, a large number of Insects reach maturity through an indefinite number of slight changes. The Humble-bee moults at least ten times before arriving at the winged state.

118 Every tissue of the larva disappears before the development of the new tissues of the imago is commenced. The organs do not change from one into the other, but the new set is developed out of formless matter. The pupa of the Moth is protected by a silken cocoon, the spinning of which was the last act of the larva; that of the Butterfly is simply enclosed in the dried skin of the larva, which is called chrysalis because of its golden spots. The pupa of the Honey-bee is called nymph; it is kept in a wax-cell lined with silk, spun by the nursing-bee, not by the larva. The time required to pass from the egg to the imago varies greatly: the Bee consumes less than twenty days, while the Cicada requires seventeen years.

119 Compare the amount of food required in proportion to the bulk of the body, and also with the amount of work done, in youth, manhood, and old age.

${ }^{120}$ Excepting, perhaps, that the new tail of a Lizard is cartilaginous.

121 The patella, or knee-pan, has no representative in the fore-limb, and, strictly, it belongs to the muscular system, rather than to the skeleton. Some anatomists contend that the great toe is homologous with the little finger, instead of the thumb.

122 "The structure of the highest plants is more complex than is that of the lowest animals; but, for all that, powcrs are possessed by Jelly-fishes of which oaks and cedars are devoid."-Mirart.

${ }^{123}$ It is, however, true that the number of cgrgs laid is proportioned to the risk in development.

${ }^{124}$ According to Mr. Darwin, the characters which naturalists consider as showing true affinity between two or more species are those which have been inlierited from a common parent; and, in so far, all truc classification is genealogical; i.e., it is not a mcre grouping of like with like, but it includes like descent, the cause of similarity. In the existing state of science, a perfect classification is impossible, for it involves a perfect knowledge of all animal structure and life's history. As it is, it is only a provisional attempt to express the real order of nature, and it comes as near to it as our laws do in explaining phenomena. It simply states what we now know about comparative anatomy and physiology. As scicuce grows, its language will become more precise and its classification more natural.

125 The tcrm type is also used to signify that form which presents all the charactcrs of the group most completely. Each genus has its typical species, each order its typical genus, etc. The word is also applied to the specimen on which a new species is founded. A persistent type is one which has continucd with very little change through a great range of time. The family of Oysters has cxisted through many geological ages.

126 The Colenterata-and Echinodermata together makc up the Radiata, the old sublingrlom of Cuvier. Echinoderma is probably more correct than Echinodermata, but we retiu the old orthography. 
${ }^{12 \pi}$ Strictly speaking, no individual is independent. Such is the dirision of labor in a live, that a single Bce, removed from the community, will soon die, for its life is bound up with the whole. An individual repeats the type of its kingdom, subkingdom, class, order, family, genus, and species, through its whole line of descent.

12S These definitions of the various groups are mainly taken from Agassiz. They are not practically very useful, as they are not used by working naturilists. The kind and degree of difference entitling a group to a particular rank varics greatly with the naturalist, and the part of the Animal Kingdom where the group is found. Some families of Insects are separatcd by gaps less than those which divide gencra of Mammals.

129 The Millepore coral, so abundant in the West Indian Sea, is the work of Hydroids. The surface is nearly smooth, with minute punctures. Gegenbaur, Hacckel, and others hold that the Acalephs have no body-cavity at all, the internal system of canals being homologous with the intestinal cavity of other animals.

130 This digestive cavity is really homologous to the proboscis of the Jellyfish, turncd in. It is lined with ectoderm. The "body-cavity" is not really such, but homologous to the digestive sac of the Hydra.

131 Among the exceptions are Tubipora, which have eight tentacles and no septa, and the extinct Cyathophylla, whosc septa arc eight or more.

132 The longer septa (called primary) are the older; the shorter, secondary ones, are developed afterwards. As a rule, sclerodcrmic corals are calcareous, and a section is star-like; the sclcrobasic are horny and solid. The latter are higlier in rank.

133 Some Star-fishes (Solaster) have twelve rays. In all Echinoderms, probably, sea-water is freely admitted into the body-cavity around the viscera.

134 The shell is not strictly external, like the crust of a Lobster, but is coated with the soft substance of the animal.

135 Six hundred pieces have been counted in the shell alone, and twelve hundred spines. The feet number about eighteen hundred. They can be protruded beyond the longest spines.

136 The classification of this edition may be compared with that of the former by the following table, in which the order of the groups is altered to show the relation more ensily:

\section{Former Edition.}

Subkingdom.

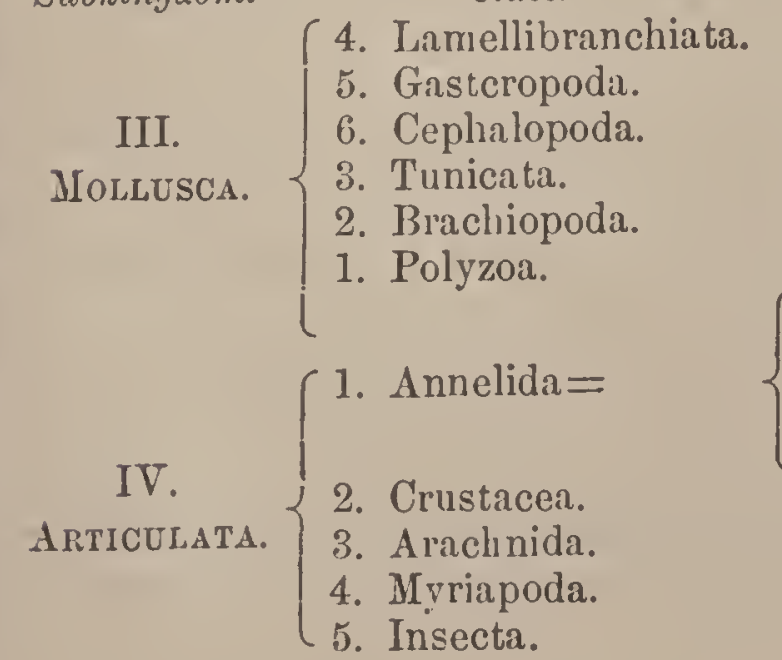

\begin{tabular}{|c|c|c|}
\hline \multicolumn{3}{|c|}{ Present Edition. } \\
\hline Class. & & Subkingdomn. \\
\hline Do. & 1. & VI. \\
\hline Do. & 2. & Molidesca. \\
\hline Do. & 3. & $\begin{array}{c}\text { VIII. } \\
\text { TuNicata. }\end{array}$ \\
\hline Do. & 4. & \\
\hline Do. & 5. & \\
\hline 1. Platyhclmir & les. & VERIES. \\
\hline 3. Rotifera. & ies. & VEKMED. \\
\hline $\begin{array}{c}\text { 6. Annelides. } \\
\text { Do. }\end{array}$ & 1. & \\
\hline Do. & 2. & VII. \\
\hline Do. & 3. & ARTHROPOD A \\
\hline Do. & 4. & \\
\hline
\end{tabular}


The two subkingdoms of the earlier edition are thus divided into four. The Classes remain tlie same, except the Annelida.

137 The most important genera are Terebralula, Rhynchonella, Discina, Lingula, Orthis, Spirifer, and Productus. The first four have representatives in existing seas. Most naturalists now admit their affinity to the worms, although some still keep them in the subkingdom Mollusea.

138 There are some exceptions: the Cyster is unequivalved, and the Pecten. equilateral.

${ }^{139}$ The clief impressions left on the shell are those made by the musclesthe dark spots called "eyes" by oystel-men; tlie pallial line made by the margin of the mantle; and tle bend in the pallial line, called pallial sinus, which exists in those shells having retractile siphons, as the Clam.

140 The Clam is tlie highest of Lamellibranchs, and the Oyster one of the lowest. The Mya arenaria, or "Soft Clam," has its shell always open a little; while Venus mercenaria, or "Hard Clam," keeps its shell closed.

141 The Slug lias no shcll to speak of, and the Chiton is covered with eight pieces. It may be remembered, as a rule, that all univalve shells in and around the United States are Gasteropods, and that all bivalves in our rivers and lakes, and along our sea-coasts (save a few Brachiopods), are Lamellibranchs.

142 Hold the shell with the apex up and the mouth towards the observer. If the mouth is on his right, the shell is riglit-handed or dextral, if on his left, sinistral. In other words, a light-handed slıcll is like a right-handed screw.

${ }^{143}$ Instead of a strong breathing-tube with a valve, answering for a forcepump and propeller, as in tlie Cuttle-fish, it has only an open gutter made by a fold in the mantle, like the siphons of the Gasteropods. The back chambers are filled with nitrogen gas.

The common Poulpe has two thousand suckers, each a wonderful little airpump, under the coutrol of the animal's will.

144 The order of the classes is one of relation rather than of rank. They cannot be arranged serially. The Myriapods liave a worm-like multiplication of parts, degrading them, and their nervous system is simpler than that of Caterpillars; yet their heads show a close relationship to Insects. The Araclsnids include some lower forms than Myriapods; on the other hand, for their wonderful instincts, $\mathrm{O}$ wen places them above the Insects. They are closely allied to Crustacenns, and stand more nearly between Crustaceans and Insects than between Mrriapods and Insects.

${ }^{145}$ Certain Crabs live on dry land, but they manage to keep their gills wet. 146 The student should remember that this threefold division is not equivalent to the like division of a vertebrate body.

${ }^{147}$ Eiach ring (called somite) is divisible into two arcs, a dorsal and ventral, and each are consists of four pieces.

147a The eyc-stalks were formerly considered to be appendages, but are no longer so regarded.

148 The four pairs of legs in Arachnids answer to the third pair of maxillæ and the threc pairs of maxillipedes in the Lobster. The grcat claws of Scorpions are the first maxilla of the Lobster, as are the pedipalpi of Spiders. 
149 The antennix are more probably altogether undeveloped, and the jaws of the Spider correspond to the mandibles of the Lobster.

${ }^{150}$ Compare the single tlırcad of the Silk-worm and other caterpillars.

151 The common Spider, Epeira, which constructs with almost geometrical precision its net of spirals and radiating threads, will finish one in forty minutcs, and just as regularly if confined in a perfectly dark place.

152 These parts do not correspond to the parts so llamed in human anatomy. See also p. 162.

153 The pupa-case is often ornamented with golden spots in Butterflies; hence the common name chrysalis.

154 In aquatic animals the posterior limbs are the ones aborted or reduced, if any; in land animals the fore-limbs are usually sacrificed.

155 The smallest corpuscles are fornd in Ruminants; the largest in Amphibians with permanent gills. The average sizc in Birds is donble that in Man, and about equal to that in the Elephant. Those of Monkeys are a trifle smaller than the human. In the embryo they are larger than in the adult. Camcls only among Mammals have oval disks.

156 The facial angle becomes of lcss and less importance as we go away from man, and for two reasons. Whcre the brains do not fill the brain-case the angle is obvionsly of little value, and if the jaws are largely developed the angle is reduced, although intelligence may not be altered.

157 Oblong human skulls, whose diameter from the frontal to the occipital greatly exceeds the transverse diameter, are called dolichocephalic; and such are usually prognathous, $i$. e., liave projecting jaws, as the negro's. Round skinls, whose extreme length docs not exceed the extreme brcadth by a greater proportion than 100 to 80 , are brachycephalic; and such are generally orthognathous, or straight-jawed.

157a It is probable that Balanoglossus and Cephalodiscus, which liave formerly been classed with Vermes, must henceforth be placed among the lowest Vertebrates, as certain structural features relating to their nervous system, notochord, and gill-slits, seem to warrant such classification. Some authorities place them in the division Hemichordata, immediately before the Urochordata.

1 1: The classes are variously grouped into the Hcomatocrya, or Coldblooded, and the Hcematotherma, or Warm-blooded; into the Branchiata and Abranchiata; into the Allantoidea and Anallantoidea.

${ }^{159}$ It would be safe to say that any living Vertebrate witlı side fins supported by fin-rays is a Fish; but the extinct Reptile Ichthyosaurus also had them.

160 "The capacity for growing as long as life lasts, which some Fislies are said to possess, may be explaincd by the facts that their bodies are, firstly, of very nearly the same specific gravity as the water in which they live, and, sccondly, of a temperature which is but a very little higher than that which they are there exposed to. Thus the force which in other animals is expended in the way of opposition to that of gravity and in the way of producing heat is available for sustaining continuous growtlı."-RoLLEsTon.

161 Amplibians with a moist skin arc also remarkable for their cutaneons respiration. They will live many days after the lungs are removed. Their 
vertebræ vary in form: in the lowest they are biconcave, like those of Fishes; in Salamanders they are opisthocolous: in the Frogs and Toads they are usually procolous.

162 Salamanders are often taken for Lizards, but differ in having gills in early life and a naked skin. The Proteus and Siren resemble a tadpole arrested in its development.

163 The Surinam Toad has no tongue.

164 The posterior pair of limbs is sometimes represented by a pair of small bones; and the Boas and Pythons show traces of external hind-limbs.

165 There are some notable exceptions. The Slow-worm is legless, and the Chamcleon has a soft skin, with minute scales.

166 According to Owell ; but Huxley insists that the plastron belongs to the exoskeleton.

167 Knees always bend forward, and heels always bend backward.

$168 \mathrm{Wc}$ cannot claim that this airy skeleton is necessary for flight. The bones of the Bat are free from air, yet it is able to keep longer on the wing than the Sparrow. The common Fowl has a hollow humerus; while some Birds of long flight, as the Snipe and Curlew, have airless bones.

169 The fossil Archoopteryx, a lizard-like Bird, is placed in a separate division, Saururce. Birds have also been divided according to their degree of development at birth into (1) Hesthogenous, as Fowls, Ostriches, Plovers, Suipes, Rails, Divers, and Ducks, whose chick is hatched completely clothed, has perfect senses, runs about, and feeds itself. When full grown, it uses its first opportunity to settle on land or water, not on trees; the male is polygamous and pugnacious; the female mạkes little or no nest; and neither sex sings. This group is of the best use to man, and approaches more nearly to Mammals, the habitual use of the legs and preference for land or watcr degrading it as a Bird and raising it in the list of animals; (2) Gymnogenous, as Gulls, Pelicans, Birds of Prey, Herons, Sparrows, Woodpeckers, and Pigeons, whose chick comes helpless, blind, and naked; it can neither walk nor feed itself, but gapes for food; the adult is monogamous, and builds elaborate nests in trees and perches; many sing; all are habitual flyers. These are birds par excellence, gifted with higher intelligence than the others, and are never domesticated for food.

170 Hopping is characteristic of and confined to the Perchers; but many of them, as the Meadow-lark, Blackbird, and Crow, walk.

171 This order is artificial. But it is better to retain it until ornithologists agree upon some natural arrangement. The classification of birds is taken from Coues's "Key to North American Birds," as being the work on ornithology in most general use.

172 The whales arc hairy during foetal life only.

173 The Manatee has 6; Hoffmann's Sloth 6; and two species of three-toed Sloth have respectively 8 and 9 .

${ }^{174}$ As in the Whale, Porpoise, Seal, and Mole. Teeth are wanting in the Whalebone Whales, Ant-eaters, Manis, and Echidna.

175 The Monotremes resemble Marsupials in liaving marsupial bones, but have no pouch. They differ from all other Mammals in having no distinct nipples. 
176 The pouch is wanting in some Opossums and the Dasyurus.

${ }^{177}$ For the best aceount of the Elephant, see Tennant's "Ceylon" or Brehm's "Thierleben."

178 The fore-feet of the Tapir have four toes, but one does not toueh the ground.

179 The extinct lorse (Hipparion) has three toes, two small hoofs dangling behind. The foot of the Horse is of wonderful structure. The bones are construeted and plieed with a view to speed, liglitness, and strengtlı, and bound togetler by ligaments of marvellous tenaeity. There are elastie pads and cartilages to prevent jarring; and all the parts are eovered by a living membrane whicl is exquisitely sensitive, and endows the foot with the sense of toueh, without whiel the aninal eould not be sure-footed. The hoof itself is a world of wonders, being made of parallel fibres, each a tube composed of thousands of minute cells, the tubular form giving strength. There are three parts, "wall," "sole," and "frog" - the triangular, elastie piee in the middle, which aets as a cushion to prevent eoncussion and also slipping.

180 The Camel and Llama are exeeptional, having two upper incisors and canines, are not strietly cloven-footed, having pads rither than hoofs, and are hornless.

381 The Hyena alone of the Carnivores has only four toes on all the limbs, and the Dog has four hind-toes. The Lion is the king of beasts in majesty, but not in strength. Five men ean easily hold down a Lion, while it requires nine to eontrol a Tiger.

182 The eye-orbits of the Lemurs are open behind. The Flying Lemur (Galeopithecus) is eonsidered an Inseetivore.

183 The old term Quadrumana is rejeeted, because it misleads, for Apes, as well as Men, liave two fect and two hands. There is as much anatomical differenee between the feet and hands of an Ape as between the feet and hands of Man. Owen, however, with Cuvier, eonsiders the Apes truly "fourhanded."

${ }^{184}$ It fails to eover in the Howling Monkey and Siamang Gibbon; but in the Squirrel Monkey it more than eovers, overlapping more than in Man. As to the eonvolutions, there is every grade, from the almost smootl brain of the Marmoset to that of the Chimpanzee or Orang, which falls but little below Man's.

185 The tailed Apes of the Old World have longer legs than arms, and generally have "cheek-pouehes," which serve as poekets for the temporary stowage of food.

186 In the lumman infant, the sole naturally turns inward; and the arms of the embryo are longer than the legs.

${ }^{187}$ The Aye-aye, the lowest of the Lemurs, is remarkable for the large proportion of the eranium to the face.

188 This feature was shared by the extinct Anoplotherium, and now to some extent by one of the Lemurs (Tarsius).

189 We have treated Man zoologically only. His place in Nature is a wider question than his position in Zoology; but it involves metaphysieal and psychologieal considerations which do not belong here. 
190 See Lewes's charming "Studies of Animal Life." 'Doubtless an examination of all the strata of the earth's crust would disclose forms immensely outnumbering all those at present known. And even had we every fossil, we would have but a fraction of the whole, for many deposits have been so altered by heat that all traces have been wiped out. Animal life is much more diversified now than it was in the old geologic ages; for several new types have come into existence, and few have dropped out.

191 Among the types characteristic of America are the Gar-pike, Snappingturtle, Hummers, Sloths, and ILusk-rat. Many of our most common animals are importations from the Old World, and therefore are not reckoned with the American fauna; such as the Horse, Ox, Dog and Sheep, Rats and Mice, Honer-bee, House-fly, Weevil, Currant-worm, Meal-worm, Clieese-maggot, Cockroach, Croton-bug, Carpet-moth, and Fur-moth. Distribution is complicated by the voluntary migration of some animals, as well as by Man's intervention. Besides Birds, the Bison and Seals, some Puats, certain Fisles, as Salmon and Herring, and Locusts and Dragon-flies among Insects, are migratory.

192 When the cable between France and Algiers was taken up from a depth of eighteen hundred fathoms, there came with it an Oyster, Cockle-shells, Annelid tubes, Polyzoa, and Sea-fans. Ooze brought up from the Atlantic plateau (two thousand fathoms) consisted of ninety-seven per cent. of Foraminifers. 


\section{THE NATURALIST'S LIBRARY.}

THE following works of reference, accessible to the American student, are recommended :

General Works and Text-books.

Agassiz, Methods of Study in Natural History.

Agassiz and Gour.t, Principles of Zoology.

Rolleston, Forms of Animal Life.

Lkwrs, Studies of Animal Life.

Huxcky and Marírin, Elementary Practical Biulogy.

Owns, Comparative Auatomy of Invertebrates and Vertebrates.

Wood, Illustrated Natmal History.

Niciorson, Manual of Zoslogy.

TrNnkr, Elements of Zoolory.

Morse, First Book of Zoology.

Packalis), Zoology.

Gegenja Ur, Comparative Auatomy.

PArkm, Zootomy.

Pakkrr, Elementary Biolngy.

Kingsiry, 'Ilie Riverside Natural History.

'THomson, Outlines of Zuology.

Cha vis and Skingwiok, Text-book of Zoulogy.

Thonson, The Sindy of Animal Life.

Lanזrster, Zoolugical Articles.

Ma ksilate and Hurst, Junior Course in Prictical Zoology.

Lang, Comparative Anatomy.

\section{Invertebrates.}

Huxury, Auatomy of Invertebrated Ani. mals.

Macalrister, Introduction to Animal Mrorphology.

Brooks, Handlook of Invertebrate Zoology.

Sirboln, Anatomy of Invertebrates.

Sinpiry, Zoology of the Invertebrata.

\section{Vertebrates.}

Hoxr.fy, Anatomy of Vertebrated Animals.

Ma Ca I.ISTI:R, Morphology of Vertebrates.

Huxlry and Ilawkiss, Atlas of Comparative Osteology.

Frowrr, Osteolugy of Mammalia.

Cuadrrau, Comparative Auatomy of Domesticated Animals.

Mivart, Lessons in Elementary Anatomy.

Wirneisurin, Comparative Auitomy of Vertebrates.

Mivart, The Cat.

Gray, Anatomy, Descriptive and Surgical.

STriokrr, Handbook of Human and Comparative Histology.

Qua In, Human Anatomy.

\section{Embryology.}

Batrour, Comparative Embryology.

Fustrir and Balfour, Elements of Embryology.

Packard, Life Histories of Aninals.

Minot, Human Embryology.

Malsuali, Vertebrate Embryology.

Heitwia, 'l'ext-book of Embryology ; Man and Mammals.

\section{Physiology.}

Carpignter, Comparative Physiology.

Hoxiny, Lessons in Elementary Pliysiology.

Fosti:r, Text-book of Physiology.

Martin, The Humin Body.

Fr.int, Physiology.

Griffiris, Physiology of the Invertebiates.

Laviois and Sruit.ing, Human Physiol. ogy. 
Geographical Distribution.

WALt.aOe, Geographical Distriluution of Allimals.

Mulray, Geographical Distribution of Mammals.

\section{Microscopy.}

Carpentri, The Microscope and its Revelations.

Griffitus and Henfrey, The Micrographic Dictiouary.

\section{Darwinism.}

Schмid, Descent and Darwinism.

HaEOK IL, History of Creation.

Darwin, Origin of Species.

Huxley, Lay Sermons, etc.

Mivart, Lessons fiom Nature.

Romanus, Darwin aud After Darwin: I. The Darwinian Theory.

Romanes, The Scientific Evidences of Orgauic Evolution.

\section{Special Works.}

Crark, Mind in Nature.

Agassiz, Sea-side Studies in Natural Histoly.

TAY Lok, Half-hours at the Seaside.

Ii wN M Manual of the Infusoria.

Gremen, Manuals of Sponges and Cœlenterata.
Dana, Corals and Coral Islands.

Darwin, Vegretable Mould and Earthworms.

VRritr. and Switu, Invertebrates of Vineyard Sound.

Godr1) and Binnty, Invertebrata of Massachusetts.

Woonwant, Manual of Mollusca.

HYAT'T, Insecta.

Packa l:D, Guide to the Study of Insects.

Duncas, 'I'rallsformation of Iusects.

Storel, Fishes and Reptiles of Massachusetts.

Couks, Key to North American Birds.

Joruan, Popular Key to the Birds, etc., of Northern United States.

BaIRd, Brewer, and Ridgway, Birds of North America.

Bairn, Mammuls of North America.

AI.LIN, Mammalia of Massachusetts.

FLower and LydukKer, Mammals, Living and Extinct.

Soammon, Marine Mammals of North Pacific.

Hartmann, Anthropoid Apes.

Prsouel, The Races of Mau.

Marsir, Man aud Nature.

Truor, Primitive Culture.

Nicholson, Palæeontology.

Poulton, The Colors of Animals.

Of serial publications, the student should have access to the American Naturalist, American Journal of Science, Popular Science Monthly, Smithsonian Contributions and Miscellaneous Collections, Bulletins and Proceedings of the various societies, Annals and Magazine of Natural History, and Nature.

The following German works are recommended as having no English equivilents:

Clads, Grundzüge der Zoologie. Payensteohrr, Allgemeine Zoologie. Brovs, Classen und Orduungeu des Thier-

Also the periodicals-

$$
\text { Zoologischer Anzeiger. }
$$

reichs (unfinished and expensive, bnt indispensable to the working zoologist).

\section{Biologisches Centralblatt.}




\section{APPENDIX.}

The following directions for experiments and dissections are given for the purpose of enabling the teacher and pupil to make direct observation of the structure and functions of certain animals which may be considered to represent in a general way the groups to which they belong. The tendency of modern teaching of Zoology is to have the student learn as much as possible by personal investigation. In a general course of Zoology, for which this book is designed, it is not practicable to introduce very much study of the specimens themselves. However, enough such observational work should be performed to give the pupil knowledge of the general structure of the more important groups of animals, as well as of the functions of their bodily organs.

The experiments and dissections are purposely chosen with a view to their simplicity, and to the ease with which they may be performed. Constant reference is made to figures which will both guide and illustrate the dissections. More extended studies may be carried out with the aid of the various works mentioned on pages 397,398 .

\section{CHAPTER II.}

The difficulty of distinguishing by ocular observation alone the lower animals from the lower plants may be illustrated by making a microscopic examination of drops of sediment from the bottom of a stagnant ditch. The water will probably be teeming with unicellular organisms, both animal and vegetable, which cannot be differentiated by characters of form, size, color, motion, etc., alone. 


\section{CHAPTER IV.}

It is especially important that the student become as familiar as possible with protoplasm by a personal study of its structure and physiology. For this purpose the most favorable objects are the Protozoa, which are readily obtained and easily prepared for examination. Directions are given on page 410. Compare with these the protoplasm seen in the cells of the water-plants, as Nitella, Chara (end-cells of leaves, and in the colorless rhizoids), and Anacharis; in the stamen hairs of Tradescantia; in Spirogyra; in the cells of the bulb scales of the Onion, etc.

\section{CHAPTER V.}

In studying protoplasm, many kinds of cell will probably be seen. Those mentioned are especially large, and in them the protoplasm is likely to be in quite active motion. To illustrate cell structure use not only the lowest organisms, but also isolated cells from higher animals and plants-for example, blood cells from the frog and from the human body. Frog's blood may be obtained by killing the animal in a box in which has been placed a small wad of cotton saturated with chloroform; as soon as the frog is dead cut into its skin to make the blood flow, then on a glass slide mix a drop of the blood with a drop of a.75 per cent. solution of salt in water, put on a cover-glass and examine under a one-fourth to one-sixth inch objective (Figs. 63, 64). Human blood may be obtained by pricking the finger and mounting the drop in the same manner (Fig. 62). Study also the cells seen in a drop of saliva. Some of these, the salivary corpuscles, are small and usually spherical in shape; others, the epithelium cells, come mainly from the lining membrane of the mouth, are pulygonal in outline, have a large nucleus, and are frequently found in groups consisting of several cells. Ciliated cells are easily obtained by placing in a drop of water on a slide a small portion of the gill of a live oyster or clam, and picking it to pieces with dissecting needles (ordinary cambric needles fixed by the eye-end into wooden pen-holders). Examine under a one-fourth or one-fifth inch objective. Some 
of the pieces will probably be seen swimming about by means of their cilia (Fig. 2). With these animal cells compare such vegetable cells as pollen grains, spores of fungi, the cells composing the bodies of some of the fresh-water alga, etc.

As the satisfactory preparation of the tissues requires skill obtained only by long training in manipulation and in the use of hardening fluids, stains, etc., in many cases it will be preferable to buy prepared specimens. These may be obtained at slight expense from dealers in microscopic supplies. Such specimens, as well as sections of various organs, are very necessary, as it is only by a clear comprehension of the structure of the different tissues and of the organs which they compose that the student can understand the functions of the various parts.

\section{CHAPTER $\mathrm{X}$.}

The principal chemical changes taking place during digestion in the higher animals may be illustrated with very simple apparatus, and at the cost of but little time. It is not necessary that the student possess any knowledge of chemistry. The object of digestion-viz., the changing of substances which are incapable of absorption into substances which may be absorbed, may be made plain even to the youngest student. The chemicals needed may be obtained of any druggist.

The following experiments deal with the three principal digestive fluids-viz., saliva, gastric juice, and pancreatic juice; and with the main kinds of foods-i.e., starchy, albuminous, and fatty substances.

\section{Salivary Digestion.}

(1) The microscopical appearance of undigested starch and its reaction with iodine.

Into a test-tube about one-fourth full of water put a pinch of corn-starch and shake the tube. Notice that the starch does not dissolve. Examine a drop of the mixture under a microscope and note the starch grains floating about in the water. Add a drop or two of dilute iodine solution to the mixture in the tube and note that it turns a deep blue. Examine a drop 
of this mixture under the microscope and note that each starch grain has turned blue.

Prepare another test-tube with water and starch, and boil the mixture in the flame of an alcohol lamp or of a Bunsen burner, keeping the tube agitated all the time in order to prevent the starch from sticking to the inside of the tube. Note that the starch swells up and forms a paste, but does not actually dissolve. Cool the paste by holding the test-tube inscold water. When sufficiently cool add a drop or two of iodine and note that the starch turns blue. This change of color serves as a test for starch whether uncooked or cooked. Hence we see that undigested starch is in the form of granules which do not dissolve in water, but which turn blue when treated with iodine.

(2) The chemical test for digested starch-i.e., grape-sugar.

Into a test-tube about one-fourth full of water put a pinch of grape-sugar, shake the tube and note that the grape-sugar dissolves. Test the solution with iodine and note that the blue color does not appear.

Prepare another solution and to it add about one-fifth its volume of a strong solution of sodium hydrate, then to this mixture add a drop or so of a one-per-cent. solution of cupric sulphate. Shake the tube to mix the contents thoroughly. Note the light-blue color. Boil the contents of the tube and the color changes, varying from light yellow to orange or brick red. Hence it is seen that digested starch (grape-sugar) dissolves in water, does not turn blue with iodine, but turns yellow or reddish when boiled with a mixture of sodium hydrate and cupric sulphate.

(3) The digestion of starch by saliva.

Collect about a third of a test-tube full of saliva, the flow of which may be promoted by chewing a piece of rubber or a button. Dip a piece of red litmus paper into the saliva and note that the paper becomes faintly blue, indicating that the saliva is slightly alkaline in its chemical reaction. In another test-tube make a mixture of about equal parts of saliva and water, and to this add a few drops of cool starch paste. Hold the tube containing this mixture in the hand for five or ten minutes in order to keep it at the temperature of the body. After a few minutes pour a portion of the mixture in another tube and test with iodine, which will probably give the blue color indicating the pres- 
ence of starch. Pour a second portion into another tube, add sodium hydrate and copper sulphate, and boil. If the yellow color appears it indicates that some of the starch has already been digested by the saliva-i.e., has been changed to grapesugar, which remains dissolved in the fluid in the test-tube. If the yellow color does not appear on the first trial, make another after an interval of a few minutes.

(4) To show that digested starch is capable of absorption, while undigested starch is not.

Prepare two dialyzers. The parchment, or parchment paper, which in each dialyzer separates the contents of the inner from the contents of the outer jar may be considered to represent roughly the membrane lining the alimentary canal, through which membrane substances are absorbed into the system. Into the inner jar of one dialyzer put a solution of grape-sugar; into the inner jar of the other put some thin starch-paste. After an hour or two test the water in the outer jar of the first dialyzer for the presence of grape-sugar; that in the outer jar of the other dialyzer for starch. It will be found that grapesugar-i.e., digested starch-dialyzes, while undigested starch does not. In other words, undigested starch cannot be absorbed. The experiment may be varied by putting both grapesugar and starch-paste into the same dialyzer. Or, a mixture of starch-paste and saliva may be put into the one, while starchpaste alone is put into the other dialyzer.

\section{Gastric Digestion.}

(1) Some of the chemical reactions of undigested albuminous substances (proteids).

Into a bowl or beaker break the white of an egg, cut it to pieces with a pair of scissors, add fifteen or twenty tımes its bulk of water, mix thoroughly by stirring, but do not beat it, then strain through muslin to remove the fine flakes of coagulated matter.

(a) Fill a test-tube one-fourth full of the mixture and boil. The albumen coagulates.

(b) Prepare another tube and add a few drops of nitric acid. The albumen coagulates. Boil. The coagulated mass turns yellow. Cool the tube and add ammonia. 'The.color deepens to orange. 
(c) Prepare another tube and add a few drops of Millon's reagent. The albumen is coagulated, and, on boiling, turns reddish. If only a little proteid is present no coagulation will occur, but the mixture will redden when boiled.

(d) Make the contents of another tube strongly acid with acetic acid, then add a few drops of potassium ferrocyanide, and a white precipitate will form.

(2) Some of the chemical reactions of digested proteids (peptones).

Make a peptone solution by dissolving some of Merck's peptone in water. Repeat the experiments given for proteids. Results similar to those in $(b)$ and $(c)$ will be obtained, but the peptone does not coagulate on boiling, nor does it give the white precipitate with acetic acid and potassium ferrocyanide.

(3) To show that peptones are diffusible through membranes, while proteids are not.

Prepare the two dialyzers as for the experiments with starch and grape-sugar. Into the inner jar of one dialyzer put some of the white-of-egg mixture, and into the other some peptone solution. After a few hours test the water in the outer jar of each dialyzer. It will be found that the peptone passes througl the membrane, while the proteid does not.

(4) To show that the gastric juice digests proteids-i.e., changes them to peptones.

Prepare some artificial gastric juice as follows: Malie some .2 per cent. hydrochloric acid by mixing 5.5 cubic centimetres of hydrochloric acid (sp. gr. 1.16) with enough distilled water to make one litre. In $100 \mathrm{cc}$. of this acidulated water put 100 milligrammes of a 6000 pepsin, or $150 \mathrm{mg}$. of a 4000 , or 300 of a 2000 pepsin. Any commercial pepsin may be used. Prepare the proteid by boiling an egg, and then cutting the white into small cubes or shreds. In place of the boiled egg some of Merck's prepared fibrin may be used.

With litmus paper test the reaction of the artificial gastric juice. It will turn blue litmus paper red, thus showing that its reaction is acid.

Fill a test-tube about one-fourth full of the artificial gastric juice, and add a few pieces of coagulated white of egg or of fibrin; then set the tube in a warm place, as in a water bath 
regulated to about $37^{\circ} \mathrm{C}$, , or near a stove. Examine the tube from time to time. The cubes of egg will be seen to be disintegrating and dissolving.

A quantity of digested white of egg may be prepared in a cup or bowl and emptied into the inner jar of a dialyzer. After a time the water in the outer jar will give the peptone tests, showing that the digested albumen is diffusible.

\section{Pancreatic Digestion.}

Procure some of the commercial pancreatic preparations and make an artificial pancreatic juice according to the directions furnished with each preparation. Test the reaction with litmus paper. It will be found to be alkaline. Try the effect of the artificial preparation on starchy and on albuminous substances in the manner given above for each. The pancreatic juice will be found to change starch to grape-sugar and proteids to peptones. Try its effect also on oil by adding a few drops of olive oil to some pancreatic juice in a test-tube. At first the oil will float on the surface of the liquid. Shake the tube vigorously to mix the two substances. The oil will be broken up into fine droplets, giving the contents of the tube a milky appearance. On standing for a time it will be seen that the oil does not separate from the digestive juice and collect at the surface as it would if shaken up with water, but the two fluids remain intimately mixed, forming an emulsion. Under a microscope examine a drop of the emulsion. It will be seen to consist of innumerable fine drops of oil, which remain separate from one another. If oil be shaken up with saliva or with artificial gastric juice no emulsion will be formed, the oil soon separating.

\section{CHAPTER XII.}

Directions for obtaining and studying blood-corpuscles are given in the notes on Chapter V. Sufficient blood to show the phenomena of clotting may be obtained by chloroforming a rabbit or a fowl, cutting one of the veins in the neck, and catching the blood in small tumblers or beakers. 


\section{CHAPTER XIII.}

The beat of the heart is very conveniently studied in the frog. Put a live frog into a glass bowl with a piece of cotton batting or of cloth saturated with chloroform and cover the bowl. In a few minutes the animal will have become motionless and insensible. Remove it from the bowl; with a sharp knife divide the skin and cartilage at the base of the skull, thus making an opening into the brain cavity; into the latter thrust a wire, and by twisting it about destroy the brain. The frog will probably struggle, but its motions are reflex, and it has no consciousness of pain. The heart may now be exposed by making an incision through the skin and muscles of the upper part of the abdomen and removing the cartilaginous part of the breastbone. The heart will be seen beating inside the pericardium. The latter may be removed and the heart freely exposed. After studying the movements of the organ it may be removed from the body by cutting the blood-vessels close to their junction with the heart, and placed on a plate of glass or in a watch glass containing .75 per cent. salt solution. Its movements will continue a long time after its removal from the body. The heart may afterward be opened and the relation of its ventricle, auricles, and the connecting veins and arteries studied (Fig. 76).

The heart of the pig, sheep, or calf may be used to show the structure of the mammalian heart. It is best to procure at the meat-shop several "plucks" - i.e., heart, lungs, and trachea all attached together. Instructions should be given the butcher that the parts are to be left intact, otherwise they will be found to be punctured with knife cuts. Dissect out the blood-vessels for some little distance from the heart in order to get their relations. Open some of the hearts lengthwise, other's crosswise, to show the internal structure (Fig. 74). Pour water into the cavities to show the action of the valves. The flow of blood through the heart may be illustrated by connecting the aorta with the venæ cavæ by means of rubber or glass tubing to represent the systemic circulation, and the pulmonary artery with the pulmonary veins to represent the pulmonary circulation, then filling the heart with water or a colored fluid and compressing the organ with the hand (Fig. 76).

The circulation may be studied in the web of the frog's hind- 
foot. Procure a thin board large enough to lay the frog upon; in one end make a hole about a half-inch in diameter, over' which the web may be stretched; anæsthetize the frog witl ether or chloroform; as soon as the animal becomes insensible lay it on the board, with its body covered with a moist cloth; over the larger toes of the foot to be examined slip nooses of thread, and fasten these in slits around the edge of the board in such positions as to spread the web between two of the toes over the hole in the board. I'ut a drop of water on the web, lay on the cover-glass, place the board on the microscope, and examine with a one-fifth or a one-sixth objective. The anæsthetic must be renewed from time to time, otherwise the struggles of the anımal will interfere with observation (Fig. 66).

\section{CHAPTER XIV.}

The gross structure of the frog's lung may be studied in specimens which have been removed from the body, inflated with air blown through a small glass tube inserted through the glottis, and placed in alcohol a few loours to harden. When cut open the lung will be seen to be a hollow sac with corrugated walls (Fig. 85).

"Plucks" obtained from a butcher will illustrate the structure of the mammalian larynx, trachea, bronchial tubes, etc. If fresh and not punctured with the knife they may be inthated. To work well they should be kept moistened (Fig. 86).

The presence of carbon-dioxide in the air exhaled from the lungs may be shown by using lime-water or baryta-water, with either of which carbon-dioxide forms an insoluble precipitate, which at first floats as a delicate white film on the surface of the liquid. Pour some of the fluid irto a saucer or watch-glass, then breathe heavily upon it a few times through the mouth, and the film will be formed.

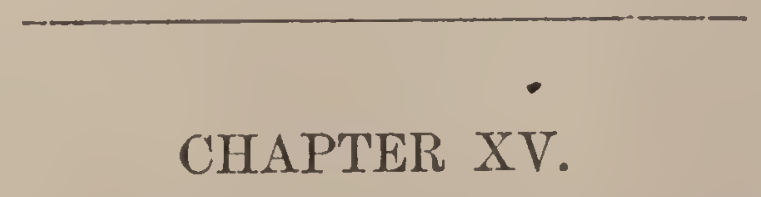

The structure of the kidneys is well illustrated by the kid- 
ney of the sheep. Several of these should be procured and opened in various directions to show the structure (Fig. 93).

\section{CHAPTER XVI.}

With little trouble skeletons of frogs, birds, and mammals with bones connected by flexible attachments may be prepared. Carefully cut away all of the muscles and other soft parts, leaving only the ligaments connecting the bones. Then place the roughly prepared specimen for one or two weeks in Wickersheimer's fluid, which is prepared as follows: In three litres of boiling water dissolve 100 grammes of alum, 60 grammes of caustic potash, 25 grammes of salt, 12 grammes of saltpetre, and 10 grammes of arsenic. Cool and filter the liquid. Then to each litre of the fluid add 400 cubic centimetres of glycerine and 100 cubic centimetres of alcohol. The ligaments of skeletons soaked in this fluid will remain flexible during many months of exposure to the air. Should the ligaments become stiffened, their flexibility may be restored by a few hours' immersion in the fluid.

\section{CHAPTER XVII.}

Muscle fibres for microscopic examination may be obtained from the leg of a frog, or even from the body of a recently killed animal at the meat-shop. Lay a small piece of muscle in a drop of .75 per cent. salt solution on a glass slide, and with a pair of dissecting-needles carefully pick the muscle to pieces. Some of the smallest shreds, upon examination with a one-fourth or a one-sixth inch objective, will be seen to be single or grouped muscle fibres, which will show the striations and the sarcolemma (Figs. 11, 12).

\section{CHAPTER XVIII.}

Nerve fibres are readily obtained from the sciatic nerve in the 
frog. This nerve may be found by removing the skin from the back of a frog's thigh and carefully separating the underlying muscles. Among them will be seen the sciatic nerve, covered in places with dark gray or black pigment spots. Remove a quarter to a half inch of the nerve, being careful to stretch it as little as possible; lay it on the glass slide in a few drops of .75 per cent. salt solution; cautiously tear it to pieces in the direction of its length with dissecting needles; then put on a coverglass and examine under a high power. The nerve will be found to consist of a number of nerve fibres, some of which will show the primitive sheath, medullary sheath, and axis cylinder (Fig. 13).

The relation between the stimulation of a nerve and the contraction of the muscle to which the nerve runs may be shown as follows: Expose the sciatic nerve as directed above; then with the quick stroke of a sharp scalpel sever the upper end of the nerve as near the body as possible. At the moment of doing this the muscles of the leg and foot will probably contract. Allow the nerve to rest for a few minutes; then pinch its upper end with a pair of forceps. Again the muscles will contract. The stimulation may be repeated at intervals if the nerve be allowed to rest for a few minutes between successive stimulations. Try also the effect of touching the nerve with a hot wire and with a drop of dilute acid or alkali.

\section{CHAPTER XX.}

The structure of the egg may be studied in the Starfish or Sea-urchin, Frog or Fowl. Starfish eggs preserved in various stages of segmentation may be purchased from the Department of Laboratory Supply of the Marine Biological Laboratory at Wood's Holl, Mass. Frogs' eggs may be found in ponds and ditches in early spring. If transferred to the laboratory and kept supplied with fresh water they may be watched through their various stages of segmentation to the formation of the tadpole, its liberation from the egg, and its later development. Compare with Fig. 174. To watch the development of a chick, eggs may be incubated by a hen or in an artificial incubator, one egg being removed each day, and opened by breaking away 
a circular piece of the shell on one side. If kept submerged in a dish of .75 per cent. salt solution, warmed to the temperature of the body, the embryo chick may be kept alive for several hour's to show the beating of the heart, etc. (Figs. 169, 170).

\section{CHAPTER XXI.}

Protozon.-As representatives of the Protozoa, Amoba, Paramecium, and Vorticella may be used. They are usually to be found in the slimy coating of water-plants-e. g., pond-lilies, etc. They occur in great abundance in aquarium-jars in which the water is becoming tainted from the decay of algr. They may be cultivated artificially by allowing a dish of marsh grass or hay, cut into fine bits and covered with water, to stand in a warm place for a few days. To prepare them for observation they may be transferred in a drop of water to the glass slide by means of a pipette and covered with the cover-slip, with its edge resting on a small scrap of tissue-paper or a piece of a hair to prevent crushing the specimens. The structure of each organism should be studied-its body mass of protoplasm, a single cell, containing the nucleus, particles of food, and contracting vacuoles; the psendopodia of Amoba (Fig. 185), and the cilia of the other forms; the cuticular corering of Paramecium (Fig. 188), and Vorticella (Fig. 160), and the muscle-like stalk of the latter. Study also their habits; motions of the protoplasm and methods of locomotion; feeding; note within the body the gradual disintegration of food particles (digestion); look for specimens in the process of division (reproduction, Fig. 160); notice the sensitiveness of their bodies to contact. If a prolonged examination of any specimen be made the animal must be kept supplied with water. As rapidly as the water under the cover-glass evaporates its loss may be made good by touching a drop to the edge of the cover-glass. Capillary attraction will draw the water between the slide and the cover, and the death of the specimen may thus be prevented. Many other forms than those mentioned are likely to be found, almost any of which will illustrate the essential features of the structure of Protozoa.

SPonges. - Because of the delicacy of their tissues, the study 
of sponges is difficult for the beginner. The arrangement of the canals and the microscopic structure of the skeleton of the Toilet Sponge should be studied (Fig. 190). Specimens for this work may be purchased at any drug-store. Then alcoholic specimens of similar sponges, in which the flesh has been preserved, may be sectioned in various directions with a sharp knife, and the difference between the skeleton and the complete body noted. Sections of Grantia, or of some other simple sponge prepared in such a way as to show the canals and ciliated chamber's, as well as the young in various stages of development, may be purchased from any dealer in microscopic supplies (Fig. 189).

Coelenterates. - If the fresh-water Hydra (Fig. 191) can be obtained it will be found useful to illustrate the structure of the Coelenterata. It lives in clear water in ponds and ditches, and attaches itself to the stems and roots of floating plants, as duck-weed, various algx, etc. Either the green or the brown form may be used. The animal may first be examined in the aquarium, and the movements of its body and tentacles noted; also its method of locomotion. Then it may be placed in a watch-glass, and studied under the low power of the microscope; small scraps of fresh meat not so large as a pin-head may be given it, and its method of feeding watched. If placed on a slide in a drop of water and covered it may be examined with a higher power and the structure noted-the body-wall consisting of cells arranged in two well-defined layers, many of the cells containing green particles; the digestive cavity extending throughout the body and into the tentacles; from some of the cells the nettling-threads may be seen to shoot out. Look for specimens bearing buds. Prepared slides showing longitudinal and cross sections of the body may be purchased.

If hydras cannot be obtained, some of the marine hydroids, either living, alcoholic, or stained specimens mounted on slides should be studied. The campanularian hydroids are suitable. These are colonial forms, and in each colony both feeding and reproductive zoöids will be found, as well as young zoöids in various stages of development from the first formation of the bud to the full-grown zoöid. Study live specimens in sea-water, noting particularly their movements, and, if possible, their method of feeding. Examine alcoholic specimens in a watch-glass containing fifty per cent. alcohol. Note the plant-like aspect 
of a colony. Microscopic examination will show the fleshy part of the colony to be protected by a transparent covering. Each nutritive zoöid will be found to have a circle of tentacles surrounding the mouth which leads to the digestive cavity, the lower end of the latter being continued into a fleshy tube which runs to the tube traversing the main stem. The cell-layers are usually well defined. The reproductive zoöids are without tentacles, and will probably contain young in various stages of development.

If Sea-anemones (Figs. 38, 199) can be obtained their structure and habits should be studied and compared with those of the hydra and the hydroids. Alcoholic specimens are most satisfactorily studied by making both transverse and longitudinal sections about a fourth of an inch in thickness. Float the sections in disles of fifty per cent. alcohol (Fig. 198).

Echinoderms. - As representatives of these the Starfish, Sea-urchin, and Sea-cucumber are useful. They may be studied in the fresh condition or preserved in alcohol. After examining the shape and the external features of the body, as spines, ambulacral grooves and areas, ambulacral feet, mouth, eyes, etc. (Fig. 212), the body may be opened, in the case of the Starfish and Sea-cucumber, by slitting with a knife or scissors, and the internal organs examined. Cut some of the rays of the Starfish crosswise; from others remove the top. The digestive system in the Starfish consists mainly of a short œsophagus leading to a set of five wrinkled pouches, at whose outer ends will be found band-like retractor and protractor muscles, the pouches forming the cardiac portion of the stomach, which is farther continued into a pentagonal sac, at whose corners enter ducts from the lobes of the "liver" or hepatic cœca, the latter being attached to the roof of the ray by a mesentery (Fig. 126). At the point of union of two adjacent rays will be found the grape-like clusters of sexual glands. On each side of the middle line - vertebral ridge - of the ray will be seen rows of water-sacs or ampulla, each of which supplies an ambulacral foot. Other sacs will be found surrounding the mouth.

The Sea-cucumber differs in several respects from the Starfish as regards internal structure. The digestive system consists mainly of a long tube, bent once or twice upon itself, at the lower end of which is attached the much branched respiratory tree. Longitudinal muscles run from near the base of the ten- 
tacles down the side of the body. Near the upper end of the intestine will be found two large Polian vesicles, which form part of the water-vascular system. The ovary is a bunch of tubes attached to the end of the oviduct.

It will be best to study the test or skeleton of the Sea-urchin before examining the internal parts. The test may be freed from the soft parts by soaking it for a few hours in a weak solution of potash, then brushing away the softer portions with a bristle brush (Figs. 96, 97). The arrangement of the ambulacral and interambulacral areas, the structure of the mouth-parts, the position of the ovarian and ocular plates, and the arrangement of the skeletal plates should be studied. Note also the tubercles on the plates and on the complete animal; note the shape, position, and arrangement of the spines and ambulacral feet (Fig. 214). For the study of the internal organization one shell may be opened longitudinally and another transversely (Fig. 28), or specimens may be soaked for a day or two in two per cent. nitric or chromic acid, which will remove the lime from the test, leaving it soft and pliable (Fig. 39).

Vermes.-The Earthworm may be taken to represent this group. Use the largest specimens obtainable. They may usually be found in the warm evenings of early summer, stretched out of the burrows, on the lawn or in the garden. Study their method of locomotion, the manner in which the burrow is made, also how the food is grasped and swallowed. If not convenient to do this out-of-doors, put several specimens in a flowerpot or box of earth and study them in the laboratory. Read the account of their habits in Darwin's "Vegetable Mould and Earth worms."

Earthworms may be killed by being put for a few minutes into lukewarm water. Then transfer to flat dishes, which are long enough to allow of extending the specimens at full length. Pour over them two to four times their bulk of fifty per cent. alcohol and leave for six or eight hours, then place in seventy-five per cent. alcohol for the same length of time. If desired, they may be still further hardened by treatment with stronger alcohol. With regard to their external anatomy, note their shape, the slight distinction between the anterior and posterior ends, the segmented structure, the grouping of the segments into regions-anterior, girdle, and posterior-the fairly constant number of segments in the first two regions, the cuticle covering the 
body, the bristles on the ventral side, the orifices-mouth, reproductive, and anal-also the dorsal pores along the middle line of the back. Note the red line which marks the course of the dorsal blood-vessel. To examine the internal anatomy, lay the worm in a dish having a layer of beeswax in the bottom, slit open the body along the middle line of the dorsal surface, and separate the muscular walls of the body from the parts lying within, fastening back the flaps by pinning them to the beeswax. Keep the specimen covered with water if the worm be freshly killed, or with fifty per cent. alcohol if it be a preserved specimen. Note the membranous partitions which subdivide the large cavity of the body: the dorsal blood-vessel, lying along the top of the digestive system, around the anterior part of which are circular blood-vessels; the digestive system, consisting of the following principal parts: pharynx, gullet, crop, stomach, intestine, and along the top of the latter the socalled "liver." Along the sides of the anterior part of the digestive system look for the osophageal glands and the reproductive glands. Slit open the alimentary canal and study its structure and contents. Look on the top of the anterior end of the pharynx for the brain. Remove the digestive system and, lying below it, look for the nerve chain of ganglia connected to the brain by nerve-threads encircling the pharynx. Make crosssections of various parts of the body of hardened specimens and examine the structure.

Mollusca. - The examination of a Snail is not easy, consequently the student would best use one of the Lamellibranchs, as the Clam or the Fresh-water Mussel. Put live clams in dishes of sea-water or mussels in fresh-water, the bottoms of the dishes being covered with a layer of sand three or four inches deep. Watch the animal crawl about and finally bury itself in the sand. Note the streams of water entering and leaving the siphons. Touch the tentacles at the margin of the siphons and note their sensitiveness. Of the anatomy, study first the shell -its shape as seen from various directions, the covering or socalled "epidermis," the position of the hinge. Separate the two valves and remove the soft part of the body, noting where and how this is attached to the shell, how the valves are held together; examine the hinge-ligament and hinge-teeth, the margin of the valves, and their thickness in various places. Note the scars left by the adductor muscles and the siphons (Fig. 
99). Examine the soft parts in a dish of water or of fifty per cent. alcohol. Note the mantle lobes, the gills, the foot, the mouth parts, etc. Cut open the body, and trace the digestive, nervous (Fig. 134), and the principal parts of the circulatory systems (Fig. 46). Harden specimens in alcohol, and make transverse sections through the body, and examine the sections again in dishes of fifty per cent. alcohol, tracing in this way the course taken by the digestive system (Fig. 78).

Arthropoda.- $(A)$ Crustacea.-Use the Lobster or Crayfish. Study living specimens in jars of water. Examine the manner of walking and swimming; of grasping food and chewing it; of defending themselves; the motions of the antennary organs, the eyes, and the appendages of the abdomen. Note the segmented structure, the segments being grouped into well-defined regions forming the cephalo-thorax and the abdomen. Note the presence of a pair of appendages on each of the abdominal segments; the similarity of structure of all these appendages except the last, and the extreme specialization of this one. On the cephalothorax look for segments; note here also the arrangement of the appendages; remove them in order from one side, and trace the modification of the same fundamental plan of structure. Open one of the large claws and study the contained muscles. Note especially the arrangement and structure of the mouth parts, eyes, and antennary organs (Fig. 250). With a pair of strong shears cut through the "shell" along each side and remove the roof of the abdomen, thus exposing the muscles witlin, and the posterior part of the circulatory and digestive system. Note the arrangement of the muscles and blood-vessels. In the same manner remove the top of the cephalo-thorax, examining the chamber in which the gills lie and their arrangement. The heart and stomach will now be exposed, also the "brain" (Fig. 70). Examine all these. Remove the digestive system, and, lying below it, find the ventral nerve chain. Look also for nerves running to the eyes.

(B) Insecta.-The large Locust or Grasshopper will be useful to study the general characteristics of insects. It is difficult to keep living specimens confined for any great length of time, consequently the best observations of their habits must be made out-of-doors. Recently caught or alcoholic specimens may be used for studying the anatomy. Notice here, as in the Lobster and the Earthworm, that the body is segmented; but the seg- 
ments are more definitely grouped into regions - viz., head, thorax, and abdomen-than in the other two animals (Fig. 98). Study the structure of each region, together with its appendages, noting that the organs of locomotion are confined to the thorax, those of special sense mainly to the head (Fig. 262). Examine the outer wings, noting their structure, their position on the body when at rest, their point of attachment to the body, and compare with the second pair. Study also the legs-their position, structure, direction in which the joints bend (Fig. 131). Examine the foot closely, noting the pads and claws (Fig. 127). Look for spiracles along the side of the abdomen (Fig. 79), and in the females note the ovipositor at the end of the abdomen. Examine the head and its appendages, and compare with Figs. 22, 24. Study the mouth parts, and compare with Fig. 21. Examine the antennæ (Fig. 147) and eyes (Figs. 155, 156). With sharp scissor's or scalpel cut open the body, and examine under water the arrangement of the internal organs, comparing with Figs. 41, 42. Harden specimens in alcohol; then accurately cut them in halves along the middle line of the body (Fig. 43). If recently killed specimens be opened under water, the larger air-sacs and tubes may be distinguished by their glistening appearance.

Vertebrata. - A very good idea of the general structure of vertebrates may be obtained by the examination of a fish, a bird, and a mammal. As in the case of other animals, as much as possible should be learned from the living organism with regard to its habits, etc. If minnows are not obtainable, let the student have "goldfish," which may be purchased at any bird-store, and which, with little trouble, may be kept in a small aquarium. Likewise, canaries and sparrows may be watched to learn some of the more obvious habits of birds. As for the mammal, a cat, dog, or rabbit may serve.

Almost any scaly fish of moderate size, as a perch, may be used for dissection. The specimen may be laid upon a thick paper, a board, or a platter. Before opening the body, note the external characters: the shape of the body; its parts - viz., head, trunk, and tail, and their connection to each other; the color of the body and its covering, consisting of scales coated with a slime-like epidermis; the arrangement of the scales (Fig. 102); the number and position of the fins (Fig. 123), their structure, and method of folding; the shape of the head; the 
position and structure of the mouth, eyes, and nostrils; the number, position, and structure of the gills; their covering, the operculum. Open the mouth, and examine the tongue and teeth. With a sharp scalpel remove the skin from one side, and study the arrangement of the plates of muscle lying underneath the skin, noting their segmental arrangement. Lay open the body-cavity by a cut extending forward from just in front of the anus. Remove one-half of the body-wall, thus exposing the internal organs. Study their position and arrangenent (Fig. 48). Cut open the digestive organs, and study their structure. Examine the heart, and note its structure and its relation to the main blood-vessels (Figs. 71, 75). With strong scissors cut away the top of the skull and expose the brain (Fig. 139). The skeleton may be roughly exposed by picking away the flesh (Fig. 112).

A pigeon or a fowl may be used to illustrate the anatomy of the bird. Examine the general shape of the body and its division into head, neck, trunk, and limbs. Note the feathers, studying particularly their variation in shape, size, color, and structure (Fig. 105), also the covering of the beak and feet. Pluck off all the feathers and note the areas over which they were distributed, and the difference in the shape of the body before and after the removal of the feathers. Study again the head, neck, trunk, and limbs. On the head note the shape of the mouth, and the position and shape of the nostrils, eyes, and ears. Make an incision through the skin, extending from the vent to the throat, and turn back the flaps thus formed. This will expose the gullet, trachea, jugular veins, and the muscles of the breast and abdomen. The crop may be inflated by means of a blow-pipe thrust down the gullet. Dissect away the outer breast muscle, and note a smaller breast muscle beneath it. Open the abdomen, and examine the arrangement and structure of the digestive organs (Fig. 50). Slit open the gullet, crop, gizzard, and intestine, wash out their contents, lay them in a dish of water, and study their structure. Inflate the lungs through the trachea, and note their elasticity. The blood-vessels (Fig. 76) and nerves are so large that they may easily be traced. The shape and attachments of the principal muscles of the wings and legs should also be studied. The bones are so firmly connected that a serviceable skeleton may be ronghly prepared by dissecting away the muscles, and other soft parts, leaving only the ligaments (Fig. 116). 
The study of the brain is best made on properly hardened specimens. These may be prepared as follows: Remove the head from the body, and cut away enough of the roof of the skull freely to expose the brain; then put the skull with the contained brain in a bowl and cover with a saturated, watery solution of chloride of zinc. Leave the brain (fowl or rabbit) in this solution from five to seven days; then replace the zinc solution with fifty per cent. alcohol for twenty-four hours, then with sixty, seventy, and eighty per cent. alcohol each for the same length of time. The brain will then be sufficiently hardened to bear careful handling without injury, and may be removed from the skull (Fig. 141). The brain may be cut into longitudinal and transverse sections about an eighth to a quarter of an inch thick, to show the internal structure.

As the representative of the mammals, a rabbit or a cat may be used. The order of study is quite the same as that given for the bird, viz. : Examine first the general external features, as shape, integument, limbs, head, etc.; then remove the skin and study the underlying muscles; after which open the body and examine the digestive, respiratory, and circulatory systems, and the more superficial parts of the nerrous system. Open the skull, and study the brain and its coverings. Use should, as before, be made of the appropriate figures, of which there are many, illustrating the structure of mammals. 


\section{N D E X.}

In the Index the numbers in Roman type (21) refer to pages; those in bold-faced type (40) refer to cuts. No attempt is made to analyze the statements uade for each group in Part II. Reference is made for each class or prominent order to those cuts in Part I. which illustrate the group.

Amomases, 89, 56.

Absorption, Invertehrates, 94 .

$$
\text { “Vertebrates, 94, 60, } 61 .
$$

Acalephæ, 247, 178.

$$
\text { " see Jelly-fish. }
$$

Acarina, 288, 258.

Acurus, $28 s$.

Acetabulnm, $14 \pi$.

Acipenser, 315, 290.

Acorn-shell, 284, 254.

$$
\text { " see Bilrnacle. }
$$

Acrania, 305, 310, 282.

Acrydium, 297.

Actinaria, 251, 198-207.

Actinoid Polyp, 251, 199.

“ anatorny of, 38, 95, 198.

" blood of, 97.

“ development of, 205, $20 \mathrm{~s}$.

" liver-celis of, 123.

" month of, 55, 38, 198.

" nettle-cells of, 51 .

“ prehension of, 51 .

" reproduction of, 192.

" respiration of, 112.

“ skeleton of, $130,95$.

، skin of, 127.

Adder, 320, 298.

Adipose Tissue, 35, 10.

Eulis, 274.

Epyoruis, 327.

Air-bladder, 117.

Air-sac, 117.

Albatross, 330 .

Albumen, 19.

Alcyonaria, 256, 200, 207, 208.

Alcyonium, 256, 208.

Alimentary Canal, 74.

$\begin{array}{lll}\text { " } & \text { " } & \text { Colenterata, } 76 . \\ \text { " } & \text { " } & \text { Crustacen, 77. } \\ & & \end{array}$

Alimentary Canal, duodenum, 90.

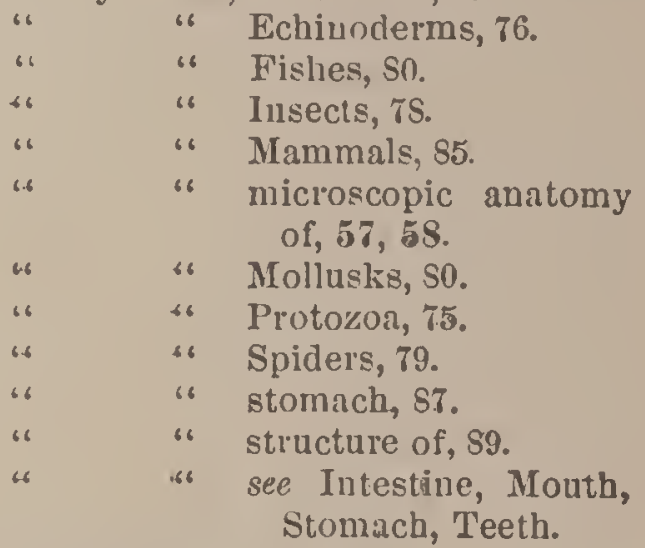

Allantoidea, 393.

Allantois, 117, 203, 169-171.

Alligator, 67, 324, 181, 303.

$$
\text { "“ nest, } 196 .
$$

Alternate generation, 211.

Ambulacra, 131, 262.

Ammonite, 279.

Amuion, 202, 170, 171.

Amœba, 50, 168, 240, 187.

" conjugation of, 196.

" ectosarc of, 75 .

" feeding of, 55 .

"locomotion of, 154, 157.

Amphibia, 317, 63-65, 76, 85, 87, 294 297.

“ blood of, 99, 63-65.

" brain of, $170,140$.

" circulation of, 108,76 .

" lungs of, 118.

" month of, 61.

"see Frog.

Amphicelous, 356 .

A mphioxus, 97, 138, 233, 310, 282.

" feeding of, 50 .

“ skeleton of, 139 .

Amphithö̈, 2S4, 252. 
INDEX.

Auallantoiclen, 393.

Analogy, 21S.

Anฉs, 311.

Auntomy, 12.

Auchylosis, 144.

Animal, defined, 22.

Animalcule, see Protozoa.

Annelides, 26S, 17, 223.

Anodon, 78 ; see Clam.

Aliourn, 31s.

Ant, 304.

Ant-eater, 344, 333.

Antennæ, 177, 147.

Anthozoa, 250, 35, 95, 195-20S.

Aorta, 104.

A pe, 6S, 356, 120, 303-357.

Aphis, 297.

A pis, 304, 42, $27 \%$.

A plysia, 274, 134.

A pteryx, 327.

Arachnid , $2 S S$.

" see Centipede, Scorpion, Spidel:

Araueina, 2S9, 18, 25, 260, 261.

" see Spider.

Ardea, 332, 313.

A revicola, 113, 77.

A reolar 'Tissne, 35, 3.

Argonauta, 280, 249.

Armadillo, 135, 344, 101, 334

A rtemia, 254.

Artery, 104, 68.

Arthropoda, 281.

$\begin{array}{ll}\text { “ } & \text { blood of, } 99 . \\ \text { " } & \text { nevelopment of, } 205 . \\ \text { " } & \text { slin of, } 127 . \\ \text { see Crab, Insect, Lobster, My. } & \text { riapoda, Spider. }\end{array}$

Ascidian, 309, 278, 279.

" circulation of, 107.

" month of, 60 .

" slinll of, 125.

Astacus, 25\%, 250.

Asterias, 260.

" see Starfish.

Asteroiden, 253, 126, 133, 210, 212, 213.

Astrien, 252, 203.

Astrophytou, 260.

A tavism, 216.

Atlas, 145.

Attacus, 30S, 274.

Auger-shell, 276, 235.

A $11 \mathrm{k}, 329$.

Aurelin, 249, 195.

" see Jelly-fish.

Aves, $325,50,65,76,105,116,125,162$, $169,170$.

A vicula, 272.

A xis, 145.

Axolotl, 232.
Bajirusa, 69, 34.

Buboon, 359.

Balæna, see Whale.

Balaunglossus, 233, 393.

Balauns, 2S4, 254.

Baruacle, 2S4, 253, 254.

" metamorphosis of, 210.

Bisket-fish, 260.

Batrachia, 315, 63-65, 76, 85, 87, 296, 297. " see Frong.

Buts, 346, 182, 339, 340.

Bear, foot of, 128.

Beaver, 346, 337.

Bed-bilg, 297.

Bec, 304, 277.

" alimentary canal of, 42.

6 eggs of, 195 .

" eye of, 155.

" instiucts of, 185 .

" mode of feeding of, 50 .

" mouth of, 59, 22 .

is section of, 81 .

" temperature of, 121.

" see Hymenoptera, Insecta.

Beetle, 297, 131, 267, 268.

" alimentary caual of, 41 .

“ development of, $297,267,268$.

" eye of, 1 1S2, 1 56.

" mouth of, 5 .

" skeletou of, $292,262$.

" see, Coleoptera, Insecta.

Belemuite, 251.

Bernicia, 310.

Beroë, 257.

Bile, 93.

Biolngy, 11.

Bird-of-Paradise, 339.

Birds, 325, 304-328.

" alimentary canal of, $\$ 4,50$.

" anatomy of, 50,304 .

" beak of, 54 .

" blood corpuscles of, 100,65 .

" brain of, 141.

" breathing of, 119.

" circulation of, 109,76 .

" distribution of, 375 .

" drinling of, 50 .

" egg of, $193,162$.

" embryo of, 169, 170.

" eye of, 184.

" feather of, 137, 204, 105.

" flight of, $160,125$.

" gizzald of, 84, 3S4, 50 .

" heart of, 109 .

"Incomotion of, 166.

" luugs of, 118, 50, S2.

" month of, 62 .

"skeleton of, 144-14\%, 116.

"sinell of, 17S. 
Birds, temperature of, 121.

" voice of, 159.

" wings of, 160,304 .

Bivalve, see Clam, Lamellibranchiata, Oyster.

Blacklird, 339.

Blastema, 33.

Blastula, 19S, 165.

Blatta, 297.

Blood, 97.

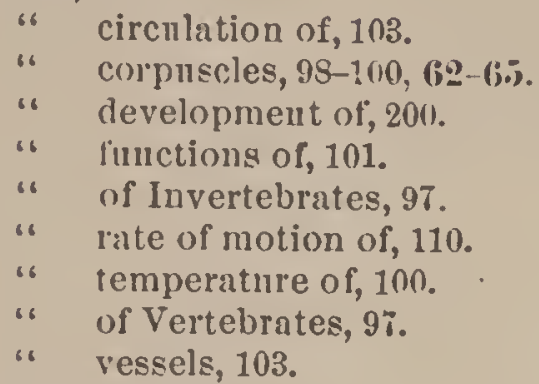

Blubber, 348.

Bluefish, 312, 284.

Bon, 54, 73, 37.

Joinblis, 304.

Bombyx, 303.

Bolle, composition of, 147.

"development of, 203.

"s structure of, $36,7,8$.

Bos, see Ox, Cow.

Brachiopoda, 266, 221, 222.

Brachycephalic, 393.

Bradypis, 344.

Braill, 170, 132, 137-145.

" case of, see Sknll.

" development of, 204.

“ functions of, 173.

" parts of, 170 .

“ weight of, 170 .

Brain-coral, 252, 204.

Brine-shrimp, 284.

Bronchns, 119, 82-\$8, \$6.

Bryozon, see Polyzon.

Bubble-shell, 274, 231.

Bnccinum, 27s, 29, 228.

Budding, 192.

see Whell.

Bufo, 318.

" see Toad.

Bugrs, mouth of, 59 .

" see Hemiptera.

Bnlimus, 275, 233.

Bulla, 274, 231.

Butterfly, 300, 273.

" austomy of, 43

" metamorphosis of, 208, 172.

" mimicly of, 217.

" mouth of, 59, 23.

" scales of, $\mathbf{2 7 1 .}$

Byssus, 271.

CAINIS-FI.Y, 295.

Cæcilia, 31S.
Cæecum, 51.

Calcispongia, 246.

Camel, 100, 352, 65.

Cameo-shell, 27S, 237.

Canaliculi, 37, 8.

Cancer, 257.

Canine teeth, 69, 34, 35.

Capillaries, 104, 66, 68.

Caprimulgus, 335, 323.

Capybara, $346,335$.

Carabus, 29S.

Carapace, 322, 11 b.

Cardinm, 227, 227.

Cariuatre, 328.

Carnivora, 553, 90, 92, 106, 105-110, 128, 142, 346-350.

" feet of, 128.

"6 teetl of, 70 .

Carp, 48, 102.

Cartilage, 36, 5,6 .

Cassis, 278, 237.

Cassowary, 327.

Castor, 346, 337.

Cat, 63, 355.

"brain of, 142

ss teeth of, 70 .

Catelpillar, 301, 975.

\begin{tabular}{|c|c|}
\hline "6 & anatomy of, $7 S, 40$. \\
\hline "6 & circulation in, 105,69 . \\
\hline “ & false legs of, 172 \\
\hline "6 & head of, $3 \cap 3, \mathbf{2 7 6}$. \\
\hline “ & heart of, 105,69 \\
\hline " & jaws of, 53,276 . \\
\hline “6 & locomotion of, 162. \\
\hline 6 & muscles of, 156 \\
\hline "6 & nervous system of, 169,136 . \\
\hline "6 & $\begin{array}{l}\text { see Butterfly, Iısecta, Lepi- } \\
\text { doptera. }\end{array}$ \\
\hline
\end{tabular}

Catfish, 316, 291.

Cebns, 35\%, 352.

Cell, 31, 1.

Cement, 38: 66, 31.

Centipede, 53, 257, 2 59.

Centrum, 140 .

Cephalization, 225.

Cephalodiscus, 398 .

Cephalopoda, 278, 16, 47, 161, 247-249. " see Cuttlefish, Squid.

Cephalo-thorax, 131, 2 S2.

Ceratodıs, 317.

Cerebellım, 171, 173, 137-144.

Cerebrum, 170, 173, 137-145.

Ceryle, 335, 327.

Cetacea, 348, 30, 341, 342.

$$
\text { "s see Whale. }
$$

Chalaza, 193, 162.

Chalk, 11, 242.

Chan eieon, 54, 322.

$$
\text { " tollgile of, } 61 \text {. }
$$

Cheiroptera, 346, 339, 340 .

Chelœ, 253. 
Chelonia, 322, $115,801,302$.

" see Turtle.

Chelydra, 323.

Chiloguatha, 257.

Chilopoda, 257, 259.

Chimæra, 314.

Chimpanzee, 357, 354, 356.

" $\quad$ skeleton of, 120.

Chitin, 132, 252.

Chitov, 278, 240.

Chlorophyl, 23.

Choriou, 203.

Choroid, 1s3, 157.

Chrysalis, 20s, 391. 393, 172, 275.

Chrysaora, 213, 178.

Chyle, 92, 102, 59.

Chyme, 92.

Cicada, 297, 266.

Cicatricnla, 194, 162.

Cicindela, 295.

Cidaris, 262, 96, 97.

Cilia, 34, 154, 2.

Ciliata, 243, 185.

Cimex, 297.

Circulation in Arthropoda, 106.

" in Ascidians, 107.
"in Birds, 109.
" development of, 200.
" in Echinodermata, 105.
" in Insects, 105.
" in Mammalia, 109.
"in Mollusca, 106.
in Vermes, 106.
in Vertebrata, 107, 306, 281.
see Heart.

Cirripedia, 254, 253, 204.

$$
\text { " see Baruncle. }
$$

Clam, 272.

" adductors of, $\mathbf{4 6}$.

" alimentary caual of, s0, 46.

" auatomy of, 46 .

" circulation in, 106.

" ear of, $178,150$.

"foot of, 161, 46 .

" gills of, 113,78 .

" heart of, $106,46$.

" hinge of, 270 .

"Incomotion of, 161.

" mouth of, 56 .

“ uervous system of, $168,135$.

" prehellsion of, 50 .

" shell of, 133, 99.

" siphous of, 46 .

" see Lamellibranchiata, Molluscr, Oyster.

Clamatores, 33S, 322.

Class, 235.

Classification, 231.

$$
\text { "6 syunsis of, } 362 .
$$$$
\text { " I'able, } 239 .
$$

Claws, 136.

Clio, 56.

Cloaca, 85.

Clothes moth, 303.

Clyperster, 262.

Coagnlation, $9 \mathrm{~S}$.

Coccus, 297.

Cochinenl, 297.

Cockle, 272, 227.

Cockroach, 297.

Cod, 316, 292.

" eggs uf, 195.

Colenterata, 246.

$$
\begin{aligned}
& \text { " number of, } 221 . \\
& \text { see Actinoid Polyp, Hydra, } \\
& \text { Jelly-fish. }
\end{aligned}
$$

Cœnosarc, 252.

Coleopterı, 297, 41, 156, 267, 268.

$$
\text { “ see Beelle. }
$$

Colias, 303.

Colnmbr, 333, $\mathbf{3 1 6 .}$

Colldor, 335.

Condyle, 144.

Cone-shell, 278, 289.

Conjugatiou, 196.

Connective Tissue, 34, 3, 4.

Contractility, 154.

Coral, 130, 251, 95, 200-20S.

" see Actinoid Polyp.

Corallium, 256, 207.

Coral reef, 254.

Cormorant, \$4, 330, 309.

Cornea, 1S3, 157

Corpuscles, see Blood.

Correlation, 218.

Corydalns, 295.

Cow, skeleton of, 11 .

Cowry, 275, 234.

Clab, 64, 2S7, 257.

"locomotion of, 162.

" vocal organs of, $15 S$.

"s see Lobster.

Cralle, 332.

Crangon, 256.

Craniota, 310.

Cranium, 141.

Cray-fish, 287, 250.

Cricliet, 297, 264.

Crinoidea, 25s, 210, 211.

Crocodilia, 323, 303.

$\begin{array}{ll}\text { " } & \text { exoskeleton of, } 135 . \\ \text { " } & \text { locomotion of, } 163 . \\ \text { " } & \text { mouth of, } 61, \mathbf{9 6 .} \\ \text { " slieleton of, } 149,118 . \\ \text { " stomach of, } 82,49 . \\ \text { see Reptilin. }\end{array}$

Crop, 75, S4, 50. see Reptilia.

Crow, 339.

Crinstacea, 282.

" $\quad$ nanplius of, $211,177$. 
Crustacea, see Crab, Lobster.

Ctenactis, 253, 202.

Ctenophora, 257, 209.

Cuckoo, 335.

Cuculi, \$35, 321.

Culex, 300, 173, 269.

"sce Mosquito.

rurassow, 333.

('nrculio, 300.

Cinrsores, 327, 305.

Cuticle, 34, $12 \mathrm{~S}$.

Cuttletish, 113, 2S0, 248.

" alimentary canal of, $80,47$.

" anatomy of, 47.

" beak of, 52,47 .

" brain of, $168,151$.

" circulation in, 107.

" ear of, 151.

" eye of, $182,151$.

“ heart of, $10 \pi$.

" ink-bag of, 47.

، mouth of, 57.

" pancreas of, 123.

"6 preheusion of, 52.

" skeleton of, 134

“ snckers of, 16.

Cyanea, 249. see Cephnlopoda, Sepia, Squid.

Cyclas, 150.

Cyclops, 2S4, 255.

Cypræa, 274-276, 234.

Cypris, 284, 25 5.

Cypseli, 335, 323.

Cytherea, 99.

DAMLY-LONG-ILEGS, 2S9, 300.

Diphnia, 284, 255.

Dasypus, 344, 334 .

Dasyurus, 343 .

Decapoda (Crustacea), 2\$6, 70, $250, \mathbf{2 5 6}$, 257.

Decussation, 154

Deel, $345, \mathbf{3 4 5}$.

Deglutition, 72.

Delphinus, 349, 343.

$$
\text { "6 see Dolphin. }
$$

Demodex, 2S7, 258.

Dental Formule. 70.

$$
\text { "6 Tissue, } 38, \mathbf{3 1} .
$$

Dentine, $35,66.31$.

Dermis, 12S, 148.

\begin{tabular}{|c|c|}
\hline & \\
\hline 16 & of Bird, 199. \\
\hline 6. & of blastula, $198, \mathbf{1 6 5}$. \\
\hline ، & of embryouic forins, 207. \\
\hline “ & of wastrula, 19S, 166. \\
\hline ‘. & of Invertebrates, 205 . \\
\hline & by inetamolphosis, 207 . \\
\hline 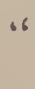 & $\begin{array}{l}\text { by metamorphosis, retr } \\
\text { grade, } 210 \text {. }\end{array}$ \\
\hline
\end{tabular}

Development, 197.
Development. oviparous, $30 \mathrm{~s}$.

$$
\begin{aligned}
& \text { “ } \\
& \text { " } \\
& \text { ovogmentation of egg, } 197 . \\
& \text { of Vertebrates, } 205 . \\
& \text { viviparous, } 30 \text { s. } \\
& \text { see Metamorphosis, Repro- } \\
& \text { duction. }
\end{aligned}
$$

Devil's darning-needle, 295.

Diuphragm, s7, 120, 88 .

Diupoph ysis, 141.

Diastema, 70, 383 .

Dibrauchiata, 2S0, 16, 47, 151, 245, 249 .

Didelphia, 342.

Differentiation, 31.

Digestion, chemical, 92.

$$
\begin{aligned}
& \text { "6 of Inverteblate, ?. } \\
& \text { " of Mall, } 93 . \\
& \text { " object of, } 91 . \\
& \text { "6 of Vertebrate, } 92 .
\end{aligned}
$$

Digitigrade, $237,355,128$.

Diuornis, 325 .

Diploria, 254, 204.

Dipnoi, 316, 322. 293.

Diptern, 300, $\mathbf{2 4 , 1 : 7 , 1 7 8 , ~ 2 6 9 , ~} \mathbf{2 7 0}$.

"see Fly, MIosquito.

\section{Discophora, 260 .}

Distoma, 265.

Distribution, 371-379.

Divers, 325 .

Dog, 355, 90, 92, 108-110.

" brain of, 171.

" skull of, 143.

"6 teeth of, 69 .

Dolichocephalic, 393.

Dolphin, 349, $\mathbf{3 4 3}$.

$$
\text { "s teeth of, } 68 \text {. }
$$

Doris, 274.

Dove, 50, 333, 316.

Drug()n-fly, 294, 263.

Duck, 331, 311.

Duck-mole, 65, 342, 331 .

" see Oruithorhyuchus.

Dugnong, 207, 350 .

$$
\text { " } 3 \text { heart of, } 73 .
$$

Duodeum, 9 .

Dytiscus, 295, 127.

EAarx, 335, 319.

\section{Ear, 178, 204, 387, 150-152.}

Ear-shell, 278, 235, 246.

Earth-worm, 269.

\section{Ecderoll, 127.}

alimentary canal of, 77.

6 circulation in, 106.

" locomotion of, 162.

" nervous system of, $16 \mathrm{~S}$.

"، prehension of, 52.

Echidna, 342.

Echinndermata, 257.

$$
\text { " number of species of, }
$$
221. 
Echinoidea, 261, 28, 39, 96, 97, 214.

Echinus, 262, 214.

" see Sen-urclin.

Ectoder'm, 246, 166.

Edentata, 344, 101, 333, 334 .

Egg, fertilization of, 197.

" form of, 195 .

"6 number of, 195.

" segmentation of, 197, $160 \overline{\text {. }}$

" structure of, 192, 161-164.

Elasmobranchii, 314, 287, 288.

Elater, 299.

$$
\text { " see Ray, Shark. }
$$

Elephant, 350, 65.

" brain of, 170 .

" foot of, 164, 129.

" skeleton of, 119.

“ teeth of, $69, \mathbf{3 6}$.

“ trunk of, 50.

“ tusks of, $71,66,119$.

" voice of, 190

Elytra, 160, 297.

Embryology, 12, 197.

Emu, 327.

Ennmel, 3S, 66, 31.

Encephalon, 174.

Enderon, 127.

Endoder'm, 246, 166.

Endosarc, 75.

Endoskeleton, 127, 137.

Entomostracn, 254, 17\%, $25 \overline{5}$.

Ephemera, 295.

Epiblast, 199, 169.

Epidermis, 34.

Epiglottis, 119, 27, 159.

Epithelium, 33, 2.

Equus, see Horse.

Euplectella, 246.

Eustachian tube, 179, 15:

Excretion, 121.

Excretory olgans, 125.

Exoskeleton, 127, 129.

Eye, of Invertebrates, 1S0, 153-156.

" of Vertebrates, 133.

" development of, 204 .

Fadint Angle, 30 .

Falcon, 335.

Family, 235.

Fat, 38, 384, 10.

Feathers, 137, 105.

"6 developnent of, 204.

Felis, 355, 106.

" see Cat, Linn.

Fertilization of Egrg, 197.

Fibriu, 98.

Fishes, 310 .

" air-bladder of, $117,48$.

" alimentary canal of, SO, $4 \mathrm{~s}$.

“ blood of, $99,100, \mathbf{6} \dot{0}$.

“ brain of, 172,139 .
Fishes, circulation in, 10ৎ, 71, 75.

" eye of, 184

" fins of, 15s, 123.

" gills of, 114,48 .

" heart of, 108, 48.

" locomotion of, 159, 124.

" mouth of, 61 .

" muscles of, $157,48$.

" number of species of, 313 .

" ovary of, 48.

" pancreas of, 123.

“ prehellsion of, 54 .

“ scales of, $135,102,283$.

“ skeleton of, 112.

“ skull of, 138, 112.

" teeth of, $61,67,32$.

Fish-hawk, 335, 318.

Fission, 191, 160.

Flagella, 154, 18.

Flagellata, 243, 187.

Flamillgo, 331, 125.

Flea, 300.

Flight of Bats, 161.

" of Birds, 160.

" of Insects, 159 .

Fluke, 265.

Fly, 300 .

" bnzzing of, 1 SS.

" foot of, 127.

" metamolphosis of, 270.

" mode of feeding of, 50 .

" mouth of, 59, 24 .

" see Diptera, Moミquito.

Fly-catcher, 33s, 322.

Flying Fox, 346 .

Follicle, 123, 90.

Food, 47-49.

Foramen, 141, 26ז, 291.

$$
\text { " magnin, } 172 .
$$

Fornminifera, 51, 129, 241, 15, 1 s。.

Formica, 304.

Forms of animals, 222.

Fowl, 85, 50.

Fox, 355, 349.

Frog, 54, 318, 140, 297.

" alimentary canal of, $\mathrm{S2}$.

“ blood-corpunscles of, 99, (68-65.

" breathing of, 119.

" circulation in, 108, 76 .

" food of, 49 .

" heart of, $10 \mathrm{~s}$.

“" lings of, 118,85 .

" lymph-heart of, 96.

“ metamorphosis of, 209.

" respiration in, 117-119.

" skeleton of, 119, 140, 145, 87.

" tongue of, 61.

" vertebre of, 140,87 .

Fruit-moth, 303, 270.

Function, 41.

Fungia, 252, 202. 
TALT-MTADDER, 124,92

Gall-fly, 304.

Gammarus, 256.

Ganglion, 166, 14, 146.

Gannet, 331.

Ganoidei, 315, 259, 290.

Gar-pike, 315, 289.

Gasteropoda, 20, 29, 45, 100, 134, 154, 176,272 .

“ see Snail.

Gastric glands, 123,90 .

" jnice, 93.

Gastrula, 19S, 166.

Gavial, 324.

Gecko, 322.

Gelatin, 36.

Genus, 235.

Germinal vesicle, 192 .

Gibbon, 357.

Gill-cover, 114.

Gills, 114, 125, 48 .

Gir:affe, 352.

Gizzard of Invertebrates, 70,77-80 " of Vertebrates, \$2-85.

Gland, 122, 89.

" gastric, 123, 90.

" liver, 123, 92.

" pancreas, $123,91,92$.

“ salivary, 122.

" sweat, 126,94 .

Globigeriua, 242.

Glottis, 119.

Glycogen, 23.

Goatsucker, 335, 323.

Goniaster, 260, 212.

Gonse, 331, 310.

Gordius, 265.

Gorgonia, 256, 208.

Gorilln, 357, 357.

Grallatores, 332, 312-314.

Grasshopper, 297.

$\begin{array}{ll}\text { " } & \text { development of, 20s. } \\ \text { “ } & \text { ear of, 17ৎ. } \\ \text { " } & \text { mizzard of, 79. } \\ \text { " } & \text { stridulation, 1SS. }\end{array}$

Grebe, 329.

Gregarinida, 242, 184, 186.

Gristle, 36.

Grouse, 333, 315.

Growth, 214.

Grubs, 359 .

Gryllins, 297, 264.

Guinea pig, 346 .

Gulls, 329.

H fimatoorsa, 393.

Hæinatother'ma, 393.

Hæmocyaniu, 102.

Hxmoglobin, 102.

Hag-fisl, 54, 66, 314.
- Hair, 136, 94, 104.

Hair-worll, 265.

Haliotis, 27S, 235, 246.

Hand, 359, 148.

Hare, $346,336$.

Harvest-man, $2 S 9$.

Haversian Canals, 37, 7 .

IIawk, 335, 318.

IIearing of Invertebrates, $1 \%$ S.

" of Vertebrates, $17 \%$.

Heart, Arthropoda, 105, 69, 70.

"development of, 200, 16\$, 169

" of Mollusks, 106.

" of 'Tnuicites, $107, \mathbf{2 7 9}$.

"s of Vertebrates, 107-109, 71-74.

Heat, 121.

Hedgehon, 345 .

Helix, 275, 20, 232.

Hemiptera, 297, 265, $\mathbf{2 6 6}$.

$$
\text { “ month of, } 59 .
$$

Herol, 332, 313.

Herring, 316.

Heterocercal, 159, 123, $\mathbf{2 8 5}$.

Heteromya, 272.

II ippopotamns, 164, 352.

Hirnudก, 339.

Histolngy, 12.

Hog, 352.

" teeth of, 68 .

Holothuroiden, 262, 210, 215 .

Homarus, see Lobster.

Homo, see Man.

Homocercal, 159, 123.

Homology, 217, 179-182.

$$
\text { “ serial, } 215 .
$$

Homomorphism, 217.

IIoney-bag, 79.

Hoofs, 136, 103.

IIorned pont, 291.

IIolnel'r, 267, 220.

Horns, 136.

Horse, brain of, 171, 138.

"6 hoof of, $136,164,103,199$.

“ skeleton of, 151, 117.

" skin of, 94.

" skull of, 144, 111.

" splint-bones of, 207.

" stomach of, $88, \overline{\mathbf{5 3}}$.

Horse-fly, month of, 60, 24.

Horseshoe-crab, 57, 284.

$$
\text { "6 " " jaws of, } 53 .
$$

House-fly, 127.

Hummer, 99, 335, 65.

Hyalea, 274, 229.

Hybrid, 235.

Hydra, 246, 191.

" budding of, 192, 191.

" digestive cavity of, 75 .

" nerve-cells of, 168 . 
INDEX.

Hydra, l'epair of, 215.

Hydroid, see Hydrozoa.

Hydrozoa, 246, 178, 191-196.

“ alternate generations, 212.

“ development of, 205.

“ see Jelly-fish.

Hyena, 355.

Hymenoptera, 303, 22, 42, 81, 277.

$$
\text { " see Bee. }
$$

Hypoblast, 199, 169.

I1318, 332.

Ichneumon-fly, 304 .

Ich thyopsida, 30 .

Ichthyo saurus, $232,324$.

Idotia, 286, 251.

Iguana, 322.

Iguanodon, 324.

Ileum, 58.

Imago, 20S, 172, 173, 267, 270, 275.

Incisors, 68 .

Individual, 220, 235.

Infusoria, 16s, 243, 160.

“ digestion in, 75, 92.

" fission, 191, 160.

“ mode of feeding of, 50.

" motion of, 154 .

“ mouth of, 55.

“ respirationl of, 112 .

“ skin of, 127.

Inheritauce, 215.

Insectivora, 346.

Insecta, 291.

“ absorption of, 94 .

“ alimentary canal of, 7S, 41-43.

" auatomy of, $\mathbf{4 3}, 81$.

" antemux of, $\mathbf{1 4 7}$.

" chrysalis of, $\mathbf{1 7 2}$.

" circulation in, 105, 293.

“ development of, 205 .

“ ear of, 179

" eye of, $181, \mathbf{1 5 5}, \mathbf{1 5 6}$.

" feet and legs of, $162,127,131$.

“ flight of, 159.

" gizzard of, 79.

" heart of, 105,69 .

" liduey of, $126,41,42$.

“ liver of, 123.

" locomotion of, $159,163$.

" metamorphosis of, 207, 172, 173, 264-270, 274, 275.

" mouth of, 57.

" mouth-parts of, 53, 21-24.

“ muscles of, 156, 131.

“ nervous system of, 169, 43, 136.

"respiration in, 114, 291.

" salivary glands of, $122, \mathbf{4 0}$.

" silk glands of, $\mathbf{4 0}$.

“ skeleton of, 132, 292, 98, 262.

“ smell of, 178.

“ spiracle of, 114, 79.

Insecta, touch of, 176, 147 .

" tuacheæ of, 114, 40, 80, 81.

" wings of, 159.

Insessores, 337, 322-328.

Inspiration, modes of, 115, 119, 120 .

Instinct, 154.

Intelligence, 187.

Intestine of Amphibian, 82.

" of Bird, 84.

" of Fish, S0.

“ of Mammal, 55,58 .

" of Reptile, 82.

Iris, 183,157

see Alimentary Camal.

Isomya, 272.

Ivory, 38,66 .

Ixodes, $2 S 8$.

JAws, 53-74.

Jay, 339.

Jelly-fish, 247, 193-197.

" blood of, 97 .

“ development of, 212, 178, 195.

" eye of, 180.

" mode of feeding of, 51.

" mouth of, 55.

“ nerves of, 165 .

“ nettle-cells of, 51 .

“ reproduction of, 212 .

Joints, 147.

Julns, 287.

June-bug, 267.

KANGAROO, 88, 343 .

Kiduey, 126, 41, 42, 46, 52, 93.

King-crab, see Horseshoe-crab.

Kingfisher, 335, 327.

Kite, 335 .

Kiwi-kiwi, 327.

Labium and Lajrum, 53, 5S, 21.

Labyrinthodontia, $31 \mathrm{~s}$.

Lacerta, 321, 300.

Lacertilia, 321.

Lachnosterna, 297, 267.

Lacteals, 94, 60 .

Lacunæ, $37,8$.

Lamellibranchiata, $270,44,46,78,99,13 อ$, $150,0204-2020$.

" $\quad$ see of, $1 \$ 1,153$.

Lamellirostres, 331, 310, 811.

Lamprey, 314, 286 .

Lamp-shell, 266, 221, 202.

Lancelet, $310,28 \%$.

Land-snail, 275, $23:$.

Lark, 340.

Larva, 208, 172, 173, 267, 274, 975.

Laryinx, 159, 159.

Lasso-cells, 51.

Leech, 26 S. 
Ieech, alimentary canal of, 77 .

" jaws of, 64.

" locomution of, 161.

" mode of feeding of, 50.

Lemur, 355, 851 .

Lepas, 2s4, 2 ј3.

Lepidoptera, $300, \mathbf{4 0}, \mathbf{4 3}, \mathbf{1 7 2}$ "6 see Butterfly.

Lepidosiren, $31 \%$.

Lepidostens, $315, \mathbf{2 S 9}$.

J ibellula, 295, 263.

I,ife, distribution of, 372 .

" duration of, 226.

" wature of, $2 S$.

" struggle for, 226 .

Ligrhtuing-bug, 299.

Ligula, 5s, 21 .

Likeness, 215.

Limax, 275, 232.

Limbs, development of, 204.

" slieleton of, 146, 179-182.

Limuxa, 275, 232.

Limpet, 27S, 245.

Limulus, 254.

" see Horseshoe-crab.

Lion, 68, 355.

" foot of, 128.

"6 skeleton of, 106.

" stomich of, SS, 05.

Liver, $123,92$.

Lizard, 141.

" see Lacertilia.

Lubster, 1116, 70, ๑506.

" alimentary caual of, $7 \mathrm{TS}$.

“ atuatomy of, $2 S 2$.

" circulation in, 106, 70.

" eill of, 179.

6 eggs of, 196.

" gills of, 114 .

"6 gizzard of, 64 .

" locomotion of, 155 .

" moultiug of, 132.

" mouth of, 57.

" preheusion of, 53,57 .

" respiration in, 114.

" skeleton of, 131.

Lub-worn, 77.

Lucolnotion of Arthropoda, 162.

$\begin{array}{ll}6 & \text { of Birds, } 160 . \\ 6 & \text { of Fishes, } 155 . \\ 6 & \text { of Iusects, } 159 . \\ 6 & \text { of Mollusks, } 161 . \\ 6 & \text { of Starfish, 161. } \\ 6 & \text { of Wortebrites, } 163 .\end{array}$

Locust, 297.

Loligo, see Squid.

Longipennes, 329, 30 S.

Joon, 329, :307.

Louse, 50, 297.

Luceruaria, 197.
Lumbricus, see Earth-worm.

Lungs, function of, 125 .

" of Suail, 116.

" of Vertebrates, 117.

Lupus, 347.

Lyıph, 102.

Lymphatics, 94, 61.

Lymph-heart, 96.

Mantra, 271, 46, 226.

Madrepore, 252, 201, 206.

Madreporic plate, 25s, 39.

Maggots, 389.

Manımalia, 340.

“ alimentary canal of, $\$ 5$.

" blond-colpuscles of, 10n, 6 .

" brain of, $171,133,142-145$.

" circulation in, $109,76,281$.

" digestion of, 92,51 .

"6 drinking of, 50.

“ eal of, 179, 152.

"6 egg of, 19S, 16 .

" embryo of, 202, 171.

" eye of, 1S3, 157.

" hair of, $136,104$.

" heart of, $109,73,74$.

" locomotion of, 163.

" lungs of, 115,86 .

" mouth of, 62 .

" palate of, $56, \mathbf{2 7}, 51$.

“ placenta of, 196, 203, 171.

" respiration in, 119.

" skeleton of, 139.

" smell of, 17S, 149.

" teeth of, 68 .

" touch of, 177.

" voice of, $189,159$.

Mau, 359, 179, 329, 330.

" blood-corpuscles of, 99, 62, (6.).

"brain of, 170, 171, 137, 144, 145.

"6 digestive tract of, $\mathbf{5 1}$.

" ear of, 179, 152.

" eye of, 198, 157, 158.

" month of, $86, \mathbf{2 7}$.

" muscles of leg of, $165,130$.

" llose of, 178, 149.

Manatee, $350,343$.

Maudibles, 58, 145, 21.

Mautis, 53.

Mantle, 127, $\mathbf{1 6 .}$

Manyplies, 59, $\mathbf{5 6}$.

Marsh-hen, 314.

Marsipobranchii, 314, 286.

Marsupialia, 342, 332.

Mastodon, 350.

May-fly, 295.

Maxillae, 58, 21.

Meandrina, 252.

Medulla oblongata, 172, 174, 187-142, 144.

Medusa, see Jelly-fisl. 
Megalosaurus, 324.

Megatherimu, 344 .

Melauia, 278.

Meloloutha, 131, 156.

Mesentery, 83.

Mesoblast, 199, 169.

Mesoder'm, 246.

Metamorphosis, 207.

" of Crab, $20 \%$.
of Frog, 209.
of Grasshopper, 20s.
of Insect, 20 S.
of Starfish, 20 S.

Metazon, 244.

Metridium, 251.

$$
\text { "6 see Actinoid Polyp. }
$$

Millepede, see Myriapoda.

Millepole, 391.

Mimicry, 217.

Minerals and Organisms, 19.

Mite, 2S5, $\mathbf{2 5 8}$.

Mon, 328.

Molar Teeth, 69, 70, 9, 31, 35, 36 .

Mole, 346.

Mollusca, 269.

\begin{tabular}{|c|c|}
\hline ، & absorption of, 94 . \\
\hline ، & auatomy of $45,46,47,78$. \\
\hline “. & circulation in, 106. \\
\hline “6 & development of, 205 . \\
\hline “6 & digestion of, 92 . \\
\hline ، & distribution of, 376 . \\
\hline 6 & ear of, $17 s, 150$. \\
\hline " & glowth of, 214 . \\
\hline "6 & lidney of, 126,75 . \\
\hline "6 & liver of, 124. \\
\hline “ & locomotion of, 161. \\
\hline “ & metamorphosis of, 211 . \\
\hline “ & mode of feeding of, 52 . \\
\hline “6 & month of, 56 . \\
\hline
\end{tabular}

" nervous system of, 16S, 13t, 135, 151-154.

" number of species of, 221 .

" respiration $\mathrm{il1}, 113,45,46,47$, 78.

" salivary glands of, 122.

"s shell of, 133, 385, 99, 100.

" skin of, 127.

" see Clam, Cuttle-fish, Snail, Squid.

Monad, 243, 187.

Monera, 240, 183.

Monkey, 356, 19, 352.

Monodelphia, 344.

$$
\text { " see Primate:. }
$$

Mollomyr, 271.

Monotremata, 342, 331.

Morpholngy, 12 motuh of, 62 .

Mosquito, 59, 300, $\mathbf{6 9 9 .}$

\footnotetext{
" metamorphosis of, 208, 173.

" mode of feeding of, 50 .
}

Moth, 300, 272, 274-276.

"6 anilomy of, 79,43 .

" metamorphosis of, 275 .

" see Butterfly, Lepidoptern.

Mother-of-pearl, 123.

Motion, 154.

Motol' Nel'ves, 167.

Moulting, 12S, 131, 209.

Monse, 346, 6 5.

Month, 55.
" of Arthropoda, 57.
" of Ascidia, 60.
" of Birds, 62 .
" of Cœlenterata, 55 .
" of Echinodermata, 56.
" of Fishes, 61.
" of Iufusoria, 55 .
"r of Mammals, 62 .
" of Mollusks, 56.
" of Parasites, 55.
" of Reptilia, 61 .
" of Vermes, 5i.
"6 of Vertebrata, 60.

Mucous Membraue, $5 ., 55$.

Mud-fish, 315.

Minex, 275.

MI $15,346$.

" see Mrouse.

Mnsca, see Diptera, Fly, Honse-fly.

Muscle, 39, 11, 12, 121, 12:, 130, 131.

" development of, 204.

" of Invertebrates, 156.

" kinds of, 155.

" of Vertebrates, 156.

Mushronm-coral, 252, 20:.

Musk-deer, 99, 65.

Mnssel, 270, 225.

Myriapoda, 287, 959.

$$
\begin{aligned}
& \text { “ alimentary canal of, } 77 . \\
& \text { " mouth of, } 57 . \\
& \text { "espiration in, } 116 . \\
& \text { see Centipede. }
\end{aligned}
$$

Myrmecoph:ıa, 344, 333.

Mytilus, 272, 225.

Myxine, 67.

$$
\text { "see Hag-fish. }
$$

NAIt.S, 135.

Narwhal, 6S, 223.

Natatores, $32 S$.

Natica, 27s.

Natural Selection, 227.

Nauplius, 211. 177.

Nautilus, so, 133, 279, $\mathbf{2 4}$.

Necturus, 318, 994.

Nematelminthes, $265,: 13$.

Nereis, 52, 269, 17

Nerve-cells, $40,13:$.

" fibres, 40, 13.

" kinds of, 167

" velocity of impulse of, 167. 


\section{INDEX.}

Nervous System, 166.

\begin{tabular}{|c|c|c|}
\hline " & 16 & $\begin{array}{l}\text { of Arthropoda, } 169 \text {. } \\
\text { Brain, } 170 .\end{array}$ \\
\hline 6، & “ & development of,199,167. \\
\hline « & 6 & of Mollusks, 168 \\
\hline 86 & “6 & Spinal Cord, 175. \\
\hline s. & "6 & of Startish, 168. \\
\hline “6 & “6 & Sympathetic, $175,146$. \\
\hline 66 & 66 & of Vertebrates, 169. \\
\hline 86 & "6 & of Worms, 16 s. \\
\hline
\end{tabular}

Neurapophysis, 140.

Neurilemma, 40, 13.

Neuroptera, 294, 263.

$$
\text { “ see Dragon-fiy. }
$$

Neuroskeleton, 141.

Newt, 31s, 174, 296.

Nomeuclature, Zoological, 236.

Notuchord, 200, 167.

Notonecta, 297, 296 .

Nucleulus, $31,1$.

Nucleus, 31, 1.

Nutrition, 45.

Nymph, 377.

OCRILI, 1S1, 292, 155.

Octopus, 280.

(Esophagus, 77-S9.

CEstrus, 300.

Olfactory Lobes, 172, 204. "Nerves, 178, 149.

Oligochœtæ, 269.

Olive-shell, 2\%S.

Oniscus, 256.

Operculum, 114, 131, 273, 313, 112, 228.

Ophidia, 320.

$$
\text { "6 see Snalie. }
$$

Ophiura, 260, 210, 213.

Opisthobrancls, 274, 352, 230. $\mathbf{2 : 3 1}$

Opisthocœlons, 386 .

Opossum, 343, 332.

Optic Lobes, 172, 204, 143.

Or:ung-uta11, 357, 353, 355.

Order, 235.

Organ, 41.

Organism, 20, 22-2s.

Organization, 30.

Organ-pipe Coral, 251, 200.

Oriole, 339.

Oruithodelphia, 341.

Oruithogruathous, 293.

Oruithorhynchus, $342,331$.

Orthoceras, "2s7.

Orthoptera, 217, 295, 21, 264 .

$$
\text { " see Grasshopper. }
$$

Orycteropus, 344.

Oscines, 33S.

Osculum, 245.

Osseous Tissue, see Bune.

Ossitication, 36, 203.

Ostrea, 272.

$$
\text { " see Oyster. }
$$

Ostrich, 99, 327, 65. 305.

Otoliths, 17s, 150, 151.

Ovipusitor, 293, 98.

Owls, 335, 317.

Ox, alimentary canal of, 90 .

'6 foot of, 164, 352, 129.

" mouth of, 63 .

" preheusion of, 5 ก, 54 .

" teeth of, $69,71,352$.

" see Ungulata.

Oyster, allatomy of, Sn, 44.

" circulation of, 106.

" development of, 205.

" eggs of, 195.

" heart of, $106,44$.

"s mouth of, 56 .

" preheusion of, 50 .

" respiration in, 113.

" see Clam, Lamellibranchiata.

Pai.ath, 61.

Pallial Sinus, 271, 99.

Palpi, 5S, 21.

Paludina, 278, 232, 244.

Pancreas, 123, 91.

Pancreatic Juice, 93.

Pangoli 11, 314.

Paper Nautilus, 2S0, $2+9$.

Papilio, 303.

Papillæ, 63, 128, 94, 148.

Paramecium, 32, 191, 243, 1 SS.

$$
\text { "sce Infusuria. }
$$

Parrot, 337, 320.

$$
\text { " tongue of, } 62,189 .
$$

Partridge, 333.

Patella, 147, 27S, 29, 106.

"see Limpet.

Pavement-teeth, 67, 32.

Pearl-oyster, 272, 224.

Pecteu, 181, 272, 153.

Pectol'al Arch, 146.

Pedicellariæ, 77, 97.

Pediculus, 297.

Pedipalpi, 2S8, 259.

Pelias, 320, 298. see Scorpion.

Pelicau, S4, 331.

Pelvic Arch, 146.

Penguin, 329, 306.

Peuuatula, 250, 208.

Pentacrinus, 25S, 211.

Pepsin, 93.

Peptone, 93.

Perch, skeletou of, $67,135,172,65,112$, 139, 283.

Perchers, 337.

Periosteum, 138, 157.

Peristaltic Movemeut, 89.

Peritoneum, 89.

Periwinkle, $27 \mathrm{~S}$.

Petrel, 330. 
Petromyzon, 314, 256.

Plialangium, 2 s9.

Pharyugrobrauchii, 310, 28:.

Phasynx, 72, 85.

Phasma, 297.

Phensaut, 333.

Phoca, 354.

Physalia, 246, 194.

Physeter, 34S, 3+1.

" see Whale.

Physiology, 12.

Picariæ, 335.

Pici, 335, 320.

Picris, 303.

Pigeoln, 84, 333, 65, 316.

Pike, 6ō.

Pinlligrade, 354, 125.

Pisces, 310, 45, 65. 71, 75, 102, 112, 123, 124, 139, 283-293.

"s sec Fish.

Placenta, 196, 171.

Planaria, 264, 217.

Plauorbia, 273, 275, 232.

Plant, 22.

" food of, 25.

"6 functions of, 24 .

Plantiglade, 237, 355, 128.

Plant-louse, 297.

Plasma of blood, 9 S.

Plastrou, 323.

Platyhelminthes, 264, 216, 217.

$$
\text { " see Tape-wotm. }
$$

Platyonychus, 257, 257.

Plesiosaurus, 324.

Pleurapophysis, 141.

Pleurobrachia, 257, 209.

Plover, 332.

Poisoll-fangs, 6S, 33.

Polychætæ, 269.

Polycistina, 129, 242, 185.

Polyp, 251.

"6 see Actinia.

Polypterns, 315.

Polyzon, 266, 220.

Pond-snail, 275, 232.

Porcupine, 346.

Porifera, see Sponge.

Porites, 252.

Polpoise, 8S, 348, 349, 54.

Purtal circulation, 306, 3S5, 281.

Portuguese man-of-war, $246,194$.

Potato-worm, 303.

Poulue, 2 So.

Prairie Chicken, 333, 315.

Primates, 356, 35, 120, 143-145, 352-360.

" brain of, 143-145.

"skeleton of Chimpanzee, 120

" teeth of Chimpanzee, 35.

"see Ape, Man, Monkey.

Probosciden, 350, 36, 119, 129.

Proboscis of Butterfly, 59, 23.
Proboscis of Eleplaut, 62, 119.

Procœlons, 356.

Prognathous, 393.

Prosimii, 355.

Prosobranchs, 278, 234-246.

Proteus, 318, 295.

"6 blood-corpuscle of, $99,65$.

Protista, 21.

Protoplasm, 19, 29, 31, 154.

Protopterus, 317, 293.

Protozoa, $23 \mathrm{~s}$.

" number of species of, 221.

" see Amœba, Iufusoria.

Psalterium, S9, 56.

Pseudopodia, 51, 154, 240, 15.

Pseudotriton, 31S, 296.

Psittaci, 337, 320.

Pterodactyle, 324.

Pteropoda, 273, 229.

" mollth of, 56 .

Pulex, 300.

Pulmonates, 274, 232, 233.

Pulse, 355.

Pupa, 20S, 172, 267, 270.

Plipil, 1S3, 157.

Pygopodes, 32s, 306, 307.

Pyropholus, 299.

Quaniumana, 356, 395. " see Monkey.

Quohog, 272.

Raccuox, 355, 346.

Radiates, 233.

Radiolalia, 242, 185.

Rail, 332, 314.

Rana, see Frog.

Rauge of Animals, 373.

Rank of Animals, 223.

Rnptores, 334, 116, 317-319.

Rasores, 332, 315.

Rat, 346.

Ratitre, 327, 305.

Rattlesnake, 6S, 136, 33.

Raven, 339.

Ray, 2s3, 314, 288.

" teeth of, $67,32$.

Razol-shell, 272.

Redstait, 338, 325.

Repair, 215.

Reproduction, 191.

$\begin{array}{ll}\text { " } & \text { nsexual, } 191 . \\ \text { " } & \text { ch budding, } 192 . \\ \text { " } & \text { by division, } 191 . \\ \text { " } & \text { rapidity of, } 226 .\end{array}$

Reptilia, 319.

" alimentary canal of, $\$ 2$.

“ braiu of, 172, 141.

“ cilculation in, 108, 76. 
Reptilia, corpuscles of, 99, 6.5.

" distribution of. $37 \%$

" lungs of, 115,84 .

" mouth of, 61 .

"6 prehension of, 61 .

scales of, 135

teeth of, 67.

voice of, 189.

see Crocodile, Lizard, Suake, Turtle.

Respiration, 111.

$\begin{array}{ll}\text { " } & \text { in Crustacen, } 114 . \\ \text { " } & \text { in Echinoderms, } 112 . \\ \text { " } & \text { in Insects, 114. } \\ \text { " } & \text { in Mollusks, 113. } \\ \text { " } & \text { rate of, 120. } \\ \text { in Vertebrates, } 117 . \\ \text { in Worms, 113. }\end{array}$

Rete mucosum, 128.

Reticulum, SS, 56 .

Retina, 1S3, $157,158$.

Rhen, 327.

Rhinoceros, 136, 164, 351, 129, 344.

Rhizopoda, 240, 15, 184, 185.

‘ skeleton of, 129.

Rodentia, 345, 335, 336, 337.

$$
\text { "teeth of, } 71,335,336 \text {. }
$$

Rostrum, 292.

Rotifera, 266, $\mathbf{2 1 9}$.

$$
\text { " jaws of, } 64 \text {. }
$$

Rudimentary organs, 207.

Rumen, ss, 56.

Ruminantia, 352.

$$
\begin{aligned}
& \text { " stomach of, } 85,56 . \\
& \text { see } \mathrm{Ox}, \text { Ungulata. }
\end{aligned}
$$

\section{SAUrdm, 146.}

Silamander, 315, 296.

$$
\text { "6 metamorphosis of, } 17 t \text {. }
$$

Saliva, function of, 93 .

Salivary Glands, 122.

Salmon, 316, 28:, 285.

Sand-flea, 2S4, 25:.

Sindpiper, 332, 31:.

Sarcode, 281.

Sarcolemnia, 39, 204.

Sauropsida, 30 s.

Sanruræe, 394.

Scales of Butterflies, 300, $271,272$.

"s of Fishes aud Reptiles, 135, 102,

$$
\text { 283. }
$$

Scallop, eye of, $151,153$.

$$
\text { "6 shell of, } 272 .
$$

Scapular Arch, 146.

Scurabrus, 299.

Scarf-skin, $128,336$.

Sclerobase, 130.

Scleroderm, 130.

Sclerotic, 183, 157.

Scolopendra, 2S7, 259.
Scorpiou, 53, 289, 259.

" mouth of 60 .

" respiration in, 116.

Sea-anemone, see Polyp.

Sea-blubber, 249.

Sea-butterfly, 273, 229.

Sen-fan, 256, 208.

Sea-hare, 274.

Seal, 355, 128, 181.

Sea-lemon, 2it.

Sea-lily, 25S, 211.

Sea-lion, 355, 350.

Sen-pen, 208.

Sea-slug, 262, 27t, 215.

Sea-11 rchin, 261, 210, 21 t.

" absorption by, 94.

alimentary cinal of, $76,39$.

anatomy of, 39.

circulation in, 105.

digestion in, 92 .

growth of, 214.

mode of feeding, 52 .

month of, 56 .

respiration in, 112.

shell of, 28.

skeleton of, 130.96 .

spives of, 130,97 .

teeth of, 64,28 .

Sea-worm, 268, 17, 223.

Secretion, 121.

" see Gland.

Secretory organs, 122.

Segmentation of $\mathrm{egg}, 197, \mathbf{1 6 5}$.

Self-division, 191, 160.

Sellsation, 176.

Sense of hearing, 178 .

"6 of sight, 180.

"6 of smell, $17 \%$.

" of taste, 177.

" of touch, 176.

Sense-organs, see Sense.

Sensibility, 176. development of, 204.

Sepia, 2so, 248.

Selpent, see Snake.

Sertularia, 247, 192.

Serum, 98.

Setæ, 269.

Setophaga, 340, 325.

Seventeen-year locust, 297, 266.

Shark, 67, 314, 65, 287.

"6 eugs of, $195,164$.

" gills of, $114, \mathbf{2 5 7}$.

"s skeleton of, 137, 145, 140 .

Sliells of Crustacea, 131.

" of Echilloderms, 130.

" of Mollusks, 133.

Shoulder-girdle, 146.

Shrew, 63, 346, 338.

Shrimp, 286.

Sight, of Arthropods, 181. 
Sight, of Colenterates, 180.

" of Molluske, 181.

" of Vertebrates, 183.

Silk-gland, 40.

Silk-wor'm, 303 .

Simia, 357, 353, 355, 356.

Sinuses, 138.

Siphon, 113, 226.

Siplionophora, 248, 194.

Siphuncle, 279, 247.

Silenia, 349, 73, 343.

"sce Dilgoll r.

Size of Auimals, 221.

slieleton, of Arthropoda, 131.

" of Birds, 144, 116.

of Cœlenterntes, 130.

of Crocudile, 113.

of Echinoderms, 130.

of Fish, 13S, 144, 145, 112. of limbs, 146.

Lion, 139, 106.

"Mammals, $139,106,114,117-$ 120.

“ Mollusks, 133.

“ Reptiles, 113, 115.

of of skull, 141, 108-111.

" of Tortuise, $\mathbf{1 1 5 .}$

" of Vertebrates, 134.

" of Vulture, 116.

" of Whale, 114.

" see Exoslieleton.

Skin of Iuvertebrates, 127.

" of Vertebrates, 123.

Skin-nu!scle, 156.

Skull, 141, 37, 108-111, 353, 354, 359, 360.

Slater, 2S6, 251.

Slug, 275, 232.

Smell, 177.

Suail, 272.

" alimentary canal of, 80,45 .

‘ anatomy of, $\mathbf{4 5}$.

" circulation in, 106, 45.

" eye of, 181, 15t.

" gills of, 113 .

" gizzard of, 64 .

“ heart of, 45 .

". jaw of, $56, \mathbf{2 0}$.

" larva of, 176.

" locornotion of, 161.

" lung of, $116,274,45$.

" mode of feeding, 52.

" mouth of, 56 .

" nervous system of, 169, 134, 154.

" operculnm of, $114,134, \mathbf{2 2 8}$.

" respiration iu, 116, 45.

"shell of, 133, 100, 225, 231-246.

" siphon of, 228 .

" sinell of, 178.

"teeth of, $\mathbf{6 5}, \mathbf{2 9}$.

"teutacles of, 176, 15t, 225 .
Snail, see Gasteropoda.

Snake, 320, 65, 298, 299.

" deglutition of, $7 \%$.

" locomotion of, 162.

" lungs of, 119,84 .

" poison apparatus of, $6 \mathrm{~S}, 33$.

" scales of, 135.

" skull of, 37.

" stomach of, S2.

" tougue of, 62.

"Vertebræ of, 140.

" voice of, 189.

" see Boa, Ophidia, Reptilia.

Snapping-bugr, 299.

Suipe, 332.

Solaster, 260

Somite, 392.

Songsters, 338.

Sorex, 346, 338.

Sow-l)ug, 286.

sparrow, 339.

Species, detined, 235.

" nilmuer of, 221.

Sperm-cells, 196.

Sperm-whale, see Whale.

Splinx-moth, 303, 43, 136.

Spider, classitication of, $289,260$.

" alimentary caunl of, $7 \%$.

" appendages of, $162, \mathbf{2 5}$.

" circulation in, 106.

"fangs of, 53, 18, 25.

" month of, 60, 25.

"respiratiou in, 116.

" spinnerets of, $259,25,261$.

“ web of, $289,260$.

Spinal colum n, 141.

“ cold, $175,137$.

Spiudle-shell, 236.

Spinneret of Spider, 289, 25, 261.

" of Caterpillar, 301,276 .

Spiracle, 114, 293, 79.

Splint-bone, 147.

Sponge, 244, 189, 190.

" alimentary canal of, 76 .

" anatomy of, 189 .

" egg of, 194, 163.

" feeding of, 50, 159.

" respiration in, 112.

" skeleton of, 129, 190.

Squash-bng, 297.

Squid, 250.

"locomotion of, $15 \mathrm{~S}$.

"see Cuttle-tish.

Squirrel, 346.

Stag, 352, 345.

Star-fish, alimentary canal of, $76, \mathbf{1 2 6}$, 210.

" anatomy of, 126.

" circulation iı, 105.

" classitication of, 255 .

" dievelopment of, 2 (1s. 
Star-fish, digestion in, 92.

" locomotion of, 161, 126.

“ metamorphosis of, $20 \mathrm{~s}$.

" mode of feediug of, 51 .

" mouth of, 56.

" mervous system of, $165,133$.

"respiration in, 112.

6 see Ecliuodermata.

Sternum, 145, SS.

Stilt, 332.

Stomach, 82-59.

Stork, 332.

digestion in, 93.

Stridulation, $15 S$.

Strombus, 2is, 243.

Struggle for Life, 226.

Struthio, 327, 305.

Sturgeoll, 61, 315, 290.

Subkingdom, 235, 250.

Sun-stir, 260.

Survival of Fittest, $22 \%$.

Suture, 147.

Swallow, 340, 328.

Swan, 331.

Sweetbread, 123.

Swift, 335 .

Swimmeret, 282.

Symmetry, 222.

Sympathetic nervous system, 175, 146.

Synovia, $1 \pm 7$.

\section{Tactur. Corpuscles, 177.}

Tæuia, see 'T'ape-worm.

Tanager, 332

Tapetum, 184.

Tape-worm, 264, 216.

Tapir, 62, 351, 180.

$$
\text { " feeding of, } 49 .
$$

Taste, 177.

Teeth, of A mphibia, 67.

" of Fishes, 61, 66, 67.

" of Invertebrates, 63.

"6 of Mammals, 6S, 70.

"6 of Reptiles, 67.

"structure of, $35,66,9, \mathbf{3 1}$.

Teleostei, 315, 254, 2S5, 291, 292.

Telson, 2 S2.

Temperature of Animals, 121.

Tendoll, 36, 15 \%.

Tentacle, 51.

Tentaculifera, 243.

Teut-caterpillal, 303.

Termes, 295.

Terebra, 278, 238.

'Terebratula, 267, 222.

Terebratulina, 267, 221.

Termite, 295.

'T'ern, 330, 305.

'I'est, 261, 96.

Testudo, sec Turtie.

Tetrabranchs, 279, 247.
Tetradecapods, 255, 251, 252.

Thoracic duct, 95, 61.

Thorax, 119, 145, 88.

Thormback, 314, 285.

Thousand-legged Worm, see Julus.

'T'hrush, 340.

Thylacinus, 343 .

Thyroid Cartilage, 159, 159.

Ticks, 2 SS.

Tissue, 32.

Toad, 54, 61, 318, 6.5.

Tongue, of Batrachians, 61.

" of Birds, 62 .

" of Fishes, 61.

" of Insects. 50. 55.

" of Mammals, 63.

" of Man, 27.

" of Mollusks, 52.

" of Spiders, 60.

Top-shell, 278, 242.

'Tortoise, 323, 302.

" see Turtle.

Totipalmates, 330, 309 .

Toucan, 335.

Touch, 176.

Trachea, 119, 86.

Tracher, 114, 40, 79, s0, 81 .

Trichina, 265, 218.

Tridacne, 272.

Trilobite, 2S4.

Trionyx, 322.

Triton, 278, 318, 110, 296 .

Tritonian, 274, 230 .

Trochosphere, 211, 175, 176.

Trochus, 278.

" embryo of, 211,176 .

Troglodytes, $\mathbf{3 5}$.

$$
\text { "6 see Chimpinzee. }
$$

Trogon, 335, 321.

Tubipora, 252, 200.

Tuuicata, 309, $278,279$.

"see Ascidians.

Turbo, 27S, 242.

Turkey, 84, 333, 141.

'Turritella, 278.

'Turtle, 322, 301, 302.

" alimentary cillal of, $\$ 2$.

" breathing of, 11?.

" mouth of, 61.

" shell of, 135.

" skeleton of, 115.

" teeth of, 65 .

" see Chelonia.

Tusks, 383.

'Iympannm, 179, 152.

Types, 233.

UNGULATA, 351, 53, 56, 103, 111, 117,118

$129,138,344,345$.

Unio, 133, 272. 
Unio, egrs of, 196.

Univilve, see Snail.

Urochordat:i, 30!!

Urodela, 31s, 295, 296

VANE88A, 303, 278.

Variation, 216.

Variety, 235.

Veins, 68, 104, 67.

Veliger, 211, 176.

Veua cava, 104.

Venus, 272.

Venus'-basket, 246.

Vermes, 263, 17, 77, 175.

" see Earth-worm, Wolnm.

Vertebr'x, development of, 203.

" hinds of, 141, 106, 107.

6 11 minber of, 141 .

Vertebrata, 305.

“ absorptiou in, 94.

" alimentary catual of, 80-91.

6. bluod of, 97.

" brain of, 170.

“ circulation in, 75,76 .

" develoument of, 205 .

" digestion in, 92.

" ear of, 179

" exoskeleton of, 134

" eye of, 183.

" gastric glands of, 123.

"6 heart of, 107.

" kidney of, $126,93$.

c: liver of, 124.

Gi lungs of, 117.

" mode of feeding of, 54 .

"6 mouth of, 60 .

" muscles of, 156 .

6 uervous system of, 16. .

b: uumler of species of, 221.

6i pancleas of, 123.

" salivary glands of, 122.

" skeleton of, 137.

" skin of, 128.

" stomach of, 80 .

" teeth of, 66 .

" tongue of, 61 .

" see Bird, Crocodile, Fish,

Vespa, 304.

Frog, Mammal, Reptile.

Villi, 95, 58

Vinegar-eel, 265.

Viper, 320, $\mathbf{2 9 8}$.

Vireo, 340, 326.

Vitality, 29.

Vitelline Memlorane, 193.

Viviparous, $30 \mathrm{~s}$.

Vocal Cords, 1S?.

Voice of Iuvertelrates, 1SS.

" of Vertebrates, 159.

Volute, 278, 241.
Vorticella, 243, 160.

Vulpes, 349.

Vultule, 335, 116.

WALIING-ETIOK, 297.

Walrus, 355, 383 .

Warbler, 340 .

Wasp, 304.

Water-beetle, 127.

Water-boatman, 297, 265.

Water-fleas, 2S4, 255.

Wax-wing, 340.

IT casel, 355,348 .

Weevil, 300 .

Whale, 349, 341, 342.

“ baleen of, $65,30$.

"6 brain of, 170.

"6 fat of, 39 .

" mode of feeding of, 50.

" molith of, 62 .

"6 Swimming of, 159

" teeth of, 207, 3S3.

Whale-bone, 65, 136, 30.

Wheel-auimalcnle, 266, 219 .

Whelk, 278, 228, 246, 254.

"see Suail.

White Aut, 295.

Windpipe, $119,86$.

Wings of Bats, 161, 182, 339, 340 .

" of Birds, $160,304$.

" of Insects, 159, 9s, 266.

Wolf, 355, 347.

Woodpecker, 335, 320.

Worms, 263.

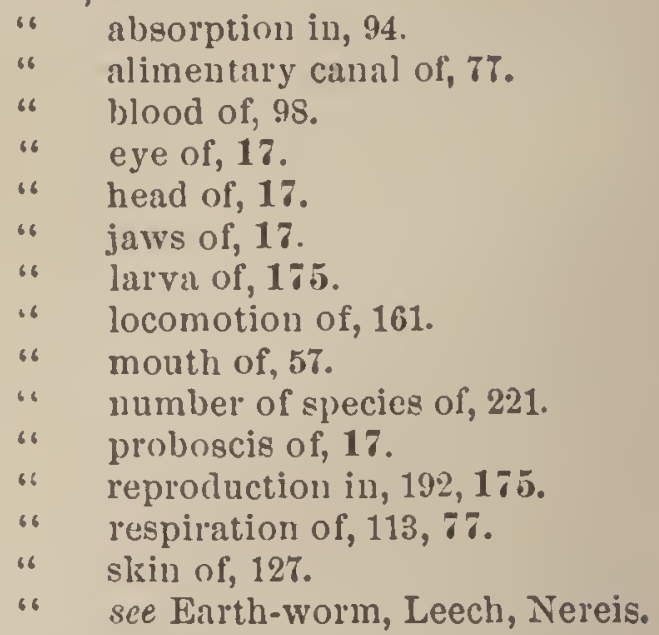

Wren, 340.

YoLK, 192

Zool1, 221.

Zoological analysis, 236.

"؛ barviers, 373

" history, 14.

" provinces, 375 .

Zoology, 12.

Zygapophyses, 140. 


\section{TEXT-BOOKS OF SCIENCE}

ASTRONOMY

\section{LOOMIS'S ELEMENTS OF ASTRONOMY}

The Elements of Astronomy. Designed for Academies and High Schools. By Elias Loomis, LL.D., Late Professor of Astronomy in Yale College. pp. 254. 12mo, Sheep, \$1 00.

The plan of this volume is essentially the same as that of the Treatise on Astronomy mentioned below, with the omission of some of the mathematical portions. Such mathematics as are included in this volume require only a knowledge of a few of the most elementary principles of algebra, geometry, and plane trigonometry. The author says in his preface: "Without some mathematical knowledge it is impossible to form an adequate idea of the substantial basis upon which the calculations of astronomy rest."

\section{LOOMIS'S PRACTICAL ASTRONOMY}

An Introduction to Practical Astronomy. With a Collection of Astronomical Tables. Designed to Supplement the Treatise on Astronomy. By Elias Loomis, LL.D., Late Professor of Astronomy in Yale College. pp. 506. 8vo, Sheep, \$1 50 .

While the attention of so many persons is earnestly directed to the inlprovement of practical astronomy, the want of suitable text-books on this subject has been extensively felt. Some work has been needed which should not only give a description of the instruments required in the outfit of an observatory, but.which should also explain the methods of employing them and the computations growing out of their uses. In this book Professor Loomis has endeavored to fill this want,

\section{LOOMIS'S TREATISE ON ASTRONOMY}

A Treatise on Astronomy. Designed for the Instruction of College Classes in the First Principles of Astronomy. By Elias Loomis, LL.D., late Professor of Astronomy in Yale University. pp. 342. 8vo, Sheep, \$1 50.

The author's aim has been to limit this book to such dimensions that it may be read without omissions, and to make such a selection of topics as 
shall embrace everything most important to the student. Wherever it could be done to adrantage, simple mathematical problems have been introduced, and at the close of the book will be found a collection of miscellaneous problems, many of them extremely simple, which may be used at the discretion of the teacher. A further discussion of various phenomena, such as the constitution of the sun, the condition of the moon's surface, the phenomena of eclipses, the law of tides, etc., has been entered into, in the hope that such discussion will enhance the interest of the subject for the class of students who might be repelled by a treatise exclusively mathematical.

\section{NEWCOMB'S POPULAR ASTRONOMY}

Popular Astronomy. By Simon Newcomb, LL.D., Superintendent American Nautical Almanac; formerly Professor U. S. Naval Observatory. With One Hundred and Twelve Engravings and Five Maps of the Stars. pp. xviii., 578. $12 \mathrm{mo}$, Clotl, $\$ 130$.

The historic and philosophic sides of the subject have been treated with greater fulness than is usual in works of this character, while the purely technical side has been proportionately condensed. Owing to the great interest which now attaches to the question of the constitution of the sun, it was deemed desirable to present the latest viers of the most distinguished investigators of this subject from their own pens. Four of these gentlemen -Rev. Father Secchi, of Rome; M. Faye, of Paris; Professor Young, of Dartmouth College; and Professor Langley, of Allegheny Observatory-hare, at the author's request, presented brief expositions of their theories, which will be found in their own language in the chapter on the sun.

\section{WARREN'S RECREATIONS IN ASTRONOMY}

Recreations in Astronomy. With Directions for Practical Experiments and Telescopic Work. By H. W. WArren, D.D. pp. xiv., 292. With 83 Illustrations and Colored Plates. $12 \mathrm{mo}$, Cloth, \$1 25.

Written not only to leveal some of the highesi achievements of the human mind, but also to let the heavens declare the glory of God. With sentiments of profound devotion, and with the calmest belief that religion gains by every advance of science, he invites the readel to scan the henveus, and there find pruofs strong as

holy writ of the truths of revealed faithChicago Times.

The explanations of difficult matter's are particularly lucid, and for readers not technically instructed in astronomy nothing could be better as a literary presentation of the attractive side of the science.N. Y. Post. 


\section{CHEMISTR Y}

\section{HOOKER'S CHEMISTRY}

Chemistry. By Worthington Hooker, M.D. pp. 430. Numerous Illustrations. New Edition, Revised and Enlarged. $12 \mathrm{mo}$, Half Leather, 90 cents.

Dr. Hooker is so well known as a stlccessful writer of books for the young that every one expects his books to be of the very best killd. His style is clenr, lively, and free from techuicalities; his method natural, and his illustratious apt,

striking, and drawn from familiar sources. I think his books, in the hands of competent teachers, will do mucli to advance the cause of true education.-Rev. WIILLiam Horominson, Principal of Lawreuce Academy, Gruton, Mass.

\section{GEOLOGY}

\section{HOOKER'S MINERALOGY AND GEOLOGY}

Mineralogy and Geology. By Worthington Hooker, M.D. pp. 360. Numerous Illustrations. $12 \mathrm{mo}$, Half Leather, 90 cts.

\section{LYELL'S GEOLOGY (Student's Series)}

The Student's Elements of Geology. By Sir Charles Lyell, Bart., F.R.S. pp. 640. Copiously Illustrated. 12mo, Cloth, \$1 25.

Notwithstanding the difficulty of reconciling brevity with the copiousness of illustration demanded by those who have not yet mastered the rudiments of the science, I have endeavored to abridge the work so as to place it within the reach of many to whom it was before inaccessible.-Extract from the Preface.

\section{HERRICK'S EARTH IN PAST AGES}

The Earth in Past Ages. A Geology for Young People. By Sophie Bledsoe Herrick. Richly Illustrated. pp. 208. Square $12 \mathrm{mo}$, Cloth, 60 cents.

The author's purpose and the scope of this book are well expressed in the New York Times, which said in its review : Beginning with the clews found in the rocks, the action of water, fire, and ice on the surface of the earth is clearly explained. It is perfectly natural to direct a child's attention to these subjects; and such subjects, treated as they are in this book, must be even more awe-inspiring to young people than are the wonderful fairy tales in which all delight." 


\section{METEOROLOGY}

\section{LOOMIS TREATISE ON METEOROLOGY}

A Treatise on Meteorology. With a Collection of Meteorological Tables. By Elias Loomis, LL.D., late Professor of Astronomy in Yale College. pp. 306. 8vo, Sheep, \$1 50.

\section{NATURAL PHILOSOPHY}

\section{HOOKER'S NATURAL PHILOSOPHY}

Natural Philosophy. By Worthington Hooker, M.D. pp. 434. Numerous Illustrations. New Edition, 12mo, Half Leather, 90 cents.

This work is designed for the use of the older scholars in grammarschools, and at the same time is suited to those advanced to a higher grade who have not gone through the previous books of the series.

\section{PHYSIOLOGY}

\section{DRAPER'S PHYSIOLOGY}

Human Physiology, Statical and Dynamical ; or, The Conditions and Course of the Life of Man. By John W. Draper, M.D., LL.D. pp. xvi., 650. With Index. Illustrated. 8vo, Cloth, \$3 50.

It is intended in this treatise to give an independent and thorough expo. sition of the Physiology of Man. The Book is divided into two parts: Statical Physiology-Conditions of Life; Dynamical Physiology-Course of Life. It is adequately illustrated, and is completed by an admirable index.

Professor Draper's work, soon after its first appearance, took a high place in popular favor. It does not include the most recent and still debatable territory won for the science of human life, but it is of permanent value for purpose of instruction.

A book that is full of interest, containing many striking views and novel and experimental illustrations. We make our sincere acknowlcdgements to the author for the fresh contributions he has furnished to our knowledge of the laws of life, and the new impulse he has imparted to the study of its mysteries.-North American Review. 


\section{DRAPER'S ANATOMY, PHYSIOLOGY, AND HY- GIENE}

A Text-Book on Anatomy, Physiology, and Hygiene. For the Use of Schools and Colleges. By John C. Draper, M.D., LL.D., Professor of Natural History and Physiology in the College of the City of New York, and Professor of Analytical Chemistry in the University of New York. pp. 260. Illustrated. 8vo, Cloth, \$2 50.

This treatise consists of the author's course of lectures on this subject be. fore the students of the College of the City of New York. Although the chief object has been to prepare a text-book for academic students, it is also designed for use in schools and families. Besides this, there are hints which will be found of use to students of medicine.

For sale by all booksellers, or will be sent to any address on receipt of price as quoted. If ordered sent by mail, 10 per cent. should be added to the price to cover cost of postage. Special rates for introduction will be furnished on above books on request. Correspondence solicited.

\section{HARPER \& BRothers, Franklin Square, N. Y.}




\section{A NEW METHOD IN BIOLOGY}

\section{DODGE'S PRACTICAL BIOLOGY}

\section{Introduction to Elementary Practical Biology. A Laboratory}

Guide for High Schools and College Students. By Charles Wright Dodge, M.S., Professor of Biology, Rochester University. Crown 8ro, Cloth, \$1 80.

Professor Dodge cxemplifics in his method the teaching of Huxley, who said: "The ideal of scientific teaching is, no doubt, a systcm by which the scholar sees every fact for himself, and the teacher sup-

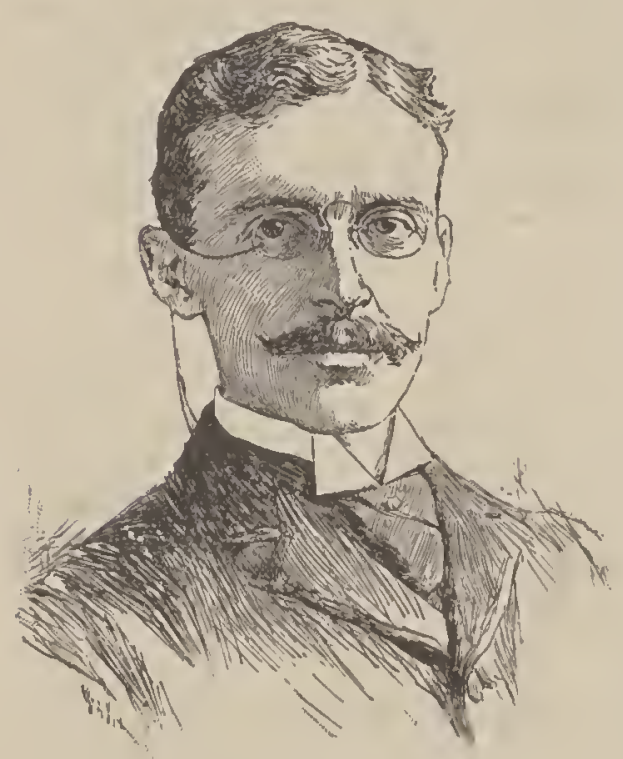

OUARLES WRIGIT DOUGL plies only the explanations." This is exactly what it is aimed to accomplish in the Introduction to Elementary Biology, just published by Harper \& Brothers. The author seats the pupil at the laboratory table, cquipped with microscope and other necessaries; then, by a series of progressive studies, manages to have the learner discover for himsclf the morphology and physiology of the cell, both animal and vegetable. Our especial point of commendation is not so much the system of exercises, though that is admirable, as it is the motive which is made to inspire the student. Under I'rofesser Dodge's guidance it would be hard indeed to resist the love of Nature.-Buffalo Express.

\section{SOME OPINIONS}

I think it is the most helpful laboratory guide in Biology that has yet appeared. I shall certainly take the occasion to try it in my class. - H. IV. Cosn, Ph.D., Professor of Biology, Wesleyan Uuiversity, Middletown, Comn.

The teaching of Biology is completely revolutionized by this method of study. And the best thing abour it is that the student becomes interested in the subject, and hence learns more than he wonld in ile old method. This book is well adapted to enable students readily and pleasantly to acquire familiarity with Elementary

Biology, - Chamlits Hrery Hrtononor, Ph.D., Dartmouth College, Hanover, N.H. The book is the work of one who is evidently not only master of his science, but also inaster of the more difficult art of the teacher. He understands how to give just what instruction is necessary, and then, by a series of skilfully selected and carefully put questions, to help the pupil to see whlat is to be seen, so that lis information as fintly gathered falls matnl'ally into systematic fol'm. - W. A. El). WARns, Principal of High School, Rocliford, IIl.

The price of Dodge's Introduction to Elementary Practical Biology is $\$ 180$, or by mail, post-paid, \$1 95 per copy. A sample copy will, however, be forwarded to any teacher who may wish to examine it with a view to class use, on reccipt of $\$ 150$.

HARPER \& BROThers, Publishers, New York.

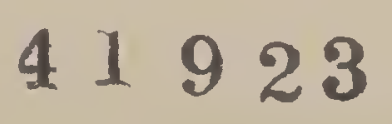



I 


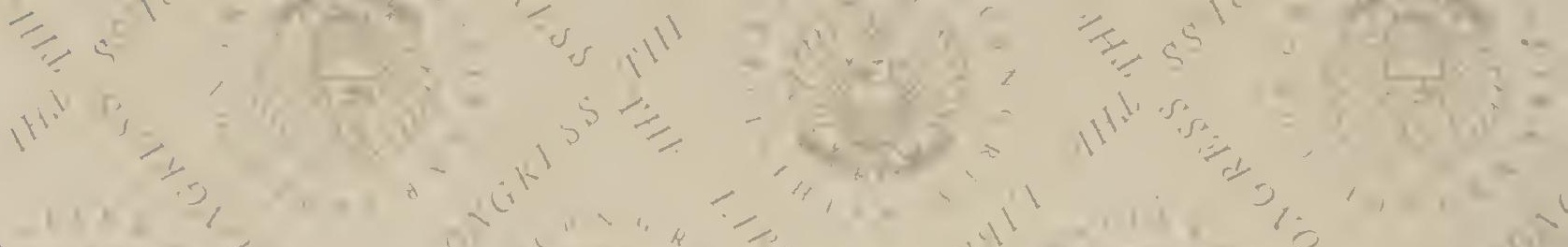

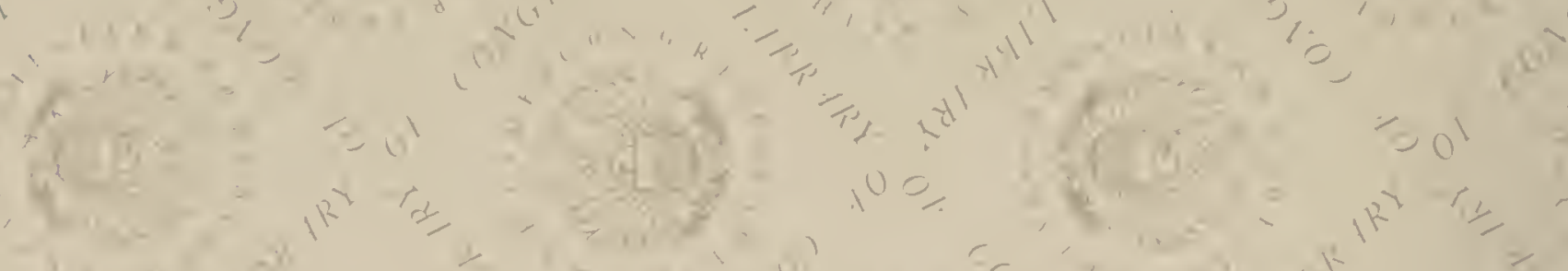

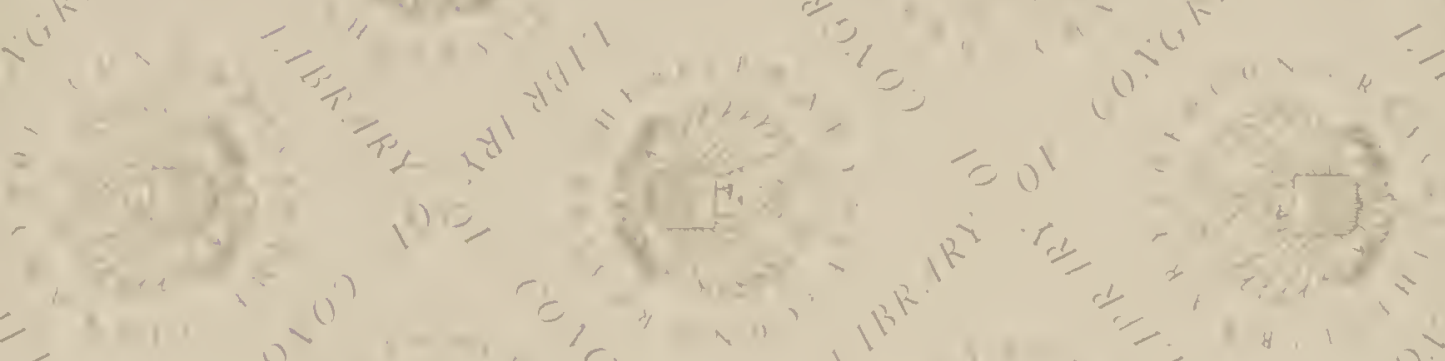
(1) $\beta^{2}-3, y=$

(1).

$\frac{11}{11}=2$ "xin

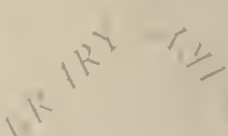




\section{LIBRARY OF CONGRESS

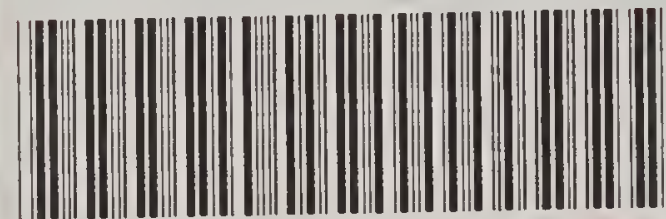 \\ 00054099277}

